\title{
Catalytic Amination of Phenols with Amines
}

Kai Chen,${ }^{\dagger}$, Qi-Kai Kang, ${ }^{\dagger, \S}$ Yuntong Li, ${ }^{\dagger}$ Wen-Qiang Wu, ${ }^{\dagger}$ Hui Zhu ${ }^{\dagger}{ }^{\dagger}$ and Hang Shi ${ }^{*},+$,

†Key Laboratory of Precise Synthesis of Functional Molecules of Zhejiang Province, School of Science, Westlake University, 18 Shilongshan Road, Hangzhou 310024, China

Institute of Natural Sciences, Westlake Institute for Advanced Study, 18 Shilongshan Road, Hangzhou 310024, China

${ }^{\S}$ These authors contributed equally: Kai Chen, Qi-Kai Kang

${ }^{*}$ Corresponding author. e-mail: shihang@westlake.edu.cn

\section{TABLE OF CONTENTS}

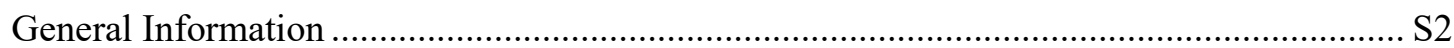

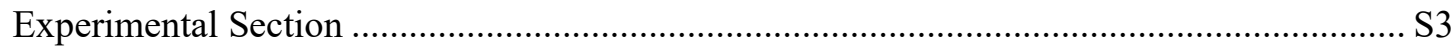

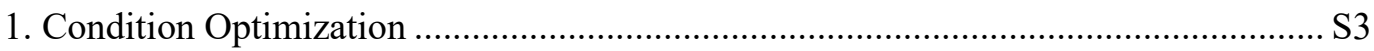

2. General Procedure for the Amination of Phenols ........................................................... S9

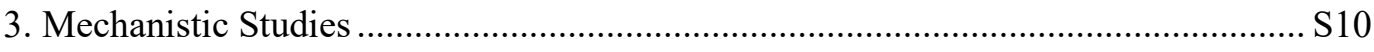

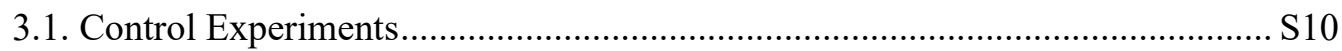

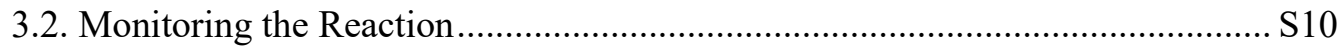

3.3. Deuterium Labeling Experiments...................................................................... S11

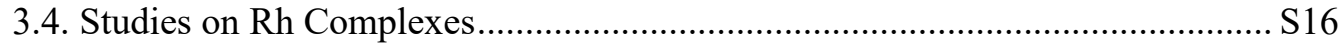

3.5. Catalyst Poisoning Experiments ................................................................... S23

4. Characterization Data of Products............................................................................... S24

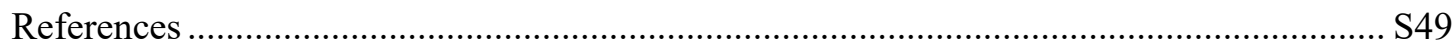

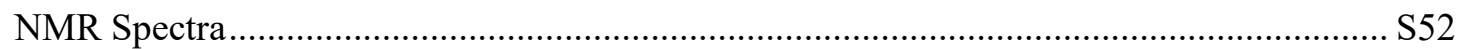




\section{General Information}

\section{Solvents}

Chloroform- $d_{1}$ (containing $0.03 \%$ TMS), acetone- $d_{6}$, benzene- $d_{6}$, and dimethyl sulfoxide- $d_{6}$, were purchased from $J \& K$ Chemical Company.

\section{Chromatography}

Analytical thin layer chromatography was performed on $0.25 \mathrm{~mm}$ silica gel $60-\mathrm{F} 254$. Visualization was carried out with UV light.

\section{Spectroscopy and Instruments}

${ }^{1} \mathrm{H}$ NMR was recorded on Bruker AVANCE NEO instrument (500 MHz). Chemical shifts were quoted in parts per million (ppm) referenced with tetramethylsilane $(\delta=$ $0.00 \mathrm{ppm})$ in chloroform- $d_{l}$ or solvent peak $(\delta=7.26 \mathrm{ppm})$ in chloroform- $d_{l}$, solvent peak $(\delta=2.05 \mathrm{ppm})$ in acetone- $d_{6}$, solvent peak $(\delta=2.50 \mathrm{ppm})$ in dimethyl sulfoxide- $d_{6}$ and solvent peak $(\delta=4.34 \mathrm{ppm})$ in $\mathrm{CD}_{3} \mathrm{NO}_{2} .{ }^{13} \mathrm{C}$ NMR spectra were recorded on Bruker AVANCE NEO instrument (125 MHz), and were fully decoupled by broad band proton decoupling. Chemical shifts were reported in ppm referenced to the center peak of a triplet at $77.00 \mathrm{ppm}$ of chloroform- $d_{1}$ or to the center peak of a heptet at $29.92 \mathrm{ppm}$ in acetone- $d_{6}$ or to the center peak of a heptet at $39.50 \mathrm{ppm}$ in dimethyl sulfoxide- $d_{6}$ or to the center peak of a heptet at $61.36 \mathrm{ppm}$ in $\mathrm{CD}_{3} \mathrm{NO}_{2}{ }^{19} \mathrm{~F}$ NMR spectra were recorded on Bruker AVANCE NEO instrument (471 MHz). The following abbreviations (or combinations thereof) were used to explain multiplicities: $\mathrm{s}=$ singlet, $\mathrm{d}=$ doublet, $\mathrm{dd}=$ doublet of doublets, $\mathrm{t}=$ triplet, $\mathrm{m}=$ multiplet, $\mathrm{br}=$ broad. Coupling constants, $J$, were reported in Hertz unit $(\mathrm{Hz})$. High-resolution mass spectra (HRMS) were recorded on a Waters Mass spectrometer using ESI-TOF (electrospray ionization-time of flight).

\section{Starting materials}

All substrates and metal catalysts were used as received from commercial suppliers, unless otherwise stated. Molecular sieves were purchased from Sigma-Aldrich and dried at $200{ }^{\circ} \mathrm{C}$ under reduced pressure for $24 \mathrm{~h} . \mathrm{Na}_{2} \mathrm{CO}_{3}$ was purchased from Shanghai Titan and dried at $180{ }^{\circ} \mathrm{C}$ under reduced pressure for 24 h. $n$-Heptane $(99 \%$, extra dry, with molecular sieves, water $\leq 50 \mathrm{ppm}$ ) was purchased from Energy Chemical. Catalyst 2-10 are known compounds and synthesized according to reported literatures. ${ }^{1}$ Catalyst $\mathbf{1 1}$ is prepared similarly according to reported literature. ${ }^{2}$ 


\section{Experimental Section}

\section{Condition Optimization}

Table S1. Effect of Ag Salts ${ }^{a}$

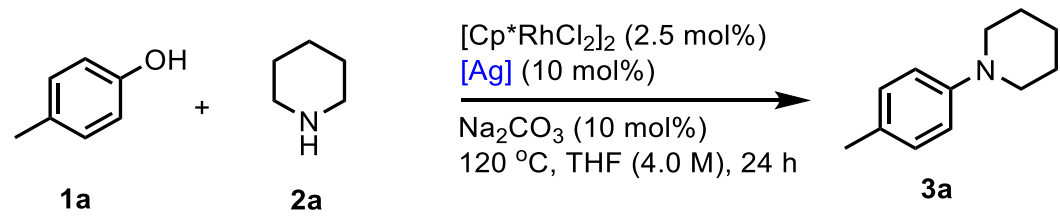

\begin{tabular}{ccc}
\hline Entry & {$[\mathrm{Ag}]$} & Yield (\%) \\
\hline 1 & $\mathrm{AgOTf}$ & 13 \\
2 & $\mathrm{AgPF}_{6}$ & 8 \\
3 & $\mathrm{AgSbF}_{6}$ & 8 \\
4 & $\mathrm{AgBF}_{4}$ & 11 \\
5 & $\mathrm{AgOAc}$ & 14 \\
6 & $\mathrm{AgNTf}_{2}$ & 7 \\
7 & --- & 17 \\
\hline
\end{tabular}

${ }^{a}$ Conditions: 1a $(0.60 \mathrm{mmol}, 1.5$ equiv. $), 2 a(0.40 \mathrm{mmol}, 1.0$ equiv. $),\left[\mathrm{Cp}^{*} \mathrm{RhCl}_{2}\right]_{2}(0.010 \mathrm{mmol})$, [Ag] $(0.040 \mathrm{mmol}), \mathrm{Na}_{2} \mathrm{CO}_{3}(0.040 \mathrm{mmol})$, THF $(0.10 \mathrm{~mL}), 120{ }^{\circ} \mathrm{C}, \mathrm{N}_{2}, 24 \mathrm{~h}$. Yield was determined by ${ }^{1} \mathrm{H}$ NMR using 1,1,2,2-tetrachloroethane as the internal standard. 
Table S2. Effect of $\operatorname{Acid}^{a}$

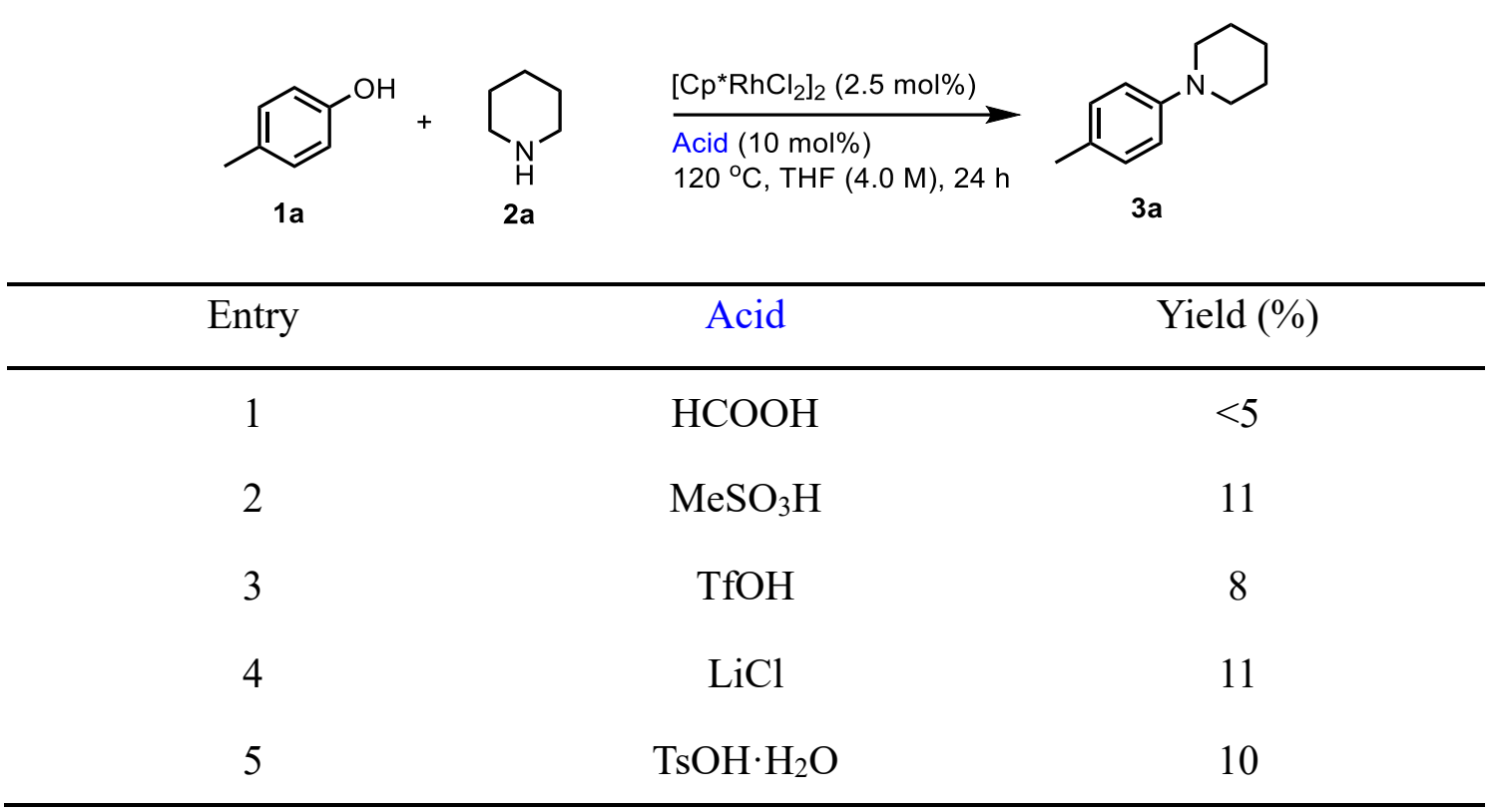

${ }^{a}$ Conditions: 1a $(0.60 \mathrm{mmol}, 1.5$ equiv. $), 2 \mathrm{a}(0.40 \mathrm{mmol}, 1.0$ equiv. $),\left[\mathrm{Cp}^{*} \mathrm{RhCl}_{2}\right]_{2}(0.010 \mathrm{mmol})$, acid $(0.040 \mathrm{mmol})$, THF $(0.10 \mathrm{~mL}), 120{ }^{\circ} \mathrm{C}, \mathrm{N}_{2}, 24 \mathrm{~h}$. Yield was determined by ${ }^{1} \mathrm{H}$ NMR using 1,1,2,2-tetrachloroethane as the internal standard. 
Table S3. Effect of Solvents and Substrate Concentration ${ }^{a}$

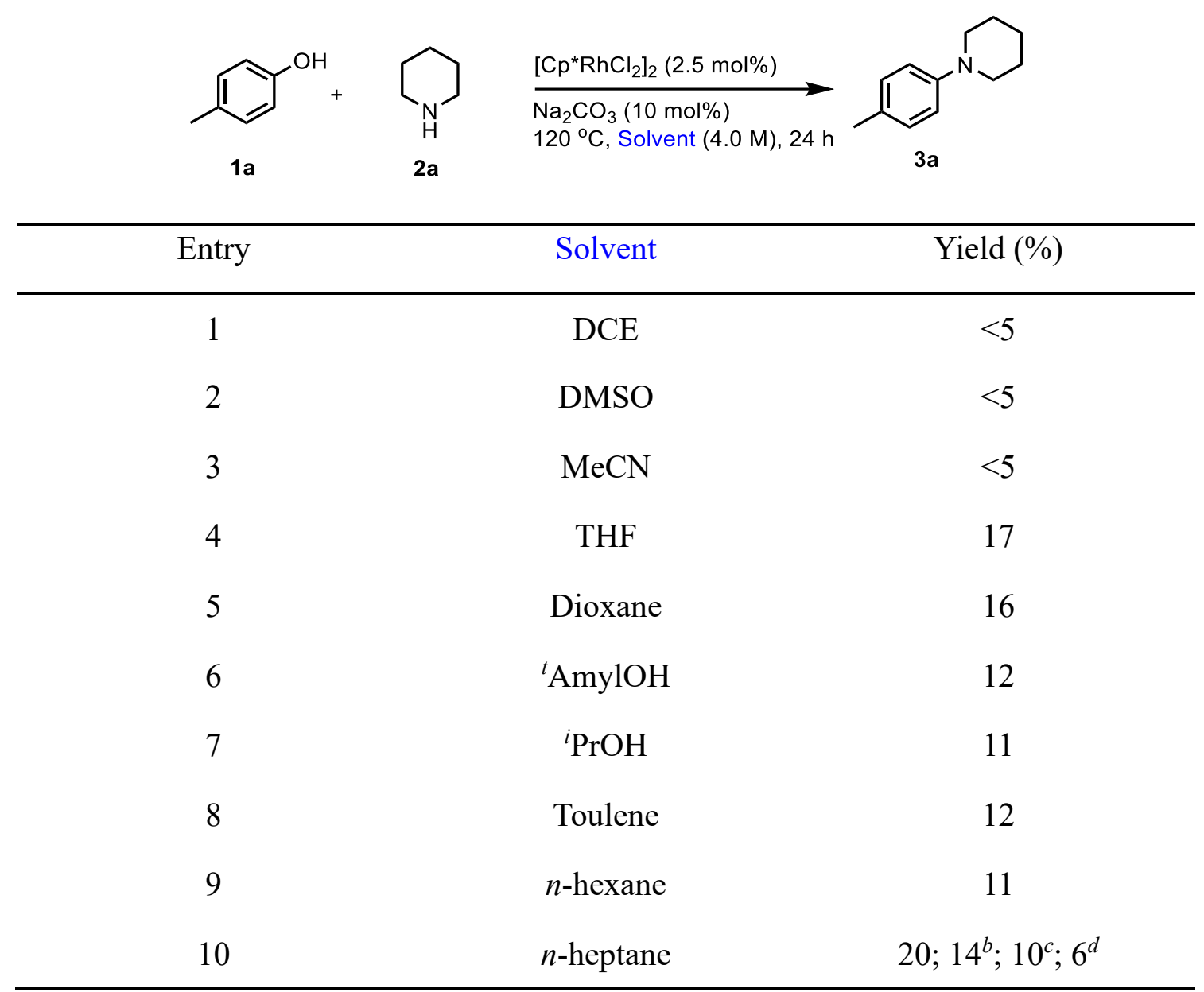

${ }^{a}$ Conditions: 1 a $(0.60 \mathrm{mmol}, 1.5$ equiv. $), 2 a(0.40 \mathrm{mmol}, 1.0$ equiv. $),\left[\mathrm{Cp}^{*} \mathrm{RhCl}_{2}\right]_{2}(0.010 \mathrm{mmol})$, $\mathrm{Na}_{2} \mathrm{CO}_{3}(0.040 \mathrm{mmol})$, Solvent $(0.10 \mathrm{~mL}), 120{ }^{\circ} \mathrm{C}, \mathrm{N}_{2}, 24 \mathrm{~h}$. Yield was determined by ${ }^{1} \mathrm{H}$ NMR using 1,1,2,2-tetrachloroethane as the internal standard. ${ }^{b}$ Solvent (2.0 M). ${ }^{c}$ Solvent (1.0 M). ${ }^{d}$ Neat. 
Table S4. Effect of Catalyst ${ }^{a}$<smiles>Cc1ccc(O)cc1</smiles>

1a<smiles>C1CCNCC1</smiles>

2a

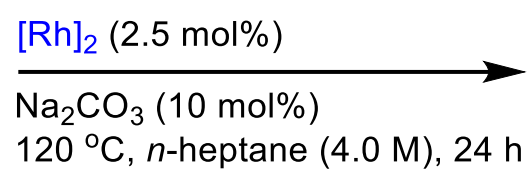

$120^{\circ} \mathrm{C}, n$-heptane $(4.0 \mathrm{M}), 24 \mathrm{~h}$

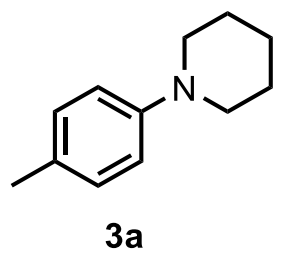

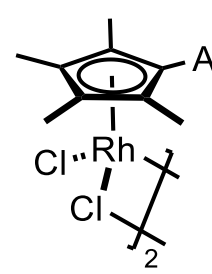

Ar Ph (cat. 6), 21\%

4- $\mathrm{MeO}-\mathrm{C}_{6} \mathrm{H}_{4}$ (cat. 7), $19 \%$

4- $\mathrm{CF}_{3}-\mathrm{C}_{6} \mathrm{H}_{4}$ (cat. 8), $28 \%$

2- $-\mathrm{CF}_{3}-\mathrm{C}_{6} \mathrm{H}_{4}$ (cat. 9), $20 \%$

3,5-di- $\mathrm{CF}_{3}-\mathrm{C}_{6} \mathrm{H}_{3}$ (cat. 10), $40 \%$

${ }^{a}$ Conditions: 1a ( $0.60 \mathrm{mmol}, 1.5$ equiv.), $\mathbf{2 a}\left(0.40 \mathrm{mmol}, 1.0\right.$ equiv.), $[\mathrm{Rh}]_{2}(0.010 \mathrm{mmol}), \mathrm{Na}_{2} \mathrm{CO}_{3}$ $(0.040 \mathrm{mmol}), n$-heptane $(0.10 \mathrm{~mL}), 120{ }^{\circ} \mathrm{C}, \mathrm{N}_{2}, 24 \mathrm{~h}$. Yield was determined by ${ }^{1} \mathrm{H}$ NMR using 1,1,2,2-tetrachloroethane as the internal standard.

Table S5. Effect of Temperature ${ }^{a}$<smiles>Cc1ccc(O)cc1</smiles>

$1 \mathbf{a}$<smiles>C1CCNCC1</smiles>

2a

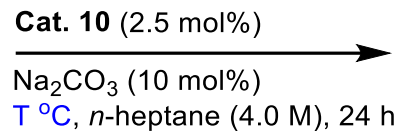

$\mathrm{T}^{\circ} \mathrm{C}, n$-heptane (4.0 M), $24 \mathrm{~h}$<smiles>Cc1ccc(N2CCCCC2)cc1</smiles>

3a

\begin{tabular}{ccc}
\hline Entry & $\mathrm{T}\left({ }^{\circ} \mathrm{C}\right)$ & Yield (\%) \\
\hline 1 & 100 & 8 \\
2 & 120 & 40 \\
3 & 130 & 60 \\
4 & 140 & 80 \\
5 & 150 & 85 \\
\hline
\end{tabular}

${ }^{a}$ Conditions: 1a $(0.60 \mathrm{mmol}, 1.5$ equiv. $), 2 a(0.40 \mathrm{mmol}, 1.0$ equiv. $)$ Cat. 10 (0.010 mmol), $\mathrm{Na}_{2} \mathrm{CO}_{3}(0.040 \mathrm{mmol}), n$-heptane $(0.10 \mathrm{~mL}), \mathrm{T}{ }^{\circ} \mathrm{C}, \mathrm{N}_{2}, 24 \mathrm{~h}$. Yield was determined by ${ }^{1} \mathrm{H}$ NMR using 1,1,2,2-tetrachloroethane as the internal standard. 
Table S6. Effect of Bases ${ }^{a}$<smiles>Cc1ccc(O)cc1</smiles>

1a<smiles>C1CCNCC1</smiles>

$2 \mathbf{a}$

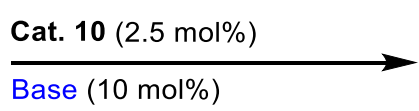

$140^{\circ} \mathrm{C}, n$-heptane $(4.0 \mathrm{M}), 24 \mathrm{~h}$<smiles>Cc1ccc(N2CCCCC2)cc1</smiles>

$3 a$

\begin{tabular}{ccc}
\hline Entry & Base & Yield (\%) \\
\hline 1 & --- & 67 \\
2 & $\mathrm{HCOONa}$ & 77 \\
3 & $\mathrm{NaOAc}$ & 73 \\
4 & $\mathrm{Na}_{2} \mathrm{CO}_{3}$ & $80 ; 76^{b} ; 80^{c}$ \\
5 & $\mathrm{~K}_{2} \mathrm{CO}_{3}$ & 80 \\
6 & $\mathrm{Cs}_{2} \mathrm{CO}_{3}$ & 76 \\
7 & $\mathrm{Li}_{2} \mathrm{CO}_{3}$ & 70 \\
8 & $\mathrm{~K}_{3} \mathrm{PO}_{4}$ & 77 \\
9 & $\mathrm{KF}$ \\
10 & $\mathrm{KO}^{t} \mathrm{Bu}$ & 79 \\
11 & $\mathrm{KOH}$ & 75 \\
12 & $\mathrm{Et}{ }_{3} \mathrm{~N}$ & 79 \\
\hline
\end{tabular}

${ }^{a}$ Conditions: 1a (0.60 mmol, 1.5 equiv.), 2 a ( $0.40 \mathrm{mmol}, 1.0$ equiv.), Cat. $10(0.010 \mathrm{mmol})$, Base (0.040 mmol), $n$-heptane $(0.10 \mathrm{~mL}), 140{ }^{\circ} \mathrm{C}, \mathrm{N}_{2}, 24 \mathrm{~h}$. Yield was determined by ${ }^{1} \mathrm{H}$ NMR using 1,1,2,2-tetrachloroethane as the internal standard. ${ }^{b}$ Base $(20 \mathrm{~mol} \%)$. ${ }^{c}$ Base $(50 \mathrm{~mol} \%)$. 
Table S7. Effect of the Ratio of Substrate ${ }^{a}$

\begin{tabular}{|c|c|c|}
\hline $\begin{array}{c}1 \mathrm{a} \\
\times \mathrm{mmol}\end{array}$ & $\begin{array}{l}\text { Cat. } 10(2.5 \mathrm{~mol} \%) \\
\underset{\mathrm{Na}_{2} \mathrm{CO}_{3}(10 \mathrm{~mol} \%)}{140^{\circ} \mathrm{C}, n \text {-heptane }(4.0 \mathrm{M}), 24 \mathrm{~h}}\end{array}$ & $Y^{N}$ \\
\hline Entry & : y $(0.4 \mathrm{mmol}$ scale $)$ & Yield (\%) \\
\hline 1 & $1.5: 1$ & $80 ; 84^{b} ; 91^{c} ; 89^{d}$ \\
\hline 2 & $1: 1$ & $78^{c}$ \\
\hline 3 & $1: 1.2$ & $76^{c}$ \\
\hline 4 & $2: 1$ & $88 ; 91^{b} ; 96^{c} ; 84^{c, e} ; 97^{d}$ \\
\hline
\end{tabular}

${ }^{a}$ Conditions: 1a (x mmol), 2a (y mmol), Cat. 10 (0.010 mmol), $\mathrm{Na}_{2} \mathrm{CO}_{3}(0.040 \mathrm{mmol}), n$-heptane $(0.10 \mathrm{~mL}), 140{ }^{\circ} \mathrm{C}, \mathrm{N}_{2}, 24 \mathrm{~h}$. Yield was determined by ${ }^{1} \mathrm{H}$ NMR using 1,1,2,2-tetrachloroethane as the internal standard. ${ }^{b} 4 \AA \mathrm{MS}(25 \mathrm{mg}) .{ }^{c} 4 \AA \mathrm{MS}(50 \mathrm{mg}) .{ }^{d} 4 \AA \mathrm{MS}(100 \mathrm{mg}) .{ }^{e}$ Isolated yield. 


\section{General Procedure for the Amination of Phenols}

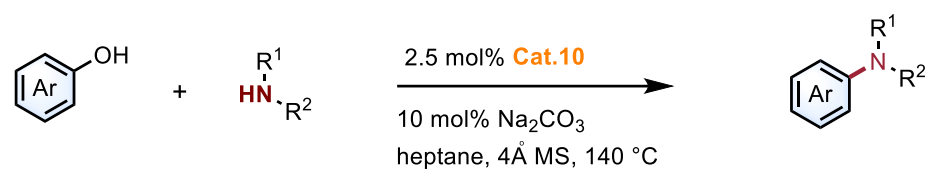

General condition A: In the glovebox ( $\mathrm{N}_{2}$ atmosphere), a $4 \mathrm{~mL}$ glass vial was charged with amine $(0.40 \mathrm{mmol})$, phenol $(0.60 \mathrm{mmol})$, Cat. $10(0.010 \mathrm{mmol}, 10.1$ $\mathrm{mg}), \mathrm{Na}_{2} \mathrm{CO}_{3}(0.040 \mathrm{mmol}, 4.2 \mathrm{mg}), 4 \AA \mathrm{MS}(50 \mathrm{mg})$ and $n$-heptane $(0.10 \mathrm{~mL})$. The vial was then sealed and reaction mixture was stirred at $140{ }^{\circ} \mathrm{C}$ for $24-36$ hours. After the reaction vial was cooled to room temperature, the mixture was purified by silica gel chromatography (ethyl acetate/petroleum ether).

General condition B: In the glovebox $\left(\mathrm{N}_{2}\right.$ atmosphere), a $4 \mathrm{~mL}$ glass vial was charged with amine $(0.40 \mathrm{mmol})$, phenol $(0.80 \mathrm{mmol})$, Cat. $10(0.010 \mathrm{mmol}, 10.1$ $\mathrm{mg}), \mathrm{Na}_{2} \mathrm{CO}_{3}(0.040 \mathrm{mmol}, 4.2 \mathrm{mg}), 4 \AA \mathrm{MS}(50 \mathrm{mg})$ and $n$-heptane $(0.10 \mathrm{~mL})$. The vial was then sealed and reaction mixture was stirred at $140{ }^{\circ} \mathrm{C}$ for $24-36$ hours. After the reaction vial was cooled to room temperature, the mixture was purified by silica gel chromatography (ethyl acetate/petroleum ether). 


\section{Mechanistic Studies}

\subsection{Control Experiments}

Scheme S1. Investigation of Possible Intermediates from H-Transfer Mechanism

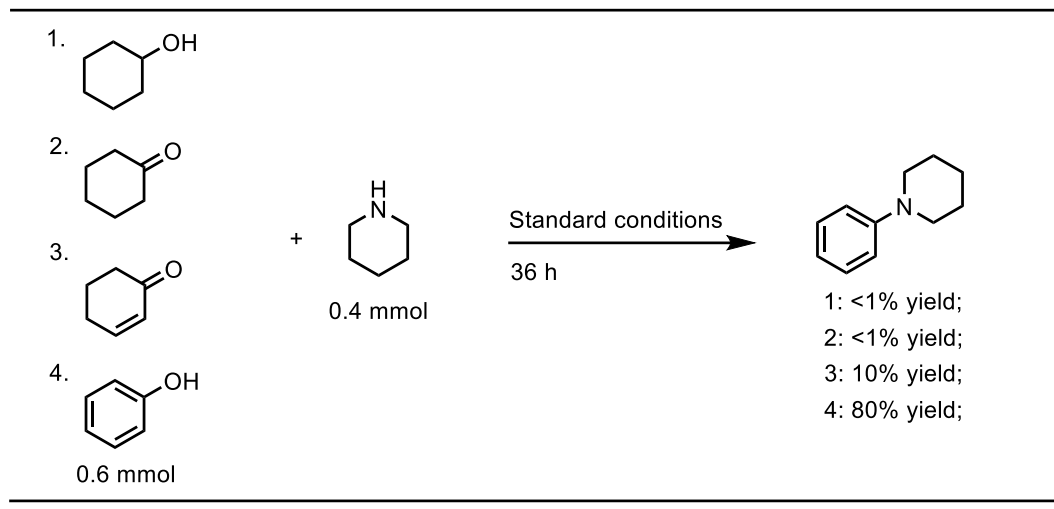

Some possible intermediates from H-transfer mechanism were investigated under the standard conditions. Cyclohexanol and cyclohexanone gave no product. Cyclohex-2-en-1-one gave very complex mixture and the desired product was observed in $10 \%{ }^{1} \mathrm{H}$ NMR yield.

\subsection{Monitoring the Reaction}

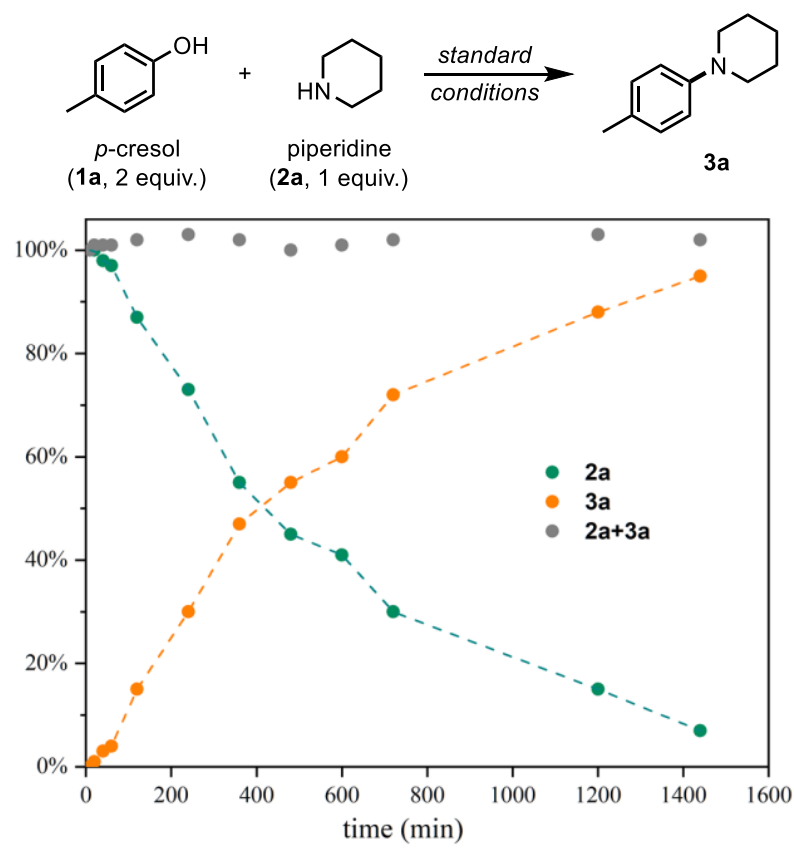

The catalytic amination of $p$-cresol with piperidine (2a) was monitored. 2a gradually converted to the aniline $\mathbf{3 a}$ without detecting any imine, cyclohexanone, or cyclohexen-1-one. 


\subsection{Deuterium Labeling Experiments}

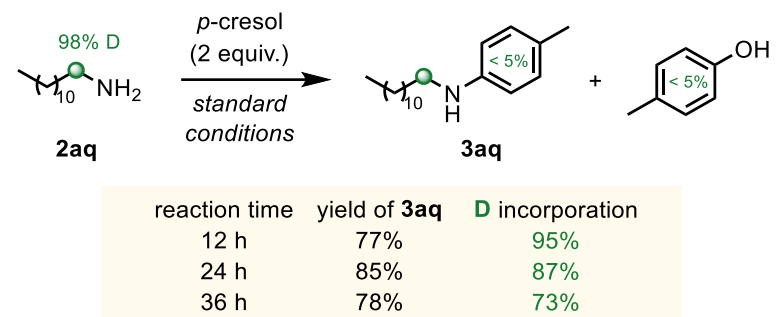

The starting material $\alpha$-deuterated dodecylamine $\mathbf{2 a q}$ was prepared as following procedure: in the glovebox $\left(\mathrm{N}_{2}\right.$ atmosphere), an oven-dried Schlenk tube was charged with $\mathrm{LiAlD}_{4}(99 \% \mathrm{D}, 3.6 \mathrm{mmol})$. Then removed the tube from the glove box, and added dry $\mathrm{Et}_{2} \mathrm{O}(6 \mathrm{~mL})$ under $\mathrm{N}_{2}$ atmosphere at $0{ }^{\circ} \mathrm{C}$. Next, added a solution of dodecanenitrile $(3.0 \mathrm{mmol})$ in dry $\mathrm{Et}_{2} \mathrm{O}(2 \mathrm{~mL})$ slowly at $0{ }^{\circ} \mathrm{C}$. The resulting mixture was allowed to warm to room temperature and stirred overnight. Quenched it slowly with water $(0.5 \mathrm{~mL})$ and $15 \% \mathrm{NaOH}(0.5 \mathrm{~mL})$ at $0{ }^{\circ} \mathrm{C}$. The white suspension was allowed to warm to room temperature before addition of $\mathrm{MgSO}_{4}(10.0 \mathrm{~g})$ and stirring for $0.5 \mathrm{~h}$. Filtration of solids and evaporation of all solvents in vacuo gave $\alpha$-deuterated dodecylamine 2 aq without further purification in $90 \%$ yield $(98 \% \mathrm{D})$.

${ }^{1} \mathrm{H}$ NMR $\left(500 \mathrm{MHz}, \mathrm{CDCl}_{3}\right) \delta 2.68-2.60(\mathrm{~m}, 0.04 \mathrm{H}), 1.43-1.37(\mathrm{~m}, 2 \mathrm{H}), 1.31-$ $1.20(\mathrm{~m}, 16 \mathrm{H}), 1.15-1.10(\mathrm{~m}, 2 \mathrm{H}), 0.86(\mathrm{t}, J=6.8 \mathrm{~Hz}, 3 \mathrm{H})$.

${ }^{1} \mathrm{H}$ NMR (500 MHz, $\left.\mathrm{CDCl}_{3}\right)$
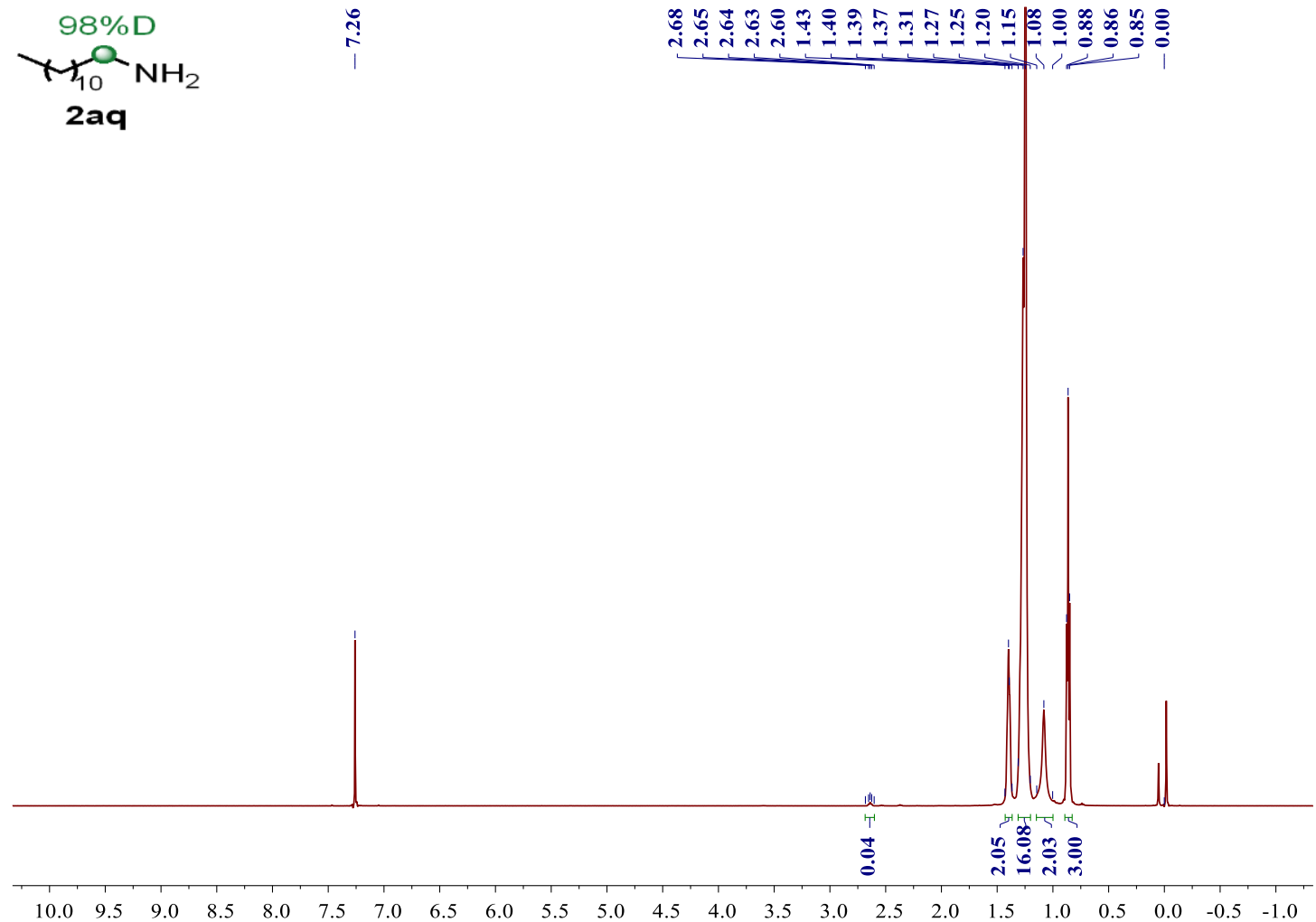
${ }^{1} \mathrm{H} \mathrm{NMR}\left(500 \mathrm{MHz}, \mathrm{CDCl}_{3}\right)$
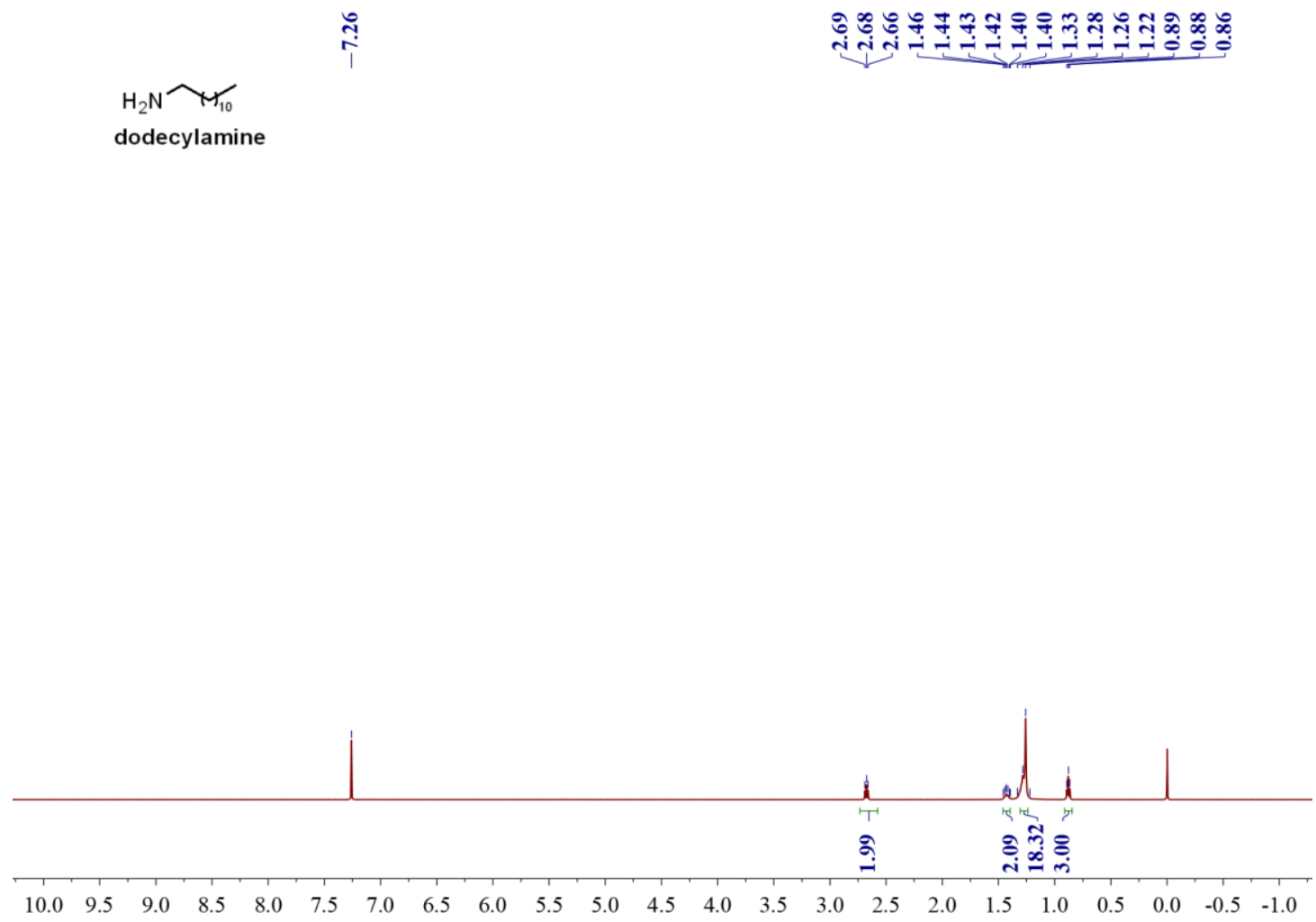

An amination with $\alpha$-deuterated dodecylamine 2 aq produced the aniline 3aq without an obvious decay of deuterium incorporation in 12 hours; while, the decay became obvious when the reaction proceeded for a longer time. Aromatic H/D exchange was not detected, indicating that a hydrogen transfer mechanism for this amination is unlikely.

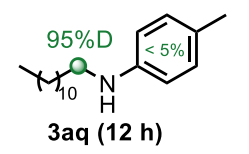

${ }^{1} \mathrm{H}$ NMR (500 MHz, DMSO-d $) \delta 6.85(\mathrm{~d}, J=8.1 \mathrm{~Hz}, 2 \mathrm{H}), 6.44(\mathrm{~d}, J=8.4 \mathrm{~Hz}, 2 \mathrm{H})$, $5.20(\mathrm{~s}, 1 \mathrm{H}), 2.94-2.88(\mathrm{~m}, 0.11 \mathrm{H}), 2.13(\mathrm{~s}, 3 \mathrm{H}), 1.49(\mathrm{t}, J=6.6 \mathrm{~Hz}, 2 \mathrm{H}), 1.36-1.16$ $(\mathrm{m}, 18 \mathrm{H}), 0.85(\mathrm{t}, J=6.8 \mathrm{~Hz}, 3 \mathrm{H}) .{ }^{13} \mathrm{C}$ NMR $\left(126 \mathrm{MHz}, \mathrm{DMSO}-d_{6}\right) \delta 146.9,129.3$, 123.5, 112.0, 43.1 - 42.1 (m, $\alpha$-Carbon of aniline 3aq), 31.3, 29.1, 29.0, 29.0, 29.0, 28.7, 28.7, 28.6, 26.7, 22.1, 20.1, 13.9 .

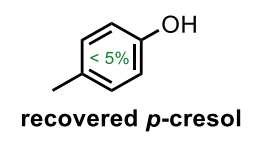

${ }^{1} \mathrm{H}$ NMR $\left(500 \mathrm{MHz}, \mathrm{CDCl}_{3}\right) \delta 7.04(\mathrm{~d}, J=8.1 \mathrm{~Hz}, 2 \mathrm{H}), 6.74(\mathrm{~d}, J=8.4 \mathrm{~Hz}, 2 \mathrm{H}), 4.21$ (s, 1H), $2.28(\mathrm{~s}, 3 \mathrm{H}) .{ }^{13} \mathrm{C} \mathrm{NMR}\left(126 \mathrm{MHz}, \mathrm{CDCl}_{3}\right) \delta 153.3,130.0,129.9,115.1,20.4$. 
${ }^{1} \mathrm{H}$ NMR (500 MHz, DMSO-d6)

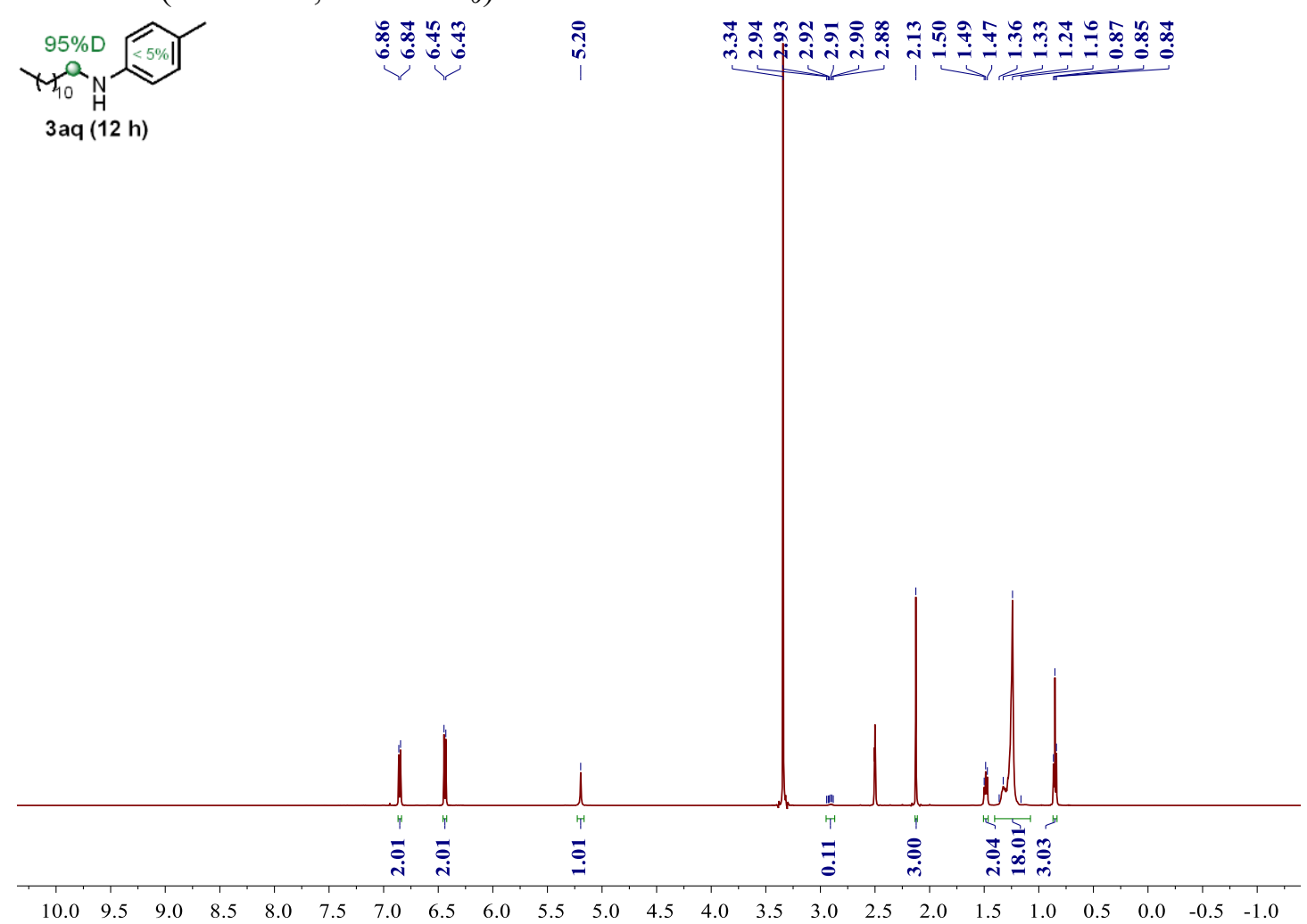

${ }^{13} \mathrm{C}$ NMR $\left(126 \mathrm{MHz}, \mathrm{DMSO}-d_{6}\right)$
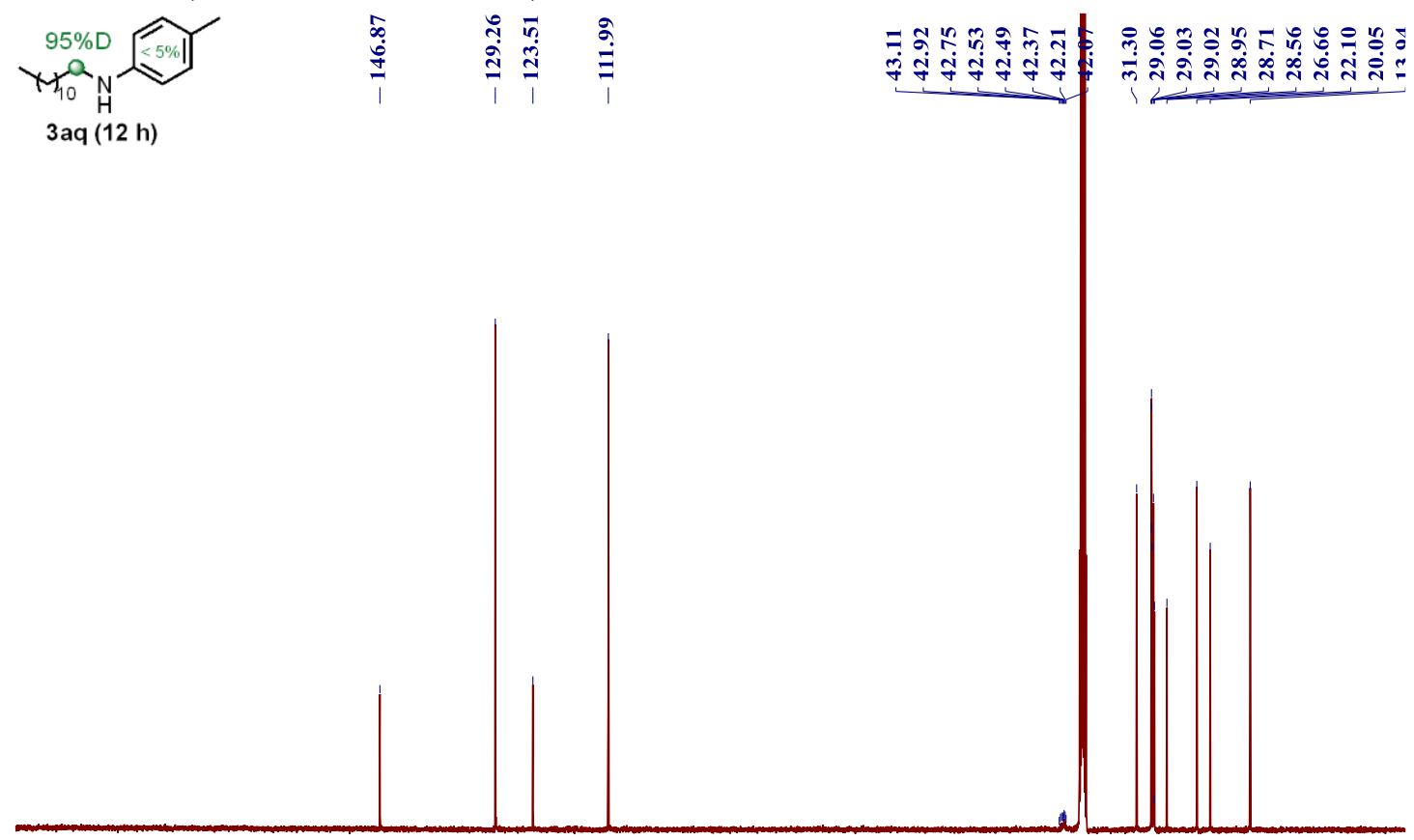

$\begin{array}{lllllllllllllllllllll}200 & 190 & 180 & 170 & 160 & 150 & 140 & 130 & 120 & 110 & 100 & 90 & 80 & 70 & 60 & 50 & 40 & 30 & 20 & 10 & 0\end{array}$ 
${ }^{1} \mathrm{H}$ NMR (500 MHz, DMSO-d6)

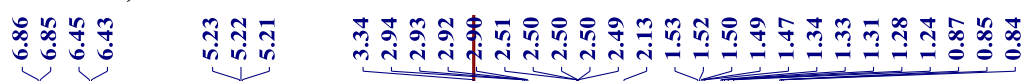
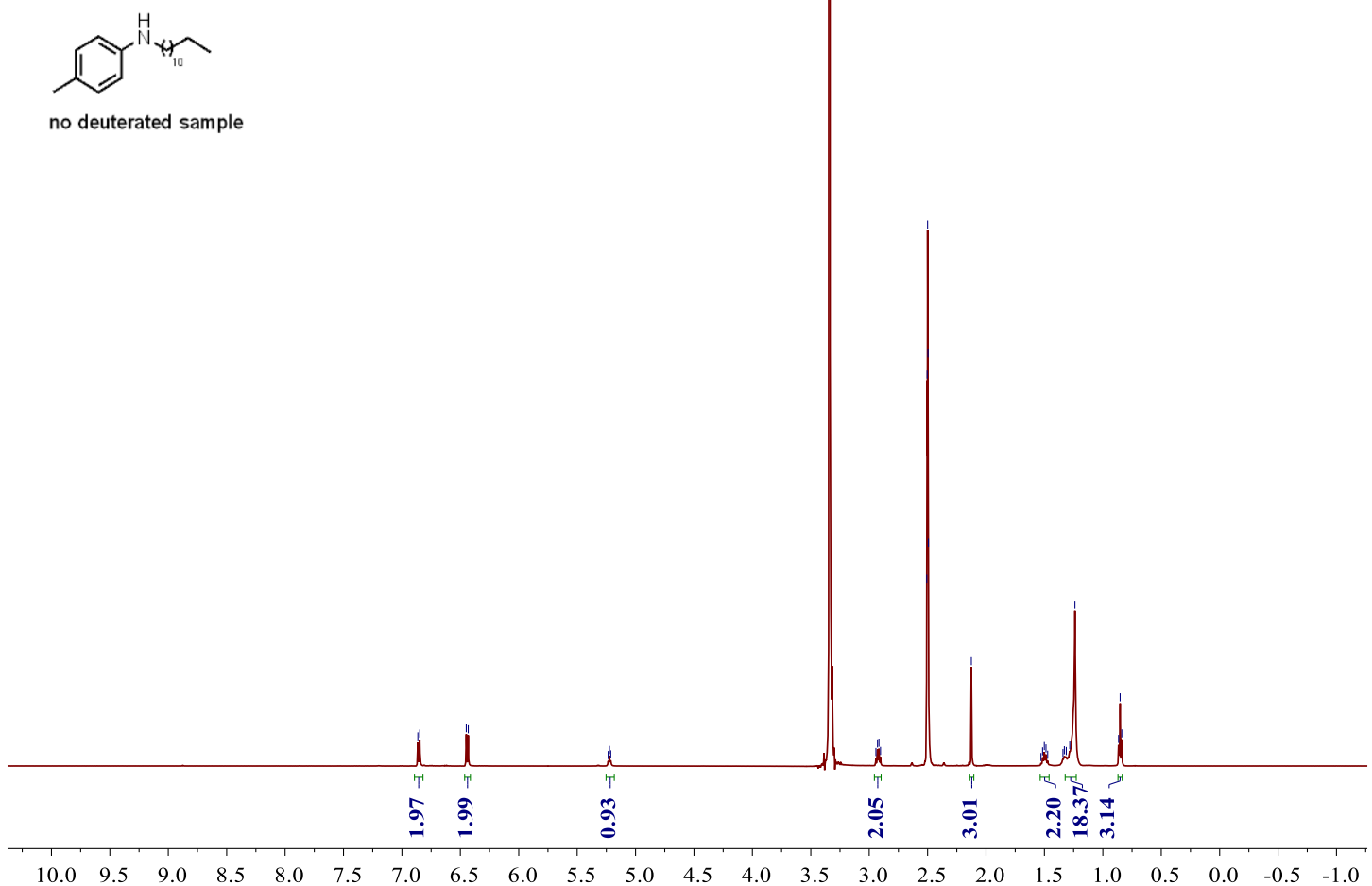

${ }^{13} \mathrm{C}$ NMR (126 MHz, DMSO- $\left.d_{6}\right)$

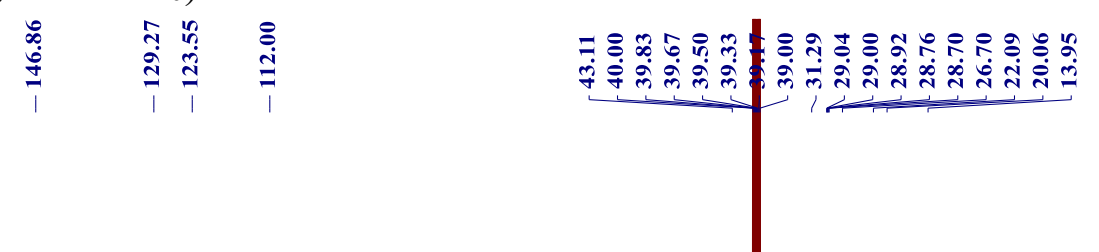

no deuterated sample

$\begin{array}{lllllllllllllllllllllllll}200 & 190 & 180 & 170 & 160 & 150 & 140 & 130 & 120 & 110 & 100 & 90 & 80 & 70 & 60 & 50 & 40 & 30 & 20 & 10 & 0 & -\end{array}$ 
${ }^{1} \mathrm{H}$ NMR $\left(500 \mathrm{MHz}, \mathrm{CDCl}_{3}\right)$

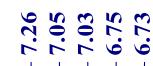

$\underset{\text { 氵 }}{\grave{1}}$

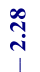

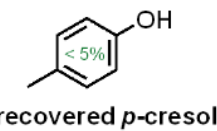

recovered $p$-cresol

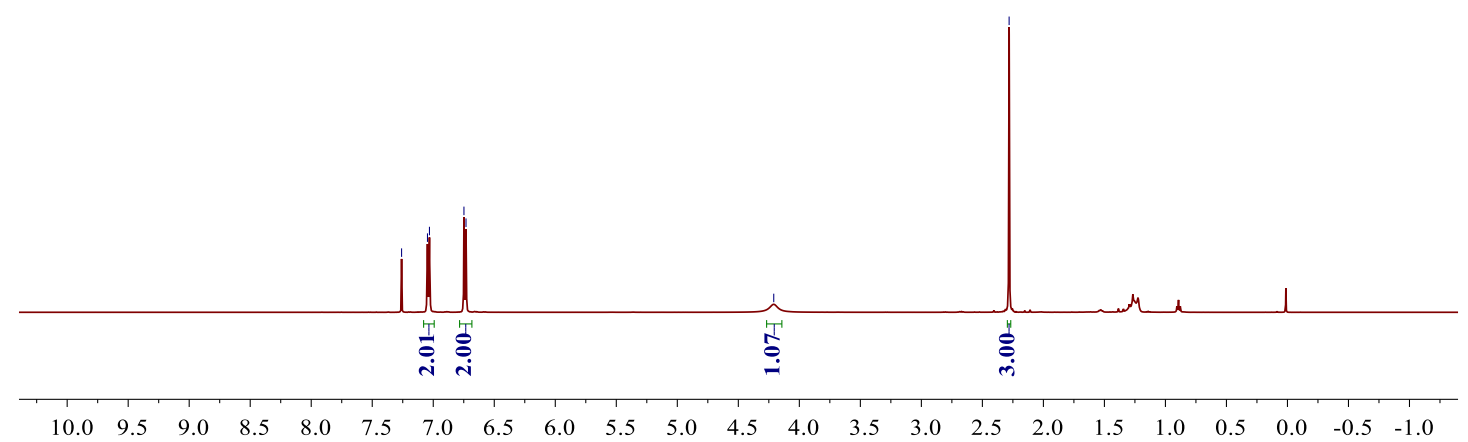

${ }^{13} \mathrm{C} \mathrm{NMR}\left(126 \mathrm{MHz}, \mathrm{CDCl}_{3}\right)$

至
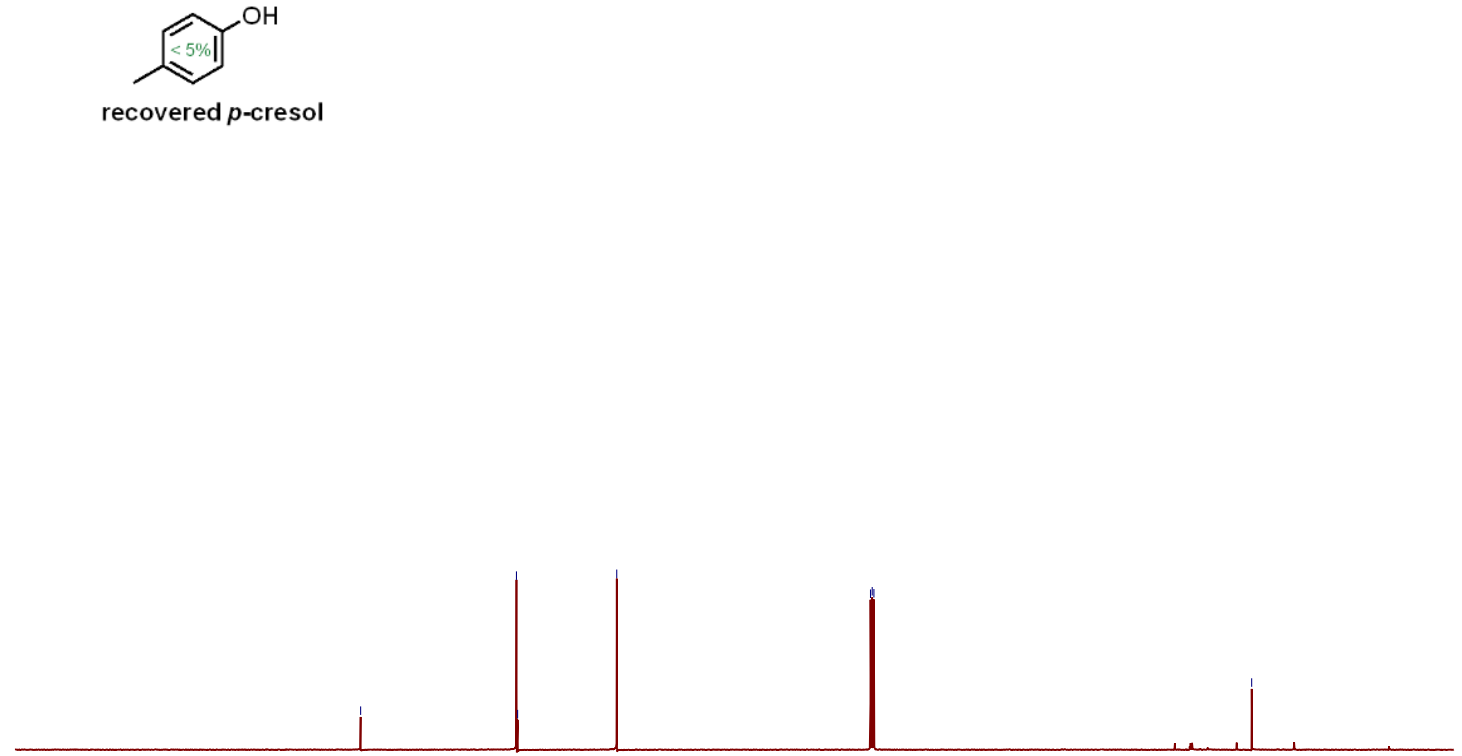

$\begin{array}{lllllllllllllllllllll}200 & 190 & 180 & 170 & 160 & 150 & 140 & 130 & 120 & 110 & 100 & 90 & 80 & 70 & 60 & 50 & 40 & 30 & 20 & 10 & 0\end{array}$ 


\subsection{Studies on Rh complexes}

Cp*Rh $\eta^{5}$-(3,5-dimethylphenoxo) complex Rh-1 was prepared as following procedure:

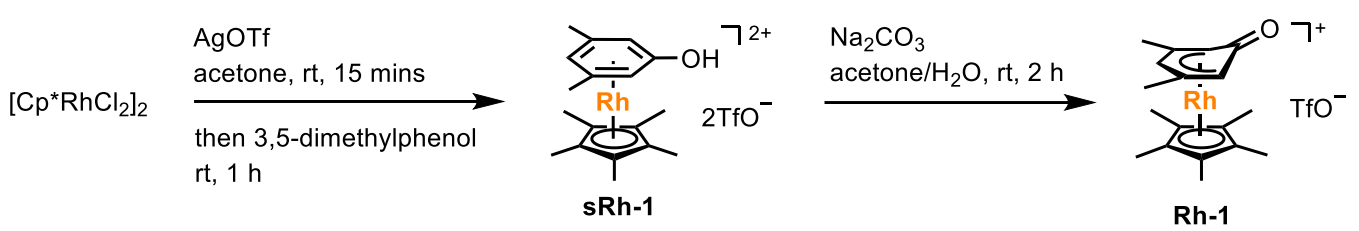

A $4 \mathrm{~mL}$ glass vial was charged with $\left[\mathrm{Cp}^{*} \mathrm{RhCl}_{2}\right]_{2}(0.10 \mathrm{mmol}), \operatorname{AgOTf}(0.40 \mathrm{mmol})$, and acetone $(2 \mathrm{~mL})$. The mixture was stirred at room temperature for 15 minutes. Then, $\mathrm{AgCl}$ was removed by filtration. 3,5-dimethylphenol $(0.22 \mathrm{mmol})$ was added into the filtrate. The mixture was stirred at room temperature for 1 hour. Then, the solvent was removed under reduced pressure. The crude product was washed by $\mathrm{Et}_{2} \mathrm{O}$ for 3 times and dried under vacuum to afford $C p^{*} R h \eta^{6}$-(3,5-dimethylphenol) complex sRh-1. Next, the collecting Cp*Rh $\eta^{6}$-(3,5-dimethylphenol) complex sRh-1 was dissolved in acetone $/ \mathrm{H}_{2} \mathrm{O}(200: 1,2 \mathrm{~mL})$, and then added $\mathrm{Na}_{2} \mathrm{CO}_{3}(0.40 \mathrm{mmol})$. After the mixture was stirred at room temperature for 2 hours, solid was removed by filtration and the filtrate was dried under reduced pressure. The crude product was washed by $\mathrm{Et}_{2} \mathrm{O}$ for 3 times and dried under vacuum to afford Rh-1 as yellow solid (three steps, $78 \%$ yield).

Cp* $R \mathbf{h} \boldsymbol{\eta}^{6}$-(3,5-dimethylphenyl piperidine) complex $\mathbf{R h - 2}$ was prepared similar to Rh-1 using 3,5-dimethylphenyl piperidine instead of 3,5-dimethylphenol without step 3 .

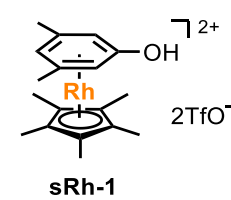

${ }^{1} \mathrm{H}$ NMR (500 MHz, Acetone- $\left.d_{6}\right) \delta 7.22(\mathrm{~s}, 1 \mathrm{H}), 7.05$ (s, 2H), $2.60(\mathrm{~s}, 6 \mathrm{H}), 2.26$ (s, $15 \mathrm{H}) .{ }^{13} \mathrm{C}$ NMR $\left(126 \mathrm{MHz}\right.$, Acetone- $\left.d_{6}\right) \delta 146.9(\mathrm{~d}, J=2.1 \mathrm{~Hz}), 123.2(\mathrm{~d}, J=4.9 \mathrm{~Hz})$, 121.9 (q, $J=321.0 \mathrm{~Hz}), 110.9$ (d, $J=7.9 \mathrm{~Hz}), 101.0(\mathrm{~d}, J=5.0 \mathrm{~Hz}), 93.5$ (d, $J=4.5$ $\mathrm{Hz}), 18.6,9.9 .{ }^{19} \mathrm{~F}$ NMR (471 MHz, Acetone- $\left.d_{6}\right) \delta-79.0$.

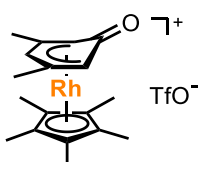

Rh-1

${ }^{1} \mathrm{H}$ NMR (500 MHz, Acetone- $\left.d_{6}\right) \delta 6.51(\mathrm{~s}, 1 \mathrm{H}), 5.52(\mathrm{~s}, 2 \mathrm{H}), 2.28(\mathrm{~s}, 6 \mathrm{H}), 2.11$ (s, $15 \mathrm{H}) .{ }^{13} \mathrm{C}$ NMR $\left(126 \mathrm{MHz}\right.$, Acetone- $\left.d_{6}\right) \delta 162.5,122.3(\mathrm{q}, J=322.0 \mathrm{~Hz}), 119.4(\mathrm{~d}, J$ $=5.7 \mathrm{~Hz}), 105.7(\mathrm{~d}, J=7.6 \mathrm{~Hz}), 91.8(\mathrm{~d}, J=5.9 \mathrm{~Hz}), 91.3(\mathrm{~d}, J=6.2 \mathrm{~Hz}), 18.8,9.6$. ${ }^{19} \mathrm{~F}$ NMR (471 MHz, Acetone- $\left.d_{6}\right) \delta$-77.9. HRMS-ESI $(\mathrm{m} / \mathrm{z})$ : $[\mathrm{M}]^{+}$calcd. for $\left[\mathrm{C}_{18} \mathrm{H}_{24} \mathrm{ORh}\right]^{+}, 359.0882$; found, 359.0880. 


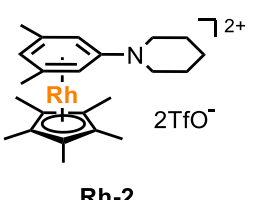

${ }^{1} \mathrm{H}$ NMR $\left(500 \mathrm{MHz}\right.$, Acetone- $\left.d_{6}\right) \delta 6.97(\mathrm{~s}, 2 \mathrm{H}), 6.90(\mathrm{~s}, 1 \mathrm{H}), 4.10-3.99(\mathrm{~m}, 2 \mathrm{H})$, $3.87-3.74(\mathrm{~m}, 2 \mathrm{H}), 2.51(\mathrm{~s}, 6 \mathrm{H}), 2.25(\mathrm{~s}, 15 \mathrm{H}), 1.82-1.72(\mathrm{~m}, 6 \mathrm{H}) .{ }^{13} \mathrm{C}$ NMR $(126$ MHz, Acetone- $\left.d_{6}\right) \delta 144.5,122.3(\mathrm{q}, J=322.0 \mathrm{~Hz}), 119.8(\mathrm{~d}, J=5.5 \mathrm{~Hz}), 109.0(\mathrm{~d}, J$ $=7.8 \mathrm{~Hz}), 96.1(\mathrm{~d}, J=5.4 \mathrm{~Hz}), 80.6(\mathrm{~d}, J=5.2 \mathrm{~Hz}), 49.1,26.8,24.0,18.9,10.3 .{ }^{19} \mathrm{~F}$ NMR (471 MHz, Acetone- $\left.d_{6}\right) \delta$-78.9.

${ }^{1} \mathrm{H}$ NMR (500 MHz, Acetone- $\left.d_{6}\right)$

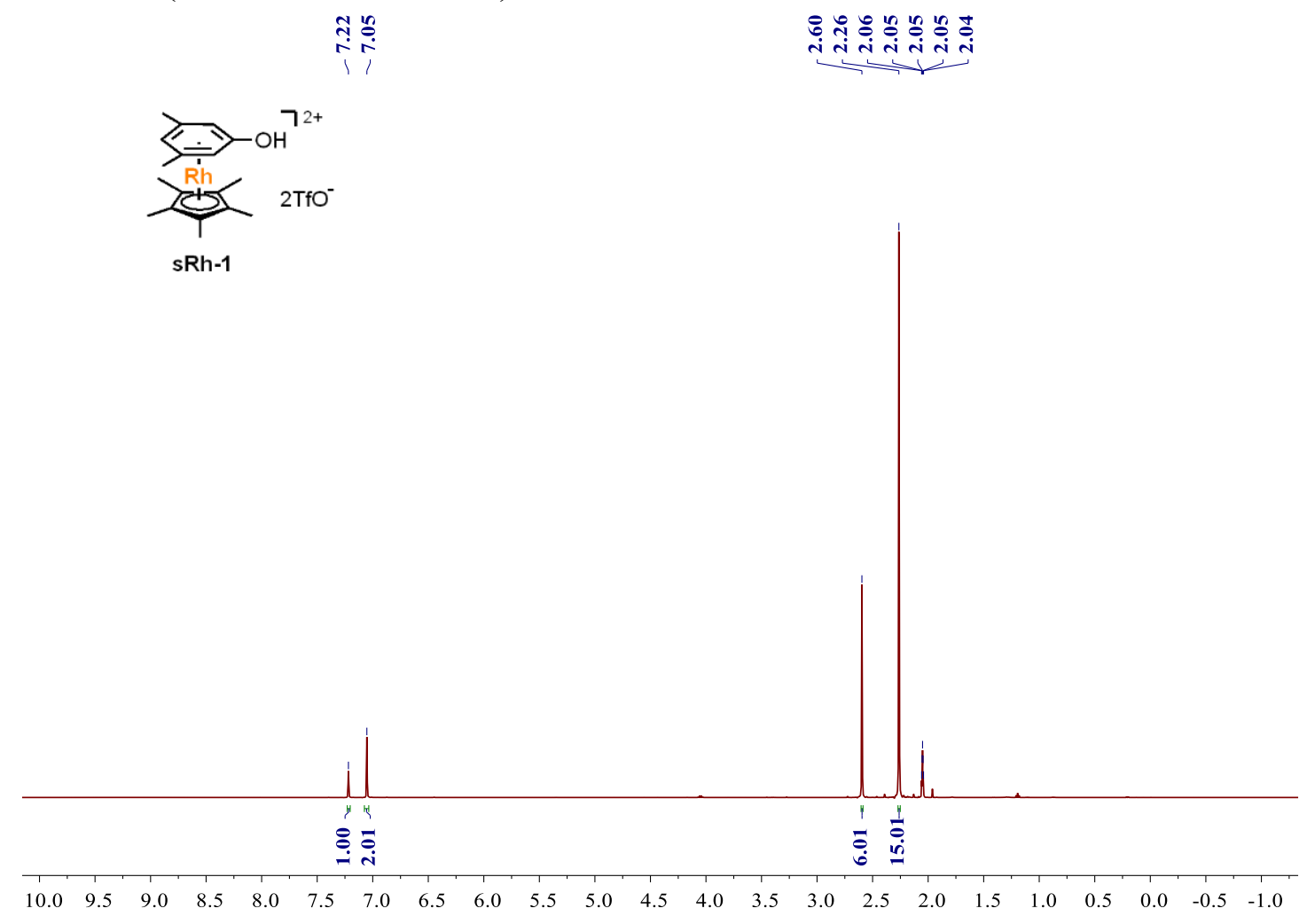


${ }^{13} \mathrm{C}$ NMR (126 MHz, Acetone- $\left.d_{6}\right)$

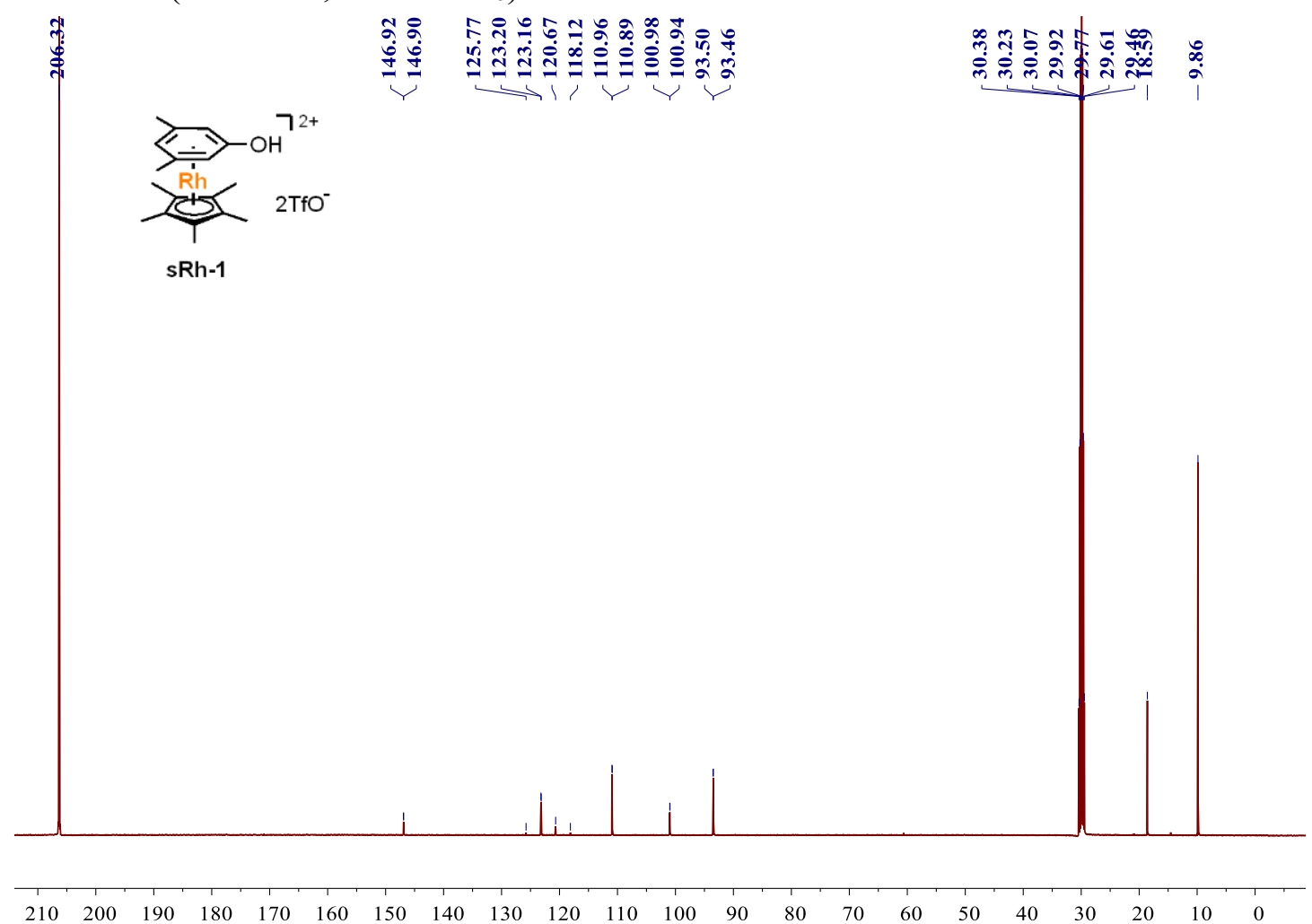

${ }^{19} \mathrm{~F}$ NMR (471 MHz, Acetone- $d_{6}$ )
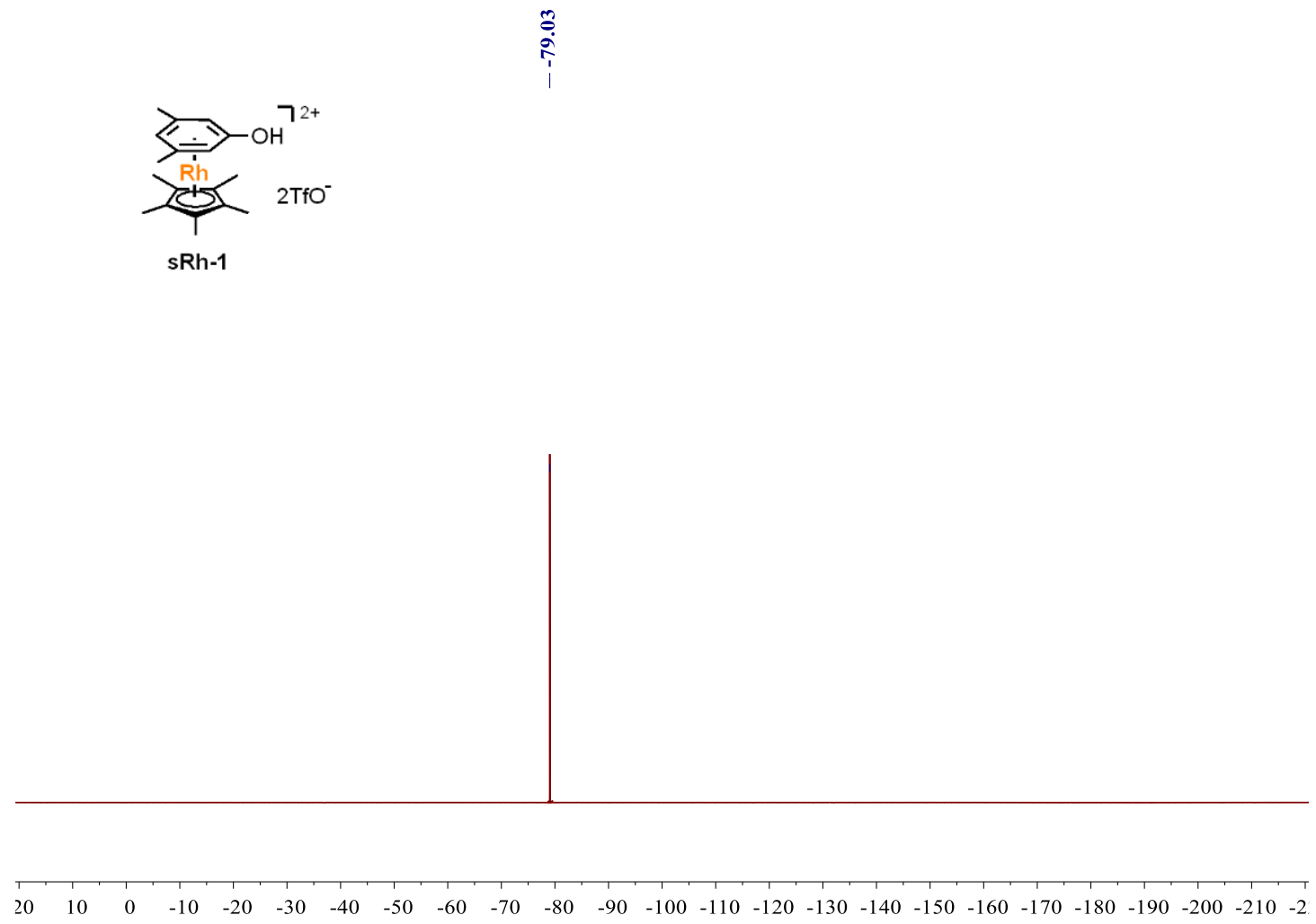
${ }^{1} \mathrm{H}$ NMR (500 MHz, Acetone- $\left.d_{6}\right)$

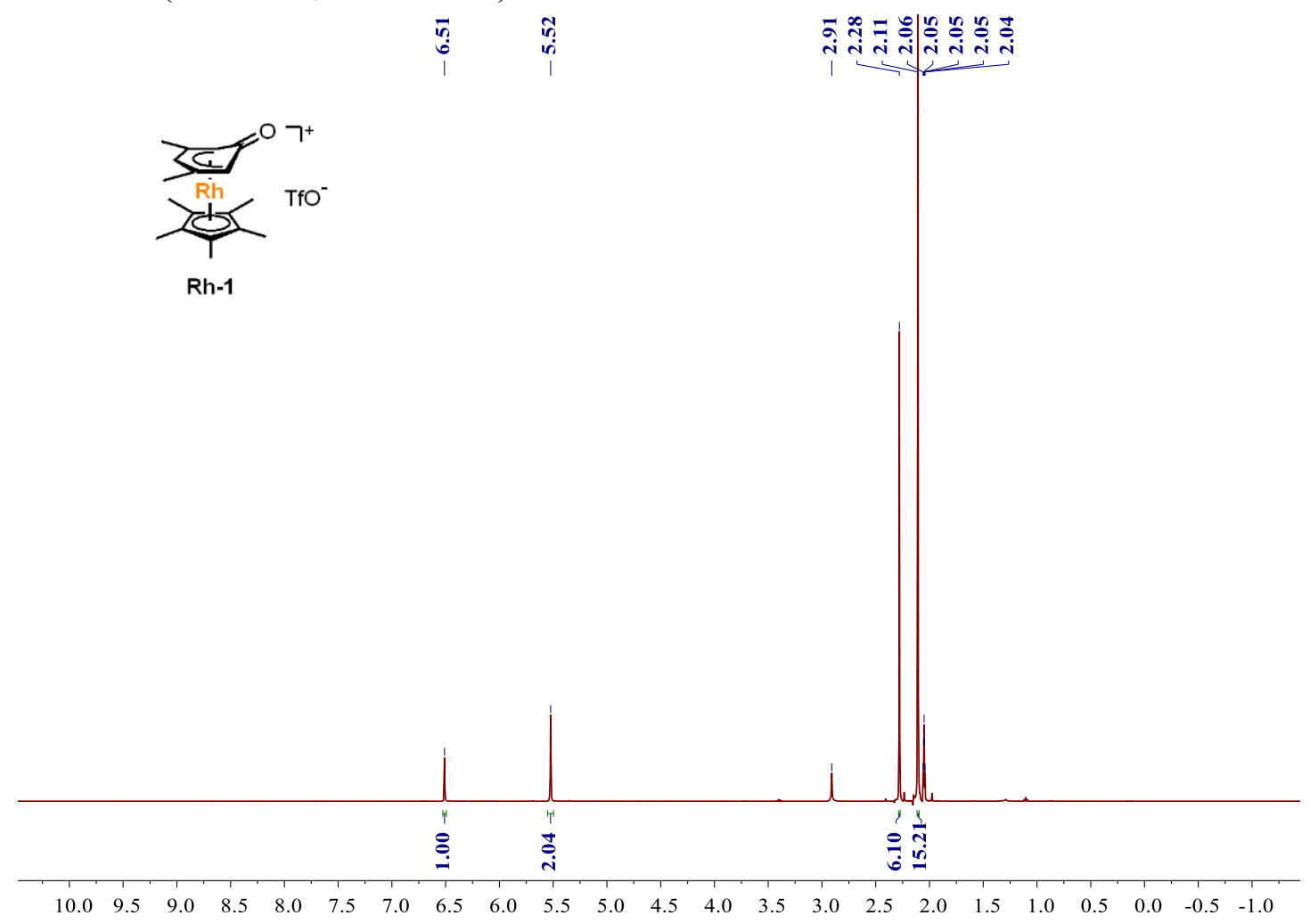

${ }^{13} \mathrm{C}$ NMR (126 MHz, Acetone- $\left.d_{6}\right)$

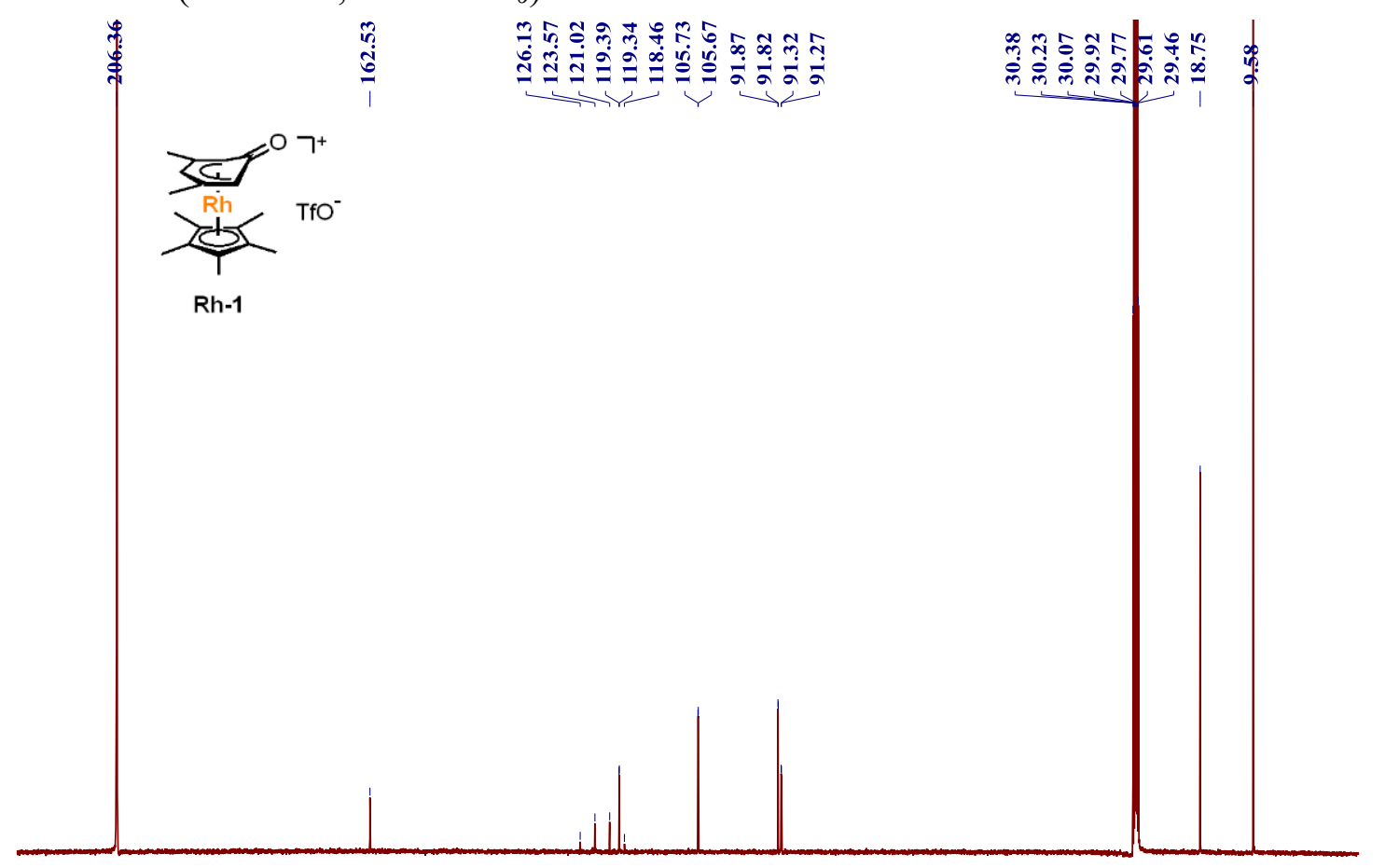

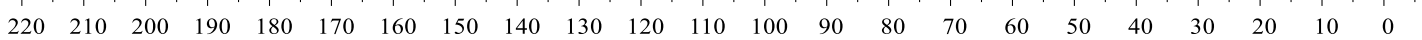


${ }^{19} \mathrm{~F}$ NMR (471 MHz, Acetone- $d_{6}$ )
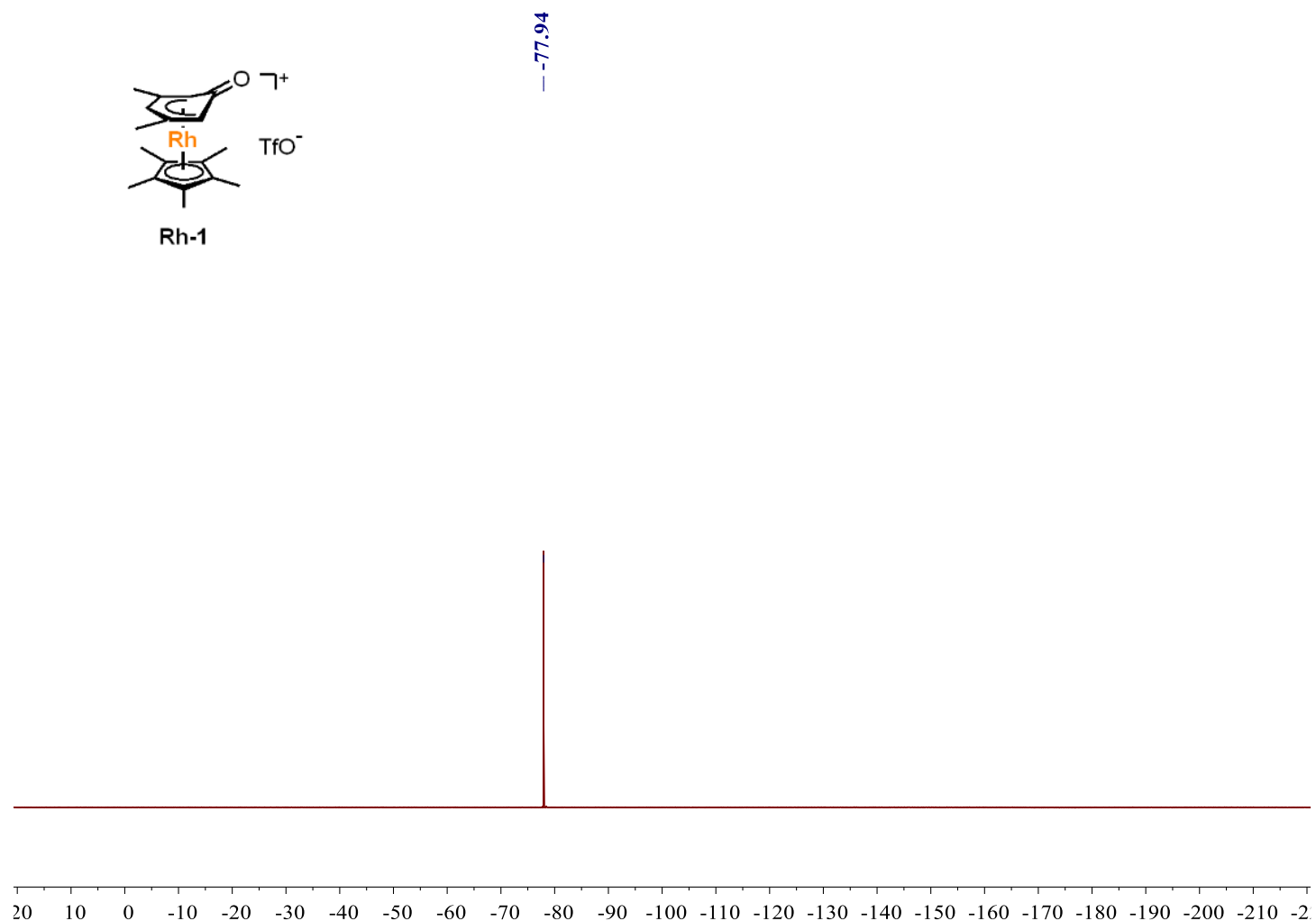

${ }^{1} \mathrm{H}$ NMR (500 MHz, Acetone- $\left.d_{6}\right)$

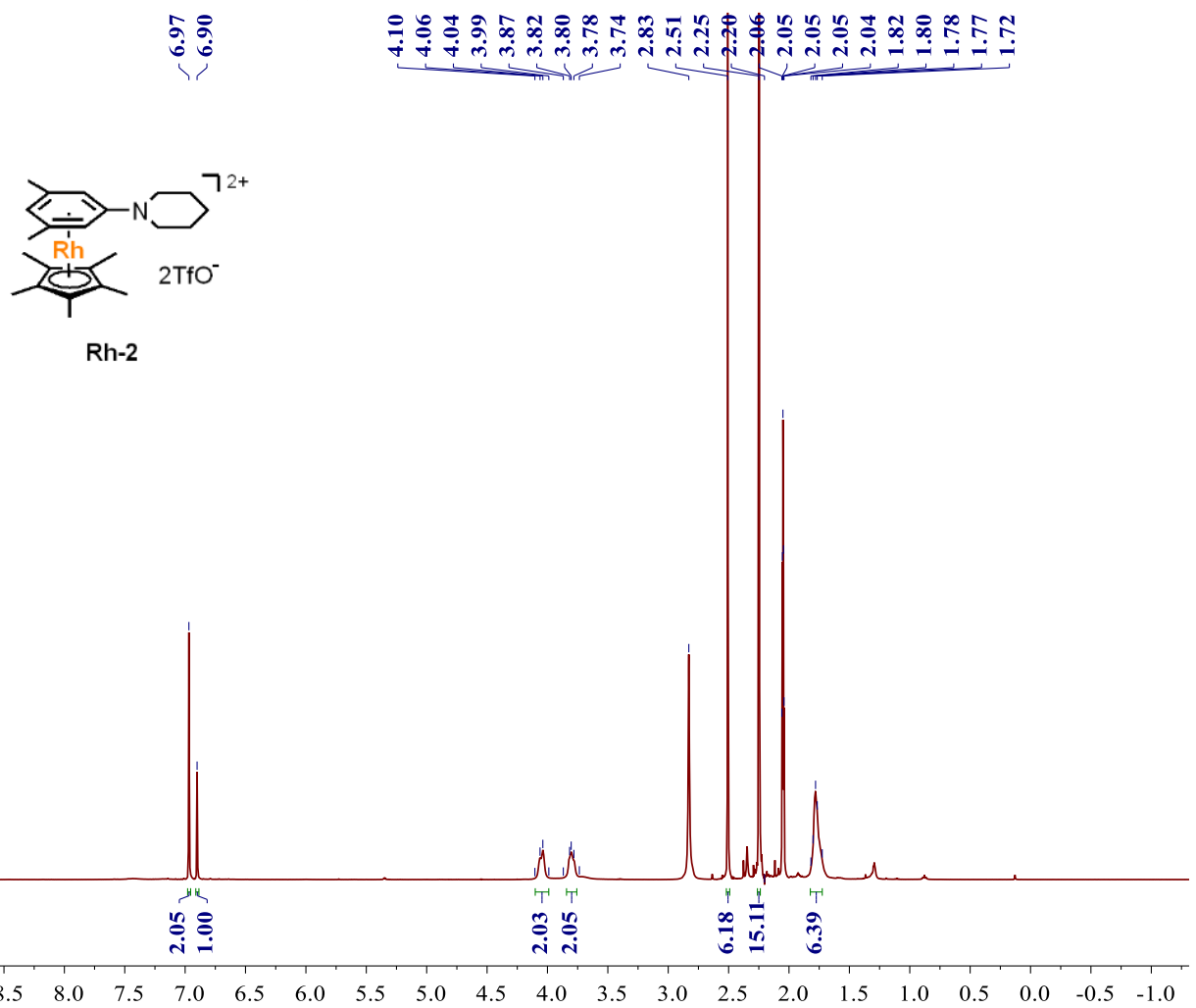


${ }^{13} \mathrm{C}$ NMR (126 MHz, Acetone- $\left.d_{6}\right)$

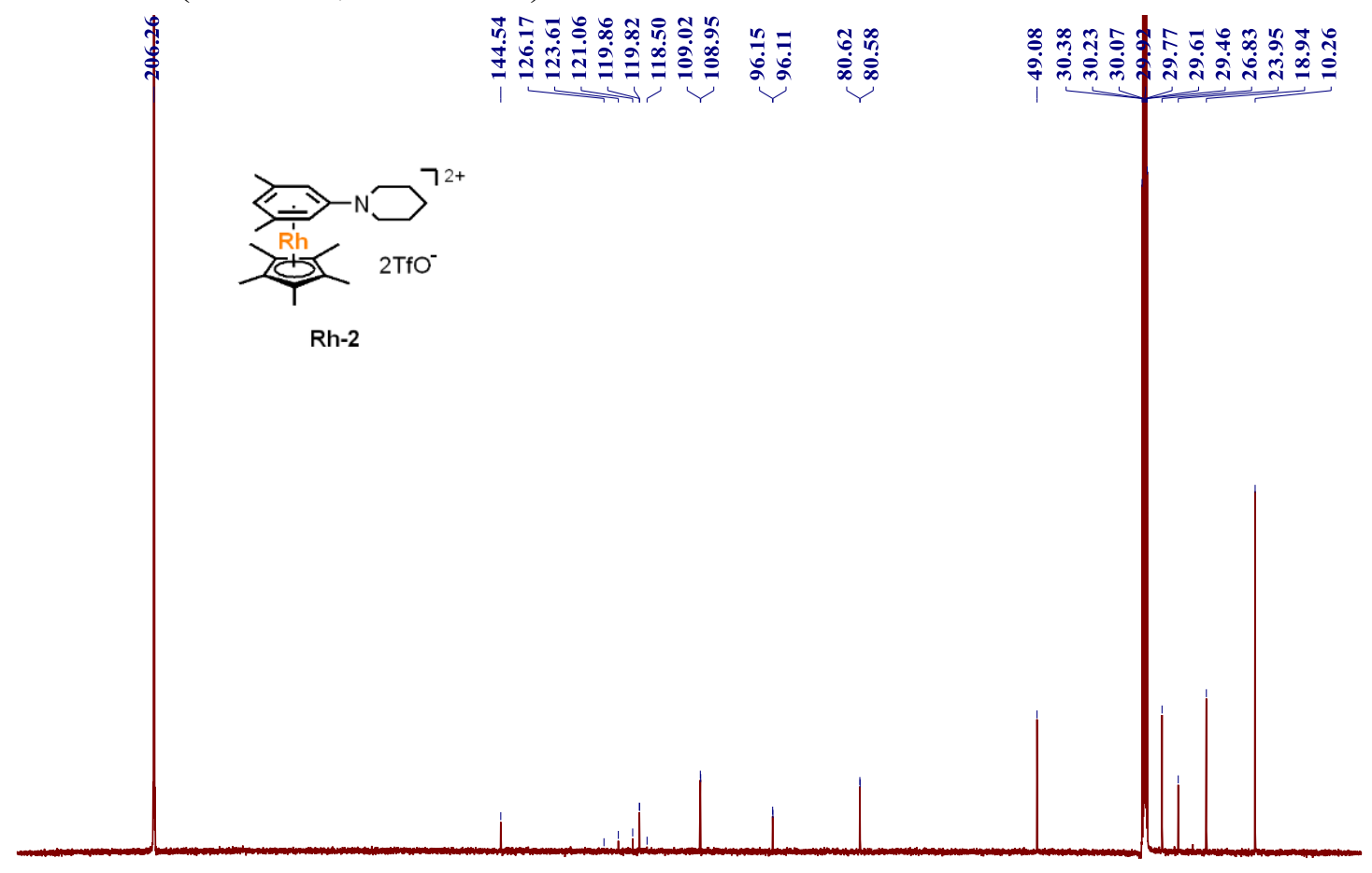

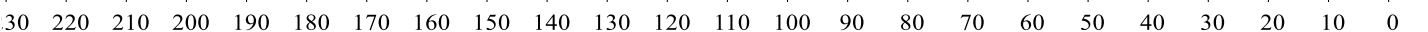

${ }^{19} \mathrm{~F}$ NMR (471 MHz, Acetone- $d_{6}$ )

\section{$\infty$
$\infty$
$\infty$
1
1}

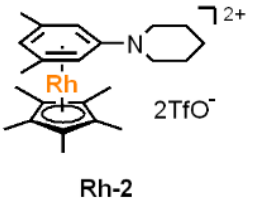

Rh-2

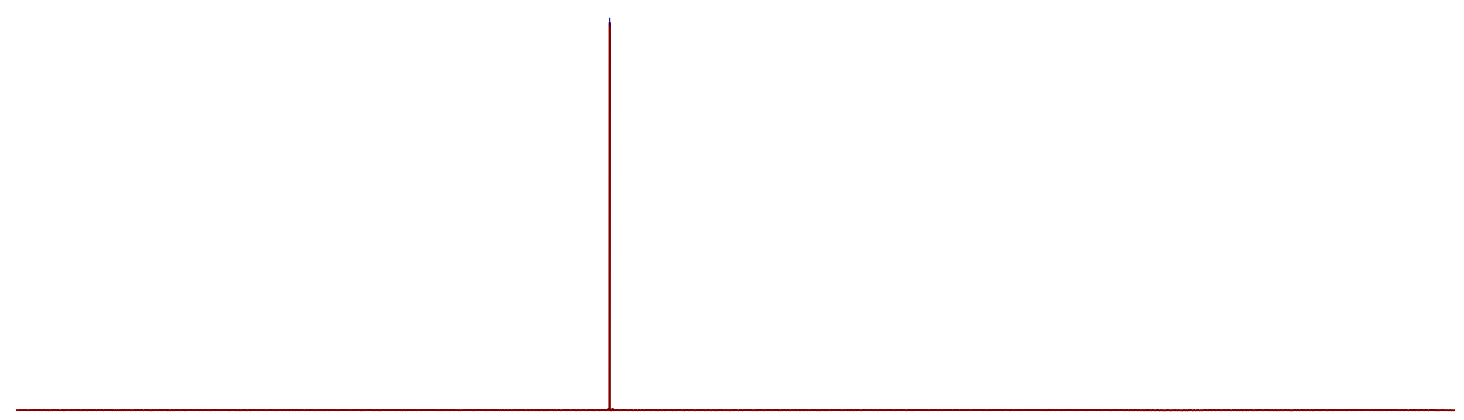

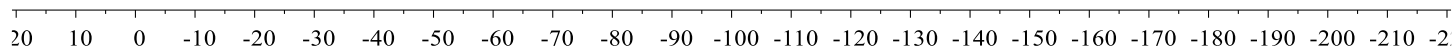


Scheme S2. Reaction of Rh Complexes

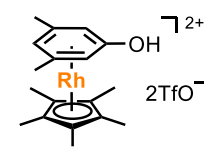

sRh-1 (0.02 mmol)

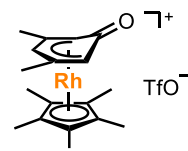

Rh-1 (0.04 mmol)

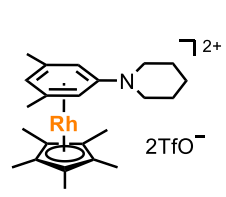

Rh-2 (0.02 mmol)

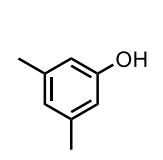

1af $(0.6 \mathrm{mmol})$

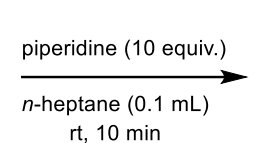

rt, $10 \mathrm{~min}$

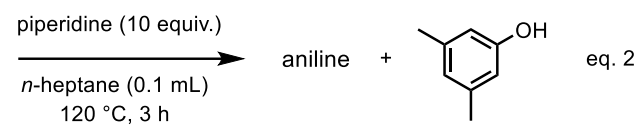

$120^{\circ} \mathrm{C}, 3 \mathrm{~h}$

3af, $55 \%$

1af, $40 \%$

eq. 1

Rh-1, 97\%

2
$\mathrm{Na}_{2} \mathrm{CO}_{3}$ (2 equiv.)

$n$-heptane $(0.1 \mathrm{~mL})$

$120^{\circ} \mathrm{C}, 2 \mathrm{~h} \quad 94 \% \quad 3$ af, $95 \%$

$\underset{\text { standard conditions }}{\stackrel{5 \mathrm{~mol} \%[\mathbf{R h}]}{\text { piperidine }(0.4 \mathrm{mmol})}}$

$\left[\mathrm{Cp}^{*} \mathrm{RhCl}_{2}\right]_{2}: 58 \%$ yield

Rh-1: $60 \%$ yield

sRh-1: $60 \%$ yield

We treated the $\eta^{6}$-phenol complex $\mathbf{s R h}-\mathbf{1}$ with piperidine at room temperature, and found that the deprotonation completed in 10 minutes, and $\eta^{5}$-phenoxo complex Rh-1 was observed in $97 \%{ }^{1} \mathrm{H}$ NMR yield by using 1,1,2,2-tetrachloroethane as the internal standard in $\mathrm{CD}_{3} \mathrm{NO}_{2}$ (eq. 1); Further heating the mixture at $120{ }^{\circ} \mathrm{C}$ for 3 hours yielded the aniline 3af (55\% ${ }^{1} \mathrm{H}$ NMR yield) together with the free phenol (eq. 2); Arene exchange of an $\eta^{6}$-aniline complex (Rh-2) with phenol 1af produced $\eta^{5}$-phenoxo complex (Rh-1) and the free aniline 3af in nearly quantitative yields (eq. 3); Moreover, we compared effects of mother $\mathrm{Rh}$ dimer $\left(\left[\mathrm{Cp}^{*} \mathrm{RhCl}_{2}\right]_{2}\right)$ with $\eta^{6}$-phenol complex (sRh-1) and $\eta^{5}$-phenoxo complex (Rh-1) in the catalytic amination of phenol 1af with piperidine. These three $\mathrm{Rh}$ complexes gave comparative results. These results support the proposed $\pi$-coordination-activation mechanism depicted as follows:

\section{Scheme S3. Proposed Mechanism}

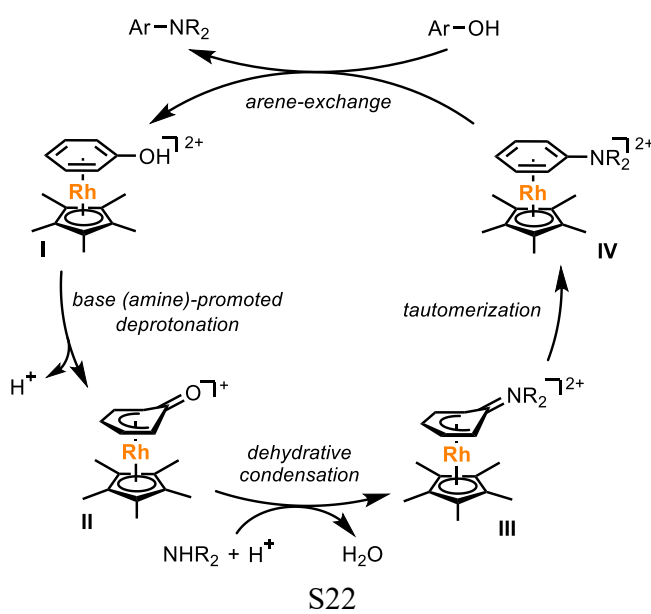




\subsection{Catalyst Poisoning Experiments}

\section{Scheme S4. Hg Poisoning Experiments ${ }^{a}$}

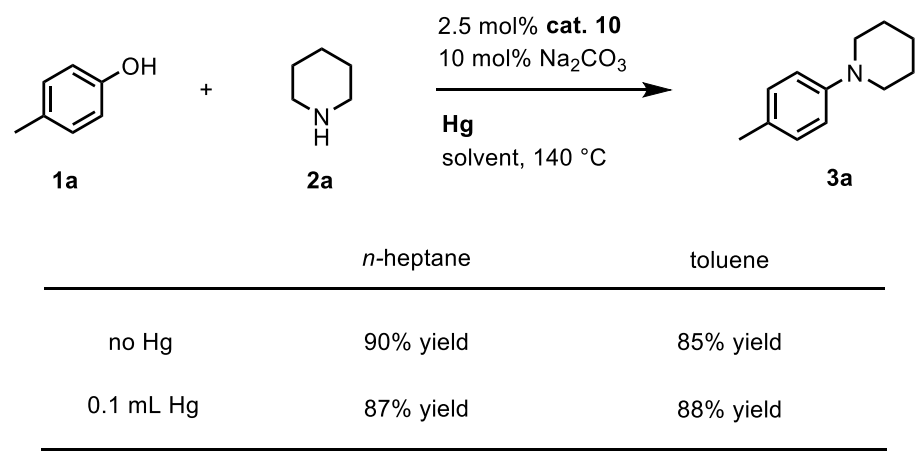

a Conditions: $1 \mathbf{a}(0.6 \mathrm{mmol}), \mathbf{2 a}(0.4 \mathrm{mmol})$, cat. $10(0.010 \mathrm{mmol}), \mathrm{Na}_{2} \mathrm{CO}_{3}(0.040 \mathrm{mmol})$, $4 \AA \mathrm{MS}(50 \mathrm{mg}), \mathrm{Hg}(\mathrm{x} \mathrm{mL})$, solvent $(0.10 \mathrm{~mL}), 140^{\circ} \mathrm{C}, \mathrm{N}_{2}, 24 \mathrm{~h}$. Yield was determined by ${ }^{1} \mathrm{H}$ NMR using $1,1,2,2$-tetrachloroethane as the internal standard.

$\mathrm{Hg}$ can poison metal-particle heterogeneous catalysts by amalgamating the metal or adsorbing on the metal surface; therefore, $\mathrm{Hg}$ could be used to suppress the heterogeneous catalysis. In the presence of $\mathrm{Hg}(0.1 \mathrm{~mL}, 337.5$ equiv. to $\mathrm{Rh}$ catalyst $)$, the amination still proceeded smoothly under the standard conditions, providing the desired aniline product in comparable yields.

\section{Scheme S5. DCT Poisoning Experiments ${ }^{a}$}

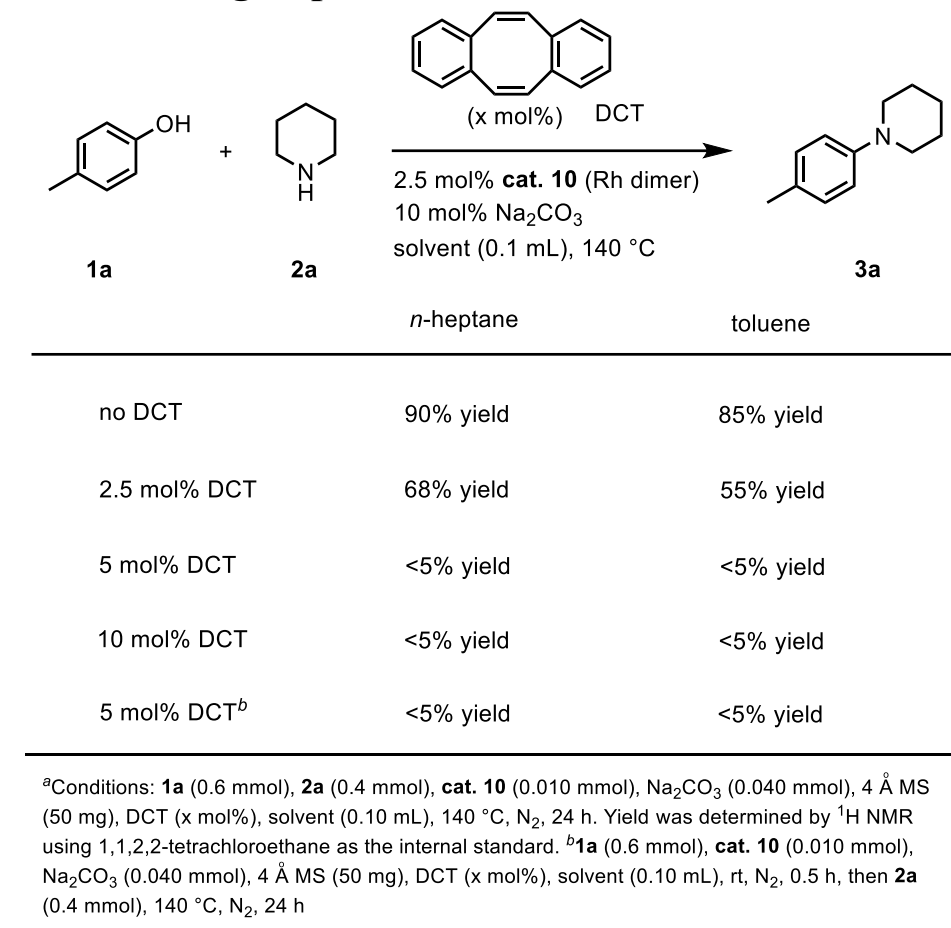

DCT (dibenzo[a,e]cyclooctatetraene) poisons at least some Group VIII homogeneous catalysts by binding strongly to metals, but has little or no effect on at least $\mathrm{Rh}$ colloids, Pd colloids and $\mathrm{Pd} / \mathrm{C}$ heterogeneous catalysts. ${ }^{3} \mathrm{We}$ found that $5 \mathrm{~mol} \% \mathrm{DCT}$ (1.0 equiv. to $\mathrm{Rh}$ ) inhibited the amination of phenols totally and only trace amount of desired product was observed in either heptane or toluene. 


\section{Characterization Data of Products}

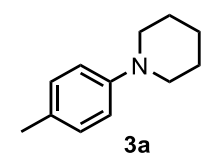

Colorless oil, $58.9 \mathrm{mg}, 84 \%$ yield, known compound ${ }^{4}$ (General condition B, 24 hours); ${ }^{1} \mathrm{H}$ NMR $\left(500 \mathrm{MHz}, \mathrm{CDCl}_{3}\right) \delta 7.10(\mathrm{~d}, J=8.3 \mathrm{~Hz}, 2 \mathrm{H}), 6.90(\mathrm{~d}, J=8.6 \mathrm{~Hz}$, $2 \mathrm{H}), 3.13(\mathrm{t}, J=5.4 \mathrm{~Hz}, 4 \mathrm{H}), 2.31(\mathrm{~s}, 3 \mathrm{H}), 1.77-1.73(\mathrm{~m}, 4 \mathrm{H}), 1.62-1.57(\mathrm{~m}, 2 \mathrm{H})$. ${ }^{13} \mathrm{C}$ NMR $\left(126 \mathrm{MHz}, \mathrm{CDCl}_{3}\right) \delta 150.3,129.5,128.7,116.9,51.3,25.9,24.3,20.4$.<smiles>c1ccc(N2CCCCC2)cc1</smiles>

Colorless oil, $50.2 \mathrm{mg}, 78 \%$ yield, known compound ${ }^{5}$ (General condition A, 36 hours); ${ }^{1} \mathrm{H}$ NMR (500 MHz, $\left.\mathrm{CDCl}_{3}\right) \delta 7.25-7.22(\mathrm{~m}, 2 \mathrm{H}), 6.93(\mathrm{~d}, J=7.9 \mathrm{~Hz}, 2 \mathrm{H})$, $6.81(\mathrm{t}, J=7.3 \mathrm{~Hz}, 1 \mathrm{H}), 3.14(\mathrm{t}, J=5.4 \mathrm{~Hz}, 4 \mathrm{H}), 1.72-1.68(\mathrm{~m}, 4 \mathrm{H}), 1.59-1.54(\mathrm{~m}$, $2 \mathrm{H}) .{ }^{13} \mathrm{C} \mathrm{NMR}\left(126 \mathrm{MHz}, \mathrm{CDCl}_{3}\right) \delta 152.2,129.0,119.2,116.5,50.7,25.8,24.3$.<smiles>CCCc1ccc(N2CCCCC2)cc1</smiles>

Colorless oil, $54.1 \mathrm{mg}, 67 \%$ yield (General condition A, 36 hours); ${ }^{1} \mathrm{H}$ NMR (500 $\left.\mathrm{MHz} \mathrm{CDCl}_{3}\right) \delta 7.09(\mathrm{~d}, J=8.5 \mathrm{~Hz}, 2 \mathrm{H}), 6.90(\mathrm{~d}, J=8.6 \mathrm{~Hz}, 2 \mathrm{H}), 3.13(\mathrm{t}, J=5.5 \mathrm{~Hz}$, 4H), 2.53 (t, $J=7.5 \mathrm{~Hz}, 2 \mathrm{H}), 1.76-1.71(\mathrm{~m}, 4 \mathrm{H}), 1.67-1.56(\mathrm{~m}, 4 \mathrm{H}), 0.96(\mathrm{t}, J=7.3$ $\mathrm{Hz}, 3 \mathrm{H}) .{ }^{13} \mathrm{C} \mathrm{NMR}\left(126 \mathrm{MHz}, \mathrm{CDCl}_{3}\right) \delta 150.4,133.6,128.9,116.7,51.1,37.1,26.0$, 24.7, 24.3, 13.8. HRMS-ESI $(\mathrm{m} / \mathrm{z})$ : $[\mathrm{M}+\mathrm{H}]^{+}$calcd. for $\left[\mathrm{C}_{14} \mathrm{H}_{22} \mathrm{~N}\right]^{+}, 204.1752$; found, 204.1750 .<smiles>CC(C)c1ccc(N2CCCCC2)cc1</smiles>

Colorless oil, $58.3 \mathrm{mg}, 72 \%$ yield, known compound ${ }^{5}$ (General condition A, 36 hours); ${ }^{1} \mathrm{H}$ NMR $\left(500 \mathrm{MHz}, \mathrm{CDCl}_{3}\right) \delta 7.15(\mathrm{~d}, J=8.6 \mathrm{~Hz}, 2 \mathrm{H}), 6.93(\mathrm{~d}, J=8.6 \mathrm{~Hz}$, $2 \mathrm{H}), 3.14(\mathrm{t}, J=5.5 \mathrm{~Hz}, 4 \mathrm{H}), 2.91-2.83(\mathrm{~m}, 1 \mathrm{H}), 1.77-1.72(\mathrm{~m}, 4 \mathrm{H}), 1.61-1.56(\mathrm{~m}$, $2 \mathrm{H}), 1.26(\mathrm{~d}, J=7.0 \mathrm{~Hz}, 6 \mathrm{H}) .{ }^{13} \mathrm{C} \mathrm{NMR}\left(126 \mathrm{MHz}, \mathrm{CDCl}_{3}\right) \delta 150.4,139.7,126.8$, 116.7, 51.1, 33.1, 26.0, 24.3, 24.1.<smiles>CC(C)(C)c1ccc(N2CCCCC2)cc1</smiles>

Colorless oil, $51.8 \mathrm{mg}, 60 \%$ yield, known compound $^{5}$ (General condition A, 36 hours); ${ }^{1} \mathrm{H}$ NMR $\left(500 \mathrm{MHz}, \mathrm{CDCl}_{3}\right) \delta 7.29(\mathrm{~d}, J=8.8 \mathrm{~Hz}, 2 \mathrm{H}), 6.91(\mathrm{~d}, J=8.8 \mathrm{~Hz}$, $2 \mathrm{H}), 3.14(\mathrm{t}, J=5.4 \mathrm{~Hz}, 4 \mathrm{H}), 1.75-1.71(\mathrm{~m}, 4 \mathrm{H}), 1.60-1.56(\mathrm{~m}, 2 \mathrm{H}), 1.32(\mathrm{~s}, 9 \mathrm{H})$. ${ }^{13} \mathrm{C}$ NMR $\left(126 \mathrm{MHz}, \mathrm{CDCl}_{3}\right) \delta 150.0,141.9,125.7,116.2,50.9,33.9,31.4,26.0$, 24.3. 


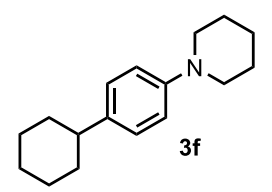

White solid, $74.7 \mathrm{mg}, 77 \%$ yield (General condition A, 36 hours); ${ }^{1} \mathrm{H}$ NMR (500 $\left.\mathrm{MHz}, \mathrm{CDCl}_{3}\right) \delta 7.13(\mathrm{~d}, J=8.6 \mathrm{~Hz}, 2 \mathrm{H}), 6.91(\mathrm{~d}, J=8.6 \mathrm{~Hz}, 2 \mathrm{H}), 3.14(\mathrm{t}, J=5.4 \mathrm{~Hz}$, $4 \mathrm{H}), 2.47-2.43(\mathrm{~m}, 1 \mathrm{H}), 1.90-1.85(\mathrm{~m}, 4 \mathrm{H}), 1.78-1.72(\mathrm{~m}, 5 \mathrm{H}), 1.61-1.56(\mathrm{~m}$, $2 \mathrm{H}), 1.46-1.37(\mathrm{~m}, 4 \mathrm{H}), 1.33-1.24(\mathrm{~m}, 1 \mathrm{H}) .{ }^{13} \mathrm{C} \mathrm{NMR}\left(126 \mathrm{MHz}, \mathrm{CDCl}_{3}\right) \delta 150.4$, 139.0, 127.2, 116.6, 51.0, 43.6, 34.6, 27.0, 26.2, 26.0, 24.3. HRMS-ESI (m/z): $[\mathrm{M}+\mathrm{H}]^{+}$calcd. for $\left[\mathrm{C}_{17} \mathrm{H}_{26} \mathrm{~N}\right]^{+}, 244.2065$; found, 244.2065 .

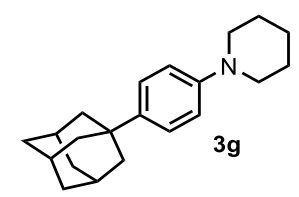

White solid, $73.4 \mathrm{mg}, 62 \%$ yield (General condition A, 36 hours); ${ }^{1} \mathrm{H}$ NMR (500 $\left.\mathrm{MHz}, \mathrm{CDCl}_{3}\right) \delta 7.23(\mathrm{~d}, J=8.8 \mathrm{~Hz}, 2 \mathrm{H}), 6.89(\mathrm{~d}, J=8.8 \mathrm{~Hz}, 2 \mathrm{H}), 3.11(\mathrm{t}, J=5.4 \mathrm{~Hz}$, $4 \mathrm{H}), 2.09-2.03(\mathrm{~m}, 3 \mathrm{H}), 1.91-1.86(\mathrm{~m}, 6 \mathrm{H}), 1.78-1.67(\mathrm{~m}, 10 \mathrm{H}), 1.57-1.52(\mathrm{~m}$, $2 \mathrm{H}) .{ }^{13} \mathrm{C} \mathrm{NMR}\left(126 \mathrm{MHz}, \mathrm{CDCl}_{3}\right) \delta 150.0,142.2,125.3,116.2,50.8,43.3,36.8,35.4$, 29.0, 26.0, 24.3. HRMS-ESI $(\mathrm{m} / \mathrm{z}):[\mathrm{M}+\mathrm{H}]^{+}$calcd. for $\left[\mathrm{C}_{21} \mathrm{H}_{30} \mathrm{~N}\right]^{+}, 296.2378$; found, 296.2379.<smiles>c1ccc(N2CCCC2)cc1</smiles>

Colorless oil, $84.6 \mathrm{mg}, 84 \%$ yield, known compound ${ }^{6}$ (General condition A, 36 hours); ${ }^{1} \mathrm{H}$ NMR $\left(500 \mathrm{MHz}, \mathrm{CDCl}_{3}\right) \delta 7.33-7.30(\mathrm{~m}, 2 \mathrm{H}), 7.26-7.21(\mathrm{~m}, 3 \mathrm{H}), 7.12$ $(\mathrm{d}, J=8.5 \mathrm{~Hz}, 2 \mathrm{H}), 6.92(\mathrm{~d}, J=8.6 \mathrm{~Hz}, 2 \mathrm{H}), 3.95(\mathrm{~s}, 2 \mathrm{H}), 3.15(\mathrm{t}, J=5.4 \mathrm{~Hz}, 4 \mathrm{H})$, $1.77-1.72(\mathrm{~m}, 4 \mathrm{H}), 1.63-1.60(\mathrm{~m}, 2 \mathrm{H}) .{ }^{13} \mathrm{C} \mathrm{NMR}\left(126 \mathrm{MHz}, \mathrm{CDCl}_{3}\right) \delta 150.6,141.8$, $131.8,129.4,128.8,128.3,125.8,116.7,50.9,41.0,25.9,24.3$.<smiles>CC(C)(c1ccccc1)c1ccc(N2CCCC2)cc1</smiles>

Colorless oil, $85.3 \mathrm{mg}, 76 \%$ yield (General condition B, $5 \mathrm{~mol} \%$ Cat. 10, $10 \mathrm{~mol} \%$ $\mathrm{Cs}_{2} \mathrm{CO}_{3}$ instead of $\mathrm{Na}_{2} \mathrm{CO}_{3}, 36$ hours); ${ }^{1} \mathrm{H}$ NMR $\left(500 \mathrm{MHz}, \mathrm{CDCl}_{3}\right) \delta 7.32-7.29(\mathrm{~m}$, 4H), $7.22-7.19(\mathrm{~m}, 1 \mathrm{H}), 7.17$ (d, $J=8.8 \mathrm{~Hz}, 2 \mathrm{H}), 6.90$ (d, $J=8.8 \mathrm{~Hz}, 2 \mathrm{H}), 3.17$ (t, $J$ $=5.4 \mathrm{~Hz}, 4 \mathrm{H}), 1.77-1.73(\mathrm{~m}, 4 \mathrm{H}), 1.71(\mathrm{~s}, 6 \mathrm{H}), 1.63-1.59(\mathrm{~m}, 2 \mathrm{H}) .{ }^{13} \mathrm{C} \mathrm{NMR}(126$ $\left.\mathrm{MHz}, \mathrm{CDCl}_{3}\right) \delta 151.1,149.9,141.2,127.8,127.3,126.7,125.4,115.9,50.6,42.1$, 30.8, 25.9, 24.3. HRMS-ESI $(\mathrm{m} / \mathrm{z}):[\mathrm{M}+\mathrm{H}]^{+}$calcd. for $\left[\mathrm{C}_{20} \mathrm{H}_{26} \mathrm{~N}\right]^{+}, 280.2065$; found, 280.2067 .

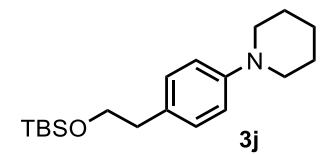

Light yellow oil, $88.1 \mathrm{mg}, 69 \%$ yield (General condition A, 36 hours); ${ }^{1} \mathrm{H}$ NMR (500 $\left.\mathrm{MHz} \mathrm{CDCl}_{3}\right) \delta 7.10(\mathrm{~d}, J=8.5 \mathrm{~Hz}, 2 \mathrm{H}), 6.88(\mathrm{~d}, J=8.6 \mathrm{~Hz}, 2 \mathrm{H}), 3.78(\mathrm{t}, J=7.4 \mathrm{~Hz}$, 2H), $3.12(\mathrm{t}, J=5.4 \mathrm{~Hz}, 4 \mathrm{H})), 2.76(\mathrm{t}, J=7.4 \mathrm{~Hz}, 2 \mathrm{H}), 1.74-1.70(\mathrm{~m}, 4 \mathrm{H}), 1.60-$ 
$1.55(\mathrm{~m}, 2 \mathrm{H}), 0.90(\mathrm{~s}, 9 \mathrm{H}), 0.02(\mathrm{~s}, 6 \mathrm{H}) .{ }^{13} \mathrm{C} \mathrm{NMR}\left(126 \mathrm{MHz}, \mathrm{CDCl}_{3}\right) \delta 150.8,129.8$, $129.6,116.7,64.9,51.1,38.7,25.9,25.9,24.3,18.3,-5.4$. HRMS-ESI $(\mathrm{m} / \mathrm{z}):[\mathrm{M}+\mathrm{H}]^{+}$ calcd. for $\left[\mathrm{C}_{19} \mathrm{H}_{34} \mathrm{NOSi}\right]^{+}, 320.2410$; found, 320.2411 .

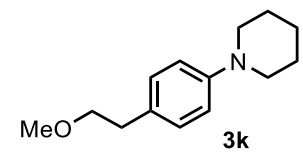

Light yellow oil, $75.0 \mathrm{mg}, 86 \%$ yield (General condition A, 36 hours); ${ }^{1} \mathrm{H}$ NMR (500 $\left.\mathrm{MHz}, \mathrm{CDCl}_{3}\right) \delta 7.12(\mathrm{~d}, J=8.6 \mathrm{~Hz}, 2 \mathrm{H}), 6.89(\mathrm{~d}, J=8.6 \mathrm{~Hz}, 2 \mathrm{H}), 3.58(\mathrm{t}, J=7.3 \mathrm{~Hz}$, $2 \mathrm{H}), 3.37(\mathrm{~s}, 3 \mathrm{H}), 3.12(\mathrm{t}, J=5.4 \mathrm{~Hz}, 4 \mathrm{H}), 2.82(\mathrm{t}, J=7.3 \mathrm{~Hz}, 2 \mathrm{H}), 1.74-1.70(\mathrm{~m}$, $4 \mathrm{H}), 1.60-1.55(\mathrm{~m}, 2 \mathrm{H}) .{ }^{13} \mathrm{C}$ NMR $\left(126 \mathrm{MHz}, \mathrm{CDCl}_{3}\right) \delta 150.8,129.4,129.3,116.7$, 73.9, 58.5, 50.9, 35.2, 25.9, 24.2. HRMS-ESI (m/z): $[\mathrm{M}+\mathrm{H}]^{+}$calcd. for $\left[\mathrm{C}_{14} \mathrm{H}_{22} \mathrm{NO}\right]^{+}$, 220.1701; found, 220.1699 .

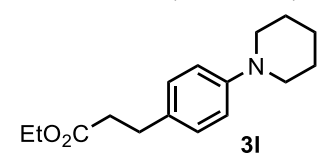

Pink oil, $46.8 \mathrm{mg}, 45 \%$ yield (General condition A, $5 \mathrm{~mol} \%$ Cat. 10, 36 hours); ${ }^{1} \mathrm{H}$ NMR $\left(500 \mathrm{MHz}, \mathrm{CDCl}_{3}\right) \delta 7.08(\mathrm{~d}, J=8.6 \mathrm{~Hz}, 2 \mathrm{H}), 6.87(\mathrm{~d}, J=8.6 \mathrm{~Hz}, 2 \mathrm{H}), 4.12(\mathrm{q}$, $J=7.1 \mathrm{~Hz}, 2 \mathrm{H}), 3.11(\mathrm{t}, J=5.4 \mathrm{~Hz}, 4 \mathrm{H}), 2.87(\mathrm{t}, J=7.9 \mathrm{~Hz}, 2 \mathrm{H}), 2.58(\mathrm{t}, J=8.2 \mathrm{~Hz}$, $2 \mathrm{H}), 1.73-1.68(\mathrm{~m}, 4 \mathrm{H}), 1.58-1.54(\mathrm{~m}, 2 \mathrm{H}), 1.24(\mathrm{t}, J=7.1 \mathrm{~Hz}, 3 \mathrm{H}) .{ }^{13} \mathrm{C} \mathrm{NMR}$ $\left(126 \mathrm{MHz} \mathrm{CDCl}_{3}\right) \delta 173.1,150.7,131.2,128.8,116.7,60.3,50.9,36.2,30.1,25.9$, 24.2, 14.2. HRMS-ESI $(\mathrm{m} / \mathrm{z})$ : $[\mathrm{M}+\mathrm{H}]^{+}$calcd. for $\left[\mathrm{C}_{16} \mathrm{H}_{24} \mathrm{NO}_{2}\right]^{+}, 262.1807$; found, 262.1805 .<smiles>c1cc(N2CCCCC2)ccc1N1CCCCC1</smiles>

White solid, $38.8 \mathrm{mg}, 79 \%$ yield, prepared from 4-methoxyphenol with piperidine, known compound ${ }^{7}$ (General condition A, 36 hours); ${ }^{1} \mathrm{H}$ NMR $\left(500 \mathrm{MHz}, \mathrm{CDCl}_{3}\right) \delta$ $6.90(\mathrm{~s}, 4 \mathrm{H}), 3.05-3.02(\mathrm{~m}, 8 \mathrm{H}), 1.74-1.69(\mathrm{~m}, 8 \mathrm{H}), 1.56-1.53(\mathrm{~m}, 4 \mathrm{H}) .{ }^{13} \mathrm{C} \mathrm{NMR}$ $\left(126 \mathrm{MHz}, \mathrm{CDCl}_{3}\right) \delta 146.3,118.1,51.9,26.1,24.2$.<smiles>CC(C)Oc1ccc(N2CCCC2)cc1</smiles>

Colorless oil, $41.0 \mathrm{mg}, 47 \%$ yield (General condition A, 36 hours); ${ }^{1} \mathrm{H}$ NMR (500 $\left.\mathrm{MHz} \mathrm{CDCl}_{3}\right) \delta 6.89(\mathrm{~d}, J=9.0 \mathrm{~Hz}, 2 \mathrm{H}), 6.82(\mathrm{~d}, J=9.1 \mathrm{~Hz}, 2 \mathrm{H}), 4.46-4.39(\mathrm{~m}, 1 \mathrm{H})$, $3.03(\mathrm{t}, J=5.4 \mathrm{~Hz}, 4 \mathrm{H}), 1.74-1.70(\mathrm{~m}, 4 \mathrm{H}), 1.56-1.52(\mathrm{~m}, 2 \mathrm{H}), 1.30(\mathrm{~d}, J=6.1 \mathrm{~Hz}$, $6 \mathrm{H}) .{ }^{13} \mathrm{C} \mathrm{NMR}\left(126 \mathrm{MHz}, \mathrm{CDCl}_{3}\right) \delta 151.7,146.9,118.6,116.9,70.6,52.2,26.1,24.2$, 22.2. HRMS-ESI (m/z): $[\mathrm{M}+\mathrm{H}]^{+}$calcd. for $\left[\mathrm{C}_{14} \mathrm{H}_{22} \mathrm{NO}\right]^{+}, 220.1701$; found, 220.1699 .<smiles>CN(C)c1ccc(N2CCCC2)cc1</smiles>

Light yellow solid, $48.1 \mathrm{mg}, 59 \%$ yield, known compound $^{8}$ (General condition A, 36 hours); ${ }^{1} \mathrm{H}$ NMR $\left(500 \mathrm{MHz}, \mathrm{CDCl}_{3}\right) \delta 6.92(\mathrm{~d}, J=9.0 \mathrm{~Hz}, 2 \mathrm{H}), 6.75(\mathrm{~d}, J=9.0 \mathrm{~Hz}$, $2 \mathrm{H}), 3.00(\mathrm{t}, J=5.4 \mathrm{~Hz}, 4 \mathrm{H}), 2.86(\mathrm{~s}, 6 \mathrm{H}), 1.74-1.70(\mathrm{~m}, 4 \mathrm{H}), 1.56-1.51(\mathrm{~m}, 2 \mathrm{H})$. 
${ }^{13} \mathrm{C} \mathrm{NMR}\left(126 \mathrm{MHz}, \mathrm{CDCl}_{3}\right) \delta 145.5,144.6,118.9,114.5,52.5,41.6,26.2,24.2$.<smiles>CCCNc1ccc(N2CCCCC2)cc1</smiles>

Brown solid, $63.4 \mathrm{mg}, 63 \%$ yield, known compound ${ }^{9}$ (General condition A, 5 mol\% Cat. 10, 36 hours); ${ }^{1} \mathrm{H}$ NMR (500 MHz, $\left.\mathrm{CDCl}_{3}\right) \delta 7.22$ (t, $\left.J=7.9 \mathrm{~Hz}, 2 \mathrm{H}\right), 7.06$ (d, $J$ $=7.6 \mathrm{~Hz}, 2 \mathrm{H}), 6.98-6.88(\mathrm{~m}, 4 \mathrm{H}), 6.83(\mathrm{t}, J=7.1 \mathrm{~Hz}, 1 \mathrm{H}), 5.49(\mathrm{br}, 1 \mathrm{H}), 3.11(\mathrm{~s}$, $4 \mathrm{H}), 1.77-1.73(\mathrm{~m}, 4 \mathrm{H}), 1.61-1.56(\mathrm{~m}, 2 \mathrm{H}) .{ }^{13} \mathrm{C} \mathrm{NMR}\left(126 \mathrm{MHz}, \mathrm{CDCl}_{3}\right) \delta 148.1$, $145.2,134.8,129.2,121.7,119.3,118.0,115.6,51.5,26.0,24.2$.

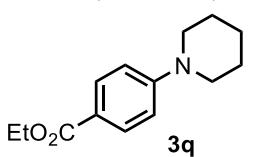

White solid, $59.6 \mathrm{mg}, 64 \%$ yield, known compound ${ }^{10}$ (General condition A, 36 hours); ${ }^{1} \mathrm{H}$ NMR $\left(500 \mathrm{MHz}, \mathrm{CDCl}_{3}\right) \delta 7.90(\mathrm{~d}, J=9.0 \mathrm{~Hz}, 2 \mathrm{H}), 6.84(\mathrm{~d}, J=9.0 \mathrm{~Hz}$, $2 \mathrm{H}), 4.32(\mathrm{q}, J=7.1 \mathrm{~Hz}, 2 \mathrm{H}), 3.31(\mathrm{q}, J=4.8 \mathrm{~Hz}, 4 \mathrm{H}), 1.68-1.61(\mathrm{~m}, 6 \mathrm{H}), 1.36(\mathrm{t}, J$ $=7.1 \mathrm{~Hz}, 3 \mathrm{H}) .{ }^{13} \mathrm{C} \mathrm{NMR}\left(126 \mathrm{MHz}, \mathrm{CDCl}_{3}\right) \delta 166.7,154.4,131.1,119.0,113.5,60.1$, $48.8,25.3,24.3,14.4$.<smiles>CC(C)CNC(=O)c1ccc(N2CCCCC2)cc1</smiles>

White solid, $41.3 \mathrm{mg}, 40 \%$ yield (General condition A, 36 hours); ${ }^{1} \mathrm{H}$ NMR (500 $\left.\mathrm{MHz}, \mathrm{CDCl}_{3}\right) \delta 7.66(\mathrm{~d}, J=8.9 \mathrm{~Hz}, 2 \mathrm{H}), 6.86(\mathrm{~d}, J=8.9 \mathrm{~Hz}, 2 \mathrm{H}), 6.14(\mathrm{~s}, 1 \mathrm{H}), 3.27-$ $3.23(\mathrm{~m}, 6 \mathrm{H}), 1.91-1.83(\mathrm{~m}, 1 \mathrm{H}), 1.69-1.65(\mathrm{~m}, 4 \mathrm{H}), 1.62-1.59(\mathrm{~m}, 2 \mathrm{H}), 0.95(\mathrm{~d}$, $J=6.7 \mathrm{~Hz}, 6 \mathrm{H}) .{ }^{13} \mathrm{C} \mathrm{NMR}\left(126 \mathrm{MHz}, \mathrm{CDCl}_{3}\right) \delta 167.3,153.7,128.2,123.7,114.3$, 49.2, 47.1, 28.7, 25.4, 24.3, 20.2. HRMS-ESI (m/z): $[\mathrm{M}+\mathrm{H}]^{+}$calcd. for $\left[\mathrm{C}_{16} \mathrm{H}_{25} \mathrm{~N}_{2} \mathrm{O}\right]^{+}$, 261.1967; found, 261.1964 .

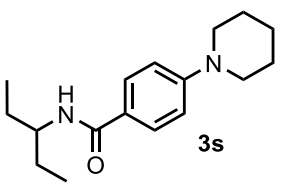

White solid, $42.3 \mathrm{mg}, 39 \%$ yield (General condition A, 36 hours); ${ }^{1} \mathrm{H}$ NMR (500 $\left.\mathrm{MHz}, \mathrm{CDCl}_{3}\right) \delta 7.66(\mathrm{~d}, J=8.8 \mathrm{~Hz}, 2 \mathrm{H}), 6.88(\mathrm{~d}, J=8.9 \mathrm{~Hz}, 2 \mathrm{H}), 5.69(\mathrm{~d}, J=8.4 \mathrm{~Hz}$, $1 \mathrm{H}), 4.03-3.96(\mathrm{~m}, 1 \mathrm{H}), 3.28-3.26(\mathrm{~m}, 4 \mathrm{H}), 1.70-1.61(\mathrm{~m}, 8 \mathrm{H}), 1.51-1.42(\mathrm{~m}$, 2H), $0.94(\mathrm{t}, J=7.4 \mathrm{~Hz}, 6 \mathrm{H}) .{ }^{13} \mathrm{C}$ NMR $\left(126 \mathrm{MHz} \mathrm{CDCl}_{3}\right) \delta 167.0,153.7,128.1$, 123.9, 114.4, 52.1, 49.3, 27.6, 25.4, 24.3, 10.3. HRMS-ESI $(\mathrm{m} / \mathrm{z}):[\mathrm{M}+\mathrm{H}]^{+}$calcd. for $\left[\mathrm{C}_{17} \mathrm{H}_{27} \mathrm{~N}_{2} \mathrm{O}\right]^{+}, 275.2123$; found, 275.2117.

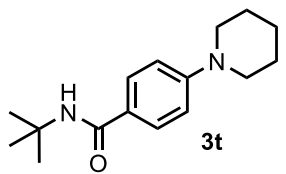

White solid, $62.3 \mathrm{mg}, 60 \%$ yield (General condition A, 36 hours); ${ }^{1} \mathrm{H}$ NMR (500 $\left.\mathrm{MHz} \mathrm{CDCl}_{3}\right) \delta 7.61(\mathrm{~d}, J=8.9 \mathrm{~Hz}, 2 \mathrm{H}), 6.85(\mathrm{~d}, J=8.9 \mathrm{~Hz}, 2 \mathrm{H}), 5.85(\mathrm{~s}, 1 \mathrm{H}), 3.24(\mathrm{t}$, $J=5.4 \mathrm{~Hz}, 4 \mathrm{H}), 1.69-1.65(\mathrm{~m}, 4 \mathrm{H}), 1.61-1.57(\mathrm{~m}, 2 \mathrm{H}), 1.44(\mathrm{~s}, 9 \mathrm{H}) .{ }^{13} \mathrm{C}$ NMR 
$\left(126 \mathrm{MHz}, \mathrm{CDCl}_{3}\right) \delta 166.6,153.6,128.0,124.8,114.4,51.2,49.3,28.9,25.4,24.2$. HRMS-ESI (m/z): $[\mathrm{M}+\mathrm{H}]^{+}$calcd. for $\left[\mathrm{C}_{16} \mathrm{H}_{25} \mathrm{~N}_{2} \mathrm{O}\right]^{+}, 261.1967$; found, 261.1964.<smiles>c1ccc(N2CCCCC2)cc1</smiles>

White solid, $85.4 \mathrm{mg}$, 90\% yield, known compound ${ }^{11}$ (General condition A, 36 hours); ${ }^{1} \mathrm{H}$ NMR $\left(500 \mathrm{MHz}, \mathrm{CDCl}_{3}\right) \delta 7.63(\mathrm{~d}, J=7.0 \mathrm{~Hz}, 2 \mathrm{H}), 7.56(\mathrm{~d}, J=8.8 \mathrm{~Hz}$, $2 \mathrm{H}), 7.46(\mathrm{t}, J=7.8 \mathrm{~Hz}, 2 \mathrm{H}), 7.33(\mathrm{t}, J=7.4 \mathrm{~Hz}, 1 \mathrm{H}), 7.05(\mathrm{~d}, J=8.8 \mathrm{~Hz}, 2 \mathrm{H}), 3.26(\mathrm{t}$ $J=5.4 \mathrm{~Hz}, 4 \mathrm{H}), 1.80-1.76(\mathrm{~m}, 4 \mathrm{H}), 1.67-1.63(\mathrm{~m}, 2 \mathrm{H}) .{ }^{13} \mathrm{C}$ NMR $(126 \mathrm{MHz}$, $\left.\mathrm{CDCl}_{3}\right) \delta 151.4,141.0,131.5,128.6,127.6,126.4,126.2,116.4,50.3,25.7,24.3$.

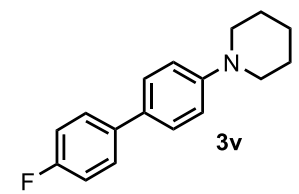

White solid, $68.5 \mathrm{mg}, 67 \%$ yield (General condition A, 36 hours); ${ }^{1} \mathrm{H}$ NMR (500 $\left.\mathrm{MHz}, \mathrm{CDCl}_{3}\right) \delta 7.53-7.50(\mathrm{~m}, 2 \mathrm{H}), 7.46(\mathrm{~d}, J=8.8 \mathrm{~Hz}, 2 \mathrm{H}), 7.10(\mathrm{t}, J=8.7 \mathrm{~Hz}, 2 \mathrm{H})$, $7.01(\mathrm{~d}, J=8.8 \mathrm{~Hz}, 2 \mathrm{H}), 3.23(\mathrm{t}, J=5.4 \mathrm{~Hz}, 4 \mathrm{H}), 1.77-1.73(\mathrm{~m}, 4 \mathrm{H}), 1.65-1.60(\mathrm{~m}$, 2H). ${ }^{13} \mathrm{C}$ NMR $\left(126 \mathrm{MHz}, \mathrm{CDCl}_{3}\right) \delta 161.9(\mathrm{~d}, J=245.4 \mathrm{~Hz}), 151.4,137.1(\mathrm{~d}, J=3.1$ $\mathrm{Hz}), 130.6,127.8$ (d, $J=7.9 \mathrm{~Hz}), 127.5,116.4,115.4$ (d, $J=21.3 \mathrm{~Hz}), 50.3,25.7,24.3$. ${ }^{19} \mathrm{~F}$ NMR (471 MHz, $\left.\mathrm{CDCl}_{3}\right) \delta$-117.2. HRMS-ESI $(\mathrm{m} / \mathrm{z}):[\mathrm{M}+\mathrm{H}]^{+}$calcd. for $\left[\mathrm{C}_{17} \mathrm{H}_{19} \mathrm{NF}\right]^{+}, 256.1501$; found, 256.1500 .

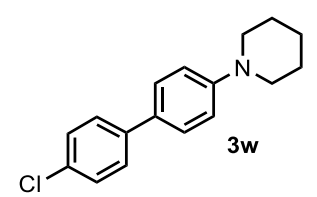

White solid, $69.8 \mathrm{mg}, 64 \%$ yield (General condition A, 36 hours); ${ }^{1} \mathrm{H}$ NMR (500 $\left.\mathrm{MHz}, \mathrm{CDCl}_{3}\right) \delta 7.49-7.44(\mathrm{~m}, 4 \mathrm{H}), 7.39-7.33(\mathrm{~m}, 2 \mathrm{H}), 7.00(\mathrm{~d}, J=8.4 \mathrm{~Hz}, 2 \mathrm{H})$, $3.23-3.21(\mathrm{~m}, 4 \mathrm{H}), 1.77-1.70(\mathrm{~m}, 4 \mathrm{H}), 1.63-1.58(\mathrm{~m}, 2 \mathrm{H}) .{ }^{13} \mathrm{C}$ NMR $(126 \mathrm{MHz}$, $\left.\mathrm{CDCl}_{3}\right) \delta 151.5,139.4,132.2,130.2,128.7,127.6,127.5,116.4,50.3,25.7,24.3$. HRMS-ESI (m/z): $[\mathrm{M}+\mathrm{H}]^{+}$calcd. for $\left[\mathrm{C}_{17} \mathrm{H}_{19} \mathrm{NCl}\right]^{+}, 272.1206$; found, 272.1202 .

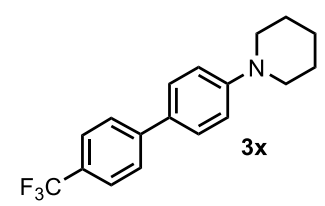

White solid, $85.4 \mathrm{mg}, 70 \%$ yield (General condition A, 36 hours); ${ }^{1} \mathrm{H}$ NMR (500 $\left.\mathrm{MHz} \mathrm{CDCl}_{3}\right) \delta 7.66(\mathrm{~s}, 4 \mathrm{H}), 7.52(\mathrm{~d}, J=8.5 \mathrm{~Hz}, 2 \mathrm{H}), 7.02(\mathrm{~d}, J=8.4 \mathrm{~Hz}, 2 \mathrm{H}), 3.26(\mathrm{t}$, $J=5.4 \mathrm{~Hz}, 4 \mathrm{H}), 1.81-1.69(\mathrm{~m}, 4 \mathrm{H}), 1.67-1.56(\mathrm{~m}, 2 \mathrm{H}) .{ }^{13} \mathrm{C}$ NMR $(126 \mathrm{MHz}$, $\left.\mathrm{CDCl}_{3}\right) \delta 151.9,144.5(\mathrm{q}, J=1.2 \mathrm{~Hz}), 129.5,128.1(\mathrm{q}, J=32.4 \mathrm{~Hz}), 127.8,126.4$, $125.6(\mathrm{q}, J=3.8 \mathrm{~Hz}), 124.5(\mathrm{q}, J=272.1 \mathrm{~Hz}), 116.2,50.0,25.6,24.3 .{ }^{19} \mathrm{~F}$ NMR $(471$ $\left.\mathrm{MHz}, \mathrm{CDCl}_{3}\right) \delta-62.2$. HRMS-ESI $(\mathrm{m} / \mathrm{z}):[\mathrm{M}+\mathrm{H}]^{+}$calcd. for $\left[\mathrm{C}_{18} \mathrm{H}_{19} \mathrm{NF}_{3}\right]^{+}, 306.1470$; found, 306.1464 .

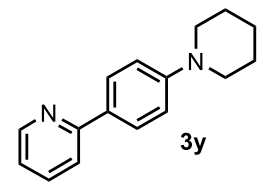


White solid, $41.5 \mathrm{mg}$, 44\% yield (General condition B, $5 \mathrm{~mol} \%$ Cat. 10, $10 \mathrm{~mol} \%$ $\mathrm{Cs}_{2} \mathrm{CO}_{3}$ instead of $\mathrm{Na}_{2} \mathrm{CO}_{3}, 36$ hours); ${ }^{1} \mathrm{H}$ NMR $\left(500 \mathrm{MHz}, \mathrm{CDCl}_{3}\right) \delta 8.63(\mathrm{~d}, J=4.7$ $\mathrm{Hz}, 1 \mathrm{H}), 7.91(\mathrm{~d}, J=8.9 \mathrm{~Hz}, 2 \mathrm{H}), 7.68-7.64(\mathrm{~m}, 2 \mathrm{H}), 7.12-7.10(\mathrm{~m}, 1 \mathrm{H}), 7.00(\mathrm{~d}, J$ $=8.9 \mathrm{~Hz}, 2 \mathrm{H}), 3.26(\mathrm{t}, J=5.4 \mathrm{~Hz}, 4 \mathrm{H}), 1.74-1.69(\mathrm{~m}, 4 \mathrm{H}), 1.63-1.58(\mathrm{~m}, 2 \mathrm{H}) .{ }^{13} \mathrm{C}$ NMR $\left(126 \mathrm{MHz}, \mathrm{CDCl}_{3}\right) \delta 157.3,152.5,149.4,136.4,129.4,127.6,120.8,119.3$, 115.7, 49.9, 25.6, 24.3. HRMS-ESI (m/z): $[\mathrm{M}+\mathrm{H}]^{+}$calcd. for $\left[\mathrm{C}_{16} \mathrm{H}_{19} \mathrm{~N}_{2}\right]^{+}, 239.1548$; found, 239.1549 .

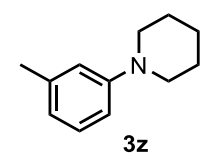

Colorless oil, $61.9 \mathrm{mg}, 88 \%$ yield, known compound $^{5}$ (General condition A, 36 hours); ${ }^{1} \mathrm{H}$ NMR $\left(500 \mathrm{MHz}, \mathrm{CDCl}_{3}\right) \delta 7.18(\mathrm{t}, J=7.7 \mathrm{~Hz}, 1 \mathrm{H}), 6.81-6.79(\mathrm{~m}, 2 \mathrm{H})$, $6.69(\mathrm{~d}, J=7.4 \mathrm{~Hz}, 1 \mathrm{H}), 3.18(\mathrm{t}, J=5.4 \mathrm{~Hz}, 4 \mathrm{H}), 2.36(\mathrm{~s}, 3 \mathrm{H}), 1.77-1.72(\mathrm{~m}, 4 \mathrm{H})$, $1.63-1.59(\mathrm{~m}, 2 \mathrm{H}) .{ }^{13} \mathrm{C} \mathrm{NMR}\left(126 \mathrm{MHz}, \mathrm{CDCl}_{3}\right) \delta 152.3,138.5,128.8,120.1,117.4$, $113.7,50.8,25.9,24.3,21.7$.<smiles>CC(C)c1cccc(N2CCCCC2)c1</smiles>

Colorless oil, $58.6 \mathrm{mg}, 72 \%$ yield (General condition A, 36 hours); ${ }^{1} \mathrm{H}$ NMR (500 $\left.\mathrm{MHz}, \mathrm{CDCl}_{3}\right) \delta 7.21(\mathrm{t}, J=7.8 \mathrm{~Hz}, 1 \mathrm{H}), 6.86(\mathrm{~s}, 1 \mathrm{H}), 6.79(\mathrm{dd}, J=8.1,2.0 \mathrm{~Hz}, 1 \mathrm{H})$, $6.75(\mathrm{~d}, J=7.5 \mathrm{~Hz}, 1 \mathrm{H}), 3.18(\mathrm{t}, J=5.4 \mathrm{~Hz}, 4 \mathrm{H}), 2.92-2.84(\mathrm{~m}, 1 \mathrm{H}), 1.77-1.73(\mathrm{~m}$, 4H), $1.62-1.58(\mathrm{~m}, 2 \mathrm{H}), 1.28(\mathrm{~d}, J=7.0 \mathrm{~Hz}, 6 \mathrm{H}) \cdot{ }^{13} \mathrm{C} \mathrm{NMR}\left(126 \mathrm{MHz}, \mathrm{CDCl}_{3}\right) \delta$ $152.4,149.7,128.8,117.5,115.2,113.9,50.9,34.4,26.0,24.3,24.0$. HRMS-ESI $(\mathrm{m} / \mathrm{z}):[\mathrm{M}+\mathrm{H}]^{+}$calcd. for $\left[\mathrm{C}_{14} \mathrm{H}_{22} \mathrm{~N}\right]^{+}, 204.1752$; found, 204.1752 .

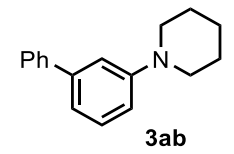

Colorless oil, $83.0 \mathrm{mg}, 87 \%$ yield, known compound ${ }^{12}$ (General condition A, 36 hours); ${ }^{1} \mathrm{H}$ NMR $\left(500 \mathrm{MHz}, \mathrm{CDCl}_{3}\right) \delta 7.67-7.65(\mathrm{~m}, 2 \mathrm{H}), 7.49(\mathrm{t}, J=7.6 \mathrm{~Hz}, 2 \mathrm{H})$, $7.41-7.37(\mathrm{~m}, 2 \mathrm{H}), 7.23(\mathrm{t}, J=2.0 \mathrm{~Hz}, 1 \mathrm{H}), 7.12(\mathrm{~d}, J=7.6 \mathrm{~Hz}, 1 \mathrm{H}), 7.00(\mathrm{dd}, J=$ 8.2, $2.4 \mathrm{~Hz}, 1 \mathrm{H}), 3.28(\mathrm{t}, J=5.5 \mathrm{~Hz}, 4 \mathrm{H}), 1.82-1.77(\mathrm{~m}, 4 \mathrm{H}), 1.68-1.63(\mathrm{~m}, 2 \mathrm{H})$. ${ }^{13} \mathrm{C}$ NMR $\left(126 \mathrm{MHz}, \mathrm{CDCl}_{3}\right) \delta 152.6,142.1,141.9,129.3,128.6,127.2,127.0,118.2$, $115.5,115.4,50.7,25.8,24.3$.

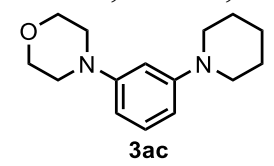

Colorless oil, $62.9 \mathrm{mg}$, 64\% yield (General condition A, 36 hours); ${ }^{1} \mathrm{H}$ NMR (500 $\left.\mathrm{MHz}, \mathrm{CDCl}_{3}\right) \delta 7.15(\mathrm{t}, J=8.4 \mathrm{~Hz}, 1 \mathrm{H}), 6.52-6.50(\mathrm{~m}, 2 \mathrm{H}), 6.44-6.42(\mathrm{~m}, 1 \mathrm{H})$, $3.85(\mathrm{t}, J=4.8 \mathrm{~Hz}, 4 \mathrm{H}), 3.16-3.13(\mathrm{~m}, 8 \mathrm{H}), 1.73-1.69(\mathrm{~m}, 4 \mathrm{H}), 1.60-1.55(\mathrm{~m}, 2 \mathrm{H})$. ${ }^{13} \mathrm{C}$ NMR (126 MHz, $\left.\mathrm{CDCl}_{3}\right) \delta 153.4,152.3,129.5,109.1,107.5,104.9,67.0,51.0$, 49.7, 25.9, 24.3. HRMS-ESI (m/z): $[\mathrm{M}+\mathrm{H}]^{+}$calcd. for $\left[\mathrm{C}_{15} \mathrm{H}_{23} \mathrm{~N}_{2} \mathrm{O}\right]^{+}, 247.1810$; found, 247.1805 . 


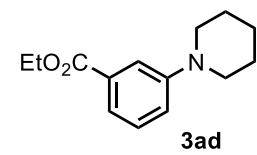

Pink oil, $39.3 \mathrm{mg}, 42 \%$ yield (General condition B, $5 \mathrm{~mol} \%$ Cat. 10, 36 hours); ${ }^{1} \mathrm{H}$ NMR $\left(500 \mathrm{MHz}, \mathrm{CDCl}_{3}\right) \delta 7.60(\mathrm{~s}, 1 \mathrm{H}), 7.48(\mathrm{~d}, J=7.6 \mathrm{~Hz}, 1 \mathrm{H}), 7.29(\mathrm{t}, J=7.9 \mathrm{~Hz}$, $1 \mathrm{H}), 7.11(\mathrm{dd}, J=8.1,2.2 \mathrm{~Hz}, 1 \mathrm{H}), 4.36(\mathrm{q}, J=7.1 \mathrm{~Hz}, 2 \mathrm{H}), 3.20(\mathrm{t}, J=5.4 \mathrm{~Hz}, 4 \mathrm{H})$, $1.74-1.69(\mathrm{~m}, 4 \mathrm{H}), 1.61-1.57(\mathrm{~m}, 2 \mathrm{H}), 1.38(\mathrm{t}, J=7.1 \mathrm{~Hz}, 3 \mathrm{H}) .{ }^{13} \mathrm{C}$ NMR $(126$ $\left.\mathrm{MHz}, \mathrm{CDCl}_{3}\right) \delta 167.1,152.1,131.2,128.9,120.7,120.0,117.1,60.8,50.4,25.7,24.2$, 14.3. HRMS-ESI (m/z): $[\mathrm{M}+\mathrm{H}]^{+}$calcd. for $\left[\mathrm{C}_{14} \mathrm{H}_{20} \mathrm{NO}_{2}\right]^{+}, 234.1494$; found, 234.1496.<smiles>CC(C)(C)NC(=O)c1cccc(N2CCCC2)c1</smiles>

White solid, $60.8 \mathrm{mg}, 58 \%$ yield (General condition A, 36 hours); ${ }^{1} \mathrm{H}$ NMR (500 $\left.\mathrm{MHz}, \mathrm{CDCl}_{3}\right) \delta 7.36(\mathrm{~s}, 1 \mathrm{H}), 7.23(\mathrm{t}, J=7.9 \mathrm{~Hz}, 1 \mathrm{H}), 7.02-6.99(\mathrm{~m}, 2 \mathrm{H}), 5.94(\mathrm{~s}$, $1 \mathrm{H}), 3.19(\mathrm{t}, J=5.4 \mathrm{~Hz}, 4 \mathrm{H}), 1.71-1.66(\mathrm{~m}, 4 \mathrm{H}), 1.60-1.55(\mathrm{~m}, 2 \mathrm{H}), 1.45(\mathrm{~s}, 9 \mathrm{H})$. ${ }^{13} \mathrm{C}$ NMR $\left(126 \mathrm{MHz}, \mathrm{CDCl}_{3}\right) \delta 167.5,152.3,136.8,128.9,118.8,116.3,115.0,51.4$, 50.3, 28.8, 25.6, 24.2. HRMS-ESI (m/z): $[\mathrm{M}+\mathrm{H}]^{+}$calcd. for $\left[\mathrm{C}_{16} \mathrm{H}_{25} \mathrm{~N}_{2} \mathrm{O}\right]^{+}, 261.1967$; found, 261.1966 .<smiles>Cc1cc(C)cc(N2CCCCC2)c1</smiles>

Colorless oil, $57.6 \mathrm{mg}, 76 \%$ yield, known compound ${ }^{5}$ (General condition A, 36 hours); ${ }^{1} \mathrm{H} \mathrm{NMR}\left(500 \mathrm{MHz}, \mathrm{CDCl}_{3}\right) \delta 6.61(\mathrm{~s}, 2 \mathrm{H}), 6.52(\mathrm{~s}, 1 \mathrm{H}), 3.15(\mathrm{t}, J=5.5 \mathrm{~Hz}$, $4 \mathrm{H}), 2.30(\mathrm{~s}, 6 \mathrm{H}), 1.75-1.70(\mathrm{~m}, 4 \mathrm{H}), 1.61-1.57(\mathrm{~m}, 2 \mathrm{H}) .{ }^{13} \mathrm{C}$ NMR $(126 \mathrm{MHz}$, $\left.\mathrm{CDCl}_{3}\right) \delta 152.4,138.4,121.2,114.6,50.9,25.9,24.4,21.6$.

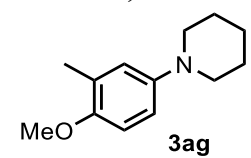

Colorless oil, $50.8 \mathrm{mg}$, 62\% yield (General condition A, 5 mol\% Cat. 10, 36 hours); ${ }^{1} \mathrm{H}$ NMR $\left(500 \mathrm{MHz}, \mathrm{CDCl}_{3}\right) \delta 6.83(\mathrm{~s}, 1 \mathrm{H}), 6.78-6.73(\mathrm{~m}, 2 \mathrm{H}), 3.78(\mathrm{~s}, 3 \mathrm{H}), 3.02(\mathrm{t}$, $J=5.4 \mathrm{~Hz}, 4 \mathrm{H}), 2.21(\mathrm{~s}, 3 \mathrm{H}), 1.75-1.70(\mathrm{~m}, 4 \mathrm{H}), 1.57-1.52(\mathrm{~m}, 2 \mathrm{H}) .{ }^{13} \mathrm{C} \mathrm{NMR}$ $\left(126 \mathrm{MHz}, \mathrm{CDCl}_{3}\right) \delta 152.0,146.5,127.0,121.2,115.2,110.7,55.7,52.4,26.2,24.2$, 16.5. HRMS-ESI (m/z): $[\mathrm{M}+\mathrm{H}]^{+}$calcd. for $\left[\mathrm{C}_{13} \mathrm{H}_{20} \mathrm{NO}\right]^{+}, 206.1545$; found, 206.1542.<smiles>Cc1cc(N2CCCCC2)ccc1N</smiles>

Gray solid, $40.8 \mathrm{mg}, 54 \%$ yield, known compound ${ }^{13}$ (General condition A, 5 mol\% Cat. 10, 36 hours); ${ }^{1} \mathrm{H}$ NMR $\left(500 \mathrm{MHz}, \mathrm{CDCl}_{3}\right) \delta 6.75-6.70(\mathrm{~m}, 2 \mathrm{H}), 6.61(\mathrm{~d}, J=$ $8.4 \mathrm{~Hz}, 1 \mathrm{H}), 3.30(\mathrm{br}, 2 \mathrm{H}), 2.98(\mathrm{t}, J=5.3 \mathrm{~Hz}, 4 \mathrm{H}), 2.16(\mathrm{~s}, 3 \mathrm{H}), 1.74-1.70(\mathrm{~m}, 4 \mathrm{H})$, $1.55-1.51(\mathrm{~m}, 2 \mathrm{H}) .{ }^{13} \mathrm{C}$ NMR $\left(126 \mathrm{MHz}, \mathrm{CDCl}_{3}\right) \delta 145.7,138.2,123.3,120.8,116.7$, $115.8,52.7,26.2,24.2,17.7$.

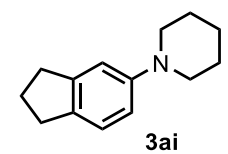


Colorless oil, $69.3 \mathrm{mg}, 86 \%$ yield, known compound ${ }^{14}$ (General condition A, 36 hours); ${ }^{1} \mathrm{H}$ NMR $\left(500 \mathrm{MHz}, \mathrm{CDCl}_{3}\right) \delta 7.15(\mathrm{~d}, J=8.2 \mathrm{~Hz}, 1 \mathrm{H}), 6.91(\mathrm{~s}, 1 \mathrm{H}), 6.81(\mathrm{dd}$, $J=8.2,2.2 \mathrm{~Hz}, 1 \mathrm{H}), 3.14(\mathrm{t}, J=5.4 \mathrm{~Hz}, 4 \mathrm{H}), 2.93-2.86(\mathrm{~m}, 4 \mathrm{H}), 2.13-2.07(\mathrm{~m}$, $2 \mathrm{H}), 1.79-1.74(\mathrm{~m}, 4 \mathrm{H}), 1.63-1.58(\mathrm{~m}, 2 \mathrm{H}) .{ }^{13} \mathrm{C} \mathrm{NMR}\left(126 \mathrm{MHz}, \mathrm{CDCl}_{3}\right) \delta 151.5$, $145.0,135.3,124.4,115.4,113.3,51.8,33.2,32.0,26.0,25.6,24.3$.

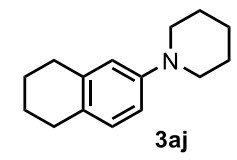

Colorless oil, $68.9 \mathrm{mg}, 80 \%$ yield, known compound ${ }^{14}$ (General condition A, 36 hours); ${ }^{1} \mathrm{H}$ NMR $\left(500 \mathrm{MHz}, \mathrm{CDCl}_{3}\right) \delta 6.99(\mathrm{~d}, J=8.3 \mathrm{~Hz}, 1 \mathrm{H}), 6.78(\mathrm{dd}, J=8.3,2.6$ $\mathrm{Hz}, 1 \mathrm{H}), 6.70(\mathrm{~d}, J=2.3 \mathrm{~Hz}, 1 \mathrm{H}), 3.12(\mathrm{t}, J=5.4 \mathrm{~Hz}, 4 \mathrm{H}), 2.77-2.73(\mathrm{~m}, 4 \mathrm{H}), 1.83-$ $1.80(\mathrm{~m}, 4 \mathrm{H}), 1.77-1.73(\mathrm{~m}, 4 \mathrm{H}), 1.62-1.57(\mathrm{~m}, 2 \mathrm{H}) .{ }^{13} \mathrm{C} \mathrm{NMR}\left(126 \mathrm{MHz}, \mathrm{CDCl}_{3}\right)$ $\delta 150.3,137.4,129.5,128.3,117.2,115.0,51.3,29.8,28.5,26.0,24.3,23.5,23.3$.<smiles>c1cc2c(cc1N1CCCCC1)OCCO2</smiles>

Colorless oil, $61.7 \mathrm{mg}, 70 \%$ yield (General condition A, 36 hours); ${ }^{1} \mathrm{H}$ NMR (500 $\left.\mathrm{MHz} \mathrm{CDCl}_{3}\right) \delta 6.77-6.75(\mathrm{~m}, 1 \mathrm{H}), 6.50-6.47(\mathrm{~m}, 2 \mathrm{H}), 4.24-4.19(\mathrm{~m}, 4 \mathrm{H}), 3.01(\mathrm{t}$, $J=5.5 \mathrm{~Hz}, 4 \mathrm{H}), 1.72-1.68(\mathrm{~m}, 4 \mathrm{H}), 1.56-1.51(\mathrm{~m}, 2 \mathrm{H}) .{ }^{13} \mathrm{C} \mathrm{NMR}(126 \mathrm{MHz}$, $\left.\mathrm{CDCl}_{3}\right) \delta 147.6,143.5,137.0,117.2,111.0,106.0,64.6,64.2,51.8,25.9,24.2$. HRMS-ESI (m/z): $[\mathrm{M}+\mathrm{H}]^{+}$calcd. for $\left[\mathrm{C}_{13} \mathrm{H}_{18} \mathrm{NO}_{2}\right]^{+}, 220.1338$; found, 220.1334 .

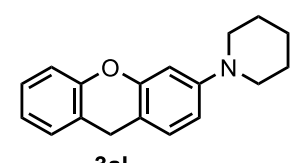

3al

Yellow oil, $95.3 \mathrm{mg}, 90 \%$ yield (General condition A, 36 hours); ${ }^{1} \mathrm{H}$ NMR (500 MHz, $\left.\mathrm{CDCl}_{3}\right) \delta 7.22-7.17(\mathrm{~m}, 2 \mathrm{H}), 7.07-7.02(\mathrm{~m}, 3 \mathrm{H}), 6.70-6.66(\mathrm{~m}, 2 \mathrm{H}), 3.99(\mathrm{~s}, 2 \mathrm{H})$, $3.18(\mathrm{t}, J=5.5 \mathrm{~Hz}, 4 \mathrm{H}), 1.76-1.72(\mathrm{~m}, 4 \mathrm{H}), 1.63-1.59(\mathrm{~m}, 2 \mathrm{H}) .{ }^{13} \mathrm{C}$ NMR $(126$ $\left.\mathrm{MHz}, \mathrm{CDCl}_{3}\right) \delta 152.3,152.0,152.0,129.0,129.0,127.4,122.6,121.0,116.3,111.8$, $110.6,103.8, \quad 50.6,27.0,25.7,24.3$. HRMS-ESI $(\mathrm{m} / \mathrm{z}):[\mathrm{M}+\mathrm{H}]^{+}$calcd. for $\left[\mathrm{C}_{18} \mathrm{H}_{20} \mathrm{NO}\right]^{+}, 266.1545$; found, 266.1541.<smiles>c1cc2c(cc1N1CCCCC1)CCCN2</smiles>

Colorless oil, $48.6 \mathrm{mg}, 56 \%$ yield (General condition A, 36 hours); ${ }^{1} \mathrm{H}$ NMR (500 $\left.\mathrm{MHz} \mathrm{CDCl}_{3}\right) \delta 6.69-6.64(\mathrm{~m}, 2 \mathrm{H}), 6.45(\mathrm{~d}, J=8.5 \mathrm{~Hz}, 1 \mathrm{H}), 3.52(\mathrm{br}, 1 \mathrm{H}), 3.25(\mathrm{t}, J$ $=4.8 \mathrm{~Hz}, 2 \mathrm{H}), 2.96(\mathrm{t}, J=4.8 \mathrm{~Hz}, 4 \mathrm{H}), 2.75(\mathrm{t}, J=6.2 \mathrm{~Hz}, 2 \mathrm{H}), 1.95-1.91(\mathrm{~m}, 2 \mathrm{H})$, $1.73-1.69(\mathrm{~m}, 4 \mathrm{H}), 1.55-1.50(\mathrm{~m}, 2 \mathrm{H}) .{ }^{13} \mathrm{C} \mathrm{NMR}\left(126 \mathrm{MHz}, \mathrm{CDCl}_{3}\right) \delta 144.8,138.9$, 122.3, 119.6, 117.3, 115.3, 52.8, 42.3, 27.2, 26.2, 24.2, 22.5. HRMS-ESI (m/z): $[\mathrm{M}+\mathrm{H}]^{+}$calcd. for $\left[\mathrm{C}_{14} \mathrm{H}_{21} \mathrm{~N}_{2}\right]^{+}, 217.1705$; found, 217.1697 .<smiles>c1cc2c(cc1N1CCCCC1)NCCC2</smiles> 
Colorless oil, $70.5 \mathrm{mg}, 82 \%$ yield (General condition A, 36 hours); ${ }^{1} \mathrm{H}$ NMR (500 $\left.\mathrm{MHz}, \mathrm{CDCl}_{3}\right) \delta 6.84(\mathrm{~d}, J=8.2 \mathrm{~Hz}, 1 \mathrm{H}), 6.30(\mathrm{dd}, J=8.2,2.4 \mathrm{~Hz}, 1 \mathrm{H}), 6.09(\mathrm{~d}, J=$ $2.3 \mathrm{~Hz}, 1 \mathrm{H}), 3.57$ (br, 1H), $3.28(\mathrm{t}, J=5.5 \mathrm{~Hz}, 2 \mathrm{H}), 3.07$ (t, $J=5.4 \mathrm{~Hz}, 4 \mathrm{H}), 2.70$ (t, $J$ $=6.4 \mathrm{~Hz}, 2 \mathrm{H}), 1.95-1.91(\mathrm{~m}, 2 \mathrm{H}), 1.72-1.68(\mathrm{~m}, 4 \mathrm{H}), 1.58-1.53(\mathrm{~m}, 2 \mathrm{H}) .{ }^{13} \mathrm{C}$ NMR (126 MHz, $\left.\mathrm{CDCl}_{3}\right) \delta 151.7,145.1,129.8,113.2,106.9,102.5,51.2,42.0,26.1$, 25.9, 24.3, 22.6. HRMS-ESI (m/z): $[\mathrm{M}+\mathrm{H}]^{+}$calcd. for $\left[\mathrm{C}_{14} \mathrm{H}_{21} \mathrm{~N}_{2}\right]^{+}, 217.1705$; found, 217.1700 .

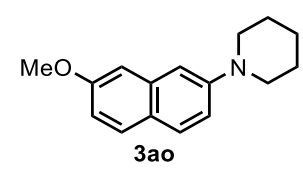

White solid, $33.1 \mathrm{mg}$, 34\% yield, known compound ${ }^{15}$ (General condition B, $10 \mathrm{~mol} \%$ $\mathrm{Cs}_{2} \mathrm{CO}_{3}$ instead of $\mathrm{Na}_{2} \mathrm{CO}_{3}, 36$ hours); ${ }^{1} \mathrm{H} \mathrm{NMR}\left(500 \mathrm{MHz}, \mathrm{CDCl}_{3}\right) \delta 7.63$ (d, $J=9.0$ $\mathrm{Hz}, 1 \mathrm{H}), 7.60(\mathrm{~d}, J=8.8 \mathrm{~Hz}, 1 \mathrm{H}), 7.13(\mathrm{dd}, J=8.9,2.3 \mathrm{~Hz}, 1 \mathrm{H}), 7.06(\mathrm{~d}, J=2.0 \mathrm{~Hz}$, $1 \mathrm{H}), 7.02(\mathrm{~d}, J=2.1 \mathrm{~Hz}, 1 \mathrm{H}), 6.95(\mathrm{dd}, J=8.8,2.4 \mathrm{~Hz}, 1 \mathrm{H}), 3.91(\mathrm{~s}, 3 \mathrm{H}), 3.26(\mathrm{t}, J=$ $5.4 \mathrm{~Hz}, 4 \mathrm{H}), 1.79-1.75(\mathrm{~m}, 4 \mathrm{H}), 1.65-1.61(\mathrm{~m}, 2 \mathrm{H}) .{ }^{13} \mathrm{C} \mathrm{NMR}\left(126 \mathrm{MHz}, \mathrm{CDCl}_{3}\right) \delta$ $158.0,150.6,135.9,128.9,128.2,123.6,117.6,115.5,109.5,105.1,55.2,50.9,25.9$, 24.4 .<smiles>CCNc1ccc(C)cc1</smiles>

Colorless oil, $92.8 \mathrm{mg}, 84 \%$ yield, known compound ${ }^{16}$ (General condition B, 36 hours); ${ }^{1} \mathrm{H}$ NMR $\left(500 \mathrm{MHz}, \mathrm{CDCl}_{3}\right) \delta 6.99(\mathrm{~d}, J=8.0 \mathrm{~Hz}, 2 \mathrm{H}), 6.55(\mathrm{~d}, J=8.4 \mathrm{~Hz}$, $2 \mathrm{H}), 3.08(\mathrm{t}, J=7.2 \mathrm{~Hz}, 2 \mathrm{H}), 2.24(\mathrm{~s}, 3 \mathrm{H}), 1.64-1.58(\mathrm{~m}, 2 \mathrm{H}), 1.42-1.27(\mathrm{~m}, 18 \mathrm{H})$, $0.89(\mathrm{t}, J=7.0 \mathrm{~Hz}, 3 \mathrm{H}) .{ }^{13} \mathrm{C} \mathrm{NMR}\left(126 \mathrm{MHz}, \mathrm{CDCl}_{3}\right) \delta 146.1,129.7,126.5,113.0$, $44.5,31.9,29.7,29.6,29.6,29.6,29.6,29.4,29.3,27.2,22.7,20.4,14.1$.

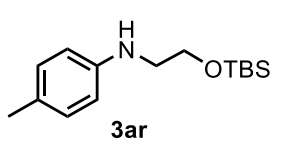

Colorless oil, $71.5 \mathrm{mg}, 67 \%$ yield (General condition B, 36 hours); ${ }^{1} \mathrm{H}$ NMR (500 $\left.\mathrm{MHz}, \mathrm{CDCl}_{3}\right) \delta 7.01(\mathrm{~d}, J=8.3 \mathrm{~Hz}, 2 \mathrm{H}), 6.59(\mathrm{~d}, J=8.3 \mathrm{~Hz}, 2 \mathrm{H}), 3.94$ (br, 1H), 3.83 (t, $J=5.4 \mathrm{~Hz}, 2 \mathrm{H}), 3.22(\mathrm{t}, J=5.4 \mathrm{~Hz}, 2 \mathrm{H}), 2.27(\mathrm{~s}, 3 \mathrm{H}), 0.94(\mathrm{~s}, 9 \mathrm{H}), 0.09(\mathrm{~s}, 6 \mathrm{H})$. ${ }^{13} \mathrm{C}$ NMR $\left(126 \mathrm{MHz}, \mathrm{CDCl}_{3}\right) \delta 146.1,129.7,126.7,113.4,61.6,46.4,25.9,20.4,18.3$, -5.4. HRMS-ESI $(\mathrm{m} / \mathrm{z}):[\mathrm{M}+\mathrm{H}]^{+}$calcd. for $\left[\mathrm{C}_{15} \mathrm{H}_{28} \mathrm{NOSi}\right]^{+}, 266.1940$; found, 266.1935 .<smiles>COCCCNc1ccc(C)cc1</smiles>

Yellow oil, $37.1 \mathrm{mg}, 52 \%$ yield, known compound ${ }^{17}$ (General condition B, 36 hours); ${ }^{1} \mathrm{H}$ NMR $\left(500 \mathrm{MHz}, \mathrm{CDCl}_{3}\right) \delta 7.01(\mathrm{~d}, J=8.1 \mathrm{~Hz}, 2 \mathrm{H}), 6.56(\mathrm{~d}, J=8.2 \mathrm{~Hz}, 2 \mathrm{H}), 3.71$ (br, 1H), 3.52 (t, $J=5.9 \mathrm{~Hz}, 2 \mathrm{H}), 3.37(\mathrm{~s}, 3 \mathrm{H}), 3.22(\mathrm{t}, J=6.6 \mathrm{~Hz}, 2 \mathrm{H}), 2.26(\mathrm{~s}, 3 \mathrm{H})$, $1.92-1.87(\mathrm{~m}, 2 \mathrm{H}) .{ }^{13} \mathrm{C} \mathrm{NMR}\left(126 \mathrm{MHz}, \mathrm{CDCl}_{3}\right) \delta 146.2,129.6,126.3,112.9,71.2$, 58.7, 42.0, 29.4, 20.3. 
<smiles>CCCCNc1ccc(C)cc1</smiles>

Yellow oil, $73.7 \mathrm{mg}, 89 \%$ yield (General condition B, 36 hours); ${ }^{1} \mathrm{H}$ NMR (500 MHz, $\left.\mathrm{CDCl}_{3}\right) \delta 7.00(\mathrm{~d}, J=8.1 \mathrm{~Hz}, 2 \mathrm{H}), 6.58(\mathrm{~d}, J=8.4 \mathrm{~Hz}, 2 \mathrm{H}), 4.18(\mathrm{br}, 1 \mathrm{H}), 3.14(\mathrm{t}, J=$ $6.0 \mathrm{~Hz}, 2 \mathrm{H}), 2.71(\mathrm{t}, J=6.0 \mathrm{~Hz}, 2 \mathrm{H}), 2.58(\mathrm{q}, J=7.1 \mathrm{~Hz}, 4 \mathrm{H}), 2.25(\mathrm{~s}, 3 \mathrm{H}), 1.04(\mathrm{t}, J$ $=7.2 \mathrm{~Hz}, 6 \mathrm{H}) .{ }^{13} \mathrm{C} \mathrm{NMR}\left(126 \mathrm{MHz}, \mathrm{CDCl}_{3}\right) \delta 146.4,129.6,126.3,113.2,51.6,46.7$, 41.6, 20.3, 11.6. HRMS-ESI (m/z): $[\mathrm{M}+\mathrm{H}]^{+}$calcd. for $\left[\mathrm{C}_{13} \mathrm{H}_{23} \mathrm{~N}_{2}\right]^{+}, 207.1861$; found, 207.1859 .

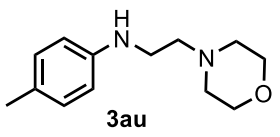

Yellow oil, $77.1 \mathrm{mg}, 88 \%$ yield (General condition B, 36 hours); ${ }^{1} \mathrm{H}$ NMR (500 MHz, $\left.\mathrm{CDCl}_{3}\right) \delta 7.00(\mathrm{~d}, J=8.2 \mathrm{~Hz}, 2 \mathrm{H}), 6.58(\mathrm{~d}, J=8.3 \mathrm{~Hz}, 2 \mathrm{H}), 4.14(\mathrm{br}, 1 \mathrm{H}), 3.72(\mathrm{t}, J=$ $4.8 \mathrm{~Hz}, 4 \mathrm{H}), 3.15(\mathrm{t}, J=5.8 \mathrm{~Hz}, 2 \mathrm{H}), 2.63(\mathrm{t}, J=5.8 \mathrm{~Hz}, 2 \mathrm{H}), 2.52-2.44(\mathrm{~m}, 4 \mathrm{H})$, $2.24(\mathrm{~s}, 3 \mathrm{H}) .{ }^{13} \mathrm{C}$ NMR $\left(126 \mathrm{MHz}, \mathrm{CDCl}_{3}\right) \delta 146.2,129.7,126.6,113.1,67.0,57.2$, 53.3, 40.4, 20.4. HRMS-ESI $(\mathrm{m} / \mathrm{z}):[\mathrm{M}+\mathrm{H}]^{+}$calcd. for $\left[\mathrm{C}_{13} \mathrm{H}_{21} \mathrm{~N}_{2} \mathrm{O}\right]^{+}, 221.1654$; found, 221.1651 .<smiles>CCCCNCCNc1ccc(C)cc1</smiles>

Light yellow oil, $56.2 \mathrm{mg}$, 73\% yield, known compound ${ }^{18}$ (General condition B, 36 hours); ${ }^{1} \mathrm{H}$ NMR $\left(500 \mathrm{MHz}, \mathrm{CDCl}_{3}\right) \delta 6.99(\mathrm{~d}, J=8.2 \mathrm{~Hz}, 2 \mathrm{H}), 6.54(\mathrm{~d}, J=8.3 \mathrm{~Hz}$, 2H), $5.98(\mathrm{~s}, 1 \mathrm{H}), 3.47(\mathrm{q}, J=5.8 \mathrm{~Hz}, 2 \mathrm{H}), 3.25(\mathrm{t}, J=5.8 \mathrm{~Hz}, 2 \mathrm{H}), 2.23(\mathrm{~s}, 3 \mathrm{H}), 1.97$ (s, 3H). ${ }^{13} \mathrm{C} \mathrm{NMR}\left(126 \mathrm{MHz}, \mathrm{CDCl}_{3}\right) \delta 170.8,145.6,129.8,127.0,112.9,44.2,39.2$, 23.2, 20.3 .<smiles>Cc1ccc(NCCc2ccccc2)cc1</smiles>

Yellow oil, $48.0 \mathrm{mg}$, 57\% yield, known compound ${ }^{4}$ (General condition B, 36 hours); ${ }^{1} \mathrm{H}$ NMR $\left(500 \mathrm{MHz}, \mathrm{CDCl}_{3}\right) \delta 7.31-7.28(\mathrm{~m}, 2 \mathrm{H}), 7.23-7.19(\mathrm{~m}, 3 \mathrm{H}), 6.98(\mathrm{~d}, J=$ $8.1 \mathrm{~Hz}, 2 \mathrm{H}), 6.53(\mathrm{~d}, J=8.4 \mathrm{~Hz}, 2 \mathrm{H}), 3.36$ (t, $J=7.1 \mathrm{~Hz}, 2 \mathrm{H}), 3.29$ (br, 1H), 2.89 (t, $J$ $=7.1 \mathrm{~Hz}, 2 \mathrm{H}), 2.23(\mathrm{~s}, 3 \mathrm{H}) .{ }^{13} \mathrm{C} \mathrm{NMR}\left(126 \mathrm{MHz}, \mathrm{CDCl}_{3}\right) \delta 145.6,139.4,129.7,128.8$, $128.5,126.7,126.3,113.2,45.4,35.5,20.4$.<smiles>Cc1ccc(NCCc2ccccc2F)cc1</smiles>

Brown oil, $49.0 \mathrm{mg}$, 53\% yield (General condition B, 5 mol\% Cat. 10, 36 hours); ${ }^{1} \mathrm{H}$ NMR (500 MHz, $\left.\mathrm{CDCl}_{3}\right) \delta 7.24-7.22(\mathrm{~m}, 2 \mathrm{H}), 7.12-7.02(\mathrm{~m}, 4 \mathrm{H}), 6.59$ (d, J=8.4 $\mathrm{Hz}, 2 \mathrm{H}), 3.41(\mathrm{t}, J=7.1 \mathrm{~Hz}, 2 \mathrm{H}), 3.29(\mathrm{br}, 1 \mathrm{H}), 2.97(\mathrm{t}, J=7.1 \mathrm{~Hz}, 2 \mathrm{H}), 2.28(\mathrm{~s}, 3 \mathrm{H})$. ${ }^{13} \mathrm{C} \mathrm{NMR}\left(126 \mathrm{MHz}, \mathrm{CDCl}_{3}\right) \delta 161.3(\mathrm{~d}, J=245.3 \mathrm{~Hz}), 145.5,131.0(\mathrm{~d}, J=5.0 \mathrm{~Hz})$, $129.8,128.1(\mathrm{~d}, J=8.6 \mathrm{~Hz}), 126.7,126.3(\mathrm{~d}, J=16.0 \mathrm{~Hz}), 124.1(\mathrm{~d}, J=3.6 \mathrm{~Hz})$, $115.3(\mathrm{~d}, J=22.1 \mathrm{~Hz}), 113.1,44.2,29.1,20.3 .{ }^{19} \mathrm{~F}$ NMR (471 MHz, $\left.\mathrm{CDCl}_{3}\right) \delta$-118.4. HRMS-ESI (m/z): $[\mathrm{M}+\mathrm{H}]^{+}$calcd. for $\left[\mathrm{C}_{15} \mathrm{H}_{17} \mathrm{FN}\right]^{+}, 230.1345$; found, 230.1343 . 
<smiles>Cc1ccc(NCC(C)O)cc1</smiles>

Yellow oil, $26.4 \mathrm{mg}, 40 \%$ yield, known compound ${ }^{19}$ (General condition B, 5 mol\% Cat. 10, 36 hours); ${ }^{1} \mathrm{H}$ NMR $\left(500 \mathrm{MHz}, \mathrm{CDCl}_{3}\right) \delta 7.00$ (d, $\left.J=8.0 \mathrm{~Hz}, 2 \mathrm{H}\right), 6.59$ (d, $J$ $=8.2 \mathrm{~Hz}, 2 \mathrm{H}), 4.01-3.98(\mathrm{~m}, 1 \mathrm{H}), 3.22-3.19(\mathrm{dd}, J=12.8,2.9 \mathrm{~Hz}, 1 \mathrm{H}), 2.99-2.95$ (m, 2H), 2.91 (br, 1H), $2.25(\mathrm{~s}, 3 \mathrm{H}), 1.26$ (d, $J=6.2 \mathrm{~Hz}, 3 \mathrm{H}) .{ }^{13} \mathrm{C}$ NMR (126 MHz, $\left.\mathrm{CDCl}_{3}\right) \delta 145.9,129.8,127.2,113.5,66.4,52.2,20.7,20.3$.<smiles>Cc1ccc(NCC(C)(C)CN)cc1</smiles>

Brown oil, $41.0 \mathrm{mg}$, 53\% yield (General condition B, $1.6 \mathrm{mmol} p$-cresol, 36 hours); ${ }^{1} \mathrm{H}$ NMR $\left(500 \mathrm{MHz}, \mathrm{CDCl}_{3}\right) \delta 6.94(\mathrm{~d}, J=8.1 \mathrm{~Hz}, 2 \mathrm{H}), 6.62(\mathrm{~d}, J=8.1 \mathrm{~Hz}, 2 \mathrm{H}), 5.60$ (br, 3H), $3.01(\mathrm{~s}, 2 \mathrm{H}), 2.84(\mathrm{~s}, 2 \mathrm{H}), 2.20(\mathrm{~s}, 3 \mathrm{H}), 1.06(\mathrm{~s}, 6 \mathrm{H}) .{ }^{13} \mathrm{C}$ NMR (126 MHz, $\left.\mathrm{CDCl}_{3}\right) \delta 146.5,129.6,126.9,113.7,53.0,48.7,34.8,23.9,20.3$. HRMS-ESI (m/z): $[\mathrm{M}+\mathrm{H}]^{+}$calcd. for $\left[\mathrm{C}_{12} \mathrm{H}_{21} \mathrm{~N}_{2}\right]^{+}, 193.1705$; found, 193.1701 .

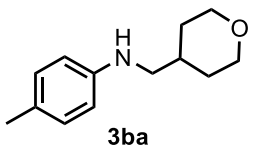

Colorless oil, $63.0 \mathrm{mg}, 77 \%$ yield (General condition B, 36 hours); ${ }^{1} \mathrm{H}$ NMR (500 $\left.\mathrm{MHz}, \mathrm{CDCl}_{3}\right) \delta 7.02(\mathrm{~d}, J=8.1 \mathrm{~Hz}, 2 \mathrm{H}), 6.56(\mathrm{~d}, J=8.1 \mathrm{~Hz}, 2 \mathrm{H}), 4.02(\mathrm{dd}, J=11.1$, $3.4 \mathrm{~Hz}, 2 \mathrm{H}), 3.65$ (br, 1H), 3.41 (td, $J=11.8,2.1 \mathrm{~Hz}, 2 \mathrm{H}), 3.03$ (d, $J=6.8 \mathrm{~Hz}, 2 \mathrm{H})$, $2.27(\mathrm{~s}, 3 \mathrm{H}), 1.89-1.81(\mathrm{~m}, 1 \mathrm{H}), 1.72(\mathrm{~d}, J=12.9 \mathrm{~Hz}, 2 \mathrm{H}), 1.42-1.30(\mathrm{~m}, 2 \mathrm{H}) .{ }^{13} \mathrm{C}$ NMR $\left(126 \mathrm{MHz}, \mathrm{CDCl}_{3}\right) \delta 145.9,129.7,126.3,112.7,67.6,50.2,34.7,31.0,20.3$. HRMS-ESI (m/z): $[\mathrm{M}+\mathrm{H}]^{+}$calcd. for $\left[\mathrm{C}_{13} \mathrm{H}_{20} \mathrm{NO}\right]^{+}, 206.1545$; found, 206.1543.

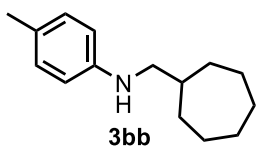

Pink oil, $76.5 \mathrm{mg}$, 88\% yield, known compound ${ }^{20}$ (General condition B, 36 hours); ${ }^{1} \mathrm{H}$ NMR $\left(500 \mathrm{MHz}, \mathrm{CDCl}_{3}\right) \delta 7.00(\mathrm{~d}, J=8.2 \mathrm{~Hz}, 2 \mathrm{H}), 6.54(\mathrm{~d}, J=8.4 \mathrm{~Hz}, 2 \mathrm{H}), 3.59$ $(\mathrm{s}, 1 \mathrm{H}), 2.94(\mathrm{~d}, J=6.6 \mathrm{~Hz}, 2 \mathrm{H}), 2.25(\mathrm{~s}, 3 \mathrm{H}), 1.85-1.43(\mathrm{~m}, 11 \mathrm{H}), 1.31-1.23(\mathrm{~m}$, 2H). ${ }^{13} \mathrm{C} \mathrm{NMR}\left(126 \mathrm{MHz}, \mathrm{CDCl}_{3}\right) \delta 146.4,129.7,126.0,112.7,51.1,39.1,32.4,28.5$, 26.4, 20.3 .<smiles>Cc1ccc(NCC23CC4CC(CC(C4)C2)C3)cc1</smiles>

Yellow solid, $79.4 \mathrm{mg}, 78 \%$ yield, known compound ${ }^{21}$ (General condition B, 36 hours); ${ }^{1} \mathrm{H} \mathrm{NMR}\left(500 \mathrm{MHz}, \mathrm{CDCl}_{3}\right) \delta 7.01(\mathrm{~d}, J=8.2 \mathrm{~Hz}, 2 \mathrm{H}), 6.58(\mathrm{~d}, J=8.4 \mathrm{~Hz}$, $2 \mathrm{H}), 3.56(\mathrm{br}, 1 \mathrm{H}), 2.81(\mathrm{~s}, 2 \mathrm{H}), 2.27(\mathrm{~s}, 3 \mathrm{H}), 2.08-2.01(\mathrm{~m}, 3 \mathrm{H}), 1.79-1.69(\mathrm{~m}$, $6 \mathrm{H}), 1.62(\mathrm{~d}, J=2.2 \mathrm{~Hz}, 6 \mathrm{H}) .{ }^{13} \mathrm{C} \mathrm{NMR}\left(126 \mathrm{MHz}, \mathrm{CDCl}_{3}\right) \delta 147.0,129.6,125.8$, 112.7, 56.6, 40.7, 37.1, 33.9, 28.4, 20.3.<smiles>Cc1ccc(NC(C)CCc2ccccc2)cc1</smiles> 
Yellow oil, $72.0 \mathrm{mg}, 75 \%$ yield, known compound ${ }^{22}$ (General condition B, 36 hours); ${ }^{1} \mathrm{H}$ NMR $\left(500 \mathrm{MHz}, \mathrm{CDCl}_{3}\right) \delta 7.33-7.30(\mathrm{~m}, 2 \mathrm{H}), 7.24-7.21(\mathrm{~m}, 3 \mathrm{H}), 7.00(\mathrm{~d}, J=$ $8.0 \mathrm{~Hz}, 2 \mathrm{H}), 6.51(\mathrm{~d}, J=8.4 \mathrm{~Hz}, 2 \mathrm{H}), 3.52-3.46(\mathrm{~m}, 1 \mathrm{H}), 3.24$ (br, 1H), $2.76(\mathrm{t}, J=$ $7.9 \mathrm{~Hz}, 2 \mathrm{H}), 2.27(\mathrm{~s}, 3 \mathrm{H}), 1.95-1.87(\mathrm{~m}, 1 \mathrm{H}), 1.82-1.75(\mathrm{~m}, 1 \mathrm{H}), 1.24(\mathrm{~d}, J=6.3$ $\mathrm{Hz}, 3 \mathrm{H}) .{ }^{13} \mathrm{C} \mathrm{NMR}\left(126 \mathrm{MHz}, \mathrm{CDCl}_{3}\right) \delta 145.1,142.0,129.8,128.4,128.3,126.2$, $125.8,113.5,48.3,38.8,32.5,20.8,20.3$.

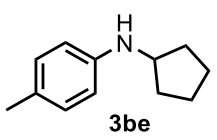

Yellow oil, $37.0 \mathrm{mg}$, 53\% yield, known compound ${ }^{23}$ (General condition B, 36 hours); ${ }^{1} \mathrm{H}$ NMR $\left(500 \mathrm{MHz}, \mathrm{CDCl}_{3}\right) \delta 7.02(\mathrm{~d}, J=8.2 \mathrm{~Hz}, 2 \mathrm{H}), 6.58(\mathrm{~d}, J=8.3 \mathrm{~Hz}, 2 \mathrm{H}), 3.83$ $-3.78(\mathrm{~m}, 1 \mathrm{H}), 3.33(\mathrm{br}, 1 \mathrm{H}), 2.28(\mathrm{~s}, 3 \mathrm{H}), 2.08-2.01(\mathrm{~m}, 2 \mathrm{H}), 1.77-1.72(\mathrm{~m}, 2 \mathrm{H})$, $1.68-1.62(\mathrm{~m}, 2 \mathrm{H}), 1.53-1.46(\mathrm{~m}, 2 \mathrm{H}) .{ }^{13} \mathrm{C} \mathrm{NMR}\left(126 \mathrm{MHz}, \mathrm{CDCl}_{3}\right) \delta 145.7,129.6$, $126.1,113.4,54.9,33.5,24.0,20.3$.

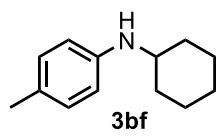

Brown solid, $59.9 \mathrm{mg}, 79 \%$ yield, known compound ${ }^{24}$ (General condition B, 36 hours); ${ }^{1} \mathrm{H}$ NMR $\left(500 \mathrm{MHz}, \mathrm{CDCl}_{3}\right) \delta 7.02(\mathrm{~d}, J=8.1 \mathrm{~Hz}, 2 \mathrm{H}), 6.57(\mathrm{~d}, J=8.4 \mathrm{~Hz}$, $2 \mathrm{H}), 3.29-3.24(\mathrm{~m}, 1 \mathrm{H}), 3.19(\mathrm{br}, 1 \mathrm{H}), 2.28(\mathrm{~s}, 3 \mathrm{H}), 2.12-2.09(\mathrm{~m}, 2 \mathrm{H}), 1.82-1.78$ $(\mathrm{m}, 2 \mathrm{H}), 1.72-1.68(\mathrm{~m}, 1 \mathrm{H}), 1.45-1.37(\mathrm{~m}, 2 \mathrm{H}), 1.31-1.25(\mathrm{~m}, 1 \mathrm{H}), 1.21-1.13$ $(\mathrm{m}, 2 \mathrm{H}) .{ }^{13} \mathrm{C} \mathrm{NMR}\left(126 \mathrm{MHz}, \mathrm{CDCl}_{3}\right) \delta 145.0,129.7,126.0,113.5,52.0,33.5,25.9$, 25.0, 20.3.

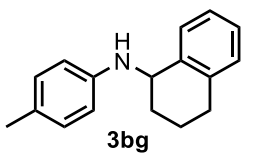

White solid, $40.0 \mathrm{mg}, 42 \%$ yield, known compound ${ }^{23}$ (General condition B, 36 hours); ${ }^{1} \mathrm{H}$ NMR $\left(500 \mathrm{MHz}, \mathrm{CDCl}_{3}\right) \delta 7.45-7.44(\mathrm{~m}, 1 \mathrm{H}), 7.24-7.15(\mathrm{~m}, 3 \mathrm{H}), 7.05$ $(\mathrm{d}, J=8.0 \mathrm{~Hz}, 2 \mathrm{H}), 6.64(\mathrm{~d}, J=8.4 \mathrm{~Hz}, 2 \mathrm{H}), 4.63(\mathrm{t}, J=5.0 \mathrm{~Hz}, 1 \mathrm{H}), 3.73(\mathrm{br}, 1 \mathrm{H})$, $2.91-2.85(\mathrm{~m}, 1 \mathrm{H}), 2.83-2.77(\mathrm{~m}, 1 \mathrm{H}), 2.30(\mathrm{~s}, 3 \mathrm{H}), 2.02-1.90(\mathrm{~m}, 3 \mathrm{H}), 1.86-$ $1.80(\mathrm{~m}, 1 \mathrm{H}) .{ }^{13} \mathrm{C} \mathrm{NMR}\left(126 \mathrm{MHz}, \mathrm{CDCl}_{3}\right) \delta 145.2,138.4,137.6,129.8,129.2,129.0$, 127.0, 126.3, 126.0, 113.0, 51.3, 29.3, 28.7, 20.3, 19.4.

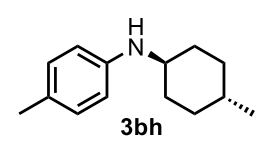

Colorless oil, $40.2 \mathrm{mg}$, 49\% yield, prepared from trans-4-methylcyclohexan-1-amine, giving the product in the ratio of trans : cis $=15.6: 1$, known compound ${ }^{25}$ (General condition B, 36 hours); ${ }^{1} \mathrm{H}$ NMR $\left(500 \mathrm{MHz}, \mathrm{CDCl}_{3}\right) \delta 6.99(\mathrm{~d}, J=8.1 \mathrm{~Hz}, 2 \mathrm{H}), 6.54$ $(\mathrm{d}, J=8.4 \mathrm{~Hz}, 2 \mathrm{H}), 3.55-3.52(\mathrm{~m}, 0.06 \mathrm{H}), 3.19-3.14(\mathrm{~m}, 0.94 \mathrm{H}), 3.04(\mathrm{br}, 1 \mathrm{H})$, $2.25(\mathrm{~s}, 3 \mathrm{H}), 2.14-2.11(\mathrm{~m}, 1.98 \mathrm{H}), 1.77-1.75(\mathrm{~m}, 2 \mathrm{H}), 1.67-1.52(\mathrm{~m}, 0.30 \mathrm{H})$, $1.44-1.37(\mathrm{~m}, 0.94 \mathrm{H}), 1.27-1.21(\mathrm{~m}, 0.12 \mathrm{H}), 1.13-1.05(\mathrm{~m}, 3.76 \mathrm{H}), 0.94(\mathrm{~d}, J=$ $6.6 \mathrm{~Hz}, 3 \mathrm{H}) .{ }^{13} \mathrm{C} \mathrm{NMR}\left(126 \mathrm{MHz}, \mathrm{CDCl}_{3}\right) \delta 145.1,145.0,129.7,126.2,126.0,113.6$, $113.4,52.5,48.4,34.1,33.6,32.3,30.2,29.7,29.2,22.2,21.3,20.5,20.3$. 


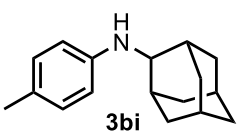

Colorless oil, $83.0 \mathrm{mg}, 86 \%$ yield (General condition B, 36 hours); ${ }^{1} \mathrm{H}$ NMR (500 $\left.\mathrm{MHz}, \mathrm{CDCl}_{3}\right) \delta 7.04(\mathrm{~d}, J=8.1 \mathrm{~Hz}, 2 \mathrm{H}), 6.60(\mathrm{~d}, J=8.2 \mathrm{~Hz}, 2 \mathrm{H}), 3.83$ (br, 1H), 3.62 $-3.55(\mathrm{~m}, 1 \mathrm{H}), 2.30(\mathrm{~s}, 3 \mathrm{H}), 2.10-2.08(\mathrm{~m}, 2 \mathrm{H}), 2.00-1.82(\mathrm{~m}, 10 \mathrm{H}), 1.65(\mathrm{~d}, J=$ $13.0 \mathrm{~Hz}, 2 \mathrm{H}) .{ }^{13} \mathrm{C} \mathrm{NMR}\left(126 \mathrm{MHz}, \mathrm{CDCl}_{3}\right) \delta 145.1,129.7,125.8,113.2,57.0,37.7$, 37.4, 31.5, 31.5, 27.5, 27.3, 20.3. HRMS-ESI $(\mathrm{m} / \mathrm{z}):[\mathrm{M}+\mathrm{H}]^{+}$calcd. for $\left[\mathrm{C}_{17} \mathrm{H}_{24} \mathrm{~N}\right]^{+}$, 242.1909; found, 242.1906 .

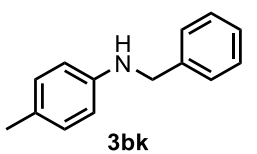

Colorless oil, $56.2 \mathrm{mg}, 71 \%$ yield, known compound ${ }^{26}$ (General condition B, 36 hours); ${ }^{1} \mathrm{H}$ NMR $\left(500 \mathrm{MHz}, \mathrm{CDCl}_{3}\right) \delta 7.42-7.36(\mathrm{~m}, 4 \mathrm{H}), 7.31(\mathrm{t}, J=7.1 \mathrm{~Hz}, 1 \mathrm{H})$, $7.03(\mathrm{~d}, J=8.1 \mathrm{~Hz}, 2 \mathrm{H}), 6.61(\mathrm{~d}, J=8.4 \mathrm{~Hz}, 2 \mathrm{H}), 4.34(\mathrm{~s}, 2 \mathrm{H}), 2.29(\mathrm{~s}, 3 \mathrm{H}) .{ }^{13} \mathrm{C} \mathrm{NMR}$ $\left(126 \mathrm{MHz}, \mathrm{CDCl}_{3}\right) \delta 145.8,139.6,129.7,128.6,127.5,127.1,126.8,113.0,48.6$, 20.4 .<smiles>Cc1ccc(NCc2c(C)cc(C)cc2C)cc1</smiles>

Brown oil, $87.3 \mathrm{mg}, 91 \%$ yield (General condition B, 36 hours); ${ }^{1} \mathrm{H}$ NMR (500 MHz, $\left.\mathrm{CDCl}_{3}\right) \delta 7.01(\mathrm{~d}, J=8.2 \mathrm{~Hz}, 2 \mathrm{H}), 6.87(\mathrm{~s}, 2 \mathrm{H}), 6.56(\mathrm{~d}, J=8.4 \mathrm{~Hz}, 2 \mathrm{H}), 4.14(\mathrm{~s}, 2 \mathrm{H})$, $3.22(\mathrm{br}, 1 \mathrm{H}), 2.33(\mathrm{~s}, 6 \mathrm{H}), 2.27(\mathrm{~s}, 3 \mathrm{H}), 2.25(\mathrm{~s}, 3 \mathrm{H}) .{ }^{13} \mathrm{C} \mathrm{NMR}\left(126 \mathrm{MHz}, \mathrm{CDCl}_{3}\right) \delta$ $146.4,137.4,137.1,132.4,129.7,129.0,126.3,112.5,42.6,20.9,20.3,19.3$. HRMS-ESI (m/z): $[\mathrm{M}+\mathrm{H}]^{+}$calcd. for $\left[\mathrm{C}_{17} \mathrm{H}_{22} \mathrm{~N}\right]^{+}, 240.1752$; found, 240.1756 .<smiles>Cc1ccc(NCc2cccc(N)c2)cc1</smiles>

Brown oil, $55.1 \mathrm{mg}$, 65\% yield (General condition B, 36 hours); ${ }^{1} \mathrm{H}$ NMR (500 MHz, $\left.\mathrm{CDCl}_{3}\right) \delta 7.14(\mathrm{t}, J=7.7 \mathrm{~Hz}, 1 \mathrm{H}), 7.00(\mathrm{~d}, J=8.2 \mathrm{~Hz}, 2 \mathrm{H}), 6.77(\mathrm{~d}, J=7.6 \mathrm{~Hz}, 1 \mathrm{H})$, $6.71(\mathrm{~s}, 1 \mathrm{H}), 6.63-6.54(\mathrm{~m}, 3 \mathrm{H}), 4.23(\mathrm{~s}, 2 \mathrm{H}), 3.72(\mathrm{br}, 3 \mathrm{H}), 2.26(\mathrm{~s}, 3 \mathrm{H}) .{ }^{13} \mathrm{C} \mathrm{NMR}$ $\left(126 \mathrm{MHz}, \mathrm{CDCl}_{3}\right) \delta 146.7,146.0,141.0,129.7,129.5,126.6,117.6,114.0,113.9$, 112.9, 48.6, 20.3. HRMS-ESI (m/z): $[\mathrm{M}+\mathrm{H}]^{+}$calcd. for $\left[\mathrm{C}_{14} \mathrm{H}_{17} \mathrm{~N}_{2}\right]^{+}, 213.1392$; found, 213.1388 .

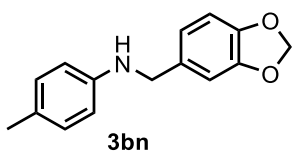

White solid, $49.8 \mathrm{mg}, 52 \%$ yield, known compound ${ }^{27}$ (General condition B, 36 hours); ${ }^{1} \mathrm{H}$ NMR $\left(500 \mathrm{MHz}, \mathrm{CDCl}_{3}\right) \delta 6.99(\mathrm{~d}, J=8.2 \mathrm{~Hz}, 2 \mathrm{H}), 6.87(\mathrm{~s}, 1 \mathrm{H}), 6.83$ (d, $J=8.0 \mathrm{~Hz}, 1 \mathrm{H}), 6.78(\mathrm{~d}, J=7.9 \mathrm{~Hz}, 1 \mathrm{H}), 6.56(\mathrm{~d}, J=8.3 \mathrm{~Hz}, 2 \mathrm{H}), 5.94(\mathrm{~s}, 2 \mathrm{H}), 4.22$ (s, 2H), $3.86(\mathrm{br}, 1 \mathrm{H}), 2.25$ (s, 3H). $\left.{ }^{13} \mathrm{C} \mathrm{NMR} \mathrm{(126} \mathrm{MHz,} \mathrm{CDCl}_{3}\right) \delta 147.9,146.7$, 145.8, 133.6, 129.7, 126.8, 120.5, 113.0, 108.3, 108.0, 100.9, 48.4, 20.4. 
<smiles>Cc1ccc(NCc2cc(C(F)(F)F)cc(C(F)(F)F)c2)cc1</smiles>

Colorless oil, $108.5 \mathrm{mg}, 81 \%$ yield (General condition B, 36 hours); ${ }^{1} \mathrm{H}$ NMR (500 $\left.\mathrm{MHz}, \mathrm{CDCl}_{3}\right) \delta 7.86(\mathrm{~s}, 2 \mathrm{H}), 7.81(\mathrm{~s}, 1 \mathrm{H}), 7.02(\mathrm{~d}, J=8.3 \mathrm{~Hz}, 2 \mathrm{H}), 6.55(\mathrm{~d}, J=8.4$ $\mathrm{Hz}, 2 \mathrm{H}), 4.46$ (s, 2H), $4.06(\mathrm{br}, 1 \mathrm{H}), 2.26(\mathrm{~s}, 3 \mathrm{H}) .{ }^{13} \mathrm{C} \mathrm{NMR}\left(126 \mathrm{MHz}, \mathrm{CDCl}_{3}\right) \delta$ 145.0, 142.8, 131.8 (q, $J=33.3 \mathrm{~Hz}), 129.9,127.8,127.3$ (q, $J=3.4 \mathrm{~Hz}), 123.3$ (q, $J=$ $273.1 \mathrm{~Hz}), 121.1$ (hept, $J=3.8 \mathrm{~Hz}), 113.1,48.0,20.3 .{ }^{19} \mathrm{~F} \mathrm{NMR}\left(471 \mathrm{MHz}, \mathrm{CDCl}_{3}\right) \delta$ -62.8. HRMS-ESI $(\mathrm{m} / \mathrm{z}):[\mathrm{M}+\mathrm{H}]^{+}$calcd. for $\left[\mathrm{C}_{16} \mathrm{H}_{14} \mathrm{NF}_{6}\right]^{+}, 334.1031$; found, 334.1033.

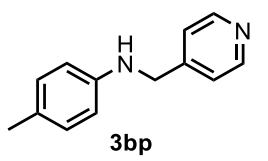

Light yellow solid, $41.2 \mathrm{mg}, 52 \%$ yield, known compound ${ }^{28}$ (General condition B, 36 hours); ${ }^{1} \mathrm{H}$ NMR $\left(500 \mathrm{MHz}, \mathrm{CDCl}_{3}\right) \delta 8.54(\mathrm{~d}, J=6.0 \mathrm{~Hz}, 2 \mathrm{H}), 7.33(\mathrm{~d}, J=5.6 \mathrm{~Hz}$, 2H), 6.97 (d, $J=8.4 \mathrm{~Hz}, 2 \mathrm{H}), 6.49$ (d, $J=8.4 \mathrm{~Hz}, 2 \mathrm{H}), 4.48$ (br, 1H), 4.37 (s, 2H), 2.23 (s, 3H). ${ }^{13} \mathrm{C} \mathrm{NMR}\left(126 \mathrm{MHz}, \mathrm{CDCl}_{3}\right) \delta 150.3,149.1,145.0,129.8,127.4,122.3$, $113.0,47.3,20.3$.

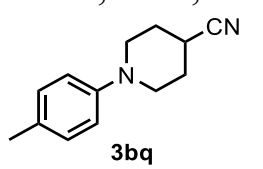

White solid, $45.0 \mathrm{mg}, 56 \%$ yield (General condition B, 5 mol\% Cat. 10, 36 hours); ${ }^{1} \mathrm{H} \mathrm{NMR}\left(500 \mathrm{MHz}, \mathrm{CDCl}_{3}\right) \delta 7.09(\mathrm{~d}, J=8.4 \mathrm{~Hz}, 2 \mathrm{H}), 6.86(\mathrm{~d}, J=8.5 \mathrm{~Hz}, 2 \mathrm{H}), 3.40$ $-3.35(\mathrm{~m}, 2 \mathrm{H}), 3.05-3.00(\mathrm{~m}, 2 \mathrm{H}), 2.79-2.74(\mathrm{~m}, 1 \mathrm{H}), 2.29(\mathrm{~s}, 3 \mathrm{H}), 2.10-2.05(\mathrm{~m}$, 2H), $2.03-1.97(\mathrm{~m}, 2 \mathrm{H}) .{ }^{13} \mathrm{C} \mathrm{NMR}\left(126 \mathrm{MHz}, \mathrm{CDCl}_{3}\right) \delta 149.0,129.9,129.7,121.5$, 117.3, 48.7, 28.6, 26.1, 20.4. HRMS-ESI $(\mathrm{m} / \mathrm{z}):[\mathrm{M}+\mathrm{H}]^{+}$calcd. for $\left[\mathrm{C}_{13} \mathrm{H}_{17} \mathrm{~N}_{2}\right]^{+}$, 201.1392; found, 210.1388 .

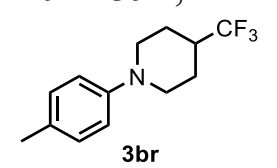

White solid, $68.9 \mathrm{mg}, 71 \%$ yield (General condition B, 36 hours); ${ }^{1} \mathrm{H}$ NMR (500 $\left.\mathrm{MHz}_{2} \mathrm{CDCl}_{3}\right) \delta 7.10(\mathrm{~d}, J=8.5 \mathrm{~Hz}, 2 \mathrm{H}), 6.88(\mathrm{~d}, J=8.5 \mathrm{~Hz}, 2 \mathrm{H}), 3.71-3.69(\mathrm{~m}, 2 \mathrm{H})$, $2.66(\mathrm{td}, J=12.4,2.2 \mathrm{~Hz}, 2 \mathrm{H}), 2.29(\mathrm{~s}, 3 \mathrm{H}), 2.19-2.10(\mathrm{~m}, 1 \mathrm{H}), 1.99-1.96(\mathrm{~m}, 2 \mathrm{H})$, $1.82-1.74(\mathrm{~m}, 2 \mathrm{H}) .{ }^{13} \mathrm{C}$ NMR $\left(126 \mathrm{MHz}, \mathrm{CDCl}_{3}\right) \delta 149.2,129.7,129.7,127.4$ (q, $J=$ $278.7 \mathrm{~Hz}), 117.2,49.5,40.3(\mathrm{q}, J=27.4 \mathrm{~Hz}), 24.6(\mathrm{q}, J=2.5 \mathrm{~Hz}), 20.4 .{ }^{19} \mathrm{~F}$ NMR $\left(471 \mathrm{MHz}, \mathrm{CDCl}_{3}\right) \delta$-73.8. HRMS-ESI $(\mathrm{m} / \mathrm{z}):[\mathrm{M}+\mathrm{H}]^{+}$calcd. for $\left[\mathrm{C}_{13} \mathrm{H}_{17} \mathrm{NF}_{3}\right]^{+}$, 244.1313; found, 244.1314.

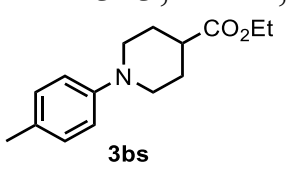

Colorless oil, $63.2 \mathrm{mg}$, 64\% yield (General condition B, 24 hours); ${ }^{1} \mathrm{H}$ NMR (500 $\left.\mathrm{MHz}, \mathrm{CDCl}_{3}\right) \delta 7.07(\mathrm{~d}, J=8.4 \mathrm{~Hz}, 2 \mathrm{H}), 6.86(\mathrm{~d}, J=8.4 \mathrm{~Hz}, 2 \mathrm{H}), 4.14(\mathrm{q}, J=7.1 \mathrm{~Hz}$, $2 \mathrm{H}), 3.60-3.56(\mathrm{~m}, 2 \mathrm{H}), 2.76-2.71(\mathrm{~m}, 2 \mathrm{H}), 2.44-2.38(\mathrm{~m}, 1 \mathrm{H}), 2.27(\mathrm{~s}, 3 \mathrm{H}), 2.04$ 
- $2.01(\mathrm{~m}, 2 \mathrm{H}), 1.93-1.85(\mathrm{~m}, 2 \mathrm{H}), 1.27(\mathrm{t}, \mathrm{J}=7.1 \mathrm{~Hz}, 3 \mathrm{H}) .{ }^{13} \mathrm{C}$ NMR (126 MHz, $\left.\mathrm{CDCl}_{3}\right) \delta 174.9,149.5,129.6,129.2,117.0,60.4,49.9,41.0,28.2,20.4,14.2$. HRMS-ESI (m/z): $[\mathrm{M}+\mathrm{H}]^{+}$calcd. for $\left[\mathrm{C}_{15} \mathrm{H}_{22} \mathrm{NO}_{2}\right]^{+}, 248.1651$; found, 248.1655.<smiles>Cc1ccc(N2CCC(O)CC2)cc1</smiles>

Light yellow solid, $34.4 \mathrm{mg}$, 45\% yield, known compound $^{29}$ (General condition B, 36 hours); ${ }^{1} \mathrm{H}$ NMR $\left(500 \mathrm{MHz}, \mathrm{CDCl}_{3}\right) \delta 7.06(\mathrm{~d}, J=8.3 \mathrm{~Hz}, 2 \mathrm{H}), 6.86(\mathrm{~d}, J=8.4 \mathrm{~Hz}$, 2H), $3.85-3.80(\mathrm{~m}, 1 \mathrm{H}), 3.51-3.47(\mathrm{~m}, 2 \mathrm{H}), 2.89-2.84(\mathrm{~m}, 2 \mathrm{H}), 2.27(\mathrm{~s}, 3 \mathrm{H}), 2.04$ - $1.99(\mathrm{~m}, 2 \mathrm{H}), 1.75-1.66(\mathrm{~m}, 2 \mathrm{H}), 1.60(\mathrm{br}, 1 \mathrm{H}) .{ }^{13} \mathrm{C} \mathrm{NMR}\left(126 \mathrm{MHz}, \mathrm{CDCl}_{3}\right) \delta$ 149.2, 129.6, 129.1, 117.0, 68.0, 47.9, 34.3, 20.4.

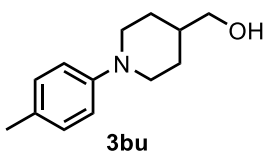

Yellow oil, $62.0 \mathrm{mg}, 76 \%$ yield (General condition B, 24 hours); ${ }^{1} \mathrm{H}$ NMR (500 MHz, $\left.\mathrm{CDCl}_{3}\right) \delta 7.07(\mathrm{~d}, J=8.3 \mathrm{~Hz}, 2 \mathrm{H}), 6.87(\mathrm{~d}, J=8.4 \mathrm{~Hz}, 2 \mathrm{H}), 3.65-3.63(\mathrm{~m}, 2 \mathrm{H}), 3.54$ $(\mathrm{d}, J=6.4 \mathrm{~Hz}, 2 \mathrm{H}), 2.67(\mathrm{t}, J=12.0 \mathrm{~Hz}, 2 \mathrm{H}), 2.27(\mathrm{~s}, 3 \mathrm{H}), 1.86-1.83(\mathrm{~m}, 2 \mathrm{H}), 1.67-$ $1.60(\mathrm{~m}, 1 \mathrm{H}), 1.51(\mathrm{br}, 1 \mathrm{H}), 1.45-1.37(\mathrm{~m}, 2 \mathrm{H}) .{ }^{13} \mathrm{C} \mathrm{NMR}\left(126 \mathrm{MHz}, \mathrm{CDCl}_{3}\right) \delta$ 149.8, 129.6, 129.0, 117.0, 67.8, 50.3, 38.5, 28.7, 20.4. HRMS-ESI (m/z): $[\mathrm{M}+\mathrm{H}]^{+}$ calcd. for $\left[\mathrm{C}_{13} \mathrm{H}_{20} \mathrm{NO}\right]^{+}, 206.1545$; found, 206.1539.<smiles>Cc1ccc(N2CCC(N3CCCCC3)CC2C)cc1</smiles>

Light yellow solid, $80.1 \mathrm{mg}, 78 \%$ yield (General condition B, 24 hours); ${ }^{1} \mathrm{H}$ NMR $\left(500 \mathrm{MHz}, \mathrm{CDCl}_{3}\right) \delta 7.06(\mathrm{~d}, J=8.2 \mathrm{~Hz}, 2 \mathrm{H}), 6.86(\mathrm{~d}, J=8.6 \mathrm{~Hz}, 2 \mathrm{H}), 3.69-3.67(\mathrm{~m}$, $2 \mathrm{H}), 2.64(\mathrm{td}, J=12.2,2.3 \mathrm{~Hz}, 2 \mathrm{H}), 2.55-2.53(\mathrm{~m}, 4 \mathrm{H}), 2.37(\mathrm{tt}, J=11.6,3.6 \mathrm{~Hz}$, 1H), 2.27 (s, 3H), $1.91-1.88(\mathrm{~m}, 2 \mathrm{H}), 1.75-1.67(\mathrm{~m}, 2 \mathrm{H}), 1.63-1.58(\mathrm{~m}, 4 \mathrm{H}), 1.48$ $-1.44(\mathrm{~m}, 2 \mathrm{H}) .{ }^{13} \mathrm{C} \mathrm{NMR}\left(126 \mathrm{MHz}, \mathrm{CDCl}_{3}\right) \delta 149.4,129.5,128.8,116.8,62.6,50.2$, 50.1, 27.8, 26.4, 24.8, 20.3. HRMS-ESI $(\mathrm{m} / \mathrm{z})$ : $[\mathrm{M}+\mathrm{H}]^{+}$calcd. for $\left[\mathrm{C}_{17} \mathrm{H}_{27} \mathrm{~N}_{2}\right]^{+}$, 259.2174; found, 259.2173 .

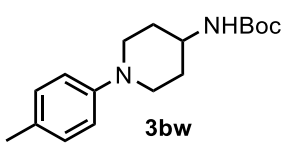

Colorless oil, $59.7 \mathrm{mg}, 51 \%$ yield (General condition B, 24 hours); ${ }^{1} \mathrm{H}$ NMR (500 $\left.\mathrm{MHz}, \mathrm{CDCl}_{3}\right) \delta 7.06(\mathrm{~d}, J=8.5 \mathrm{~Hz}, 2 \mathrm{H}), 6.85(\mathrm{~d}, J=8.5 \mathrm{~Hz}, 2 \mathrm{H}), 4.48(\mathrm{br}, 1 \mathrm{H}), 3.59$ $-3.51(\mathrm{~m}, 3 \mathrm{H}), 2.79(\mathrm{td}, J=12.6,2.4 \mathrm{~Hz}, 2 \mathrm{H}), 2.26(\mathrm{~s}, 3 \mathrm{H}), 2.03(\mathrm{~d}, J=11.0 \mathrm{~Hz}, 2 \mathrm{H})$, $1.58-1.51(\mathrm{~m}, 2 \mathrm{H}), 1.46(\mathrm{~s}, 9 \mathrm{H}) .{ }^{13} \mathrm{C} \mathrm{NMR}\left(126 \mathrm{MHz}, \mathrm{CDCl}_{3}\right) \delta 155.2,149.2,129.6$, 129.3, 117.0, 79.3, 49.3, 47.7, 32.5, 28.4, 20.4. HRMS-ESI $(\mathrm{m} / \mathrm{z}):[\mathrm{M}+\mathrm{H}]^{+}$calcd. for $\left[\mathrm{C}_{17} \mathrm{H}_{27} \mathrm{~N}_{2} \mathrm{O}_{2}\right]^{+}, 291.2072$; found, 291.2071.

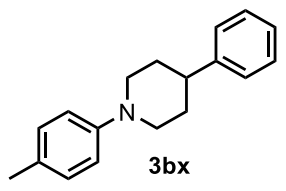


White solid, $81.7 \mathrm{mg}, 81 \%$ yield (General condition B, 24 hours); ${ }^{1} \mathrm{H}$ NMR (500 $\left.\mathrm{MHz}, \mathrm{CDCl}_{3}\right) \delta 7.30(\mathrm{t}, J=7.5 \mathrm{~Hz}, 2 \mathrm{H}), 7.24(\mathrm{~d}, J=7.1 \mathrm{~Hz}, 2 \mathrm{H}), 7.21-7.18(\mathrm{~m}, 1 \mathrm{H})$, $7.07(\mathrm{~d}, J=8.3 \mathrm{~Hz}, 2 \mathrm{H}), 6.90(\mathrm{~d}, J=8.5 \mathrm{~Hz}, 2 \mathrm{H}), 3.73-3.71(\mathrm{~m}, 2 \mathrm{H}), 2.75(\mathrm{td}, J=$ 11.8, 3.9 Hz, 2H), $2.64-2.58(\mathrm{~m}, 1 \mathrm{H}), 2.27(\mathrm{~s}, 3 \mathrm{H}), 1.94-1.84(\mathrm{~m}, 4 \mathrm{H}) .{ }^{13} \mathrm{C} \mathrm{NMR}$ $\left(126 \mathrm{MHz}, \mathrm{CDCl}_{3}\right) \delta 149.8,146.1,129.6,129.0,128.4,126.8,126.2,117.0,51.1,42.4$, 33.4, 20.4. HRMS-ESI $(\mathrm{m} / \mathrm{z}):[\mathrm{M}+\mathrm{H}]^{+}$calcd. for $\left[\mathrm{C}_{18} \mathrm{H}_{22} \mathrm{~N}\right]^{+}, 252.1752$; found, 252.1750 .

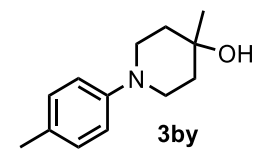

Light yellow solid, $57.0 \mathrm{mg}, 69 \%$ yield (General condition B, 24 hours); ${ }^{1} \mathrm{H}$ NMR $\left(500 \mathrm{MHz}, \mathrm{CDCl}_{3}\right) \delta 7.08(\mathrm{~d}, J=8.3 \mathrm{~Hz}, 2 \mathrm{H}), 6.89(\mathrm{~d}, J=8.5 \mathrm{~Hz}, 2 \mathrm{H}), 3.30-3.25(\mathrm{~m}$, $2 \mathrm{H}), 3.16-3.11(\mathrm{~m}, 2 \mathrm{H}), 2.28(\mathrm{~s}, 3 \mathrm{H}), 1.83-1.77(\mathrm{~m}, 2 \mathrm{H}), 1.71-1.68(\mathrm{~m}, 2 \mathrm{H}), 1.49$ (br, $1 \mathrm{H}), 1.29(\mathrm{~s}, 3 \mathrm{H}) .{ }^{13} \mathrm{C} \mathrm{NMR}\left(126 \mathrm{MHz}, \mathrm{CDCl}_{3}\right) \delta 149.3,129.5,128.9,116.9,67.8$, 46.6, 38.4, 29.7, 20.4. HRMS-ESI (m/z): $[\mathrm{M}+\mathrm{H}]^{+}$calcd. for $\left[\mathrm{C}_{13} \mathrm{H}_{20} \mathrm{NO}\right]^{+}, 206.1545$; found, 206.1542 .

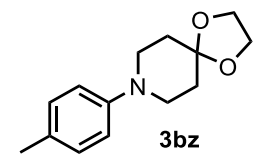

White solid, $56.2 \mathrm{mg}, 60 \%$ yield, known compound ${ }^{30}$ (General condition B, 24 hours); ${ }^{1} \mathrm{H} \mathrm{NMR}\left(500 \mathrm{MHz}, \mathrm{CDCl}_{3}\right) \delta 7.07(\mathrm{~d}, J=8.2 \mathrm{~Hz}, 2 \mathrm{H}), 6.88(\mathrm{~d}, J=8.6 \mathrm{~Hz}$, $2 \mathrm{H}), 4.00(\mathrm{~s}, 4 \mathrm{H}), 3.27(\mathrm{t}, J=5.7 \mathrm{~Hz}, 4 \mathrm{H}), 2.28(\mathrm{~s}, 3 \mathrm{H}), 1.86(\mathrm{t}, J=5.8 \mathrm{~Hz}, 4 \mathrm{H}) .{ }^{13} \mathrm{C}$ NMR $\left(126 \mathrm{MHz}, \mathrm{CDCl}_{3}\right) \delta 148.9,129.6,128.9,117.0,107.1,64.2,48.3,34.6,20.4$.

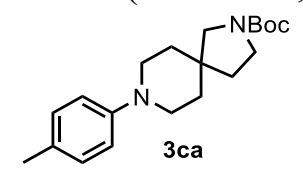

Colorless oil, $94.0 \mathrm{mg}, 71 \%$ yield (General condition B, 24 hours); ${ }^{1} \mathrm{H}$ NMR (500 $\left.\mathrm{MHz} \mathrm{CDCl}_{3}\right) \delta 7.06(\mathrm{~d}, J=8.5 \mathrm{~Hz}, 2 \mathrm{H}), 6.86(\mathrm{~d}, J=8.5 \mathrm{~Hz}, 2 \mathrm{H}), 3.44-3.38(\mathrm{~m}, 2 \mathrm{H})$, $3.26(\mathrm{~s}, 1 \mathrm{H}), 3.21-3.13(\mathrm{~m}, 3 \mathrm{H}), 3.08-2.99(\mathrm{~m}, 2 \mathrm{H}), 2.27(\mathrm{~s}, 3 \mathrm{H}), 1.76-1.66(\mathrm{~m}$, $6 \mathrm{H}), 1.48(\mathrm{~s}, 9 \mathrm{H}) .{ }^{13} \mathrm{C} \mathrm{NMR}\left(126 \mathrm{MHz}, \mathrm{CDCl}_{3}\right.$, rotamers) $\delta 154.6,154.6,149.4,149.3$, $129.5,128.9,128.9,116.8,116.6,79.0,78.9,55.6,54.9,47.6,47.5,44.1,43.8,40.2$, $39.4,36.2,35.1,34.5,34.5,28.4,20.3$. HRMS-ESI $(\mathrm{m} / \mathrm{z}):[\mathrm{M}+\mathrm{H}]^{+}$calcd. for $\left[\mathrm{C}_{20} \mathrm{H}_{31} \mathrm{~N}_{2} \mathrm{O}_{2}\right]^{+}, 331.2386$; found, 331.2387.

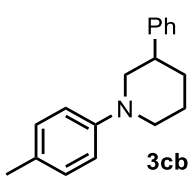

Colorless oil, $80.8 \mathrm{mg}, 80 \%$ yield (General condition B, 24 hours); ${ }^{1} \mathrm{H}$ NMR (500 $\left.\mathrm{MHz} \mathrm{CDCl}_{3}\right) \delta 7.35(\mathrm{t}, J=7.4 \mathrm{~Hz}, 2 \mathrm{H}), 7.30(\mathrm{~d}, J=6.9 \mathrm{~Hz}, 2 \mathrm{H}), 7.28-7.23(\mathrm{~m}, 1 \mathrm{H})$, $7.08(\mathrm{~d}, J=8.4 \mathrm{~Hz}, 2 \mathrm{H}), 6.90(\mathrm{~d}, J=8.6 \mathrm{~Hz}, 2 \mathrm{H}), 3.75-3.67(\mathrm{~m}, 2 \mathrm{H}), 2.97-2.91(\mathrm{~m}$, $1 \mathrm{H}), 2.75-2.69(\mathrm{~m}, 2 \mathrm{H}), 2.28(\mathrm{~s}, 3 \mathrm{H}), 2.04-2.01(\mathrm{~m}, 1 \mathrm{H}), 1.91-1.80(\mathrm{~m}, 2 \mathrm{H}), 1.66$ $-1.57(\mathrm{~m}, 1 \mathrm{H}) .{ }^{13} \mathrm{C}$ NMR $\left(126 \mathrm{MHz}, \mathrm{CDCl}_{3}\right) \delta 149.6,144.4,129.6,128.9,128.5$, $127.3,126.5,116.9,57.6,50.7,42.6,31.5,25.5,20.4$. HRMS-ESI $(\mathrm{m} / \mathrm{z}):[\mathrm{M}+\mathrm{H}]^{+}$ calcd. for $\left[\mathrm{C}_{18} \mathrm{H}_{22} \mathrm{~N}\right]^{+}, 252.1752$; found, 252.1750. 


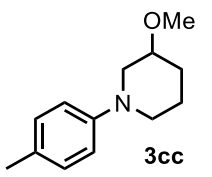

Colorless oil, $59.0 \mathrm{mg}, 72 \%$ yield (General condition B, 24 hours); ${ }^{1} \mathrm{H}$ NMR (500 $\left.\mathrm{MHz}, \mathrm{CDCl}_{3}\right) \delta 7.09(\mathrm{~d}, J=8.1 \mathrm{~Hz}, 2 \mathrm{H}), 6.89(\mathrm{~d}, J=8.2 \mathrm{~Hz}, 2 \mathrm{H}), 3.62(\mathrm{~d}, J=10.2$ $\mathrm{Hz}, 1 \mathrm{H}), 3.47-3.43(\mathrm{~m}, 4 \mathrm{H}), 3.37-3.35(\mathrm{~m}, 1 \mathrm{H}), 2.79$ (t, $J=9.9 \mathrm{~Hz}, 1 \mathrm{H}), 2.74-$ $2.70(\mathrm{~m}, 1 \mathrm{H}), 2.30(\mathrm{~s}, 3 \mathrm{H}), 2.08-2.05(\mathrm{~m}, 1 \mathrm{H}), 1.90-1.87(\mathrm{~m}, 1 \mathrm{H}), 1.72-1.65(\mathrm{~m}$, $1 \mathrm{H}), 1.46-1.39(\mathrm{~m}, 1 \mathrm{H}) .{ }^{13} \mathrm{C} \mathrm{NMR}\left(126 \mathrm{MHz}, \mathrm{CDCl}_{3}\right) \delta 149.5,129.5,129.0,117.0$, $75.8,56.1,54.6,50.5,29.6,23.0,20.3$. HRMS-ESI $(\mathrm{m} / \mathrm{z}):[\mathrm{M}+\mathrm{H}]^{+}$calcd. for $\left[\mathrm{C}_{13} \mathrm{H}_{20} \mathrm{NO}\right]^{+}, 206.1545$; found, 206.1538.

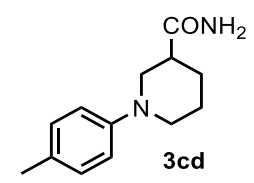

White solid, $45.6 \mathrm{mg}, 52 \%$ yield (General condition B, 36 hours); ${ }^{1} \mathrm{H}$ NMR (500 $\left.\mathrm{MHz} \mathrm{CDCl}_{3}\right) \delta 7.09(\mathrm{~d}, J=8.2 \mathrm{~Hz}, 2 \mathrm{H}), 6.90(\mathrm{~d}, J=8.5 \mathrm{~Hz}, 2 \mathrm{H}), 6.85(\mathrm{~s}, 1 \mathrm{H}), 5.94$ $(\mathrm{s}, 1 \mathrm{H}), 3.30-3.22(\mathrm{~m}, 2 \mathrm{H}), 3.15-3.04(\mathrm{~m}, 2 \mathrm{H}), 2.64-2.60(\mathrm{~m}, 1 \mathrm{H}), 2.28(\mathrm{~s}, 3 \mathrm{H})$, $1.92-1.70(\mathrm{~m}, 4 \mathrm{H}) .{ }^{13} \mathrm{C}$ NMR $\left(126 \mathrm{MHz}, \mathrm{CDCl}_{3}\right) \delta 177.3,149.4,130.5,129.7,118.0$, 53.1, 51.5, 42.0, 27.0, 23.4, 20.4. HRMS-ESI (m/z): $[\mathrm{M}+\mathrm{H}]^{+}$calcd. for $\left[\mathrm{C}_{13} \mathrm{H}_{19} \mathrm{~N}_{2} \mathrm{O}\right]^{+}$, 219.1497; found, 219.1492 .

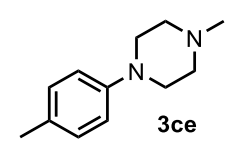

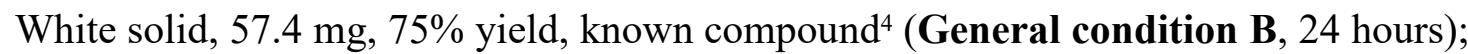
${ }^{1} \mathrm{H} \mathrm{NMR}\left(500 \mathrm{MHz}, \mathrm{CDCl}_{3}\right) \delta 7.07(\mathrm{~d}, J=8.4 \mathrm{~Hz}, 2 \mathrm{H}), 6.85(\mathrm{~d}, J=8.6 \mathrm{~Hz}, 2 \mathrm{H}), 3.17$ $(\mathrm{t}, J=5.1 \mathrm{~Hz}, 4 \mathrm{H}), 2.60(\mathrm{t}, J=5.1 \mathrm{~Hz}, 4 \mathrm{H}), 2.36(\mathrm{~s}, 3 \mathrm{H}), 2.27(\mathrm{~s}, 3 \mathrm{H}) .{ }^{13} \mathrm{C}$ NMR $(126$ $\left.\mathrm{MHz}, \mathrm{CDCl}_{3}\right) \delta 149.2,129.6,129.3,116.4,55.1,49.6,46.1,20.4$.

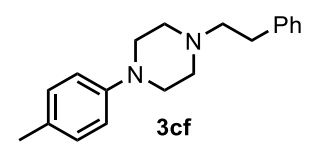

Light yellow solid, $85.4 \mathrm{mg}, 76 \%$ yield (General condition B, 24 hours); ${ }^{1} \mathrm{H}$ NMR $\left(500 \mathrm{MHz}, \mathrm{CDCl}_{3}\right) \delta 7.28(\mathrm{t}, J=7.5 \mathrm{~Hz}, 2 \mathrm{H}), 7.22-7.18(\mathrm{~m}, 3 \mathrm{H}), 7.07(\mathrm{~d}, J=8.3 \mathrm{~Hz}$, $2 \mathrm{H}), 6.85(\mathrm{~d}, J=8.5 \mathrm{~Hz}, 2 \mathrm{H}), 3.17(\mathrm{t}, J=5.1 \mathrm{~Hz}, 4 \mathrm{H}), 2.85-2.82(\mathrm{~m}, 2 \mathrm{H}), 2.69-$ $2.63(\mathrm{~m}, 6 \mathrm{H}), 2.26(\mathrm{~s}, 3 \mathrm{H}) .{ }^{13} \mathrm{C} \mathrm{NMR}\left(126 \mathrm{MHz}, \mathrm{CDCl}_{3}\right) \delta 149.2,140.2,129.6,129.2$, 128.6, 128.4, 126.0, 116.4, 60.5, 53.2, 49.6, 33.5, 20.4. HRMS-ESI (m/z): $[\mathrm{M}+\mathrm{H}]^{+}$ calcd. for $\left[\mathrm{C}_{19} \mathrm{H}_{25} \mathrm{~N}_{2}\right]^{+}, 281.2018$; found, 281.2017.

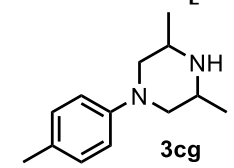

Yellow oil, $59.8 \mathrm{mg}, 73 \%$ yield (General condition B, 24 hours); ${ }^{1} \mathrm{H}$ NMR (500 MHz, $\left.\mathrm{CDCl}_{3}\right) \delta 7.05(\mathrm{~d}, J=8.3 \mathrm{~Hz}, 2 \mathrm{H}), 6.83(\mathrm{~d}, J=8.5 \mathrm{~Hz}, 2 \mathrm{H}), 3.44(\mathrm{~d}, J=11.5 \mathrm{~Hz}, 2 \mathrm{H})$, $3.10-3.03(\mathrm{~m}, 2 \mathrm{H}), 2.78(\mathrm{br}, 1 \mathrm{H}), 2.31(\mathrm{t}, J=11.5 \mathrm{~Hz}, 2 \mathrm{H}), 2.25(\mathrm{~s}, 3 \mathrm{H}), 1.16(\mathrm{~d}, J=$ $6.4 \mathrm{~Hz}, 6 \mathrm{H}) .{ }^{13} \mathrm{C}$ NMR $\left(126 \mathrm{MHz}, \mathrm{CDCl}_{3}\right) \delta 149.0,129.5,129.0,116.4,56.4,50.7$, 
20.3, 19.3. HRMS-ESI $(\mathrm{m} / \mathrm{z})$ : $[\mathrm{M}+\mathrm{H}]^{+}$calcd. for $\left[\mathrm{C}_{13} \mathrm{H}_{21} \mathrm{~N}_{2}\right]^{+}, 205.1705$; found, 205.1700 .

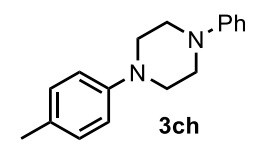

White solid, $62.0 \mathrm{mg}$, 61\% yield, known compound ${ }^{31}$ (General condition B, 24 hours); ${ }^{1} \mathrm{H}$ NMR $\left(500 \mathrm{MHz}, \mathrm{CDCl}_{3}\right) \delta 7.37-7.30(\mathrm{~m}, 2 \mathrm{H}), 7.15(\mathrm{~d}, J=8.3 \mathrm{~Hz}, 2 \mathrm{H})$, $7.03(\mathrm{~d}, J=7.9 \mathrm{~Hz}, 2 \mathrm{H}), 6.96-6.93(\mathrm{~m}, 3 \mathrm{H}), 3.39-3.37(\mathrm{~m}, 4 \mathrm{H}), 3.33-3.32(\mathrm{~m}$, $4 \mathrm{H}), 2.33(\mathrm{~s}, 3 \mathrm{H}) .{ }^{13} \mathrm{C} \mathrm{NMR}\left(126 \mathrm{MHz}, \mathrm{CDCl}_{3}\right) \delta 151.3,149.1,129.7,129.6,129.1$, 120.0, 116.7, 116.3, 49.9, 49.4, 20.4 .

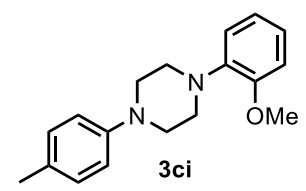

Pink solid, $67.1 \mathrm{mg}, 59 \%$ yield (General condition B, 24 hours); ${ }^{1} \mathrm{H}$ NMR (500 MHz, $\left.\mathrm{CDCl}_{3}\right) \delta 7.13(\mathrm{~d}, J=8.4 \mathrm{~Hz}, 2 \mathrm{H}), 7.07-7.01(\mathrm{~m}, 2 \mathrm{H}), 6.99-6.91(\mathrm{~m}, 4 \mathrm{H}), 3.91(\mathrm{~s}$, $3 \mathrm{H}), 3.37-3.35(\mathrm{~m}, 4 \mathrm{H}), 3.27-3.25(\mathrm{~m}, 4 \mathrm{H}), 2.32(\mathrm{~s}, 3 \mathrm{H}),{ }^{13} \mathrm{C}$ NMR (126 MHz, $\left.\mathrm{CDCl}_{3}\right) \delta 152.3,149.3,141.2,129.6,129.2,123.1,121.0,118.2,116.5,111.2,55.3$, 50.7, 50.0, 20.4. HRMS-ESI (m/z): $[\mathrm{M}+\mathrm{H}]^{+}$calcd. for $\left[\mathrm{C}_{18} \mathrm{H}_{23} \mathrm{~N}_{2} \mathrm{O}\right]^{+}, 283.1810$; found, 283.1809 .

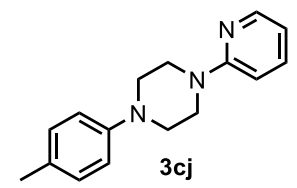

Yellow solid, $30.1 \mathrm{mg}, 30 \%$ yield, known compound ${ }^{30}$ (General condition B, 5 mol\% Cat. 10, 36 hours); ${ }^{1} \mathrm{H}$ NMR $\left(500 \mathrm{MHz}, \mathrm{CDCl}_{3}\right) \delta 8.22(\mathrm{dd}, J=5.0,1.0 \mathrm{~Hz}, 1 \mathrm{H}), 7.53$ $-7.49(\mathrm{~m}, 1 \mathrm{H}), 7.10(\mathrm{~d}, J=8.3 \mathrm{~Hz}, 2 \mathrm{H}), 6.91(\mathrm{~d}, J=8.5 \mathrm{~Hz}, 2 \mathrm{H}), 6.71(\mathrm{~d}, J=8.6 \mathrm{~Hz}$, $1 \mathrm{H}), 6.65(\mathrm{dd}, J=6.8,5.1 \mathrm{~Hz}, 1 \mathrm{H}), 3.76-3.68(\mathrm{t}, J=5.2 \mathrm{~Hz}, 4 \mathrm{H}), 3.30-3.22(\mathrm{t}, J=$ $5.2 \mathrm{~Hz}, 4 \mathrm{H}), 2.29(\mathrm{~s}, 3 \mathrm{H}) .{ }^{13} \mathrm{C} \mathrm{NMR}\left(126 \mathrm{MHz}, \mathrm{CDCl}_{3}\right) \delta 159.5,149.2,148.0,137.5$, $129.7,129.7,116.8,113.5,107.2,49.8,45.3,20.4$.

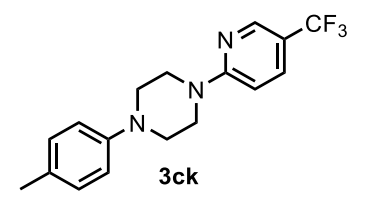

White solid, $80.8 \mathrm{mg}$, 63\% yield (General condition B, 5 mol\% Cat. 10, 36 hours); ${ }^{1} \mathrm{H}$ NMR $\left(500 \mathrm{MHz}, \mathrm{CDCl}_{3}\right) \delta 8.44(\mathrm{~s}, 1 \mathrm{H}), 7.66(\mathrm{dd}, J=9.0,2.3 \mathrm{~Hz}, 1 \mathrm{H}), 7.12(\mathrm{~d}, J$ $=8.3 \mathrm{~Hz}, 2 \mathrm{H}), 6.90(\mathrm{~d}, J=8.5 \mathrm{~Hz}, 2 \mathrm{H}), 6.69(\mathrm{~d}, J=9.0 \mathrm{~Hz}, 1 \mathrm{H}), 3.81(\mathrm{t}, J=5.1 \mathrm{~Hz}$, $4 \mathrm{H}), 3.25(\mathrm{t}, J=5.0 \mathrm{~Hz}, 4 \mathrm{H}), 2.30(\mathrm{~s}, 3 \mathrm{H}) .{ }^{13} \mathrm{C} \mathrm{NMR}\left(126 \mathrm{MHz}, \mathrm{CDCl}_{3}\right) \delta 160.3$, $149.0,145.8$ (q, $J=4.2 \mathrm{~Hz}), 134.5(\mathrm{q}, J=3.1 \mathrm{~Hz}), 129.9,129.8,124.6(\mathrm{q}, J=271.2$ $\mathrm{Hz}), 116.8,115.2$ (q, $J=33.0 \mathrm{~Hz}), 105.6,49.7,44.7,20.4 .{ }^{19} \mathrm{~F}$ NMR (471 MHz, $\left.\mathrm{CDCl}_{3}\right) \delta$-61.1. HRMS-ESI (m/z): $[\mathrm{M}+\mathrm{H}]^{+}$calcd. for $\left[\mathrm{C}_{17} \mathrm{H}_{19} \mathrm{~N}_{3} \mathrm{~F}_{3}\right]^{+}, 322.1531$; found, 322.1531 . 


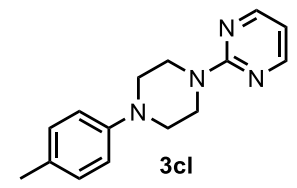

White solid, $38.9 \mathrm{mg}$, 38\% yield (General condition B, 5 mol\% Cat. 10, 36 hours); ${ }^{1} \mathrm{H}$ NMR $\left(500 \mathrm{MHz}, \mathrm{CDCl}_{3}\right) \delta 8.33(\mathrm{~d}, J=4.7 \mathrm{~Hz}, 2 \mathrm{H}), 7.10(\mathrm{~d}, J=8.1 \mathrm{~Hz}, 2 \mathrm{H}), 6.90$ $(\mathrm{d}, J=8.4 \mathrm{~Hz}, 2 \mathrm{H}), 6.51(\mathrm{t}, J=4.7 \mathrm{~Hz}, 1 \mathrm{H}), 3.98(\mathrm{t}, J=5.2 \mathrm{~Hz}, 4 \mathrm{H}), 3.20(\mathrm{t}, J=5.2$ $\mathrm{Hz}, 4 \mathrm{H}), 2.28$ (s, 3H). ${ }^{13} \mathrm{C} \mathrm{NMR}\left(126 \mathrm{MHz}, \mathrm{CDCl}_{3}\right) \delta 161.7,157.7,149.2,129.8$, 129.7, 116.9, 110.0, 49.9, 43.7, 20.4. HRMS-ESI $(\mathrm{m} / \mathrm{z}):[\mathrm{M}+\mathrm{H}]^{+}$calcd. for $\left[\mathrm{C}_{15} \mathrm{H}_{19} \mathrm{~N}_{4}\right]^{+}, 255.1610$; found, 255.1607.

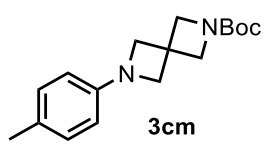

White solid, $52.2 \mathrm{mg}, 45 \%$ yield (General condition B, 36 hours); ${ }^{1} \mathrm{H}$ NMR (500 $\left.\mathrm{MHz}, \mathrm{CDCl}_{3}\right) \delta 7.03(\mathrm{~d}, J=8.1 \mathrm{~Hz}, 2 \mathrm{H}), 6.39(\mathrm{~d}, J=8.4 \mathrm{~Hz}, 2 \mathrm{H}), 4.08(\mathrm{~s}, 4 \mathrm{H}), 3.92$ $(\mathrm{s}, 4 \mathrm{H}), 2.25(\mathrm{~s}, 3 \mathrm{H}), 1.44(\mathrm{~s}, 9 \mathrm{H}) .{ }^{13} \mathrm{C} \mathrm{NMR}\left(126 \mathrm{MHz}, \mathrm{CDCl}_{3}\right) \delta 156.1,149.2,129.5$, 127.5, 112.0, 79.7, 62.5, 59.4, 33.5, 28.4, 20.4. HRMS-ESI $(\mathrm{m} / \mathrm{z}):[\mathrm{M}+\mathrm{H}]^{+}$calcd. for $\left[\mathrm{C}_{17} \mathrm{H}_{25} \mathrm{~N}_{2} \mathrm{O}_{2}\right]^{+}, 289.1916$; found, 289.1913.

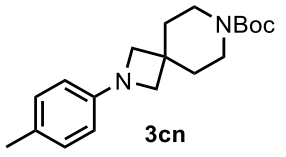

Pink solid, $115.0 \mathrm{mg}, 91 \%$ yield (General condition B, 24 hours); ${ }^{1} \mathrm{H}$ NMR (500 $\left.\mathrm{MHz} \mathrm{CDCl}_{3}\right) \delta 7.03(\mathrm{~d}, J=8.1 \mathrm{~Hz}, 2 \mathrm{H}), 6.39(\mathrm{~d}, J=8.3 \mathrm{~Hz}, 2 \mathrm{H}), 3.59$ (s, 4H), 3.40 (t, $J=5.4 \mathrm{~Hz}, 4 \mathrm{H}), 2.25(\mathrm{~s}, 3 \mathrm{H}), 1.76(\mathrm{t}, J=5.4 \mathrm{~Hz}, 4 \mathrm{H}), 1.47(\mathrm{~s}, 9 \mathrm{H}) .{ }^{13} \mathrm{C}$ NMR $(126$ $\left.\mathrm{MHz}, \mathrm{CDCl}_{3}\right) \delta 154.7,149.6,129.4,126.4,111.3,79.4,61.7,40.9,35.5,34.5,28.3$, 20.3. HRMS-ESI $(\mathrm{m} / \mathrm{z}):[\mathrm{M}+\mathrm{H}]^{+}$calcd. for $\left[\mathrm{C}_{19} \mathrm{H}_{29} \mathrm{~N}_{2} \mathrm{O}_{2}\right]^{+}, 317.2229$; found, 317.2230 .

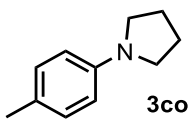

White solid, $53.1 \mathrm{mg}, 82 \%$ yield, known compound ${ }^{4}$ (General condition B, 24 hours); ${ }^{1} \mathrm{H}$ NMR $\left(500 \mathrm{MHz}, \mathrm{CDCl}_{3}\right) \delta 7.05(\mathrm{~d}, J=8.3 \mathrm{~Hz}, 2 \mathrm{H}), 6.52(\mathrm{~d}, J=8.5 \mathrm{~Hz}, 2 \mathrm{H}), 3.27$ $(\mathrm{t}, J=6.6 \mathrm{~Hz}, 4 \mathrm{H}), 2.27(\mathrm{~s}, 3 \mathrm{H}), 2.02-1.99(\mathrm{~m}, 4 \mathrm{H}) .{ }^{13} \mathrm{C} \mathrm{NMR}\left(126 \mathrm{MHz}, \mathrm{CDCl}_{3}\right) \delta$ $146.1,129.6,124.4,111.8,47.8,25.4,20.2$.

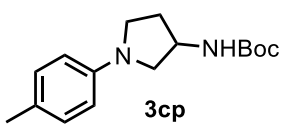

White solid, $53.1 \mathrm{mg}, 48 \%$ yield (General condition B, 24 hours); ${ }^{1} \mathrm{H}$ NMR (500 $\left.\mathrm{MHz} \mathrm{CDCl}_{3}\right) \delta 7.06(\mathrm{~d}, J=8.1 \mathrm{~Hz}, 2 \mathrm{H}), 6.50(\mathrm{~d}, J=8.2 \mathrm{~Hz}, 2 \mathrm{H}), 4.78(\mathrm{~s}, 1 \mathrm{H}), 4.36$ $(\mathrm{s}, 1 \mathrm{H}), 3.53-3.50(\mathrm{~m}, 1 \mathrm{H}), 3.43-3.39(\mathrm{~m}, 1 \mathrm{H}), 3.30-3.26(\mathrm{~m}, 1 \mathrm{H}), 3.18-3.10(\mathrm{~m}$, 1H), $2.29-2.22(\mathrm{~m}, 4 \mathrm{H}), 1.93-1.89(\mathrm{~m}, 1 \mathrm{H}), 1.47(\mathrm{~s}, 9 \mathrm{H}) .{ }^{13} \mathrm{C}$ NMR (126 MHz, $\left.\mathrm{CDCl}_{3}\right) \delta 155.4,145.6,129.7,125.3,111.9,79.5,54.1,50.4,46.0,31.9,28.4,20.2$. HRMS-ESI (m/z): $[\mathrm{M}+\mathrm{H}]^{+}$calcd. for $\left[\mathrm{C}_{16} \mathrm{H}_{25} \mathrm{~N}_{2} \mathrm{O}_{2}\right]^{+}, 277.1916$; found, 277.1917. 


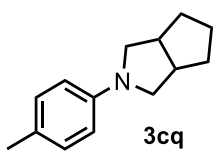

White solid, $43.3 \mathrm{mg}, 54 \%$ yield (General condition B, 24 hours); ${ }^{1} \mathrm{H}$ NMR (500 $\left.\mathrm{MHz} \mathrm{CDCl}_{3}\right) \delta 7.06(\mathrm{~d}, J=8.2 \mathrm{~Hz}, 2 \mathrm{H}), 6.58(\mathrm{~d}, J=8.4 \mathrm{~Hz}, 2 \mathrm{H}), 3.44-3.41(\mathrm{~m}, 2 \mathrm{H})$, $3.00(\mathrm{dd}, J=9.3,3.6 \mathrm{~Hz}, 2 \mathrm{H}), 2.82-2.75(\mathrm{~m}, 2 \mathrm{H}), 2.28(\mathrm{~s}, 3 \mathrm{H}), 1.90-1.83(\mathrm{~m}, 2 \mathrm{H})$, $1.77-1.71(\mathrm{~m}, 1 \mathrm{H}), 1.62-1.50(\mathrm{~m}, 3 \mathrm{H}) .{ }^{13} \mathrm{C} \mathrm{NMR}\left(126 \mathrm{MHz}, \mathrm{CDCl}_{3}\right) \delta 146.9,129.5$, 125.5, 113.3, 55.7, 42.6, 33.2, 25.8, 20.3. HRMS-ESI $(\mathrm{m} / \mathrm{z}):[\mathrm{M}+\mathrm{H}]^{+}$calcd. for $\left[\mathrm{C}_{14} \mathrm{H}_{20} \mathrm{~N}\right]^{+}, 202.1596$; found, 202.1590 .

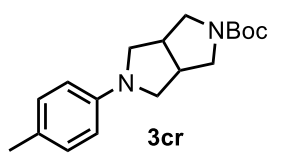

White solid, $77.9 \mathrm{mg}, 64 \%$ yield (General condition B, 24 hours); ${ }^{1} \mathrm{H}$ NMR (500 $\mathrm{MHz}^{\mathrm{CDCl}} \mathrm{CD}_{3} \delta 7.05(\mathrm{~d}, J=8.3 \mathrm{~Hz}, 2 \mathrm{H}), 6.49(\mathrm{~d}, J=8.4 \mathrm{~Hz}, 2 \mathrm{H}), 3.67-3.63(\mathrm{~m}, 2 \mathrm{H})$, $3.54-3.48(\mathrm{~m}, 2 \mathrm{H}), 3.38(\mathrm{~d}, J=9.4 \mathrm{~Hz}, 1 \mathrm{H}), 3.25-3.18(\mathrm{~m}, 3 \mathrm{H}), 2.99-2.97(\mathrm{~m}$, 2H), $2.26(\mathrm{~s}, 3 \mathrm{H}), 1.46(\mathrm{~s}, 9 \mathrm{H}) .{ }^{13} \mathrm{C} \mathrm{NMR}\left(126 \mathrm{MHz}, \mathrm{CDCl}_{3}\right) \delta 154.5,145.6,129.7$, 125.3, 112.0, 76.8, 52.4, 50.6, 50.2, 42.2, 41.3, 28.4, 20.2. HRMS-ESI (m/z): $[\mathrm{M}+\mathrm{H}]^{+}$ calcd. for $\left[\mathrm{C}_{18} \mathrm{H}_{27} \mathrm{~N}_{2} \mathrm{O}_{2}\right]^{+}, 303.2072$; found, 303.2071.

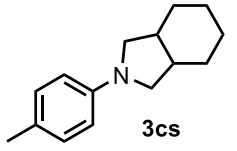

Colorless oil, $53.0 \mathrm{mg}, 62 \%$ yield (General condition B, 24 hours); ${ }^{1} \mathrm{H}$ NMR (500 $\left.\mathrm{MHz} \mathrm{CDCl}_{3}\right) \delta 7.03(\mathrm{~d}, J=8.2 \mathrm{~Hz}, 2 \mathrm{H}), 6.44(\mathrm{~d}, J=8.5 \mathrm{~Hz}, 2 \mathrm{H}), 3.28-3.26(\mathrm{~m}, 2 \mathrm{H})$, $3.16-3.13(\mathrm{~m}, 2 \mathrm{H}), 2.33-2.29(\mathrm{~m}, 2 \mathrm{H}), 2.25(\mathrm{~s}, 3 \mathrm{H}), 1.66-1.57(\mathrm{~m}, 2 \mathrm{H}), 1.53-$ $1.48(\mathrm{~m}, 4 \mathrm{H}), 1.42-1.32(\mathrm{~m}, 2 \mathrm{H}) .{ }^{13} \mathrm{C} \mathrm{NMR}\left(126 \mathrm{MHz}, \mathrm{CDCl}_{3}\right) \delta 146.2,129.6,123.7$, 111.0, 51.8, 37.3, 26.5, 23.1, 20.2. HRMS-ESI (m/z): $[\mathrm{M}+\mathrm{H}]^{+}$calcd. for $\left[\mathrm{C}_{15} \mathrm{H}_{22} \mathrm{~N}\right]^{+}$, 216.1752; found, 216.1743.

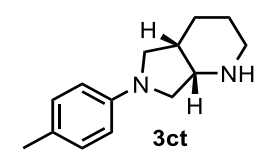

Light yellow solid, $51.0 \mathrm{mg}$, 59\% yield (General condition B, 36 hours); ${ }^{1} \mathrm{H}$ NMR $\left(500 \mathrm{MHz}, \mathrm{CDCl}_{3}\right) \delta 7.04(\mathrm{~d}, J=8.1 \mathrm{~Hz}, 2 \mathrm{H}), 6.46(\mathrm{~d}, J=8.2 \mathrm{~Hz}, 2 \mathrm{H}), 3.46-3.36(\mathrm{~m}$, $3 \mathrm{H}), 3.26(\mathrm{t}, J=8.5 \mathrm{~Hz}, 1 \mathrm{H}), 3.20(\mathrm{~d}, J=9.3 \mathrm{~Hz}, 1 \mathrm{H}), 3.02-2.99(\mathrm{~m}, 1 \mathrm{H}), 2.69-$ $2.64(\mathrm{~m}, 1 \mathrm{H}), 2.35-2.31(\mathrm{~m}, 1 \mathrm{H}), 2.26(\mathrm{~s}, 3 \mathrm{H}), 1.85-1.73(\mathrm{~m}, 2 \mathrm{H}), 1.70-1.59(\mathrm{~m}$, 2H), $1.51-1.48(\mathrm{~m}, 1 \mathrm{H}) .{ }^{13} \mathrm{C}$ NMR $\left(126 \mathrm{MHz}, \mathrm{CDCl}_{3}\right) \delta 146.0,129.5,124.2,111.2$, 56.4, 54.3, 49.3, 44.6, 36.5, 23.8, 22.3, 20.2. HRMS-ESI $(\mathrm{m} / \mathrm{z}):[\mathrm{M}+\mathrm{H}]^{+}$calcd. for $\left[\mathrm{C}_{14} \mathrm{H}_{21} \mathrm{~N}_{2}\right]^{+}, 217.1705$; found, 217.1699.

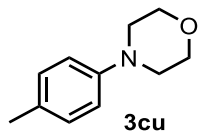

Colorless oil, $45.4 \mathrm{mg}, 64 \%$ yield, known compound ${ }^{4}$ (General condition B, 24 hours); ${ }^{1} \mathrm{H}$ NMR $\left(500 \mathrm{MHz}, \mathrm{CDCl}_{3}\right) \delta 7.10(\mathrm{~d}, J=8.4 \mathrm{~Hz}, 2 \mathrm{H}), 6.84(\mathrm{~d}, J=8.6 \mathrm{~Hz}$, $2 \mathrm{H}), 3.87(\mathrm{t}, J=4.8 \mathrm{~Hz}, 4 \mathrm{H}), 3.11(\mathrm{t}, J=4.8 \mathrm{~Hz}, 4 \mathrm{H}), 2.28(\mathrm{~s}, 3 \mathrm{H}) .{ }^{13} \mathrm{C}$ NMR $(126$ $\left.\mathrm{MHz} \mathrm{CDCl}_{3}\right) \delta 149.2,129.7,129.6,116.0,67.0,49.9,20.4$. 


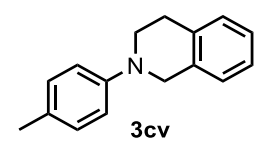

Colorless oil, $66.7 \mathrm{mg}, 75 \%$ yield, known compound ${ }^{32}$ (General condition B, 24 hours); ${ }^{1} \mathrm{H}$ NMR $\left(500 \mathrm{MHz}, \mathrm{CDCl}_{3}\right) \delta 7.16-7.10(\mathrm{~m}, 4 \mathrm{H}), 7.07(\mathrm{~d}, J=8.3 \mathrm{~Hz}, 2 \mathrm{H})$, $6.89(\mathrm{~d}, J=8.6 \mathrm{~Hz}, 2 \mathrm{H}), 4.32(\mathrm{~s}, 2 \mathrm{H}), 3.47(\mathrm{t}, J=5.9 \mathrm{~Hz}, 2 \mathrm{H}), 2.94(\mathrm{t}, J=5.8 \mathrm{~Hz}, 2 \mathrm{H})$, $2.26(\mathrm{~s}, 3 \mathrm{H}) .{ }^{13} \mathrm{C} \mathrm{NMR}\left(126 \mathrm{MHz}, \mathrm{CDCl}_{3}\right) \delta 148.6,134.7,134.5,129.7,128.5,128.3$, $126.5,126.2,125.9,115.8,51.4,47.2,29.0,20.4$.

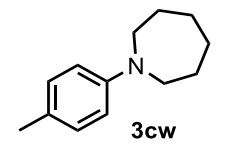

Colorless oil, $47.9 \mathrm{mg}, 63 \%$ yield, known compound ${ }^{5}$ (General condition B, 24 hours); ${ }^{1} \mathrm{H}$ NMR $\left(500 \mathrm{MHz}, \mathrm{CDCl}_{3}\right) \delta 7.02(\mathrm{~d}, J=8.5 \mathrm{~Hz}, 2 \mathrm{H}), 6.62(\mathrm{~d}, J=8.6 \mathrm{~Hz}$, $2 \mathrm{H}), 3.44(\mathrm{t}, J=6.0 \mathrm{~Hz}, 4 \mathrm{H}), 2.24(\mathrm{~s}, 3 \mathrm{H}), 1.81-1.75(\mathrm{~m}, 4 \mathrm{H}), 1.54-1.50(\mathrm{~m}, 4 \mathrm{H})$.

${ }^{13} \mathrm{C} \mathrm{NMR}\left(126 \mathrm{MHz}, \mathrm{CDCl}_{3}\right) \delta 146.8,129.8,124.1,111.2,49.2,27.8,27.2,20.1$.

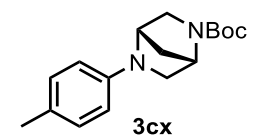

White solid, $60.6 \mathrm{mg}$, 53\% yield, known compound ${ }^{33}$ (General condition B, 24 hours); ${ }^{1} \mathrm{H}$ NMR $\left(500 \mathrm{MHz}, \mathrm{CDCl}_{3}\right.$, rotamers) $\delta 7.05 \& 7.03(\mathrm{~d}, J=8.5 \mathrm{~Hz}, 2 \mathrm{H}), 6.49$ \& $6.47(\mathrm{~d}, J=7.9 \mathrm{~Hz}, 2 \mathrm{H}), 4.61 \& 4.46(\mathrm{~s}, 1 \mathrm{H}), 4.35(\mathrm{~s}, 1 \mathrm{H}), 3.58-3.33(\mathrm{~m}, 3 \mathrm{H})$, $3.17 \& 3.08(\mathrm{~d}, J=8.6 \mathrm{~Hz}, 1 \mathrm{H}), 2.26 \& 2.25(\mathrm{~s}, 3 \mathrm{H}), 1.98-1.96(\mathrm{~m}, 1 \mathrm{H}), 1.92-1.86$ $(\mathrm{m}, 1 \mathrm{H}), 1.44 \& 1.42(\mathrm{~s}, 9 \mathrm{H}) .{ }^{13} \mathrm{C} \mathrm{NMR}\left(126 \mathrm{MHz}, \mathrm{CDCl}_{3}\right) \delta 154.1,144.6,129.8$, $125.7,112.5,79.5,57.3,57.0,56.5,50.8,37.4,28.5,20.3$.<smiles>CCN(C)c1ccc(C)cc1</smiles>

Light yellow oil, $44.7 \mathrm{mg}, 58 \%$ yield (General condition B, 36 hours); ${ }^{1} \mathrm{H}$ NMR (500 $\left.\mathrm{MHz} \mathrm{CDCl}_{3}\right) \delta 7.05(\mathrm{~d}, J=8.4 \mathrm{~Hz}, 2 \mathrm{H}), 6.65(\mathrm{~d}, J=8.6 \mathrm{~Hz}, 2 \mathrm{H}), 3.27(\mathrm{t}, J=7.6 \mathrm{~Hz}$, 2H), 2.90 (s, 3H), $2.27(\mathrm{~s}, 3 \mathrm{H}), 1.60-1.54(\mathrm{~m}, 2 \mathrm{H}), 1.40-1.28(\mathrm{~m}, 4 \mathrm{H}), 0.92$ (t, $J=$ $7.1 \mathrm{~Hz}, 3 \mathrm{H}) .{ }^{13} \mathrm{C} \mathrm{NMR}\left(126 \mathrm{MHz}, \mathrm{CDCl}_{3}\right) \delta 147.5,129.6,125.1,112.6,53.1,38.4$, 29.4, 26.2, 22.6, 20.2, 14.1. HRMS-ESI $(\mathrm{m} / \mathrm{z}):[\mathrm{M}+\mathrm{H}]^{+}$calcd. for $\left[\mathrm{C}_{13} \mathrm{H}_{22} \mathrm{~N}\right]^{+}$, 192.1752; found, 192.1747.

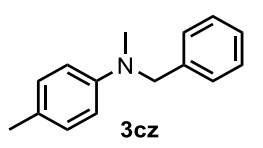

Light yellow oil, $52.0 \mathrm{mg}, 62 \%$ yield, known compound ${ }^{4}$ (General condition B, 36 hours); ${ }^{1} \mathrm{H}$ NMR (500 MHz, $\left.\mathrm{CDCl}_{3}\right) \delta 7.31-7.28(\mathrm{~m}, 2 \mathrm{H}), 7.24-7.21(\mathrm{~m}, 3 \mathrm{H}), 7.02$ $(\mathrm{d}, J=8.4 \mathrm{~Hz}, 2 \mathrm{H}), 6.68(\mathrm{~d}, J=8.6 \mathrm{~Hz}, 2 \mathrm{H}), 4.48(\mathrm{~s}, 2 \mathrm{H}), 2.96(\mathrm{~s}, 3 \mathrm{H}), 2.24(\mathrm{~s}, 3 \mathrm{H})$. ${ }^{13} \mathrm{C}$ NMR $\left(126 \mathrm{MHz}, \mathrm{CDCl}_{3}\right) \delta 147.8,139.2,129.7,128.5,126.8,126.8,125.8,112.7$, $57.0,38.6,20.2$.<smiles>Cc1ccc(N(C)CCc2ccccc2)cc1</smiles> 
Light yellow oil, $53.7 \mathrm{mg}, 60 \%$ yield, known compound ${ }^{34}$ (General condition B, 36 hours); ${ }^{1} \mathrm{H}$ NMR (500 MHz, $\left.\mathrm{CDCl}_{3}\right) \delta 7.30-7.27(\mathrm{~m}, 2 \mathrm{H}), 7.21-7.18(\mathrm{~m}, 3 \mathrm{H}), 7.06$ $(\mathrm{d}, J=8.3 \mathrm{~Hz}, 2 \mathrm{H}), 6.66(\mathrm{~d}, J=8.6 \mathrm{~Hz}, 2 \mathrm{H}), 3.52(\mathrm{t}, J=7.7 \mathrm{~Hz}, 2 \mathrm{H}), 2.85(\mathrm{~s}, 3 \mathrm{H})$, $2.82(\mathrm{t}, J=7.9 \mathrm{~Hz}, 2 \mathrm{H}), 2.26(\mathrm{~s}, 3 \mathrm{H}) .{ }^{13} \mathrm{C} \mathrm{NMR}\left(126 \mathrm{MHz}, \mathrm{CDCl}_{3}\right) \delta 146.8,139.9$, $129.8,128.8,128.5,126.1,125.4,112.6,55.0,38.6,32.7,20.2$.<smiles>COCCN(C)c1ccc(C)cc1</smiles>

Colorless oil, $50.2 \mathrm{mg}, 69 \%$ yield (General condition B, 24 hours); ${ }^{1} \mathrm{H}$ NMR (500 $\left.\mathrm{MHz}, \mathrm{CDCl}_{3}\right) \delta 7.08(\mathrm{~d}, J=8.4 \mathrm{~Hz}, 2 \mathrm{H}), 6.70(\mathrm{~d}, J=8.6 \mathrm{~Hz}, 2 \mathrm{H}), 3.58(\mathrm{t}, J=5.9 \mathrm{~Hz}$, $2 \mathrm{H}), 3.51(\mathrm{t}, J=6.0 \mathrm{~Hz}, 2 \mathrm{H}), 3.39(\mathrm{~s}, 3 \mathrm{H}), 2.98(\mathrm{~s}, 3 \mathrm{H}), 2.29(\mathrm{~s}, 3 \mathrm{H}) .{ }^{13} \mathrm{C} \mathrm{NMR}(126$ $\left.\mathrm{MHz}, \mathrm{CDCl}_{3}\right) \delta 147.3,129.6,125.6,112.6,70.1,58.9,52.7,39.0,20.1$. HRMS-ESI $(\mathrm{m} / \mathrm{z}):[\mathrm{M}+\mathrm{H}]^{+}$calcd. for $\left[\mathrm{C}_{11} \mathrm{H}_{18} \mathrm{NO}\right]^{+}, 180.1388$; found, 180.1383 .<smiles>CCCN(C)c1ccc(C)cc1</smiles>

Yellow oil, $32.0 \mathrm{mg}$, 42\% yield, known compound ${ }^{35}$ (General condition B, 36 hours); ${ }^{1} \mathrm{H}$ NMR $\left(500 \mathrm{MHz}, \mathrm{CDCl}_{3}\right) \delta 7.04(\mathrm{~d}, J=8.3 \mathrm{~Hz}, 2 \mathrm{H}), 6.65(\mathrm{~d}, J=8.4 \mathrm{~Hz}, 2 \mathrm{H}), 3.45$ (t, $J=7.5 \mathrm{~Hz}, 2 \mathrm{H}), 2.92(\mathrm{~s}, 3 \mathrm{H}), 2.52(\mathrm{t}, J=7.6 \mathrm{~Hz}, 2 \mathrm{H}), 2.33(\mathrm{~s}, 6 \mathrm{H}), 2.25(\mathrm{~s}, 3 \mathrm{H})$. ${ }^{13} \mathrm{C}$ NMR $\left(126 \mathrm{MHz}, \mathrm{CDCl}_{3}\right) \delta 147.1,129.8,125.7,112.7,55.7,51.2,45.7,38.8$, 20.2 .<smiles>Cc1ccc(N(C)CC2CC2)cc1</smiles>

Pink oil, $41.3 \mathrm{mg}, 55 \%$ yield (General condition B, 5 mol\% Cat. 10, 36 hours); ${ }^{1} \mathrm{H}$ NMR (500 MHz, $\left.\mathrm{CDCl}_{3}\right) \delta 7.04(\mathrm{~d}, J=8.5 \mathrm{~Hz}, 2 \mathrm{H}), 6.65(\mathrm{~d}, J=8.6 \mathrm{~Hz}, 2 \mathrm{H}), 3.29$ (d, $J=6.9 \mathrm{~Hz}, 2 \mathrm{H}), 2.90(\mathrm{~s}, 3 \mathrm{H}), 2.73-2.63(\mathrm{~m}, 1 \mathrm{H}), 2.26(\mathrm{~s}, 3 \mathrm{H}), 2.07-1.98(\mathrm{~m}, 2 \mathrm{H})$, $1.93-1.72(\mathrm{~m}, 4 \mathrm{H}) .{ }^{13} \mathrm{C} \mathrm{NMR}\left(126 \mathrm{MHz}, \mathrm{CDCl}_{3}\right) \delta 147.8,129.6,125.3,112.8,58.8$, 38.9, 34.4, 27.2, 20.2, 18.8. HRMS-ESI $(\mathrm{m} / \mathrm{z}):[\mathrm{M}+\mathrm{H}]^{+}$calcd. for $\left[\mathrm{C}_{13} \mathrm{H}_{20} \mathrm{~N}\right]^{+}$, 190.1596; found, 190.1593.

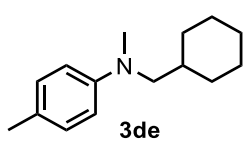

Brown oil, $48.9 \mathrm{mg}, 56 \%$ yield, known compound ${ }^{36}$ (General condition B, 5 mol\% Cat. 10, 36 hours); ${ }^{1} \mathrm{H}$ NMR $\left(500 \mathrm{MHz}, \mathrm{CDCl}_{3}\right) \delta 7.03(\mathrm{~d}, J=8.3 \mathrm{~Hz}, 2 \mathrm{H}), 6.59$ (d, $J$ $=8.6 \mathrm{~Hz}, 2 \mathrm{H}), 3.08(\mathrm{~d}, J=6.8 \mathrm{~Hz}, 2 \mathrm{H}), 2.92(\mathrm{~s}, 3 \mathrm{H}), 2.25(\mathrm{~s}, 3 \mathrm{H}), 1.75-1.66(\mathrm{~m}, 6 \mathrm{H})$, $1.22-1.14(\mathrm{~m}, 3 \mathrm{H}), 0.96-0.88(\mathrm{t}, J=11.7 \mathrm{~Hz}, 2 \mathrm{H}) .{ }^{13} \mathrm{C} \mathrm{NMR}\left(126 \mathrm{MHz}, \mathrm{CDCl}_{3}\right) \delta$ $147.7,129.6,124.5,111.9,60.0,39.7,36.9,31.3,26.6,26.0,20.1$.

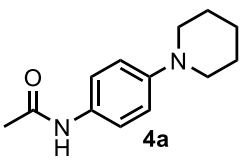

White solid, $58.4 \mathrm{mg}$, 67\% yield, known compound ${ }^{37}$ (General condition A, 5 mol\% Cat. 10, 36 hours); ${ }^{1} \mathrm{H}$ NMR $\left(500 \mathrm{MHz}, \mathrm{CDCl}_{3}\right) \delta 7.34(\mathrm{~d}, J=9.0 \mathrm{~Hz}, 2 \mathrm{H}), 7.30$ (br, $1 \mathrm{H}), 6.90(\mathrm{~d}, J=8.9 \mathrm{~Hz}, 2 \mathrm{H}), 3.10(\mathrm{t}, J=5.4 \mathrm{~Hz}, 4 \mathrm{H}), 2.13(\mathrm{~s}, 3 \mathrm{H}), 1.73-1.69$ (m, 
4H), $1.58-1.53(\mathrm{~m}, 2 \mathrm{H}) .{ }^{13} \mathrm{C} \mathrm{NMR}\left(126 \mathrm{MHz}, \mathrm{CDCl}_{3}\right) \delta 168.2,149.1,130.1,121.5$, 117.1, 51.2, 25.7, 24.3, 24.1.

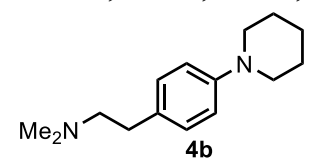

White solid, $46.4 \mathrm{mg}, 50 \%$ yield (General condition A, 36 hours); ${ }^{1} \mathrm{H}$ NMR (500 $\left.\mathrm{MHz} \mathrm{CDCl}_{3}\right) \delta 7.08(\mathrm{~d}, J=8.5 \mathrm{~Hz}, 2 \mathrm{H}), 6.87(\mathrm{~d}, J=8.6 \mathrm{~Hz}, 2 \mathrm{H}), 3.10(\mathrm{t}, J=5.4 \mathrm{~Hz}$, $4 \mathrm{H}), 2.72-2.69(\mathrm{~m}, 2 \mathrm{H}), 2.53-2.50(\mathrm{~m}, 2 \mathrm{H}), 2.30(\mathrm{~s}, 6 \mathrm{H}), 1.72-1.68(\mathrm{~m}, 4 \mathrm{H}), 1.58$ $-1.54(\mathrm{~m}, 2 \mathrm{H}) .{ }^{13} \mathrm{C} \mathrm{NMR}\left(126 \mathrm{MHz}, \mathrm{CDCl}_{3}\right) \delta 150.6,130.8,129.1,116.8,61.6,50.9$, 45.3, 33.2, 25.9, 24.2. HRMS-ESI (m/z): $[\mathrm{M}+\mathrm{H}]^{+}$calcd. for $\left[\mathrm{C}_{15} \mathrm{H}_{25} \mathrm{~N}_{2}\right]^{+}, 233.2018$; found, 233.2016.

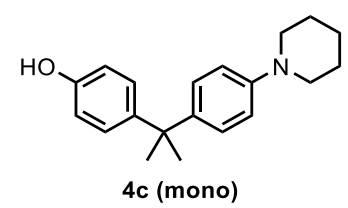

Brown yellow solid, $47.3 \mathrm{mg}, 40 \%$ yield (General condition A, $0.4 \mathrm{mmol}$ bisphenol A, $5 \mathrm{~mol} \%$ Cat. 10, 10 mol\% $\mathrm{Cs}_{2} \mathrm{CO}_{3}$ instead of $\mathrm{Na}_{2} \mathrm{CO}_{3}, 36$ hours); ${ }^{1} \mathrm{H}$ NMR (500 $\left.\mathrm{MHz} \mathrm{CDCl}_{3}\right) \delta 7.12(\mathrm{~d}, J=8.5 \mathrm{~Hz}, 2 \mathrm{H}), 7.01(\mathrm{~d}, J=8.4 \mathrm{~Hz}, 2 \mathrm{H}), 6.91(\mathrm{~d}, J=7.6 \mathrm{~Hz}$, $2 \mathrm{H}), 6.59(\mathrm{~d}, J=8.4 \mathrm{~Hz}, 2 \mathrm{H}), 6.09(\mathrm{br}, 1 \mathrm{H}), 3.13(\mathrm{t}, J=4.9 \mathrm{~Hz}, 4 \mathrm{H}), 1.78-1.71(\mathrm{~m}$, $4 \mathrm{H}), 1.60(\mathrm{~s}, 6 \mathrm{H}), 1.60-1.51(\mathrm{~m}, 2 \mathrm{H}) .{ }^{13} \mathrm{C} \mathrm{NMR}\left(126 \mathrm{MHz}, \mathrm{CDCl}_{3}\right) \delta 153.8,149.6$, $142.7,142.5,127.7,127.4,116.6,114.7,51.2,41.5,31.0,25.6,24.1$. HRMS-ESI $(\mathrm{m} / \mathrm{z}):[\mathrm{M}+\mathrm{H}]^{+}$calcd. for $\left[\mathrm{C}_{20} \mathrm{H}_{26} \mathrm{NO}\right]^{+}, 296.2014$; found, 296.2012.

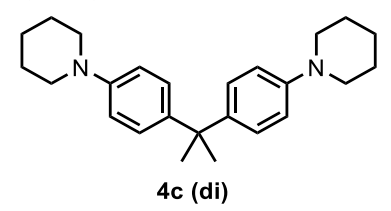

White solid, $21.5 \mathrm{mg}, 30 \%$ yield, known compound ${ }^{38}$ (General condition A, 0.4 mmol bisphenol A, 5 mol\% Cat. 10, 10 mol\% $\mathrm{Cs}_{2} \mathrm{CO}_{3}$ instead of $\mathrm{Na}_{2} \mathrm{CO}_{3}, 36$ hours); ${ }^{1} \mathrm{H}$ NMR $\left(500 \mathrm{MHz}, \mathrm{CDCl}_{3}\right) \delta 7.11(\mathrm{~d}, J=8.8 \mathrm{~Hz}, 4 \mathrm{H}), 6.84(\mathrm{~d}, J=8.8 \mathrm{~Hz}, 4 \mathrm{H}), 3.11$ $(\mathrm{t}, J=5.4 \mathrm{~Hz}, 8 \mathrm{H}), 1.73-1.68(\mathrm{~m}, 8 \mathrm{H}), 1.62(\mathrm{~s}, 6 \mathrm{H}), 1.58-1.54(\mathrm{~m}, 4 \mathrm{H}) .{ }^{13} \mathrm{C} \mathrm{NMR}$ $\left(126 \mathrm{MHz}, \mathrm{CDCl}_{3}\right) \delta 149.8,141.9,127.3,115.9,50.7,41.4,30.9,26.0,24.3$.

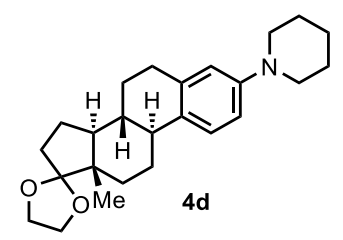

Colorless sticky oil, $73.6 \mathrm{mg}, 48 \%$ yield (General condition A, 36 hours); ${ }^{1} \mathrm{H}$ NMR $\left(500 \mathrm{MHz}, \mathrm{CDCl}_{3}\right) \delta 7.18(\mathrm{~d}, J=8.6 \mathrm{~Hz}, 1 \mathrm{H}), 6.76(\mathrm{~d}, J=8.5 \mathrm{~Hz}, 1 \mathrm{H}), 6.68(\mathrm{~s}, 1 \mathrm{H})$, $3.98-3.90(\mathrm{~m}, 4 \mathrm{H}), 3.10(\mathrm{t}, J=5.4 \mathrm{~Hz}, 4 \mathrm{H}), 2.88-2.78(\mathrm{~m}, 2 \mathrm{H}), 2.35-2.29(\mathrm{~m}, 1 \mathrm{H})$, $2.26-2.19(\mathrm{~m}, 1 \mathrm{H}), 2.03(\mathrm{t}, J=11.5 \mathrm{~Hz}, 1 \mathrm{H}), 1.91-1.31(\mathrm{~m}, 16 \mathrm{H}), 0.89(\mathrm{~s}, 3 \mathrm{H}) .{ }^{13} \mathrm{C}$ NMR $\left(126 \mathrm{MHz}, \mathrm{CDCl}_{3}\right) \delta 150.2,137.1,131.5,125.8,119.5,117.0,114.5,65.2,64.5$, 51.0, 49.4, 46.2, 43.6, 39.1, 34.2, 30.7, 29.9, 27.1, 26.0, 25.9, 24.3, 22.3, 14.3 . HRMS-ESI (m/z): $[\mathrm{M}+\mathrm{H}]^{+}$calcd. for $\left[\mathrm{C}_{25} \mathrm{H}_{36} \mathrm{NO}_{2}\right]^{+}, 382.2746$; found, 382.2762. 


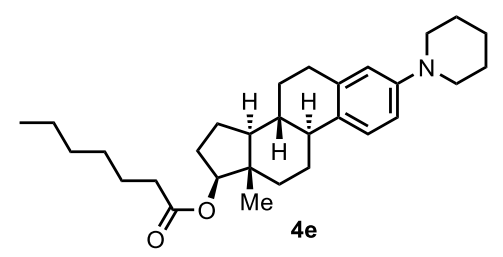

Colorless oil, $104.8 \mathrm{mg}$, 58\% yield (General condition A, 5 mol\% Cat. 10, 36 hours); ${ }^{1} \mathrm{H}$ NMR $\left(500 \mathrm{MHz}, \mathrm{CDCl}_{3}\right) \delta 7.18(\mathrm{~d}, J=8.6 \mathrm{~Hz}, 1 \mathrm{H}), 6.77(\mathrm{dd}, J=8.6,2.5 \mathrm{~Hz}, 1 \mathrm{H})$, $6.68(\mathrm{~d}, J=2.3 \mathrm{~Hz}, 1 \mathrm{H}), 4.71(\mathrm{t}, J=8.5 \mathrm{~Hz}, 1 \mathrm{H}), 3.11(\mathrm{t}, J=5.4 \mathrm{~Hz}, 4 \mathrm{H}), 2.88-2.80$ $(\mathrm{m}, 2 \mathrm{H}), 2.34-2.18(\mathrm{~m}, 5 \mathrm{H}), 1.91-1.83(\mathrm{~m}, 2 \mathrm{H}), 1.78-1.26(\mathrm{~m}, 22 \mathrm{H}), 0.92-0.90$ $(\mathrm{m}, 3 \mathrm{H}), 0.84(\mathrm{~s}, 3 \mathrm{H}) .{ }^{13} \mathrm{C} \mathrm{NMR}\left(126 \mathrm{MHz}, \mathrm{CDCl}_{3}\right) \delta 173.9,150.2,137.0,131.2$, $125.8,116.9,114.5,82.4,50.9,49.8,43.8,42.9,38.6,36.9,34.6,31.4,29.9,28.8$, 27.6, 27.4, 26.0, 25.9, 25.0, 24.3, 23.2, 22.5, 14.0, 12.1. HRMS-ESI (m/z): $[\mathrm{M}+\mathrm{H}]^{+}$ calcd. for $\left[\mathrm{C}_{30} \mathrm{H}_{46} \mathrm{NO}_{2}\right]^{+}, 452.3528$; found, 452.3532 .

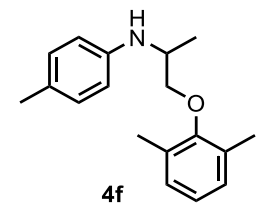

Yellow oil, $59.0 \mathrm{mg}, 55 \%$ yield (General condition B, 36 hours); ${ }^{1} \mathrm{H}$ NMR (500 MHz, $\left.\mathrm{CDCl}_{3}\right) \delta 7.04-7.02(\mathrm{~m}, 4 \mathrm{H}), 6.96-6.93(\mathrm{~m}, 1 \mathrm{H}), 6.64(\mathrm{~d}, J=8.4 \mathrm{~Hz}, 2 \mathrm{H}), 3.91-$ $3.78(\mathrm{~m}, 3 \mathrm{H}), 2.30(\mathrm{~s}, 6 \mathrm{H}), 2.28(\mathrm{~s}, 3 \mathrm{H}), 1.47(\mathrm{~d}, J=6.4 \mathrm{~Hz}, 3 \mathrm{H}) .{ }^{13} \mathrm{C}$ NMR $(126 \mathrm{MHz}$, $\left.\mathrm{CDCl}_{3}\right) \delta 155.2,144.8,130.8,129.8,128.9,126.8,123.9,113.8,74.3,49.1,20.4,18.1$, 16.2. HRMS-ESI (m/z): $[\mathrm{M}+\mathrm{H}]^{+}$calcd. for $\left[\mathrm{C}_{18} \mathrm{H}_{24} \mathrm{NO}\right]^{+}, 270.1858$; found, 270.1857 .

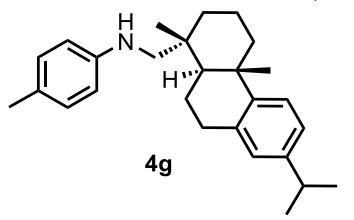

Colorless oil, $128.0 \mathrm{mg}, 85 \%$ yield, known compound ${ }^{39}$ (General condition B, 36 hours); ${ }^{1} \mathrm{H}$ NMR $\left(500 \mathrm{MHz}, \mathrm{CDCl}_{3}\right) \delta 7.20(\mathrm{~d}, J=8.2 \mathrm{~Hz}, 1 \mathrm{H}), 7.01(\mathrm{~d}, J=8.1 \mathrm{~Hz}$, $1 \mathrm{H}), 6.98(\mathrm{~d}, J=8.2 \mathrm{~Hz}, 2 \mathrm{H}), 6.91(\mathrm{~s}, 1 \mathrm{H}), 6.54(\mathrm{~d}, J=8.4 \mathrm{~Hz}, 2 \mathrm{H}), 3.48(\mathrm{br}, 1 \mathrm{H})$, $3.03(\mathrm{~d}, J=12.4 \mathrm{~Hz}, 1 \mathrm{H}), 2.94-2.81(\mathrm{~m}, 4 \mathrm{H}), 2.31(\mathrm{dt}, J=12.9,3.1 \mathrm{~Hz}, 1 \mathrm{H}), 2.24(\mathrm{~s}$, $3 \mathrm{H}), 1.86-1.70(\mathrm{~m}, 4 \mathrm{H}), 1.66-1.63(\mathrm{~m}, 1 \mathrm{H}), 1.48-1.39(\mathrm{~m}, 3 \mathrm{H}), 1.25(\mathrm{~s}, 6 \mathrm{H}), 1.24$ (s, 3H), $1.02(\mathrm{~s}, 3 \mathrm{H}) .{ }^{13} \mathrm{C} \mathrm{NMR}\left(126 \mathrm{MHz}, \mathrm{CDCl}_{3}\right) \delta 147.4,146.8,145.6,134.7,129.7$, $126.8,126.0,124.2,123.9,112.7,55.3,45.3,38.4,37.5,37.4,36.3,33.4,30.1,25.3$, 24.0, 20.3, 19.3, 18.8, 18.7 .

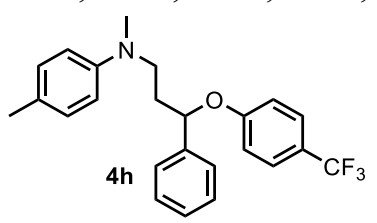

Colorless oil, $51.4 \mathrm{mg}, 32 \%$ yield (General condition B, 5 mol\% Cat. 10, $10 \mathrm{~mol} \%$ $\mathrm{Cs}_{2} \mathrm{CO}_{3}$ instead of $\mathrm{Na}_{2} \mathrm{CO}_{3}, 36$ hours); ${ }^{1} \mathrm{H} \mathrm{NMR}\left(500 \mathrm{MHz}, \mathrm{CDCl}_{3}\right) \delta 7.42$ (d, $J=8.7$ $\mathrm{Hz}, 2 \mathrm{H}), 7.34-7.29(\mathrm{~m}, 4 \mathrm{H}), 7.27-7.25(\mathrm{~m}, 1 \mathrm{H}), 6.98(\mathrm{~d}, J=8.4 \mathrm{~Hz}, 2 \mathrm{H}), 6.89$ (d, $J$ $=8.7 \mathrm{~Hz}, 2 \mathrm{H}), 6.61(\mathrm{~d}, J=8.5 \mathrm{~Hz}, 2 \mathrm{H}), 5.20(\mathrm{dd}, J=8.6,4.2 \mathrm{~Hz}, 1 \mathrm{H}), 3.57-3.44(\mathrm{~m}$, $2 \mathrm{H}), 2.87$ (s, 3H), 2.22 (s, 3H), $2.20-2.09$ (m, 2H). $\left.{ }^{13} \mathrm{C} \mathrm{NMR} \mathrm{(126} \mathrm{MHz,} \mathrm{CDCl}_{3}\right) \delta$ $160.5,147.2,140.9,129.7,128.8,127.8,126.8$ (q, $J=3.8 \mathrm{~Hz}), 125.8,125.7,124.4$ (q, 
$J=271.5 \mathrm{~Hz}), 122.8(\mathrm{q}, J=32.7 \mathrm{~Hz}), 115.7,112.9,78.0,49.4,38.7,36.0,20.1 .{ }^{19} \mathrm{~F}$ $\mathrm{NMR}\left(471 \mathrm{MHz}, \mathrm{CDCl}_{3}\right) \quad \delta$-61.5. HRMS-ESI $(\mathrm{m} / \mathrm{z}):[\mathrm{M}+\mathrm{H}]^{+}$calcd. for $\left[\mathrm{C}_{24} \mathrm{H}_{25} \mathrm{~F}_{3} \mathrm{NO}\right]^{+}, 400.1888$; found, 400.1885 .

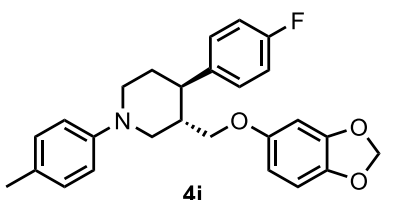

Colorless sticky oil, $88.7 \mathrm{mg}, 53 \%$ yield (General condition B, 5 mol\% Cat. 10, 36 hours); ${ }^{1} \mathrm{H}$ NMR (500 MHz, $\left.\mathrm{CDCl}_{3}\right) \delta 7.23-7.20(\mathrm{~m}, 2 \mathrm{H}), 7.13(\mathrm{~d}, J=8.2 \mathrm{~Hz}, 2 \mathrm{H})$, $7.02(\mathrm{t}, J=8.7 \mathrm{~Hz}, 2 \mathrm{H}), 6.97(\mathrm{~d}, J=8.5 \mathrm{~Hz}, 2 \mathrm{H}), 6.66(\mathrm{~d}, J=8.5 \mathrm{~Hz}, 1 \mathrm{H}), 6.40(\mathrm{~d}, J=$ $2.4 \mathrm{~Hz}, 1 \mathrm{H}), 6.19(\mathrm{dd}, J=8.5,2.5 \mathrm{~Hz}, 1 \mathrm{H}), 5.90(\mathrm{~s}, 2 \mathrm{H}), 4.07-3.94(\mathrm{~m}, 1 \mathrm{H}), 3.78-$ 3.75 (m, 1H), 3.67 (dd, $J=9.4,2.8 \mathrm{~Hz}, 1 \mathrm{H}), 3.57-3.53(\mathrm{~m}, 1 \mathrm{H}), 2.85-2.75(\mathrm{~m}, 2 \mathrm{H})$, $2.62(\mathrm{td}, J=11.6,4.2 \mathrm{~Hz}, 1 \mathrm{H}), 2.39-2.35(\mathrm{~m}, 1 \mathrm{H}), 2.32(\mathrm{~s}, 3 \mathrm{H}), 2.09-1.88(\mathrm{~m}, 2 \mathrm{H})$. ${ }^{13} \mathrm{C} \mathrm{NMR}\left(126 \mathrm{MHz}, \mathrm{CDCl}_{3}\right) \delta 162.5(\mathrm{~d}, J=245.0 \mathrm{~Hz}), 154.3,149.5,148.1,141.6$, $139.5(\mathrm{~d}, J=3.2 \mathrm{~Hz}), 129.6,129.3,128.8(\mathrm{~d}, J=7.8 \mathrm{~Hz}), 117.1,115.4(\mathrm{~d}, J=21.1$ $\mathrm{Hz}), 107.8,105.6,101.0,98.0,69.4,54.3,51.2$, 44.0, 41.8, 34.2, 20.4. ${ }^{19} \mathrm{~F} \mathrm{NMR}(471$ $\left.\mathrm{MHz}, \mathrm{CDCl}_{3}\right) \delta$-116.4. HRMS-ESI $(\mathrm{m} / \mathrm{z}):[\mathrm{M}+\mathrm{H}]^{+}$calcd. for $\left[\mathrm{C}_{26} \mathrm{H}_{27} \mathrm{FNO}_{3}\right]^{+}$, 420.1975; found, 420.1974.

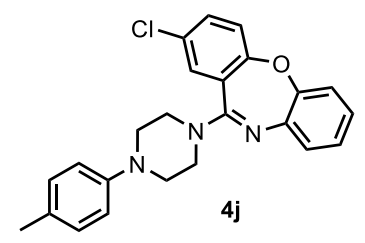

Colorless sticky oil, $49.6 \mathrm{mg}, 31 \%$ yield (General condition B, 5 mol\% Cat. 10, 36 hours); ${ }^{1} \mathrm{H}$ NMR $\left(500 \mathrm{MHz}, \mathrm{CDCl}_{3}\right) \delta 7.41(\mathrm{dd}, J=8.6,2.5 \mathrm{~Hz}, 1 \mathrm{H}), 7.37$ (d, $J=2.5$ $\mathrm{Hz}, 1 \mathrm{H}), 7.23-7.16(\mathrm{~m}, 2 \mathrm{H}), 7.12-7.09$ (m, 4H), 7.01 (t, $J=7.6 \mathrm{~Hz}, 1 \mathrm{H}), 6.90$ (d, $J$ $=8.5 \mathrm{~Hz}, 2 \mathrm{H}), 3.68(\mathrm{~s}, 4 \mathrm{H}), 3.26(\mathrm{~s}, 4 \mathrm{H}), 2.30(\mathrm{~s}, 3 \mathrm{H}) .{ }^{13} \mathrm{C} \mathrm{NMR}\left(126 \mathrm{MHz}, \mathrm{CDCl}_{3}\right) \delta$ $159.3,158.9,151.8,149.0,140.1,132.6,130.3,129.8,129.7,129.1,127.1,125.8$, 125.0, 124.6, 122.7, 120.1, 116.8, 49.8, 46.0, 20.4. HRMS-ESI $(\mathrm{m} / \mathrm{z}):[\mathrm{M}+\mathrm{H}]^{+}$calcd. for $\left[\mathrm{C}_{24} \mathrm{H}_{23} \mathrm{~N}_{3} \mathrm{ClO}\right]^{+}, 404.1530$; found, 404.1528 .

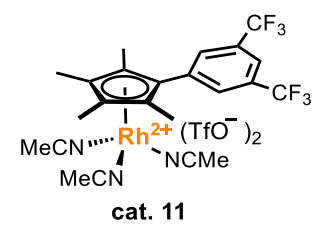

Yellow solid; ${ }^{1} \mathrm{H}$ NMR (500 MHz, DMSO- $\left.d_{6}\right) \delta 8.44(\mathrm{~s}, 1 \mathrm{H}), 8.41(\mathrm{~s}, 2 \mathrm{H}), 2.07$ (s, 9H), 1.69 (s, 6H), $1.58(\mathrm{~s}, 6 \mathrm{H}) .{ }^{13} \mathrm{C} \mathrm{NMR}\left(126 \mathrm{MHz}, \mathrm{DMSO}-d_{6}\right) \delta 131.1,131.0$ (q, $J=$ $33.4 \mathrm{~Hz}$ ), 130.3 (q, $J=3.1 \mathrm{~Hz}), 123.9$ (hept, $J=3.5 \mathrm{~Hz}$ ), 123.0 (q, $J=273.5 \mathrm{~Hz}$ ), 120.7 (q, $J=322.8 \mathrm{~Hz}), 116.8,100.9,92.3(\mathrm{~d}, J=7.3 \mathrm{~Hz}), 83.5$ (d, $J=10.1 \mathrm{~Hz}), 9.2$, 8.3, 1.1. ${ }^{19} \mathrm{~F}$ NMR (471 MHz, $\left.\mathrm{CDCl}_{3}\right) \delta-61.2,-77.8$. 


\section{References}

1. Piou, T.; Romanov-Michailidis, F.; Romanova-Michaelides, M.; Jackson, K. E.; Semakul, N.; Taggart, T. D.; Newell, B. S.; Rithner, C. D.; Paton, R. S.; Rovis, T. Correlating Reactivity and Selectivity to Cyclopentadienyl Ligand Properties in Rh(III)-Catalyzed C-H Activation Reactions: An Experimental and Computational Study. J. Am. Chem. Soc. 2017, 139, 1296-1310.

2. Li, Y.; Li, B.-J.; Wang, W.-H.; Huang, W.-P.; Zhang, X.-S.; Chen, K.; Shi, Z.-J. Rhodium-Catalyzed Direct Addition of Aryl C-H Bonds to $\mathrm{N}$-Sulfonyl Aldimines. Angew. Chem. Int. Ed. 2011, 50, 2115-2119.

3. Widegren, J. A.; Finke, R. G. A Review of the Problem of Distinguishing True Homogeneous Catalysis from Soluble or Other Metal-Particle Heterogeneous Catalysis under Reducing Conditions. J. Mol. Catal. A: Chem. 2003, 198, 317-341.

4. Liu, W. J.; Xu, J. M.; Chen, X. H.; Zhang, F. X.; Xu, Z. F.; Wang, D. P.; He, Y. Q.; Xia, X. H.; Zhang, X.; Liang, Y. CuI/2-Aminopyridine 1-Oxide Catalyzed Amination of Aryl Chlorides with Aliphatic Amines. Org. Lett. 2020, 22, 7486-7490.

5. Yang, P.; Zhang, C.; Gao, W.-C.; Ma, Y.; Wang, X.; Zhang, L.; Yue, J.; Tang, B. Nickel-Catalyzed Borrowing Hydrogen Annulations: Access to Diversified $N$-Heterocycles. Chem. Commun. 2019, 55, 7844-7847.

6. Yang, Z.; Zhu, X.; Yang, S.; Yang, Z. Iridium-Catalysed Reductive Deoxygenation of Ketones with Formic Acid as Traceless Hydride Donor. Adv. Synth. Catal. 2020, 362, 5496-5505.

7. Wei, D.; Netkaew, C.; Wu, J.; Darcel, C. Iron-Catalyzed Hydrosilylation of Diacids in the Presence of Amines: A New Route to Cyclic Amines. ChemCatChem 2020, 12, 5449-5455.

8. Hatakeyama, T.; Yoshimoto, Y.; Ghorai, S. K.; Nakamura, M. Transition-Metal-Free Electrophilic Amination between Aryl Grignard Reagents and $\mathrm{N}$-Chloroamines. Org. Lett. 2010, 12, 1516-1519.

9. Tardiff, B. J.; McDonald, R.; Ferguson, M. J.; Stradiotto, M. Rational and Predictable Chemoselective Synthesis of Oligoamines via Buchwald-Hartwig Amination of (Hetero)Aryl Chlorides Employing Mor-dalphos. J. Org. Chem. 2011, 77, 1056-1071.

10. Qian, X.; Yu, Z.; Auffrant, A.; Gosmini, C. Cobalt-Catalyzed Electrophilic Amination of Arylzincs with $\mathrm{N}$-Chloroamines. Chem. Eur. J. 2013, 19, 6225-6229.

11. Kaga, A.; Hayashi, H.; Hakamata, H.; Takita, R.; Chiba, S. Nucleophilic Amination of Methoxy Arenes Promoted by a Sodium Hydride/Iodide Composite. Angew. Chem. Int. Ed. 2017, 56, 11807-11811.

12. Girard, S. A.; Hu, X.; Knauber, T.; Zhou, F.; Simon, M.-O.; Deng, G.-J.; Li, C.-J. Pd-Catalyzed Synthesis of Aryl Amines via Oxidative Aromatization of Cyclic Ketones and Amines with Molecular Oxygen. Org. Lett. 2012, 14, 5606-5609.

13. Mikhailine, A. A.; Mannino, G. A. G.; Colacot, T. J. Catalyst-Directed Chemoselective Double Amination of Bromo-chloro(hetero)arenes: A Synthetic Route towards Advanced Amino-aniline Intermediates. Org. Lett. 2018, 20, 
2301-2305.

14. Hamura, T.; Ibusuki, Y.; Sato, K.; Matsumoto, T.; Osamura, Y.; Suzuki, K. Strain-Induced Regioselectivities in Reactions of Benzyne Possessing a Fused Four-Membered Ring. Org. Lett. 2003, 5, 3551-3554.

15. Shimasaki, T.; Tobisu, M.; Chatani, N. Nickel-Catalyzed Amination of Aryl Pivalates by the Cleavage of Aryl C-O Bonds. Angew. Chem. Int. Ed. 2010, 49, 2929-2932.

16. Hajra, A.; Ye, W.; Yoshikai, N. Palladium-Catalyzed Aerobic Dehydrogenative Aromatization of Cyclohexanone Imines to Arylamines. Org. Lett. 2012, 14, 5488-5491.

17. Yu, X.-Q.; Yamamoto, Y.; Miyaura, N. Aryl Triolborates: Novel Reagent for Copper-Catalyzed N Arylation of Amines, Anilines, and Imidazoles. Chem. Asian J. 2008, 3, 1517-1522.

18. María, V. C.; María, C. C.; Isabel, P.; Alejandra, S. An Efficient Synthesis of 1-Acyl-3-arylimidazolidines Catalyzed by Montmorillonite K-10 Clay under Microwave Irradiation. Heterocycles 2009, 78, 771-781.

19. Llabres-Campaner, P. J.; Woodbridge-Ortega, P.; Ballesteros-Garrido, R.; Ballesteros, R.; Abarca, B. Heterogeneous Borrowing Hydrogen Reactions with $\mathrm{Pd} / \mathrm{C}$ and $\mathrm{ZnO}$ : Diol Scope. Tetrahedron Lett. 2017, 58, 4880-4882.

20. Li, Z. L.; Sun, K. K.; Wu, P. Y.; Cai, C. Iron-Catalyzed Regioselective $\alpha-C-H$ Alkylation of $\mathrm{N}$-Methylanilines: Cross-Dehydrogenative Coupling between Unactivated $\mathrm{C}\left(\mathrm{sp}^{3}\right)-\mathrm{H}$ and $\mathrm{C}\left(\mathrm{sp}^{3}\right)-\mathrm{H}$ Bonds via a Radical Process. J. Org. Chem. 2019, 84, 6830-6839.

21. Voloboev, S. N.; Andreev, A. V.; Mkrtchyan, A. S.; Navrotskii, M. B.; Novakov, I. A.; Orlinson, B. S.; Son, V. V. Potential Synthetic Adaptogens 1. Synthesis and Studies of New $\quad N$-[(Adamantan-1-yl)methyl]aniline Derivatives Based on Adamantane-1-Carbaldehyde. Russ. Chem. Bull. 2016, 65, 1336-1340.

22. Abdukader, A.; Jin, H.; Cheng, Y.; Zhu, C. Rhenium-Catalyzed Amination of Alcohols by Hydrogen Transfer Process. Tetrahedron Lett. 2014, 55, 4172-4174.

23. Song, H.; Yang, Z.; Tung, C.-H.; Wang, W. Iron-Catalyzed Reductive Coupling of Nitroarenes with Olefins: Intermediate of Iron-Nitroso Complex. ACS Catal. 2020, 10, 276-281.

24. Nguyen, V. T.; Nguyen, V. D.; Haug, G. C.; Vuong, N. T. H.; Dang, H. T.; Arman, H. D.; Larionov, O. V. Visible-Light-Enabled Direct Decarboxylative N-Alkylation. Angew. Chem. Int. Ed. 2020, 59, 7921-7927.

25. Cao, D.; Zeng, H.; Li, C.-J. Formal Cross-Coupling of Diaryl Ethers with Ammonia by Dual C(Ar)-O Bond Cleavages. ACS Catal. 2018, 8, 8873-8878.

26. Lo, Q. A.; Sale, D.; Braddock, D. C.; Davies, R. P. New Insights into the Reaction Capabilities of Ionic Organic Bases in Cu-Catalyzed Amination. Eur. J. Org. Chem. 2019, 2019, 1944-1951.

27. Mastalir, M.; Stöger, B.; Allmaier, G.; Kirchner, K. Air Stable Iron(II) PNP Pincer Complexes as Efficient Catalysts for the Selective Alkylation of Amines with Alcohols. Adv. Synth. Catal. 2016, 358, 3824-3831.

28. Kouznetsov, V. V.; Vargas Méndez, L. Y.; Sortino, M.; Vásquez, Y.; Gupta, M. P.; Freile, M.; Enriz, R. D.; Zacchino, S. A.; Antifungal and Cytotoxic Activities of Some 
$N$-Substituted Aniline Derivatives Bearing a Hetaryl Fragment. Bioorg. Med. Chem. 2008, 16, 794-809.

29. Shafir, A.; Lichtor, P. A.; Buchwald, S. L. N- vs. O-Arylation of Aminoalcohols: Orthogonal Selectivity in Copper-Based Catalysts. J. Am. Chem. Soc. 2007, 129, 3490-3491.

30. Cook, A.; Prakash, S.; Zheng, Y.-L.; Newman, S. G. Exhaustive Reduction of Esters Enabled by Nickel Catalysis. J. Am. Chem. Soc. 2020, 142, 8109-8115.

31. Zhang, Y.; Lavigne, G.; Cesar, V. Buchwald-Hartwig Amination of (Hetero)Aryl Tosylates Using a Well-Defined N-Heterocyclic Carbene/Palladium(II) Precatalyst. J. Org. Chem. 2015, 80, 7666-7673.

32. Kosso, A. R. O.; Sellet, N.; Baralle, A.; Cormier, M.; Goddard, J.-P. Cyanine-Based Near Infra-red Organic Photoredox Catalysis. Chem. Sci. 2021, 12, 6964-6968.

33. Dighe, S. U.; Juliá, F.; Luridiana, A.; Douglas, J. J.; Leonori, D. A. Photochemical Dehydrogenative Strategy for Aniline Synthesis. Nature 2020, 584, 75-81.

34. Jaspers, D.; Doye, S. Potassium Hydroxide Catalyzed Addition of Arylamines to Styrenes. Synlett. 2011, 2011, 1444-1448.

35. Cawley, M. J.; Cloke, F. G. N.; Fitzmaurice, R. J.; Pearson, S. E.; Scott, J. S.; Caddick, S. Development of a Practical Buchwald-Hartwig Amine Arylation Protocol Using a Conveniently Prepared (NHC)Pd(R-allyl)Cl Catalyst. Org. Biomol. Chem. 2008, 6, 2820-2825.

36. Zhao, Y.; Zhang, S.; Jin, T.; Yamamoto, Y.; Terada, M. Heterogeneous Catalytic Reduction of Tertiary Amides with Hydrosilanes Using Unsupported Nanoporous Gold Catalyst. Adv. Synth. Catal. 2019, 361, 4817-4824.

37. Ruffoni, A.; Juliá, F.; Svejstrup, T. D.; McMillan, A. J.; Douglas, J. J.; Leonori, D. Practical and Regioselective Amination of Arenes Using Alkyl Amines. Nat. Chem. 2019, 11, 426-433.

38. Zhai, G.; Liu, X.; Ma, W.; Wang, G.; Yang, L.; Li, S; Wu, Y.; Hu, X. $\mathrm{B}\left(\mathrm{C}_{6} \mathrm{~F}_{5}\right)_{3}$-Catalyzed Tandem Friedel-Crafts and $\mathrm{C}-\mathrm{H} / \mathrm{C}-\mathrm{O}$ Coupling Reactions of Dialkylanilines. Chem. Asian J. 2020, 15, 3082-3086.

39. Sun, H.-B.; Gong, L; Tian, Y.-B.; Wu, J.-G.; Zhang, X.; Liu, J.; Fu, Z.; Niu, D. Metaland Base-Free Room-Temperature Amination of Organoboronic Acids with $N$-Alkyl Hydroxylamines. Angew. Chem. Int. Ed. 2018, 57, 9456-9460. 


\section{NMR Spectra}

${ }^{1} \mathrm{H}$ NMR (500 MHz, $\left.\mathrm{CDCl}_{3}\right)$

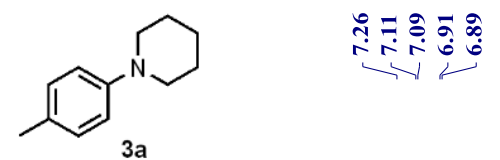

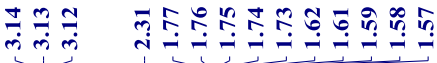

$3 a$

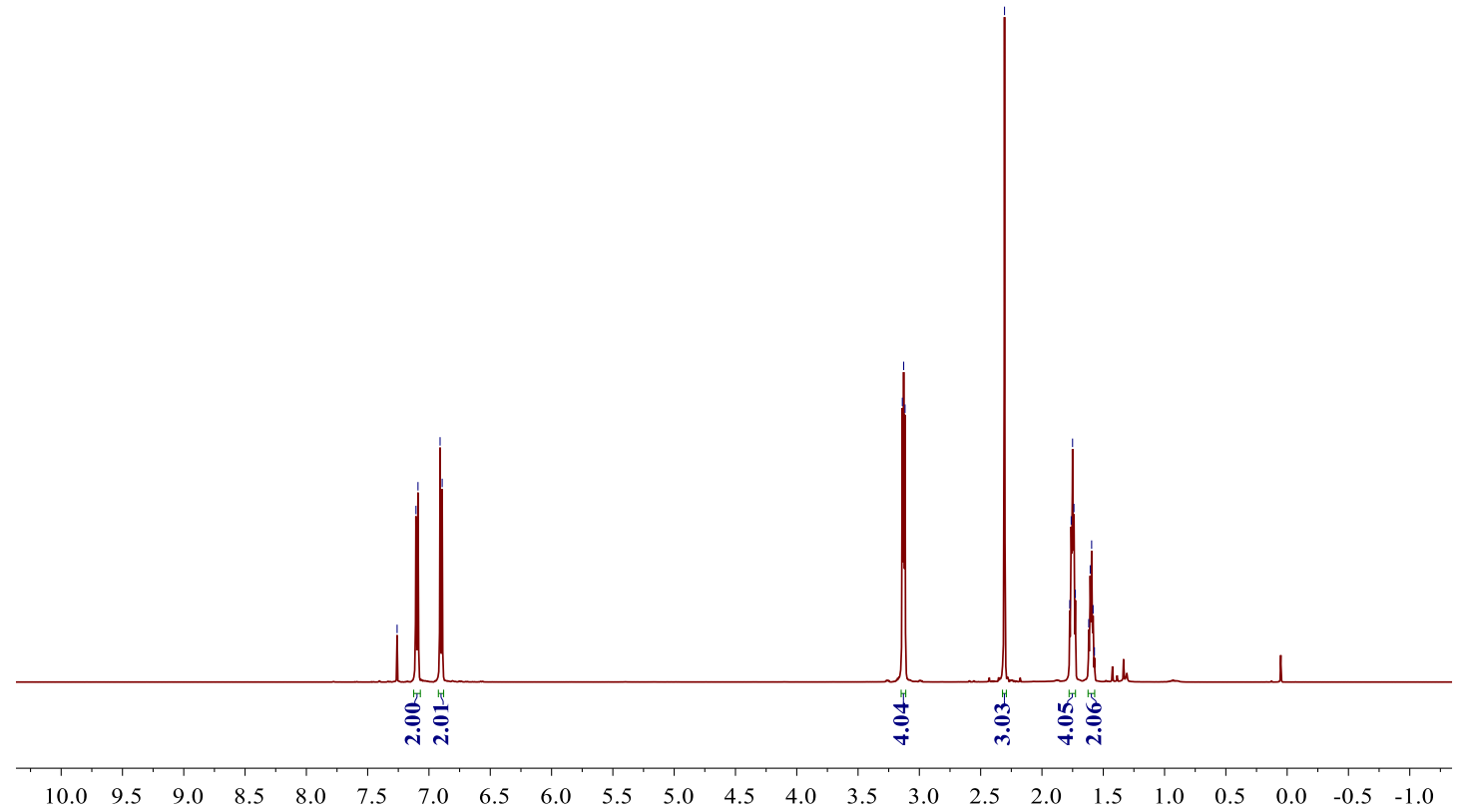

${ }^{13} \mathrm{C} \mathrm{NMR}\left(126 \mathrm{MHz}, \mathrm{CDCl}_{3}\right)$
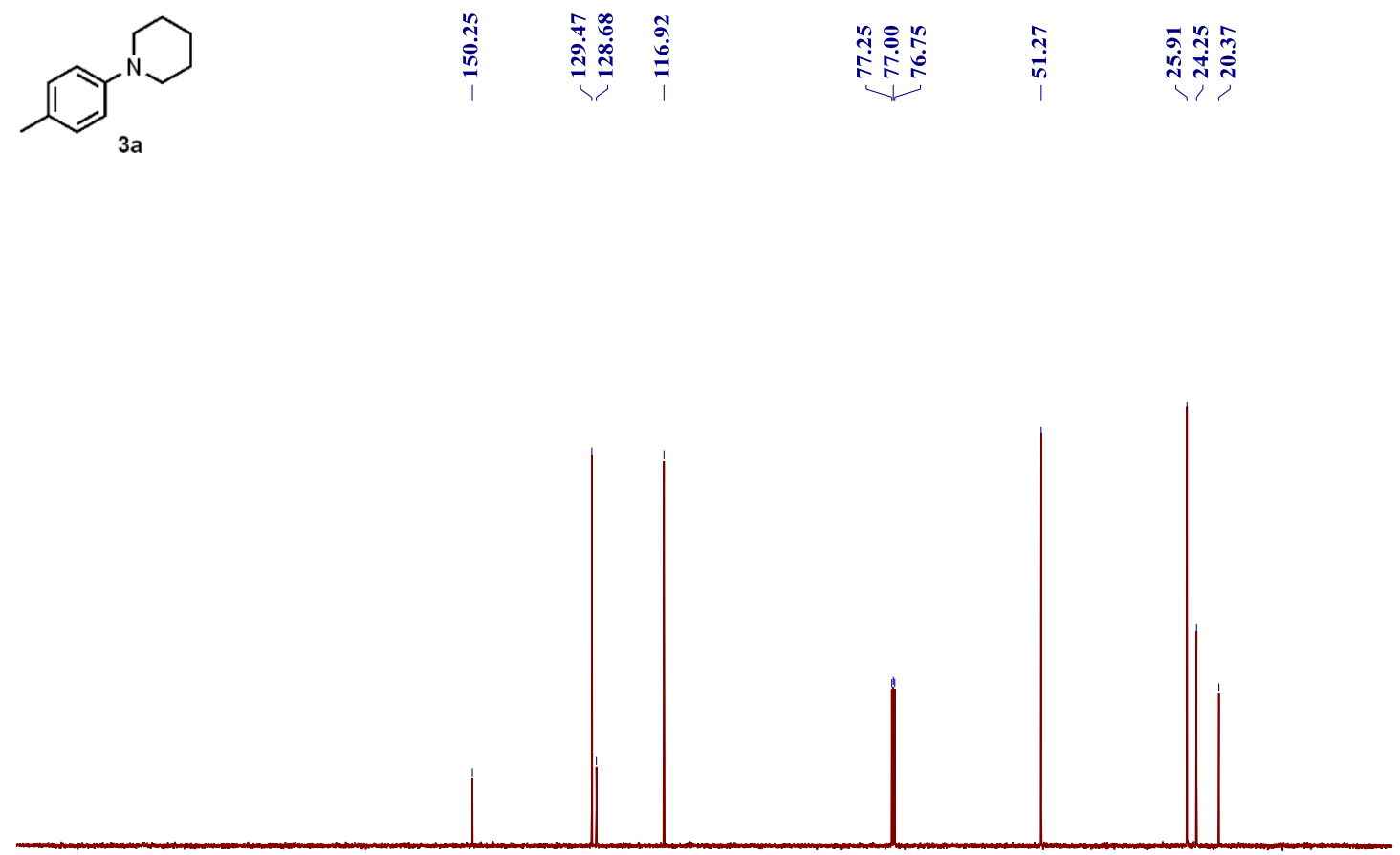

$\begin{array}{lllllllllllllllllllllll}220 & 210 & 200 & 190 & 180 & 170 & 160 & 150 & 140 & 130 & 120 & 110 & 100 & 90 & 80 & 70 & 60 & 50 & 40 & 30 & 20 & 10 & 0\end{array}$ 
${ }^{1} \mathrm{H} \mathrm{NMR}\left(500 \mathrm{MHz}, \mathrm{CDCl}_{3}\right)$

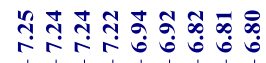

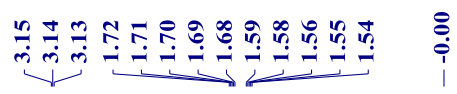<smiles>c1ccc(N2CCCCC2)cc1</smiles>

$3 b$

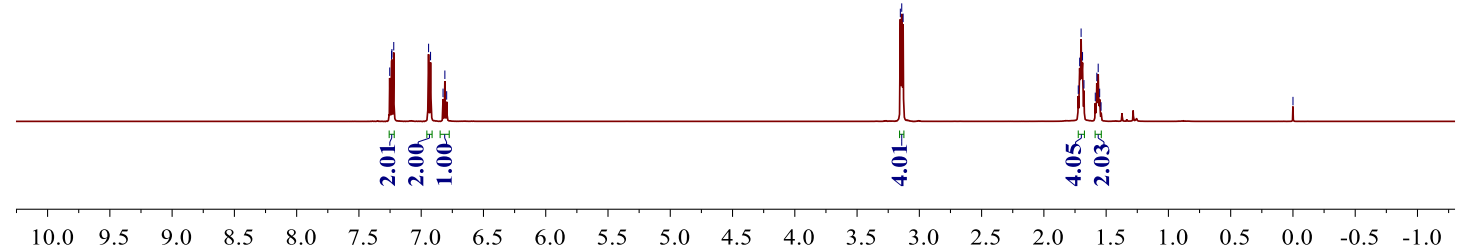

${ }^{13} \mathrm{C}$ NMR (126 MHz, $\left.\mathrm{CDCl}_{3}\right)$

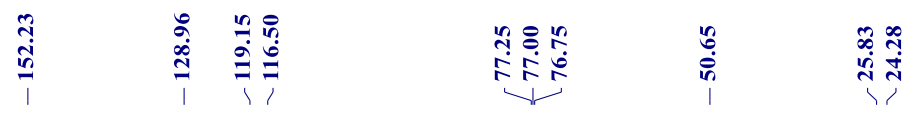<smiles>c1ccc(N2CCCCC2)cc1</smiles>

$3 b$

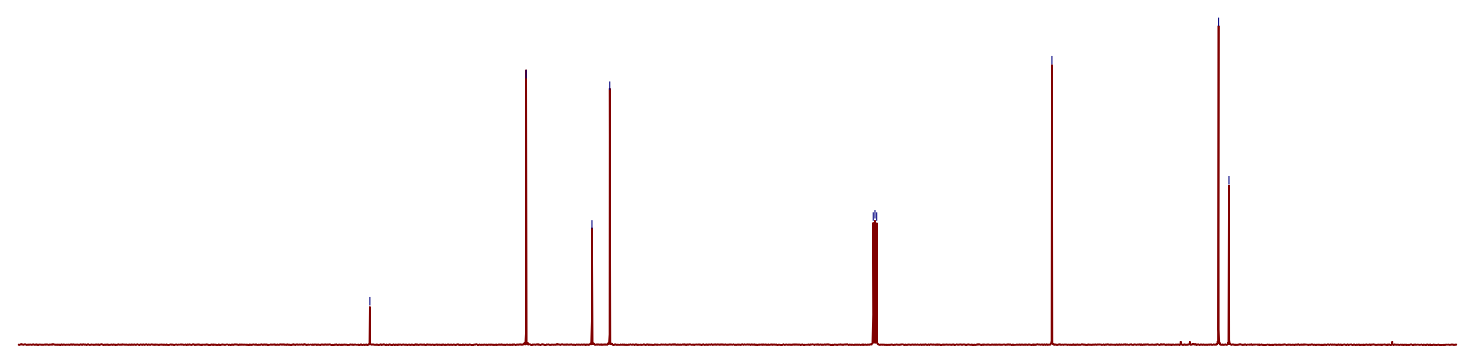

$\begin{array}{lllllllllllllllllllll}200 & 190 & 180 & 170 & 160 & 150 & 140 & 130 & 120 & 110 & 100 & 90 & 80 & 70 & 60 & 50 & 40 & 30 & 20 & 10 & 0\end{array}$ 
${ }^{1} \mathrm{H} \mathrm{NMR}\left(500 \mathrm{MHz}, \mathrm{CDCl}_{3}\right)$
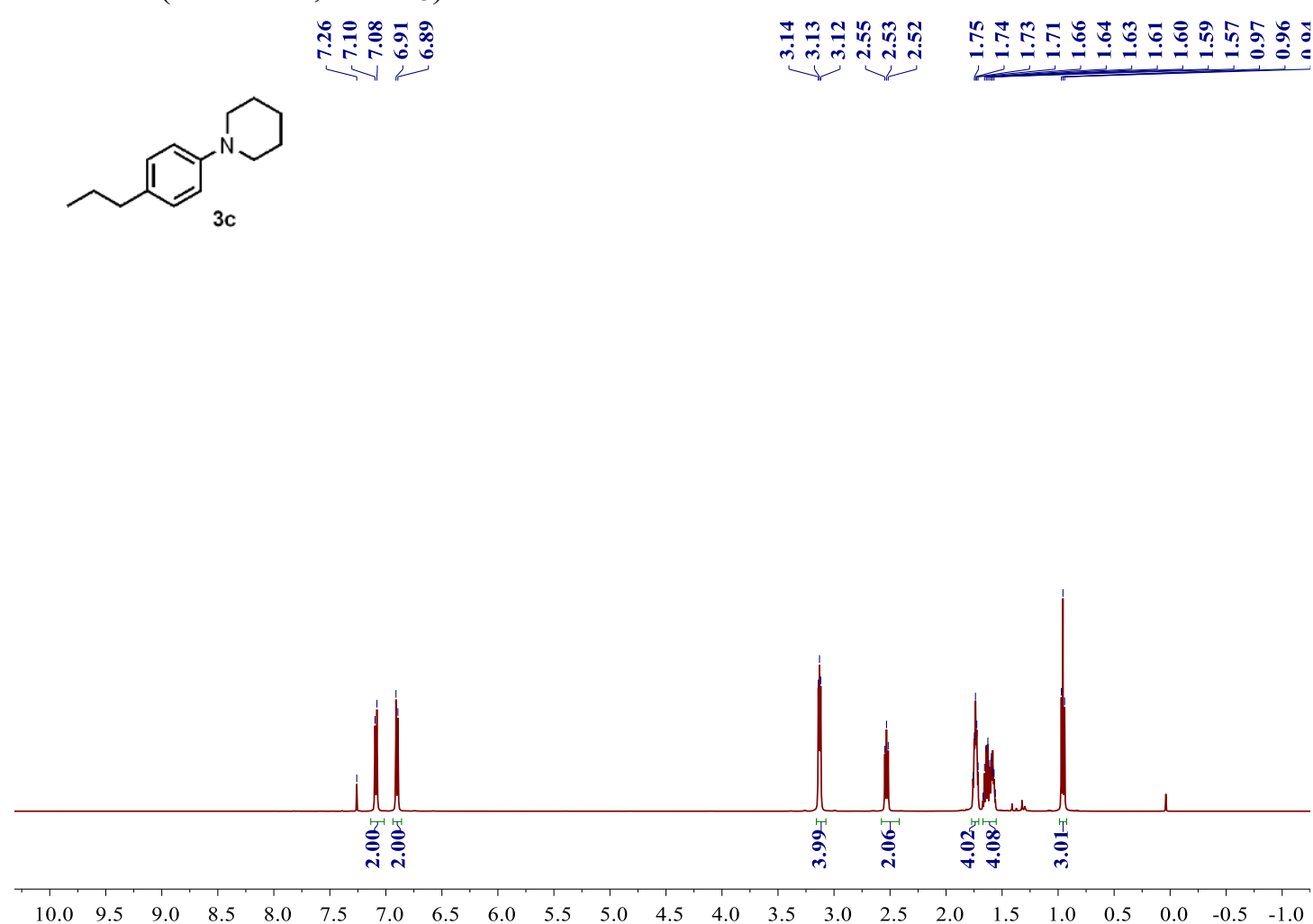

${ }^{13} \mathrm{C}$ NMR (126 MHz, $\left.\mathrm{CDCl}_{3}\right)$
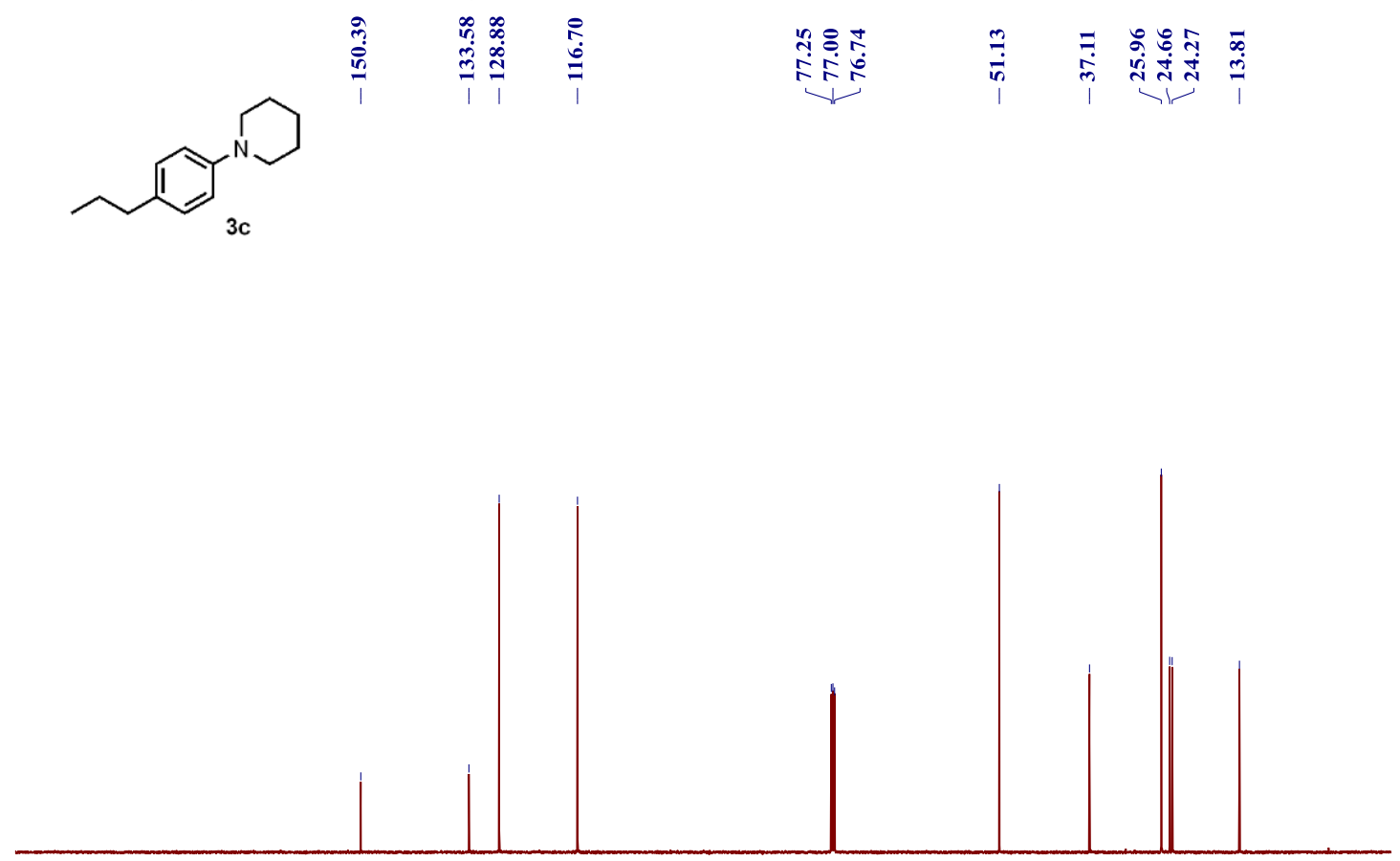

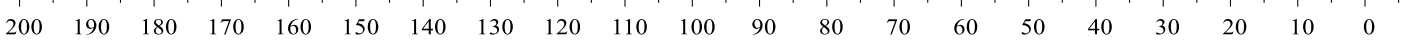


${ }^{1} \mathrm{H}$ NMR $\left(500 \mathrm{MHz}, \mathrm{CDCl}_{3}\right)$

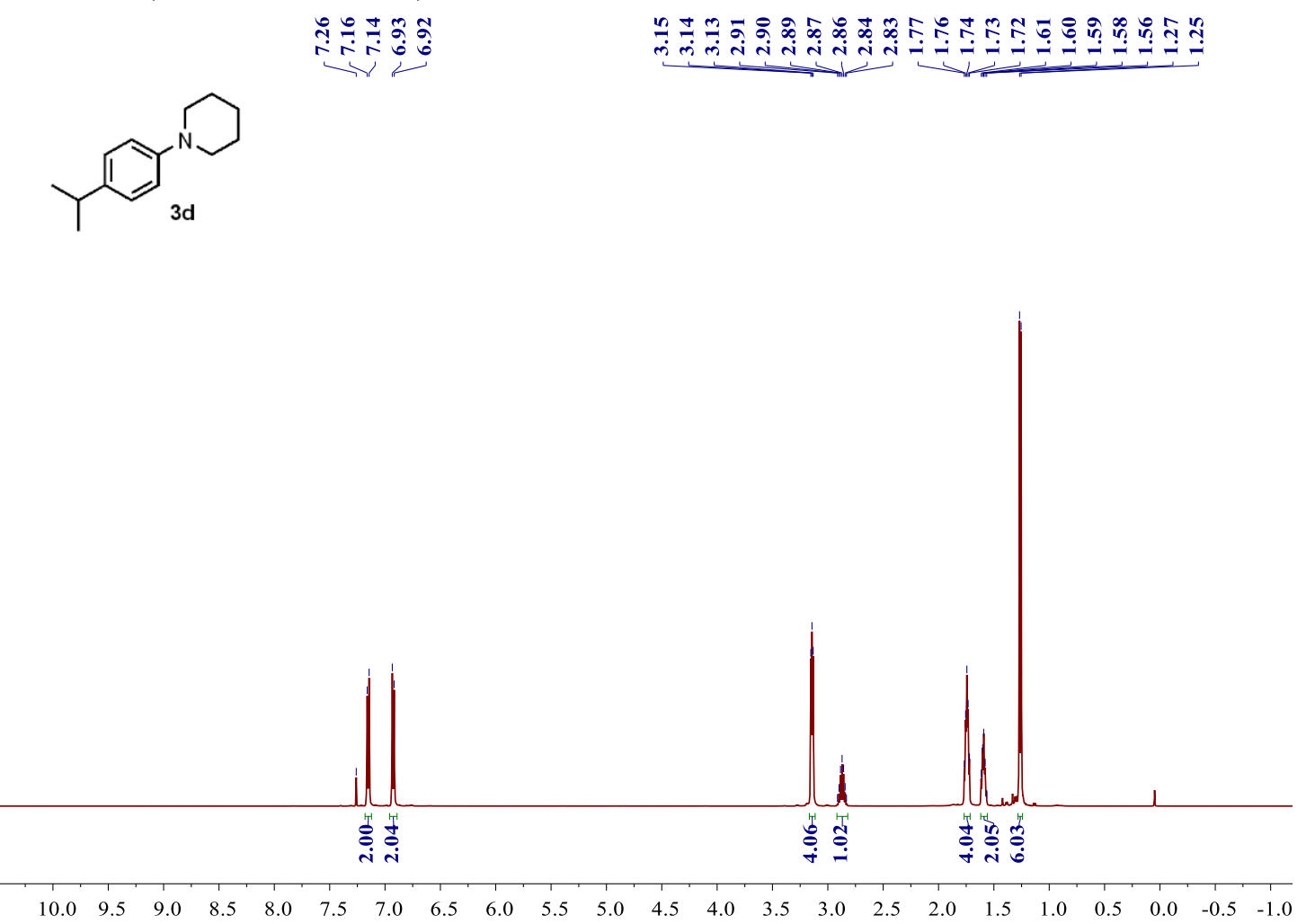

${ }^{13} \mathrm{C}$ NMR $\left(126 \mathrm{MHz}, \mathrm{CDCl}_{3}\right)$

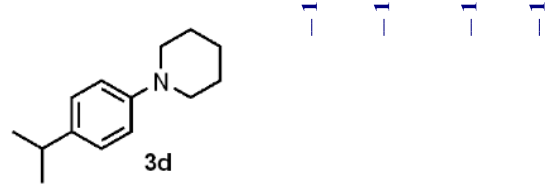

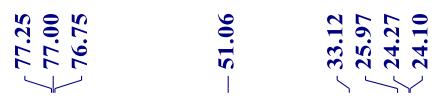

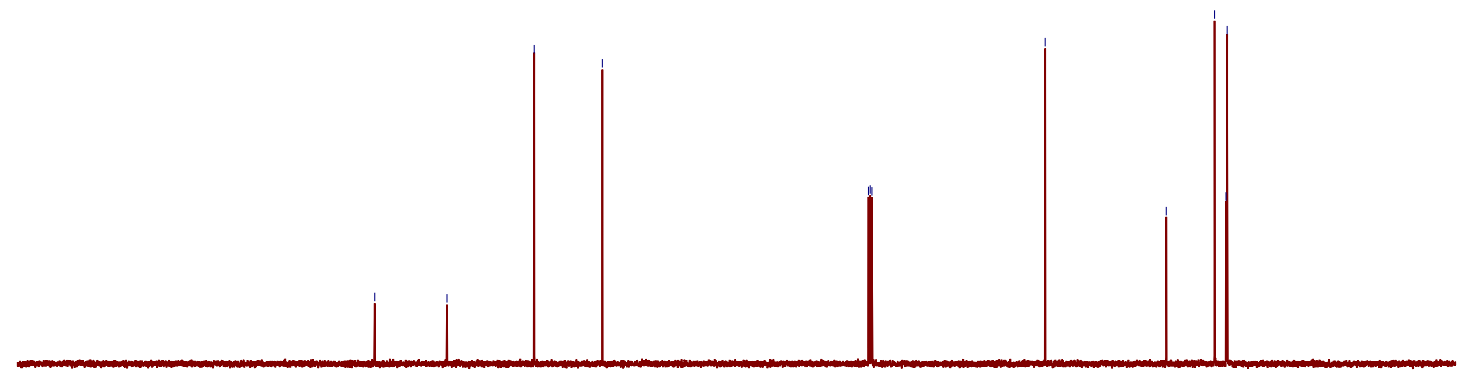

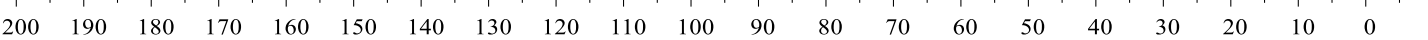


${ }^{1} \mathrm{H}$ NMR $\left(500 \mathrm{MHz}, \mathrm{CDCl}_{3}\right)$

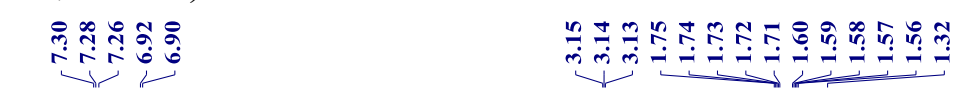
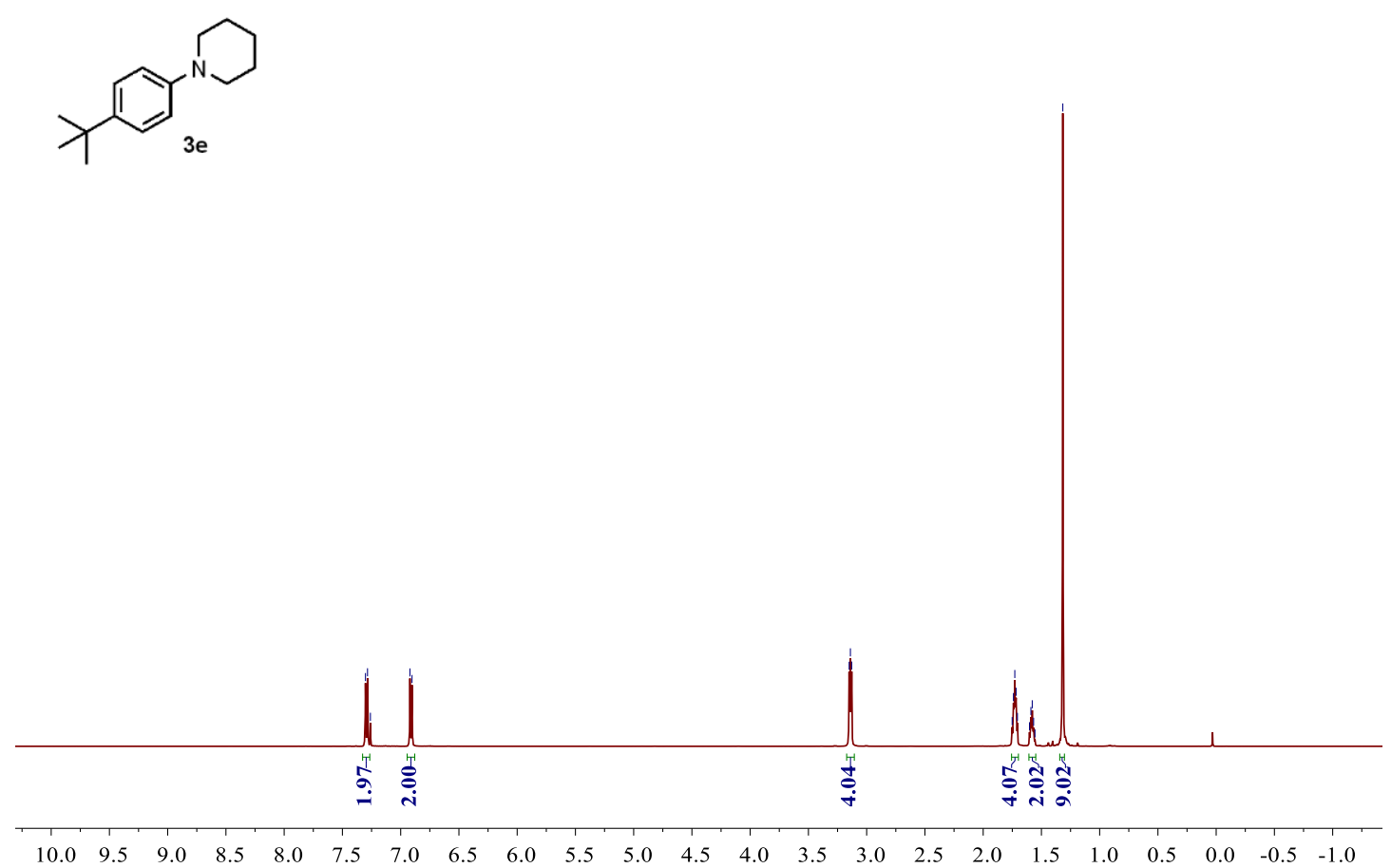

${ }^{13} \mathrm{C} \mathrm{NMR}\left(126 \mathrm{MHz}, \mathrm{CDCl}_{3}\right)$

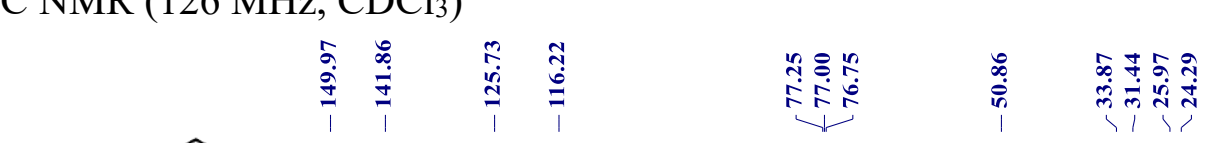<smiles>CC(C)(C)c1ccc(N2CCCC2)cc1</smiles>

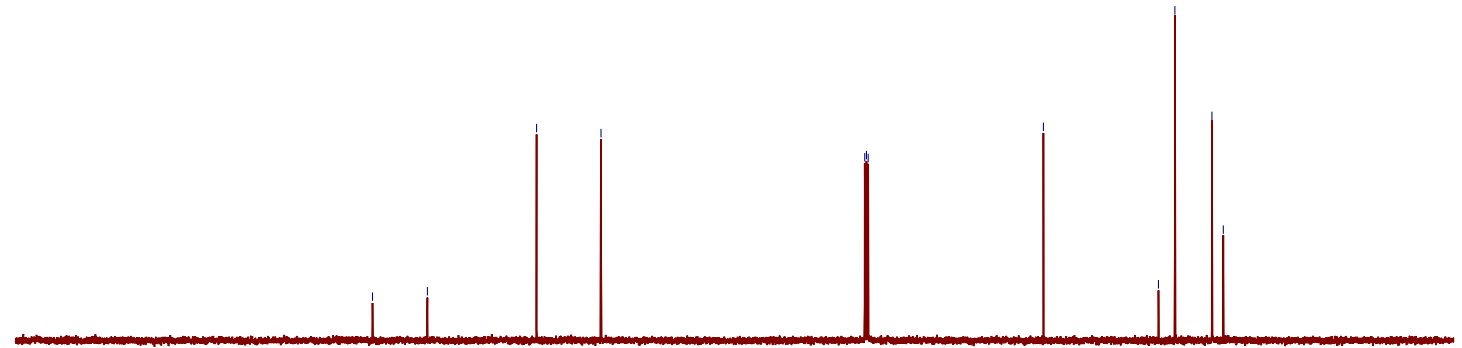

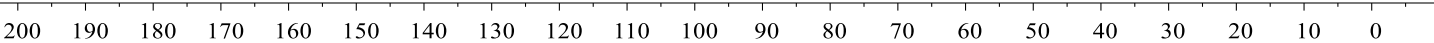


${ }^{1} \mathrm{H} \mathrm{NMR}\left(500 \mathrm{MHz}, \mathrm{CDCl}_{3}\right)$

ํํำ

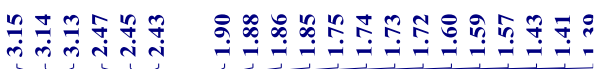<smiles>c1cc(N2CCCC2)ccc1C1CCCCC1</smiles>

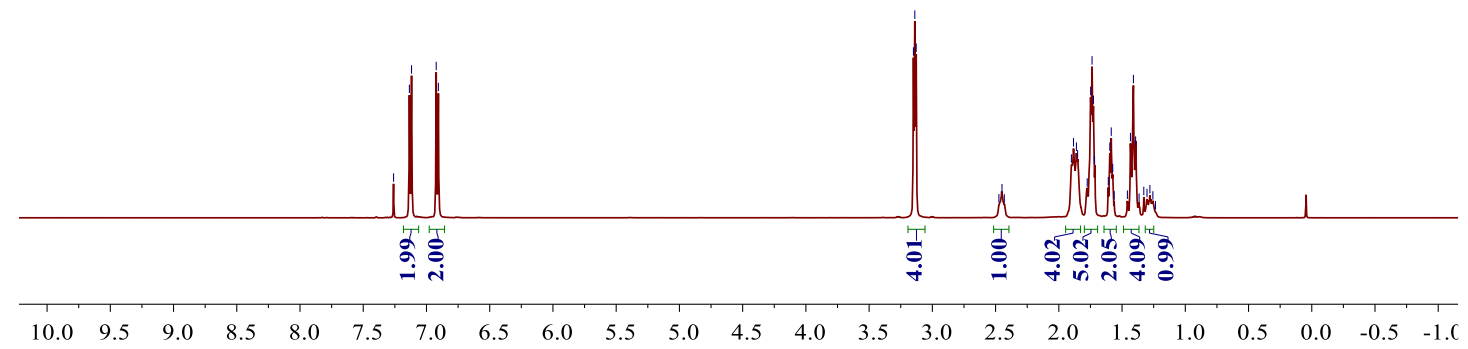

${ }^{13} \mathrm{C} \mathrm{NMR}\left(126 \mathrm{MHz}, \mathrm{CDCl}_{3}\right)$

\begin{tabular}{|c|c|c|c|c|c|}
\hline 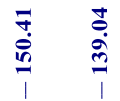 & $\frac{\sqrt{3}}{\stackrel{1}{1}}$ & 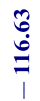 & 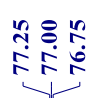 & 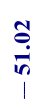 & 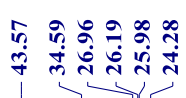 \\
\hline
\end{tabular}<smiles>c1cc(N2CCCCC2)ccc1C1CCCCC1</smiles>

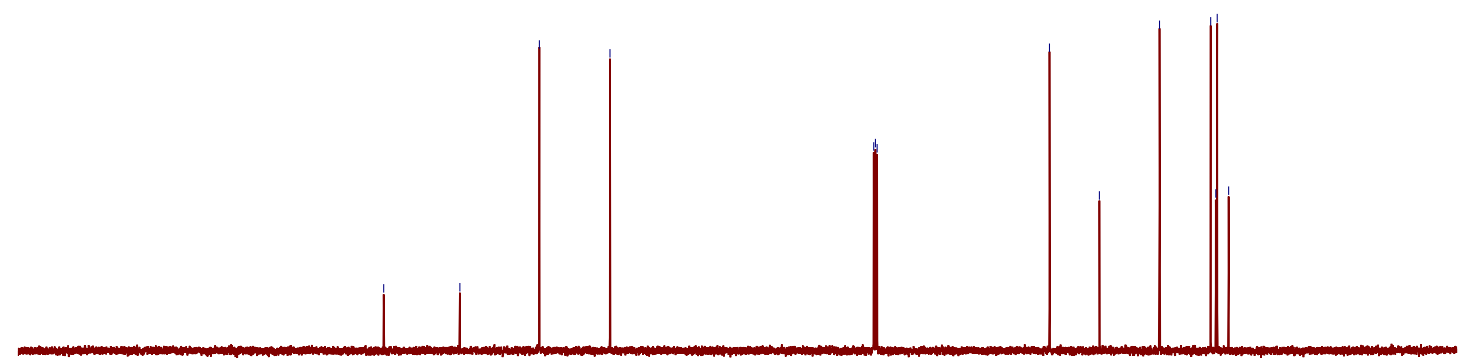

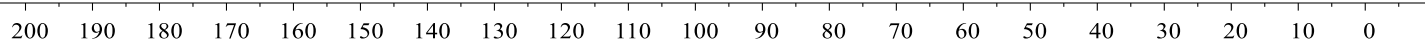


${ }^{1} \mathrm{H}$ NMR $\left(500 \mathrm{MHz}, \mathrm{CDCl}_{3}\right)$

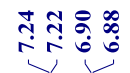
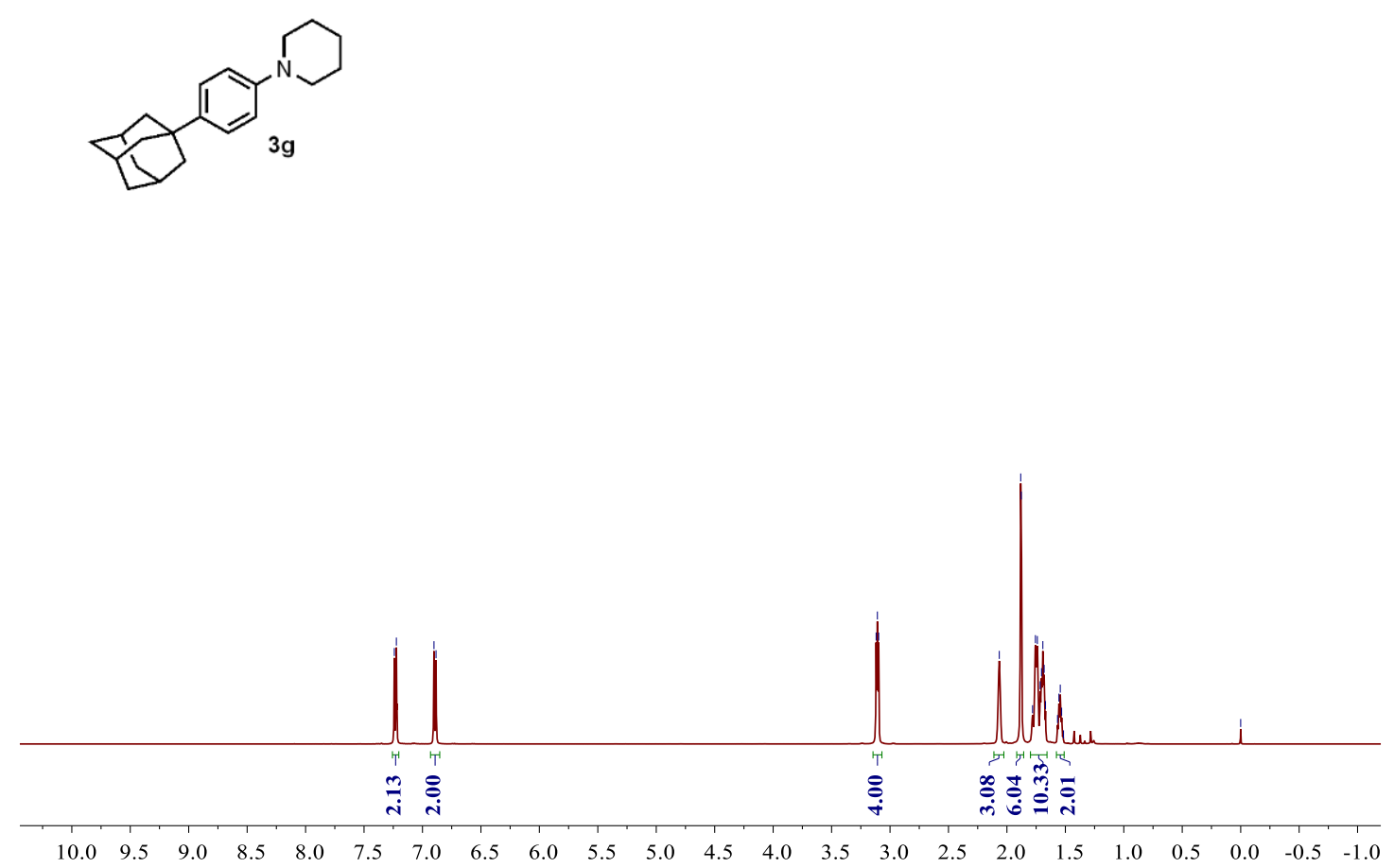

${ }^{13} \mathrm{C} \mathrm{NMR}\left(126 \mathrm{MHz}, \mathrm{CDCl}_{3}\right)$
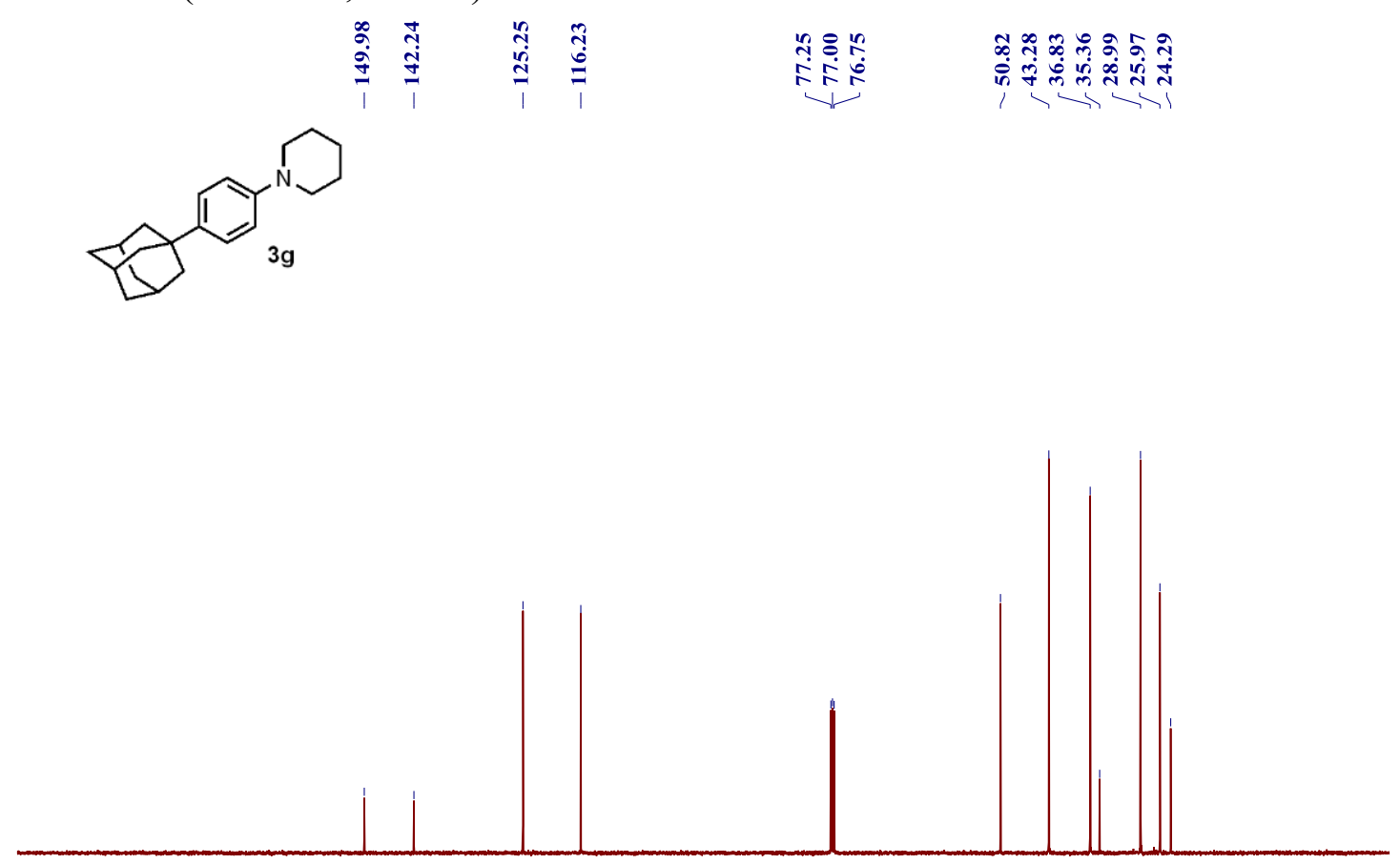

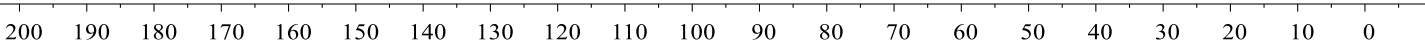


${ }^{1} \mathrm{H}$ NMR (500 MHz, $\left.\mathrm{CDCl}_{3}\right)$

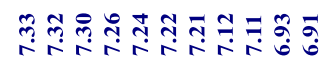<smiles>CCCc1ccc(N2CCCCC2)cc1</smiles>

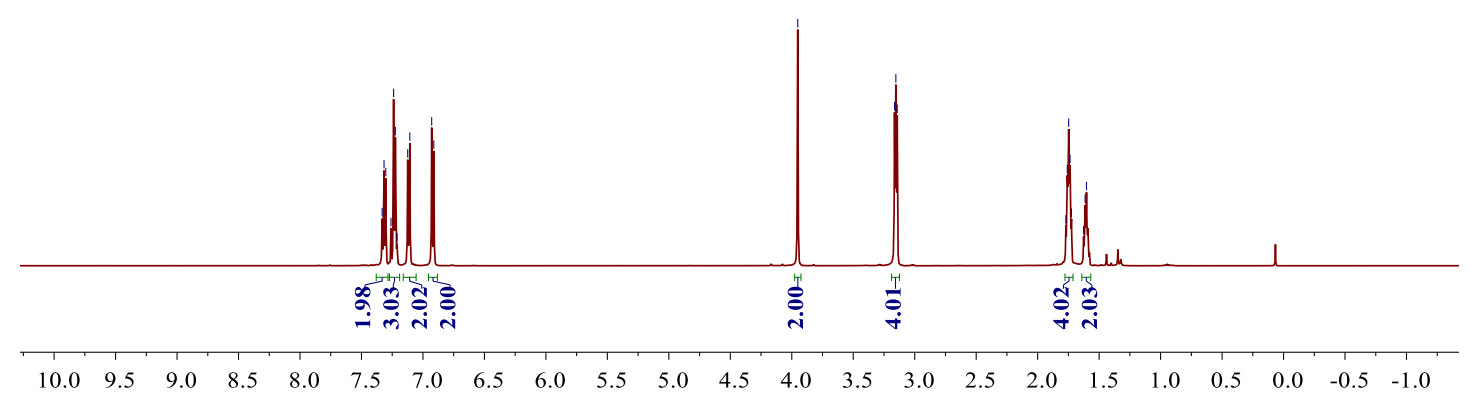

${ }^{13} \mathrm{C} \mathrm{NMR}\left(126 \mathrm{MHz}, \mathrm{CDCl}_{3}\right)$

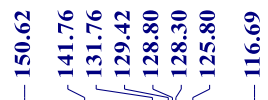

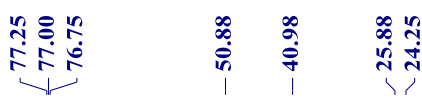<smiles>c1ccc(Cc2ccc(N3CCCCC3)cc2)cc1</smiles>

$3 \mathrm{~h}$

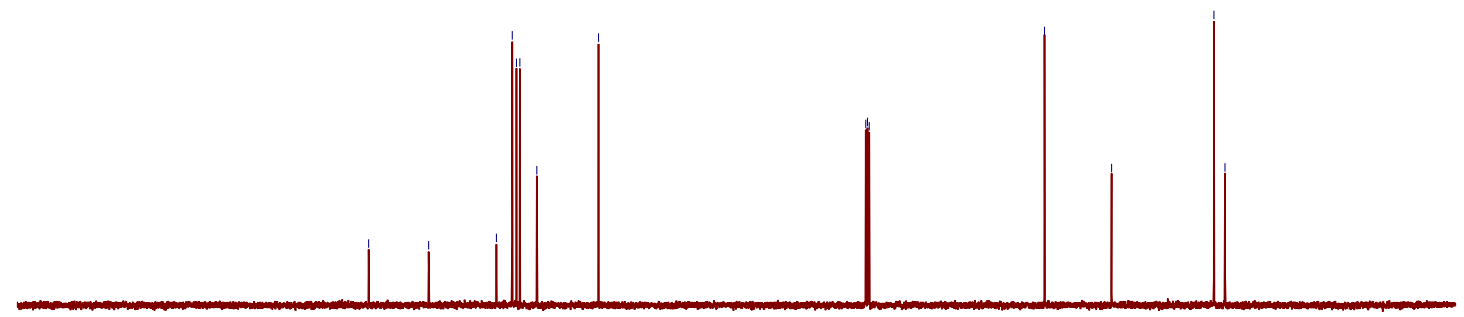

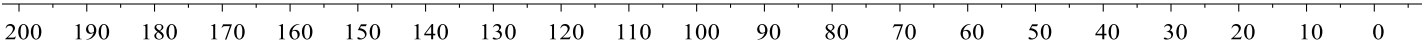


${ }^{1} \mathrm{H}$ NMR $\left(500 \mathrm{MHz}, \mathrm{CDCl}_{3}\right)$

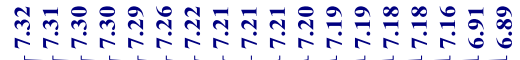

$\underbrace{\infty}$<smiles>CC(C)(c1ccccc1)c1ccc(N2CCCCC2)cc1</smiles>

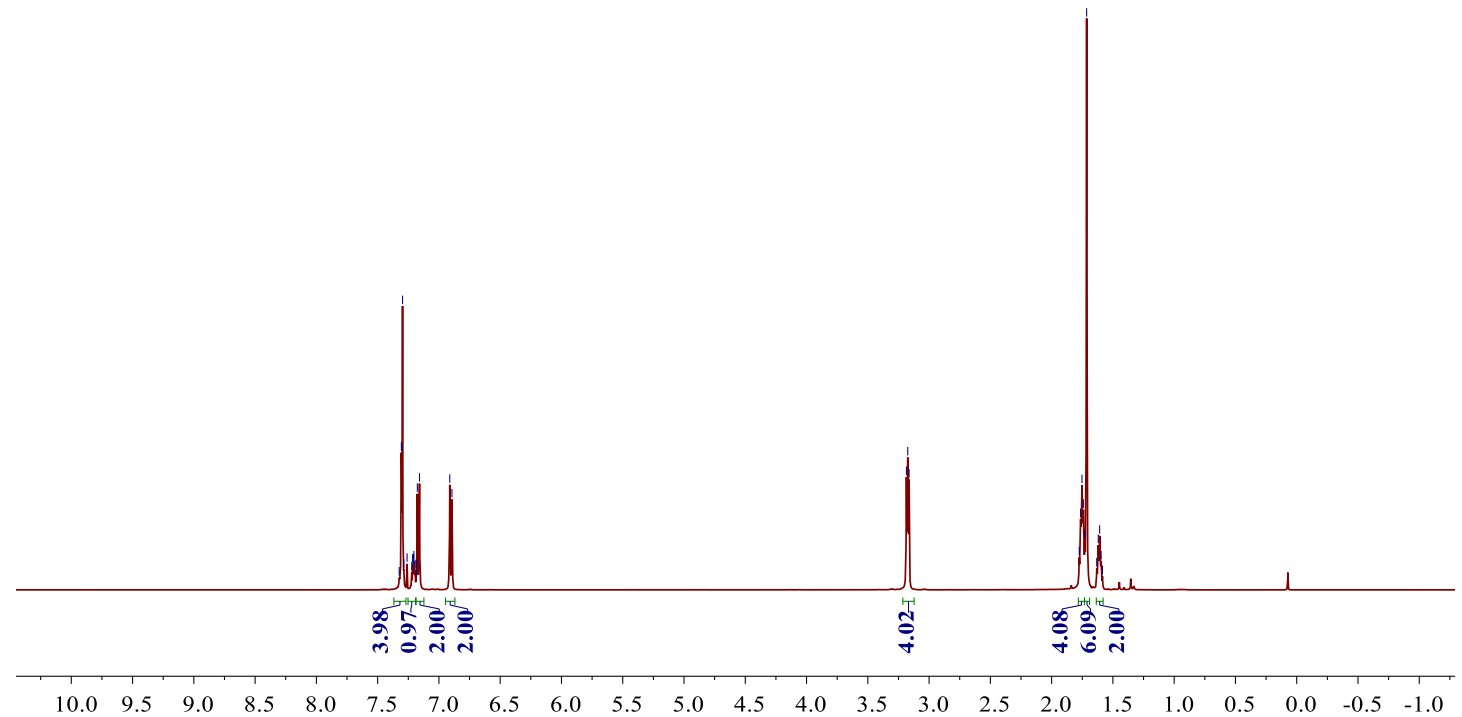

${ }^{13} \mathrm{C}$ NMR (126 MHz, $\left.\mathrm{CDCl}_{3}\right)$

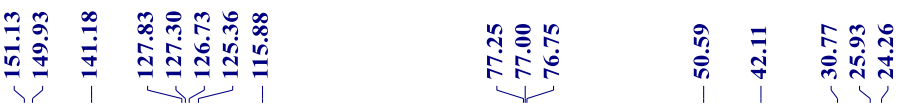<smiles>CC(C)(c1ccccc1)c1ccc(N2CCCCC2)cc1</smiles>

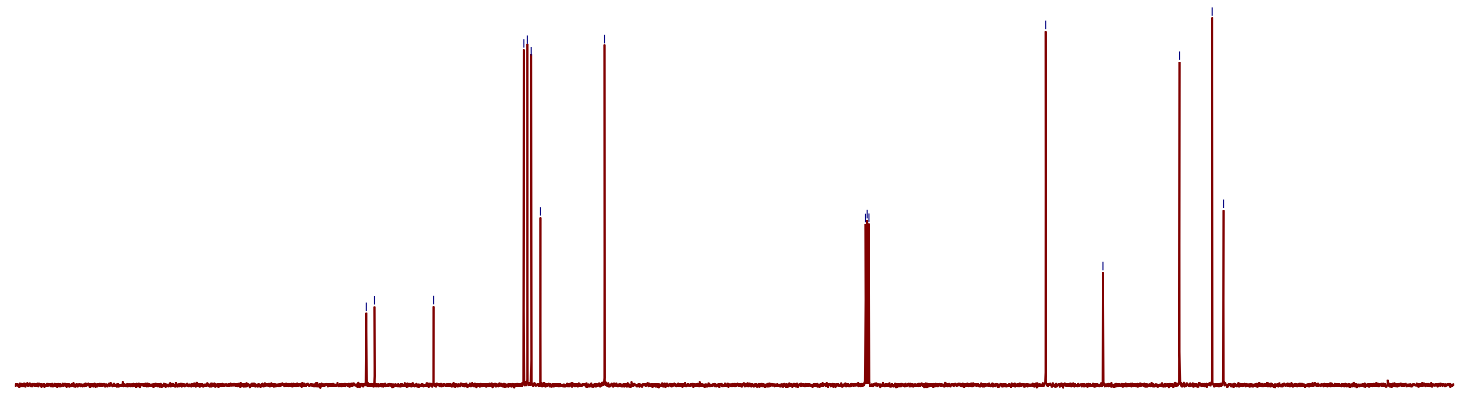

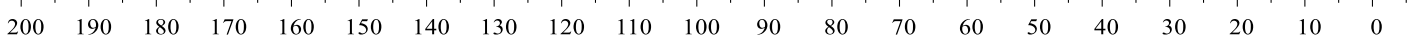


${ }^{1} \mathrm{H} \mathrm{NMR}\left(500 \mathrm{MHz}, \mathrm{CDCl}_{3}\right)$

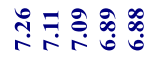

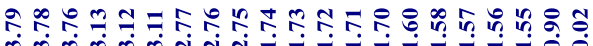

11<smiles>CCCCOCCc1ccc(N2CCCCC2)cc1</smiles>

$3 \mathrm{j}$

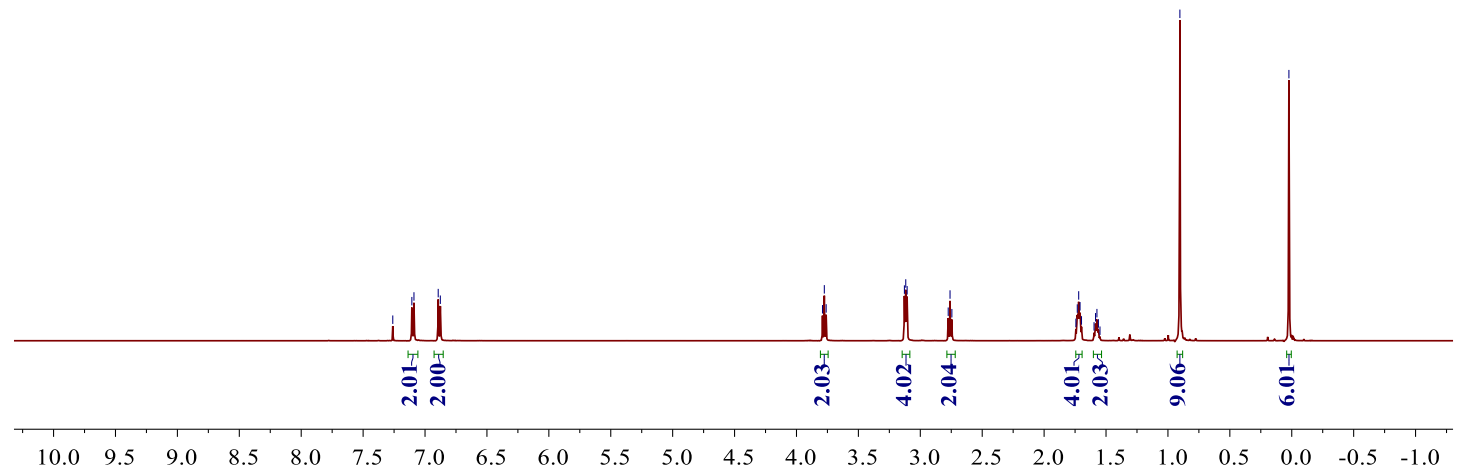

${ }^{13} \mathrm{C}$ NMR (126 MHz, $\left.\mathrm{CDCl}_{3}\right)$
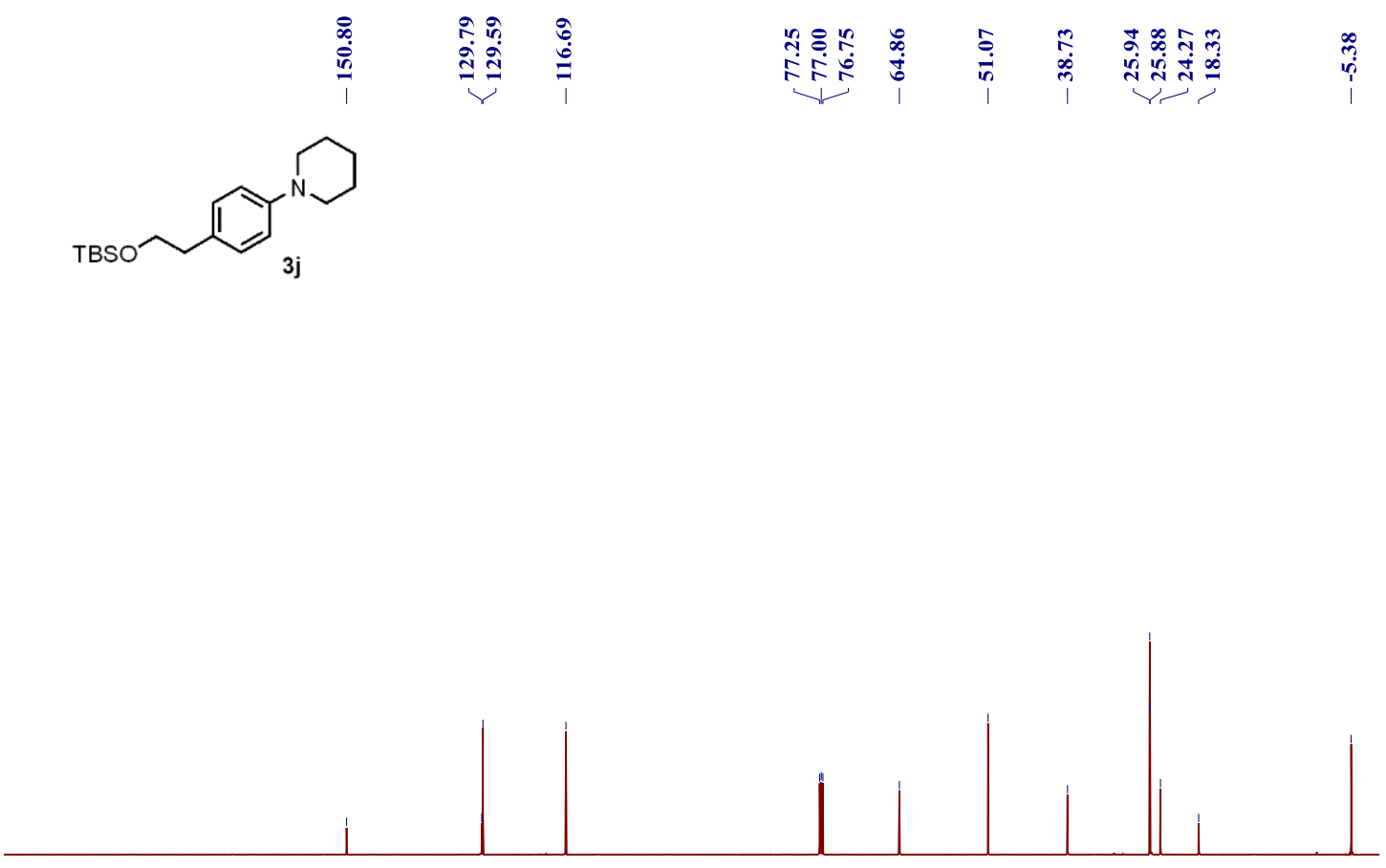

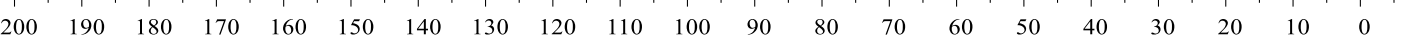


${ }^{1} \mathrm{H}$ NMR (500 MHz, $\left.\mathrm{CDCl}_{3}\right)$

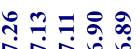

成

$\underbrace{\infty}_{\operatorname{ming}}$

${ }_{M e O}$

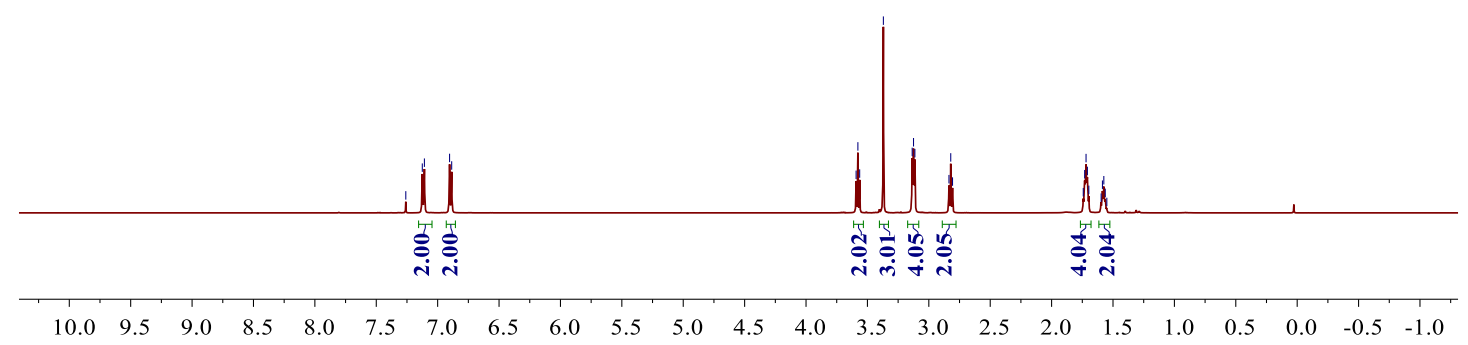

${ }^{13} \mathrm{C} \mathrm{NMR}\left(126 \mathrm{MHz}, \mathrm{CDCl}_{3}\right)$
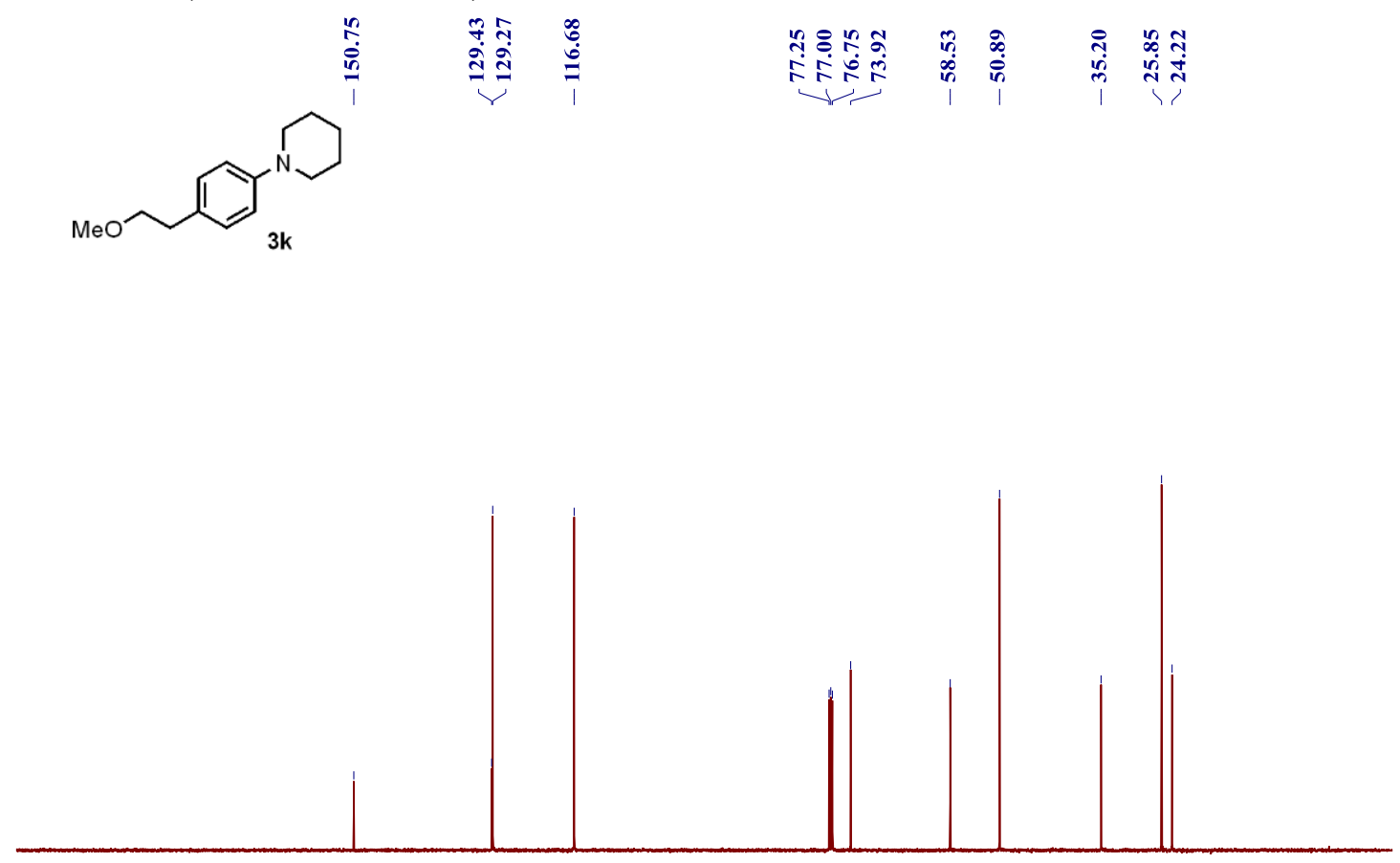

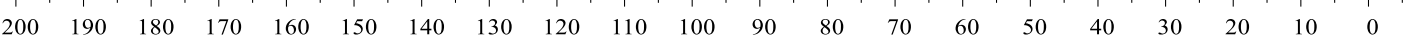


${ }^{1} \mathrm{H}$ NMR $\left(500 \mathrm{MHz}, \mathrm{CDCl}_{3}\right)$

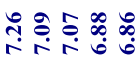<smiles>CCOCCc1ccc(N2CCCCC2)cc1</smiles>

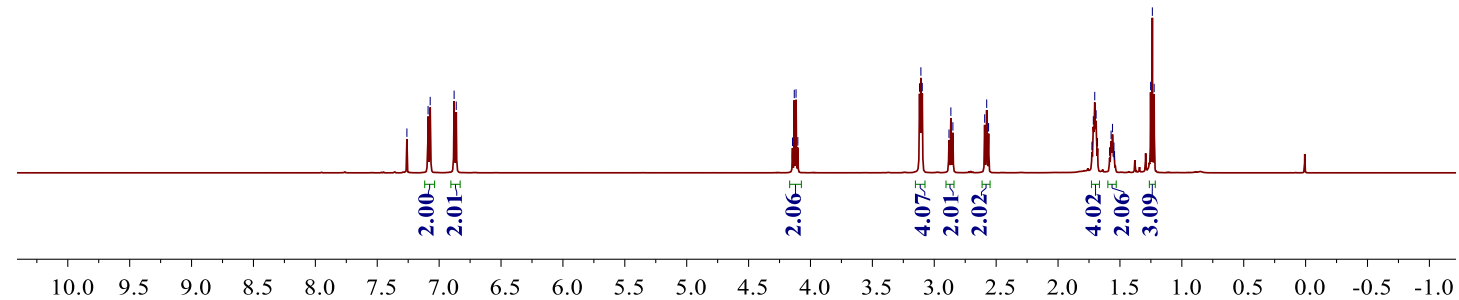

${ }^{13} \mathrm{C}$ NMR (126 MHz, $\mathrm{CDCl}_{3}$ )

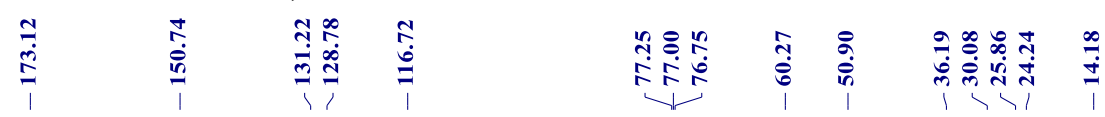<smiles>CC(=O)OCCc1ccc(N2CCCCC2)cc1</smiles>

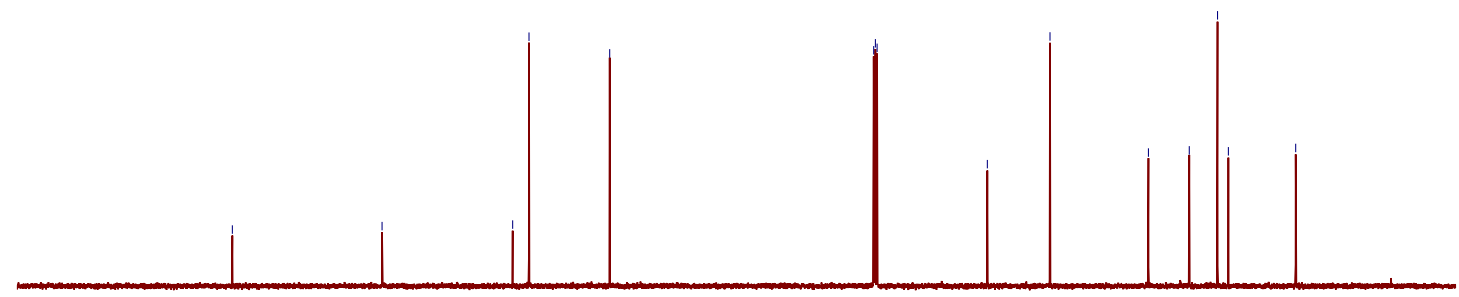

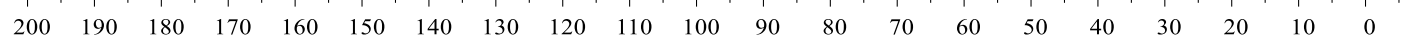


${ }^{1} \mathrm{H}$ NMR $\left(500 \mathrm{MHz}, \mathrm{CDCl}_{3}\right)$

ঙำ

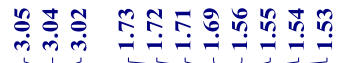<smiles>c1cc(N2CCCCC2)ccc1N1CCCCC1</smiles>

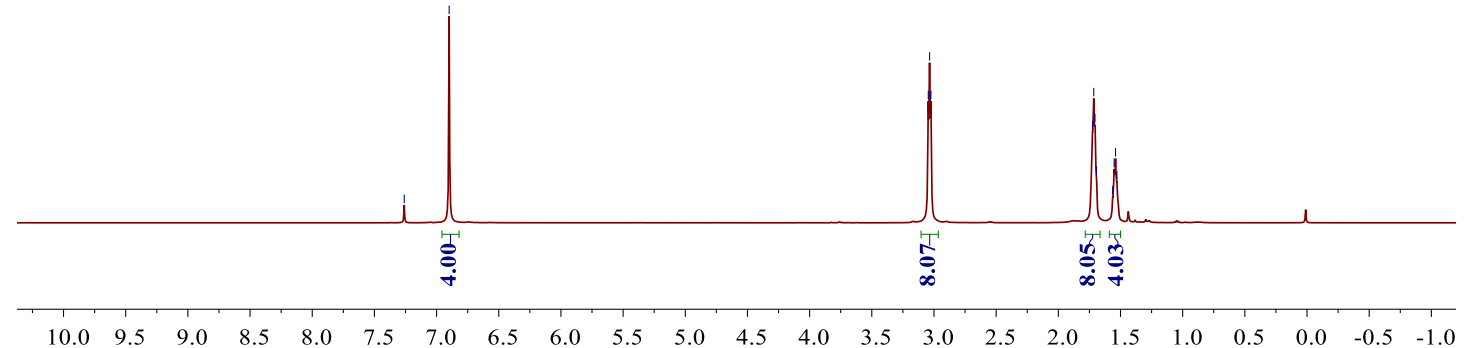

${ }^{13} \mathrm{C}$ NMR $\left(126 \mathrm{MHz}, \mathrm{CDCl}_{3}\right)$

要<smiles>c1cc(N2CCCCC2)ccc1N1CCCCC1</smiles>

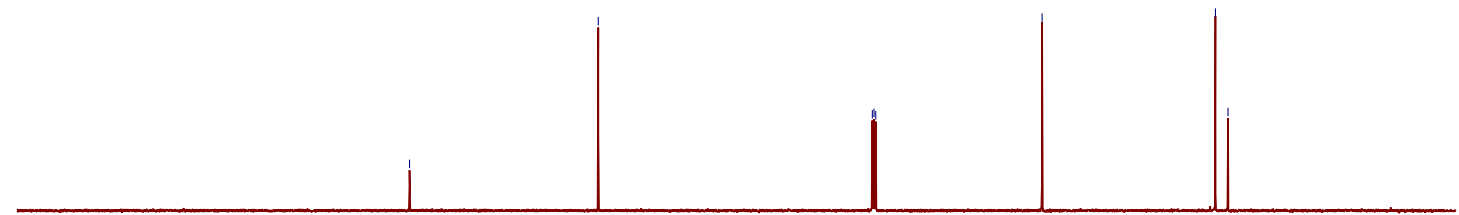

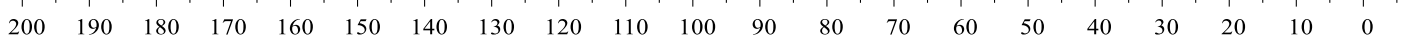


${ }^{1} \mathrm{H}$ NMR $\left(500 \mathrm{MHz}, \mathrm{CDCl}_{3}\right)$

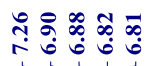

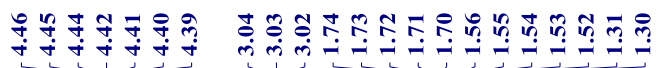
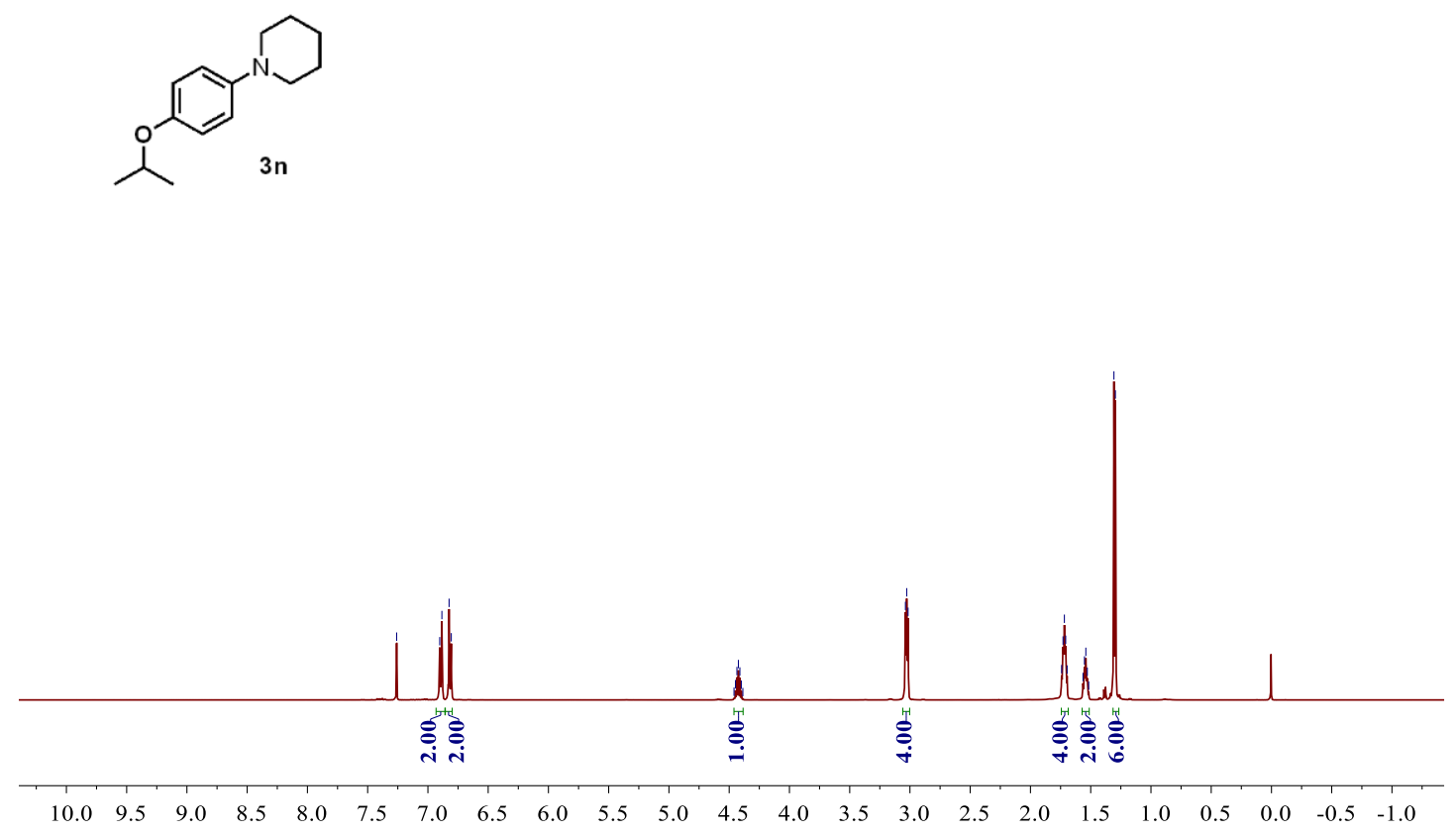

${ }^{13} \mathrm{C} \mathrm{NMR}\left(126 \mathrm{MHz}, \mathrm{CDCl}_{3}\right)$

\begin{tabular}{|c|c|c|c|c|}
\hline 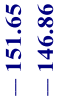 & 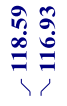 & 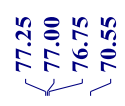 & $\begin{array}{l}= \\
\text { in } \\
\text { i }\end{array}$ & 目 \\
\hline
\end{tabular}
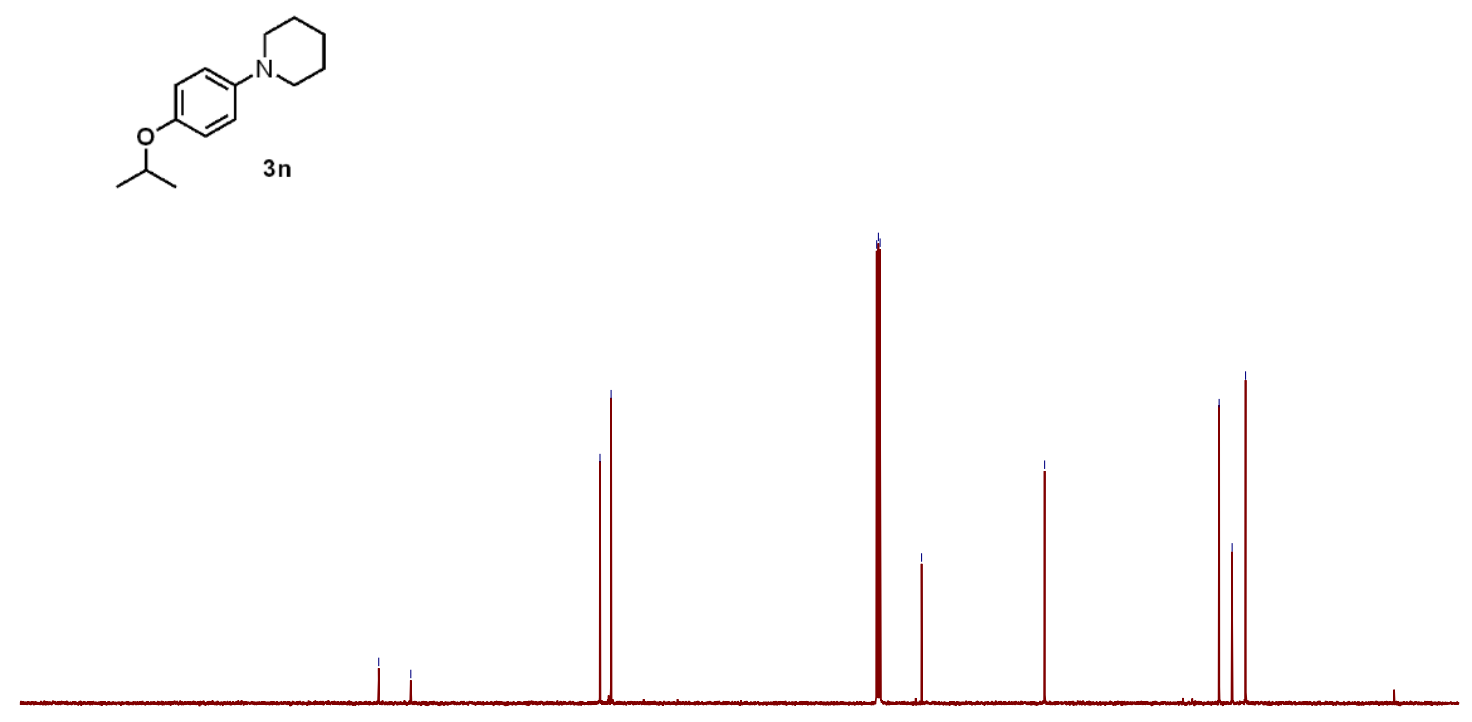

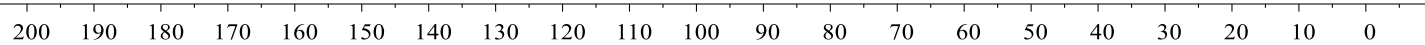


${ }^{1} \mathrm{H} \mathrm{NMR}\left(500 \mathrm{MHz}, \mathrm{CDCl}_{3}\right)$

ำ

$\therefore, 060$

อำ
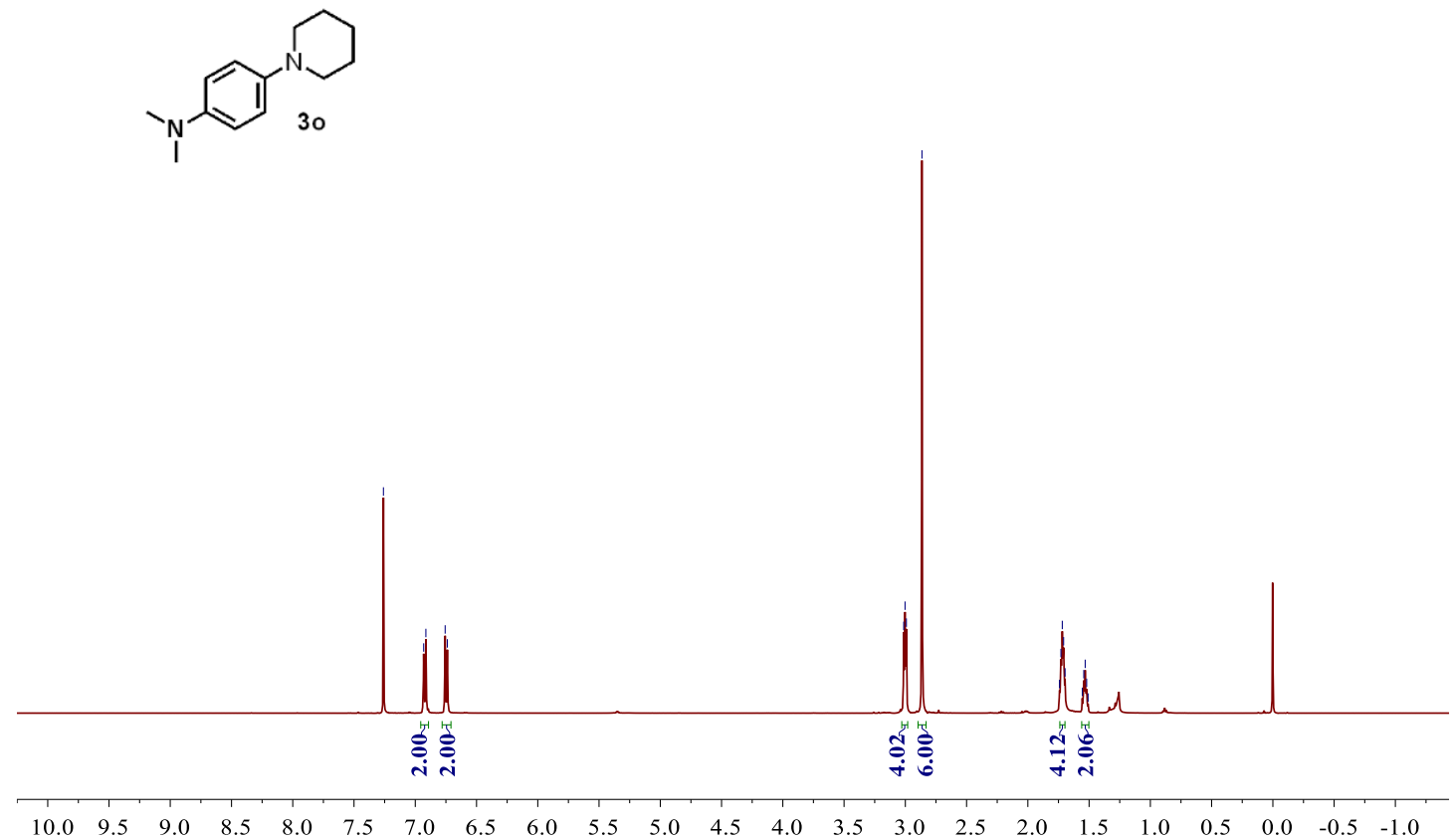

${ }^{13} \mathrm{C}$ NMR (126 MHz, $\mathrm{CDCl}_{3}$ )

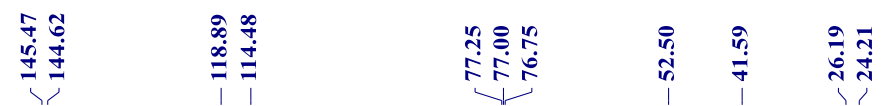

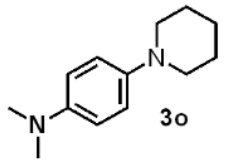

$\begin{array}{lllllllllll}200 & 190 & 180 & 170 & 160 & 150 & 140 & 130 & 120 & 110 & 100\end{array}$

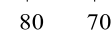

$\begin{array}{llllll}60 & 50 & 40 & 30 & 20 & 10\end{array}$ 
${ }^{1} \mathrm{H}$ NMR $\left(500 \mathrm{MHz}, \mathrm{CDCl}_{3}\right)$

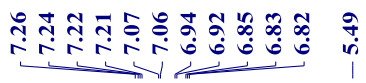

$=$<smiles>CCCNc1ccc(N2CCCC2)cc1</smiles>

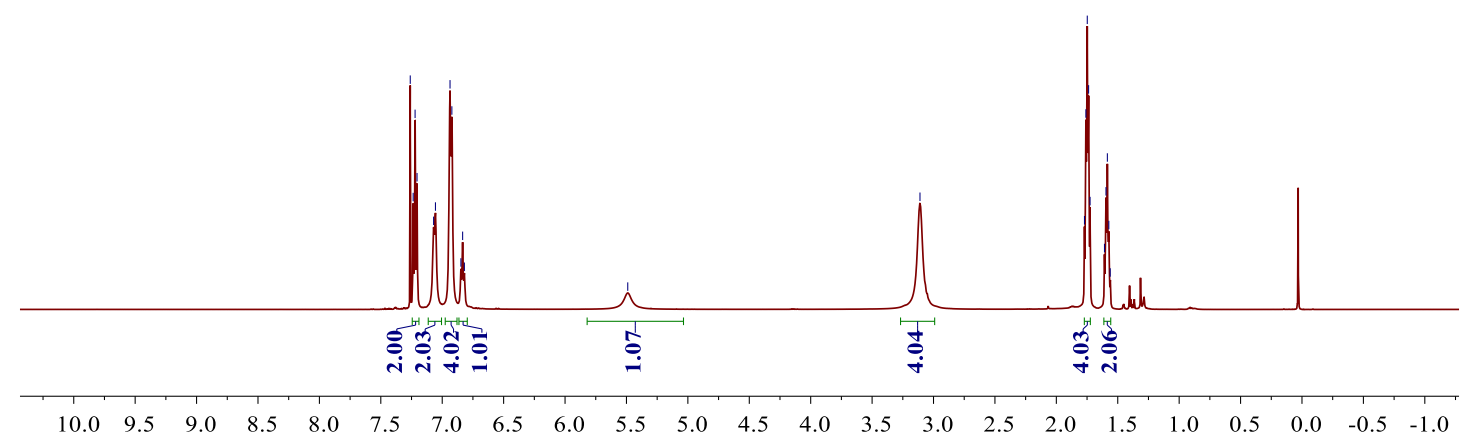

${ }^{13} \mathrm{C} \mathrm{NMR}\left(126 \mathrm{MHz}, \mathrm{CDCl}_{3}\right)$

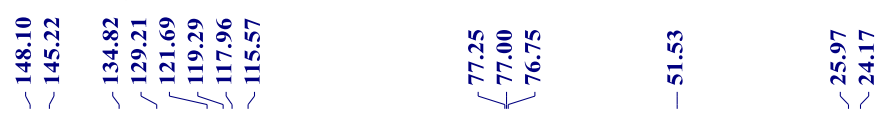<smiles>c1ccc(Nc2ccc(N3CCCCC3)cc2)cc1</smiles>

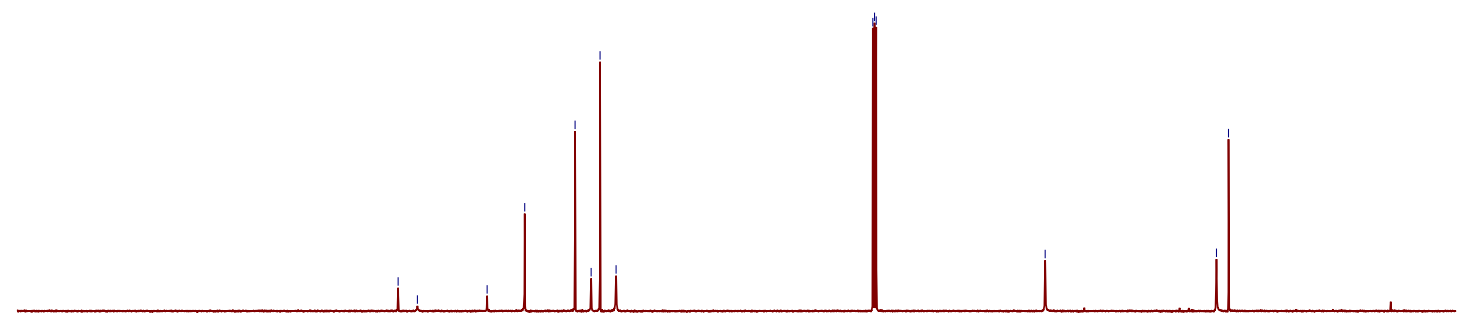

$\begin{array}{llllllllllllllllllllll}1 & 190 & 190 & 180 & 170 & 160 & 150 & 140 & 130 & 120 & 110 & 100 & 90 & 80 & 70 & 60 & 50 & 40 & 30 & 20 & 10 & 0\end{array}$ 
${ }^{1} \mathrm{H}$ NMR $\left(500 \mathrm{MHz}, \mathrm{CDCl}_{3}\right)$

苛

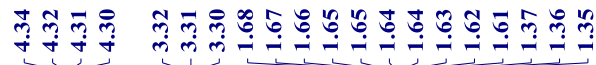
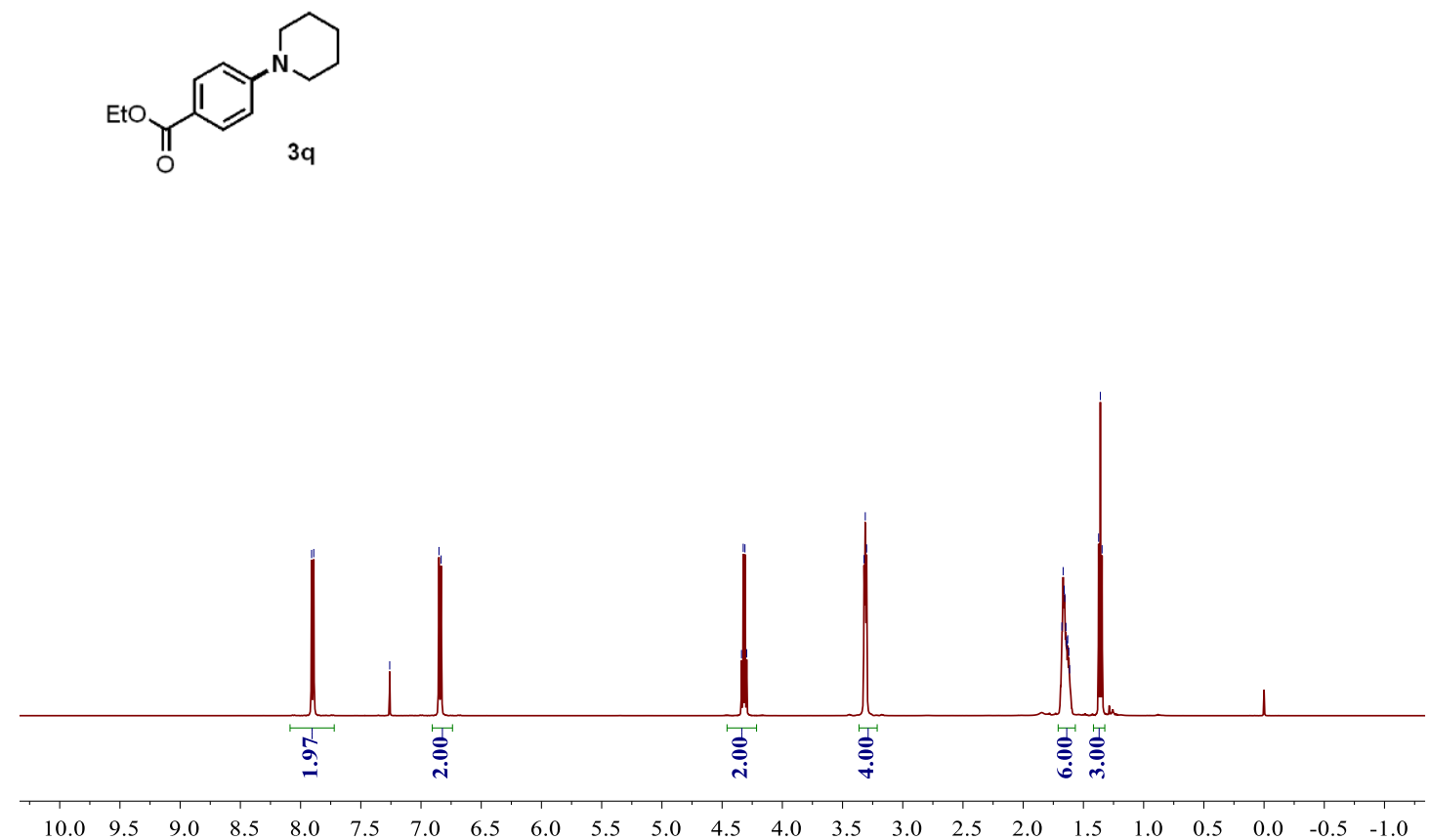

${ }^{13} \mathrm{C} \mathrm{NMR}\left(126 \mathrm{MHz}, \mathrm{CDCl}_{3}\right)$

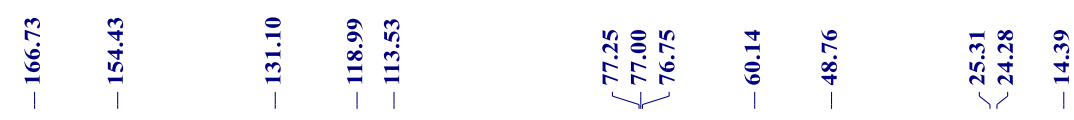<smiles>CCOC(=O)c1ccc(N2CCCCC2)cc1</smiles>

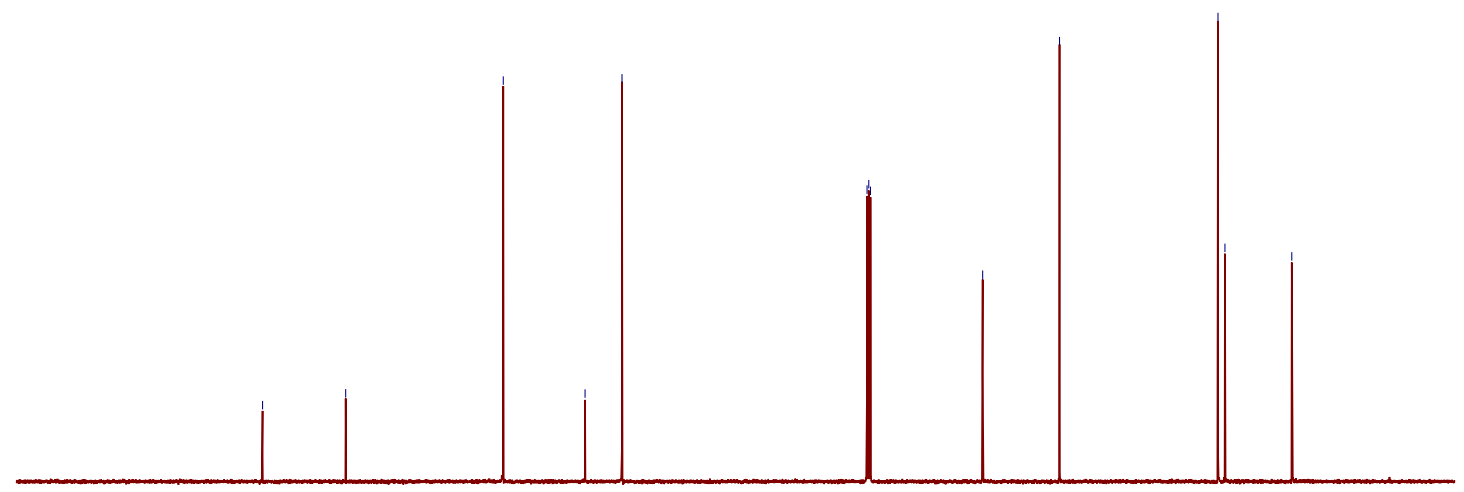

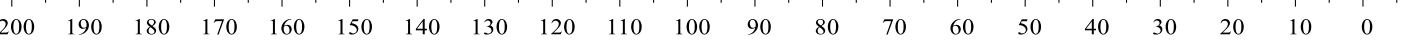


${ }^{1} \mathrm{H}$ NMR $\left(500 \mathrm{MHz}, \mathrm{CDCl}_{3}\right)$

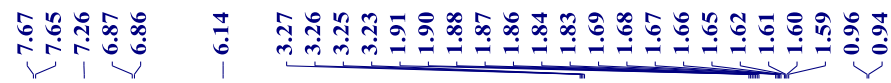<smiles>CC(C)CNC(=O)c1ccc(N2CCCCC2)cc1</smiles>

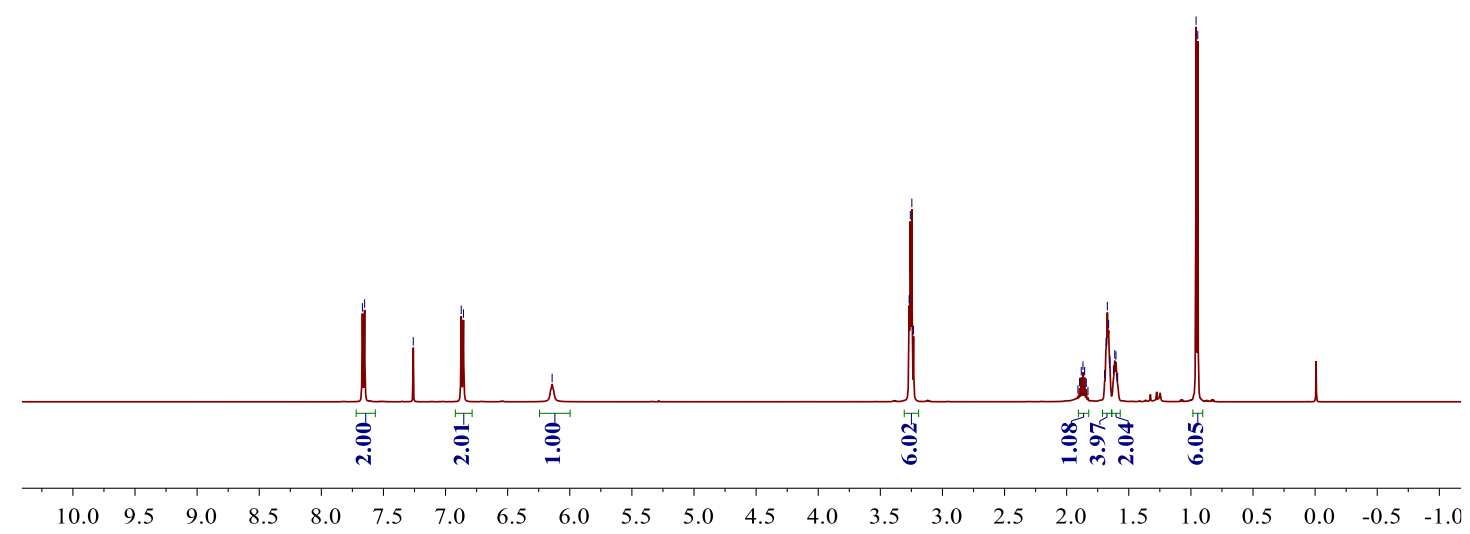

${ }^{13} \mathrm{C} \mathrm{NMR}\left(126 \mathrm{MHz}, \mathrm{CDCl}_{3}\right)$

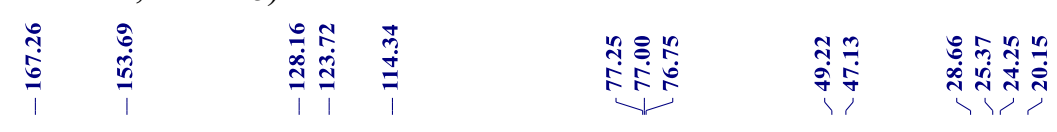<smiles>CC(C)CNC(=O)c1ccc(N2CCCCC2)cc1</smiles>

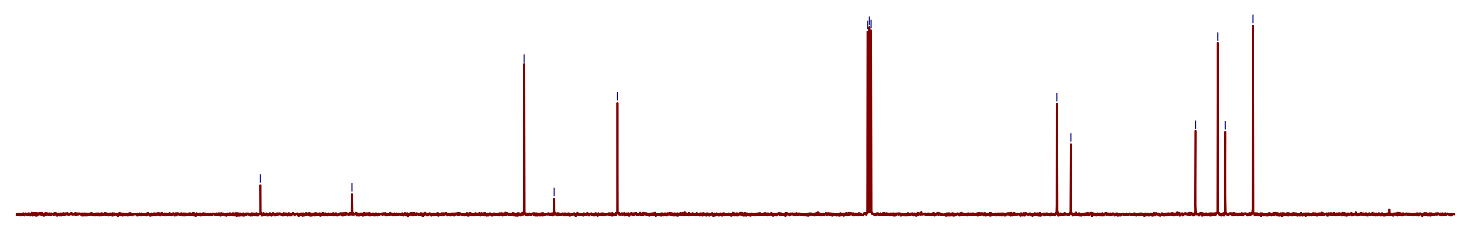

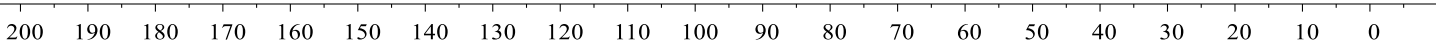


${ }^{1} \mathrm{H}$ NMR $\left(500 \mathrm{MHz}, \mathrm{CDCl}_{3}\right)$

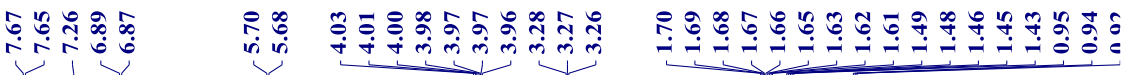<smiles>CCC(CC)NC(=O)c1ccc(N2CCCC2)cc1</smiles>

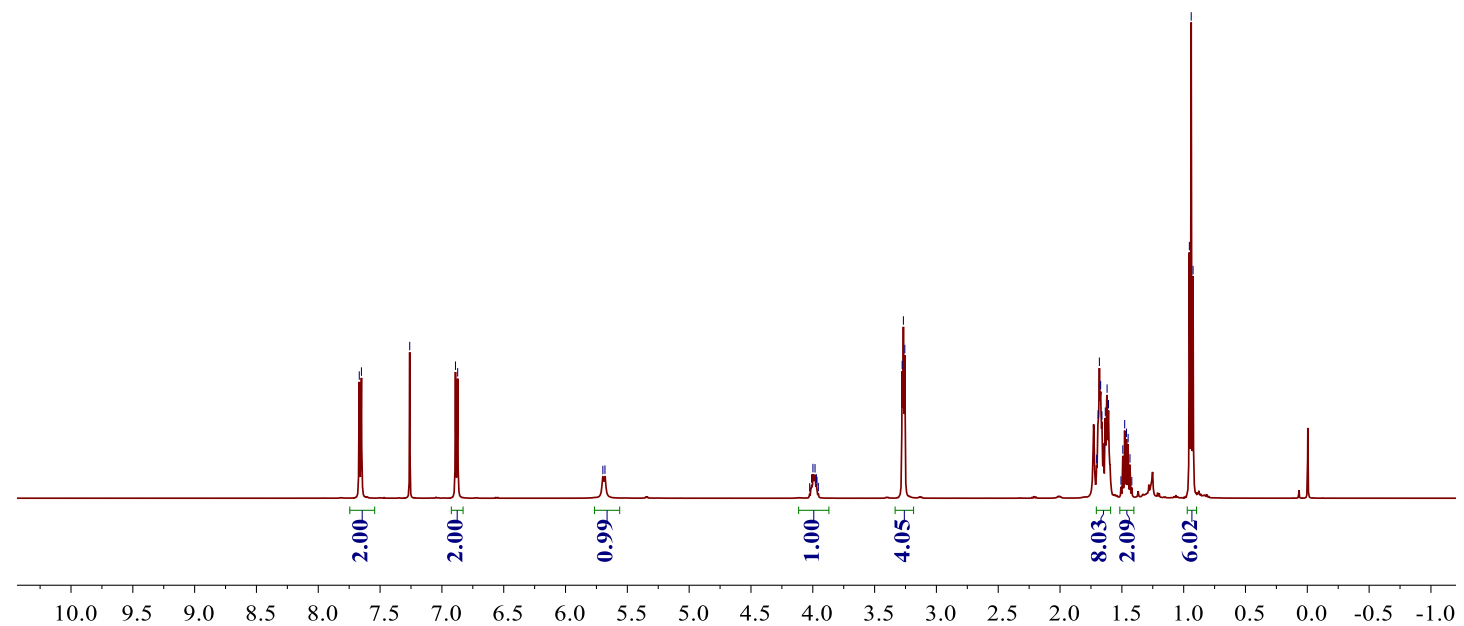

${ }^{13} \mathrm{C} \mathrm{NMR}\left(126 \mathrm{MHz}, \mathrm{CDCl}_{3}\right)$

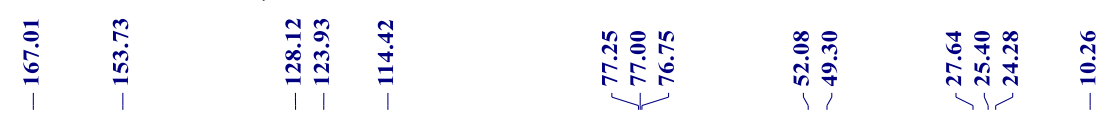<smiles>CCC(CC)NC(=O)c1ccc(N2CCCC2)cc1</smiles>

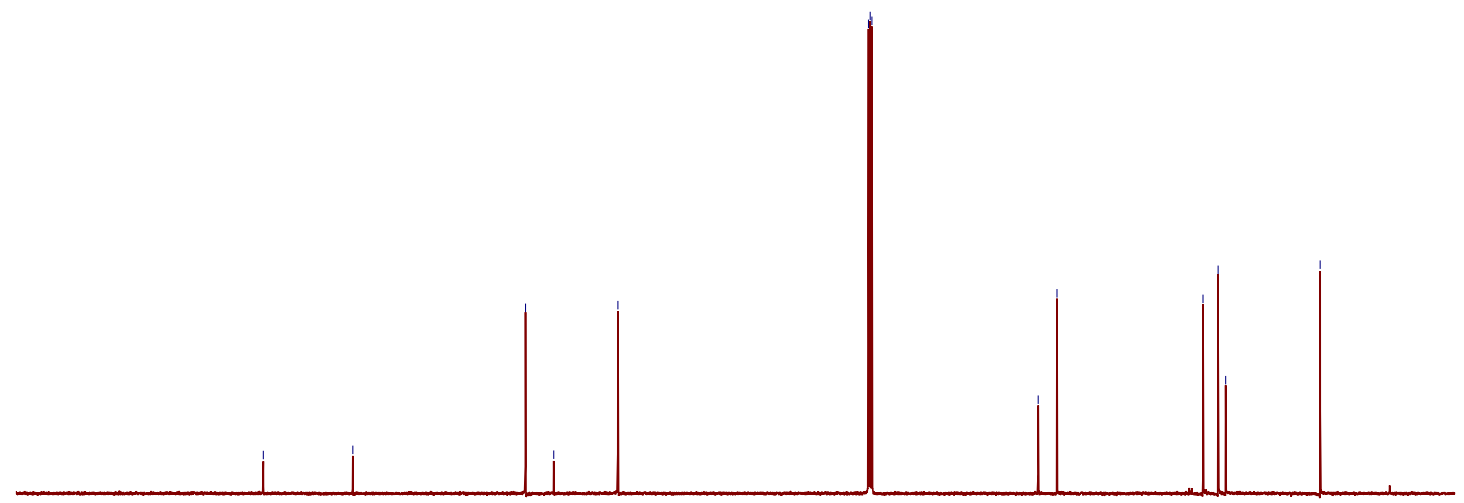

$\begin{array}{lllllllllllllllllllll}200 & 190 & 180 & 170 & 160 & 150 & 140 & 130 & 120 & 110 & 100 & 90 & 80 & 70 & 60 & 50 & 40 & 30 & 20 & 10 & 0\end{array}$ 
${ }^{1} \mathrm{H}$ NMR (500 MHz, $\left.\mathrm{CDCl}_{3}\right)$
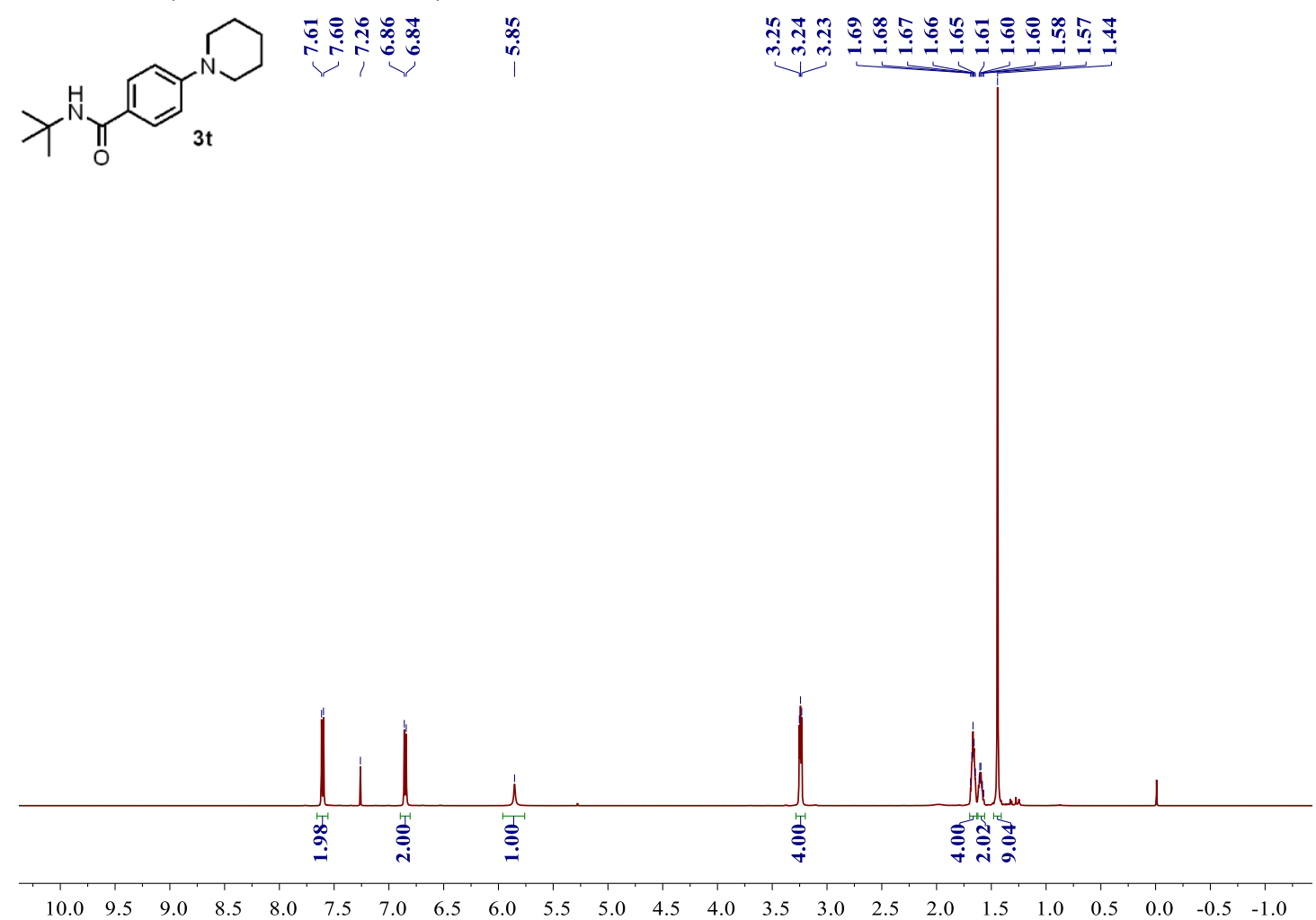

${ }^{13} \mathrm{C} \mathrm{NMR}\left(126 \mathrm{MHz}, \mathrm{CDCl}_{3}\right)$
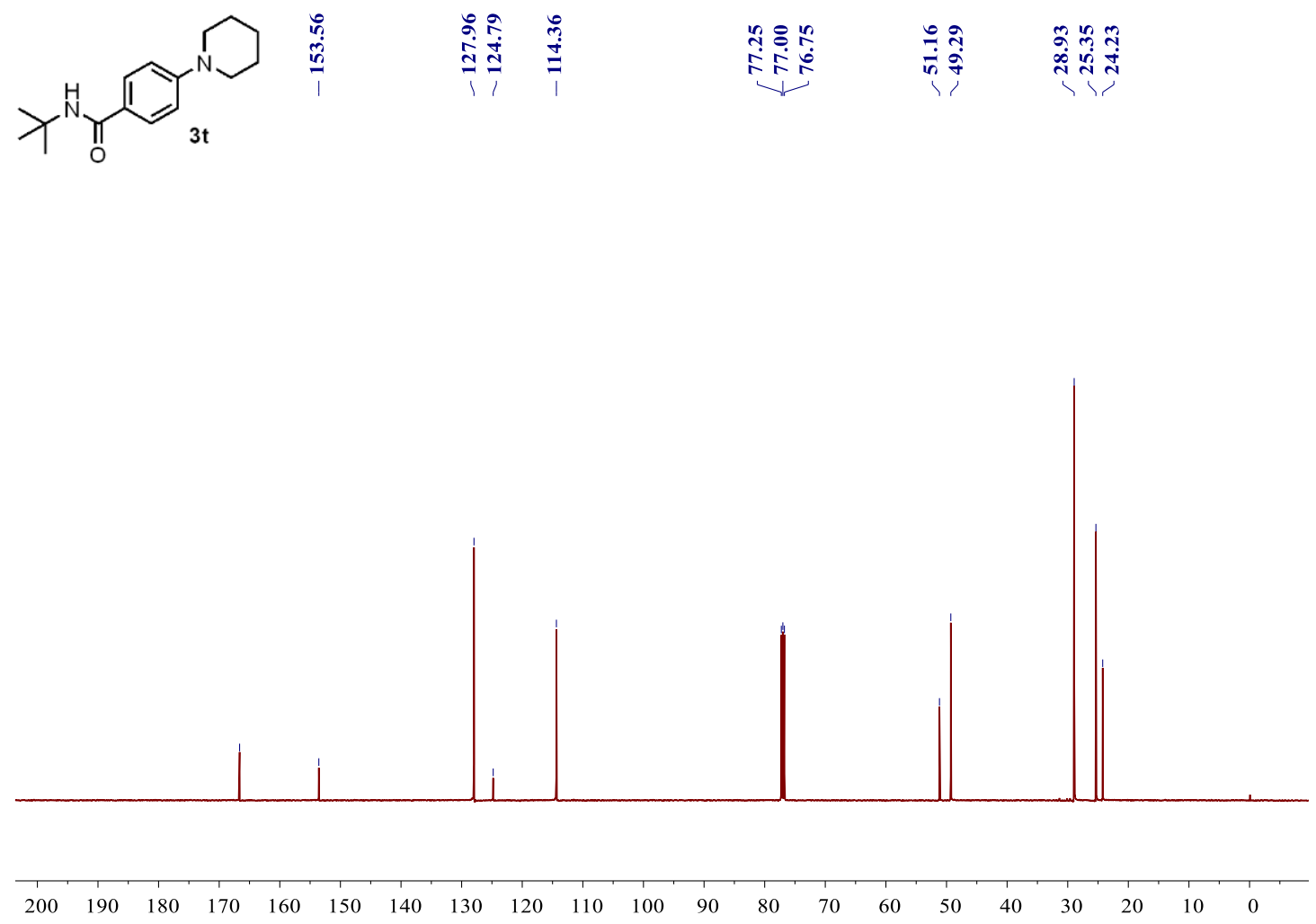
${ }^{1} \mathrm{H} \mathrm{NMR}\left(500 \mathrm{MHz}, \mathrm{CDCl}_{3}\right)$

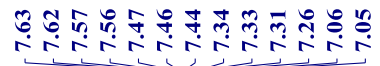

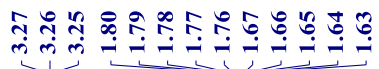<smiles>c1ccc(-c2ccc(N3CCCCC3)cc2)cc1</smiles>

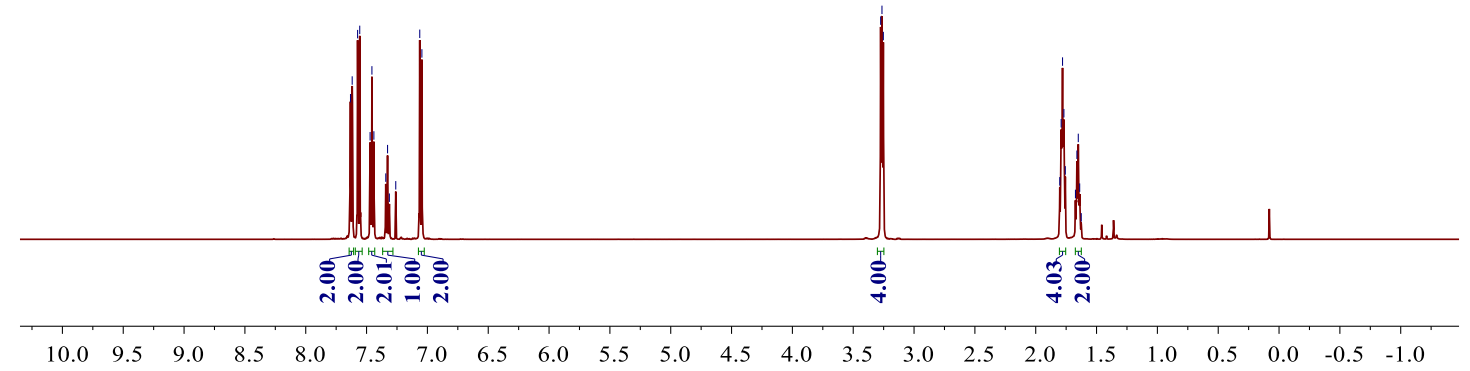

${ }^{13} \mathrm{C} \mathrm{NMR}\left(126 \mathrm{MHz}, \mathrm{CDCl}_{3}\right)$

\begin{tabular}{|c|c|c|}
\hline 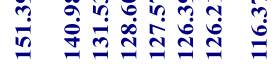 & فئم & $\tilde{\overbrace{}}$ \\
\hline
\end{tabular}<smiles>c1ccc(-c2ccc(N3CCCCC3)cc2)cc1</smiles>

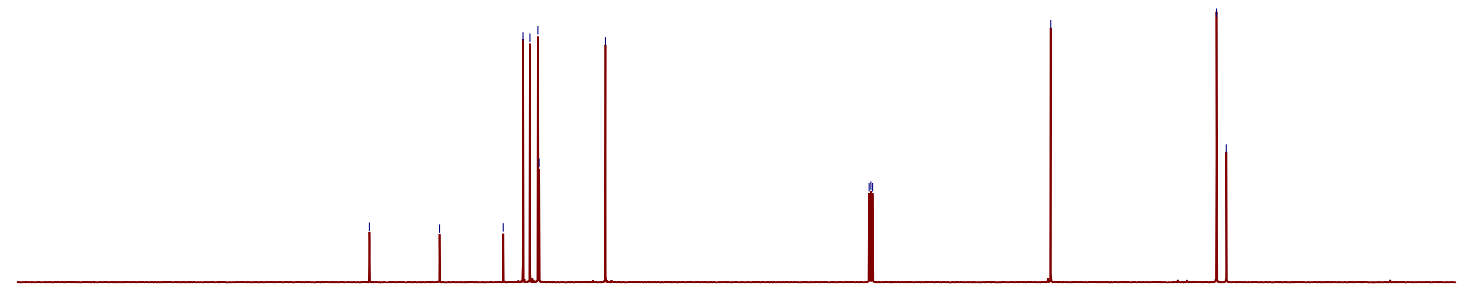

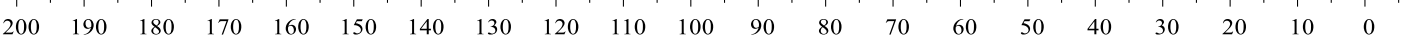


${ }^{1} \mathrm{H} \mathrm{NMR}\left(500 \mathrm{MHz}, \mathrm{CDCl}_{3}\right)$

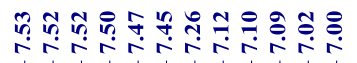

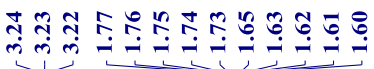
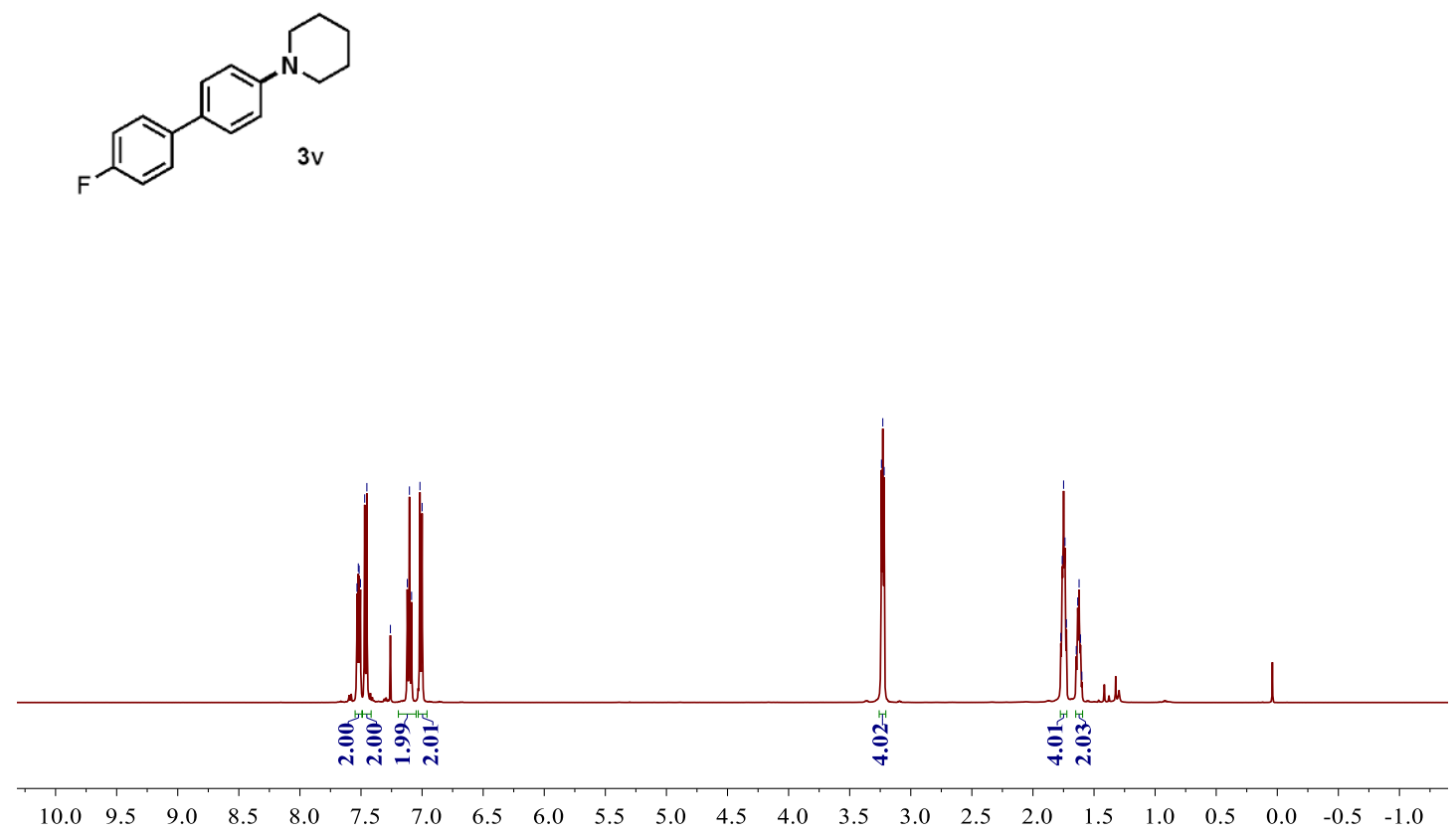

${ }^{13} \mathrm{C} \mathrm{NMR}\left(126 \mathrm{MHz}, \mathrm{CDCl}_{3}\right)$

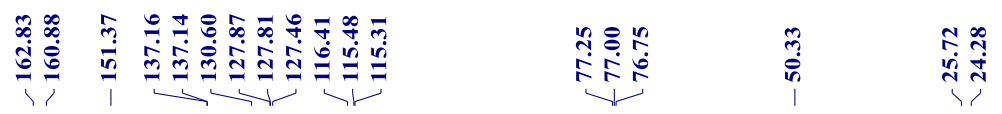<smiles>Fc1ccc(-c2ccc(N3CCCCC3)cc2)cc1</smiles>

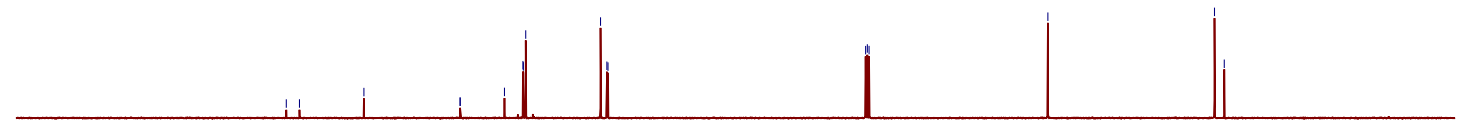

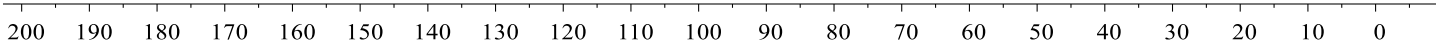


${ }^{19} \mathrm{~F} \mathrm{NMR}\left(471 \mathrm{MHz}, \mathrm{CDCl}_{3}\right)$

$\stackrel{\stackrel{3}{5}}{\frac{3}{5}}$

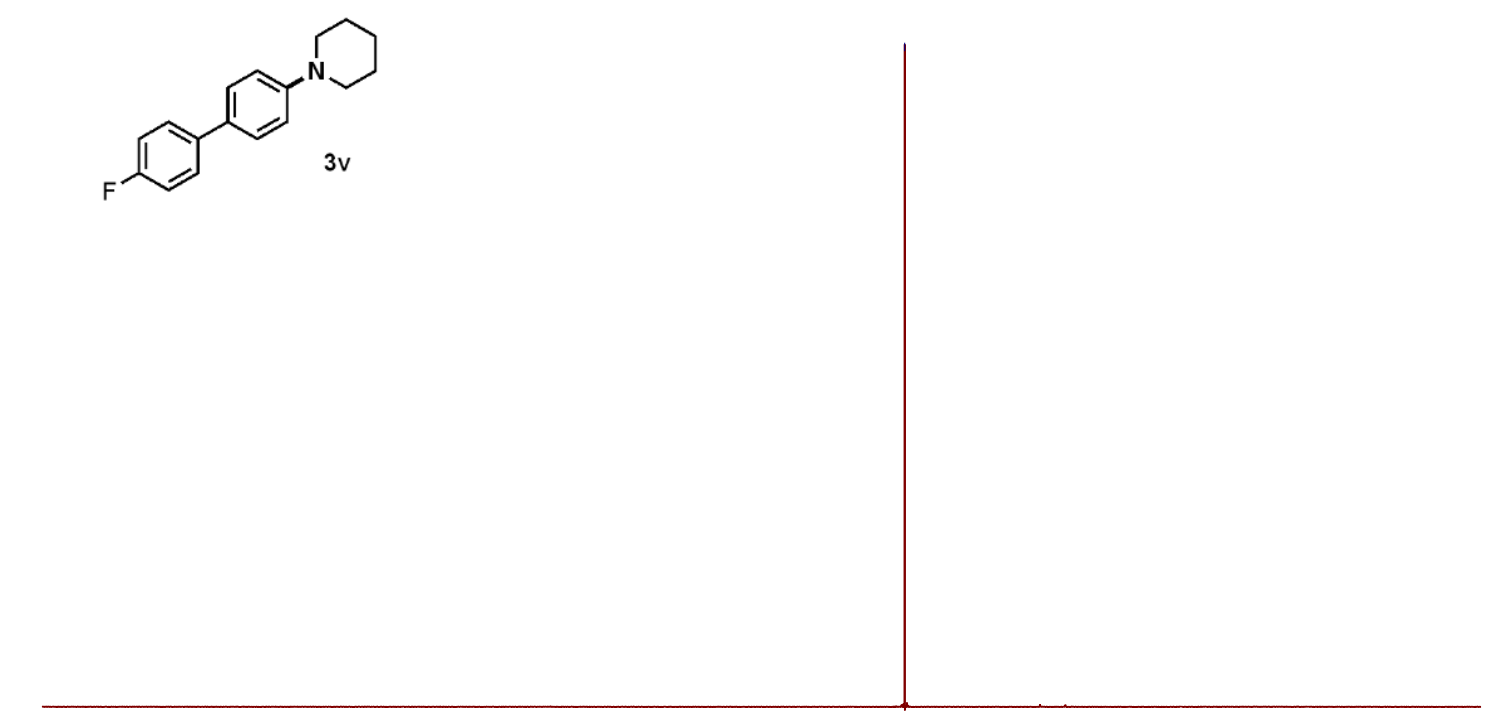

\begin{tabular}{llllllllllllllllllllll}
\hline 10 & 0 & -10 & -20 & -30 & -40 & -50 & -60 & -70 & -80 & -90 & -100 & -110 & -120 & -130 & -140 & -150 & -160 & -170 & -180 & -190 & -200
\end{tabular}

${ }^{1} \mathrm{H}$ NMR (500 $\left.\mathrm{MHz}, \mathrm{CDCl}_{3}\right)$

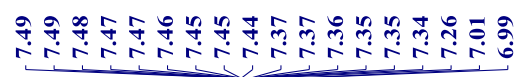

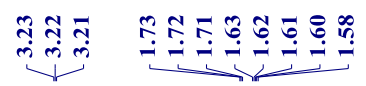<smiles>Clc1ccc(-c2ccc(N3CCCCC3)cc2)cc1</smiles>

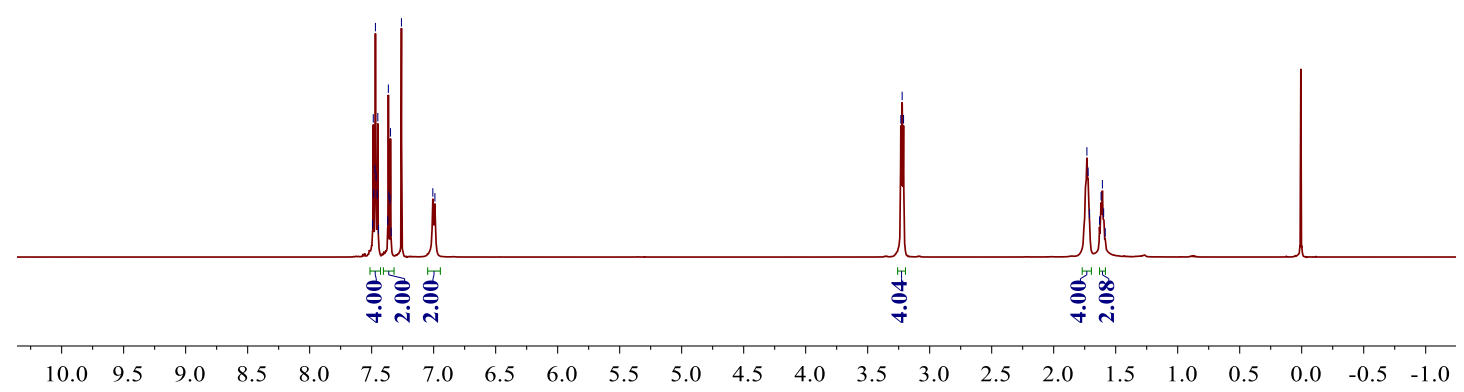


${ }^{13} \mathrm{C}$ NMR (126 MHz, $\left.\mathrm{CDCl}_{3}\right)$

至

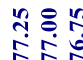

ลิ่
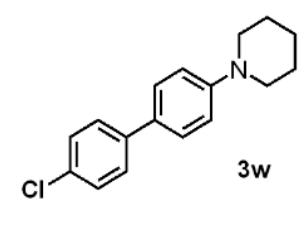

$$
\text { w }
$$
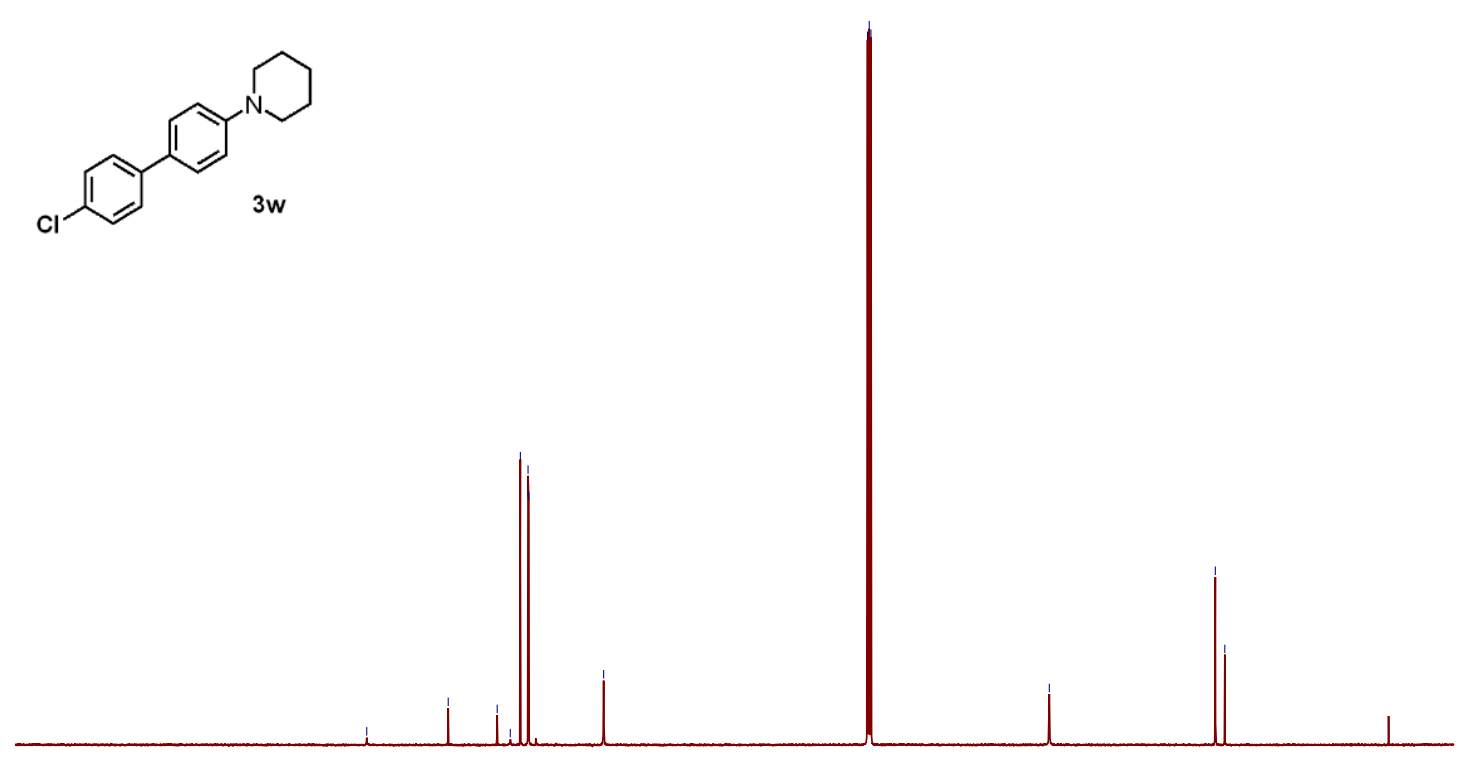

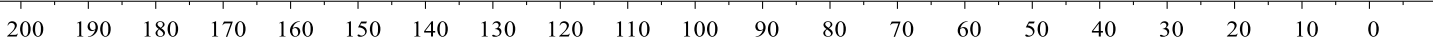

${ }^{1} \mathrm{H}$ NMR (500 MHz, $\left.\mathrm{CDCl}_{3}\right)$

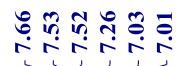

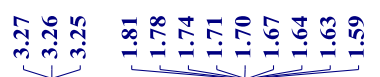
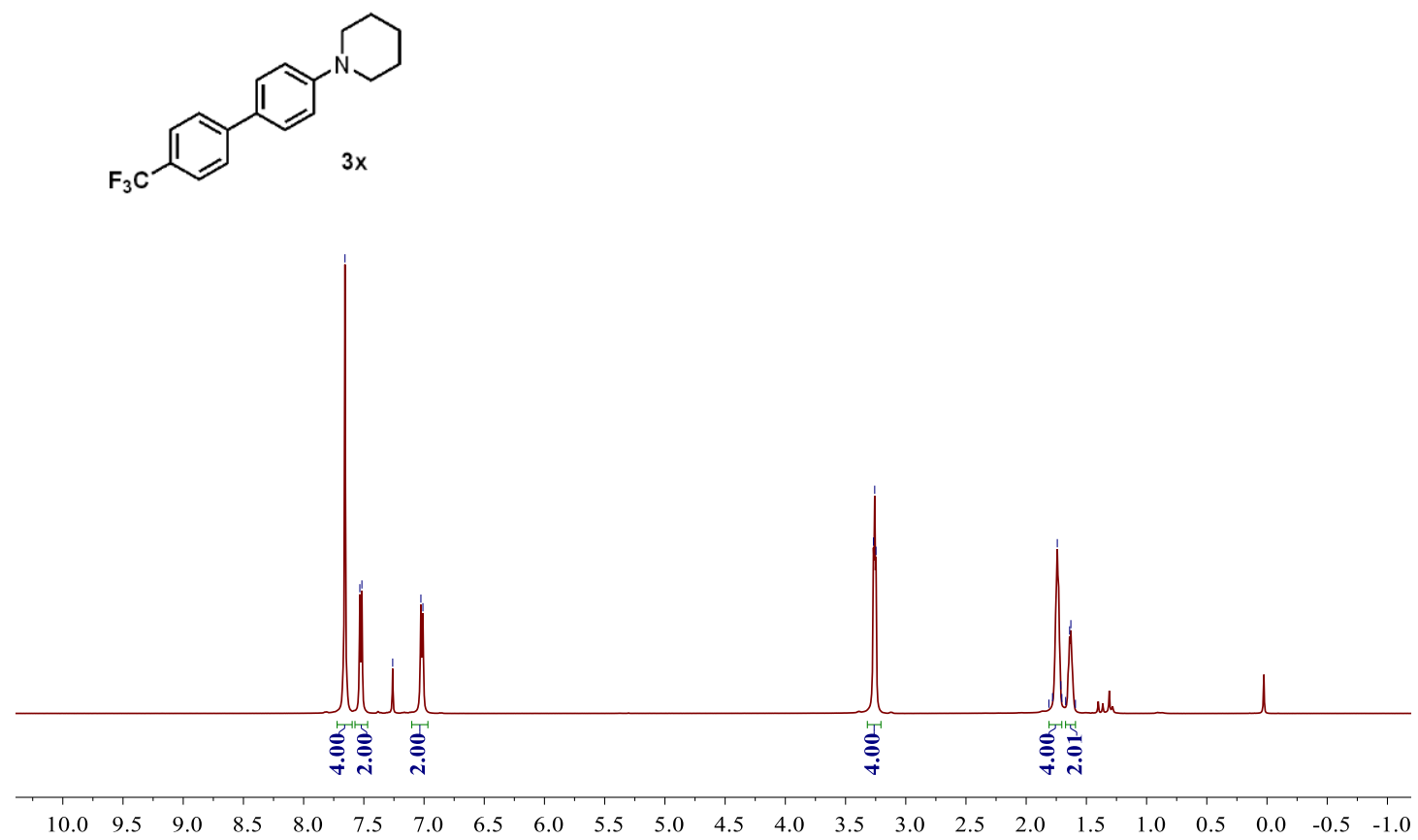
${ }^{13} \mathrm{C}$ NMR $\left(126 \mathrm{MHz}, \mathrm{CDCl}_{3}\right)$

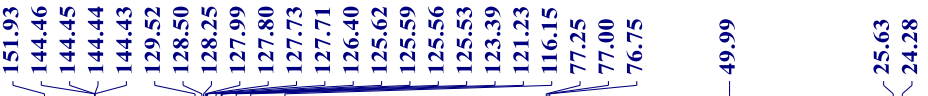
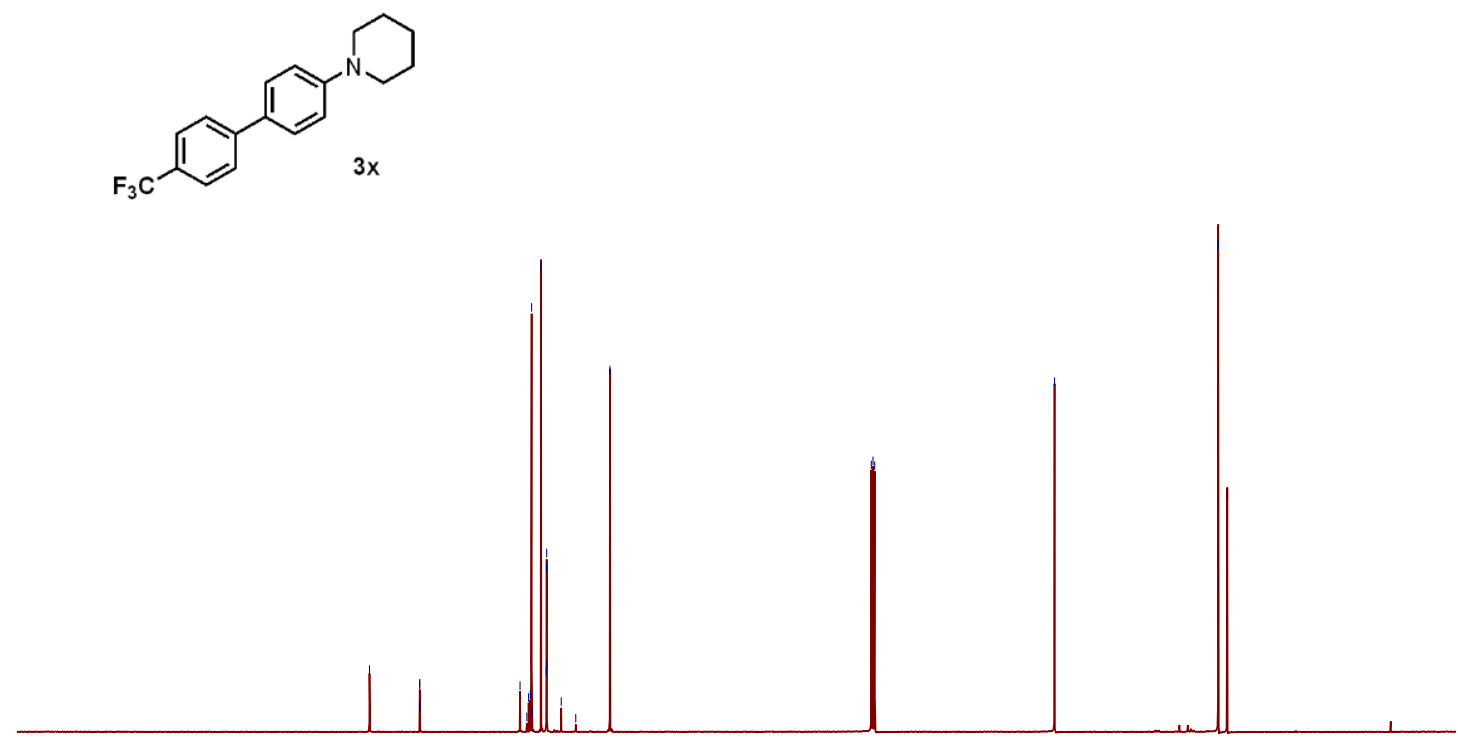

$\begin{array}{llllllllllllllllllll}200 & 190 & 180 & 170 & 160 & 150 & 140 & 130 & 120 & 110 & 100 & 90 & 80 & 70 & 60 & 50 & 40 & 30 & 20 & 10\end{array}$

${ }^{19} \mathrm{~F}$ NMR $\left(471 \mathrm{MHz}, \mathrm{CDCl}_{3}\right)$

กิ
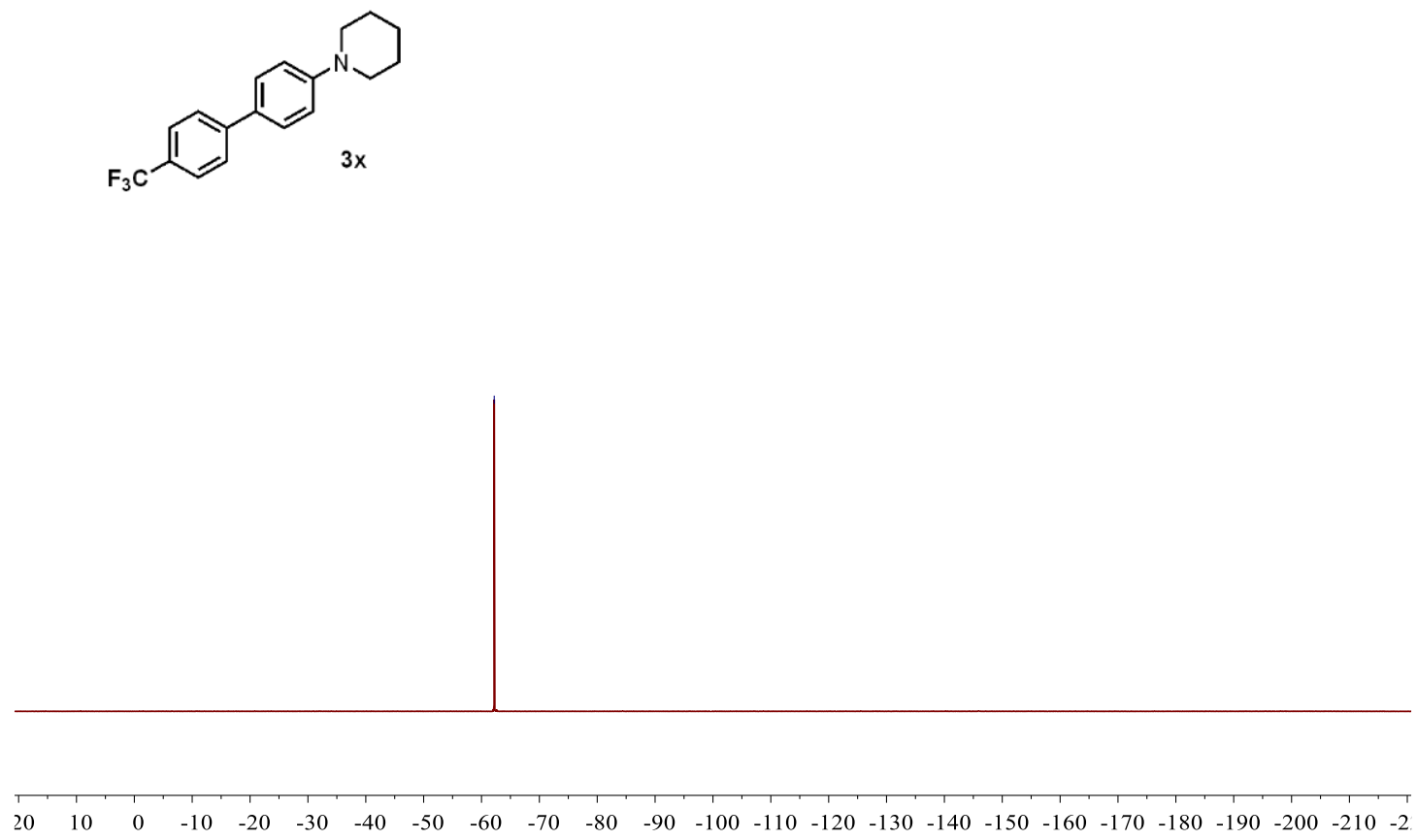
${ }^{1} \mathrm{H}$ NMR $\left(500 \mathrm{MHz}, \mathrm{CDCl}_{3}\right)$

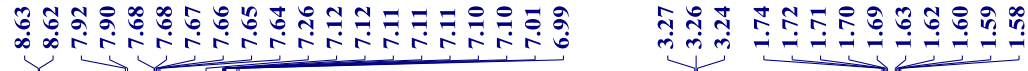<smiles>c1ccc(-c2ccc(N3CCCC3)cc2)nc1</smiles>

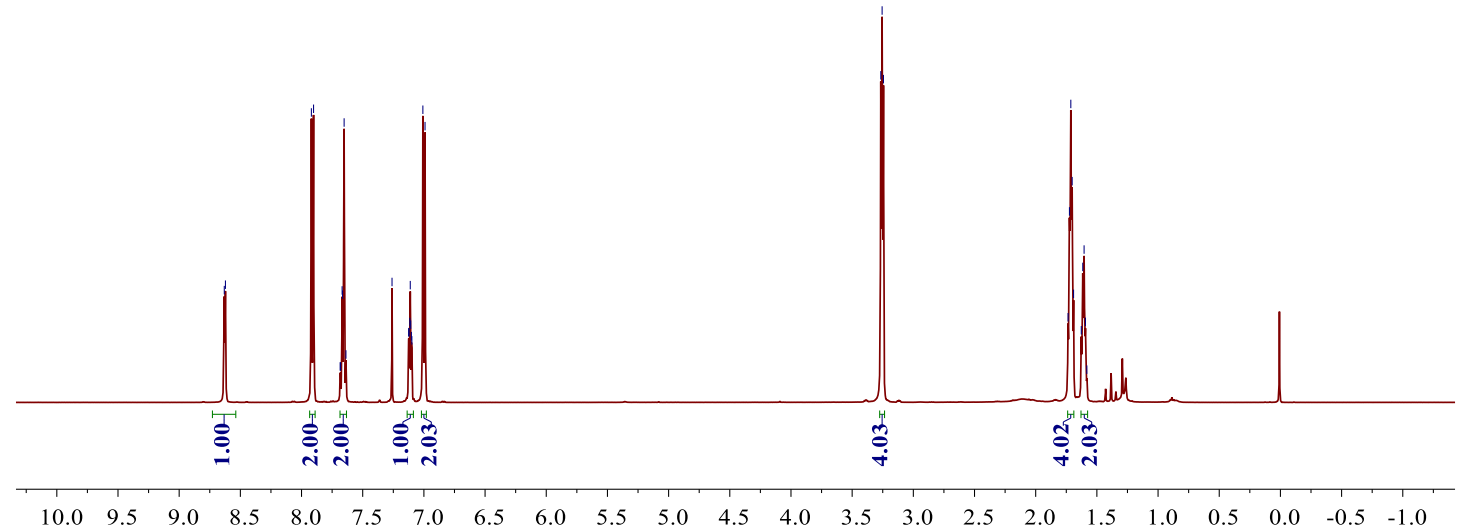

${ }^{13} \mathrm{C} \mathrm{NMR}\left(126 \mathrm{MHz}, \mathrm{CDCl}_{3}\right)$

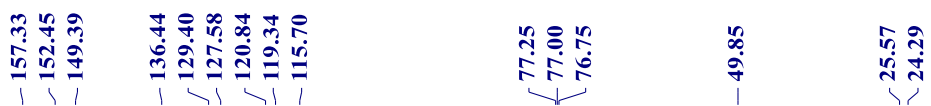<smiles>c1ccc(-c2ccc(N3CCCCC3)cc2)nc1</smiles>

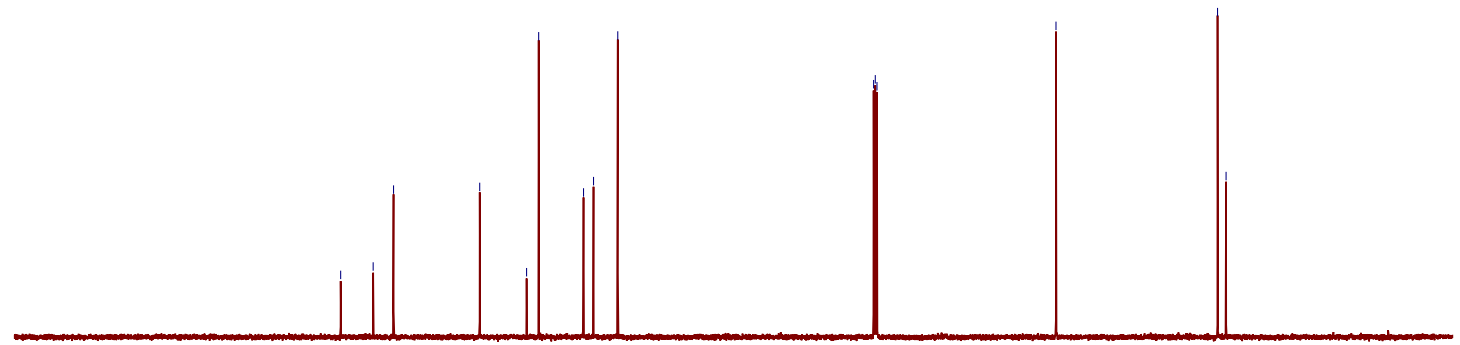

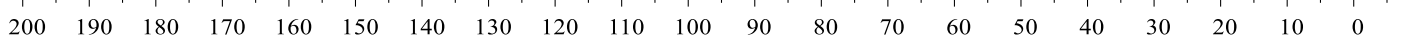


${ }^{1} \mathrm{H} \mathrm{NMR}\left(500 \mathrm{MHz}, \mathrm{CDCl}_{3}\right)$
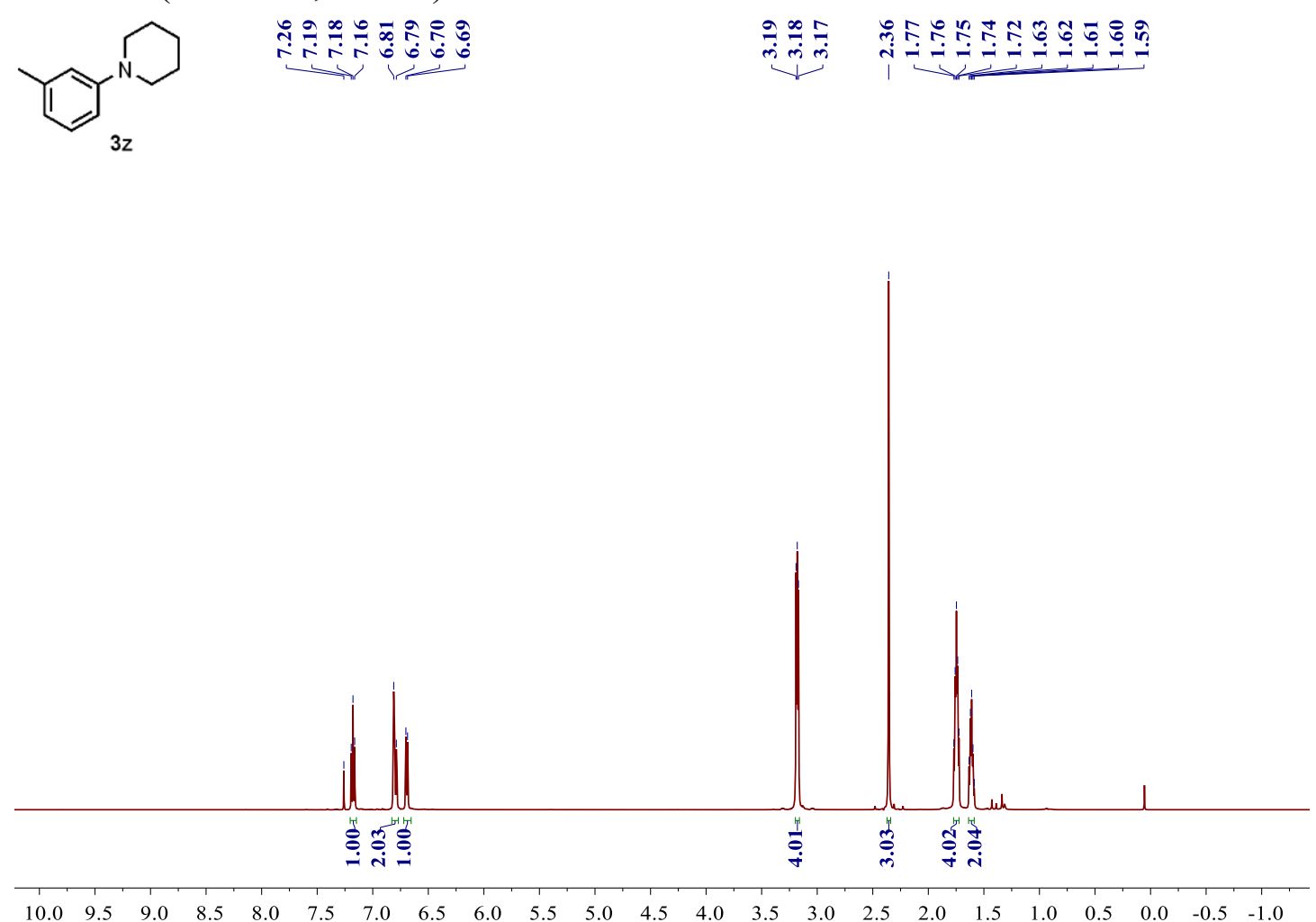

$\left.{ }^{13} \mathrm{C} \mathrm{NMR} \mathrm{(126} \mathrm{MHz,} \mathrm{CDCl}_{3}\right)$

$\underbrace{N}_{3 z} \underbrace{n}_{3 z}$

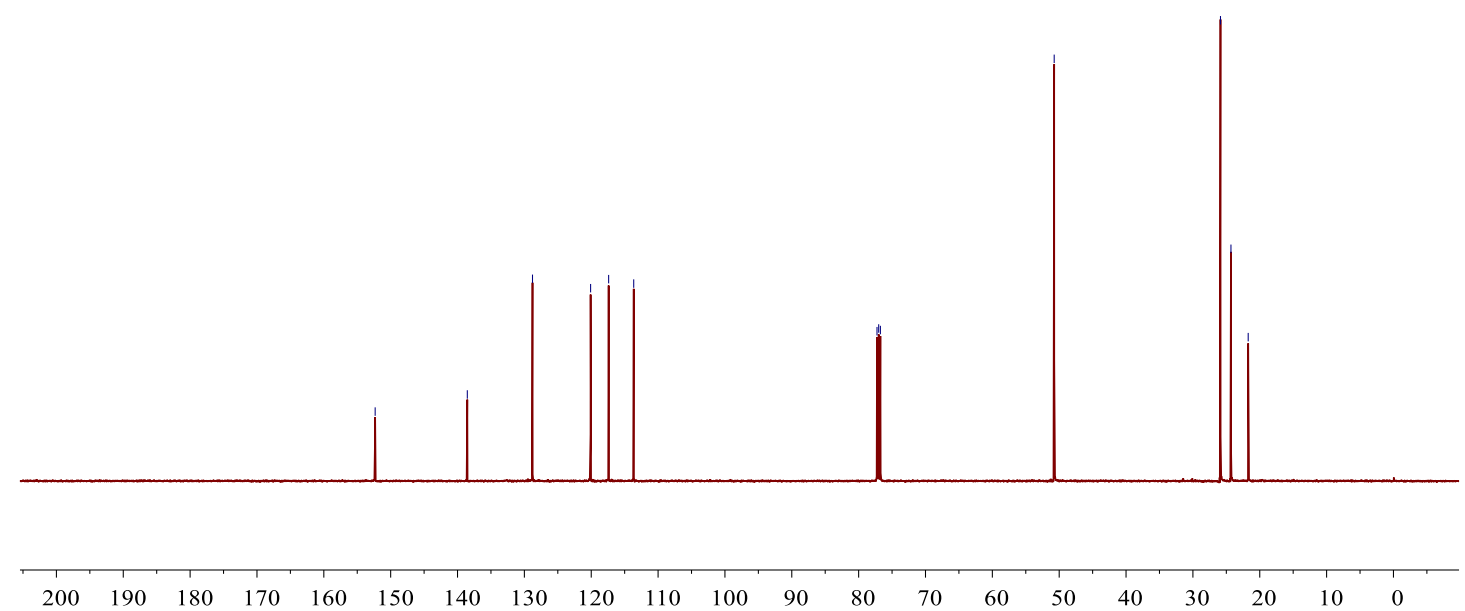


${ }^{1} \mathrm{H}$ NMR $\left(500 \mathrm{MHz}, \mathrm{CDCl}_{3}\right)$

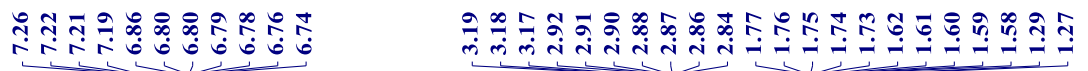

$\underbrace{N}_{3 a a}$

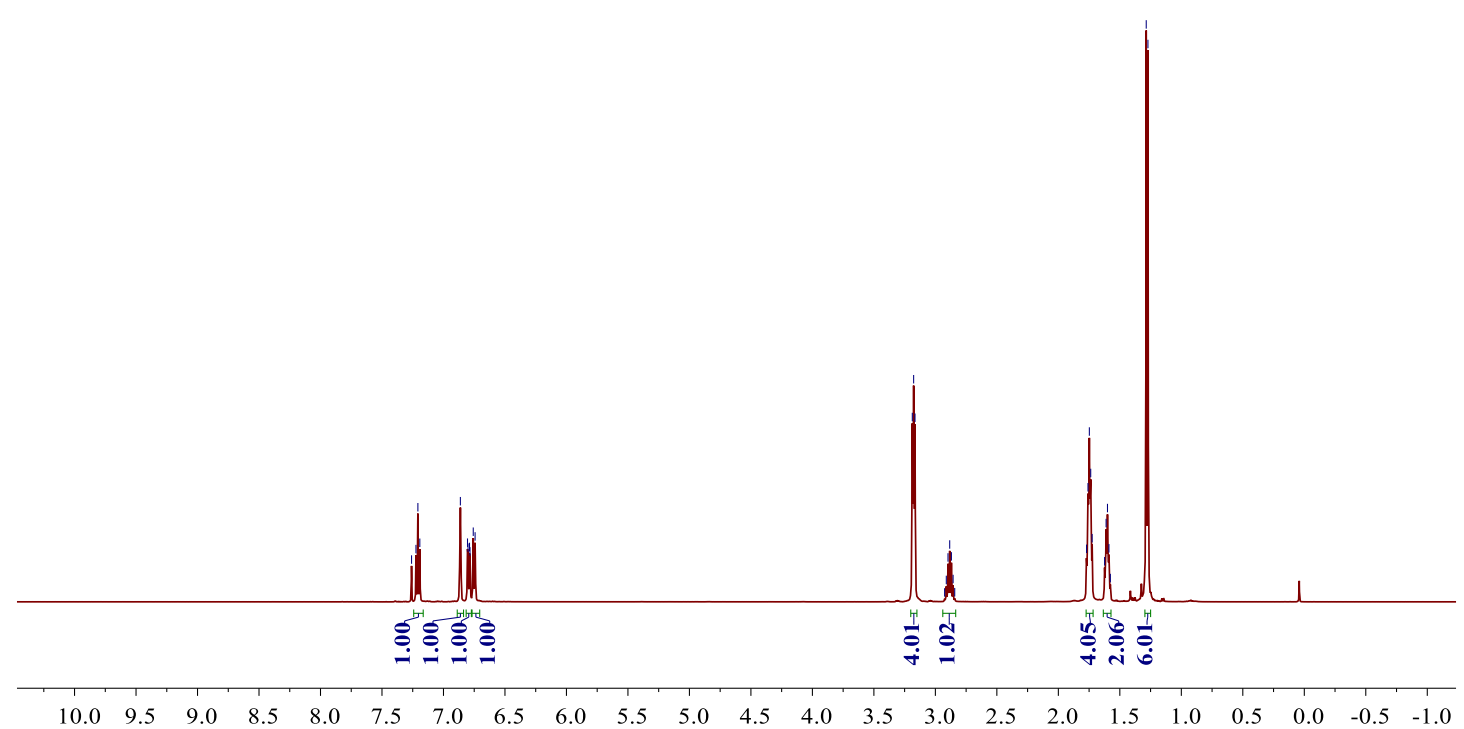

${ }^{13} \mathrm{C} \mathrm{NMR}\left(126 \mathrm{MHz}, \mathrm{CDCl}_{3}\right)$
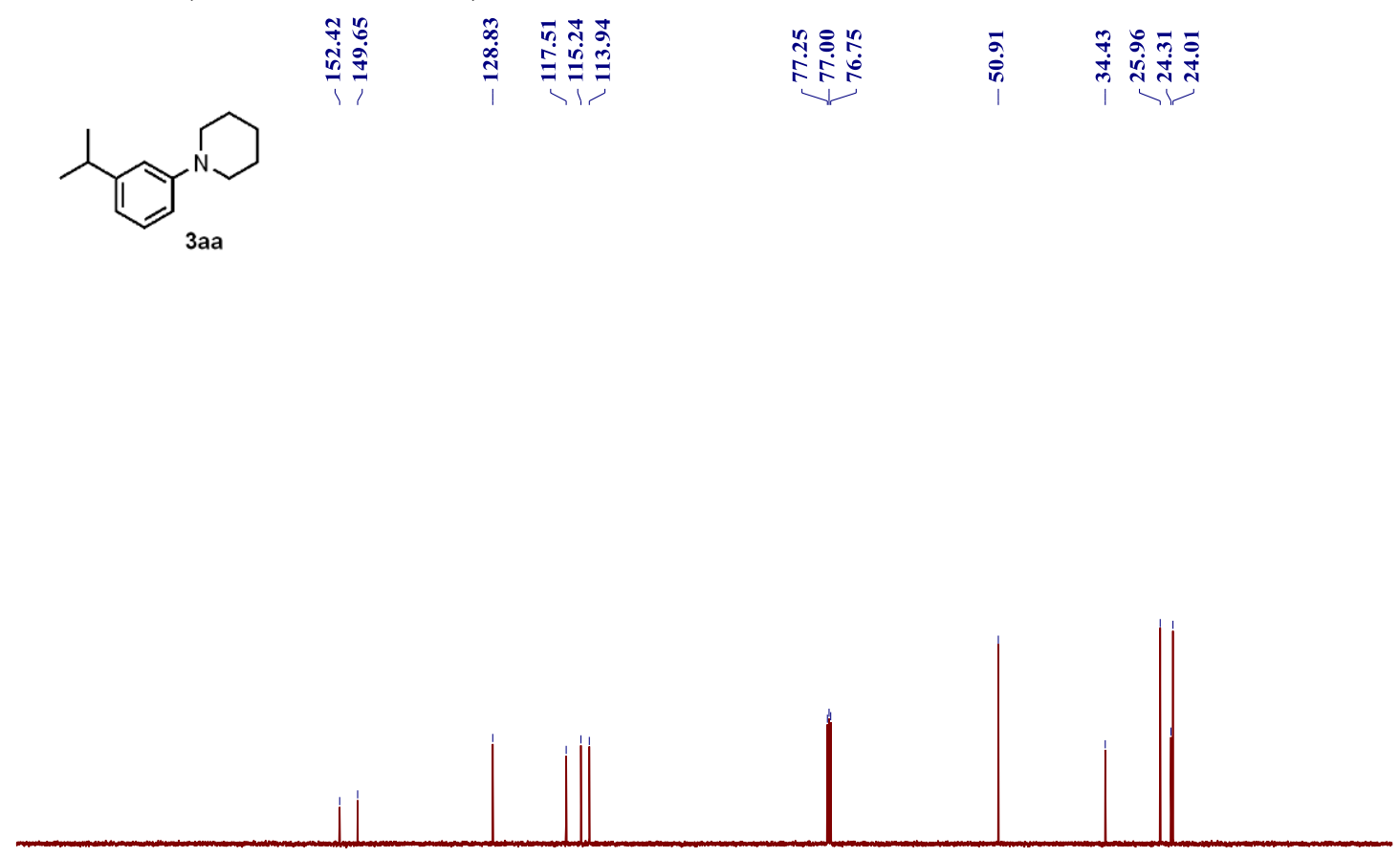

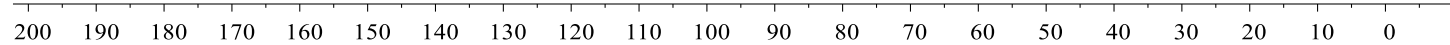


${ }^{1} \mathrm{H} \mathrm{NMR}\left(500 \mathrm{MHz}, \mathrm{CDCl}_{3}\right)$

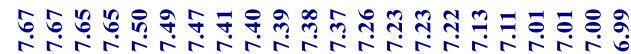

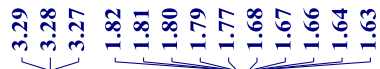
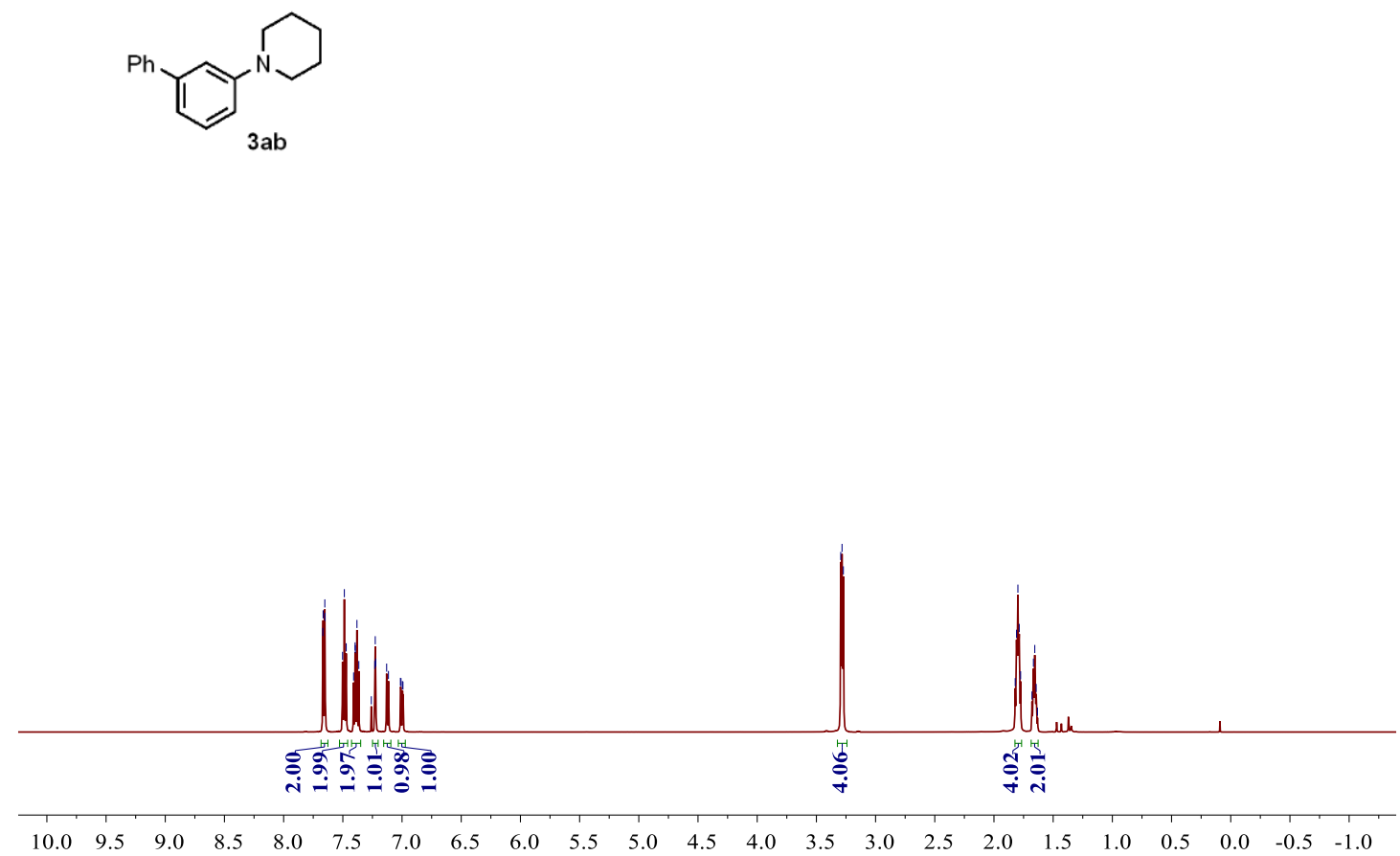

${ }^{13} \mathrm{C} \mathrm{NMR}\left(126 \mathrm{MHz}, \mathrm{CDCl}_{3}\right)$

นิำ

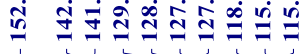

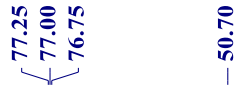

ชุำ

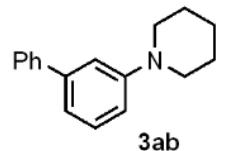

$3 a b$

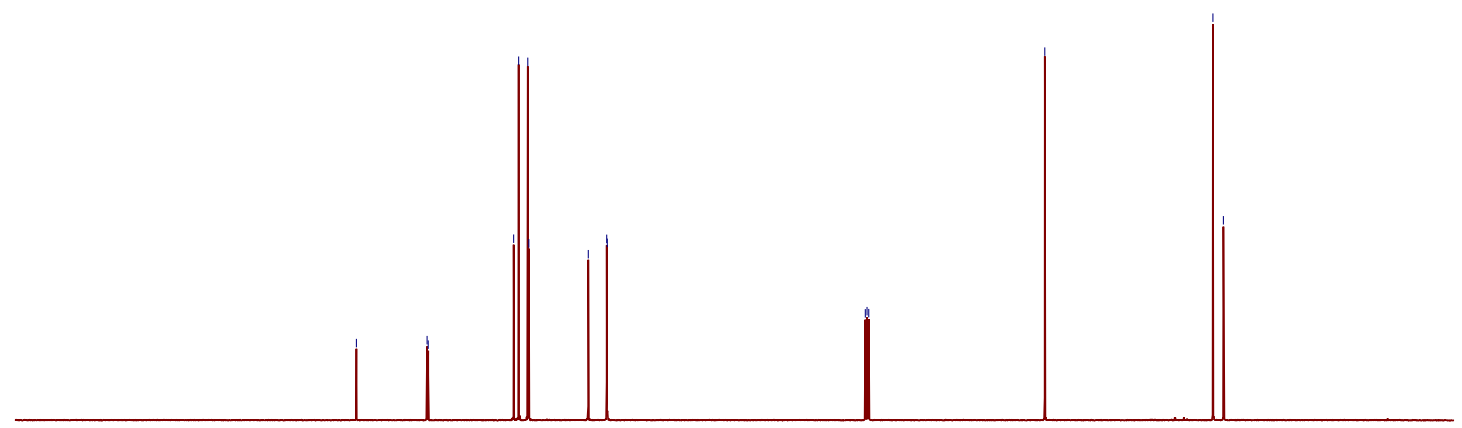

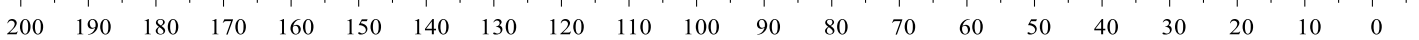


${ }^{1} \mathrm{H}$ NMR $\left(500 \mathrm{MHz}, \mathrm{CDCl}_{3}\right)$
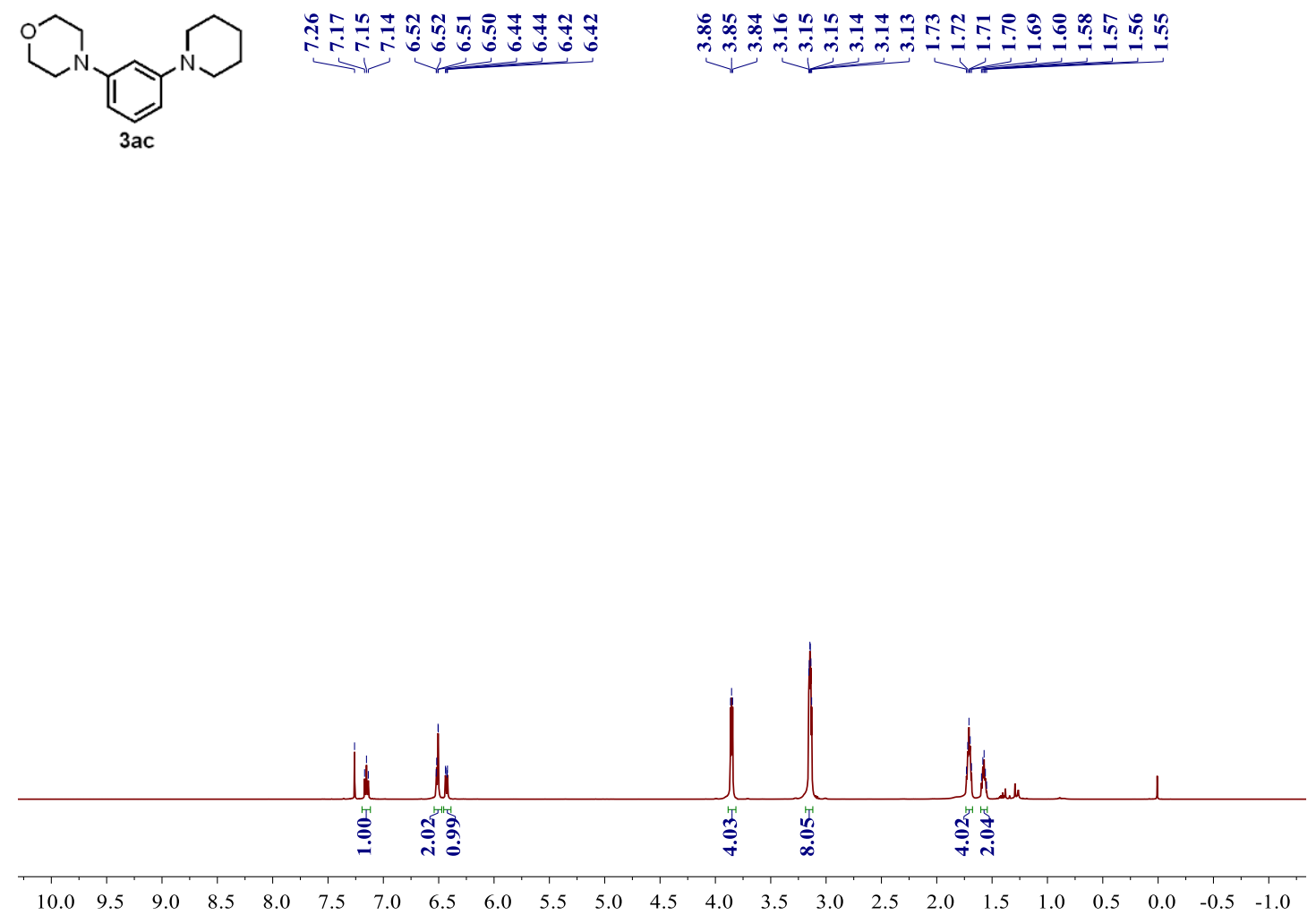

${ }^{13} \mathrm{C} \mathrm{NMR}\left(126 \mathrm{MHz}, \mathrm{CDCl}_{3}\right)$

$\underbrace{}_{3 a c}$

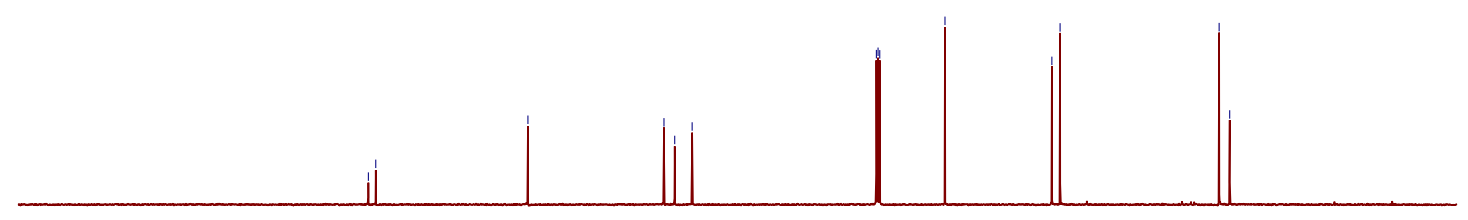

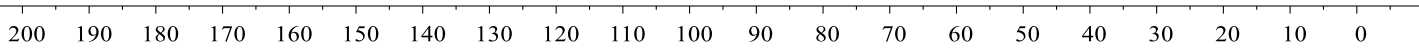


${ }^{1} \mathrm{H}$ NMR $\left(500 \mathrm{MHz}, \mathrm{CDCl}_{3}\right)$

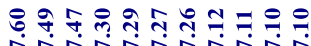

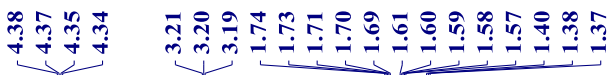<smiles>CCOC(=O)c1cccc(N2CCCCC2)c1</smiles>

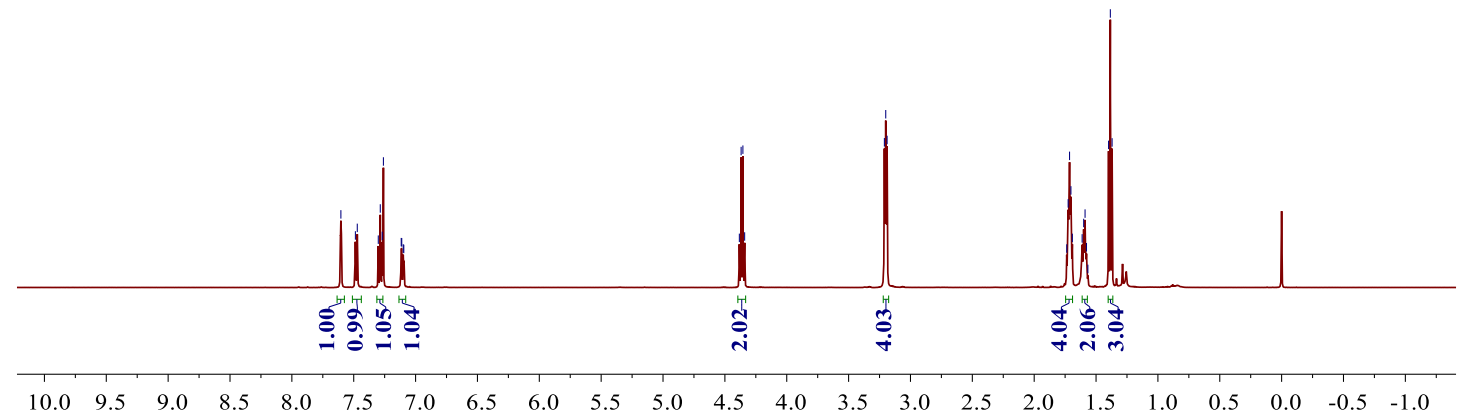

${ }^{13} \mathrm{C}$ NMR $\left(126 \mathrm{MHz}, \mathrm{CDCl}_{3}\right)$
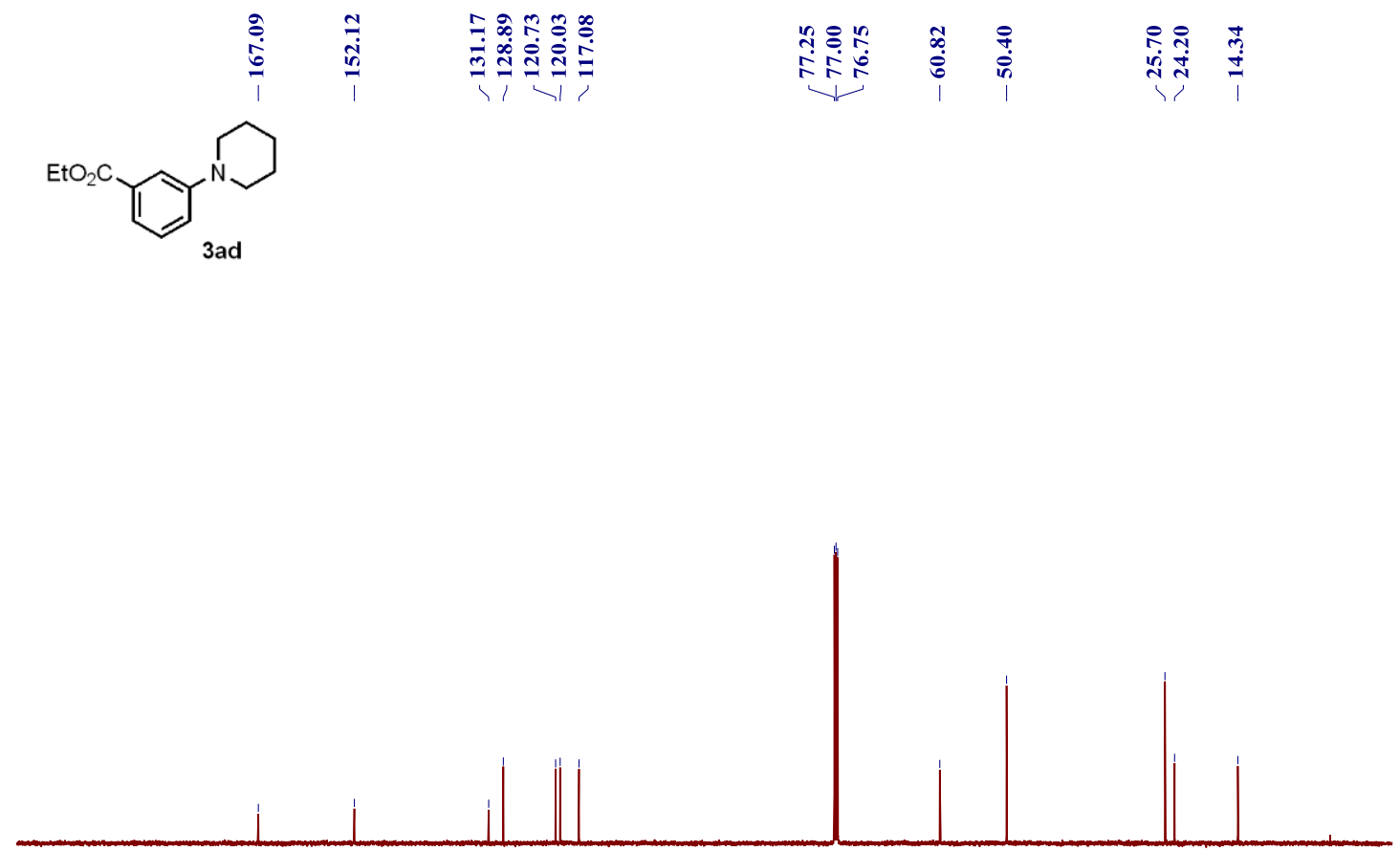

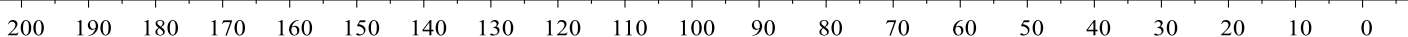


${ }^{1} \mathrm{H} \mathrm{NMR}\left(500 \mathrm{MHz}, \mathrm{CDCl}_{3}\right)$

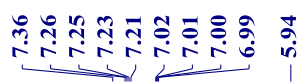

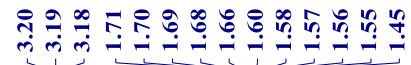

$>\underbrace{}_{3 a e}$

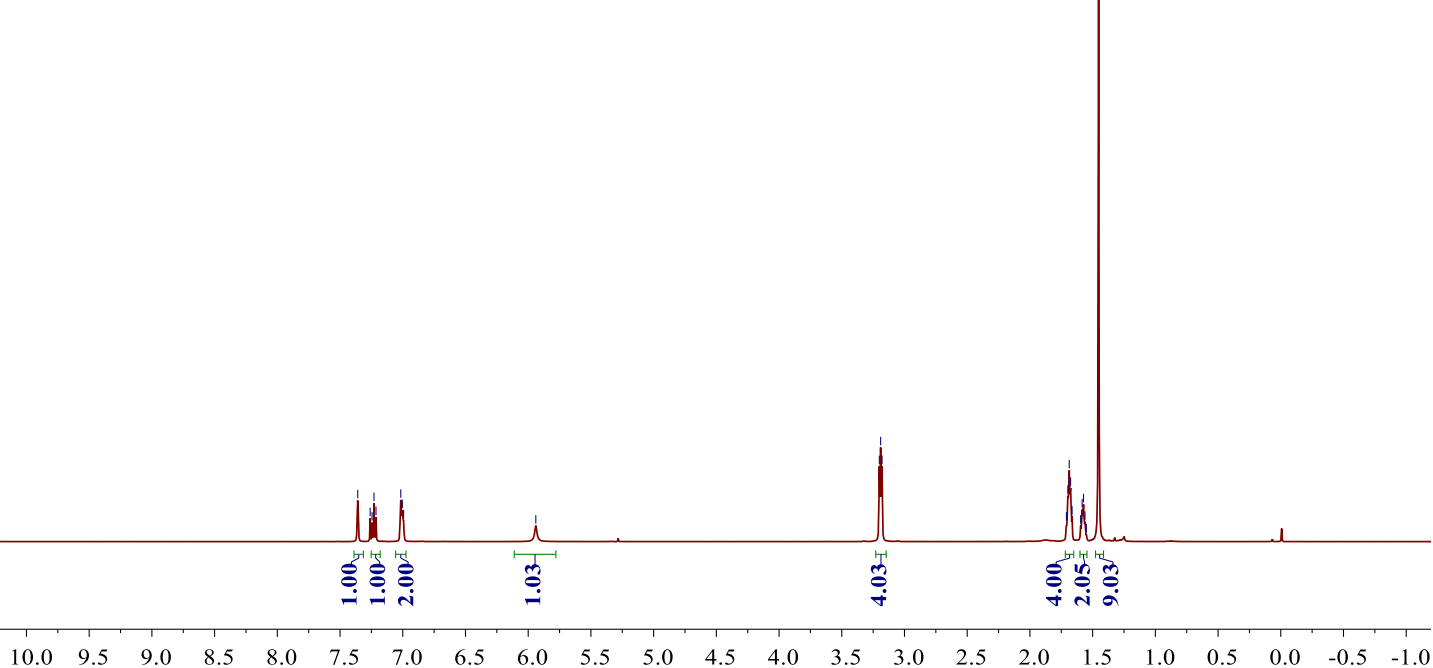

${ }^{13} \mathrm{C} \mathrm{NMR}\left(126 \mathrm{MHz}, \mathrm{CDCl}_{3}\right)$

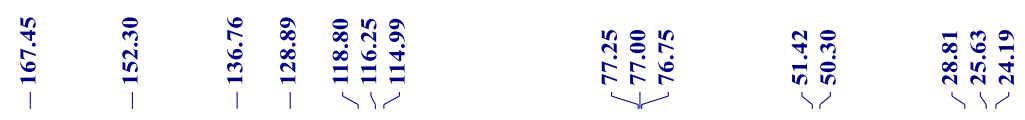<smiles>CC(C)(C)NC(=O)c1cccc(N2CCCCC2)c1</smiles>

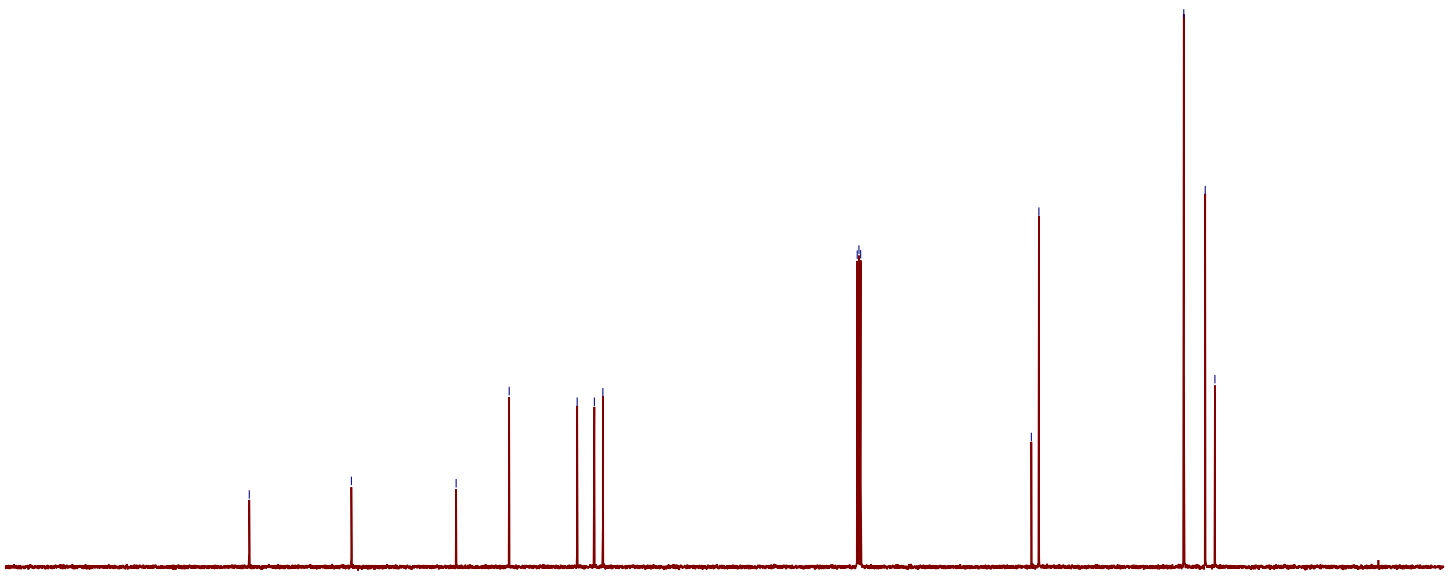

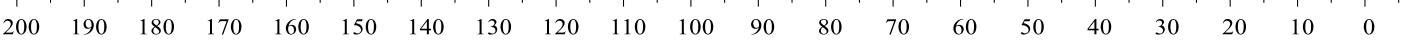


${ }^{1} \mathrm{H}$ NMR (500 MHz, $\left.\mathrm{CDCl}_{3}\right)$
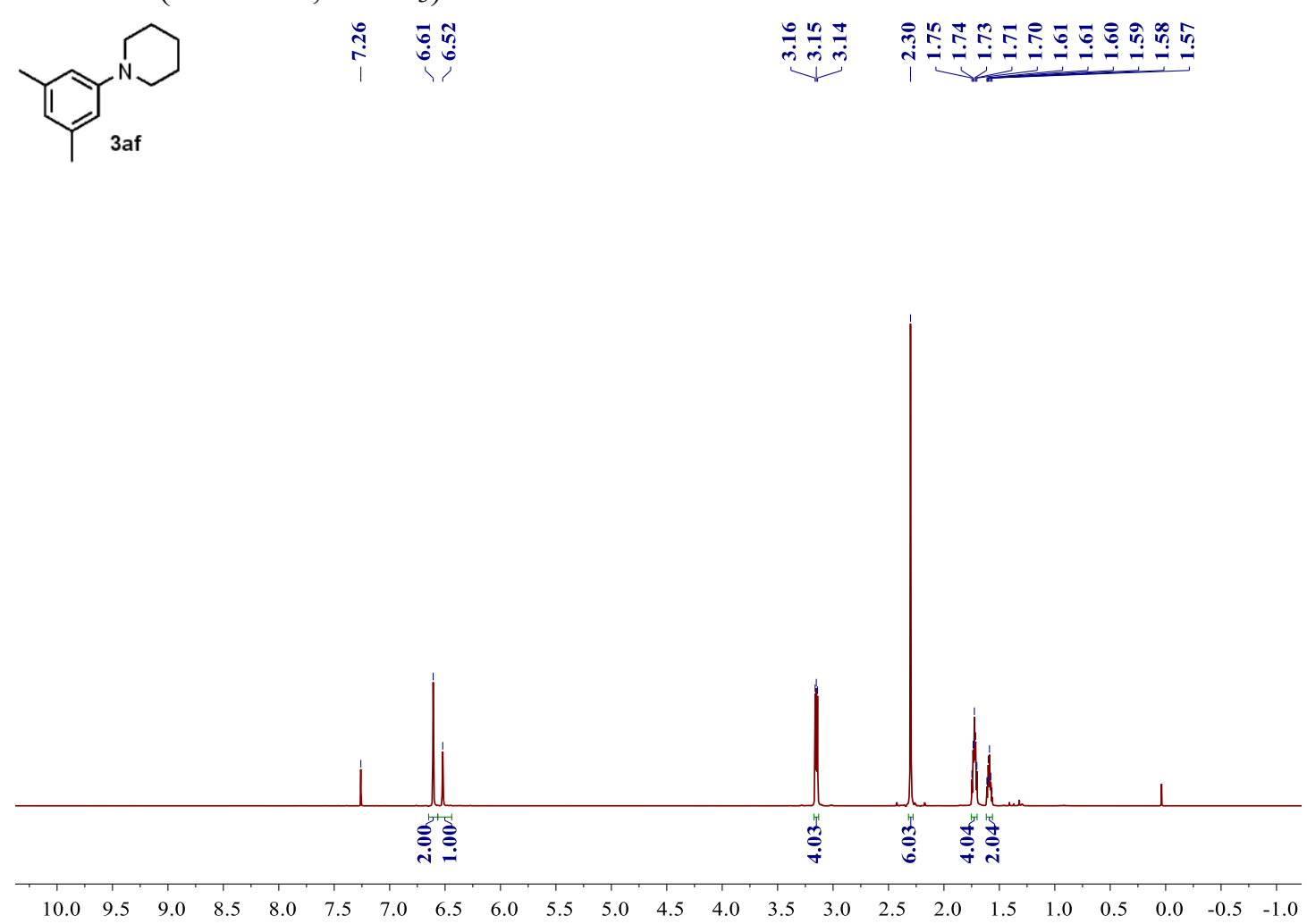

$\left.{ }^{13} \mathrm{C} \mathrm{NMR} \mathrm{(126} \mathrm{MHz,} \mathrm{CDCl}_{3}\right)$

$\underbrace{N}_{3 a f}$

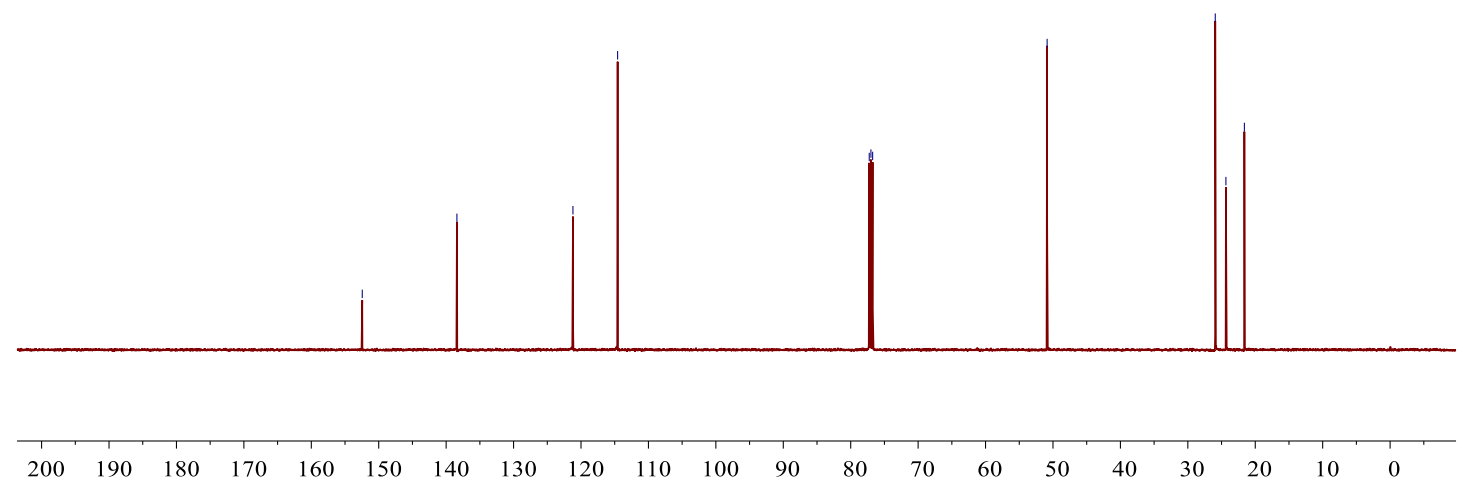


${ }^{1} \mathrm{H}$ NMR $\left(500 \mathrm{MHz}, \mathrm{CDCl}_{3}\right)$
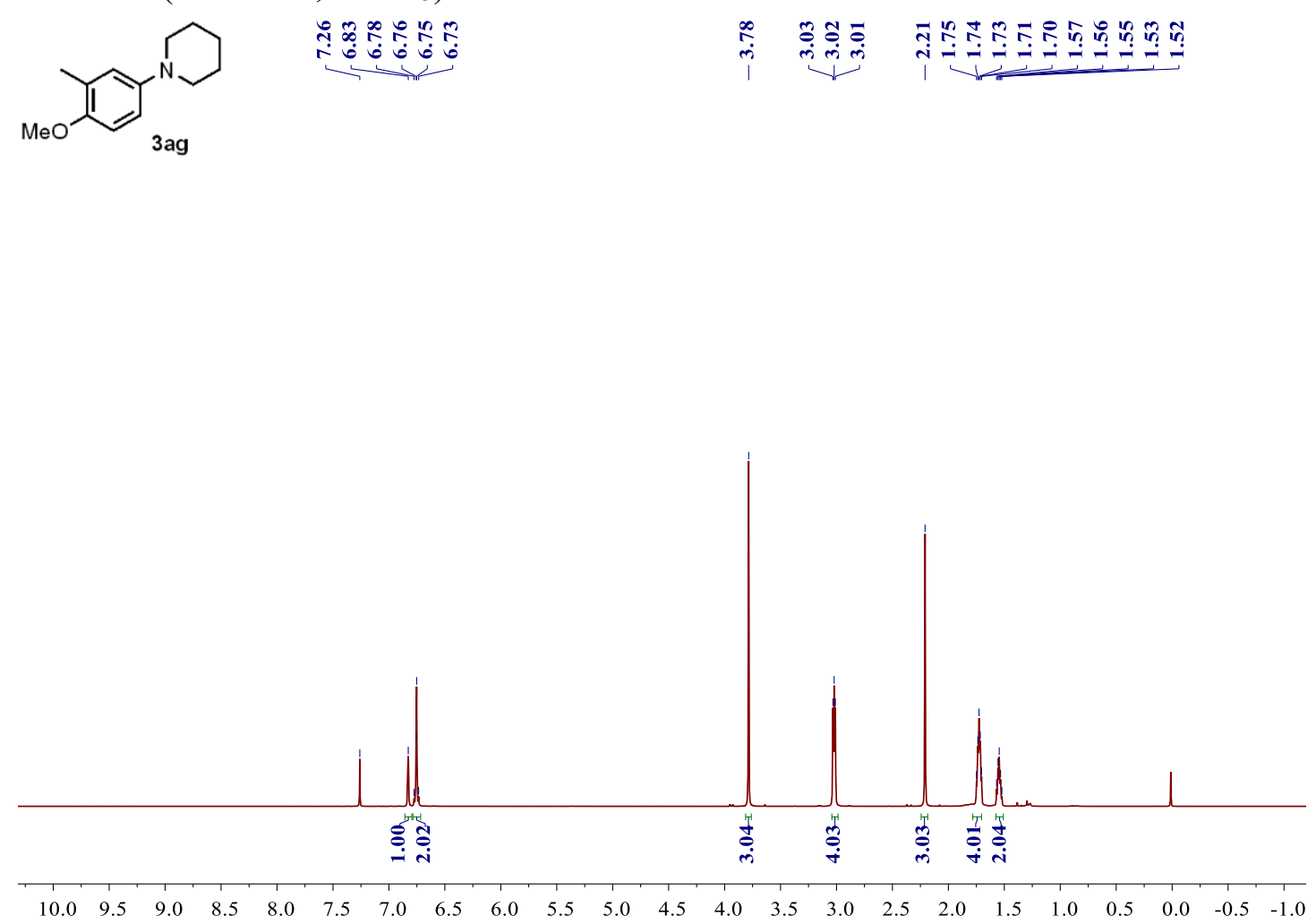

${ }^{13} \mathrm{C} \mathrm{NMR}\left(126 \mathrm{MHz}, \mathrm{CDCl}_{3}\right)$

MeO药

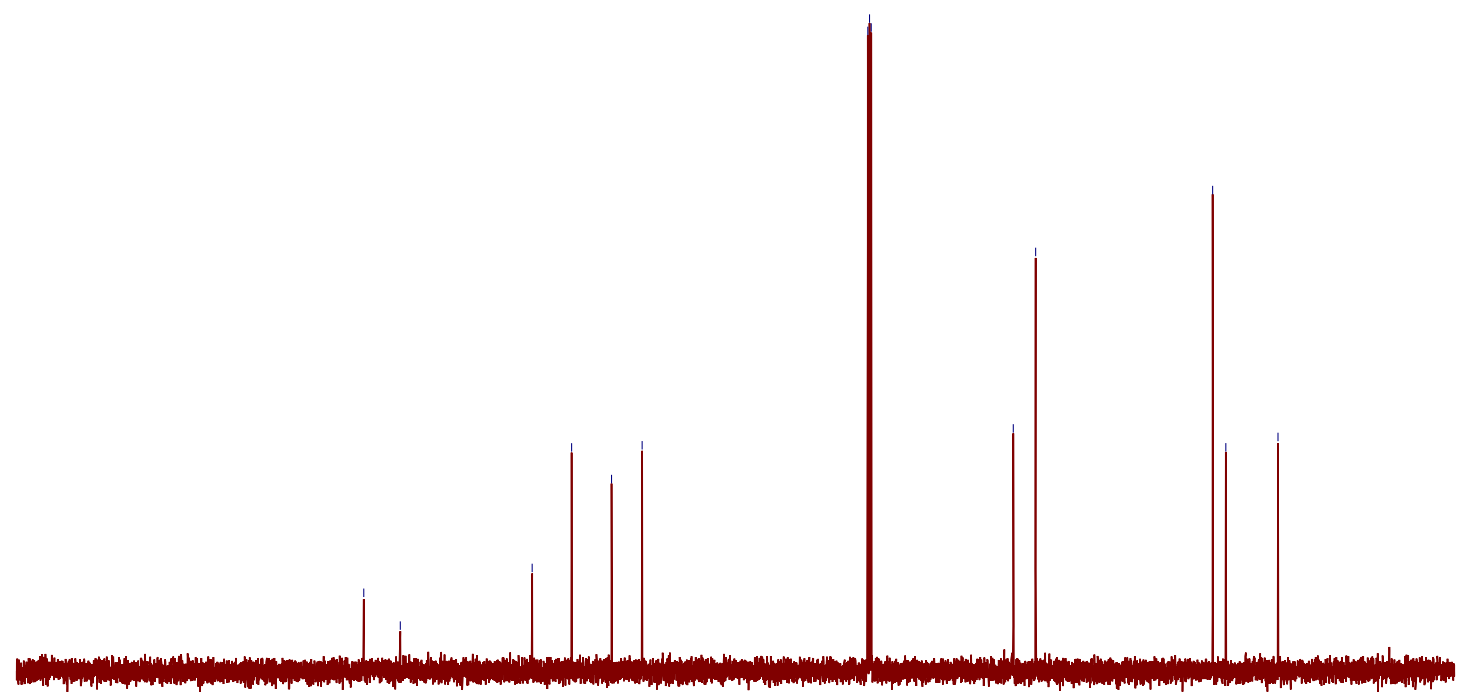

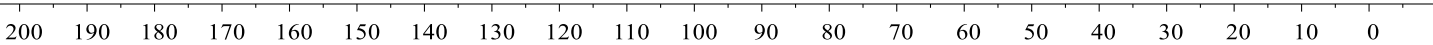


${ }^{1} \mathrm{H} \mathrm{NMR}\left(500 \mathrm{MHz}, \mathrm{CDCl}_{3}\right)$
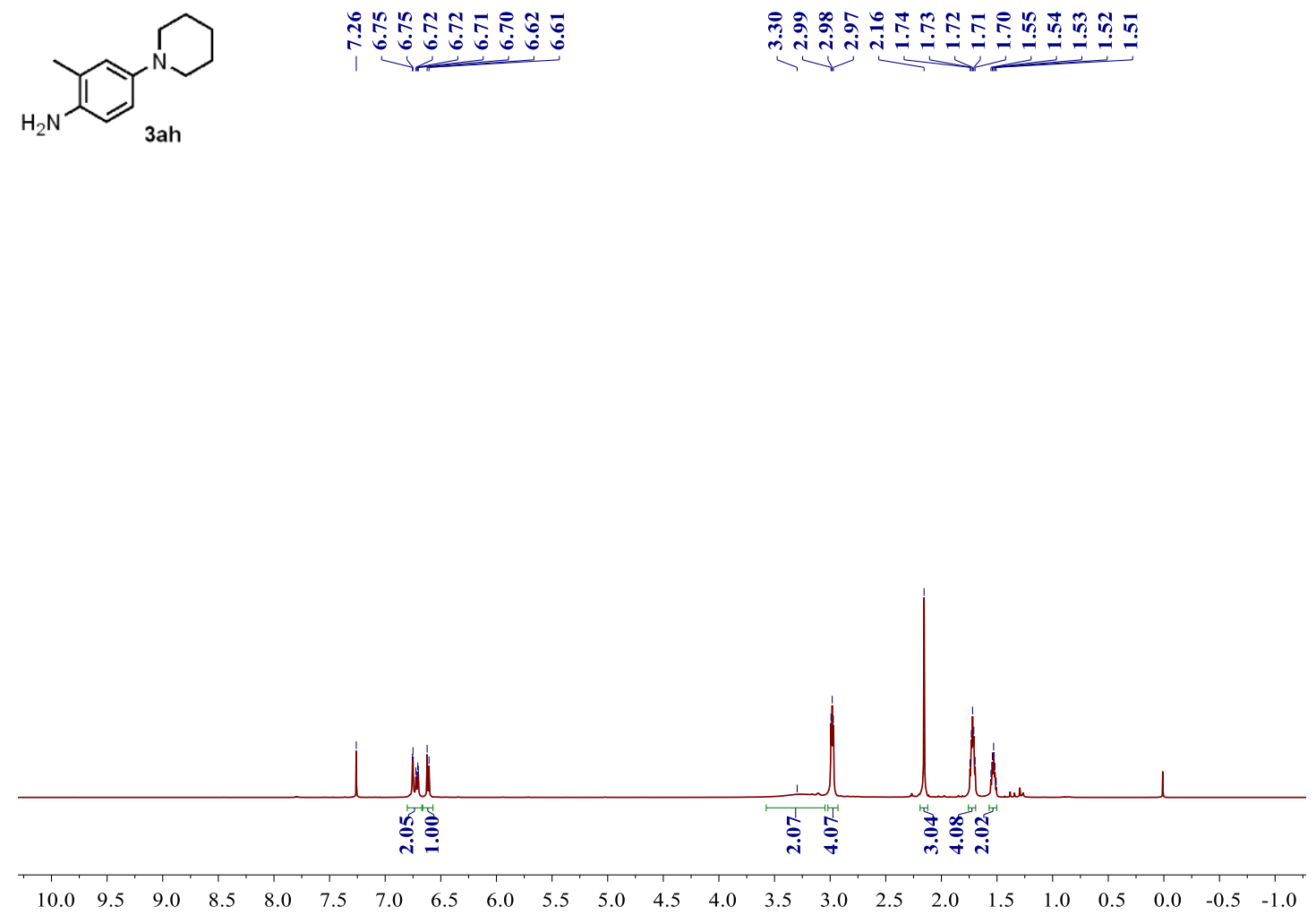

${ }^{13} \mathrm{C} \mathrm{NMR}\left(126 \mathrm{MHz}, \mathrm{CDCl}_{3}\right)$

每

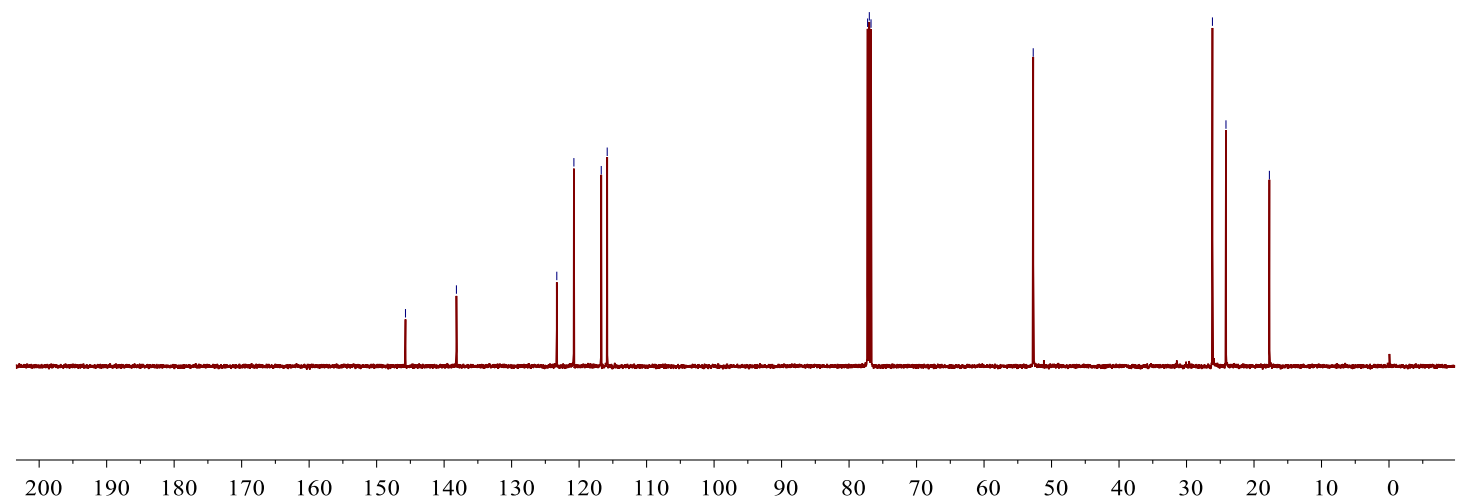


${ }^{1} \mathrm{H}$ NMR $\left(500 \mathrm{MHz}, \mathrm{CDCl}_{3}\right)$
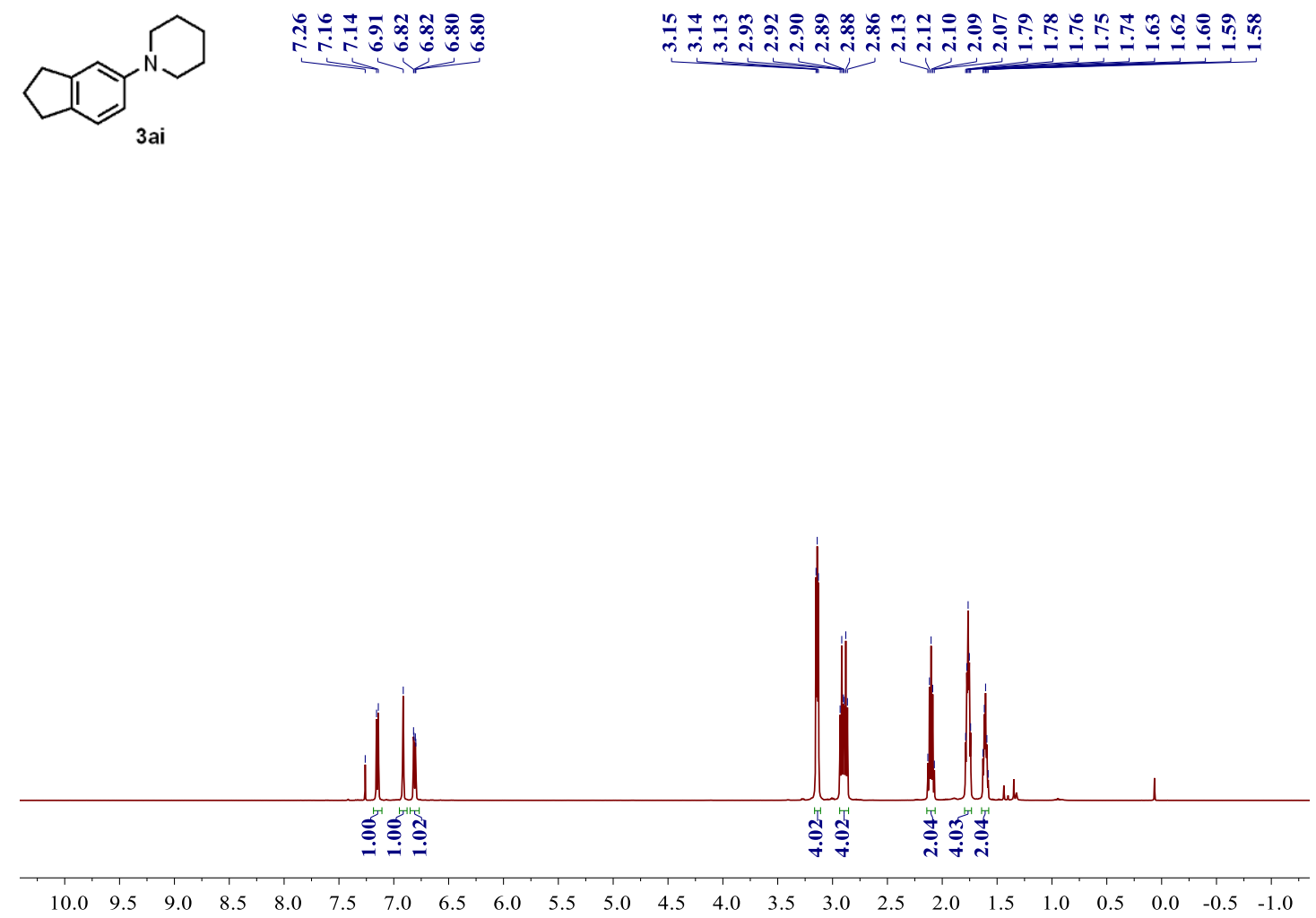

${ }^{13} \mathrm{C}$ NMR $\left(126 \mathrm{MHz}, \mathrm{CDCl}_{3}\right)$

$\underbrace{N}_{3 \text { ai }}$

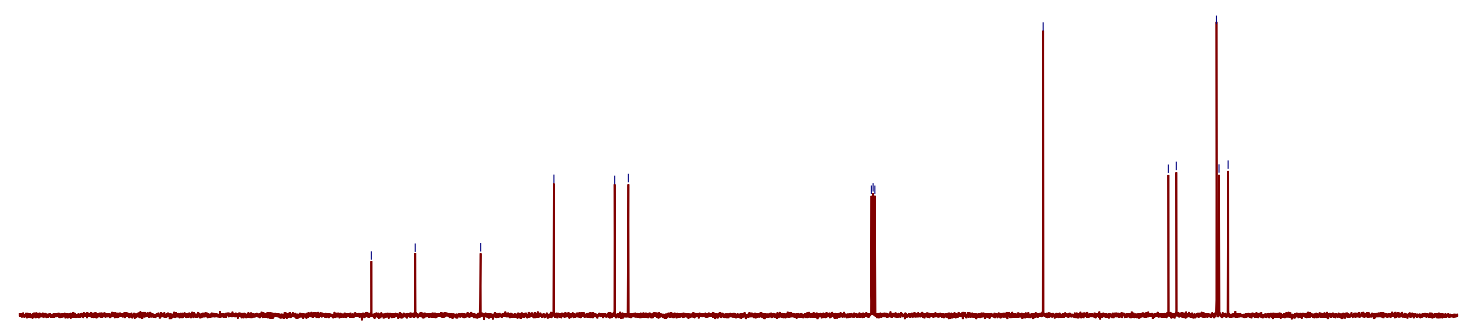

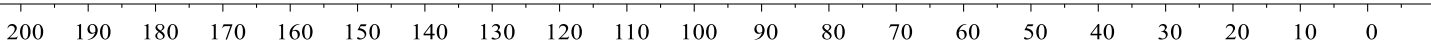


${ }^{1} \mathrm{H}$ NMR $\left(500 \mathrm{MHz}, \mathrm{CDCl}_{3}\right)$
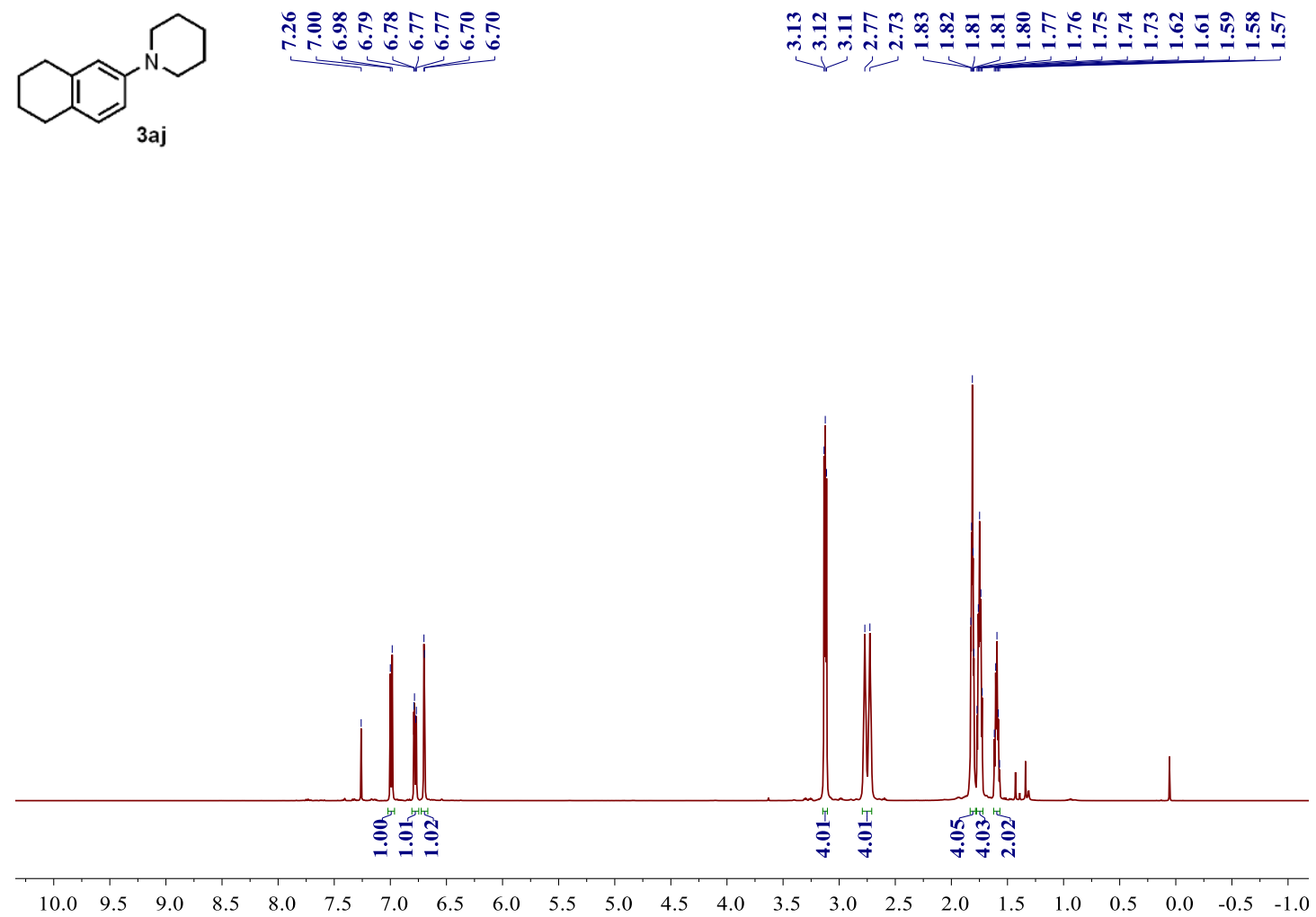

${ }^{13} \mathrm{C} \mathrm{NMR}\left(126 \mathrm{MHz}, \mathrm{CDCl}_{3}\right)$

$\underbrace{N}_{3 a j}$

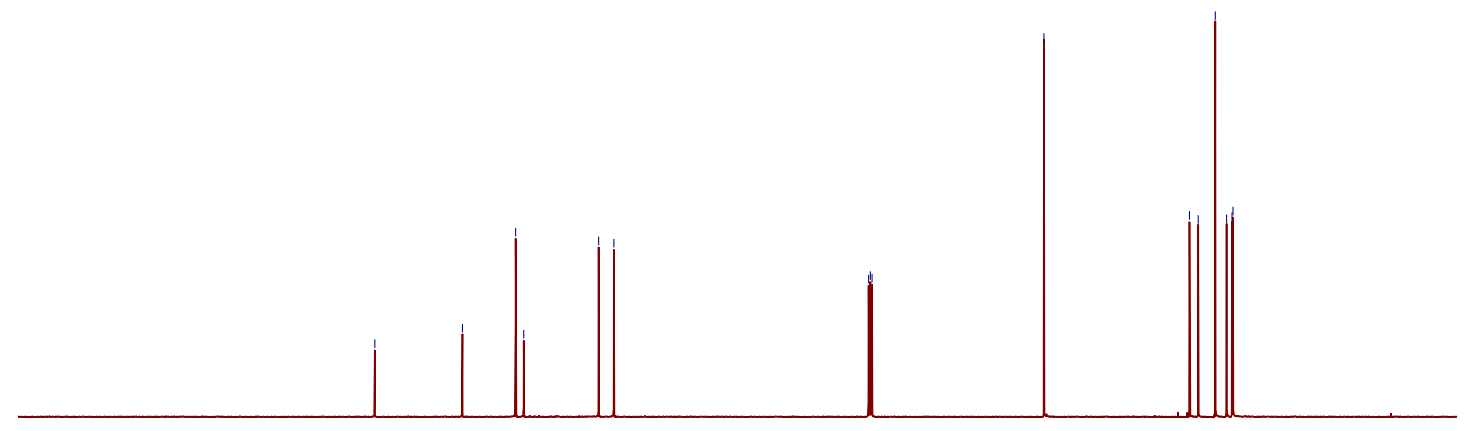

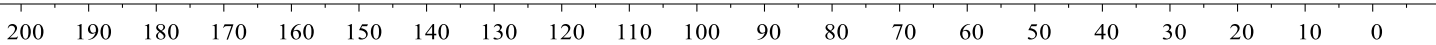


${ }^{1} \mathrm{H}$ NMR (500 MHz, $\mathrm{CDCl}_{3}$ )
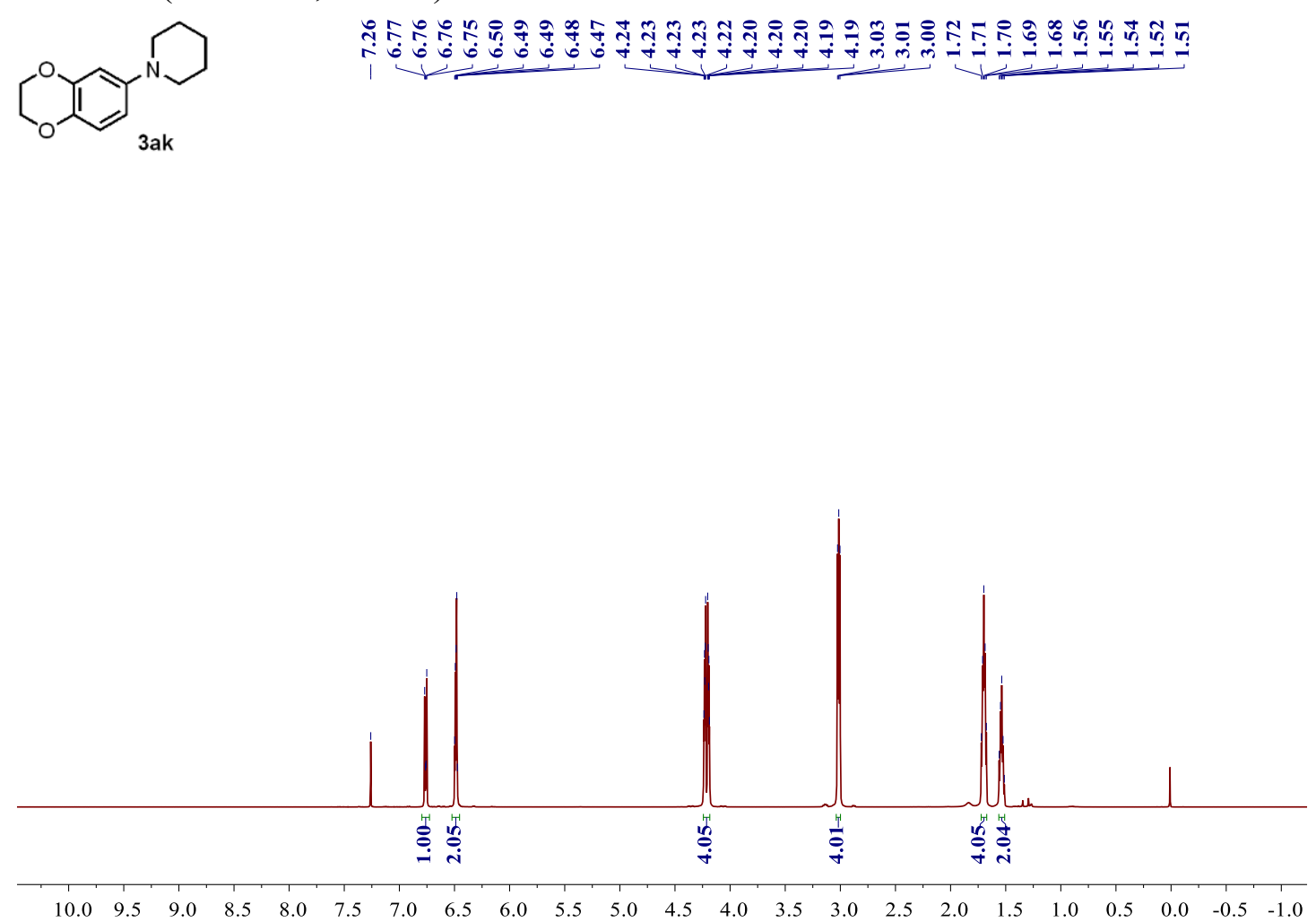

${ }^{13} \mathrm{C} \mathrm{NMR}\left(126 \mathrm{MHz}, \mathrm{CDCl}_{3}\right)$

$\underbrace{1}_{3 a k}$

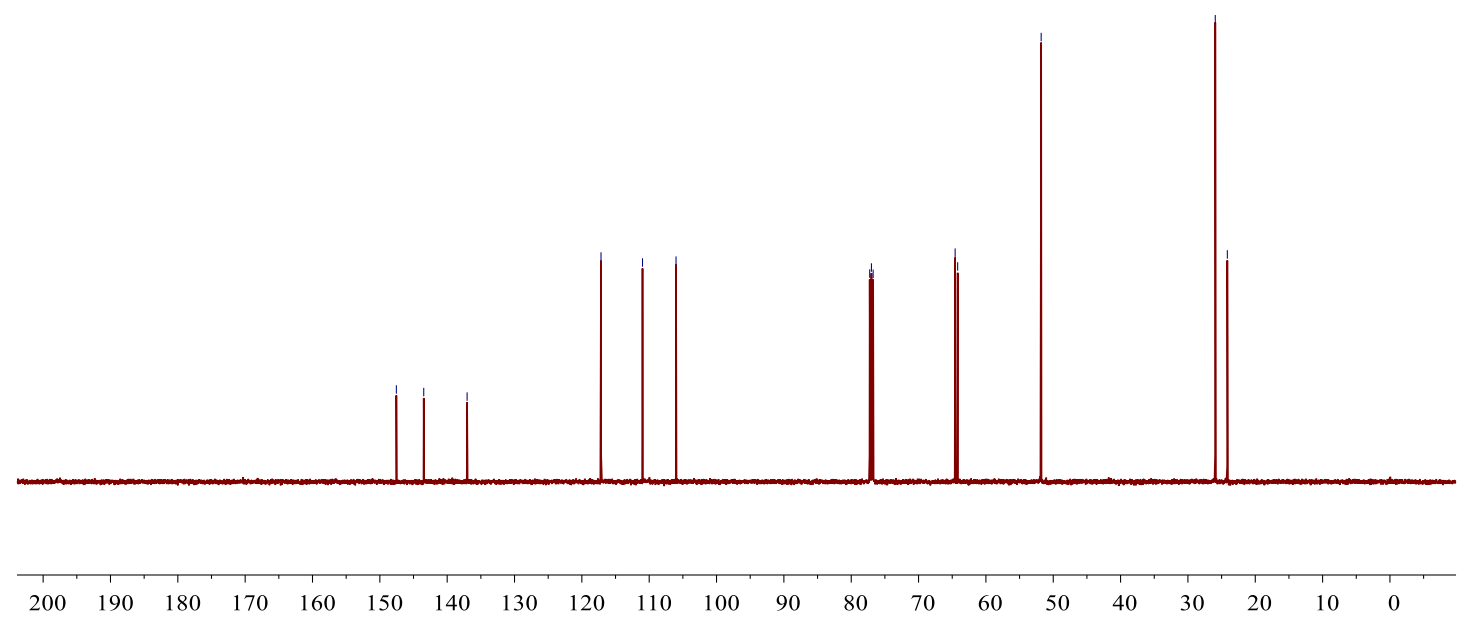


${ }^{1} \mathrm{H}$ NMR $\left(500 \mathrm{MHz}, \mathrm{CDCl}_{3}\right)$

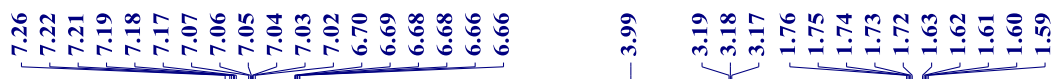<smiles>c1ccc2c(c1)CCc1cc(N3CCCCC3)ccc1O2</smiles>

3al

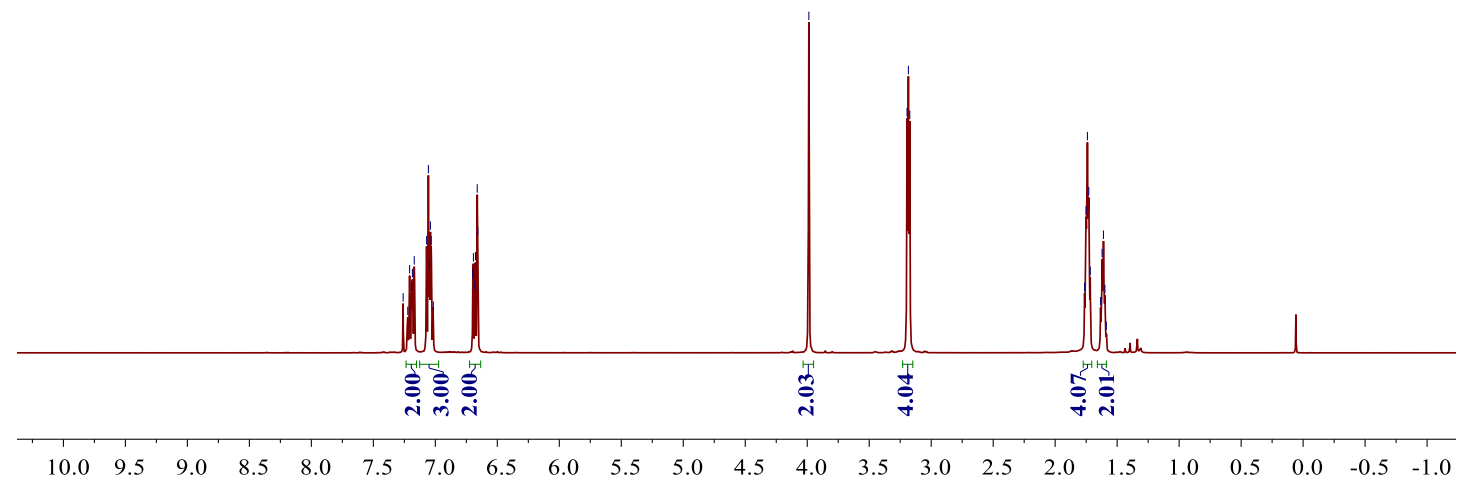

${ }^{13} \mathrm{C} \mathrm{NMR}\left(126 \mathrm{MHz}, \mathrm{CDCl}_{3}\right)$

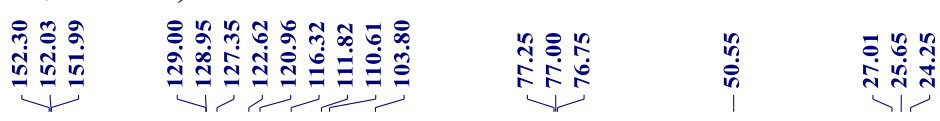<smiles>c1ccc2c(c1)Cc1ccc(N3CCCCC3)cc1O2</smiles>

3al

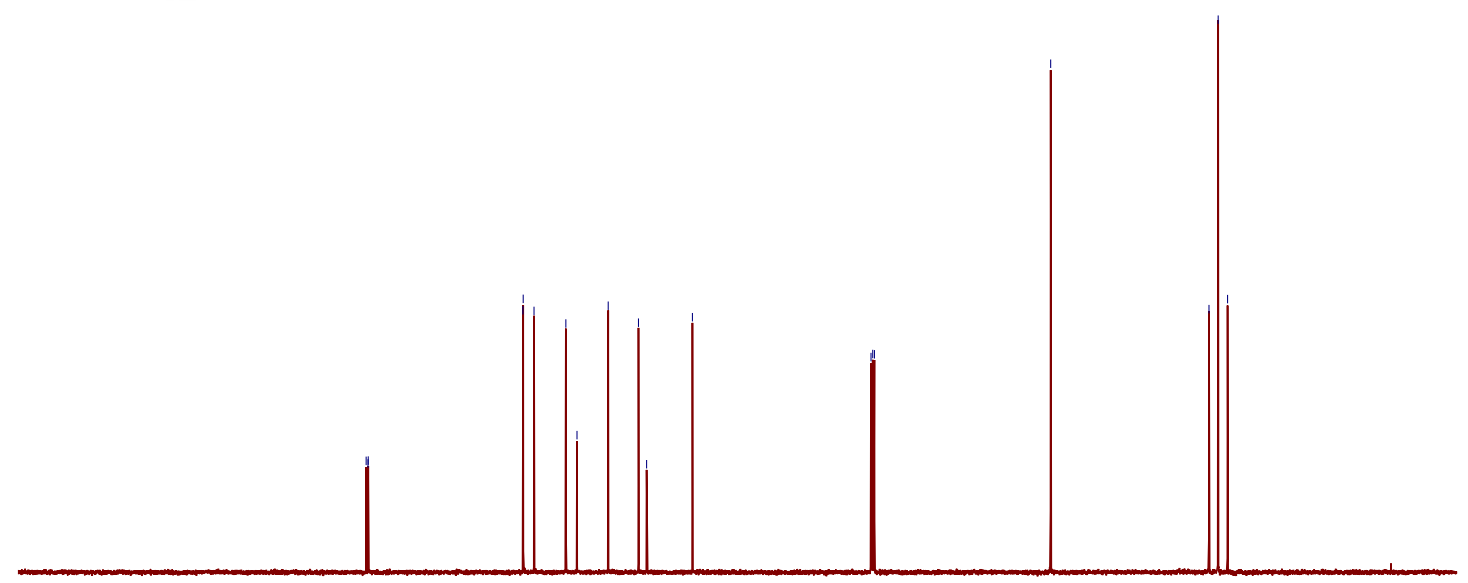

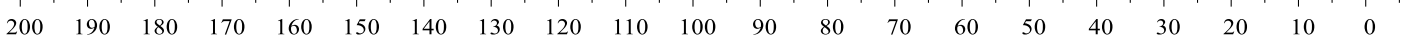


${ }^{1} \mathrm{H}$ NMR $\left(500 \mathrm{MHz}, \mathrm{CDCl}_{3}\right)$
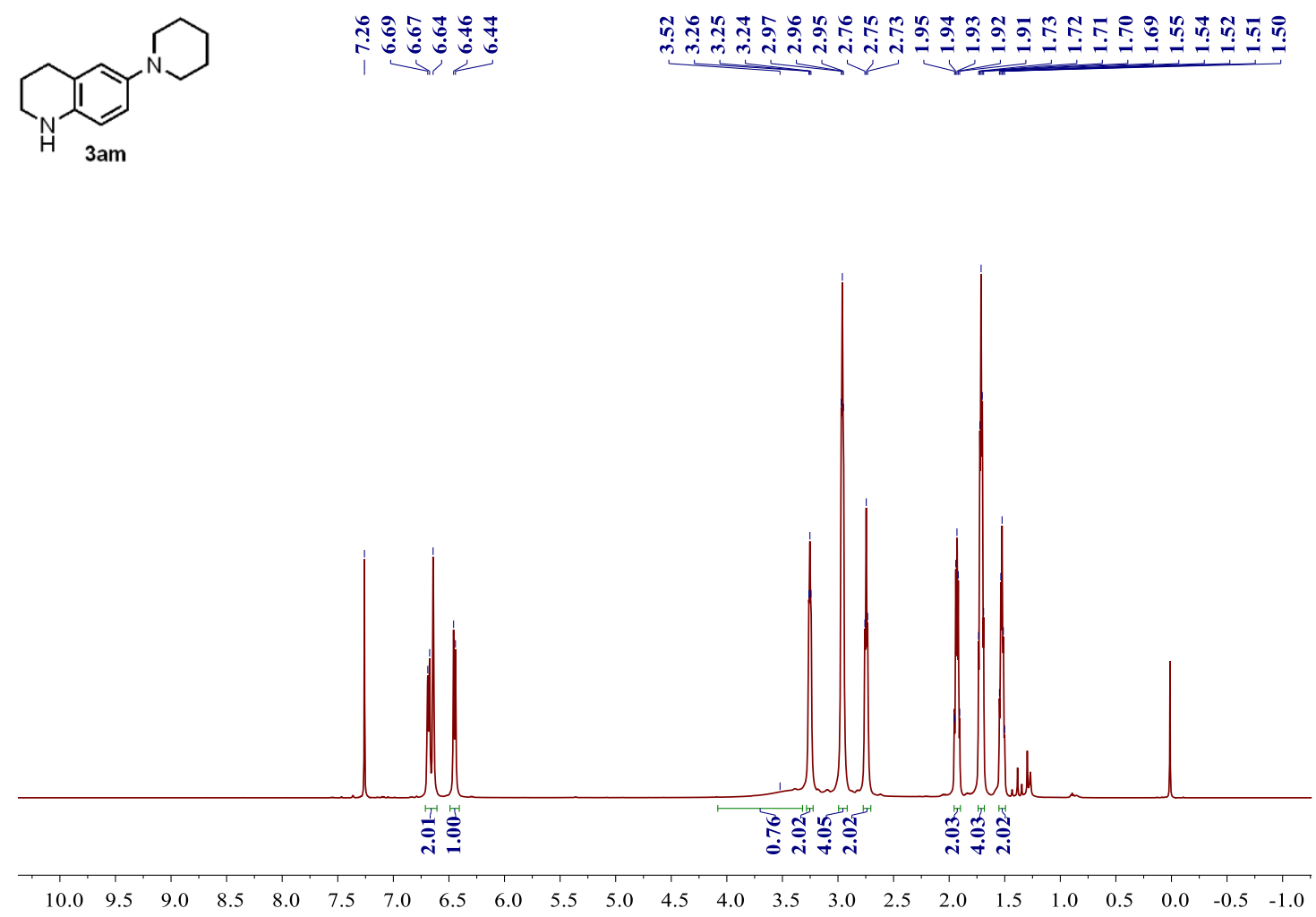

${ }^{13} \mathrm{C} \mathrm{NMR}\left(126 \mathrm{MHz}, \mathrm{CDCl}_{3}\right)$

(N)

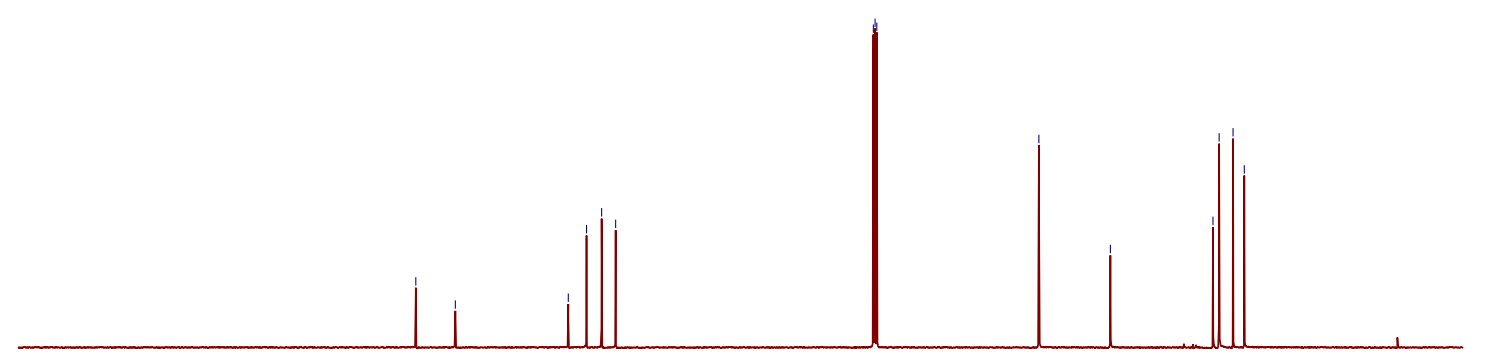

$\begin{array}{lllllllllllllllllllll}200 & 190 & 180 & 170 & 160 & 150 & 140 & 130 & 120 & 110 & 100 & 90 & 80 & 70 & 60 & 50 & 40 & 30 & 20 & 10 & 0\end{array}$ 
${ }^{1} \mathrm{H}$ NMR $\left(500 \mathrm{MHz}, \mathrm{CDCl}_{3}\right)$<smiles>c1cc2c(cc1N1CCCCC1)NCC2</smiles>

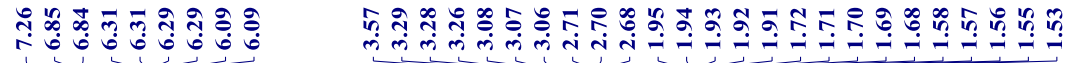

3an

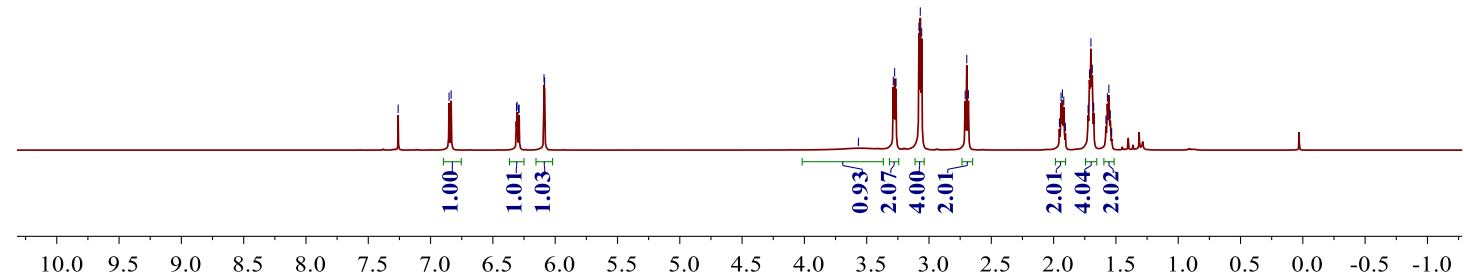

${ }^{13} \mathrm{C} \mathrm{NMR}\left(126 \mathrm{MHz}, \mathrm{CDCl}_{3}\right)$

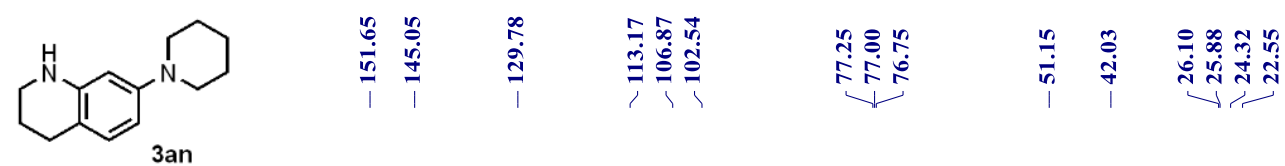

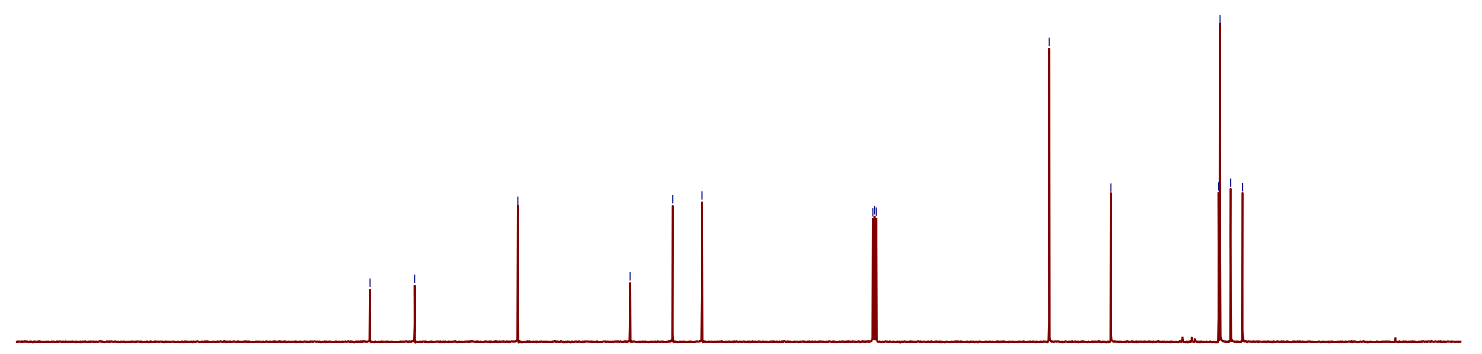

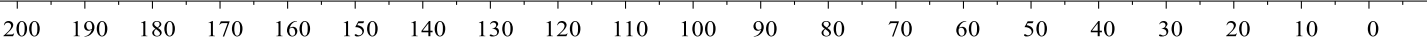


${ }^{1} \mathrm{H} \mathrm{NMR}\left(500 \mathrm{MHz}, \mathrm{CDCl}_{3}\right)$

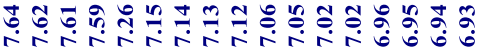

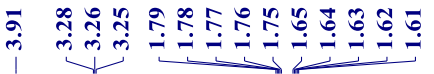<smiles>COc1ccc2ccc(N3CCCCC3)cc2c1</smiles>

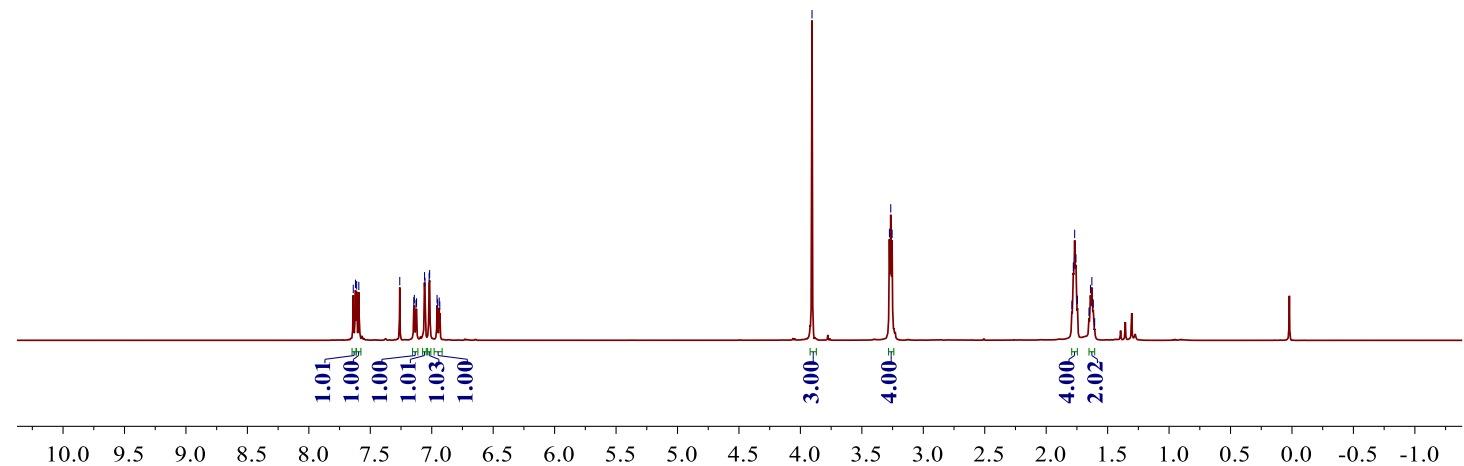

${ }^{13} \mathrm{C} \mathrm{NMR}\left(126 \mathrm{MHz}, \mathrm{CDCl}_{3}\right)$

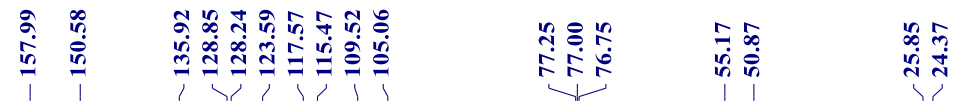<smiles>COc1ccc2ccc(N3CCCCC3)cc2c1</smiles>

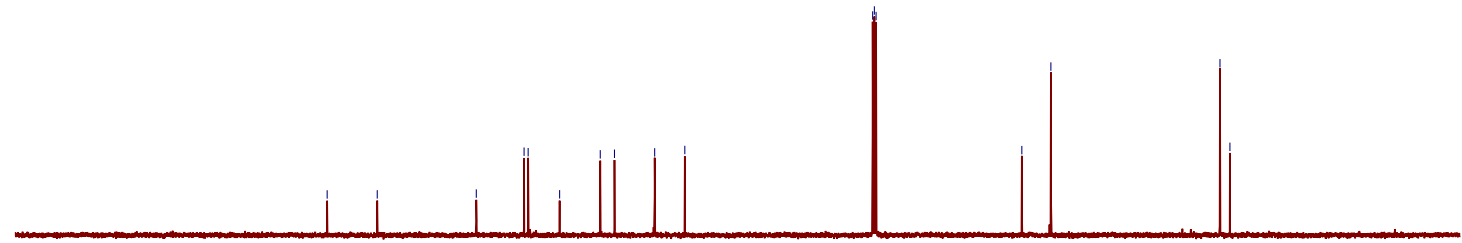

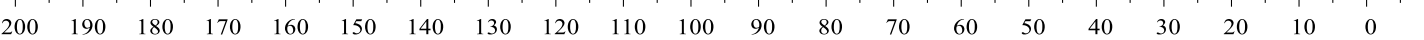


${ }^{1} \mathrm{H}$ NMR $\left(500 \mathrm{MHz}, \mathrm{CDCl}_{3}\right)$
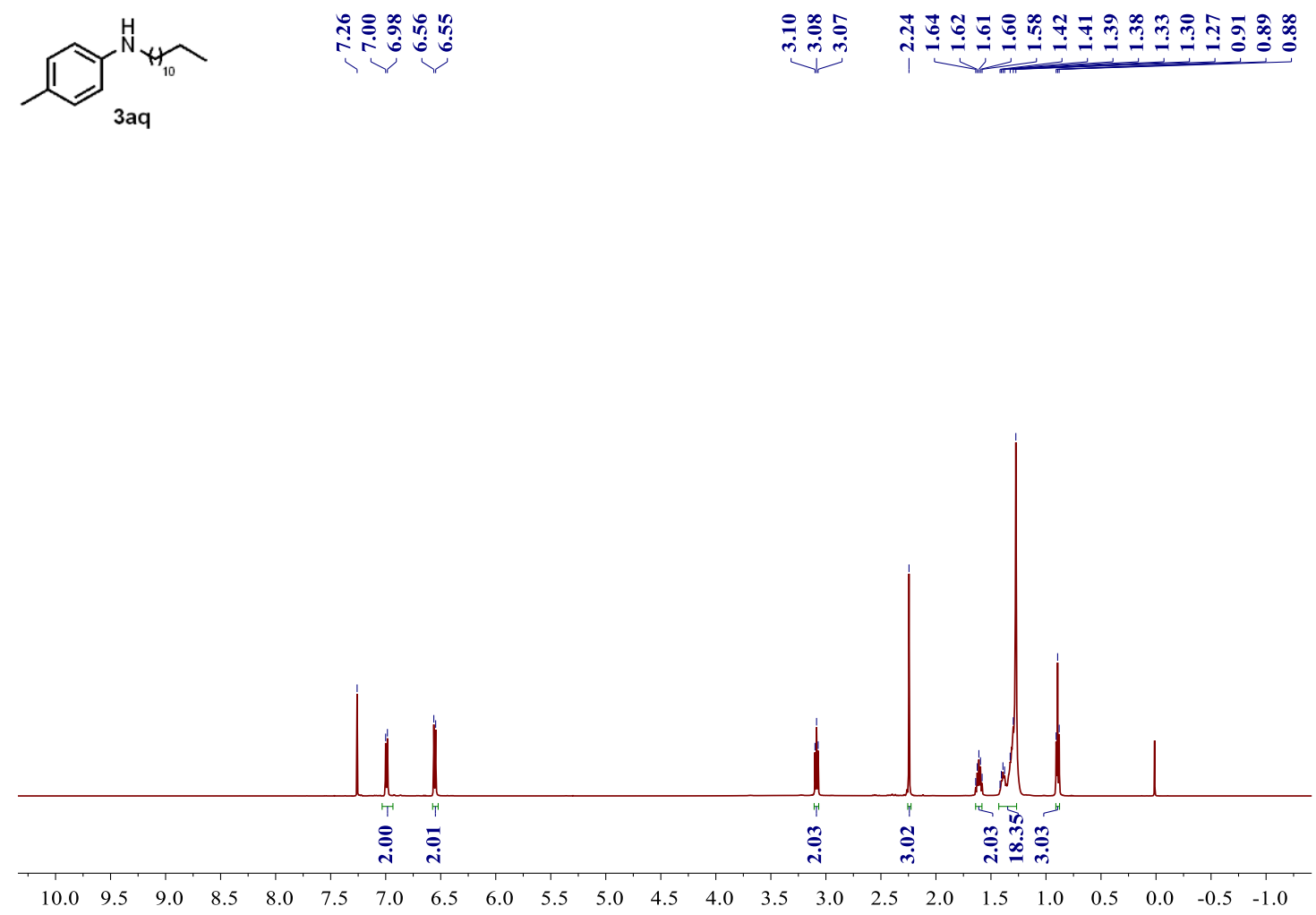

${ }^{13} \mathrm{C} \mathrm{NMR}\left(126 \mathrm{MHz}, \mathrm{CDCl}_{3}\right)$

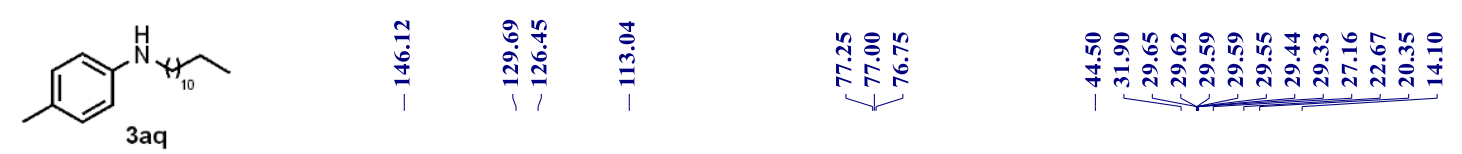

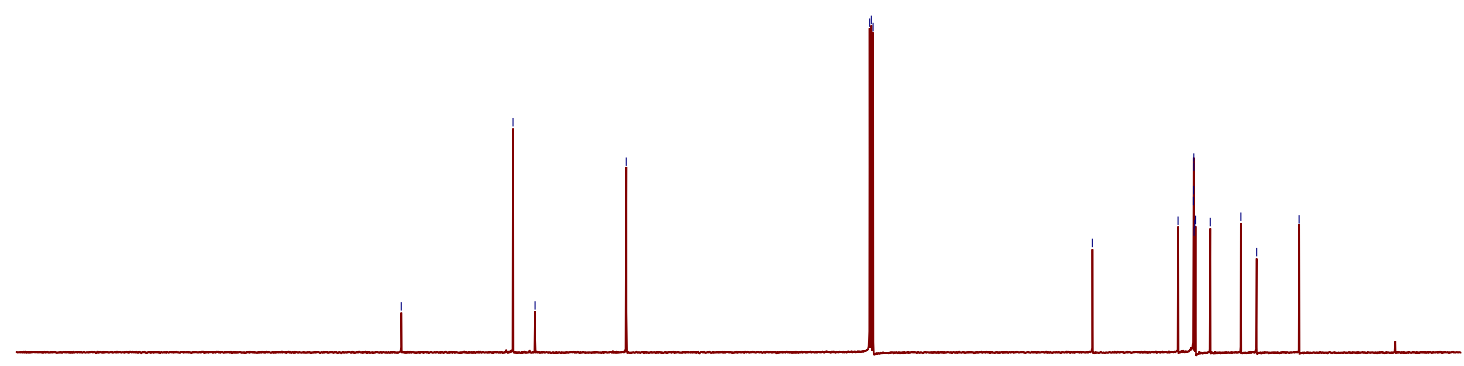

$\begin{array}{lllllllllllllllllllllll}200 & 190 & 180 & 170 & 160 & 150 & 140 & 130 & 120 & 110 & 100 & 90 & 80 & 70 & 60 & 50 & 40 & 30 & 20 & 10 & 0\end{array}$ 
${ }^{1} \mathrm{H}$ NMR $\left(500 \mathrm{MHz}, \mathrm{CDCl}_{3}\right)$

ำ

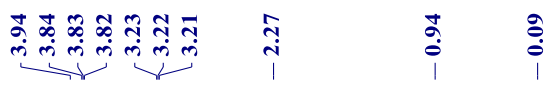

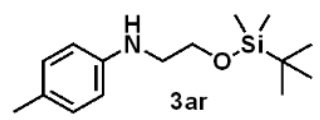

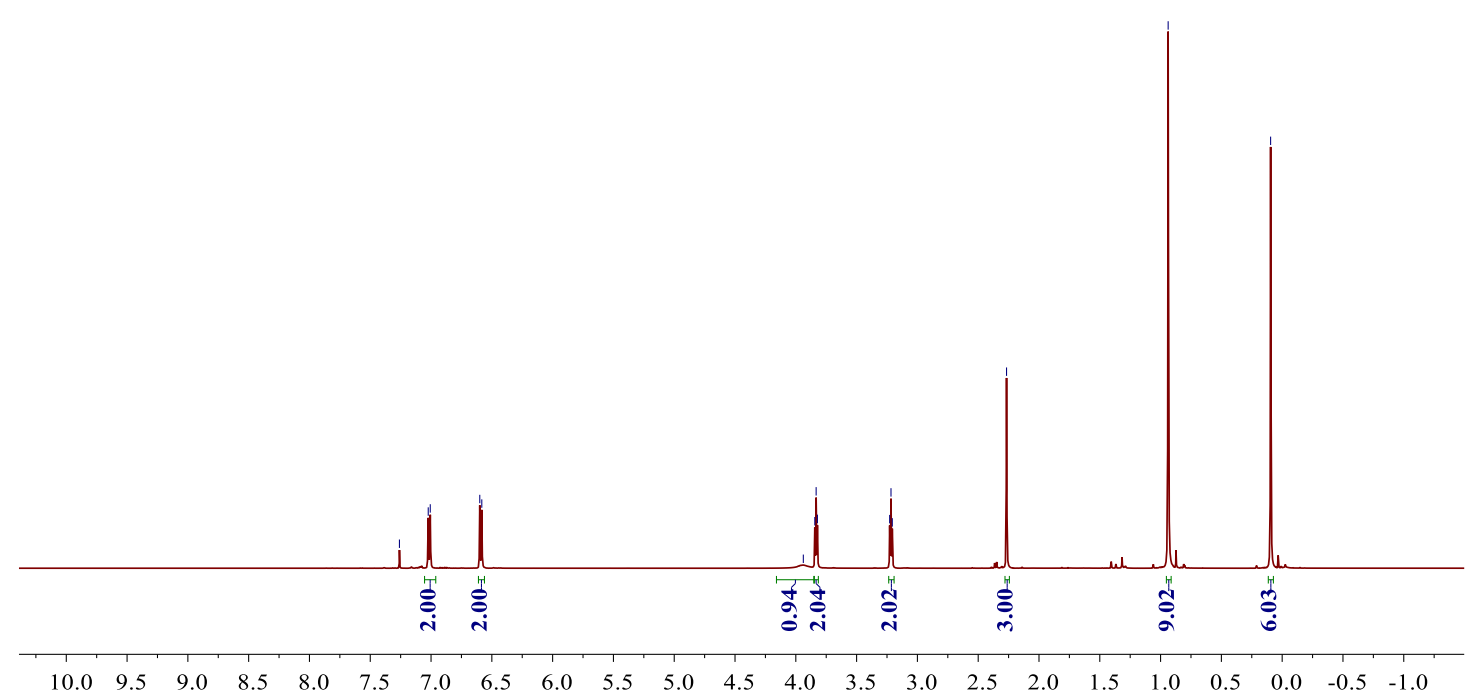

${ }^{13} \mathrm{C} \mathrm{NMR}\left(126 \mathrm{MHz}, \mathrm{CDCl}_{3}\right)$

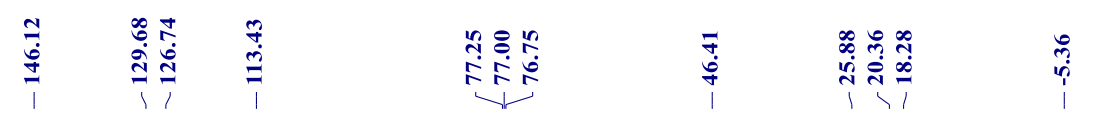<smiles>Cc1ccc(NCC[Si](C)(C)C(C)(C)C)cc1</smiles>

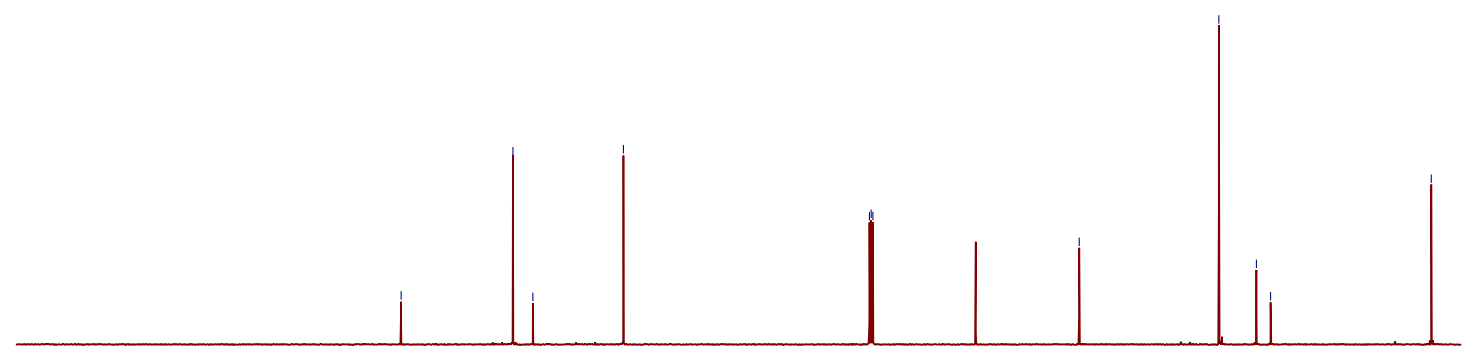

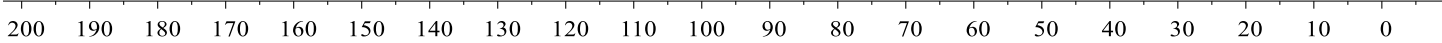


${ }^{1} \mathrm{H}$ NMR $\left(500 \mathrm{MHz}, \mathrm{CDCl}_{3}\right)$

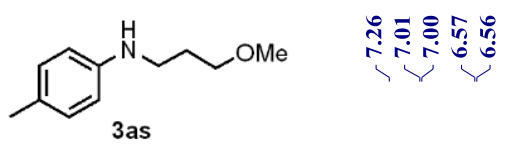

3 as

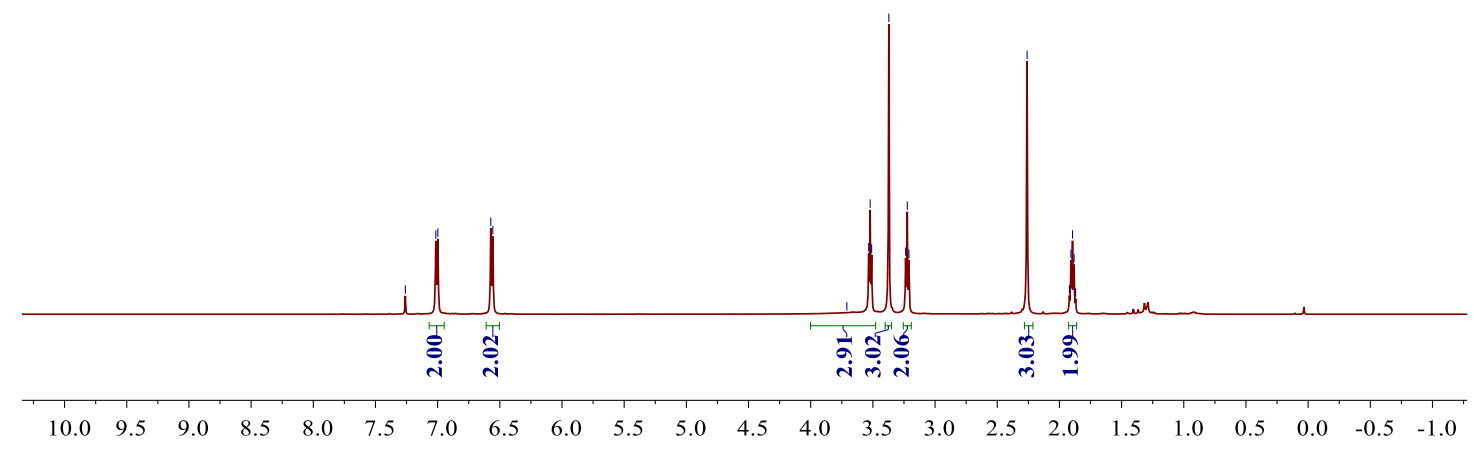

${ }^{13} \mathrm{C} \mathrm{NMR}\left(126 \mathrm{MHz}, \mathrm{CDCl}_{3}\right)$

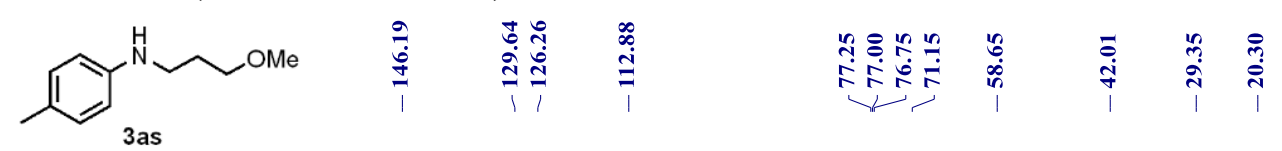

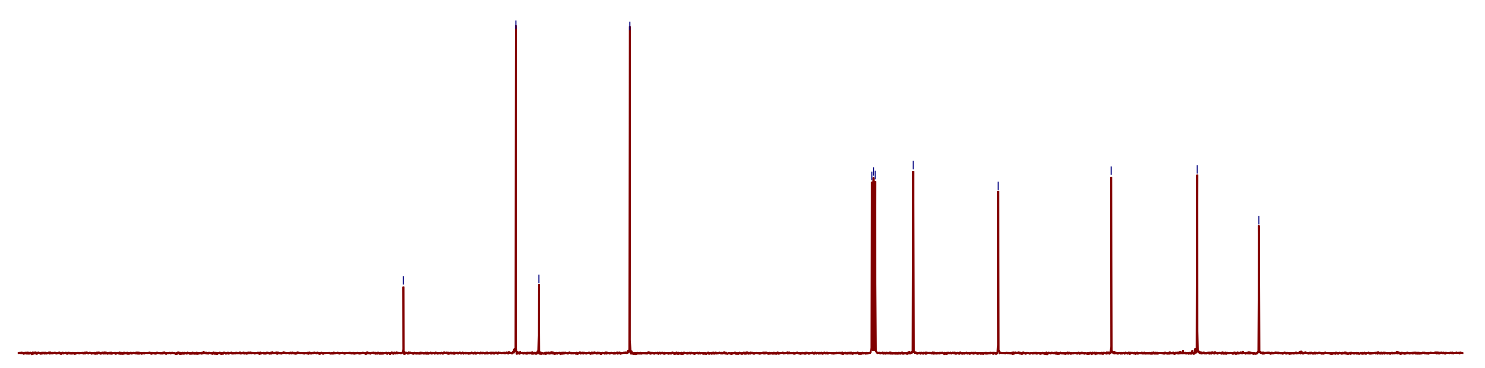

$\begin{array}{llllllllllllllllllllllll}200 & 190 & 180 & 170 & 160 & 150 & 140 & 130 & 120 & 110 & 100 & 90 & 80 & 70 & 60 & 50 & 40 & 30 & 20 & 10 & 0 & 10\end{array}$ 
${ }^{1} \mathrm{H}$ NMR $\left(500 \mathrm{MHz}, \mathrm{CDCl}_{3}\right)$

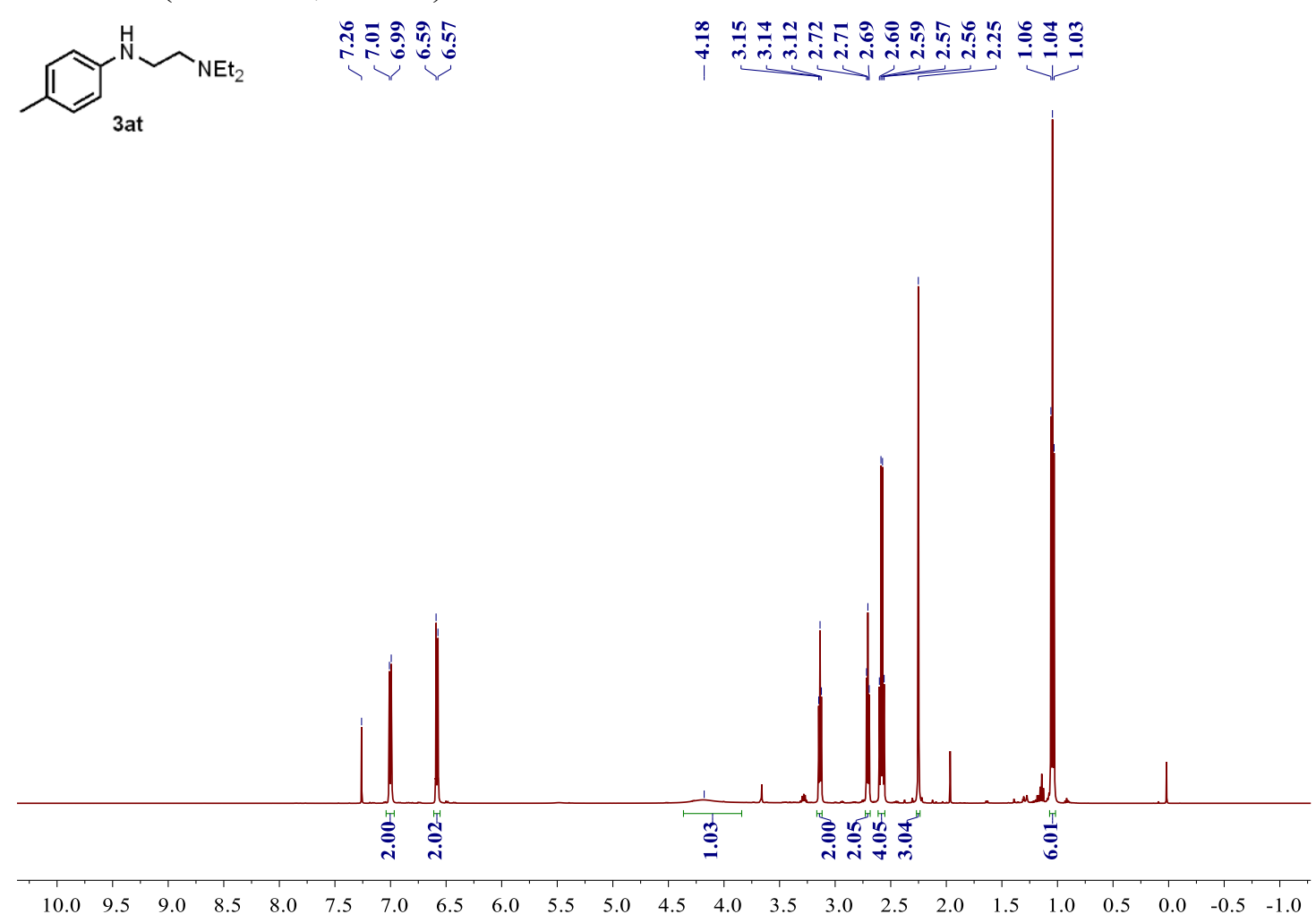

${ }^{13} \mathrm{C}$ NMR $\left(126 \mathrm{MHz}, \mathrm{CDCl}_{3}\right)$

$\overbrace{3 a t}^{\mathrm{N} \sim \mathrm{NEt}_{2}}$

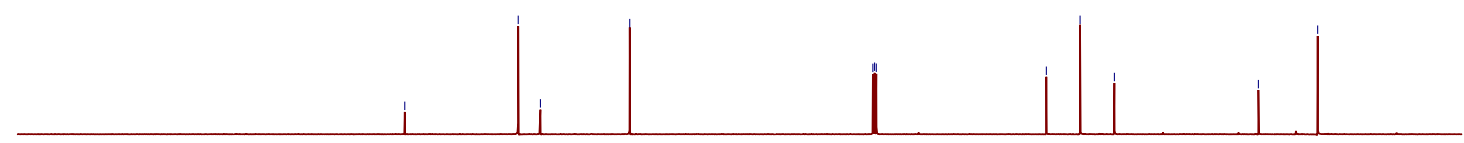

$\begin{array}{lllllllllllllllllllllll}200 & 190 & 180 & 170 & 160 & 150 & 140 & 130 & 120 & 110 & 100 & 90 & 80 & 70 & 60 & 50 & 40 & 30 & 20 & 10 & 0\end{array}$ 
${ }^{1} \mathrm{H}$ NMR $\left(500 \mathrm{MHz}, \mathrm{CDCl}_{3}\right)$
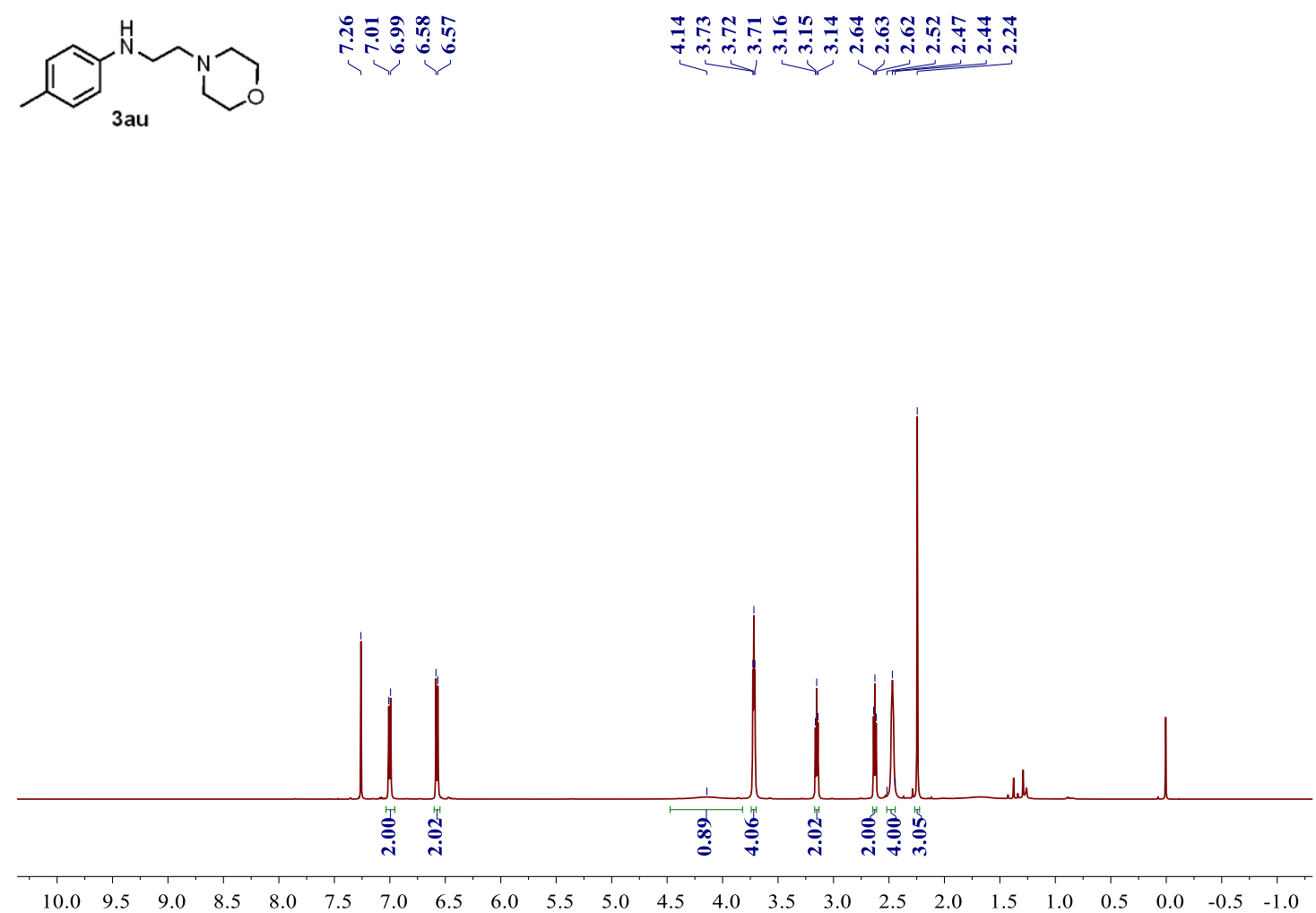

${ }^{13} \mathrm{C} \mathrm{NMR}\left(126 \mathrm{MHz}, \mathrm{CDCl}_{3}\right)$

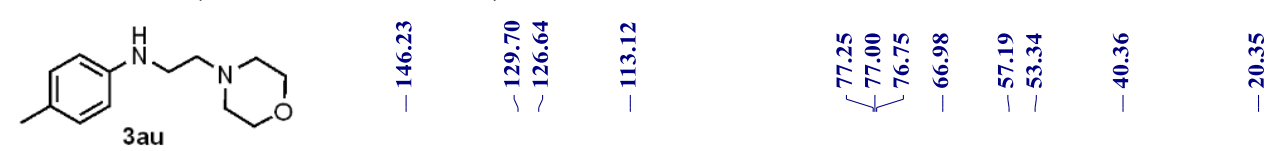

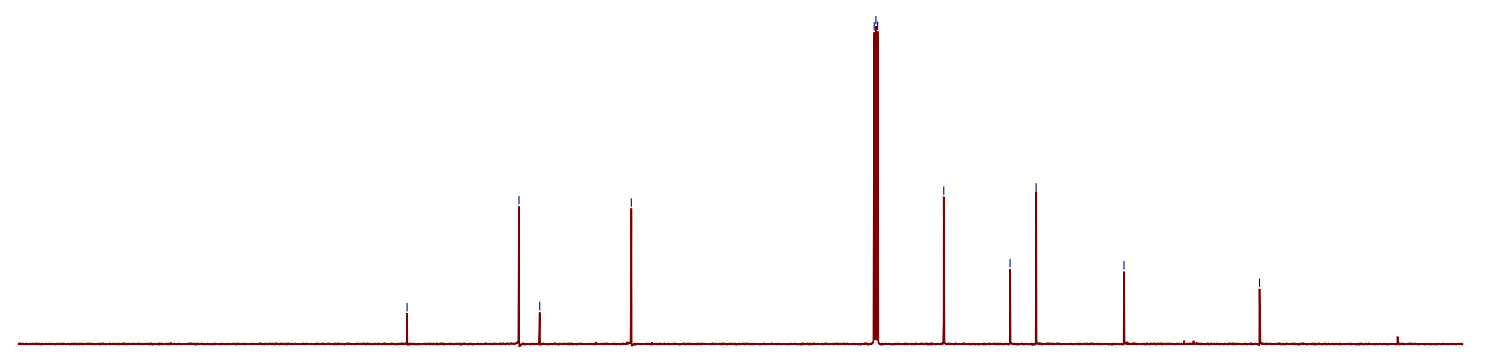

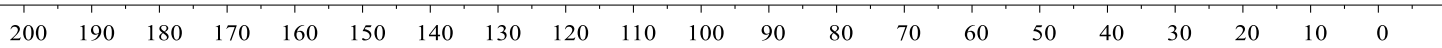


${ }^{1} \mathrm{H} \mathrm{NMR}\left(500 \mathrm{MHz}, \mathrm{CDCl}_{3}\right)$
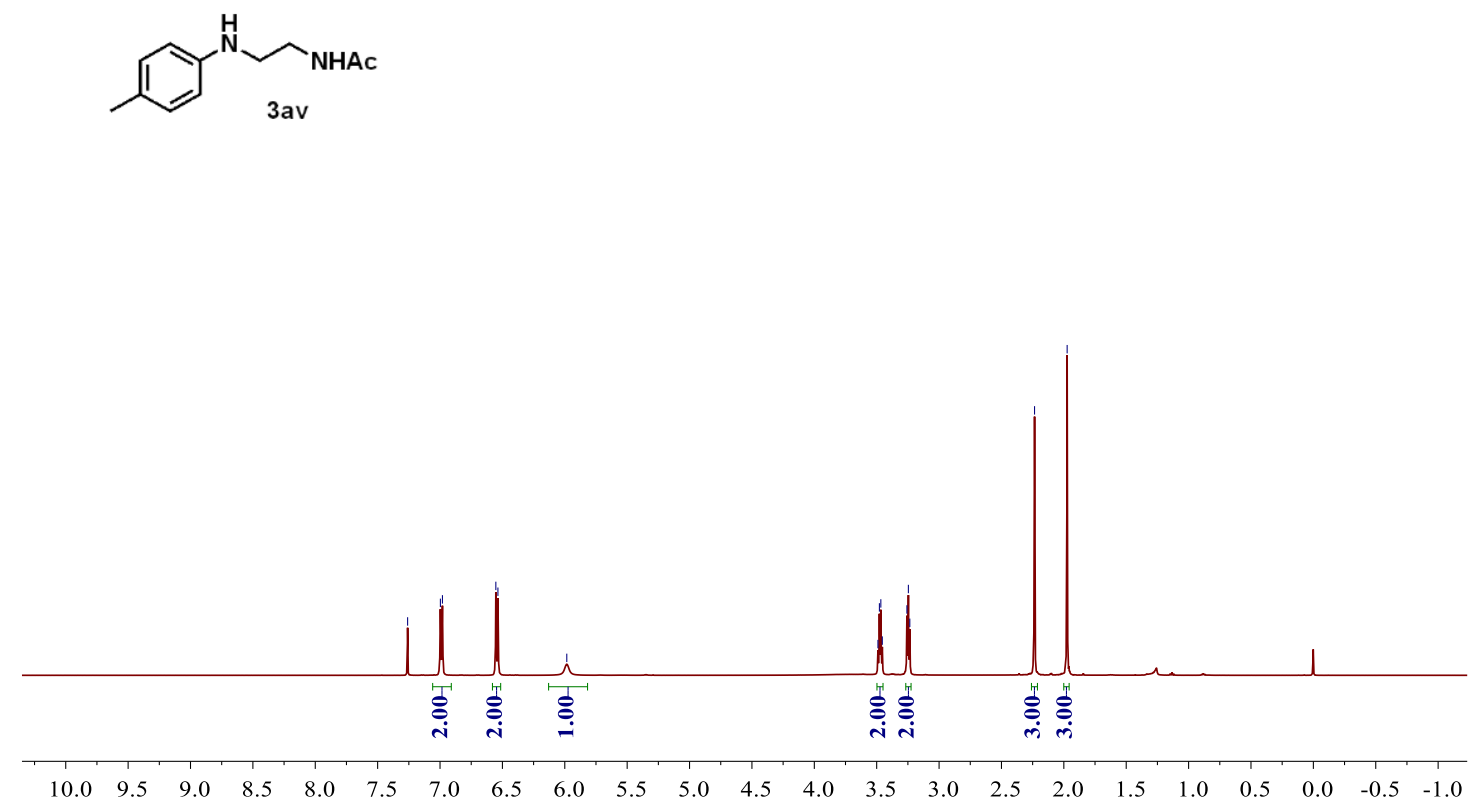

${ }^{13} \mathrm{C} \mathrm{NMR}\left(126 \mathrm{MHz}, \mathrm{CDCl}_{3}\right)$

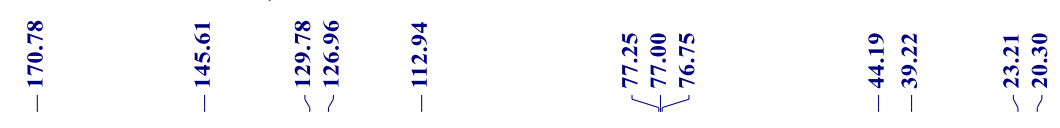<smiles>CNCCNc1ccc(C)cc1</smiles>

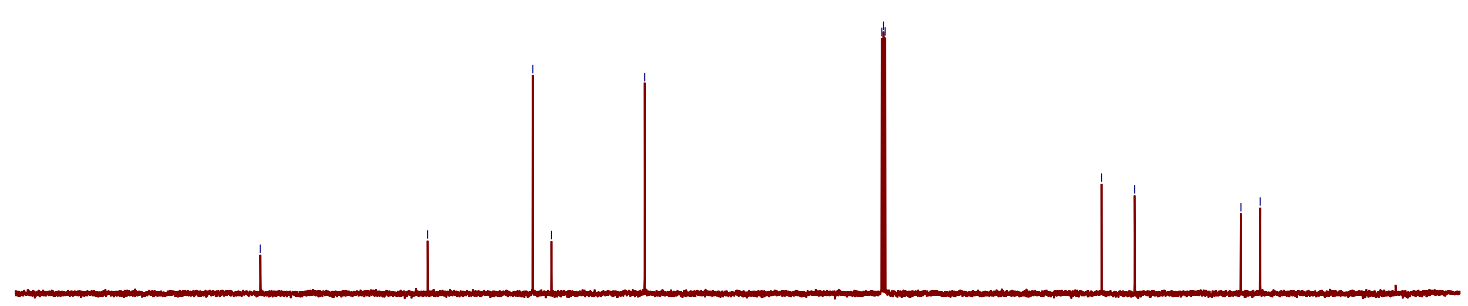

$\begin{array}{lllllllllllllllllllll}200 & 190 & 180 & 170 & 160 & 150 & 140 & 130 & 120 & 110 & 100 & 90 & 80 & 70 & 60 & 50 & 40 & 30 & 20 & 10 & 0\end{array}$ 
${ }^{1} \mathrm{H} \mathrm{NMR}\left(500 \mathrm{MHz}, \mathrm{CDCl}_{3}\right)$

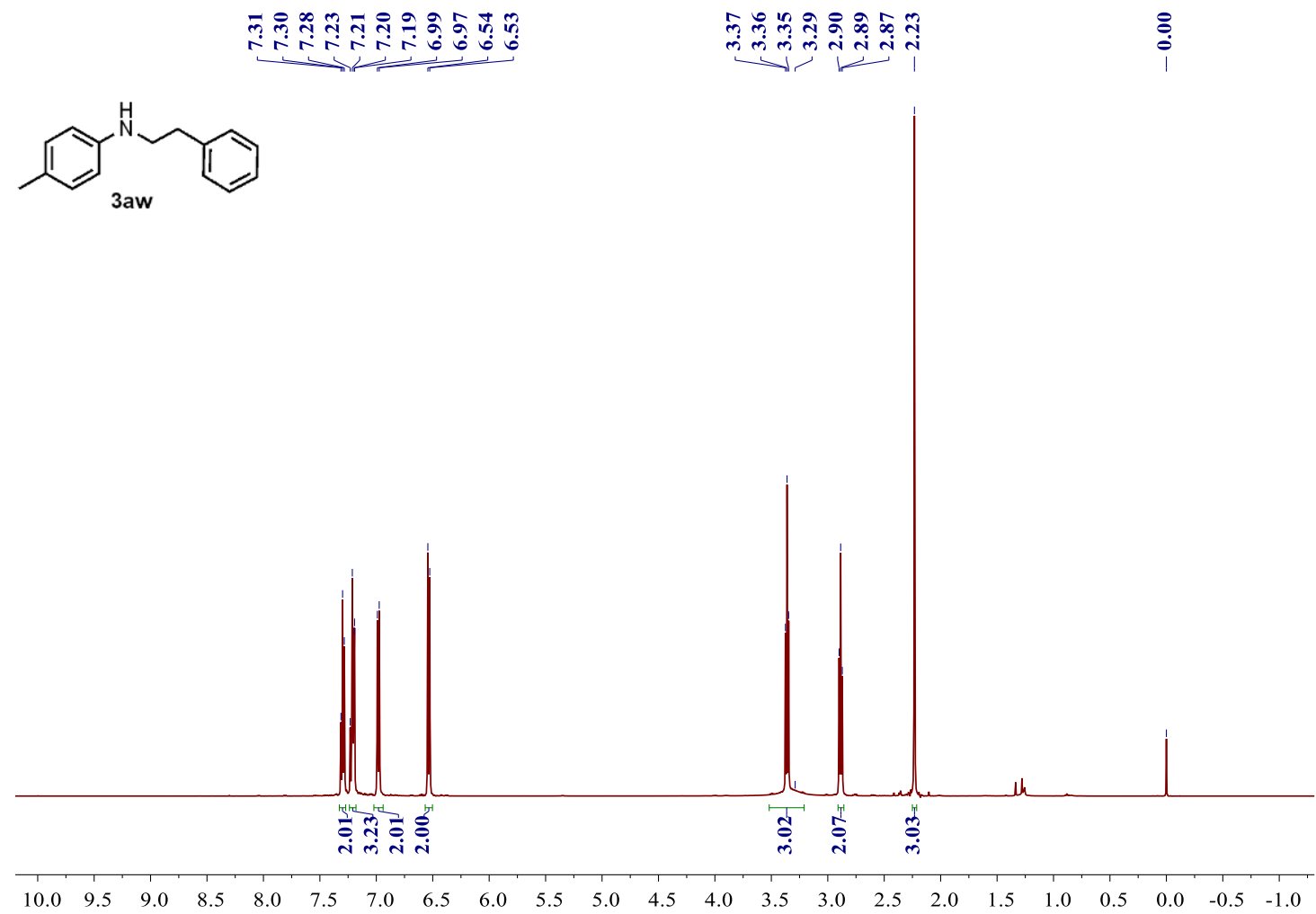

${ }^{13} \mathrm{C}$ NMR (126 MHz, $\mathrm{CDCl}_{3}$ )

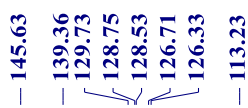

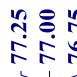

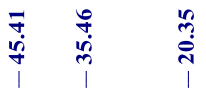<smiles>Cc1ccc(NCCc2ccccc2)cc1</smiles>

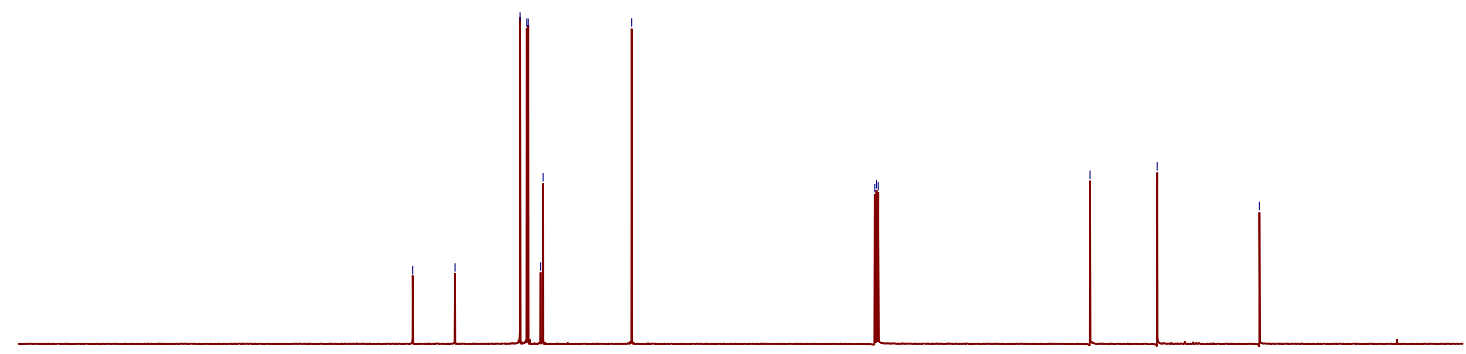

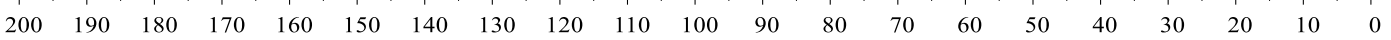


${ }^{1} \mathrm{H} \mathrm{NMR}\left(500 \mathrm{MHz}, \mathrm{CDCl}_{3}\right)$

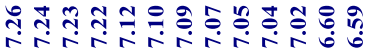

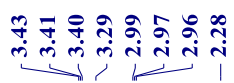

$\overbrace{3 a x}^{N}$

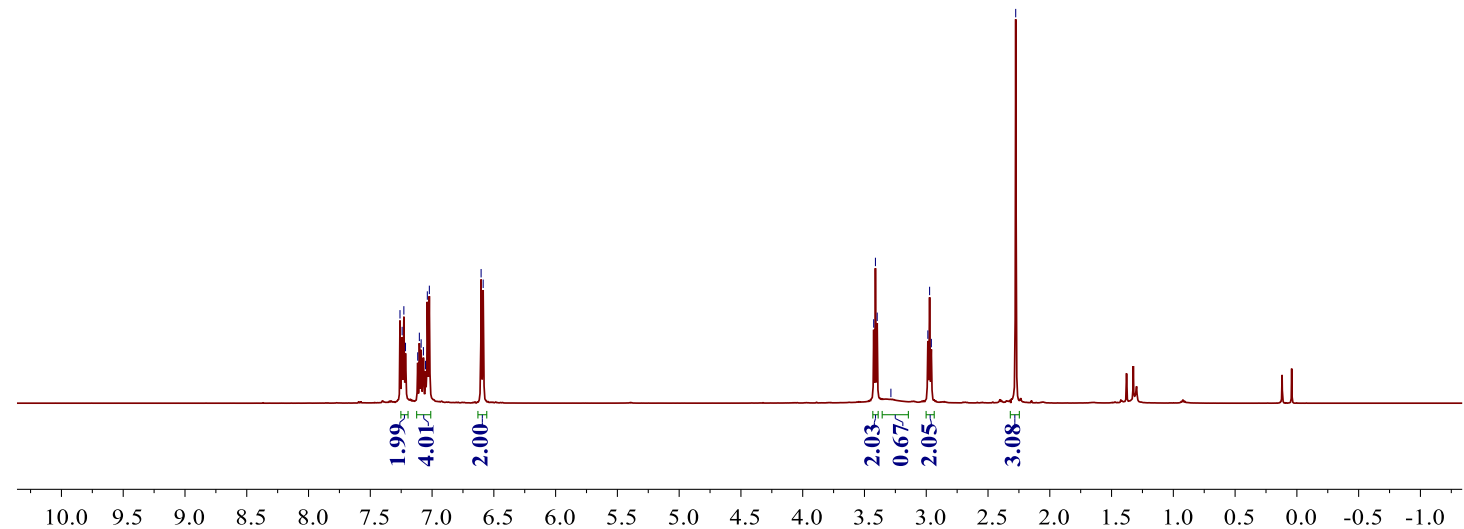

${ }^{13} \mathrm{C} \mathrm{NMR}\left(126 \mathrm{MHz}, \mathrm{CDCl}_{3}\right)$

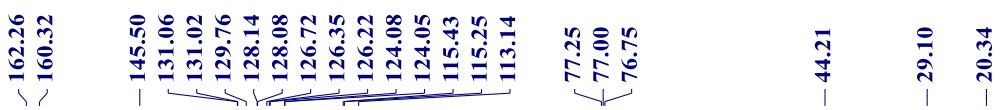<smiles>Cc1ccc(NCCc2ccccc2F)cc1</smiles>

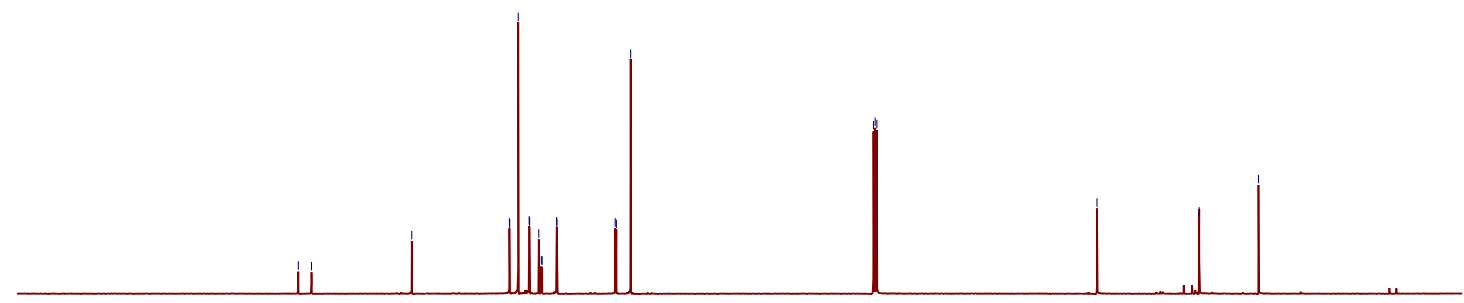

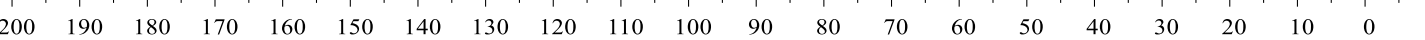


${ }^{19} \mathrm{~F}$ NMR (471 MHz, $\left.\mathrm{CDCl}_{3}\right)$

$\stackrel{\substack{0 \\ \bar{\infty}}}{i}$<smiles>Cc1ccc(NCCc2ccccc2F)cc1</smiles>

$3 a x$

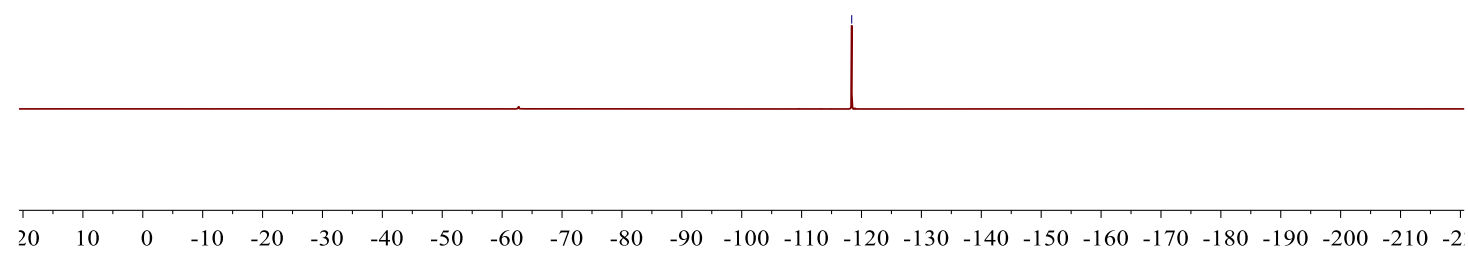

${ }^{1} \mathrm{H}$ NMR (500 MHz, $\left.\mathrm{CDCl}_{3}\right)$
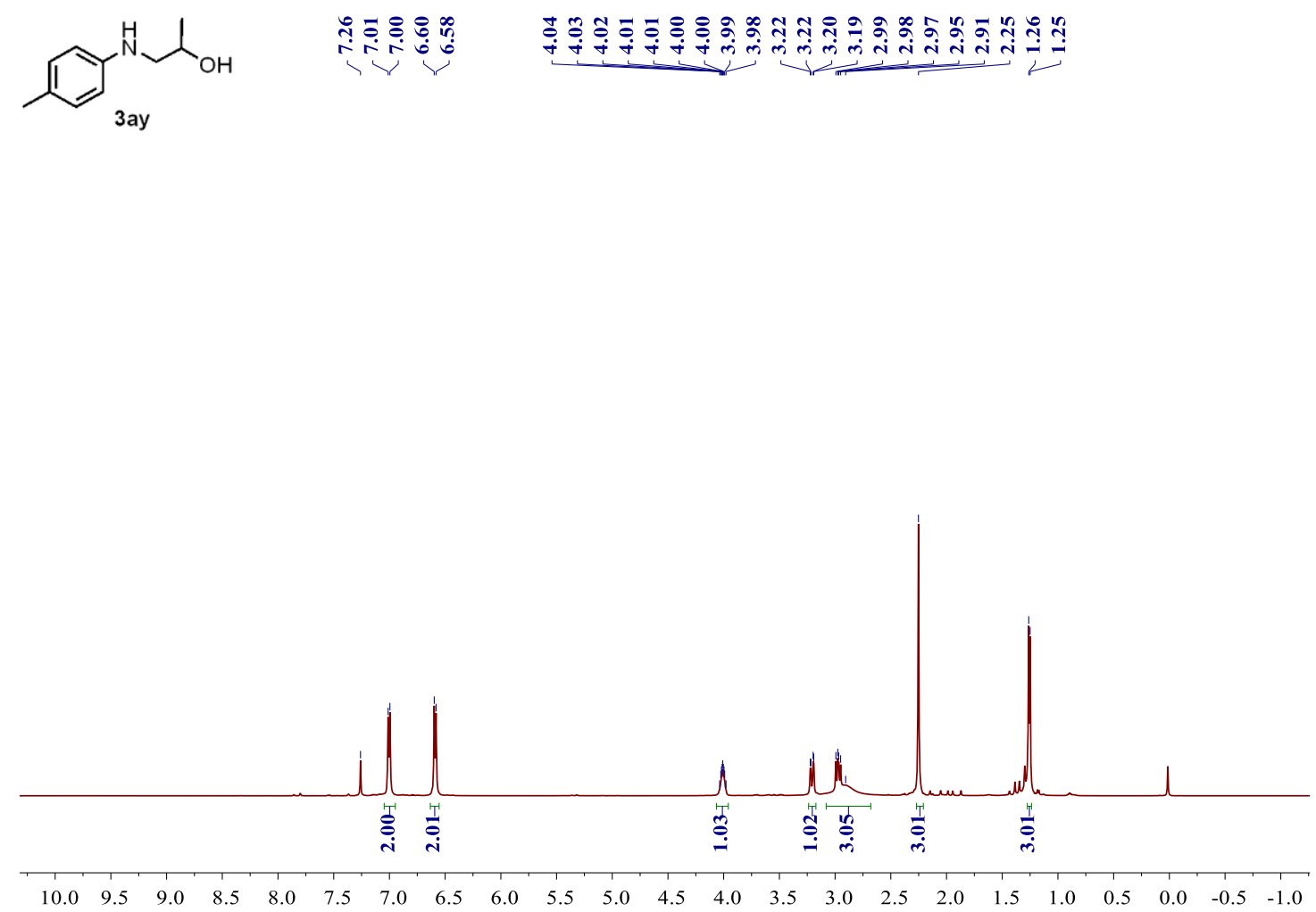
${ }^{13} \mathrm{C}$ NMR $\left(126 \mathrm{MHz}, \mathrm{CDCl}_{3}\right)$

呬

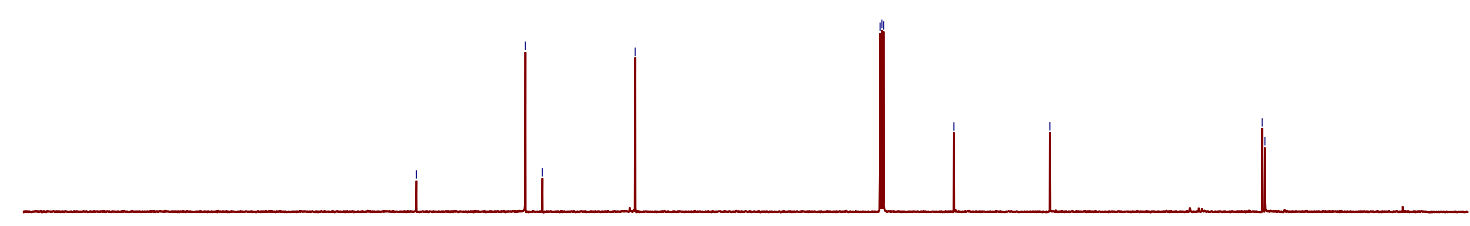

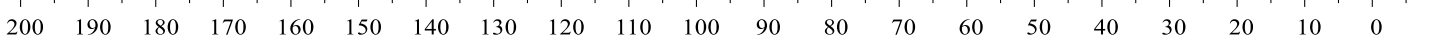

${ }^{1} \mathrm{H} \mathrm{NMR}\left(500 \mathrm{MHz}, \mathrm{CDCl}_{3}\right)$

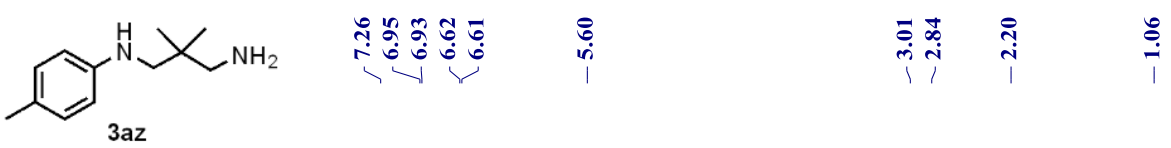

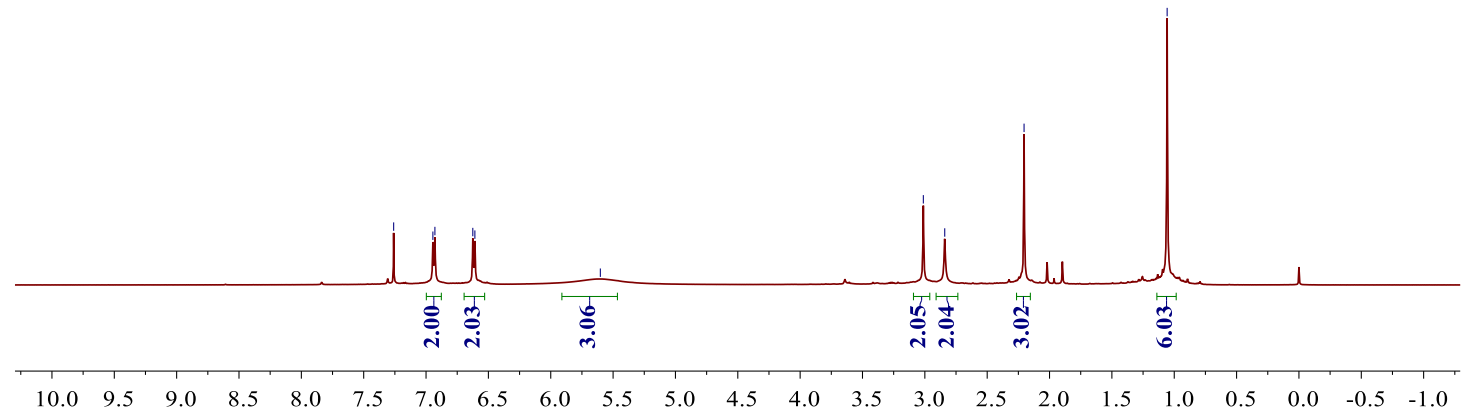


${ }^{13} \mathrm{C} \mathrm{NMR}\left(126 \mathrm{MHz}, \mathrm{CDCl}_{3}\right)$

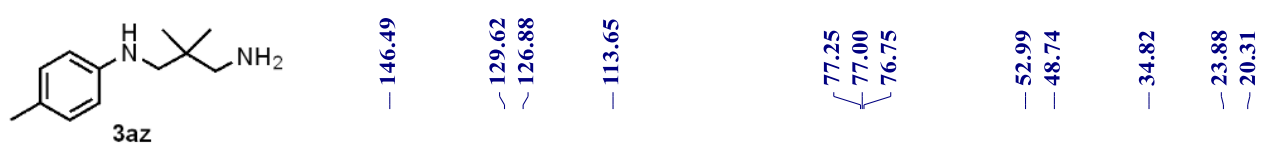

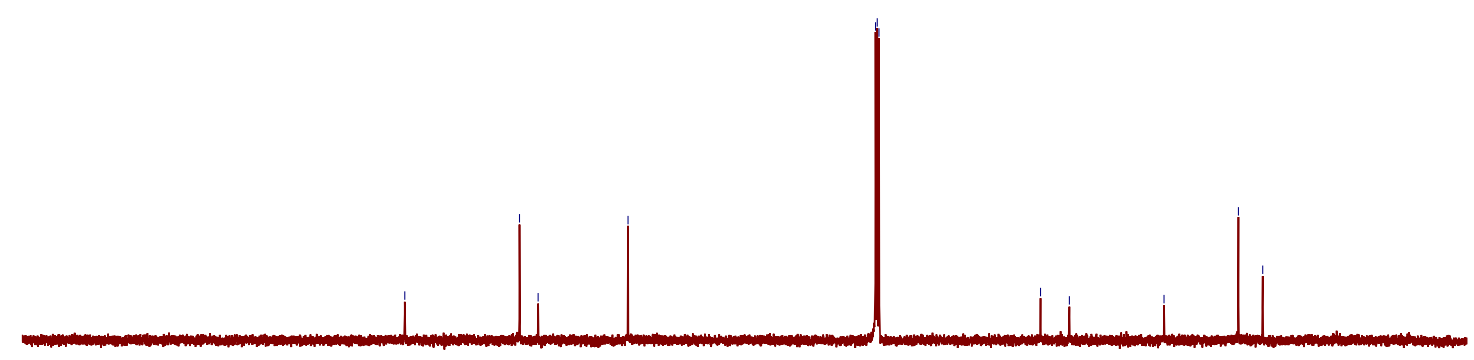

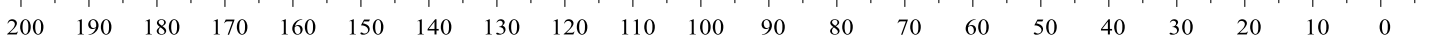

${ }^{1} \mathrm{H} \mathrm{NMR}\left(500 \mathrm{MHz}, \mathrm{CDCl}_{3}\right)$
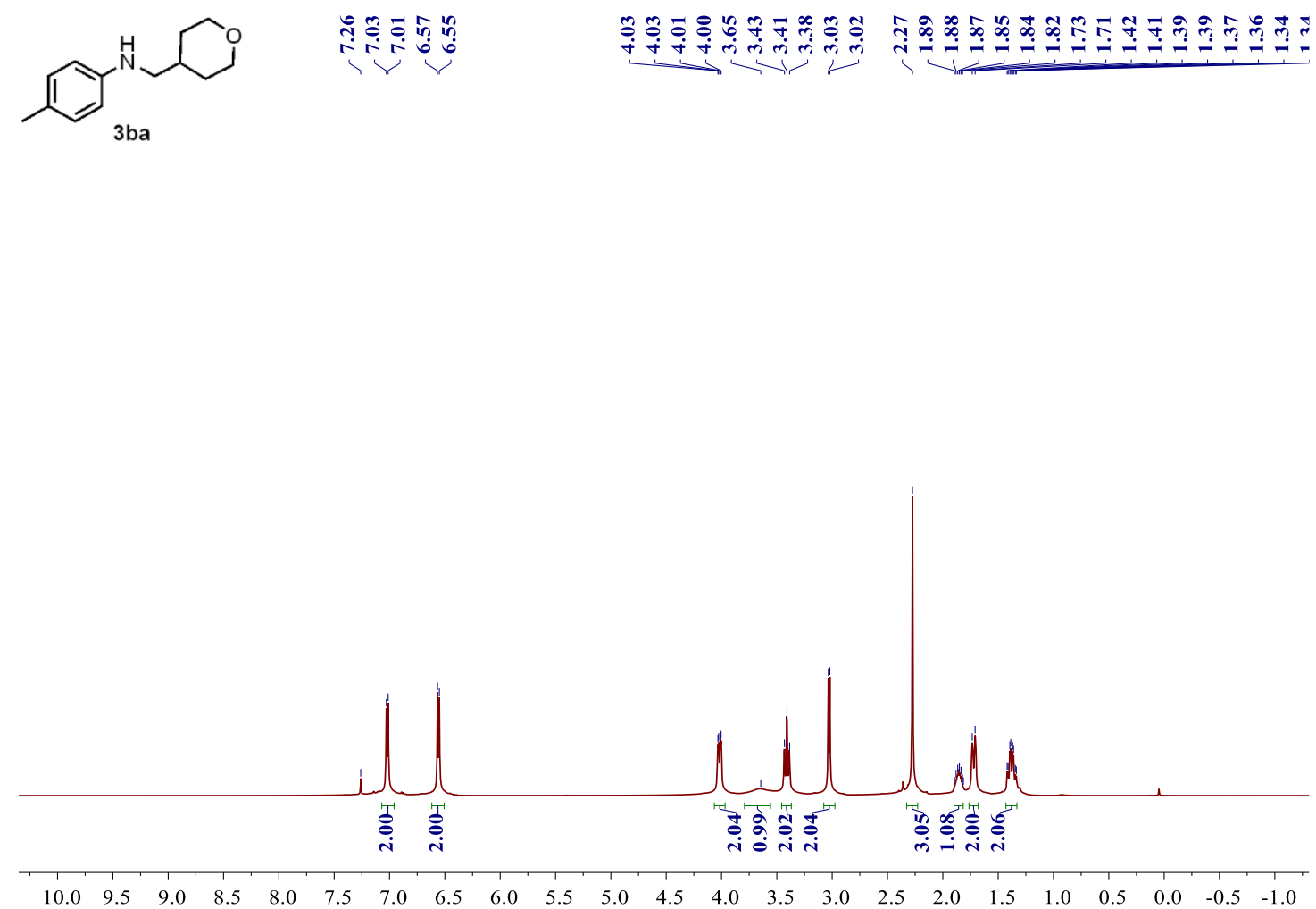
${ }^{13} \mathrm{C} \mathrm{NMR}\left(126 \mathrm{MHz}, \mathrm{CDCl}_{3}\right)$

等

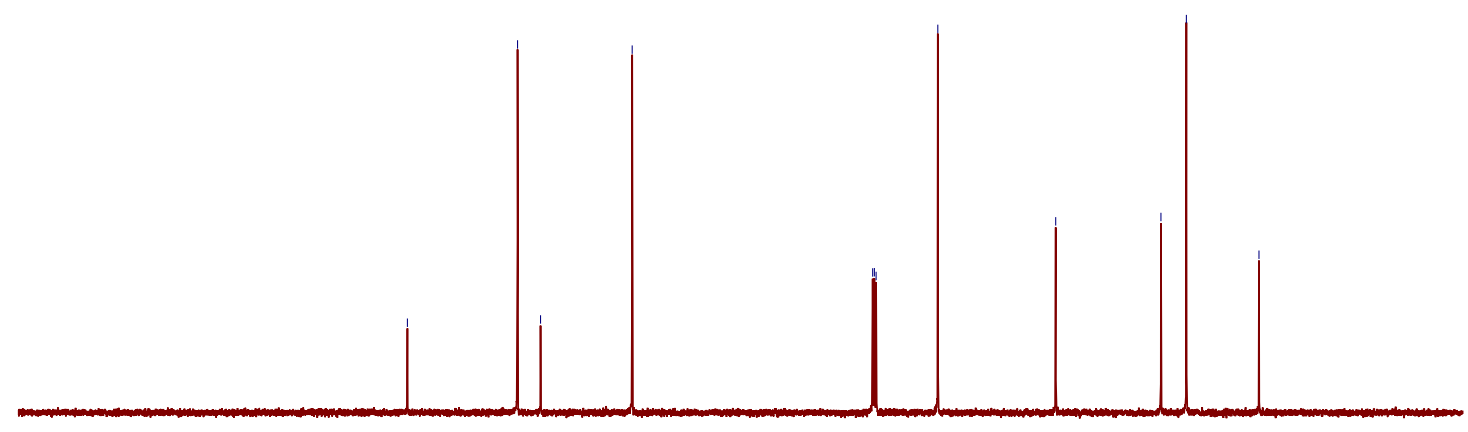

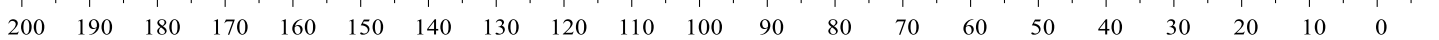

${ }^{1} \mathrm{H} \mathrm{NMR}\left(500 \mathrm{MHz}, \mathrm{CDCl}_{3}\right)$

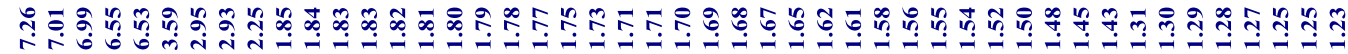<smiles>Cc1ccc(NC2CCCCC2)cc1</smiles>

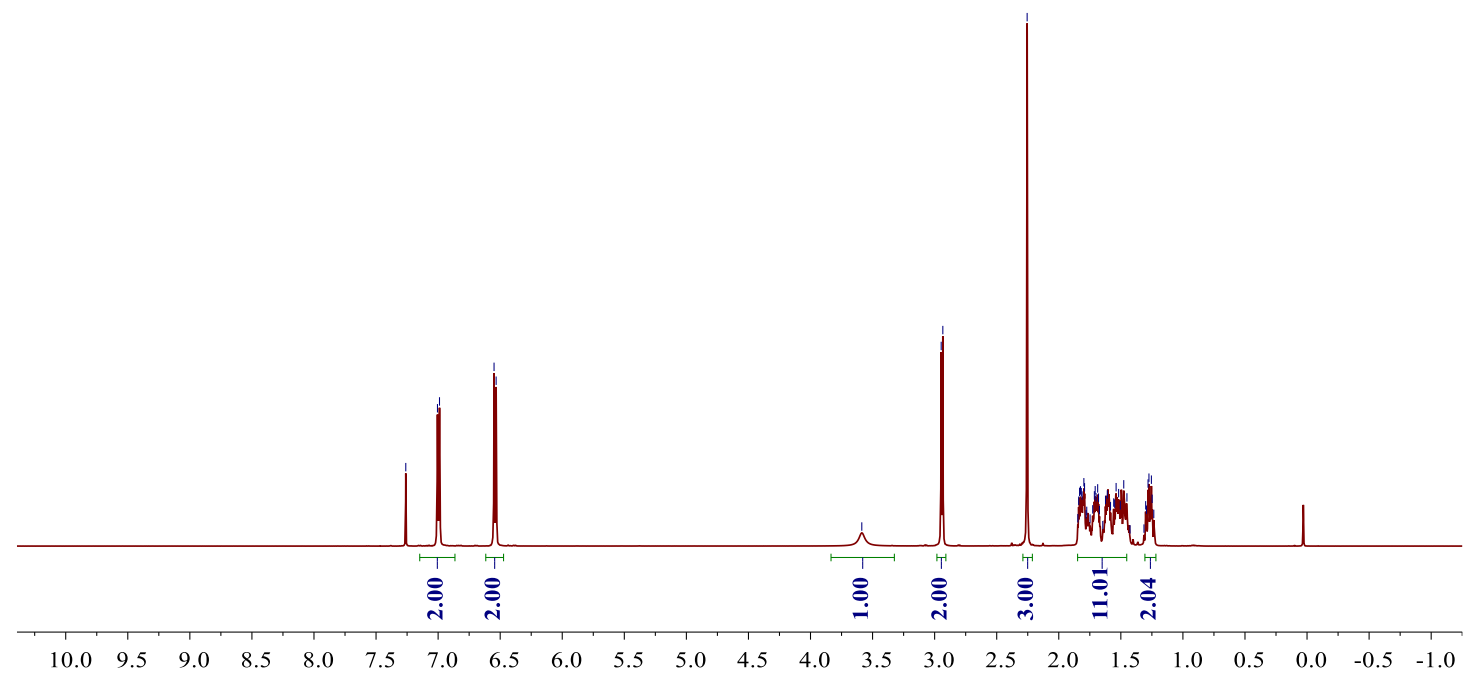


${ }^{13} \mathrm{C} \mathrm{NMR}\left(126 \mathrm{MHz}, \mathrm{CDCl}_{3}\right)$

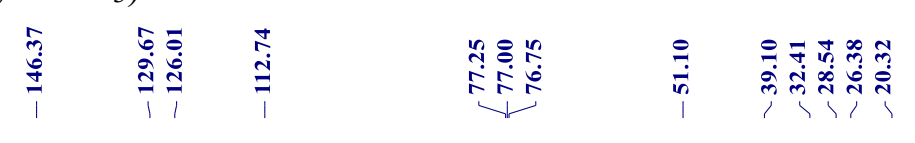<smiles>Cc1ccc(NCC2CCCCCC2)cc1</smiles>
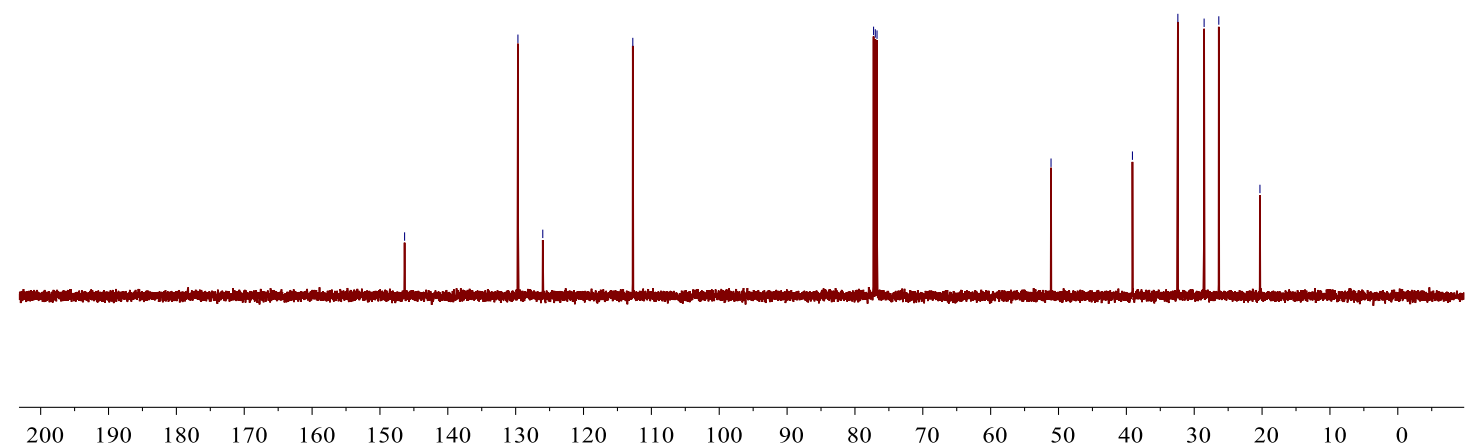

${ }^{1} \mathrm{H}$ NMR $\left(500 \mathrm{MHz}, \mathrm{CDCl}_{3}\right)$
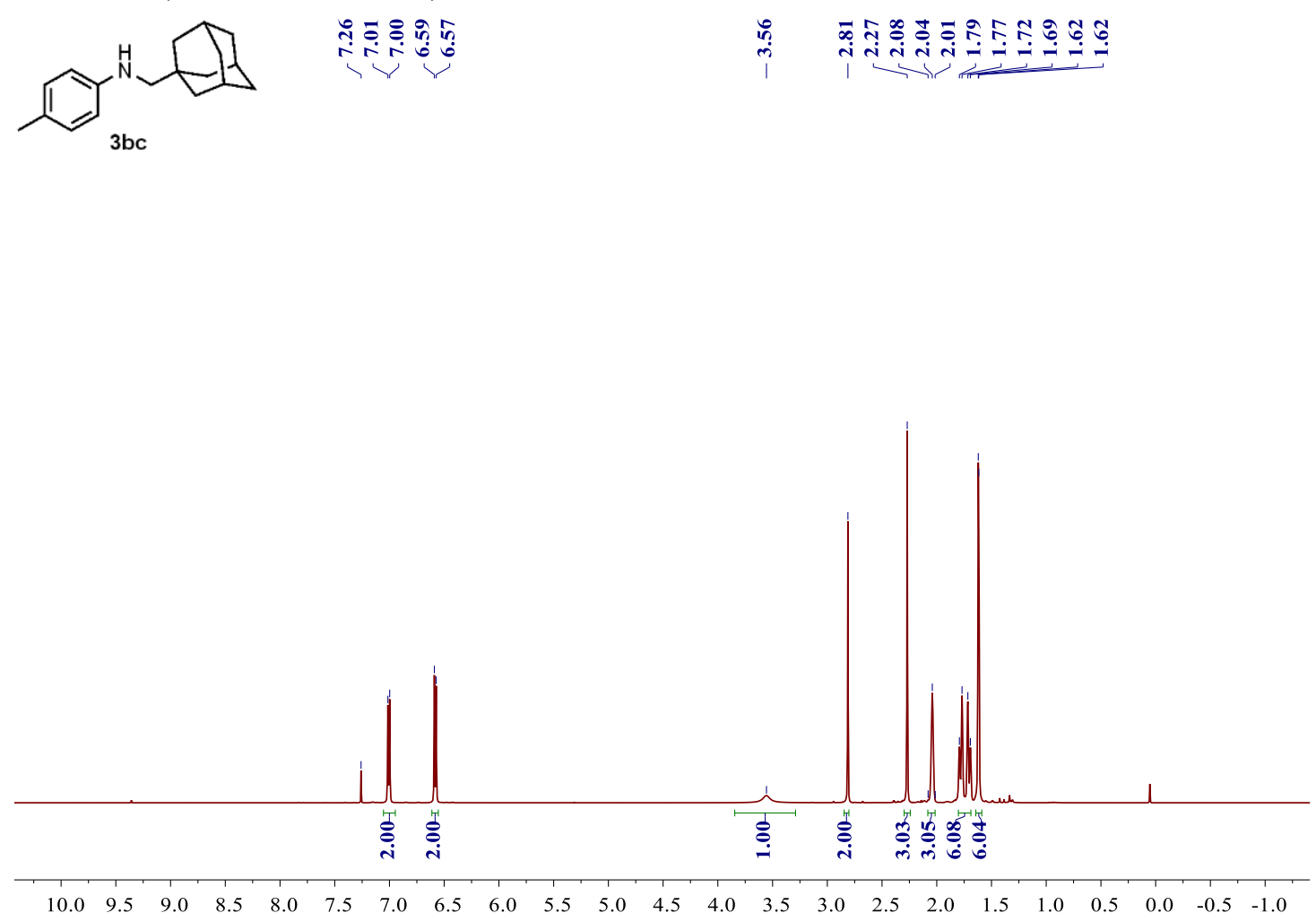
${ }^{13} \mathrm{C} \mathrm{NMR}\left(126 \mathrm{MHz}, \mathrm{CDCl}_{3}\right)$

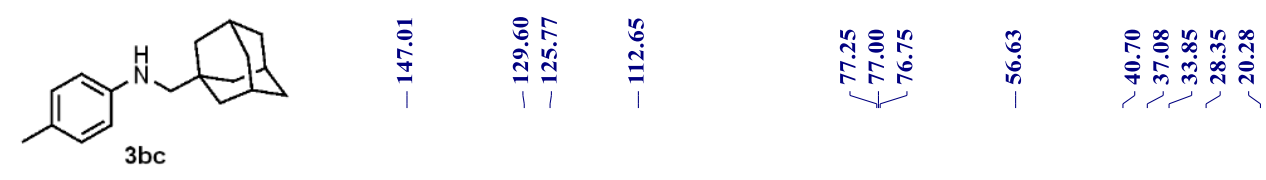

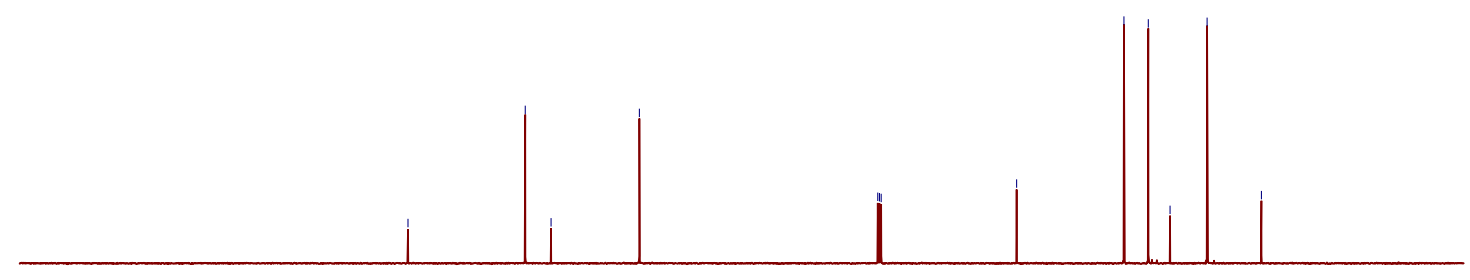

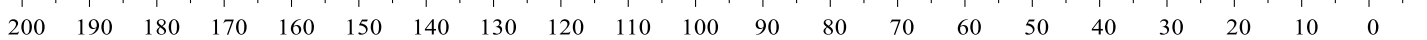

${ }^{1} \mathrm{H} \mathrm{NMR}\left(500 \mathrm{MHz}, \mathrm{CDCl}_{3}\right)$

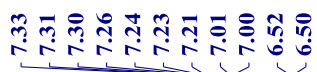<smiles>Cc1ccc(NC(C)CCc2ccccc2)cc1</smiles>

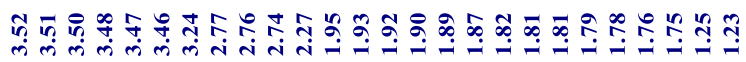

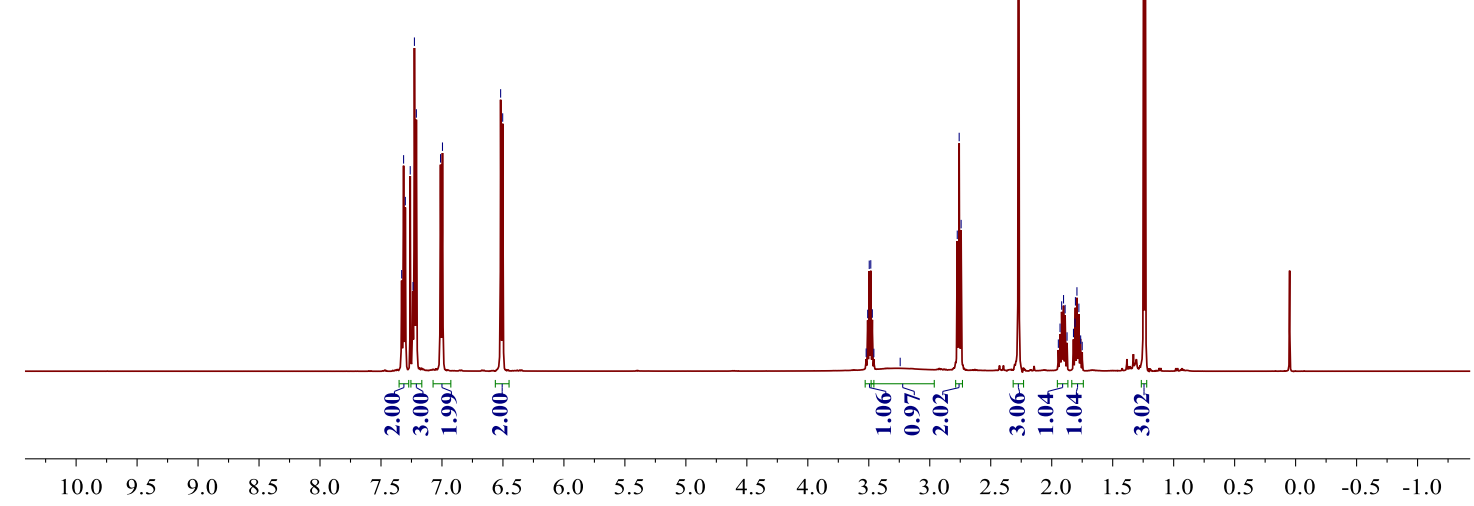


${ }^{13} \mathrm{C}$ NMR $\left(126 \mathrm{MHz}, \mathrm{CDCl}_{3}\right)$

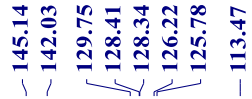

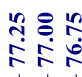

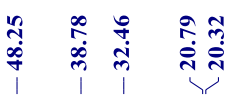<smiles>Cc1ccc(NC(C)CCc2ccccc2)cc1</smiles>

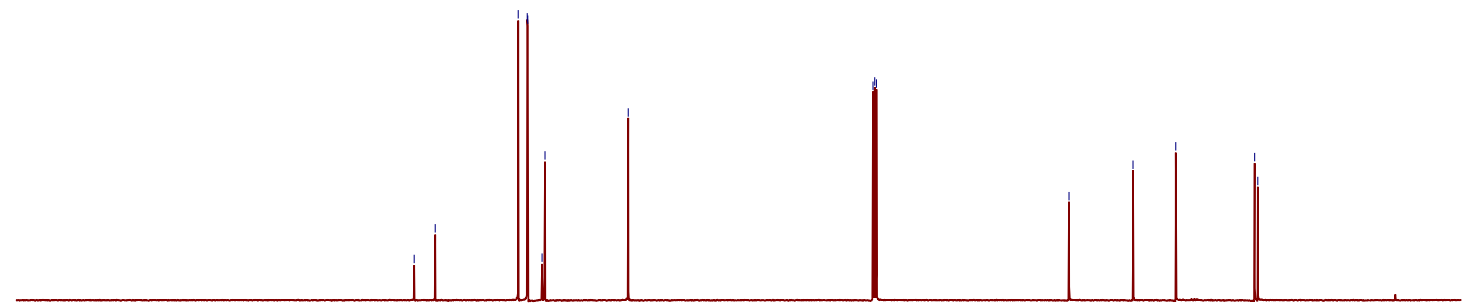

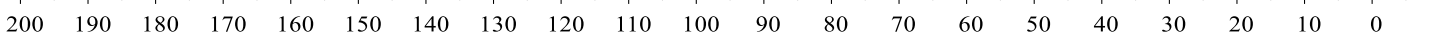

${ }^{1} \mathrm{H} \mathrm{NMR}\left(500 \mathrm{MHz}, \mathrm{CDCl}_{3}\right)$
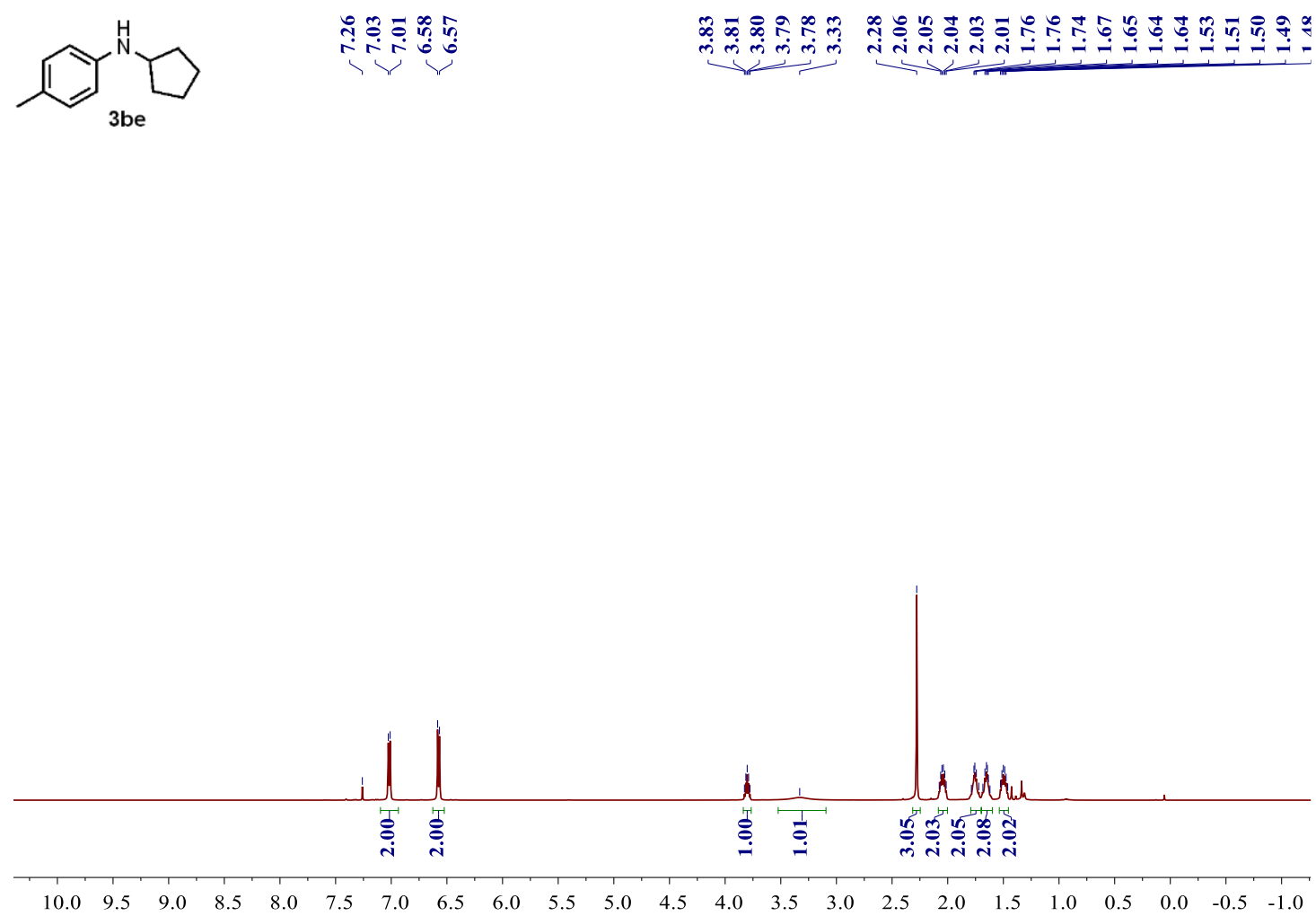
${ }^{13} \mathrm{C} \mathrm{NMR}\left(126 \mathrm{MHz}, \mathrm{CDCl}_{3}\right)$

呬

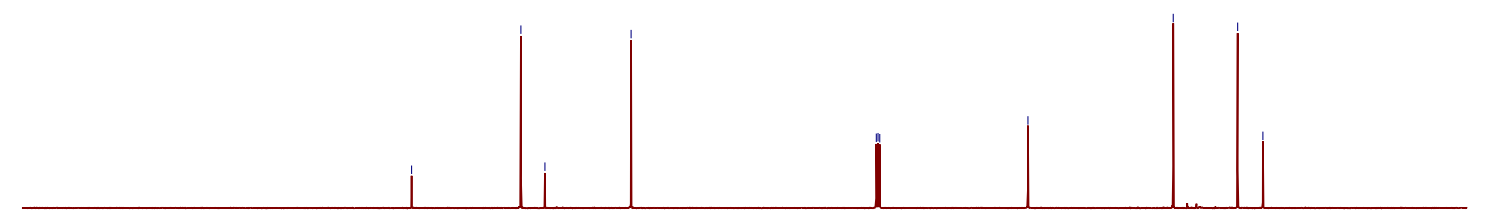

$\begin{array}{lllllllllllllllllllllllll}200 & 190 & 180 & 170 & 160 & 150 & 140 & 130 & 120 & 110 & 100 & 90 & 80 & 70 & 60 & 50 & 40 & 30 & 20 & 10 & 0 & 10\end{array}$

${ }^{1} \mathrm{H}$ NMR $\left(500 \mathrm{MHz}, \mathrm{CDCl}_{3}\right)$

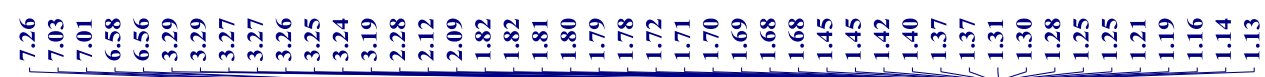<smiles>Cc1ccc(NC2CCCCC2)cc1</smiles>

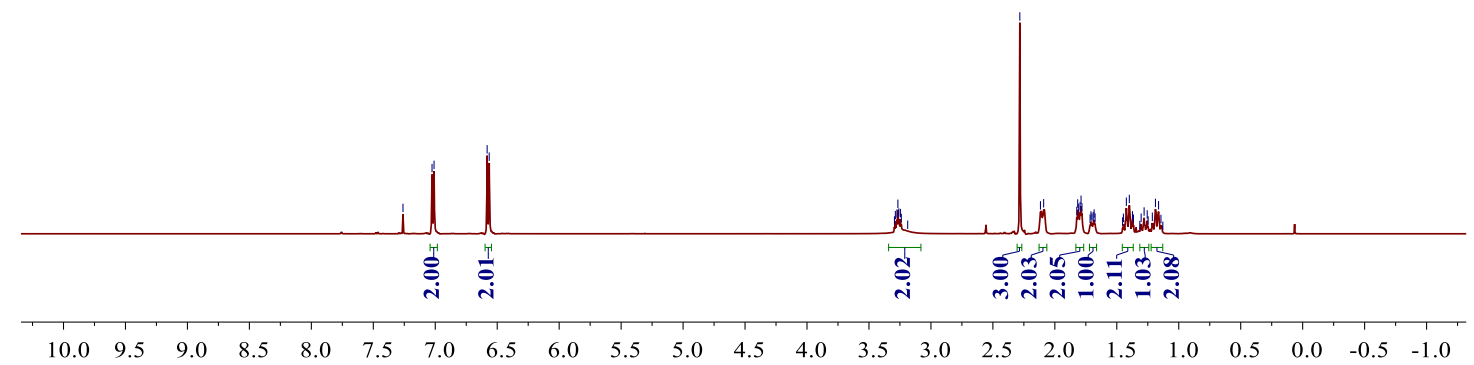


${ }^{13} \mathrm{C} \mathrm{NMR}\left(126 \mathrm{MHz}, \mathrm{CDCl}_{3}\right)$

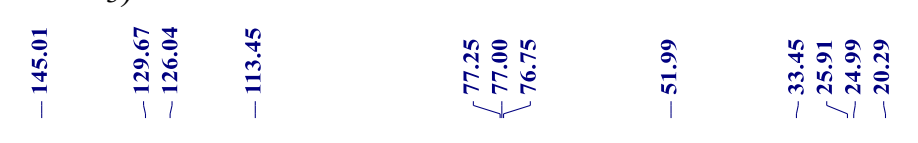<smiles>Cc1ccc(NC2CCCCC2)cc1</smiles>

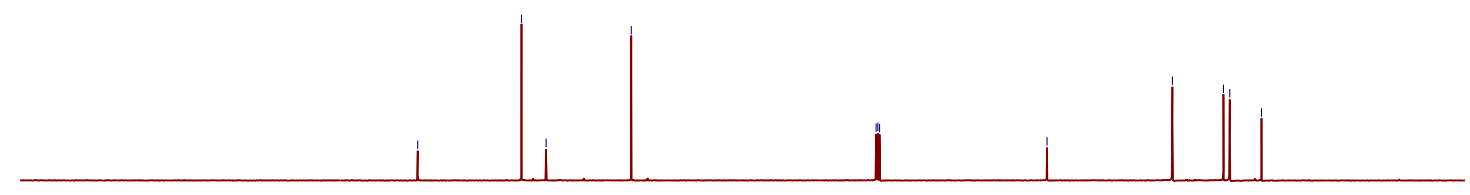

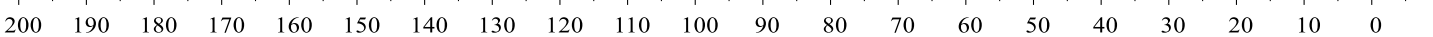

${ }^{1} \mathrm{H} \mathrm{NMR}\left(500 \mathrm{MHz}, \mathrm{CDCl}_{3}\right)$

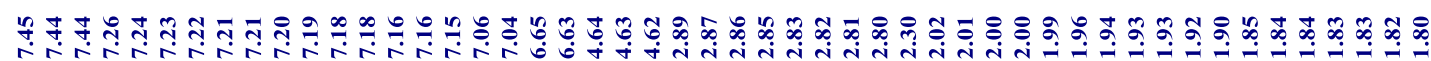<smiles>Cc1ccc(NC2CCCc3ccccc32)cc1</smiles>

$3 \mathrm{bg}$

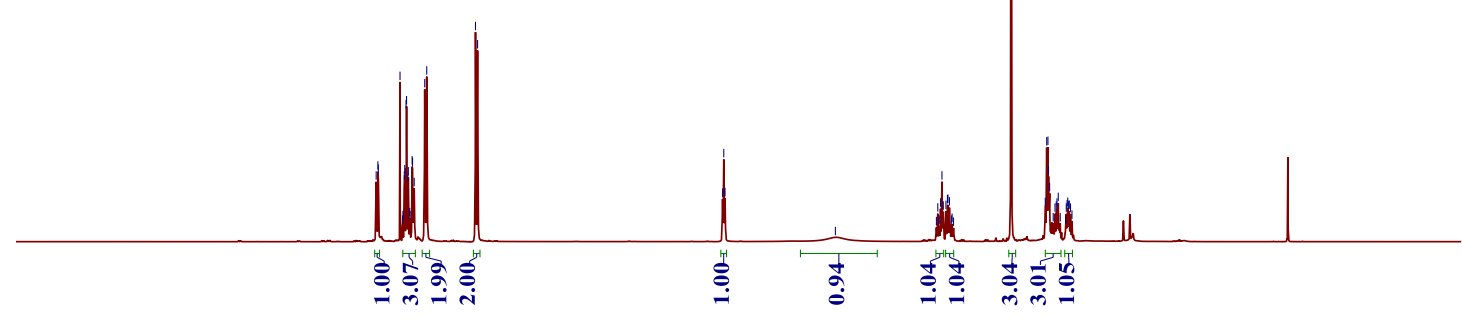

$\begin{array}{llllllllllllllllllllllll}10.0 & 9.5 & 9.0 & 8.5 & 8.0 & 7.5 & 7.0 & 6.5 & 6.0 & 5.5 & 5.0 & 4.5 & 4.0 & 3.5 & 3.0 & 2.5 & 2.0 & 1.5 & 1.0 & 0.5 & 0.0 & -0.5 & -1.0\end{array}$ 
${ }^{13} \mathrm{C} \mathrm{NMR}\left(126 \mathrm{MHz}, \mathrm{CDCl}_{3}\right)$

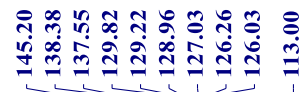

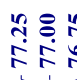

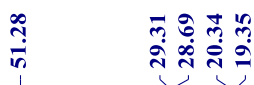<smiles>Cc1ccc(NC2CCCc3ccccc32)cc1</smiles>

$3 \mathrm{bg}$

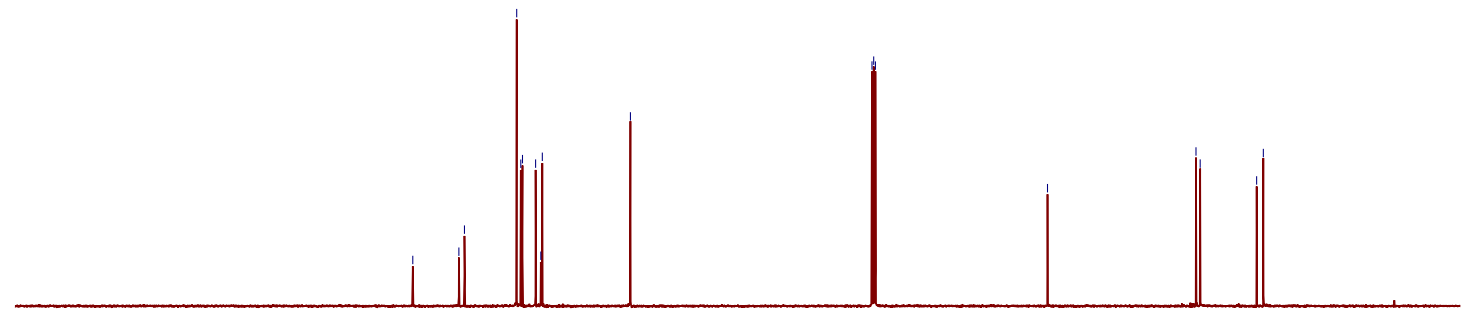

$\begin{array}{lllllllllllllllllllll}200 & 190 & 180 & 170 & 160 & 150 & 140 & 130 & 120 & 110 & 100 & 90 & 80 & 70 & 60 & 50 & 40 & 30 & 20 & 10 & 0\end{array}$

${ }^{1} \mathrm{H} \mathrm{NMR}\left(500 \mathrm{MHz}, \mathrm{CDCl}_{3}\right)$

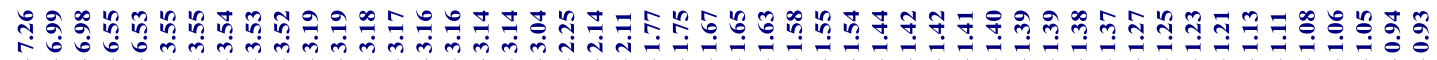<smiles>Cc1ccc(NC2CCC(C)CC2)cc1</smiles>

$3 \mathrm{bh}$

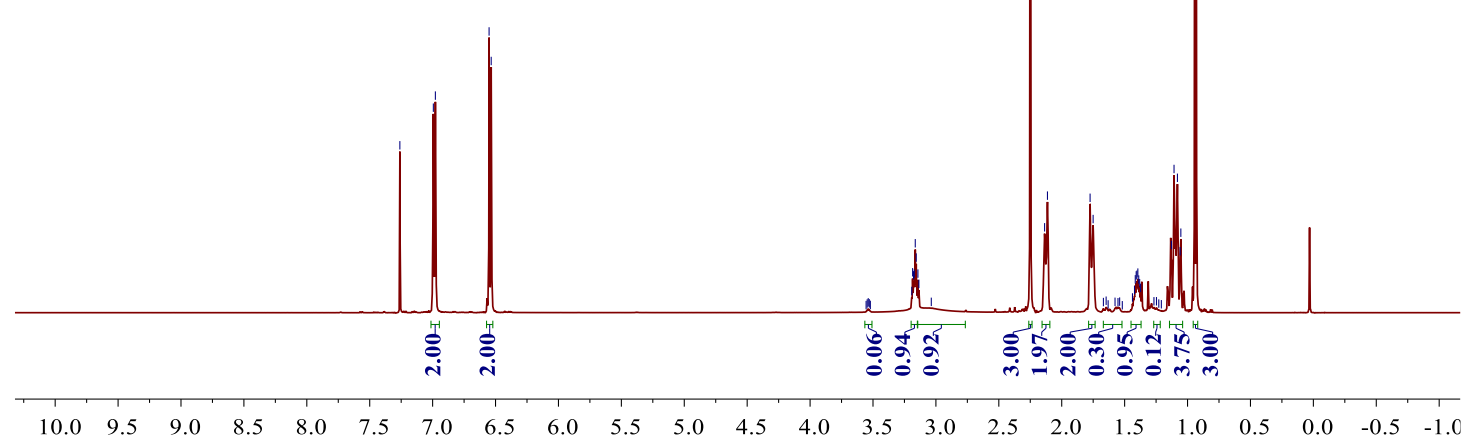


${ }^{13} \mathrm{C}$ NMR $\left(126 \mathrm{MHz}, \mathrm{CDCl}_{3}\right)$

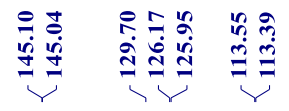

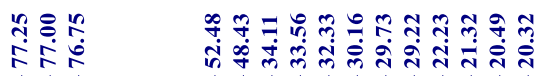<smiles>Cc1ccc(NC2CCC(C)CC2)cc1</smiles>

3bh

${ }^{1} \mathrm{H} \mathrm{NMR}\left(500 \mathrm{MHz}, \mathrm{CDCl}_{3}\right)$
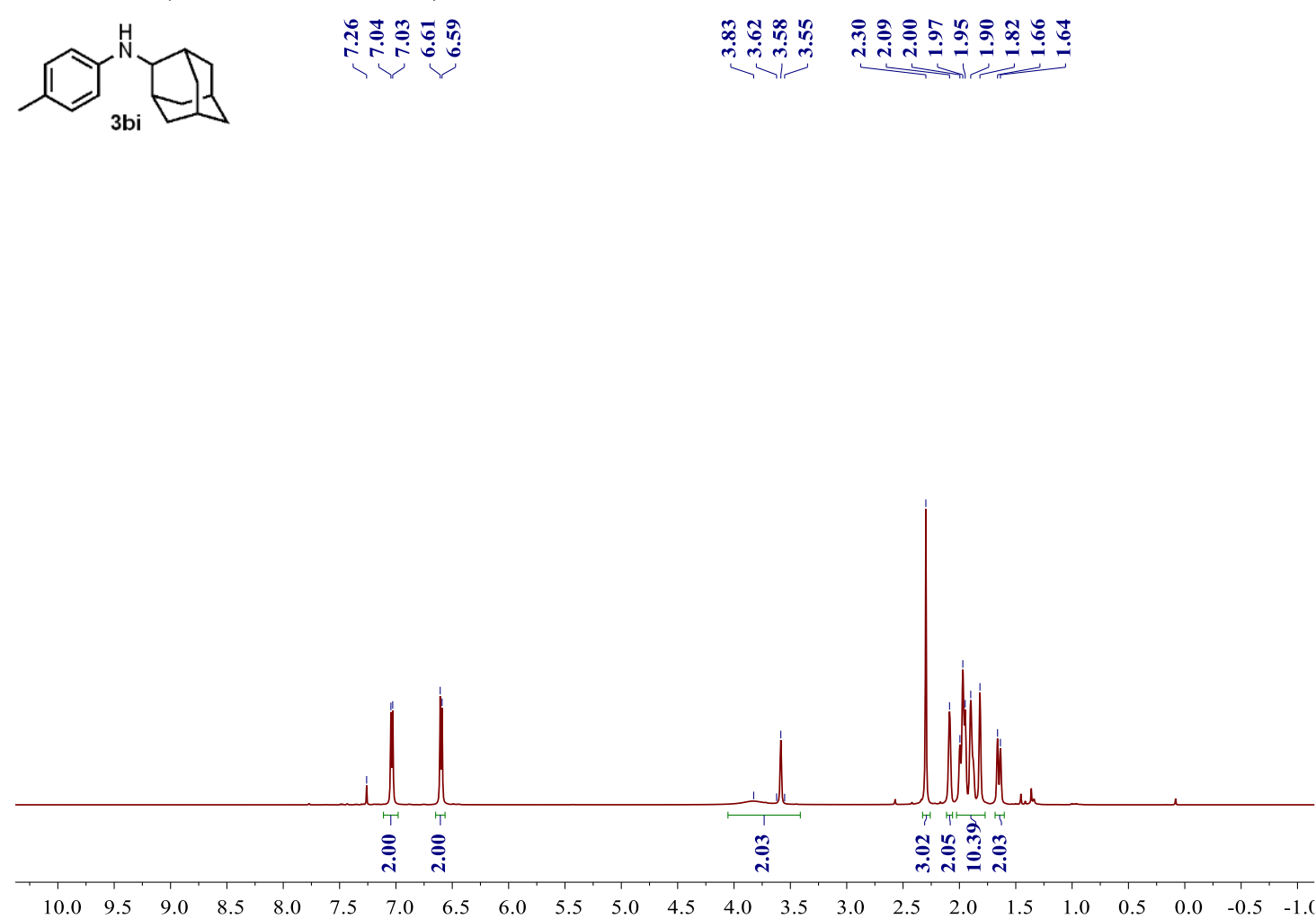
${ }^{13} \mathrm{C} \mathrm{NMR}\left(126 \mathrm{MHz}, \mathrm{CDCl}_{3}\right)$

ل身

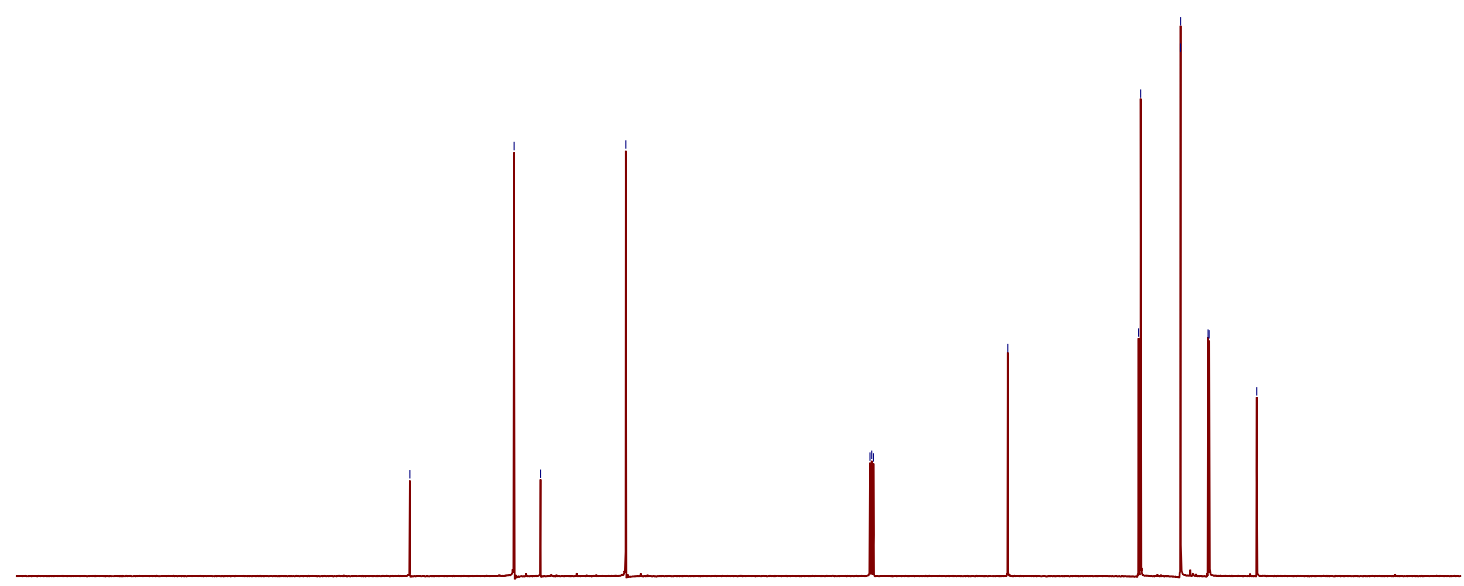

$\begin{array}{lllllllllllllllllllll}200 & 190 & 180 & 170 & 160 & 150 & 140 & 130 & 120 & 110 & 100 & 90 & 80 & 70 & 60 & 50 & 40 & 30 & 20 & 10 & 0\end{array}$

${ }^{1} \mathrm{H} \mathrm{NMR}\left(500 \mathrm{MHz}, \mathrm{CDCl}_{3}\right)$

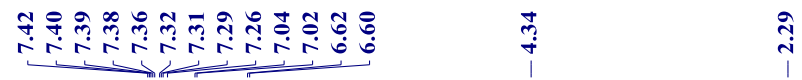<smiles>Cc1ccc(NCc2ccccc2)cc1</smiles>

$3 b k$

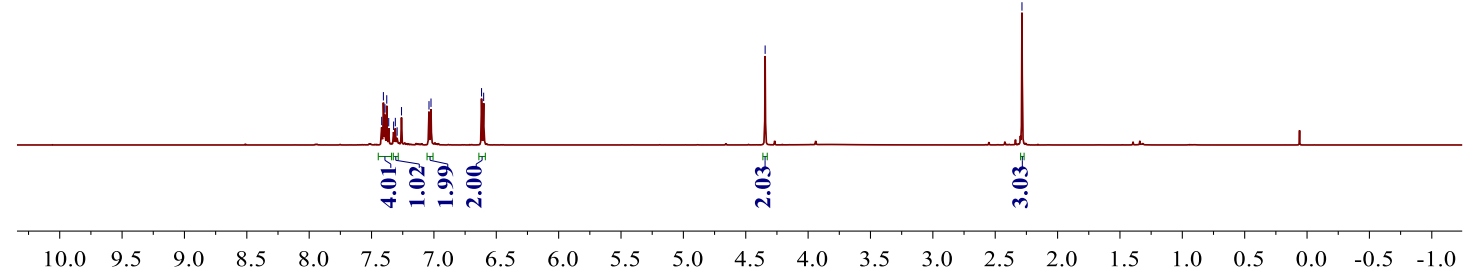


${ }^{13} \mathrm{C}$ NMR $\left(126 \mathrm{MHz}, \mathrm{CDCl}_{3}\right)$

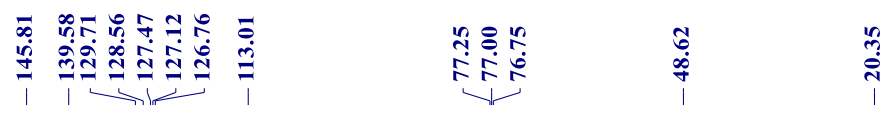<smiles>Cc1ccc(NCc2ccccc2)cc1</smiles>

3bk

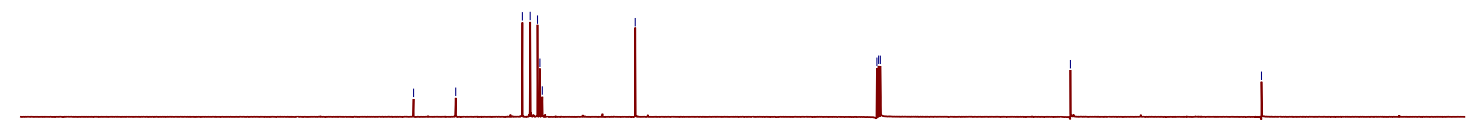

$\begin{array}{lllllllllllllllllllll}200 & 190 & 180 & 170 & 160 & 150 & 140 & 130 & 120 & 110 & 100 & 90 & 80 & 70 & 60 & 50 & 40 & 30 & 20 & 10 & 0\end{array}$

${ }^{1} \mathrm{H} \mathrm{NMR}\left(500 \mathrm{MHz}, \mathrm{CDCl}_{3}\right)$

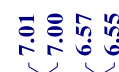

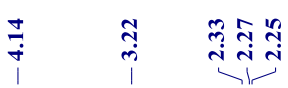

$\stackrel{8}{\circ}$<smiles>Cc1ccc(NCc2c(C)cc(C)cc2C)cc1</smiles>

$3 \mathrm{bl}$

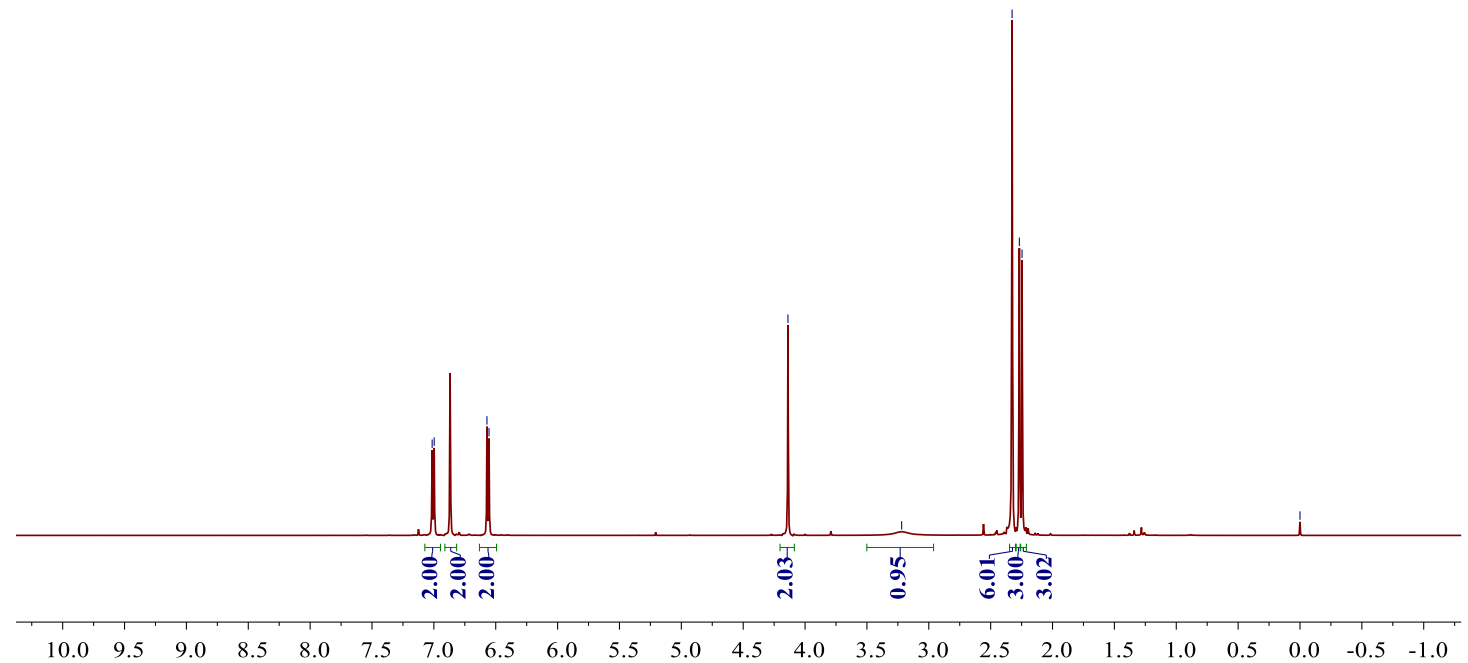


${ }^{13} \mathrm{C}$ NMR (126 MHz, $\left.\mathrm{CDCl}_{3}\right)$

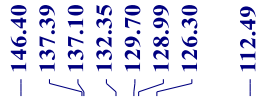

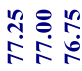

iู่<smiles>Cc1ccc(NCc2c(C)cc(C)cc2C)cc1</smiles>

$3 b$

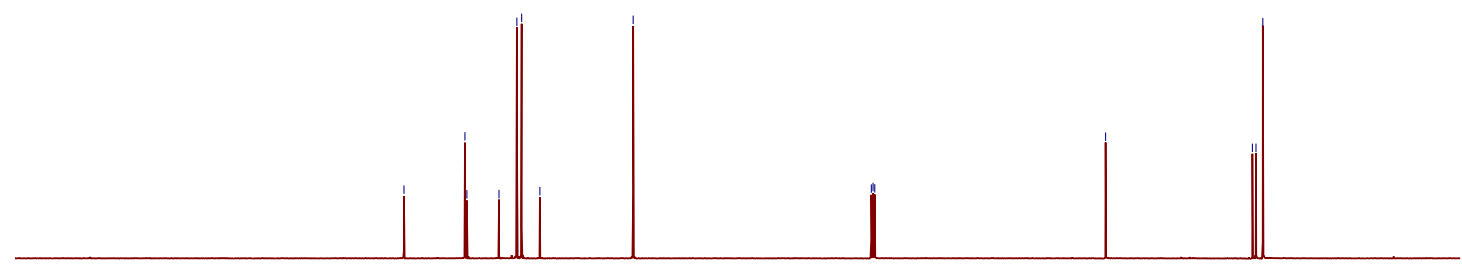

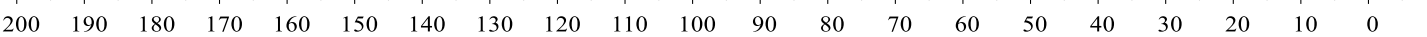

${ }^{1} \mathrm{H} \mathrm{NMR}\left(500 \mathrm{MHz}, \mathrm{CDCl}_{3}\right)$

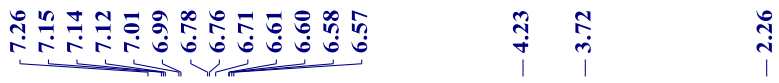<smiles>Cc1ccc(NCc2cccc(N)c2)cc1</smiles>

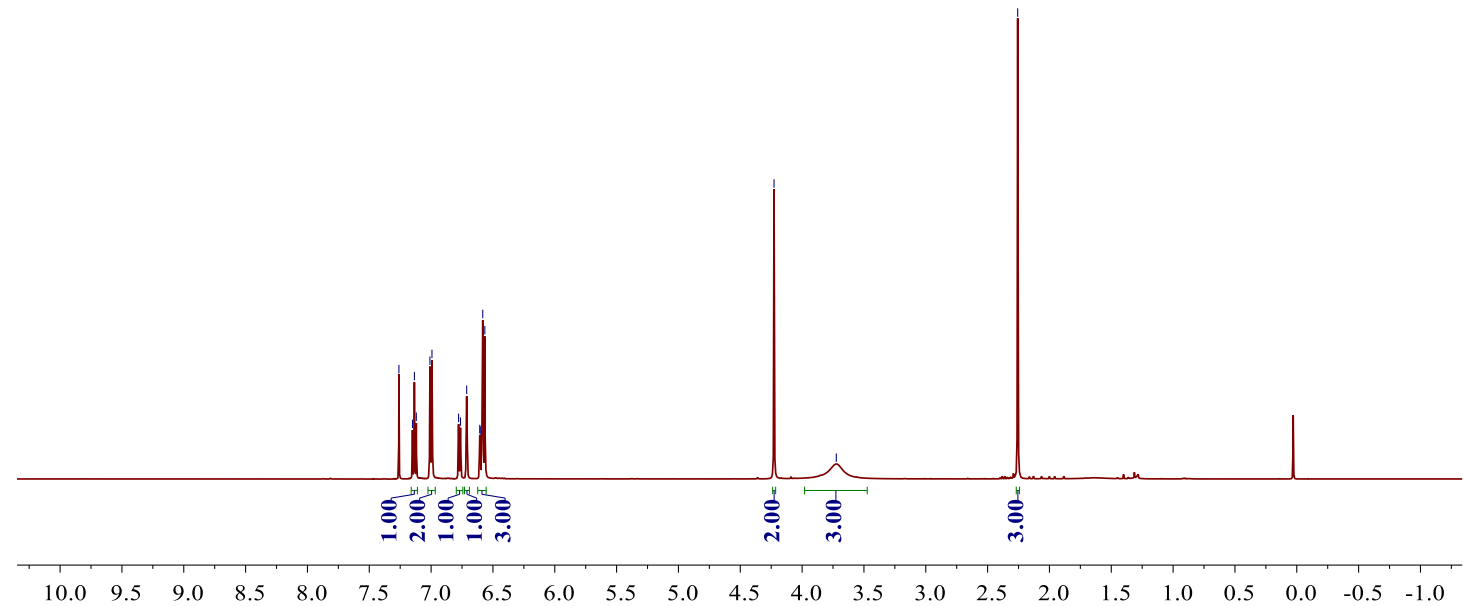


${ }^{13} \mathrm{C} \mathrm{NMR}\left(126 \mathrm{MHz}, \mathrm{CDCl}_{3}\right)$

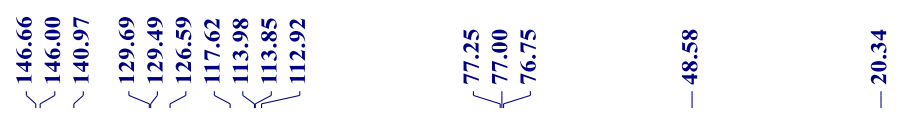<smiles>Cc1ccc(NCc2cccc(N)c2)cc1</smiles>

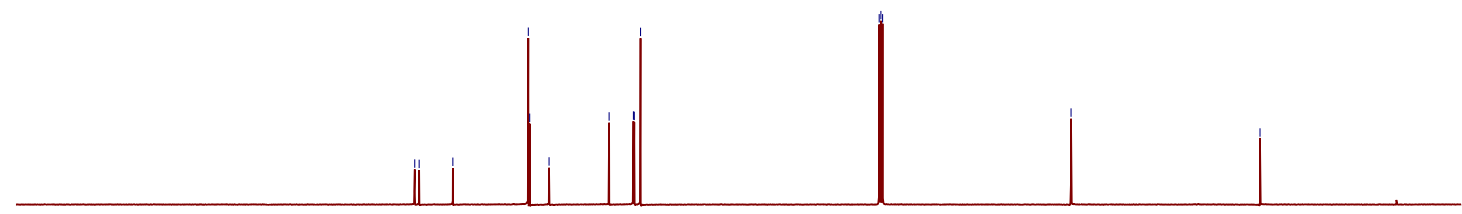

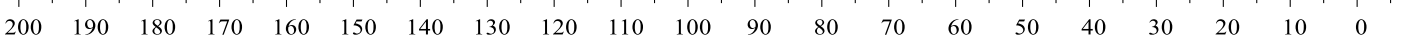

${ }^{1} \mathrm{H} \mathrm{NMR}\left(500 \mathrm{MHz}, \mathrm{CDCl}_{3}\right)$

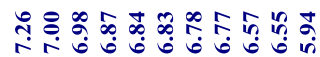

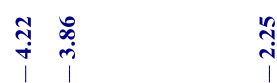

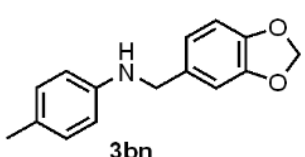

3bn

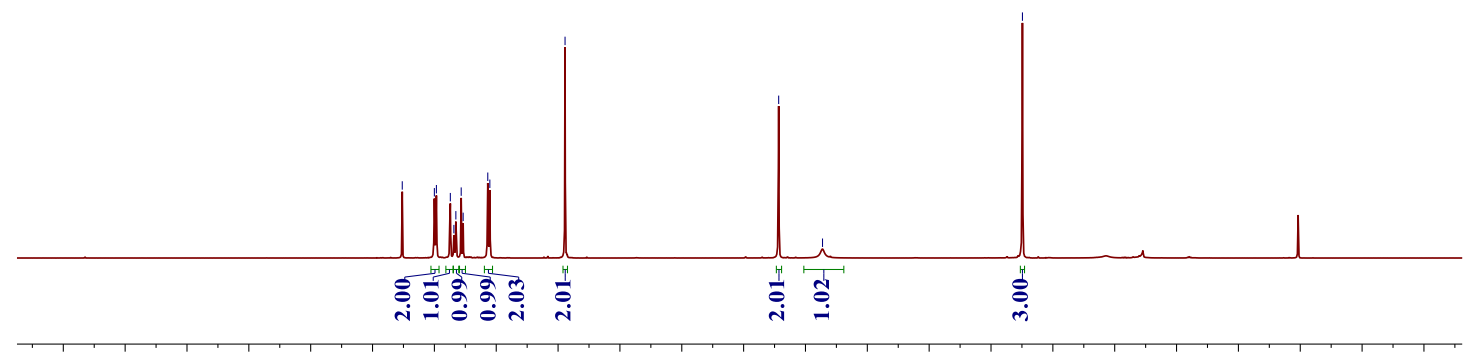

$\begin{array}{llllllllllllllllllllllll}10.0 & 9.5 & 9.0 & 8.5 & 8.0 & 7.5 & 7.0 & 6.5 & 6.0 & 5.5 & 5.0 & 4.5 & 4.0 & 3.5 & 3.0 & 2.5 & 2.0 & 1.5 & 1.0 & 0.5 & 0.0 & -0.5 & -1.0\end{array}$ 
${ }^{13} \mathrm{C}$ NMR (126 MHz, $\mathrm{CDCl}_{3}$ )

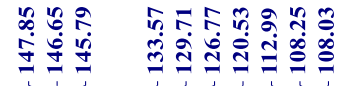

ำ

$\underset{\substack{9 \\ \infty}}{\stackrel{9}{\infty}}$

$\stackrel{\leftrightarrow}{\stackrel{ก}{3}}$<smiles>Cc1ccc(NCc2ccc3c(c2)OCO3)cc1</smiles>

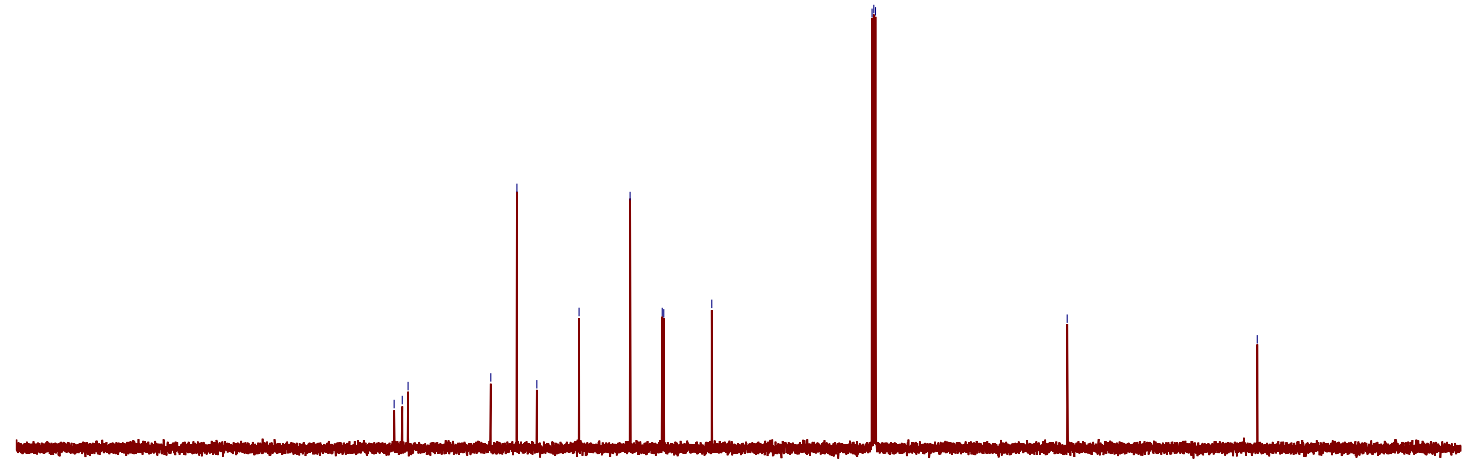

$\begin{array}{lllllllllllllllllllll}200 & 190 & 180 & 170 & 160 & 150 & 140 & 130 & 120 & 110 & 100 & 90 & 80 & 70 & 60 & 50 & 40 & 30 & 20 & 10 & 0\end{array}$

${ }^{1} \mathrm{H} \mathrm{NMR}\left(500 \mathrm{MHz}, \mathrm{CDCl}_{3}\right)$

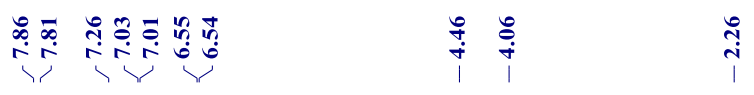<smiles>Cc1ccc(NCc2cc(C(F)(F)F)cc(C(F)(F)F)c2)cc1</smiles>

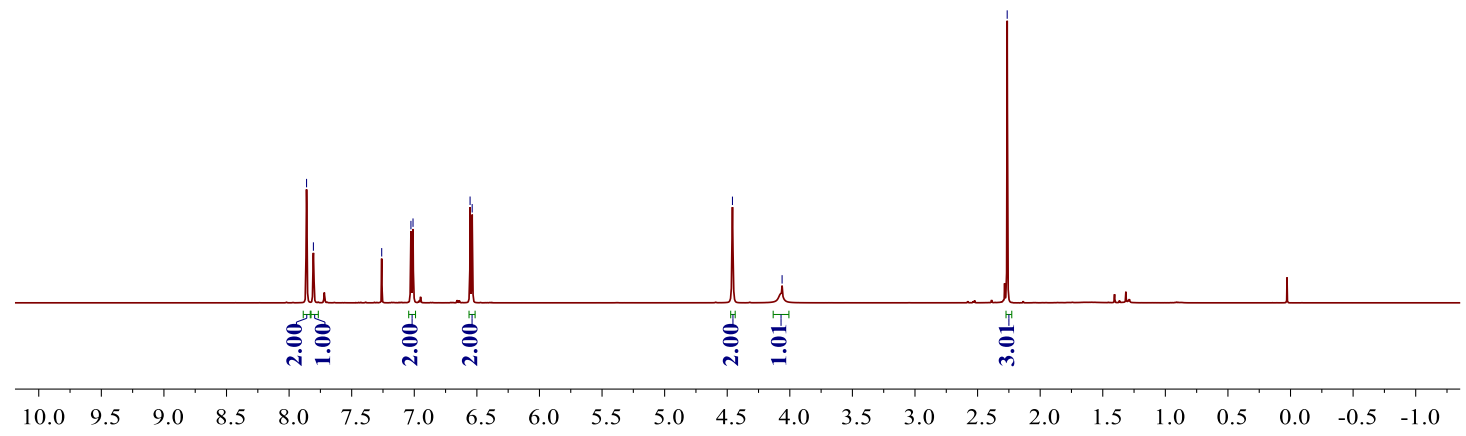


${ }^{13} \mathrm{C}$ NMR (126 MHz, $\left.\mathrm{CDCl}_{3}\right)$

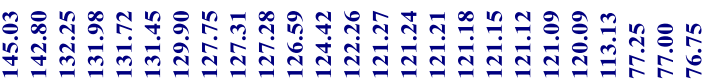

$\underset{\stackrel{\infty}{\infty}}{\stackrel{\infty}{+}}$

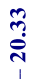<smiles>Cc1ccc(NCc2cc(C(F)(F)F)cc(C(F)(F)F)c2)cc1</smiles>

3bo

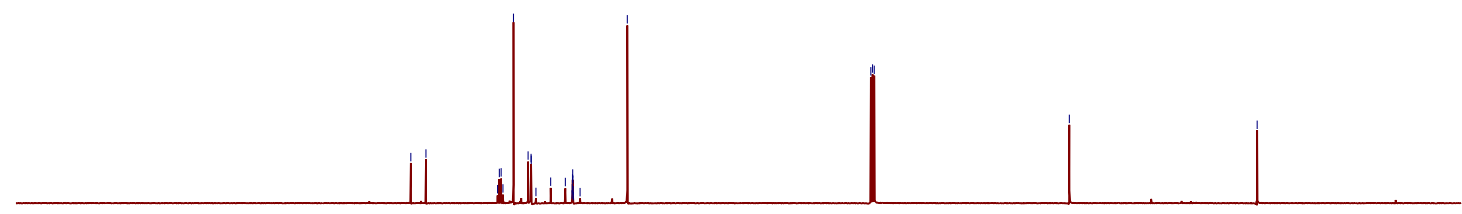

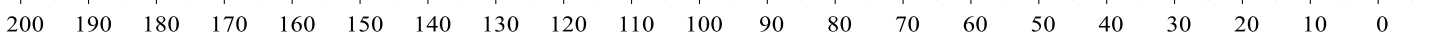

${ }^{19} \mathrm{~F}$ NMR (471 MHz, $\left.\mathrm{CDCl}_{3}\right)$<smiles>Cc1ccc(NCc2cc(C(F)(F)F)cc(C(F)(F)F)c2)cc1</smiles>

iิ

$3 \mathrm{bo}$

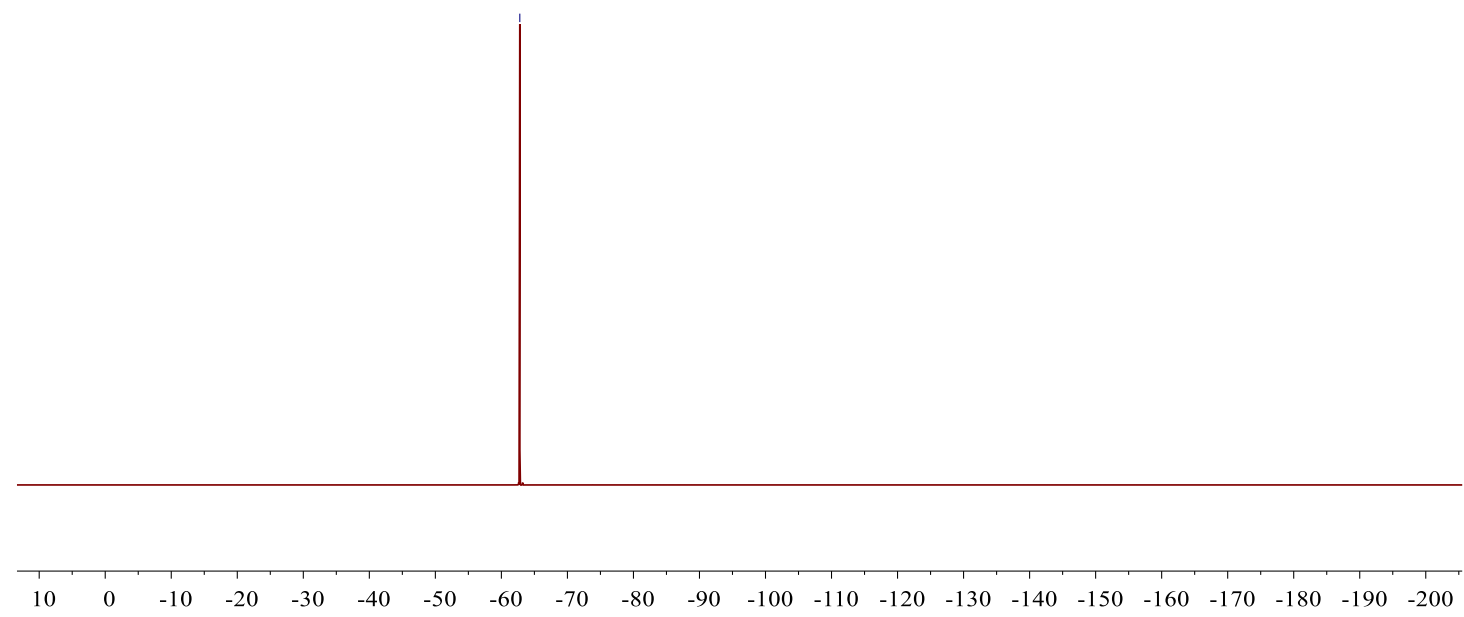


${ }^{1} \mathrm{H}$ NMR (500 MHz, $\left.\mathrm{CDCl}_{3}\right)$

话若

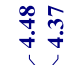
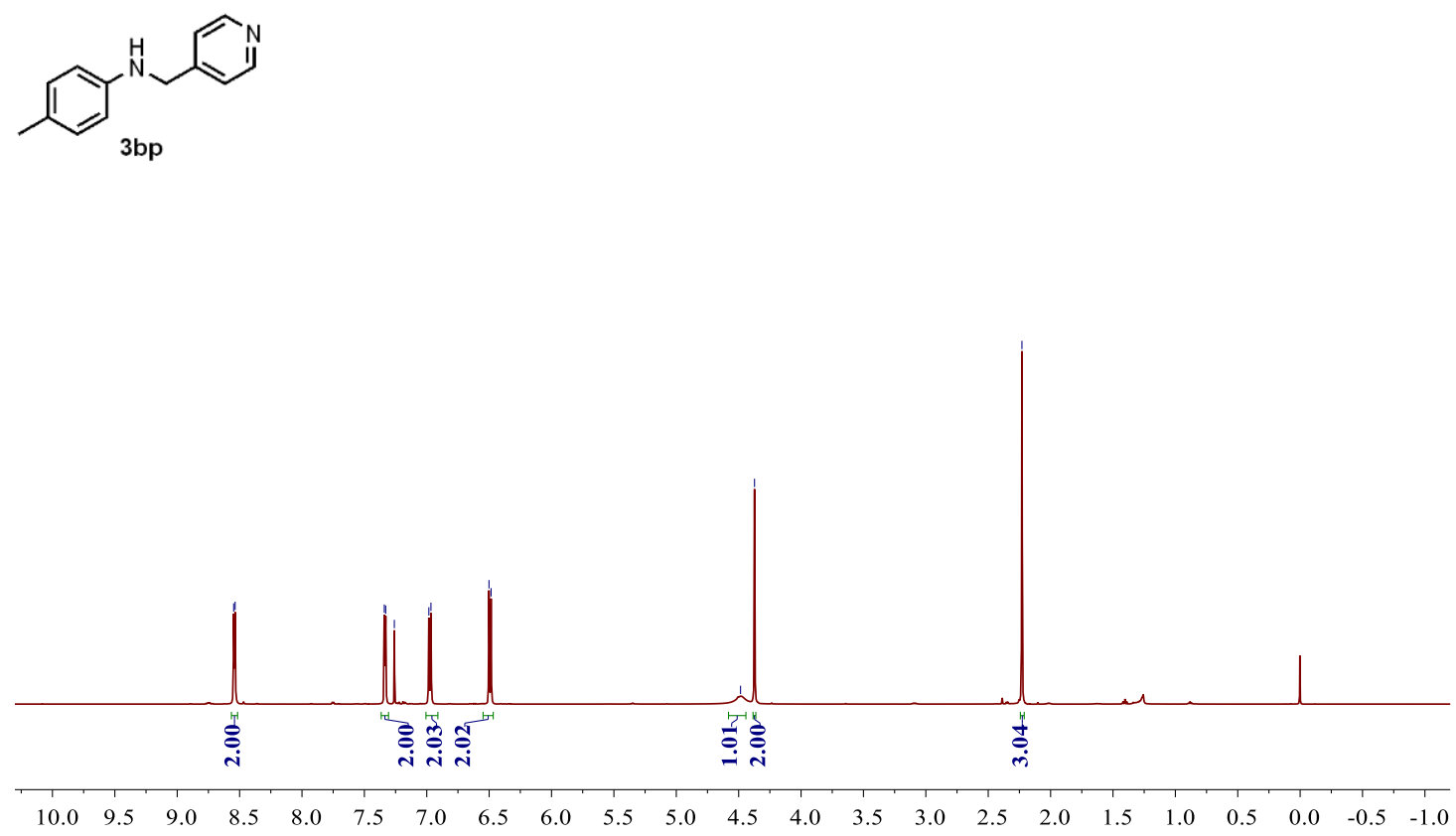

${ }^{13} \mathrm{C} \mathrm{NMR}\left(126 \mathrm{MHz}, \mathrm{CDCl}_{3}\right)$

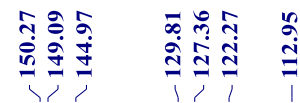

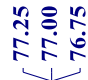

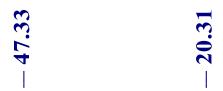<smiles>Cc1ccc(NCc2ccncc2)cc1</smiles>

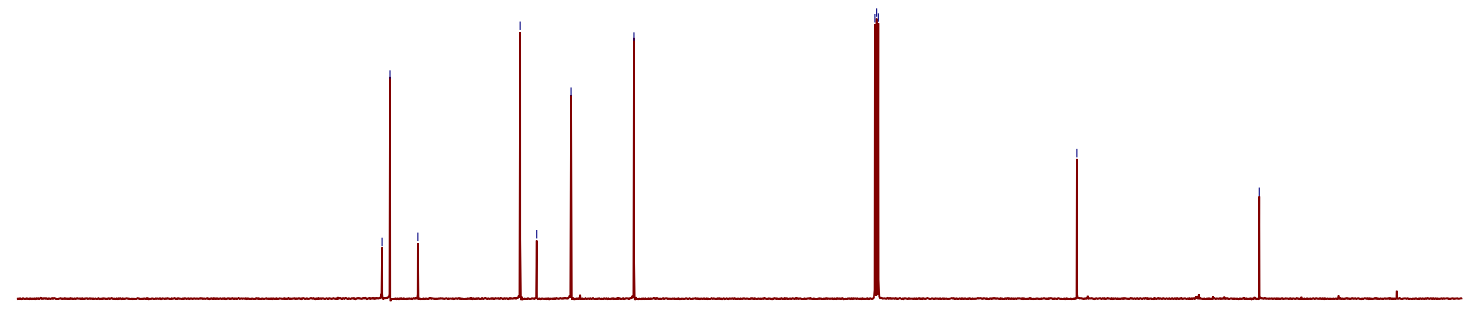

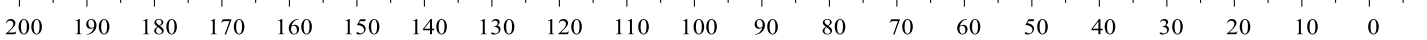


${ }^{1} \mathrm{H}$ NMR $\left(500 \mathrm{MHz}, \mathrm{CDCl}_{3}\right)$

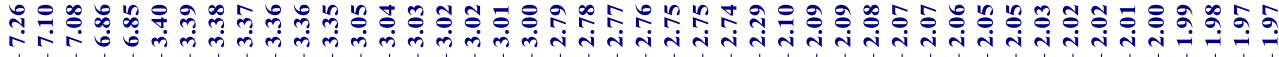<smiles>Cc1ccc(N2CCC(C#N)CC2)cc1</smiles>

$3 b q$

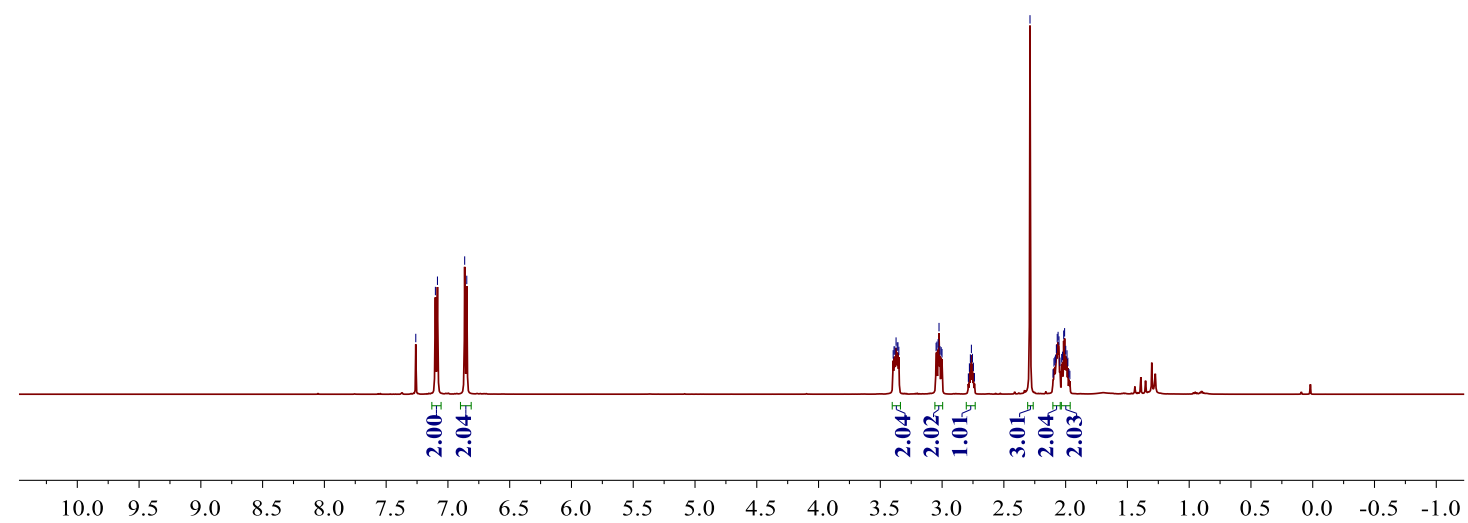

${ }^{13} \mathrm{C} \mathrm{NMR}\left(126 \mathrm{MHz}, \mathrm{CDCl}_{3}\right)$

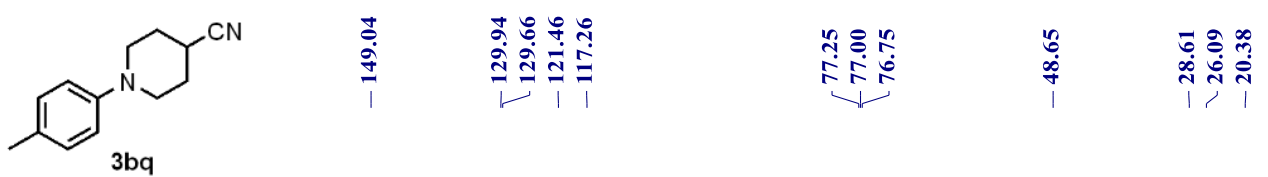

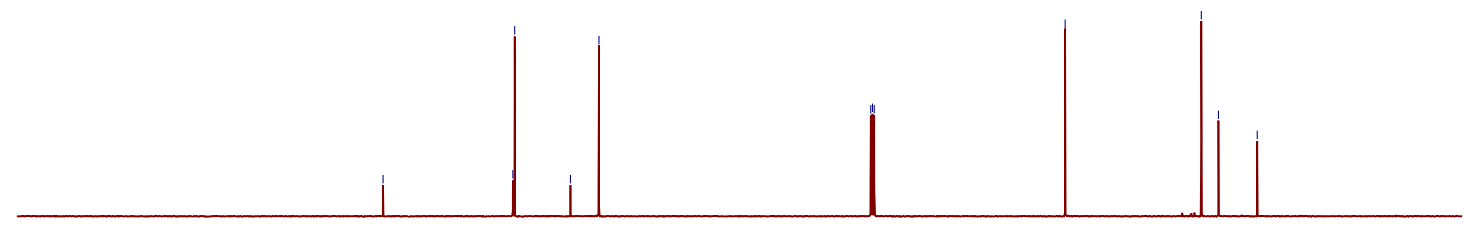

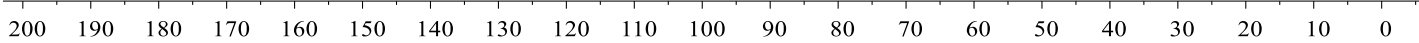


${ }^{1} \mathrm{H}$ NMR $\left(500 \mathrm{MHz}, \mathrm{CDCl}_{3}\right)$

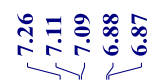

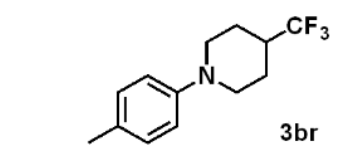

$3 \mathrm{br}$

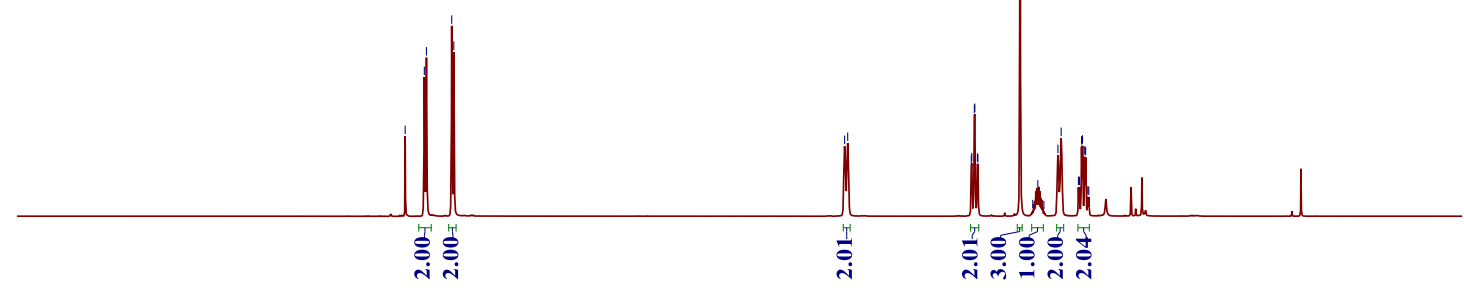

$\begin{array}{lllllllllllllllllllllllllll}10.0 & 9.5 & 9.0 & 8.5 & 8.0 & 7.5 & 7.0 & 6.5 & 6.0 & 5.5 & 5.0 & 4.5 & 4.0 & 3.5 & 3.0 & 2.5 & 2.0 & 1.5 & 1.0 & 0.5 & 0.0 & -0.5 & -1.0\end{array}$

${ }^{13} \mathrm{C} \mathrm{NMR}\left(126 \mathrm{MHz}, \mathrm{CDCl}_{3}\right)$

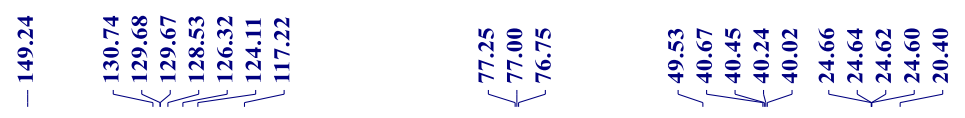<smiles>Cc1ccc(N2CCC(C(C)(F)F)CC2)cc1</smiles>

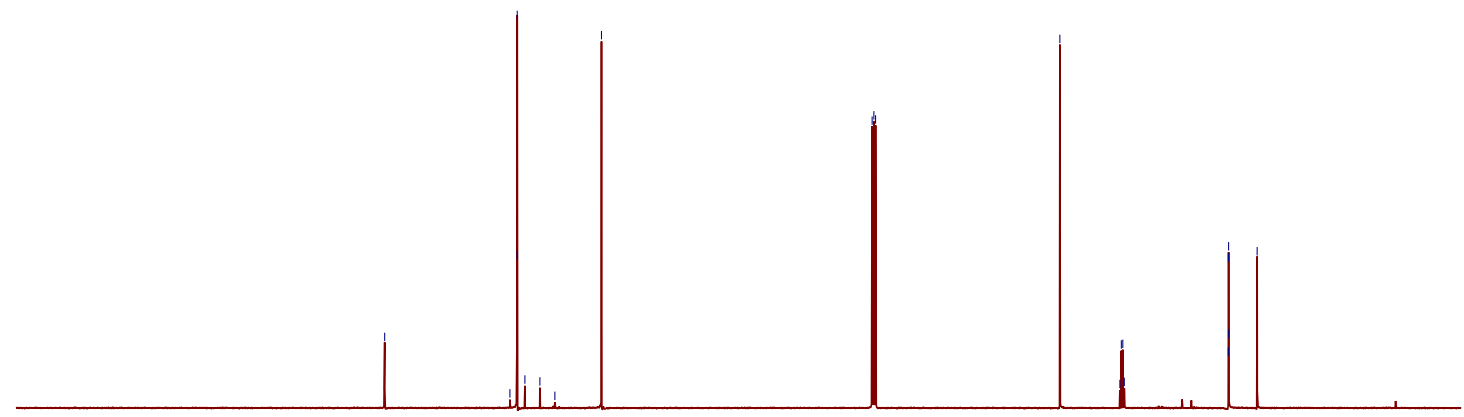

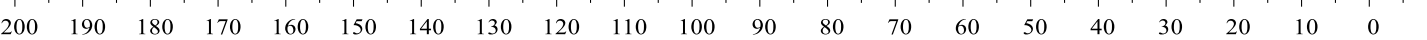


${ }^{19} \mathrm{~F}$ NMR (471 MHz, $\left.\mathrm{CDCl}_{3}\right)$

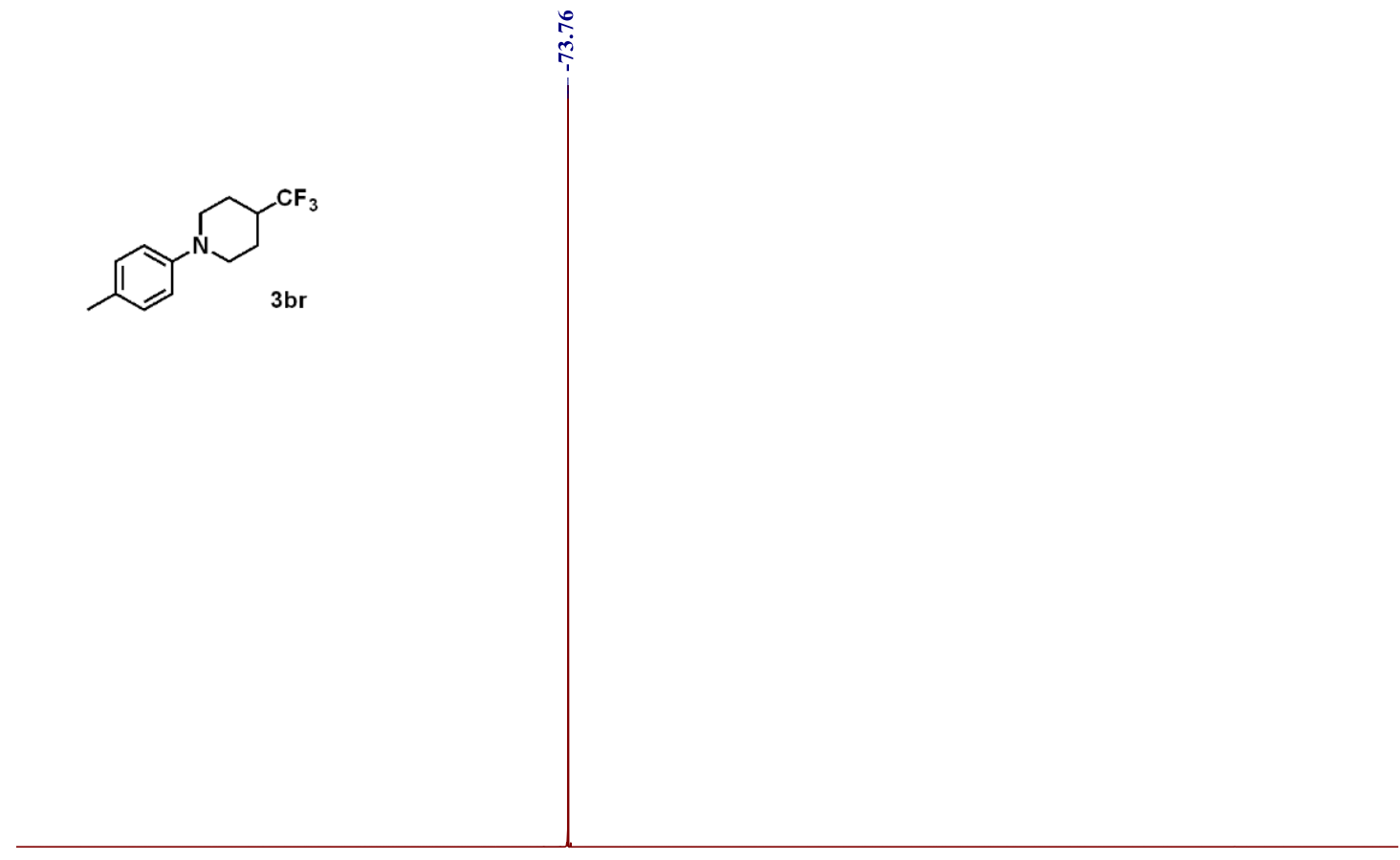

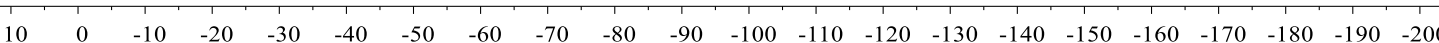

${ }^{1} \mathrm{H}$ NMR $\left(500 \mathrm{MHz}, \mathrm{CDCl}_{3}\right)$

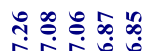

Nivo

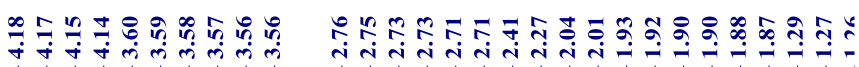<smiles>CCOC(=O)C1CCN(c2ccc(C)cc2)CC1</smiles>

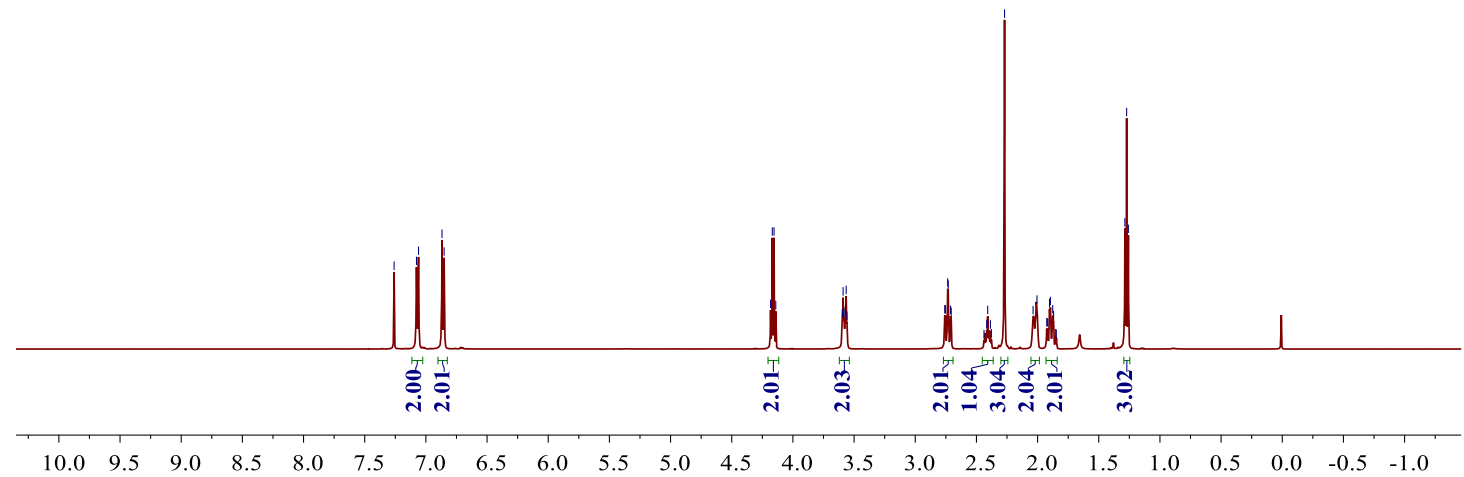


${ }^{13} \mathrm{C}$ NMR (126 MHz, $\left.\mathrm{CDCl}_{3}\right)$

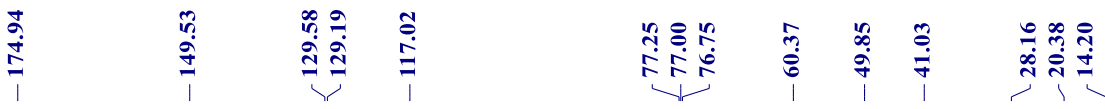<smiles>CCOC1CCN(c2ccc(C)cc2)CC1</smiles>

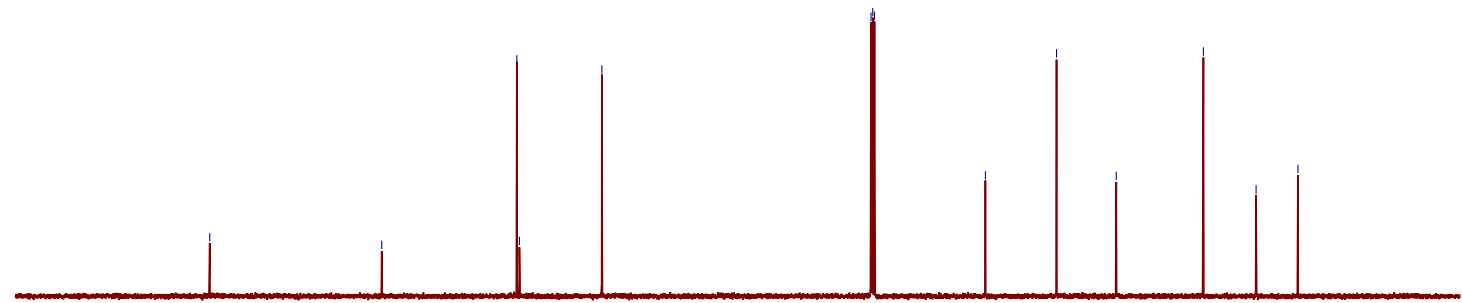

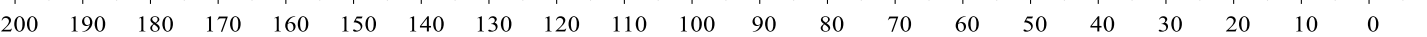

${ }^{1} \mathrm{H} \mathrm{NMR}\left(500 \mathrm{MHz}, \mathrm{CDCl}_{3}\right)$
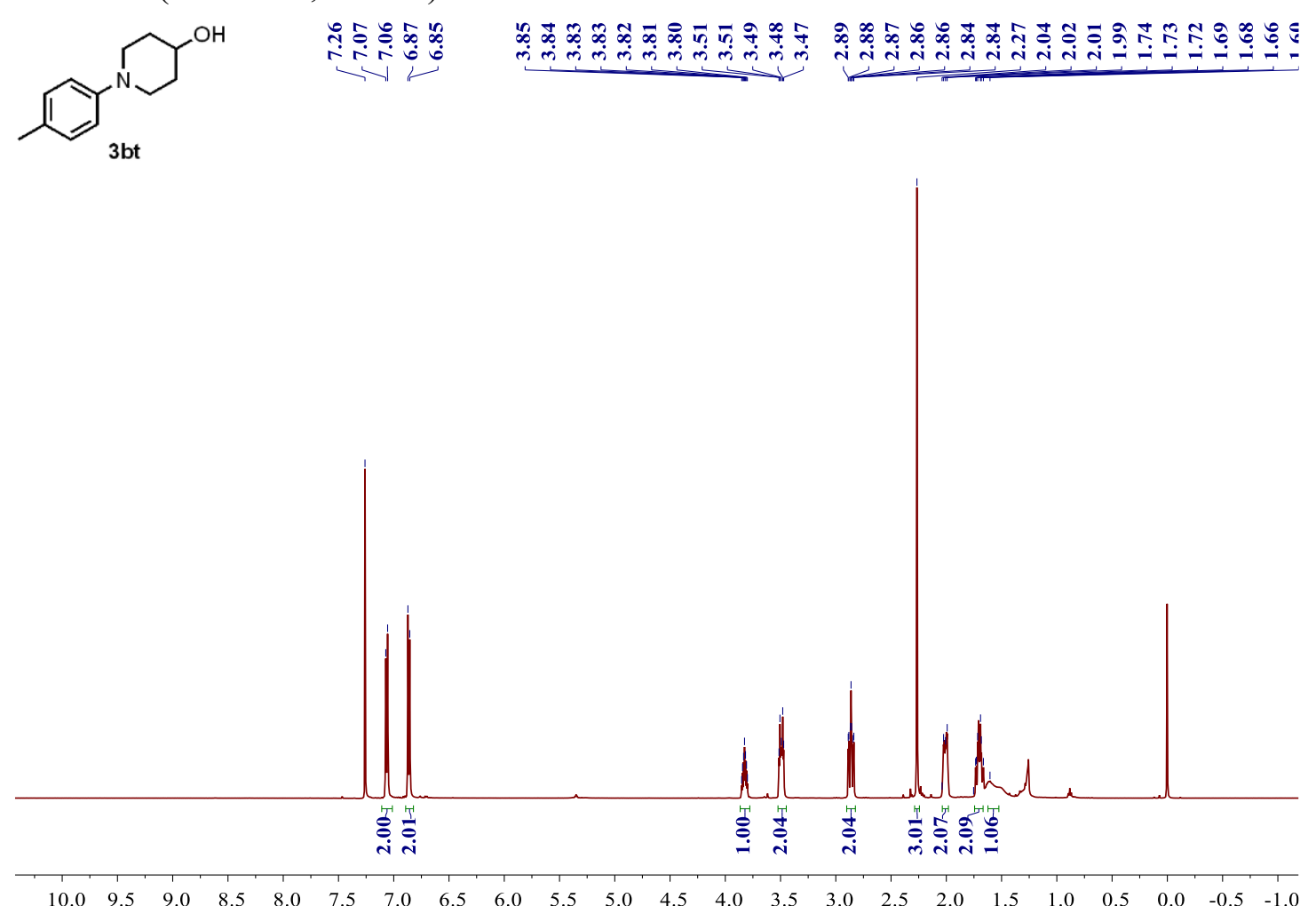
${ }^{13} \mathrm{C} \mathrm{NMR}\left(126 \mathrm{MHz}, \mathrm{CDCl}_{3}\right)$

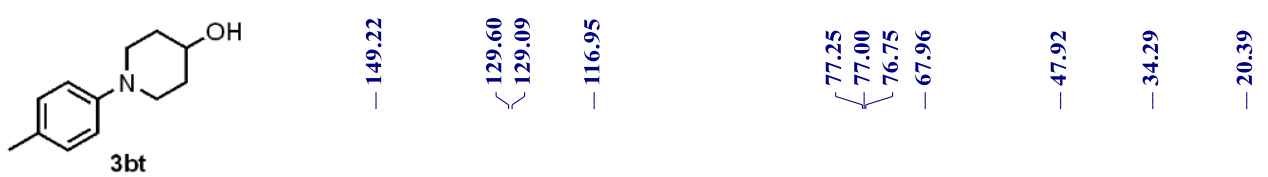

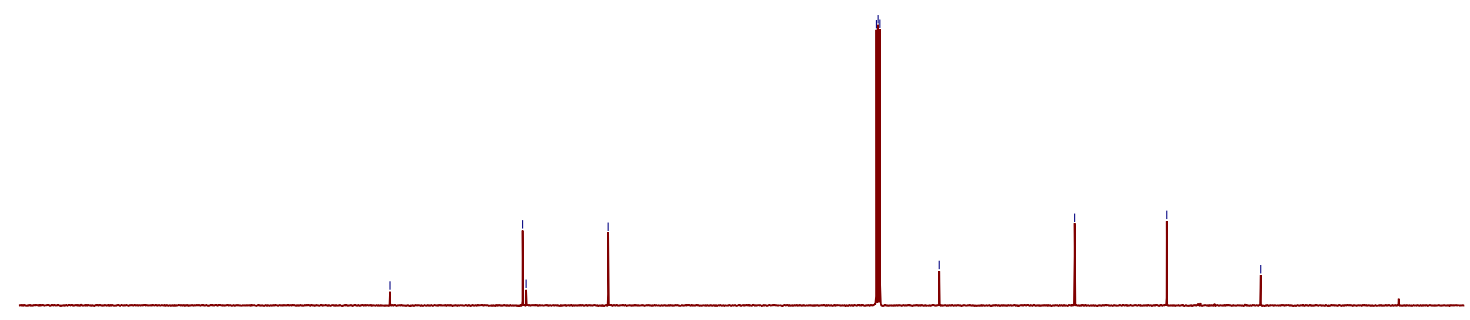

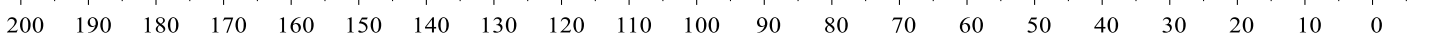

${ }^{1} \mathrm{H} \mathrm{NMR}\left(500 \mathrm{MHz}, \mathrm{CDCl}_{3}\right)$
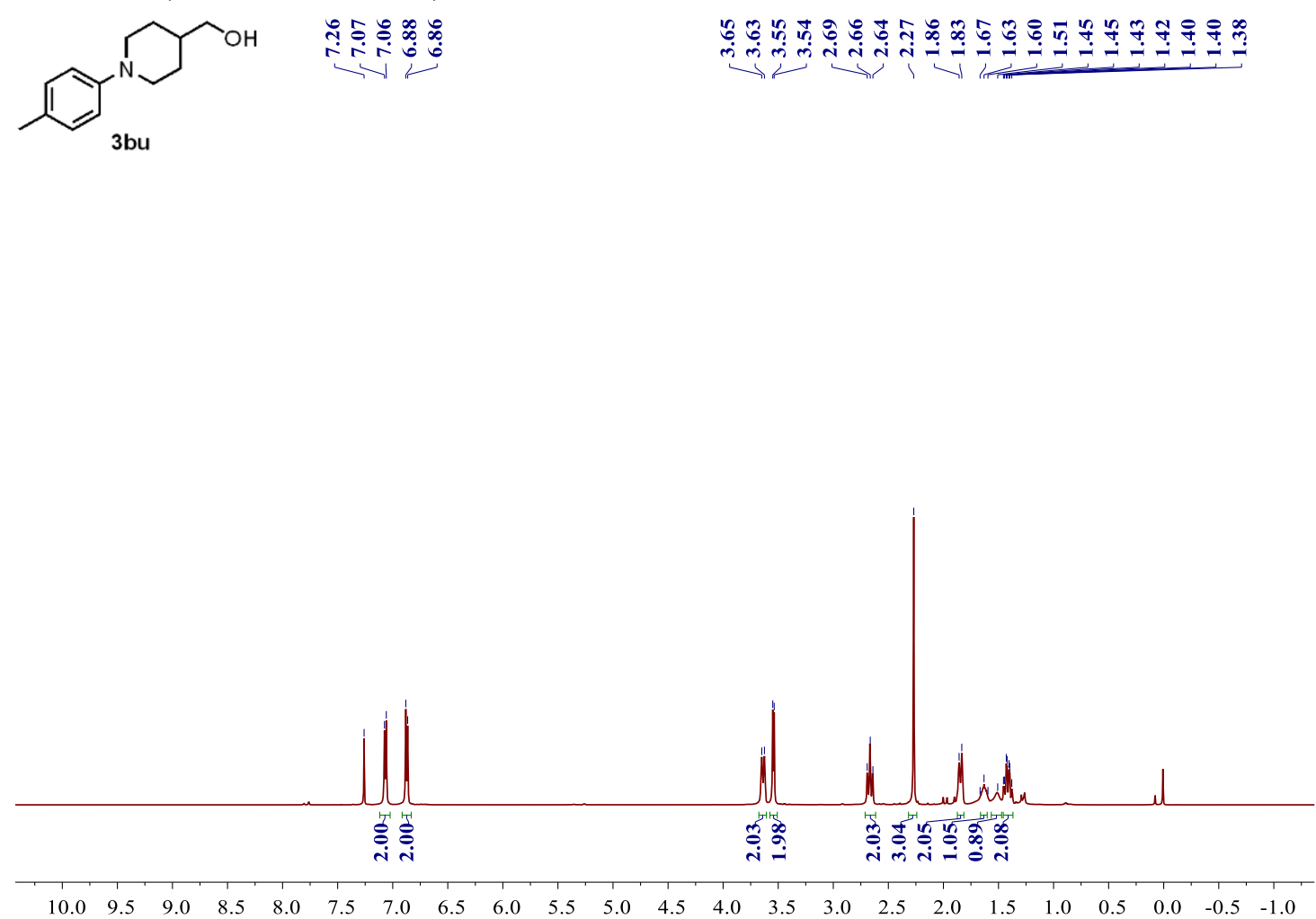
${ }^{13} \mathrm{C}$ NMR $\left(126 \mathrm{MHz}, \mathrm{CDCl}_{3}\right)$

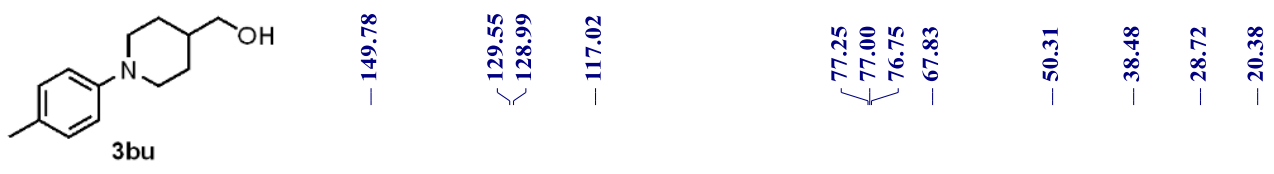

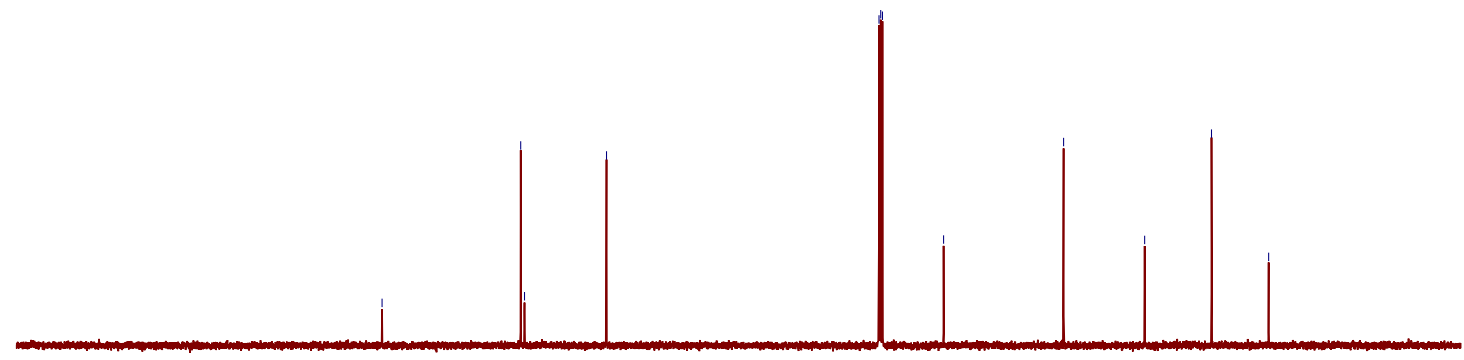

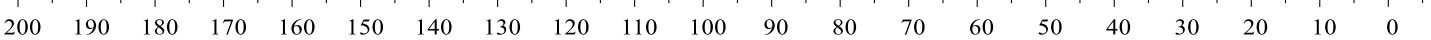

${ }^{1} \mathrm{H} \mathrm{NMR}\left(500 \mathrm{MHz}, \mathrm{CDCl}_{3}\right)$

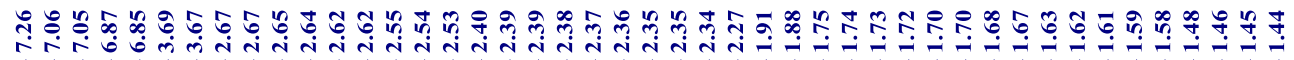<smiles>Cc1ccc(N2CCC(N3CCCC3)CC2)cc1</smiles>

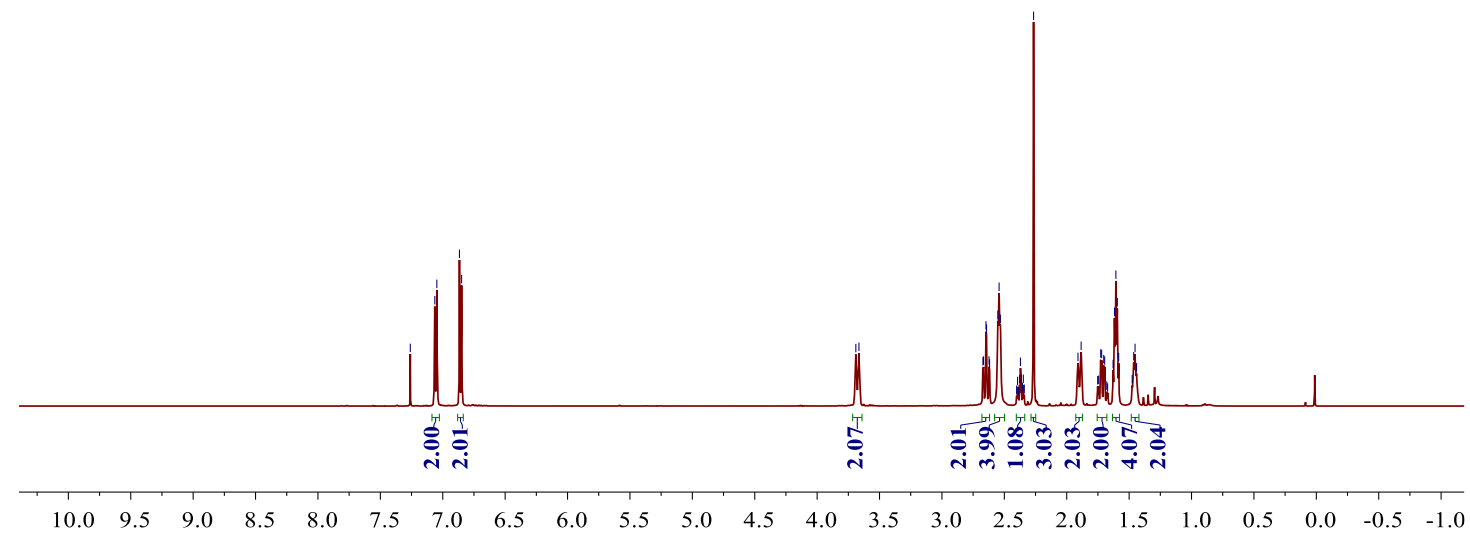


${ }^{13} \mathrm{C} \mathrm{NMR}\left(126 \mathrm{MHz}, \mathrm{CDCl}_{3}\right)$

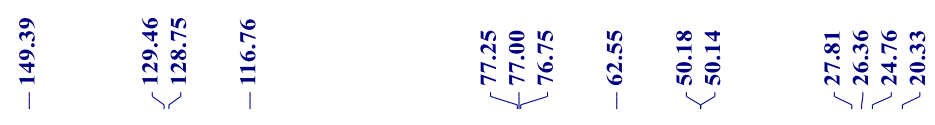<smiles>Cc1ccc(N2CCC(N3CCCCC3)CC2)cc1</smiles>

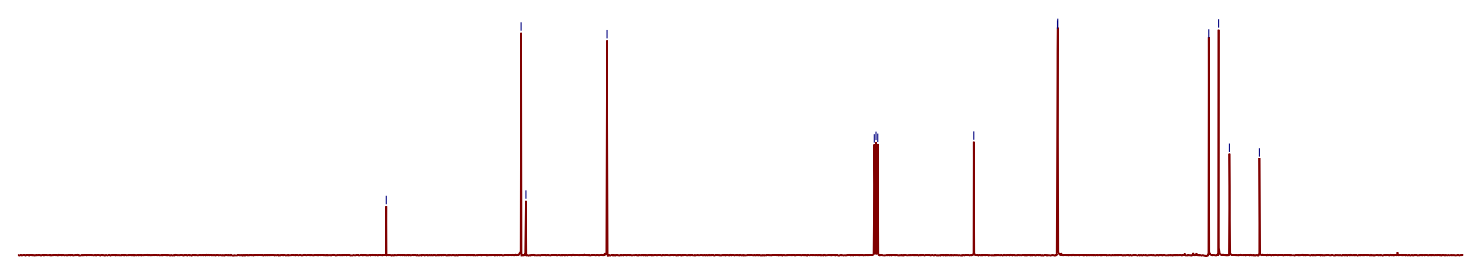

$\begin{array}{lllllllllllllllllllll}200 & 190 & 180 & 170 & 160 & 150 & 140 & 130 & 120 & 110 & 100 & 90 & 80 & 70 & 60 & 50 & 40 & 30 & 20 & 10 & 0\end{array}$

${ }^{1} \mathrm{H} \mathrm{NMR}\left(500 \mathrm{MHz}, \mathrm{CDCl}_{3}\right)$

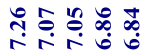

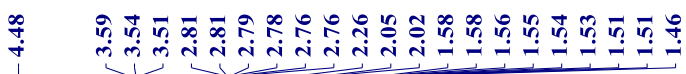
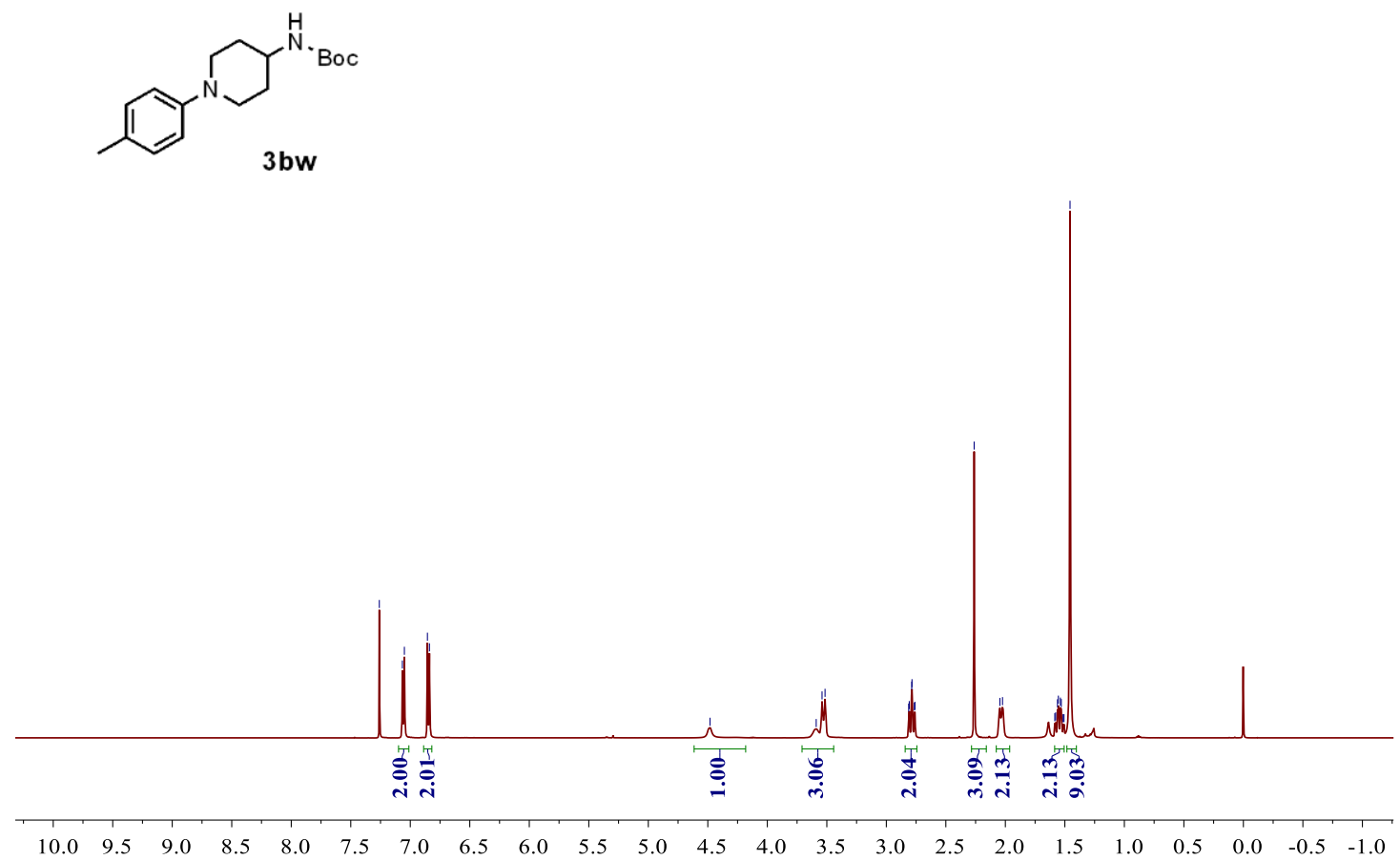
${ }^{13} \mathrm{C}$ NMR (126 MHz, $\left.\mathrm{CDCl}_{3}\right)$

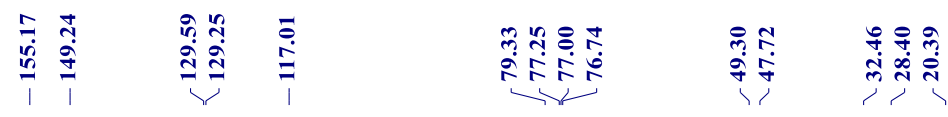<smiles>Cc1ccc(N2CCC(NC(=O)OCc3ccccc3)CC2)cc1</smiles>

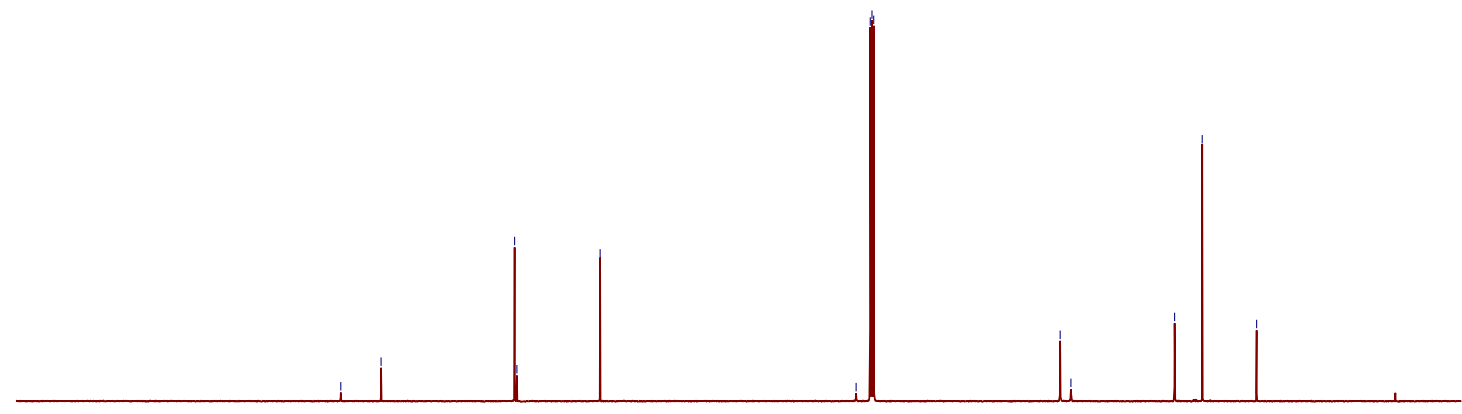

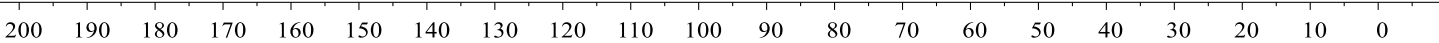

${ }^{1} \mathrm{H} \mathrm{NMR}\left(500 \mathrm{MHz}, \mathrm{CDCl}_{3}\right)$

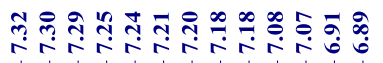

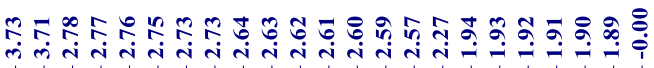<smiles>Cc1ccc(N2CCC(c3ccccc3)CC2)cc1</smiles>

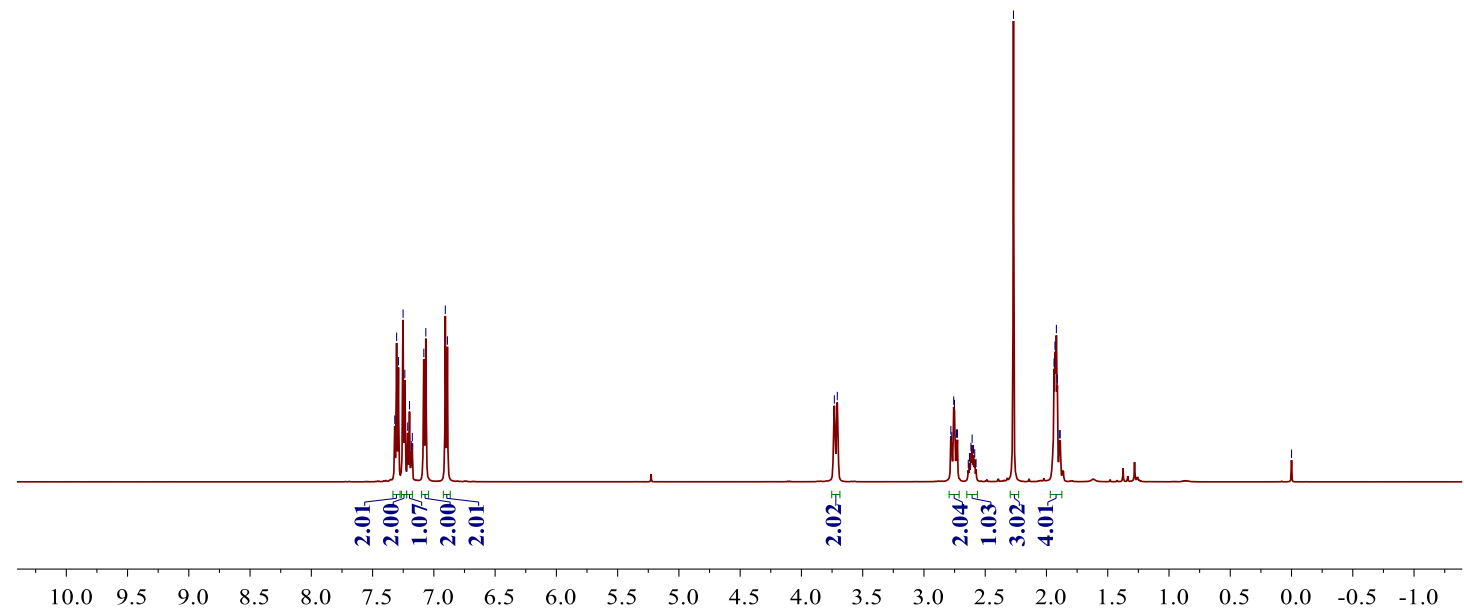


${ }^{13} \mathrm{C}$ NMR (126 MHz, $\left.\mathrm{CDCl}_{3}\right)$
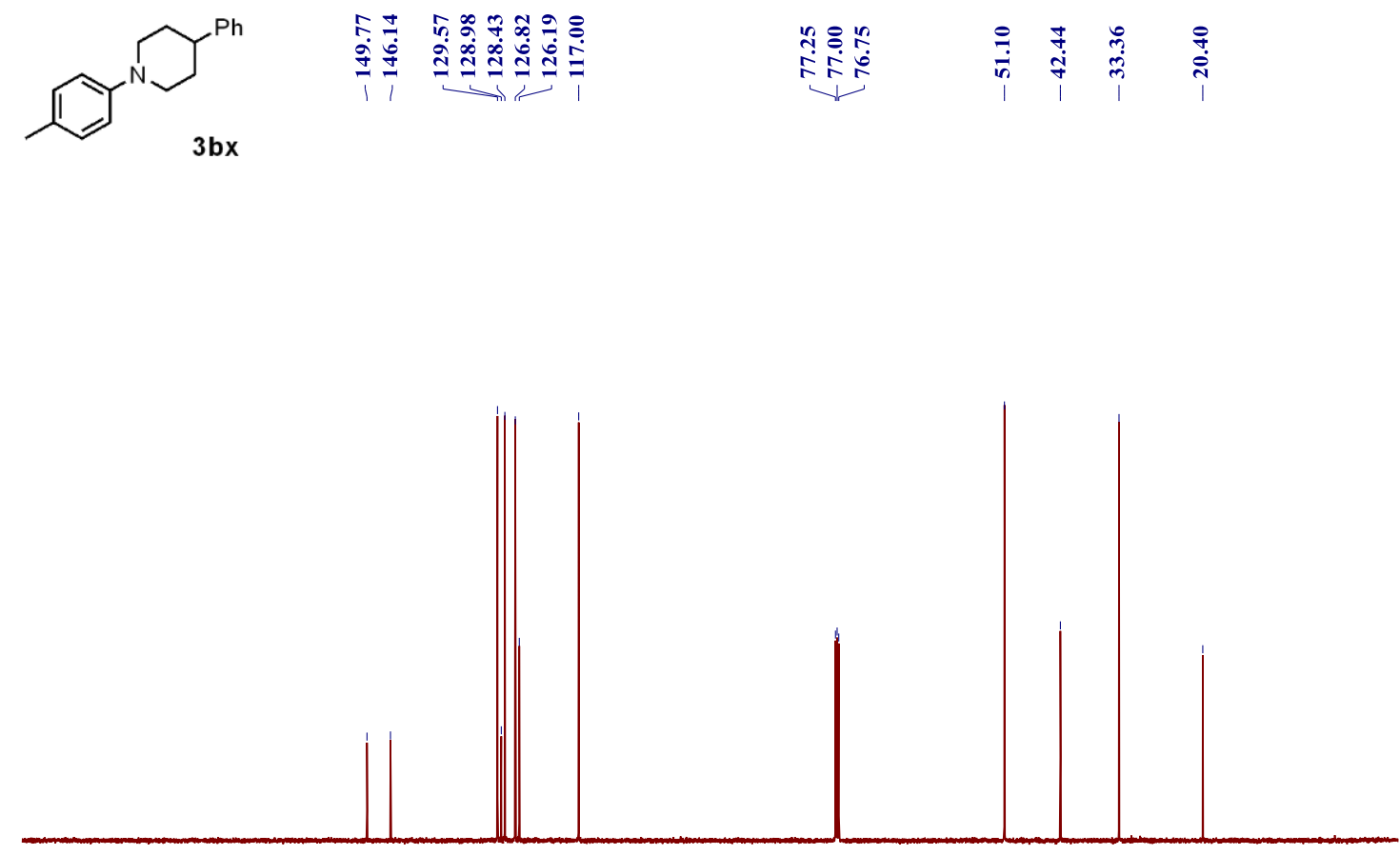

$\begin{array}{lllllllllllllllllllll}200 & 190 & 180 & 170 & 160 & 150 & 140 & 130 & 120 & 110 & 100 & 90 & 80 & 70 & 60 & 50 & 40 & 30 & 20 & 10 & 0\end{array}$

${ }^{1} \mathrm{H} \mathrm{NMR}\left(500 \mathrm{MHz}, \mathrm{CDCl}_{3}\right)$

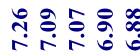

낭

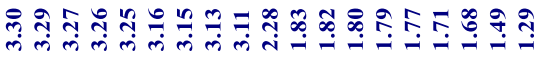
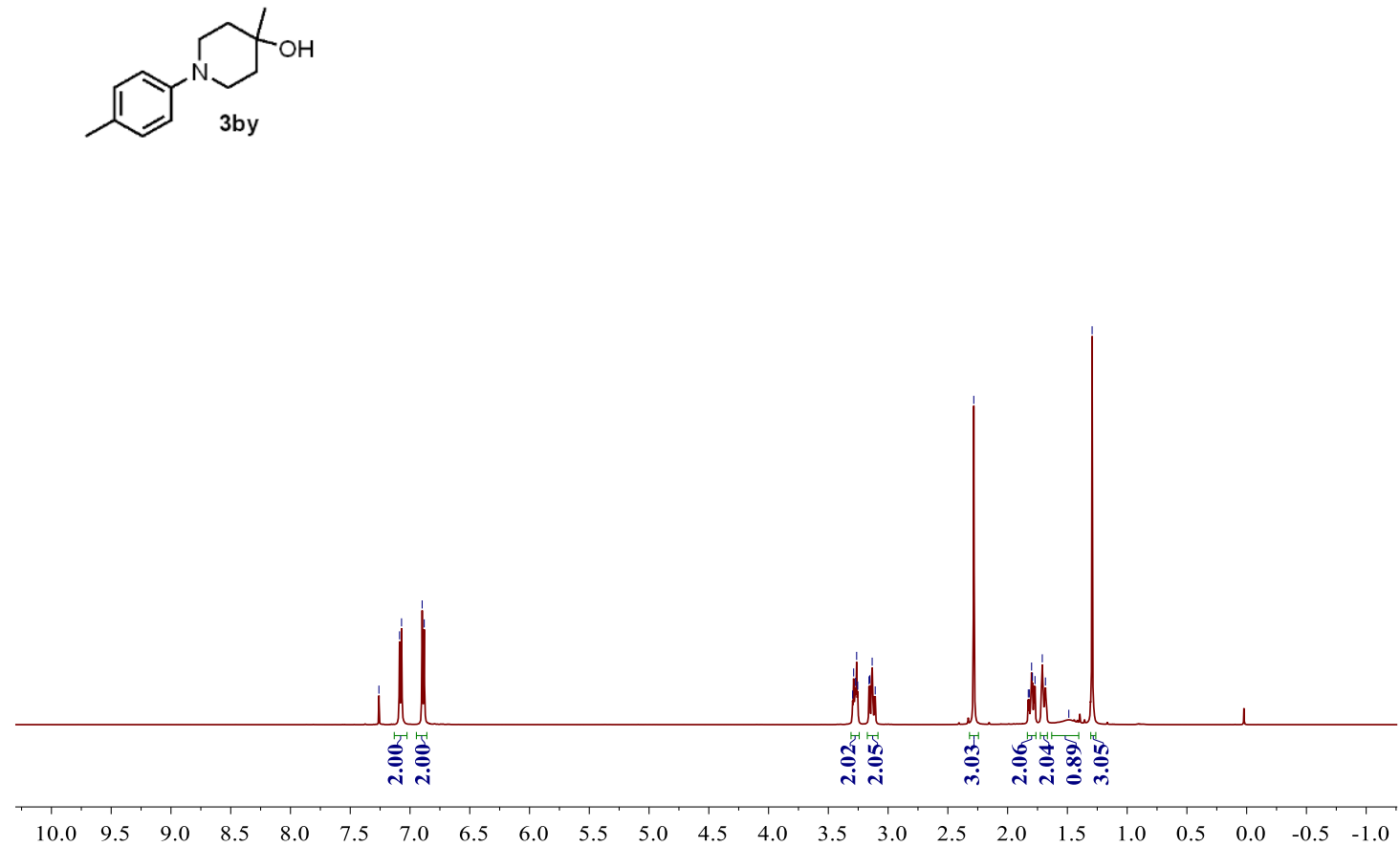
${ }^{13} \mathrm{C}$ NMR $\left(126 \mathrm{MHz}, \mathrm{CDCl}_{3}\right)$

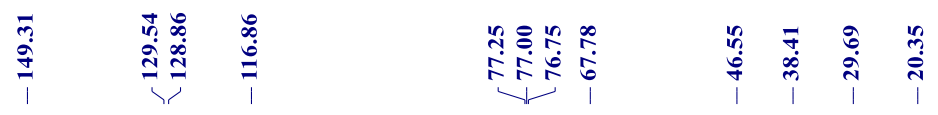<smiles>Cc1ccc(N2CCC(C)(O)C2)cc1</smiles>

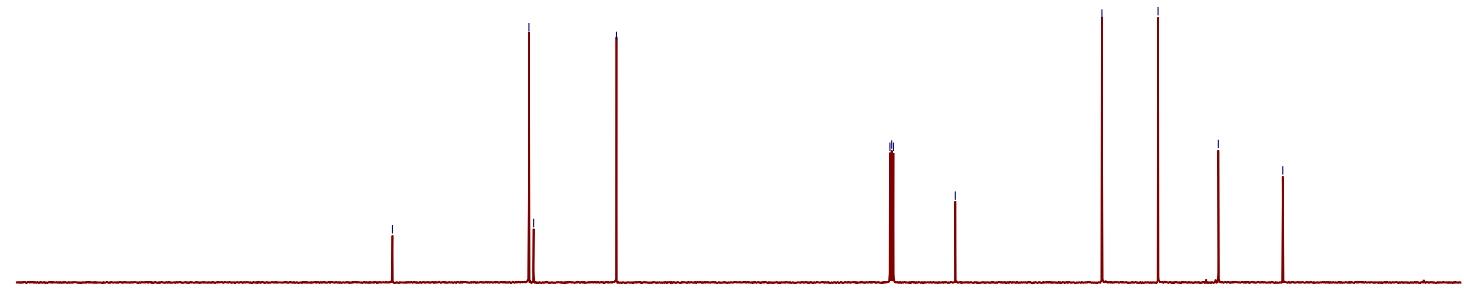

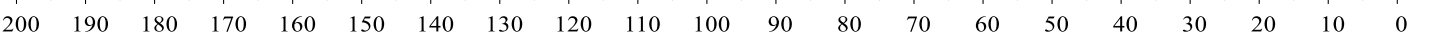

${ }^{1} \mathrm{H} \mathrm{NMR}\left(500 \mathrm{MHz}, \mathrm{CDCl}_{3}\right)$

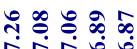

芒
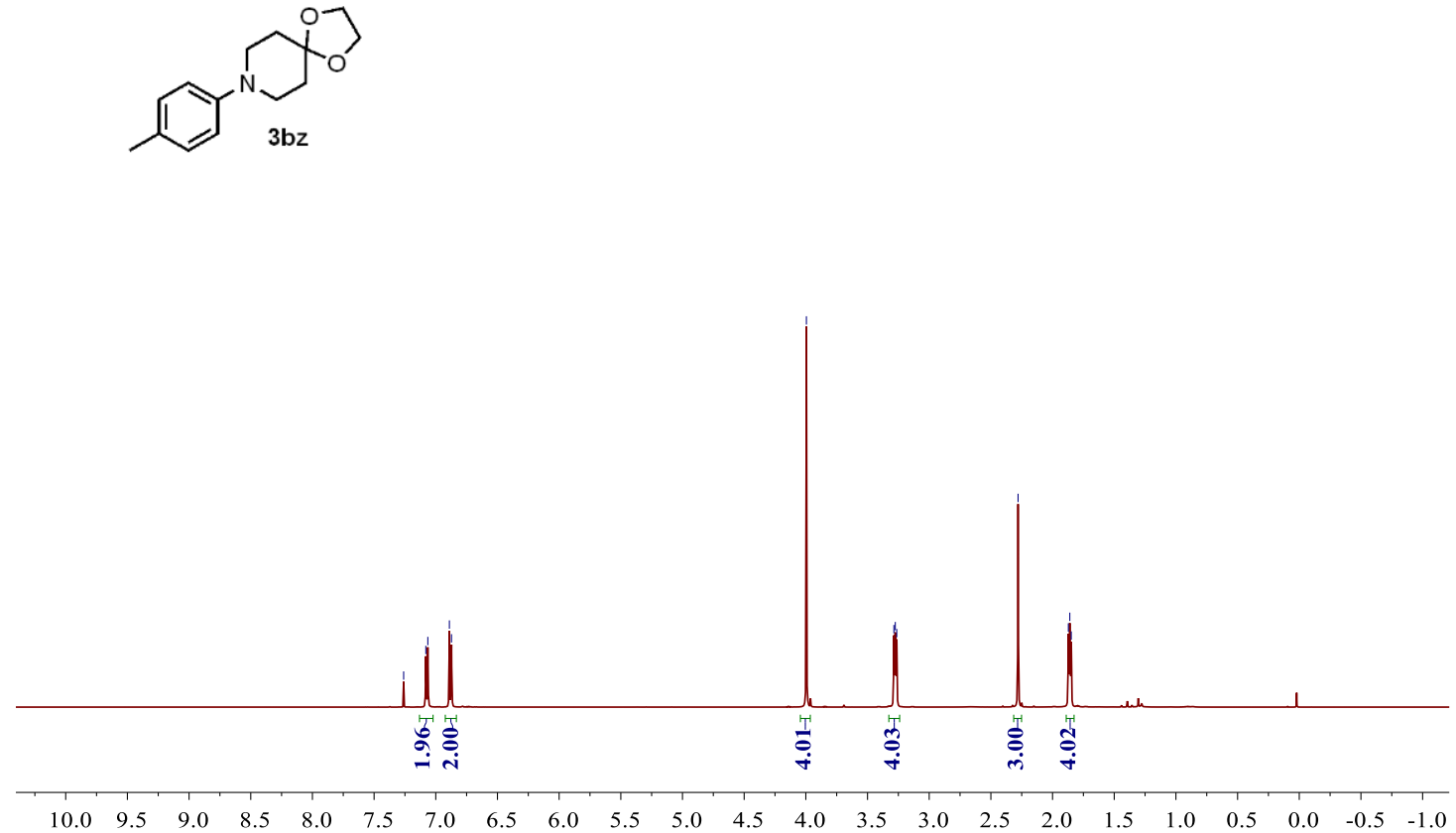
${ }^{13} \mathrm{C} \mathrm{NMR}\left(126 \mathrm{MHz}, \mathrm{CDCl}_{3}\right)$

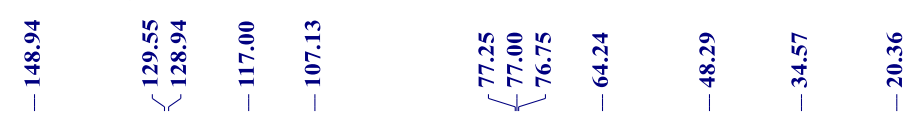<smiles>Cc1ccc(N2CCC3(CC2)OCCO3)cc1</smiles>

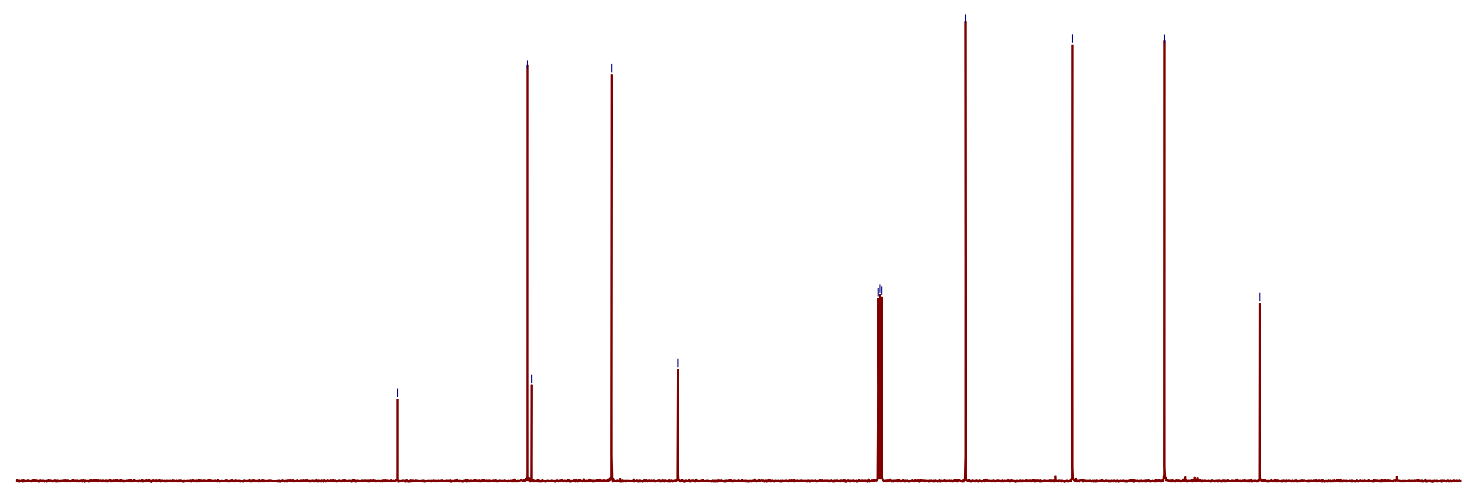

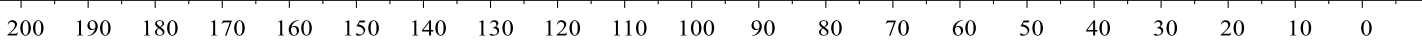

${ }^{1} \mathrm{H}$ NMR $\left(500 \mathrm{MHz}, \mathrm{CDCl}_{3}\right)$

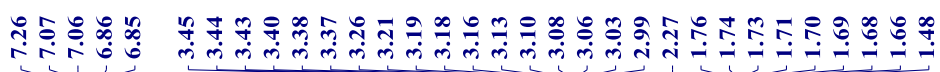
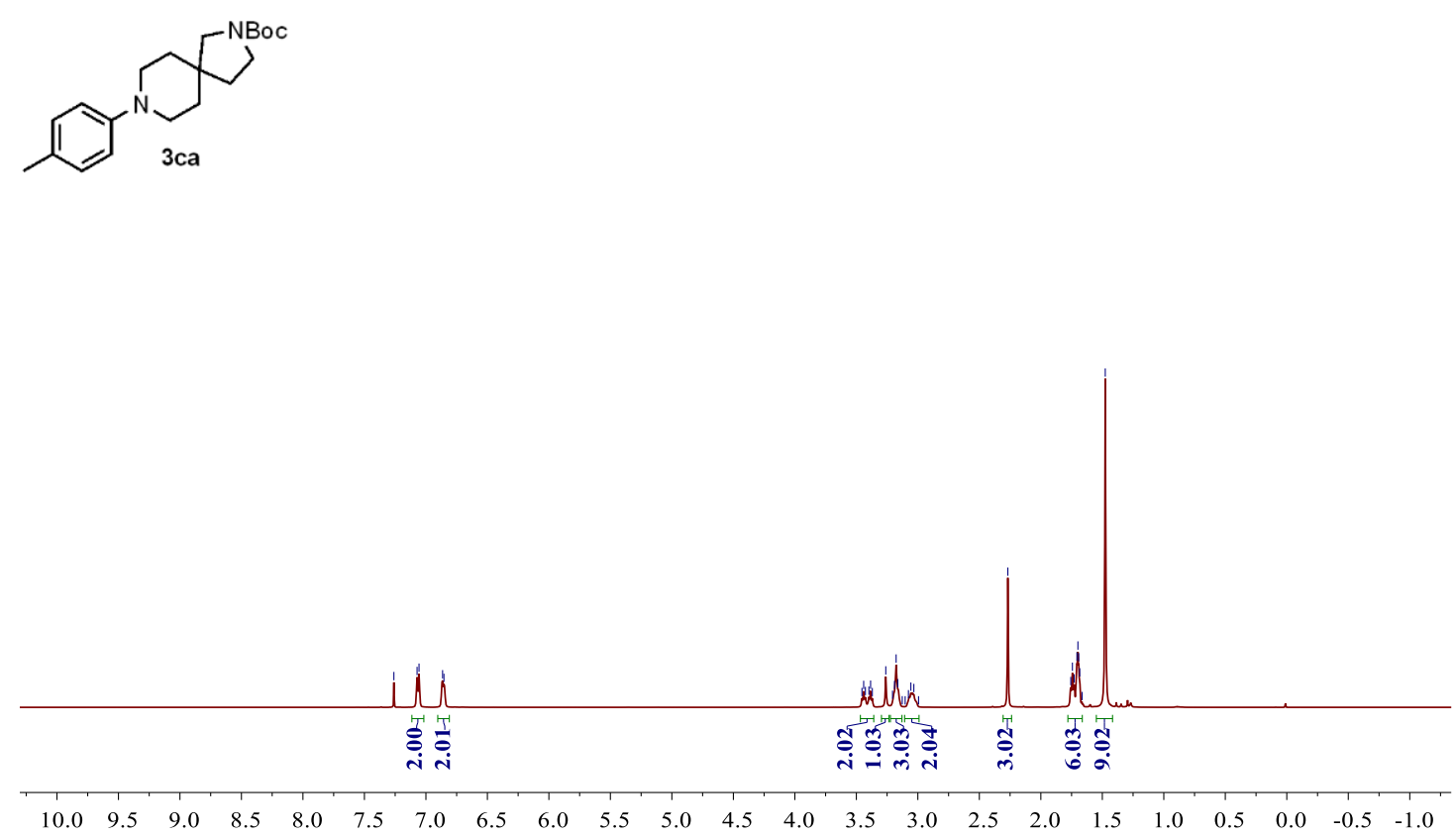
${ }^{13} \mathrm{C} \mathrm{NMR}\left(126 \mathrm{MHz}, \mathrm{CDCl}_{3}\right)$

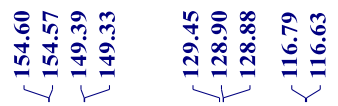

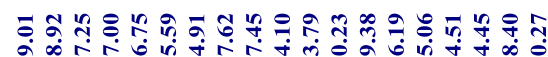
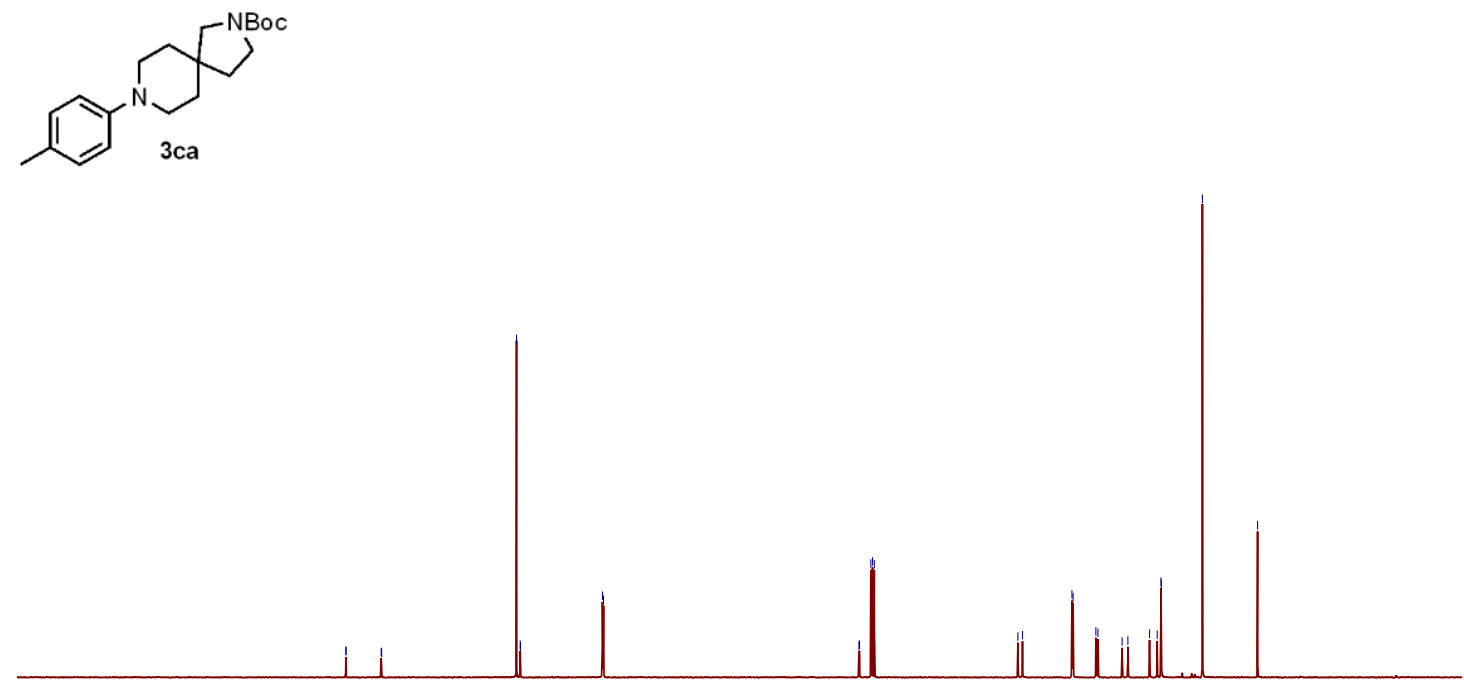

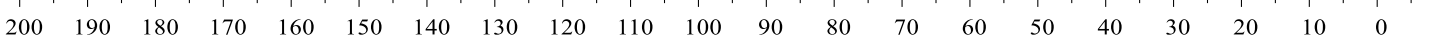

${ }^{1} \mathrm{H} \mathrm{NMR}\left(500 \mathrm{MHz}, \mathrm{CDCl}_{3}\right)$

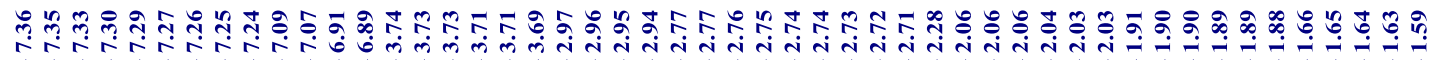<smiles>Cc1ccc(N2CCCC(c3ccccc3)C2)cc1</smiles>

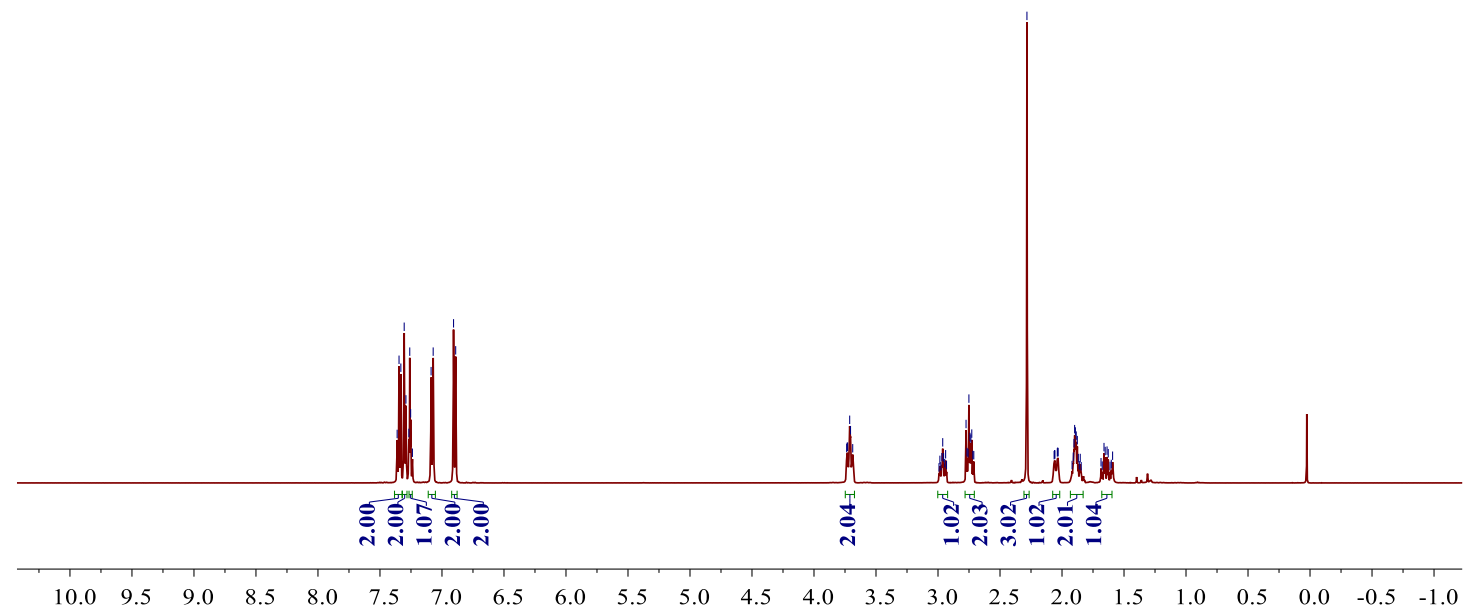


${ }^{13} \mathrm{C} \mathrm{NMR}\left(126 \mathrm{MHz}, \mathrm{CDCl}_{3}\right)$

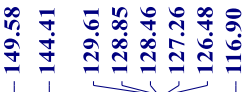

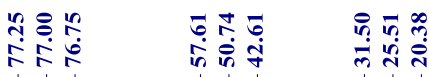<smiles>Cc1ccc(N2CCCC(c3ccccc3)C2)cc1</smiles>

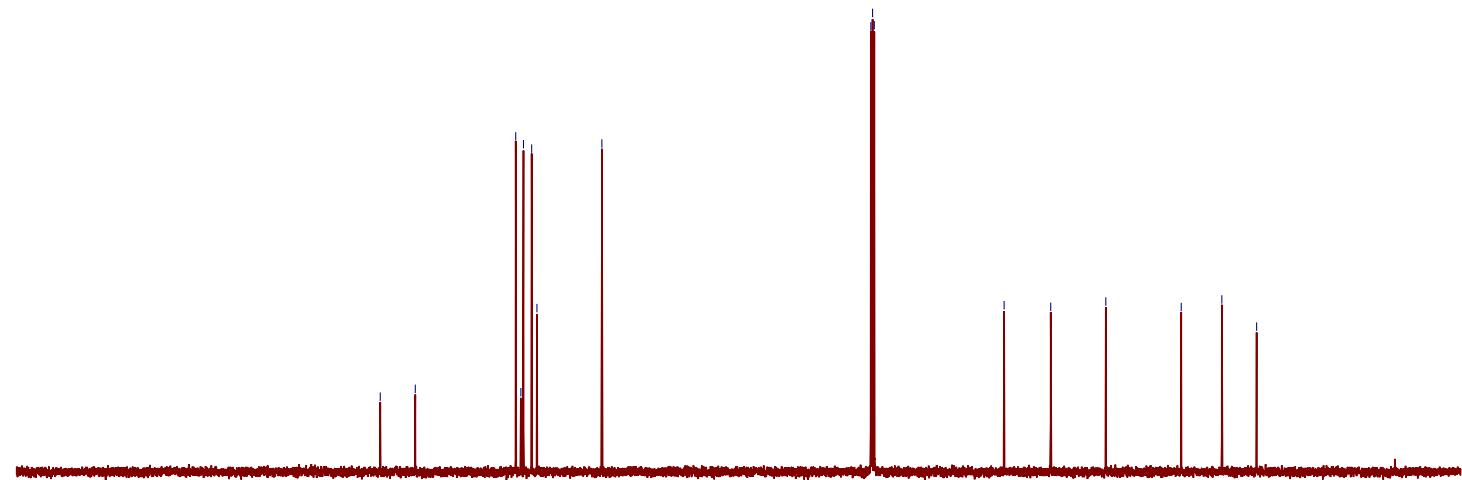

$\begin{array}{lllllllllllllllllllll}200 & 190 & 180 & 170 & 160 & 150 & 140 & 130 & 120 & 110 & 100 & 90 & 80 & 70 & 60 & 50 & 40 & 30 & 20 & 10 & 0\end{array}$

${ }^{1} \mathrm{H} \mathrm{NMR}\left(500 \mathrm{MHz}, \mathrm{CDCl}_{3}\right)$

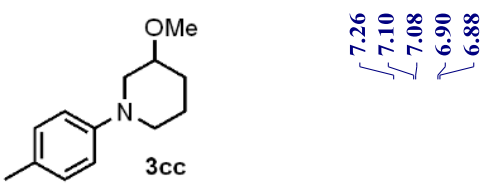

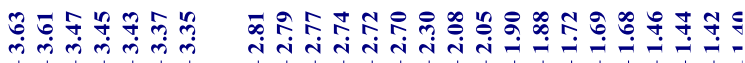

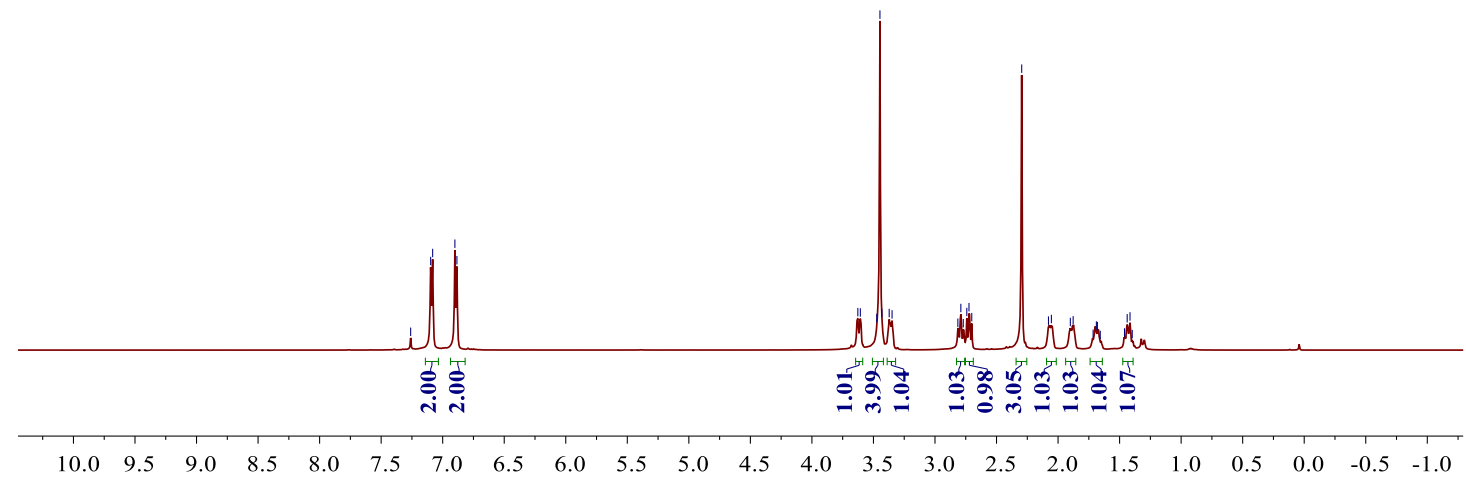


${ }^{13} \mathrm{C}$ NMR (126 MHz, $\left.\mathrm{CDCl}_{3}\right)$
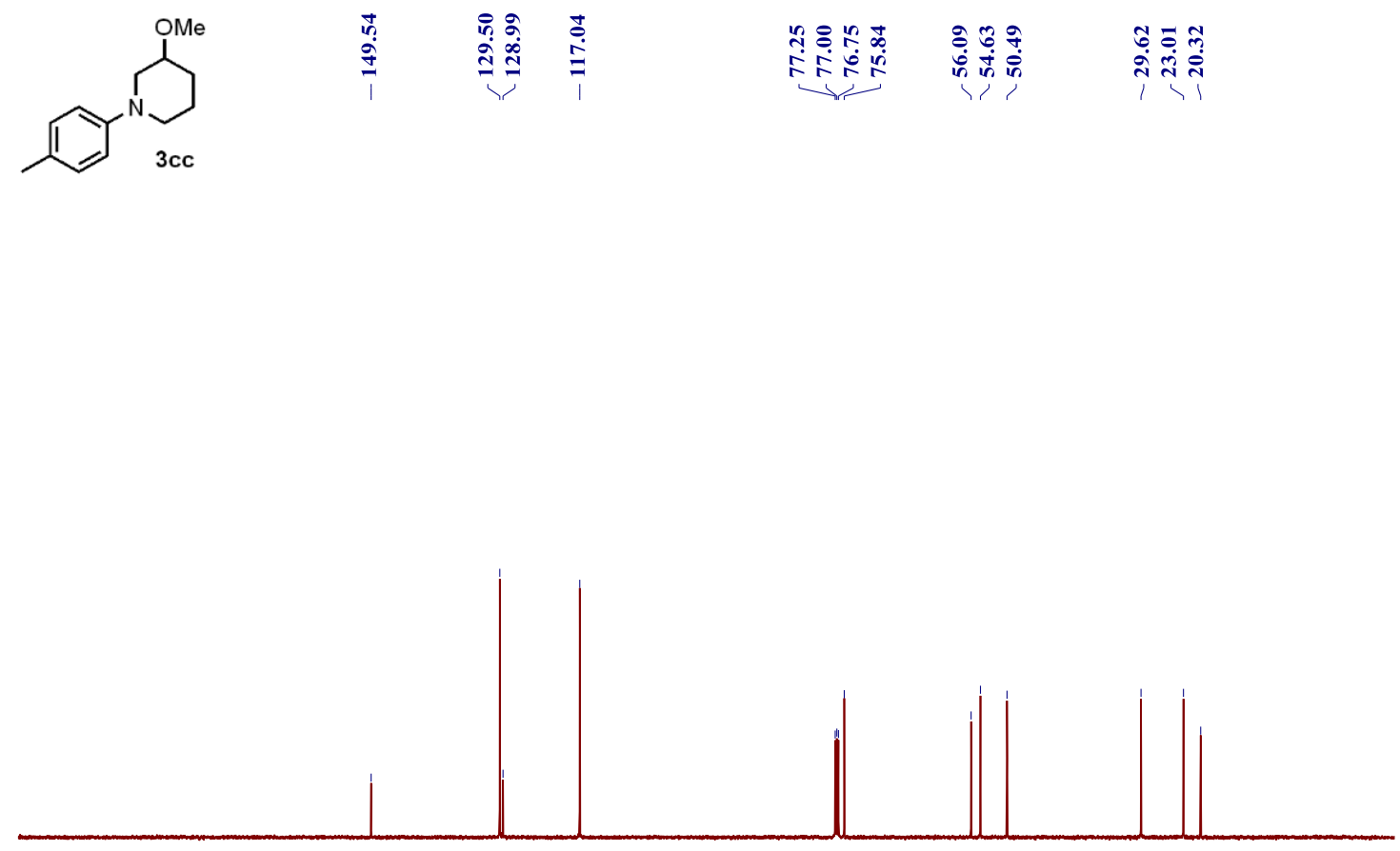

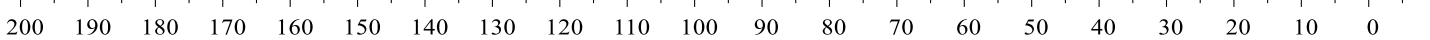

${ }^{1} \mathrm{H}$ NMR (500 MHz, $\left.\mathrm{CDCl}_{3}\right)$

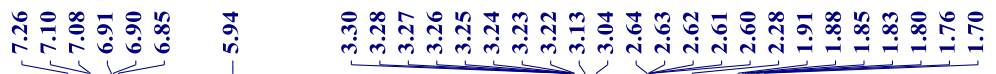<smiles>Cc1ccc(N2CCCC(C(N)=O)C2)cc1</smiles>

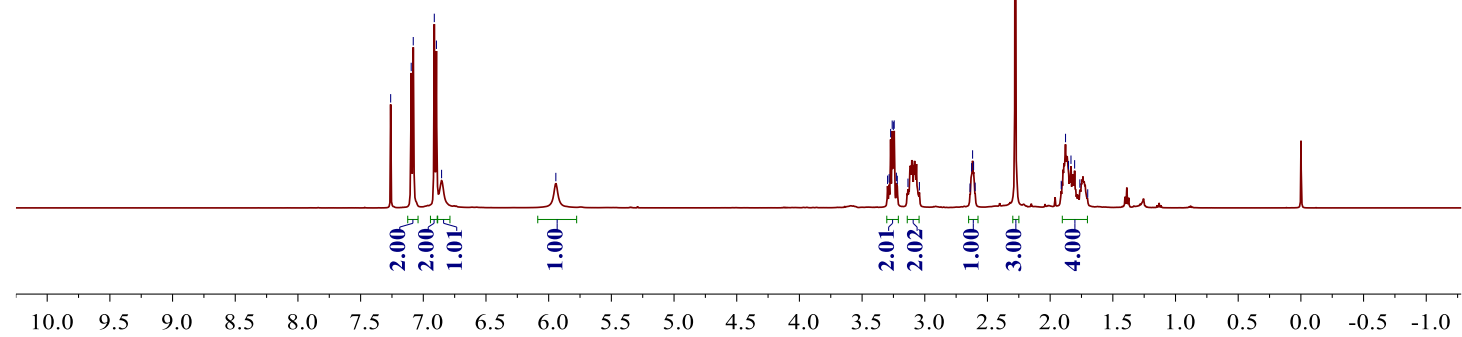


${ }^{13} \mathrm{C}$ NMR $\left(126 \mathrm{MHz}, \mathrm{CDCl}_{3}\right)$

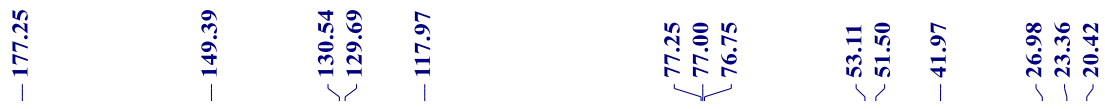<smiles>Cc1ccc(N2CCCC(C(N)=O)C2)cc1</smiles>

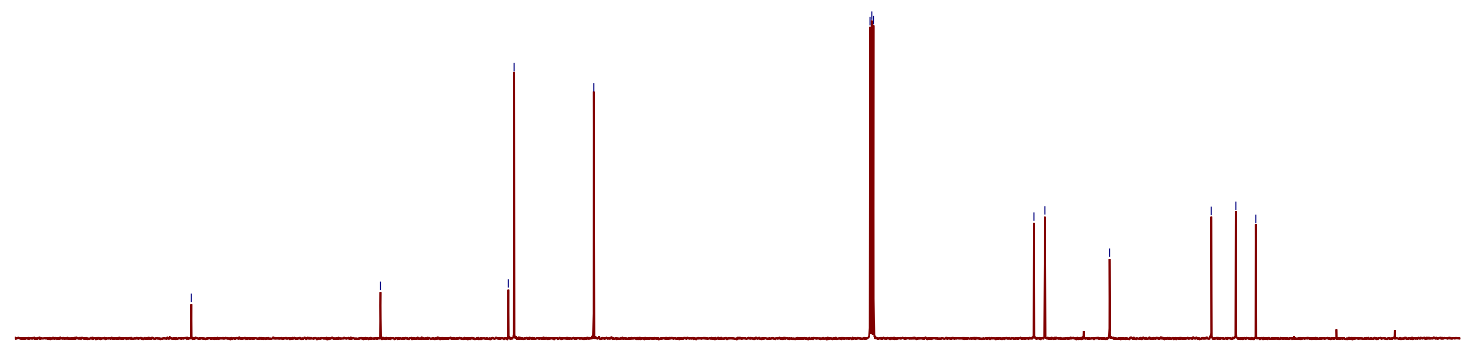

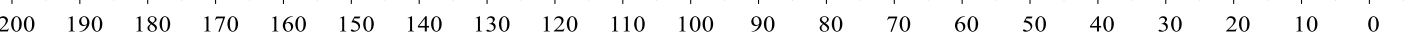

${ }^{1} \mathrm{H} \mathrm{NMR}\left(500 \mathrm{MHz}, \mathrm{CDCl}_{3}\right)$

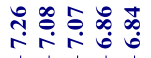

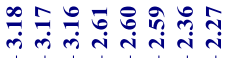<smiles>Cc1ccc(N2CCN(C)CC2)cc1</smiles>

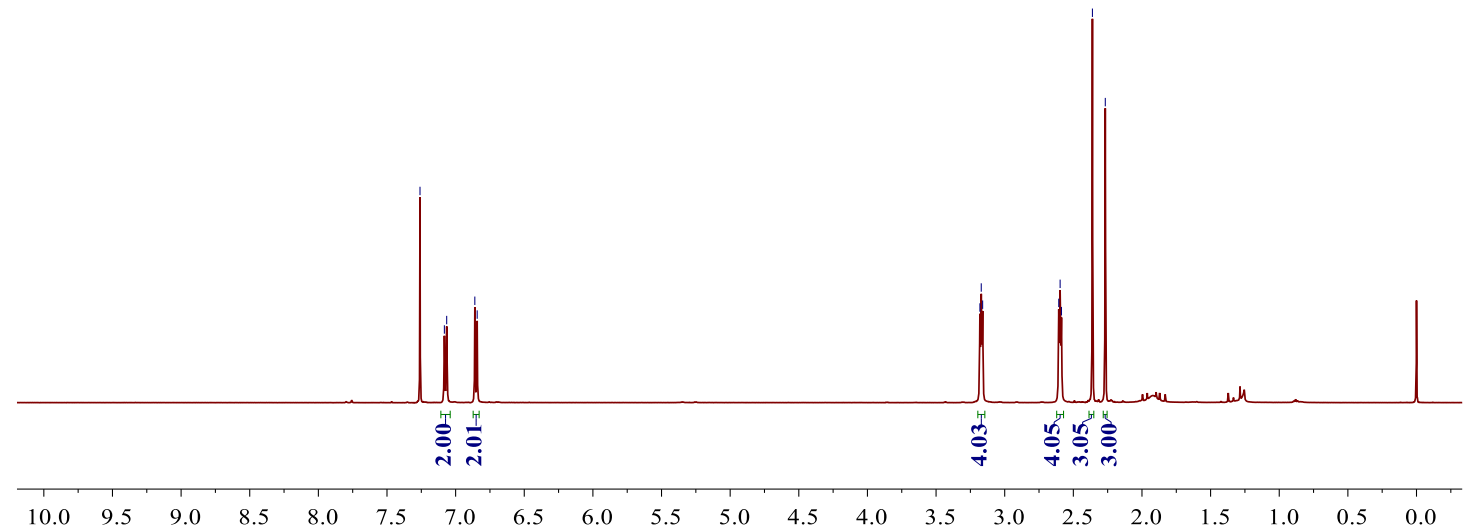


${ }^{13} \mathrm{C} \mathrm{NMR}\left(126 \mathrm{MHz}, \mathrm{CDCl}_{3}\right)$
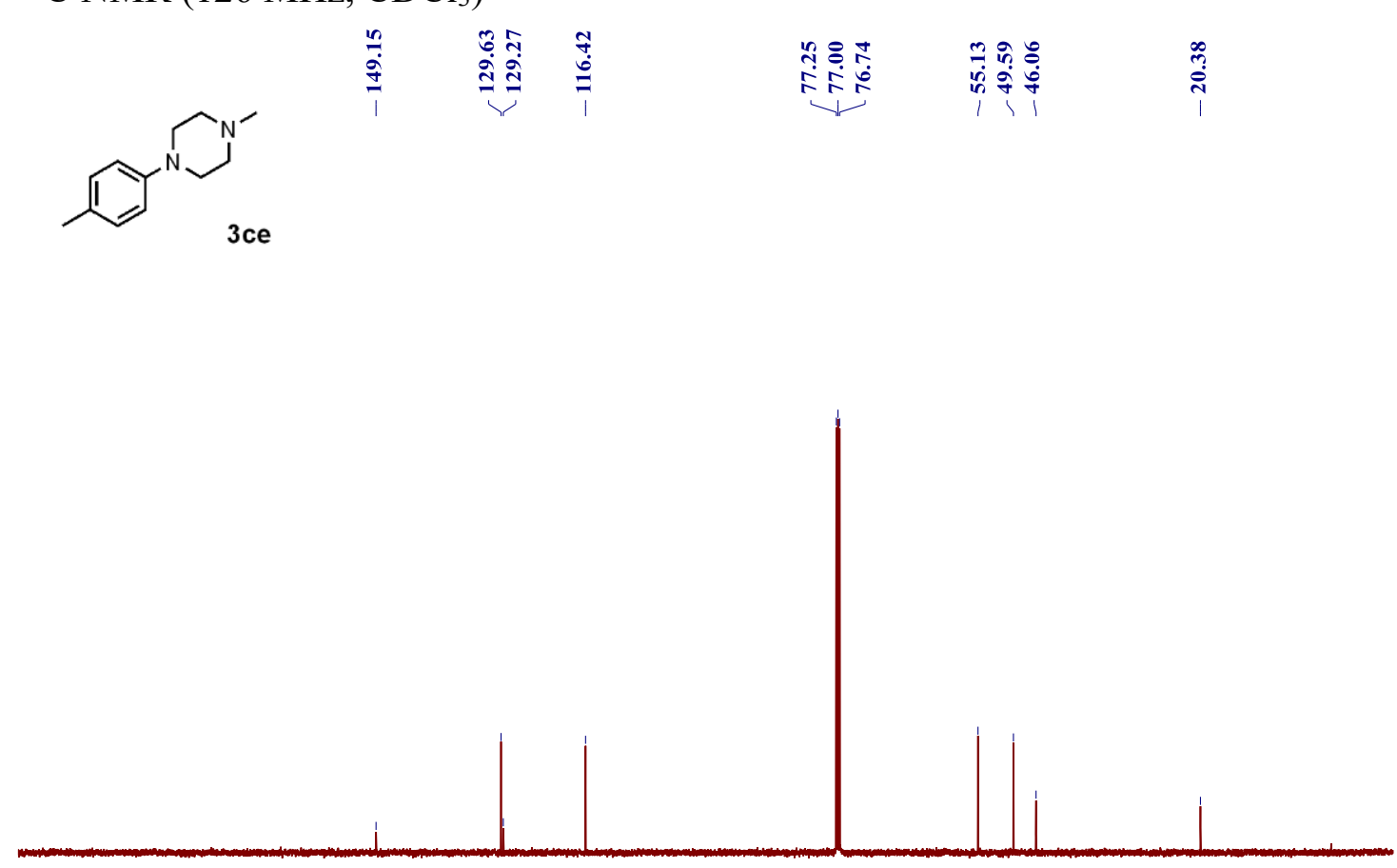

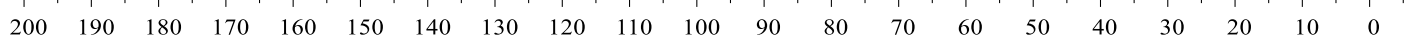

${ }^{1} \mathrm{H} \mathrm{NMR}\left(500 \mathrm{MHz}, \mathrm{CDCl}_{3}\right)$

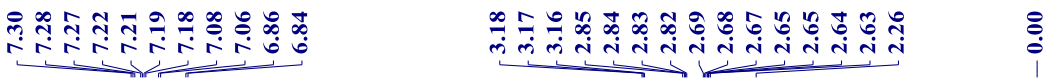<smiles>Cc1ccc(N2CCN(CCc3ccccc3)CC2)cc1</smiles>

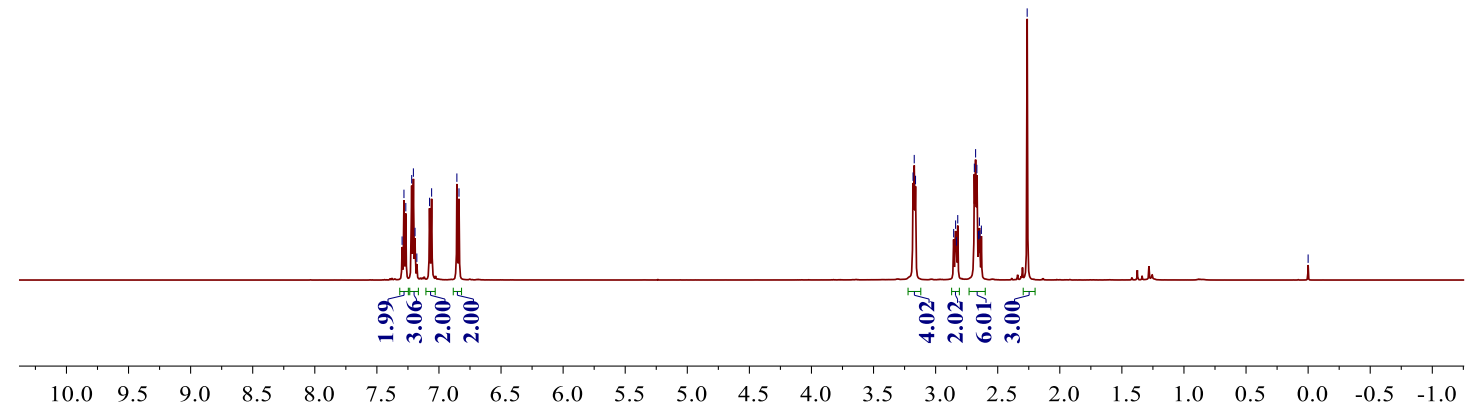


${ }^{13} \mathrm{C}$ NMR (126 MHz, $\left.\mathrm{CDCl}_{3}\right)$

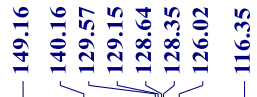

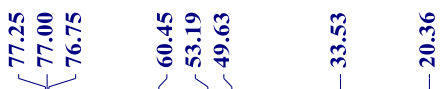<smiles>Cc1ccc(N2CCN(CCc3ccccc3)CC2)cc1</smiles>

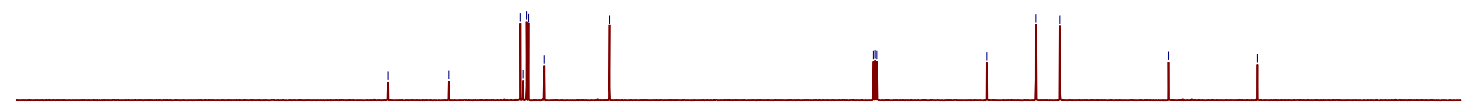

$\begin{array}{lllllllllllllllllllll}200 & 190 & 180 & 170 & 160 & 150 & 140 & 130 & 120 & 110 & 100 & 90 & 80 & 70 & 60 & 50 & 40 & 30 & 20 & 10 & 0\end{array}$

${ }^{1} \mathrm{H} \mathrm{NMR}\left(500 \mathrm{MHz}, \mathrm{CDCl}_{3}\right)$
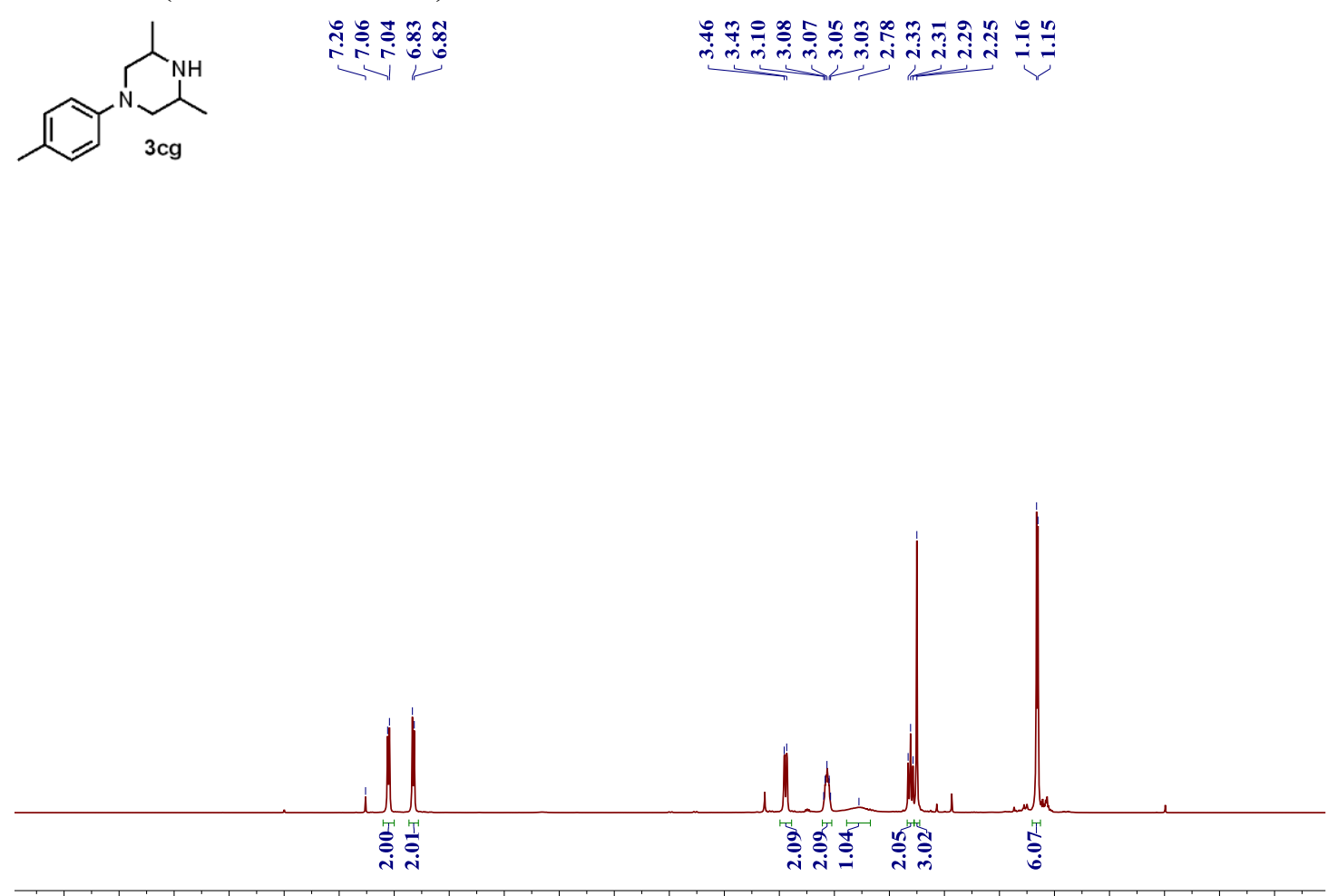

$\begin{array}{lllllllllllllllllllllllll}10.0 & 9.5 & 9.0 & 8.5 & 8.0 & 7.5 & 7.0 & 6.5 & 6.0 & 5.5 & 5.0 & 4.5 & 4.0 & 3.5 & 3.0 & 2.5 & 2.0 & 1.5 & 1.0 & 0.5 & 0.0 & -0.5 & -1.0\end{array}$ 
${ }^{13} \mathrm{C}$ NMR (126 MHz, $\left.\mathrm{CDCl}_{3}\right)$

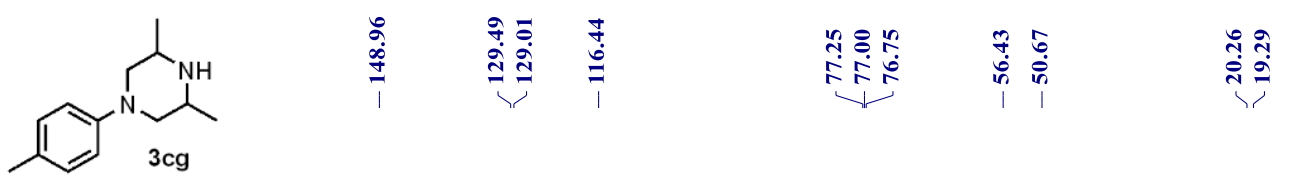

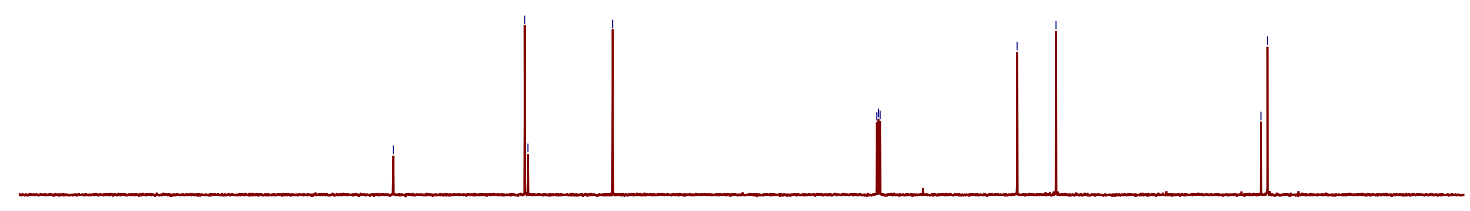

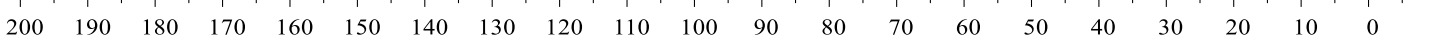

${ }^{1} \mathrm{H} \mathrm{NMR}\left(500 \mathrm{MHz}, \mathrm{CDCl}_{3}\right)$

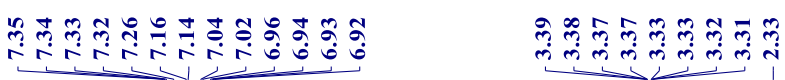

$\overbrace{3 c h}^{N}$

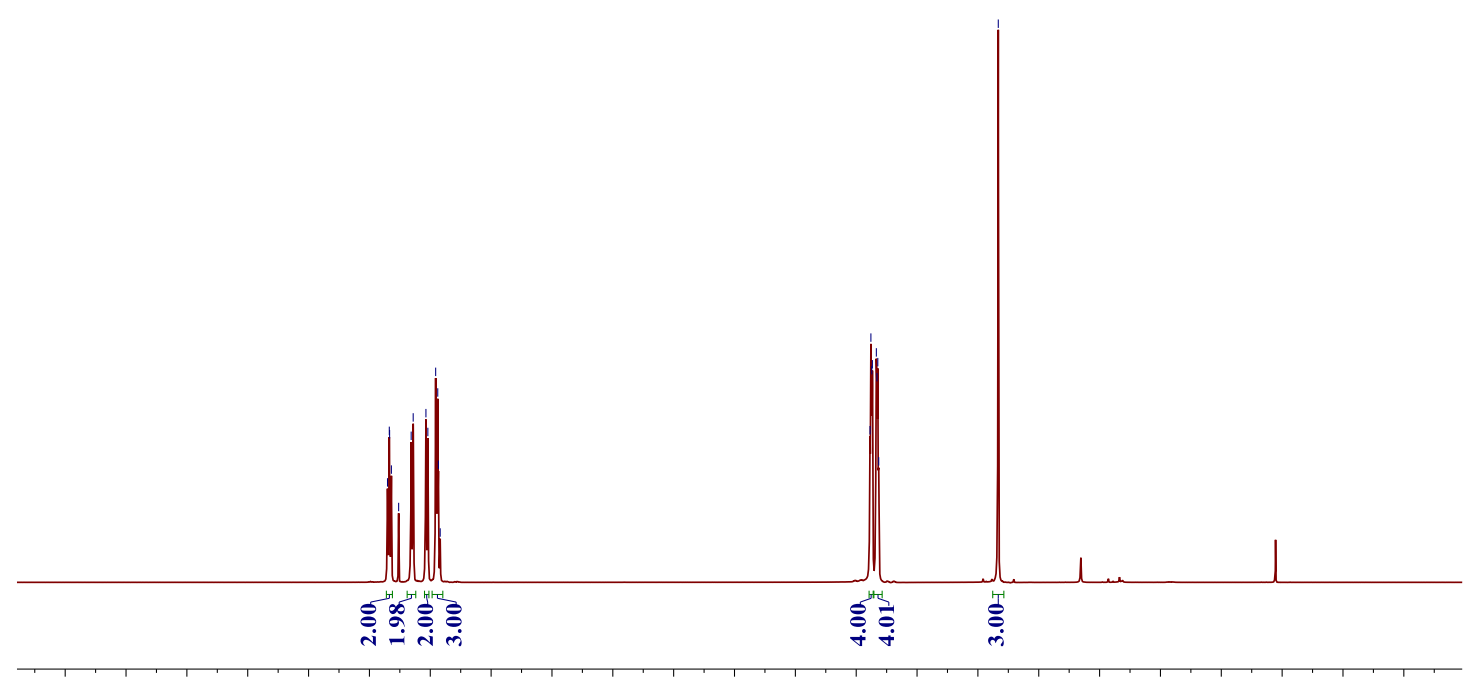

$\begin{array}{lllllllllllllllllllllllll}10.0 & 9.5 & 9.0 & 8.5 & 8.0 & 7.5 & 7.0 & 6.5 & 6.0 & 5.5 & 5.0 & 4.5 & 4.0 & 3.5 & 3.0 & 2.5 & 2.0 & 1.5 & 1.0 & 0.5 & 0.0 & -0.5 & -1.0\end{array}$ 
${ }^{13} \mathrm{C}$ NMR $\left(126 \mathrm{MHz}, \mathrm{CDCl}_{3}\right)$

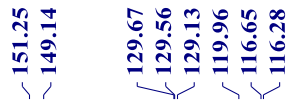

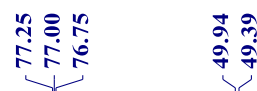

ษั่<smiles>Cc1ccc(N2CCNCC2)cc1</smiles>

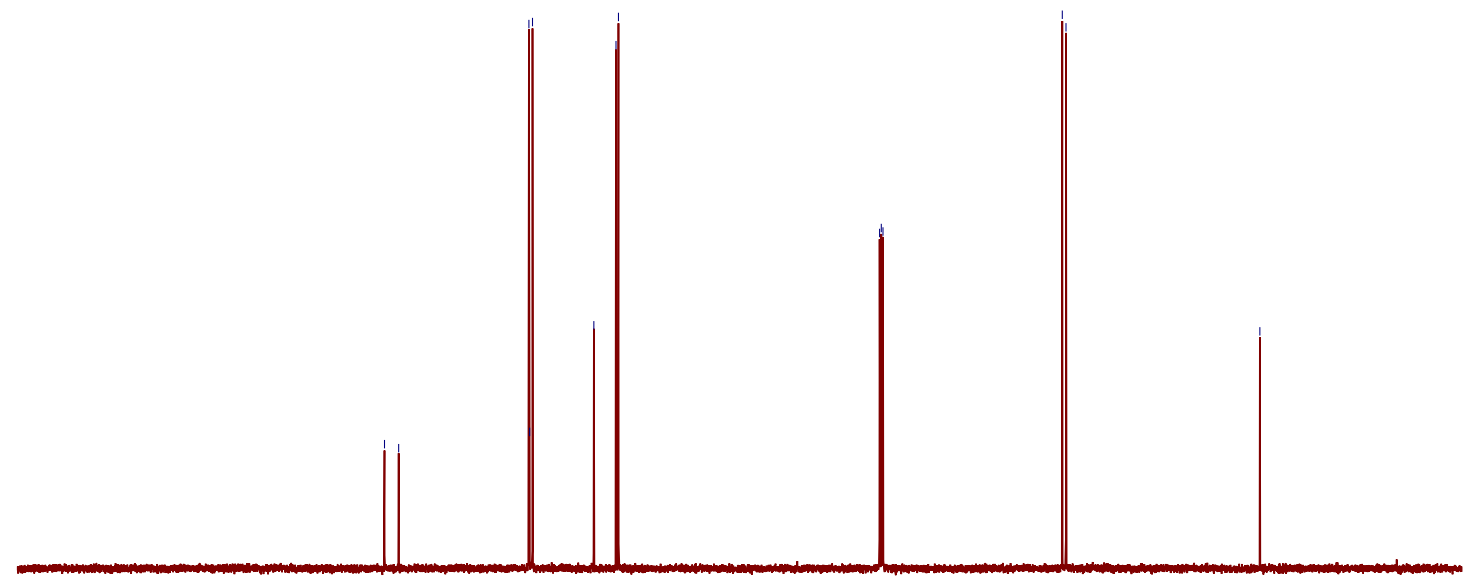

$\begin{array}{lllllllllllllllllllll}200 & 190 & 180 & 170 & 160 & 150 & 140 & 130 & 120 & 110 & 100 & 90 & 80 & 70 & 60 & 50 & 40 & 30 & 20 & 10 & 0\end{array}$

${ }^{1} \mathrm{H} \mathrm{NMR}\left(500 \mathrm{MHz}, \mathrm{CDCl}_{3}\right)$

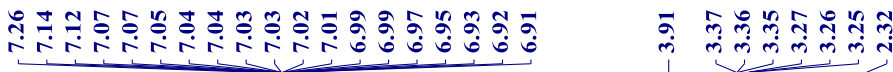
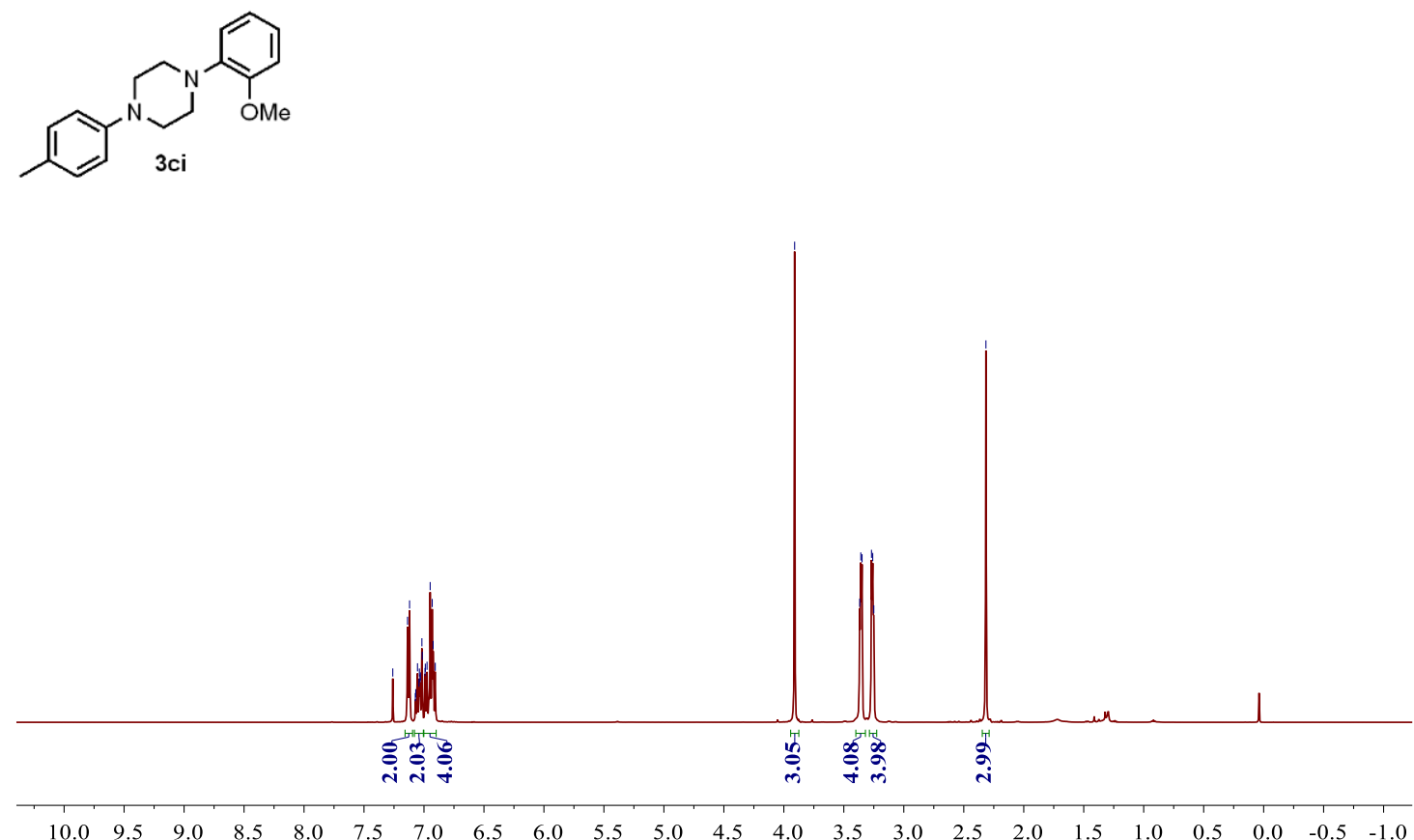
${ }^{13} \mathrm{C} \mathrm{NMR}\left(126 \mathrm{MHz}, \mathrm{CDCl}_{3}\right)$
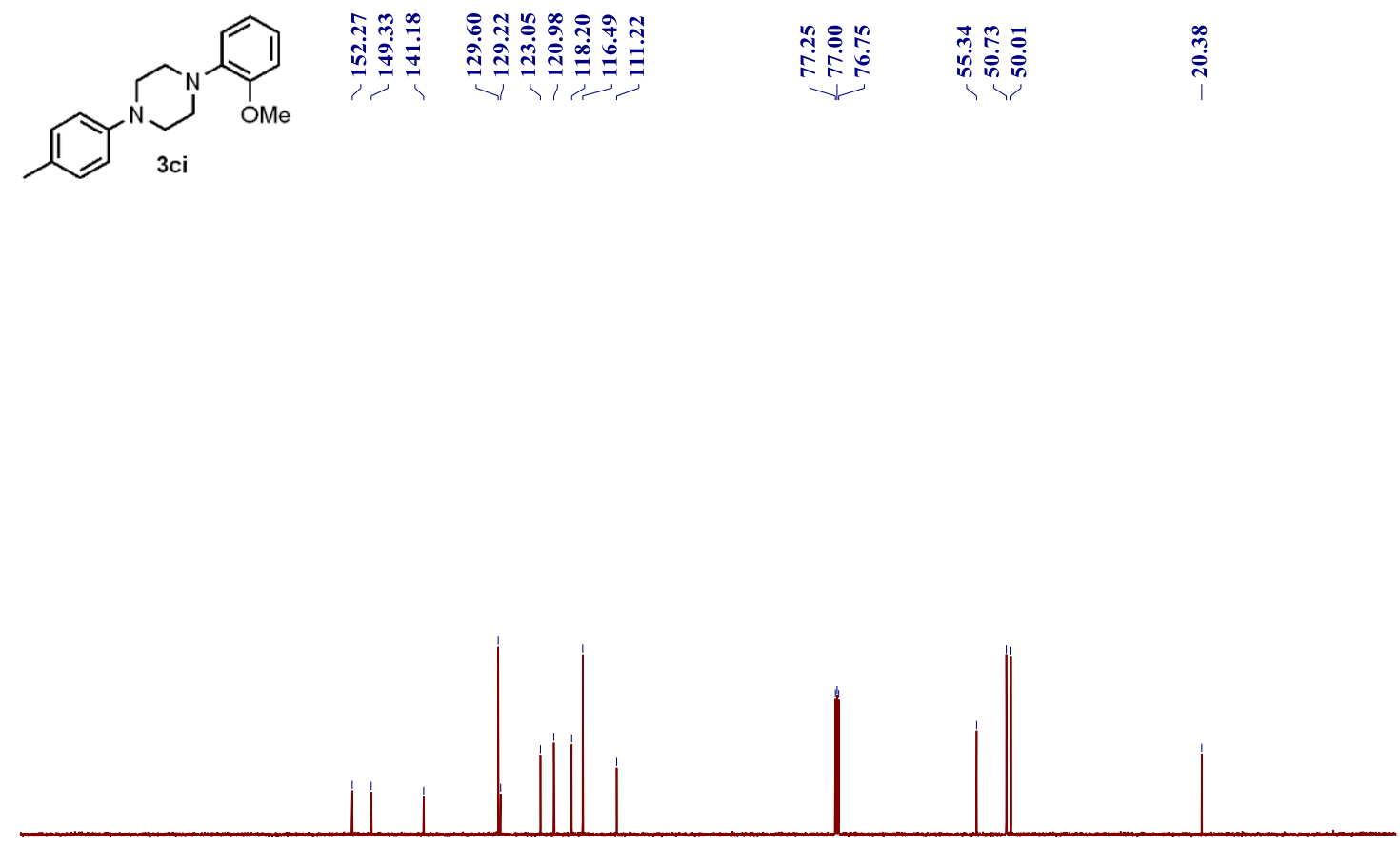

$\begin{array}{lllllllllllllllllllll}200 & 190 & 180 & 170 & 160 & 150 & 140 & 130 & 120 & 110 & 100 & 90 & 80 & 70 & 60 & 50 & 40 & 30 & 20 & 10 & 0\end{array}$

${ }^{1} \mathrm{H} \mathrm{NMR}\left(500 \mathrm{MHz}, \mathrm{CDCl}_{3}\right)$

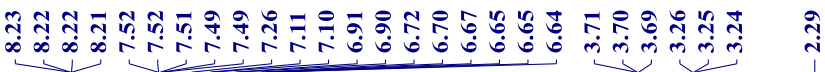<smiles>Cc1ccc(N2CCN(c3ccccn3)CC2)cc1</smiles>

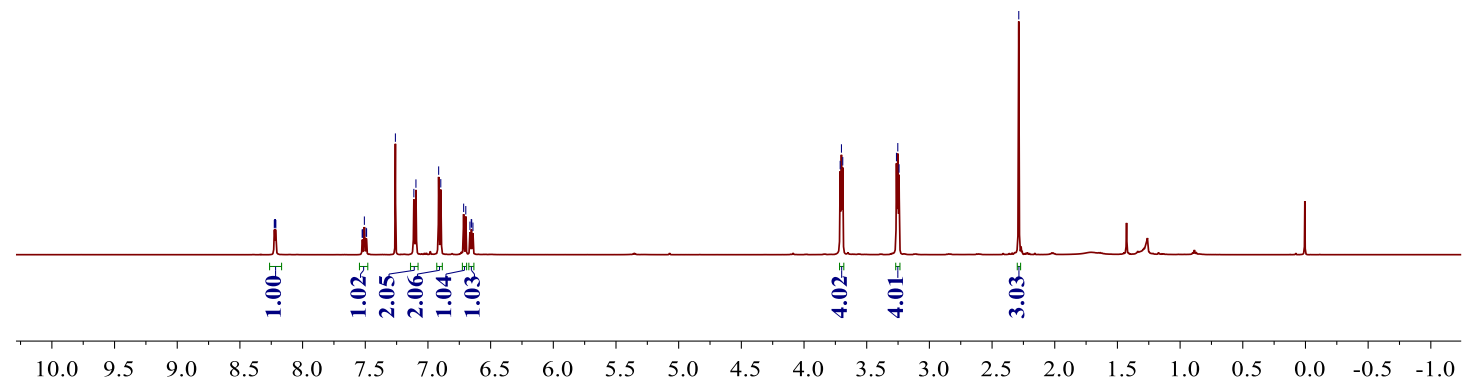


${ }^{13} \mathrm{C} \mathrm{NMR}\left(126 \mathrm{MHz}, \mathrm{CDCl}_{3}\right)$

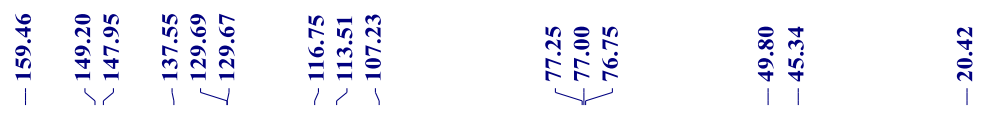<smiles>Cc1ccc(N2CCN(c3ccccn3)CC2)cc1</smiles>

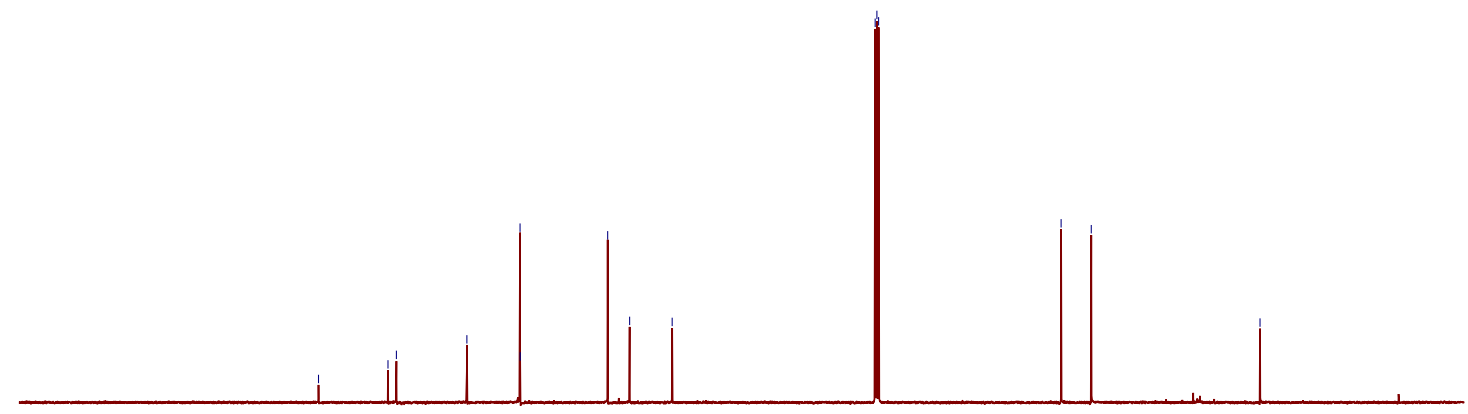

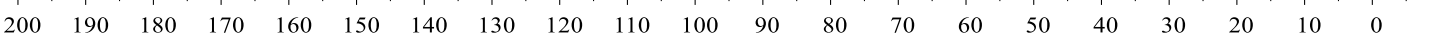

${ }^{1} \mathrm{H} \mathrm{NMR}\left(500 \mathrm{MHz}, \mathrm{CDCl}_{3}\right)$

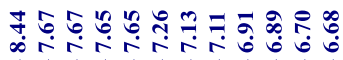

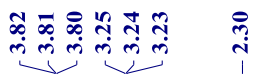
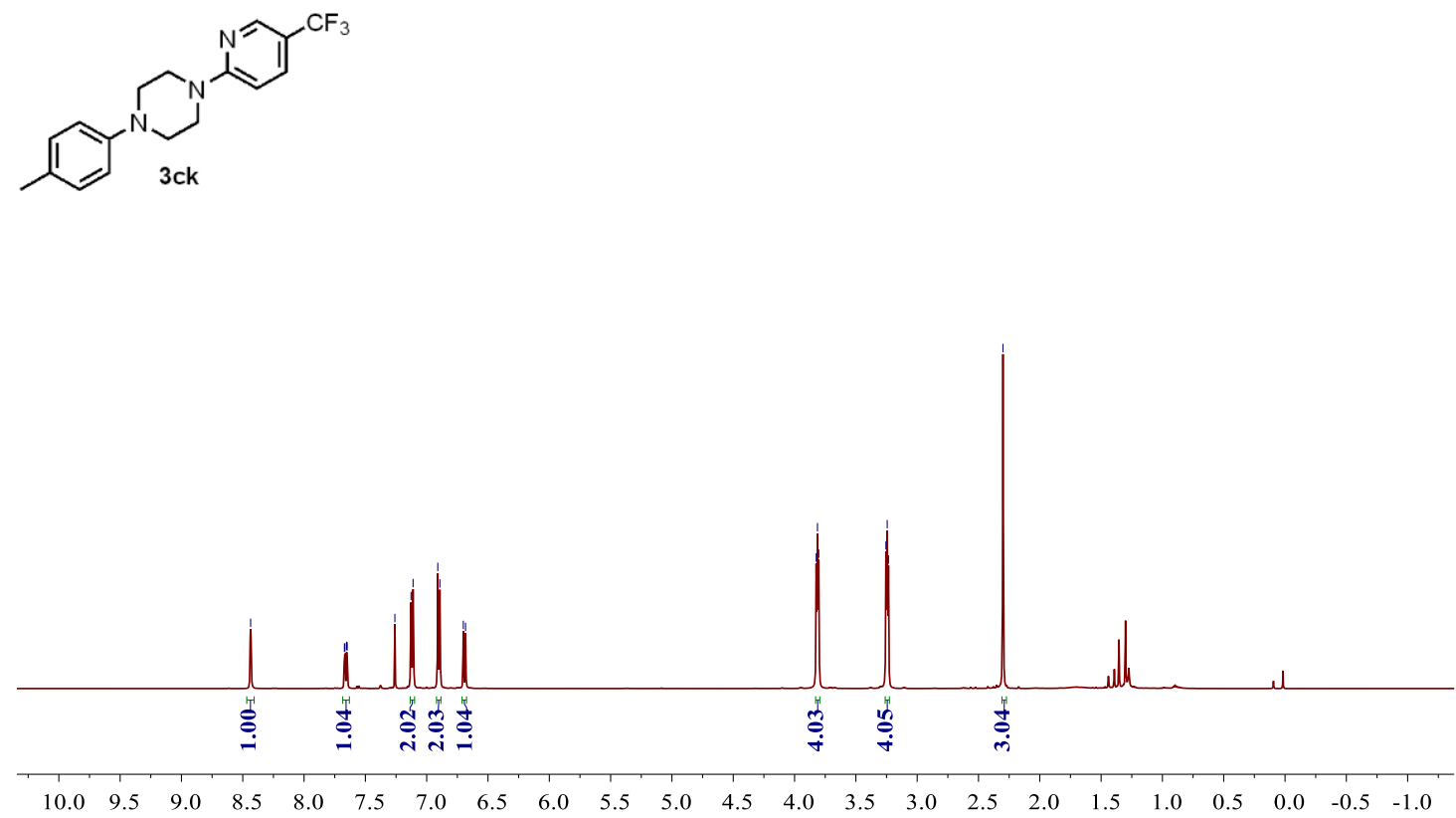
${ }^{13} \mathrm{C} \mathrm{NMR}\left(126 \mathrm{MHz}, \mathrm{CDCl}_{3}\right)$

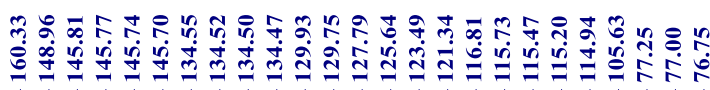

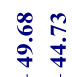

$\stackrel{\text { พั. }}{\stackrel{\sim}{1}}$<smiles>Cc1ccc(N2CCN(c3ccc(C(F)(F)F)cn3)CC2)cc1</smiles>

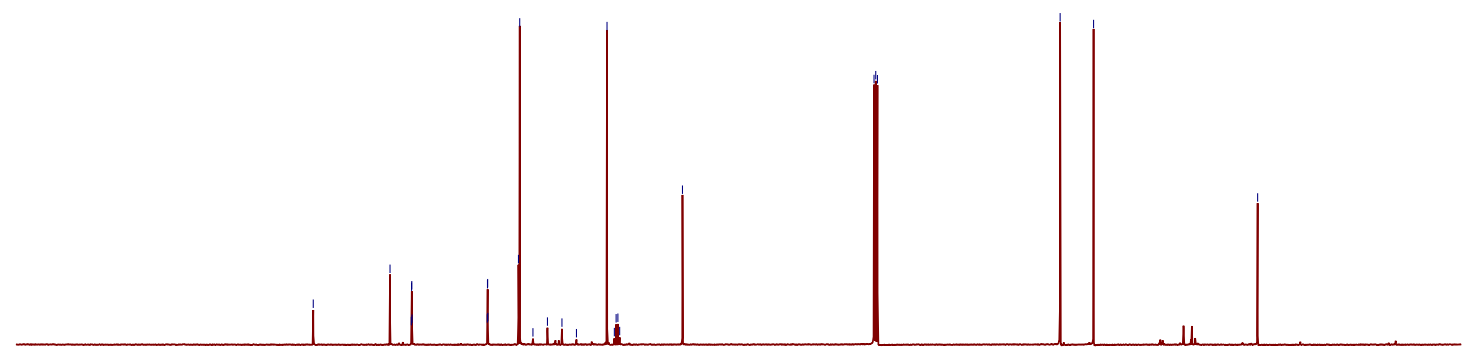

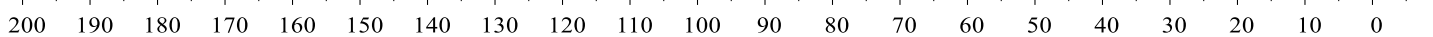

${ }^{19} \mathrm{~F}$ NMR (471 MHz, $\mathrm{CDCl}_{3}$ )

产<smiles>Cc1ccc(N2CCN(c3ccc(C(F)(F)F)cn3)CC2)cc1</smiles>

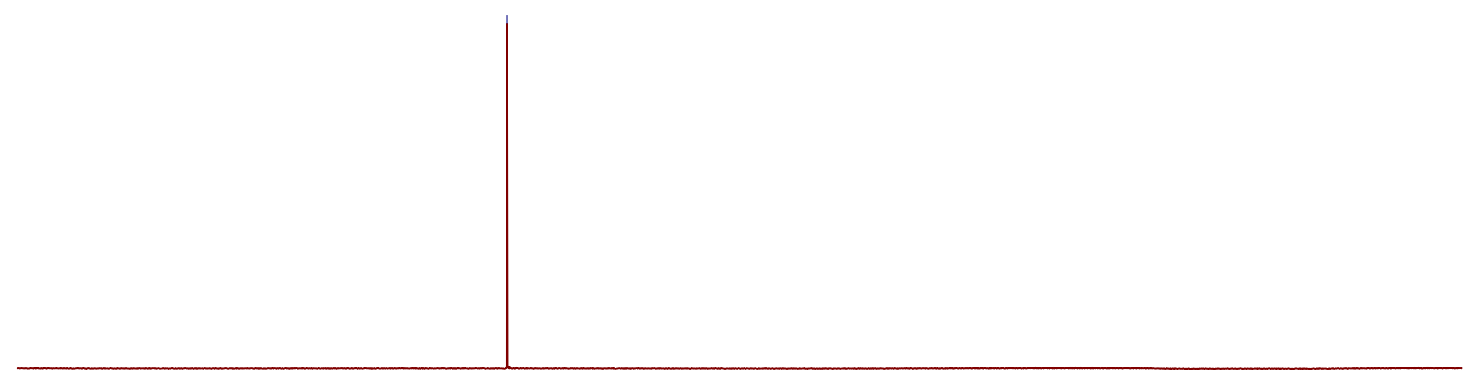

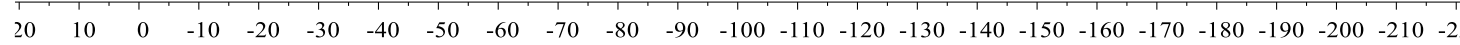


${ }^{1} \mathrm{H} \mathrm{NMR}\left(500 \mathrm{MHz}, \mathrm{CDCl}_{3}\right)$

商为<smiles>Cc1ccc(N2CCN(c3ncccn3)CC2)cc1</smiles>

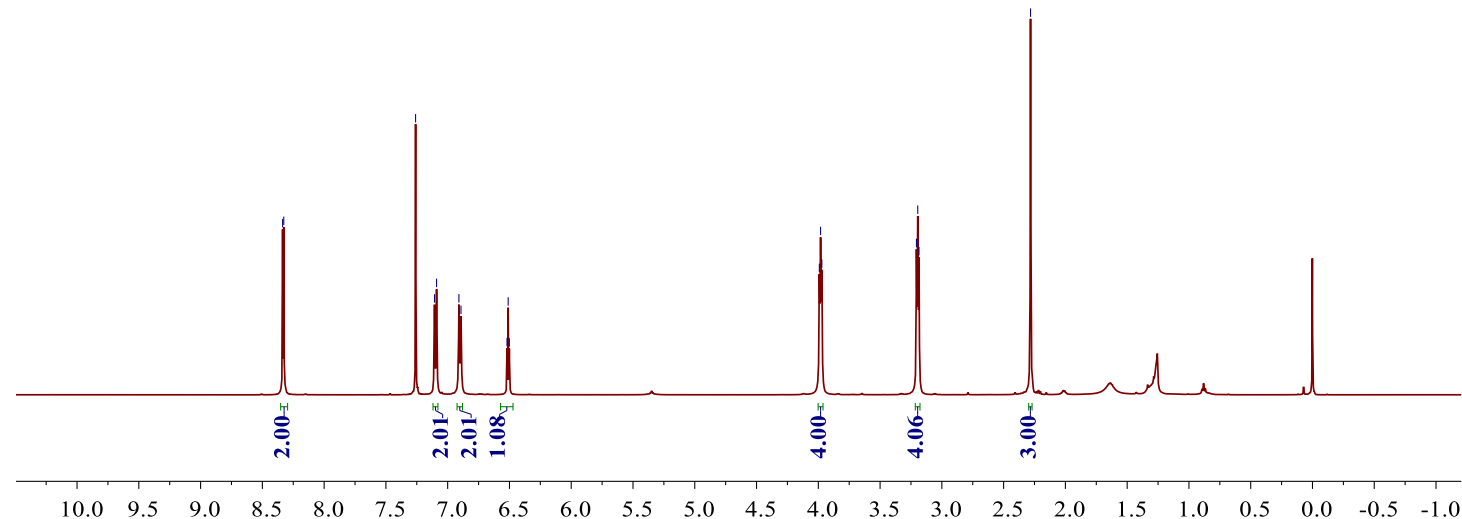

${ }^{13} \mathrm{C}$ NMR (126 MHz, $\mathrm{CDCl}_{3}$ )

\begin{tabular}{|c|c|c|c|c|c|c|}
\hline 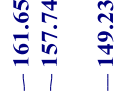 & 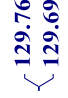 & $\begin{array}{l}\stackrel{0}{\infty} \\
\stackrel{0}{0} \\
=\end{array}$ & $\stackrel{\varrho}{\stackrel{0}{\Theta}}$ & 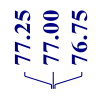 & $\begin{array}{l}\text { ڤे } \\
\dot{\sigma} \\
\end{array}$ & t \\
\hline
\end{tabular}<smiles>Cc1ccc(N2CCN(c3ncccn3)CC2)cc1</smiles>

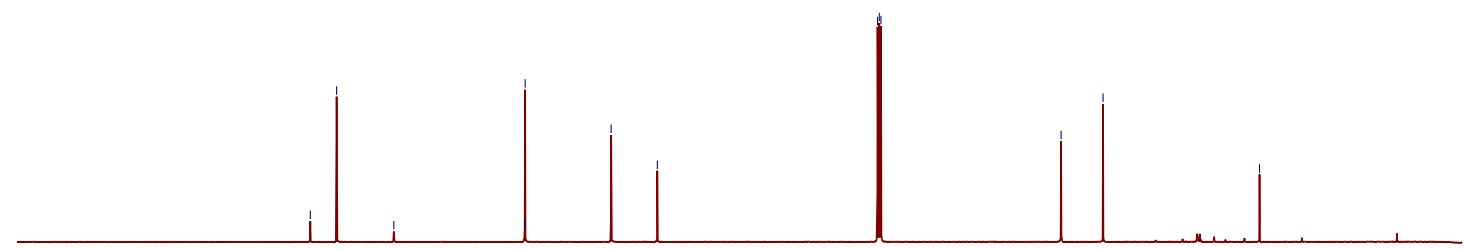

$\begin{array}{llllllllllllllllllllll}200 & 190 & 180 & 170 & 160 & 150 & 140 & 130 & 120 & 110 & 100 & 90 & 80 & 70 & 60 & 50 & 40 & 30 & 20 & 10 & 0\end{array}$ 
${ }^{1} \mathrm{H} \mathrm{NMR}\left(500 \mathrm{MHz}, \mathrm{CDCl}_{3}\right)$
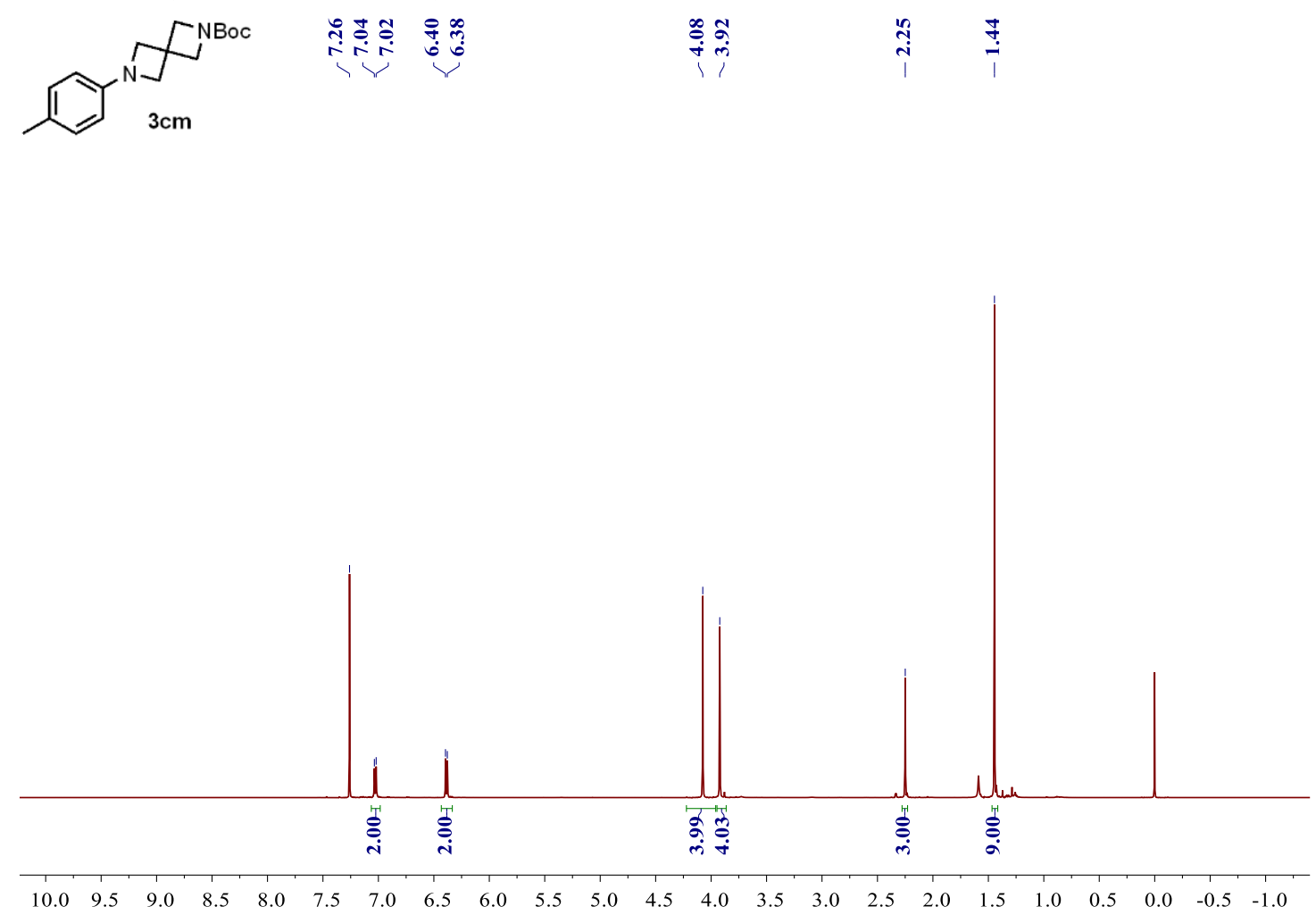

${ }^{13} \mathrm{C} \mathrm{NMR}\left(126 \mathrm{MHz}, \mathrm{CDCl}_{3}\right)$

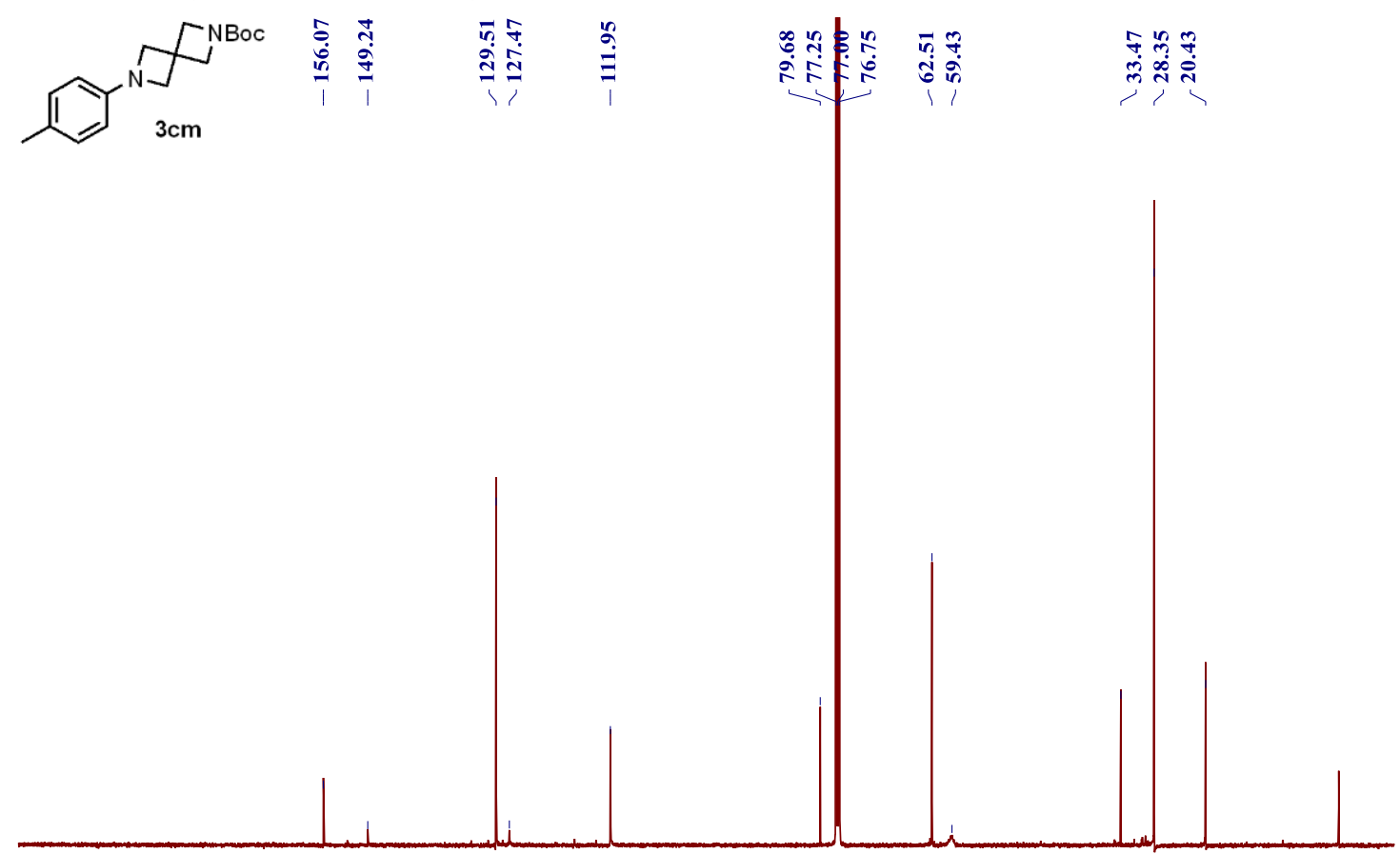

$\begin{array}{llllllllllllllllllllll}200 & 190 & 180 & 170 & 160 & 150 & 140 & 130 & 120 & 110 & 100 & 90 & 80 & 70 & 60 & 50 & 40 & 30 & 20 & 10 & 0\end{array}$ 
${ }^{1} \mathrm{H} \mathrm{NMR}\left(500 \mathrm{MHz}, \mathrm{CDCl}_{3}\right)$
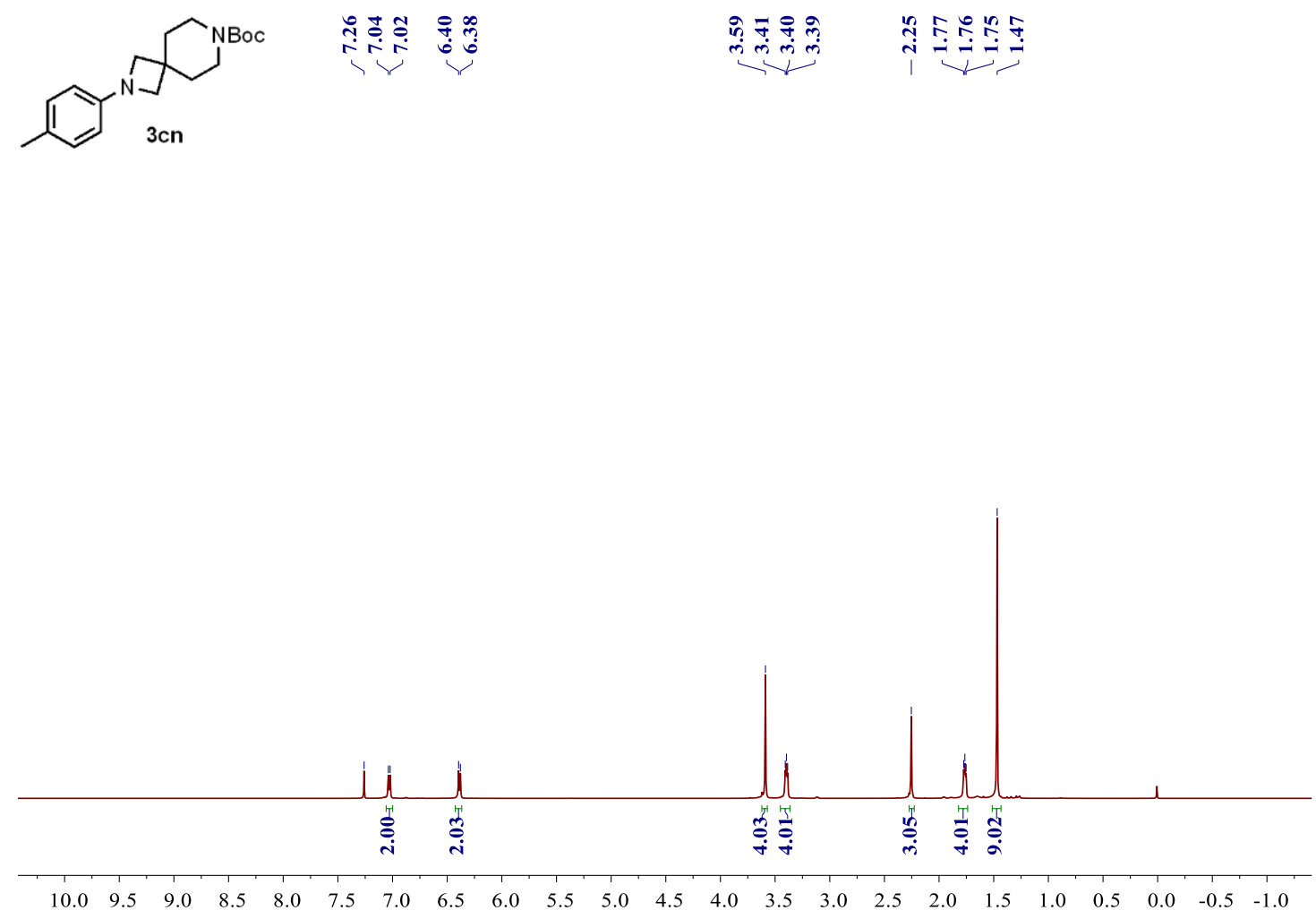

${ }^{13} \mathrm{C} \mathrm{NMR}\left(126 \mathrm{MHz}, \mathrm{CDCl}_{3}\right)$

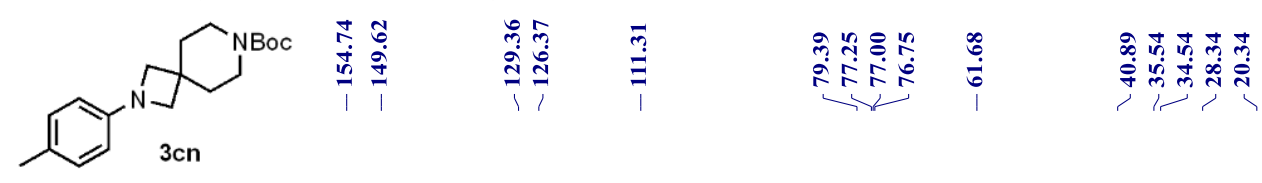

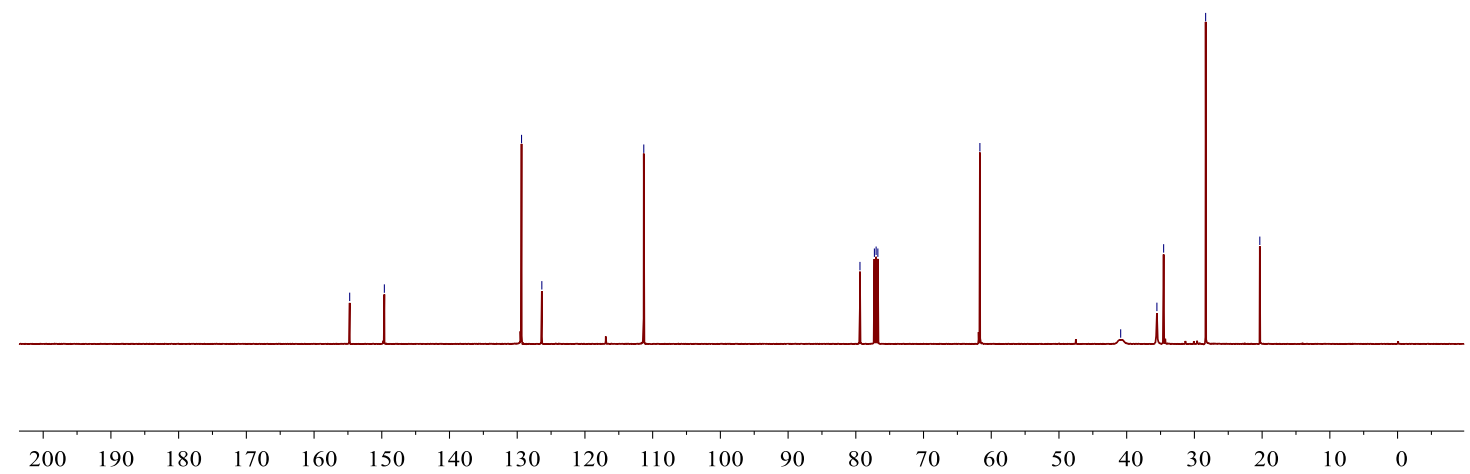


${ }^{1} \mathrm{H}$ NMR $\left(500 \mathrm{MHz}, \mathrm{CDCl}_{3}\right)$
$\overbrace{3 c 0}^{N}$

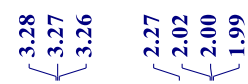
ํㅗㄴ

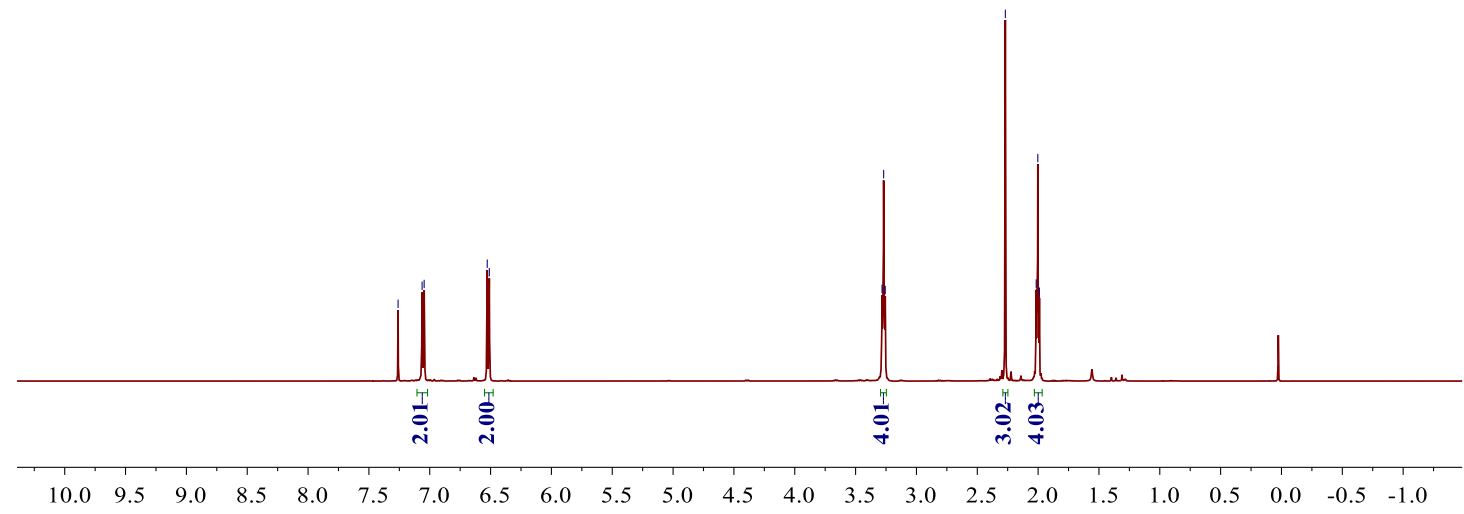

${ }^{13} \mathrm{C} \mathrm{NMR}\left(126 \mathrm{MHz}, \mathrm{CDCl}_{3}\right)$

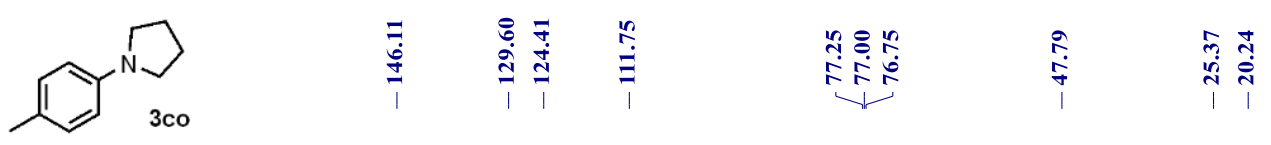

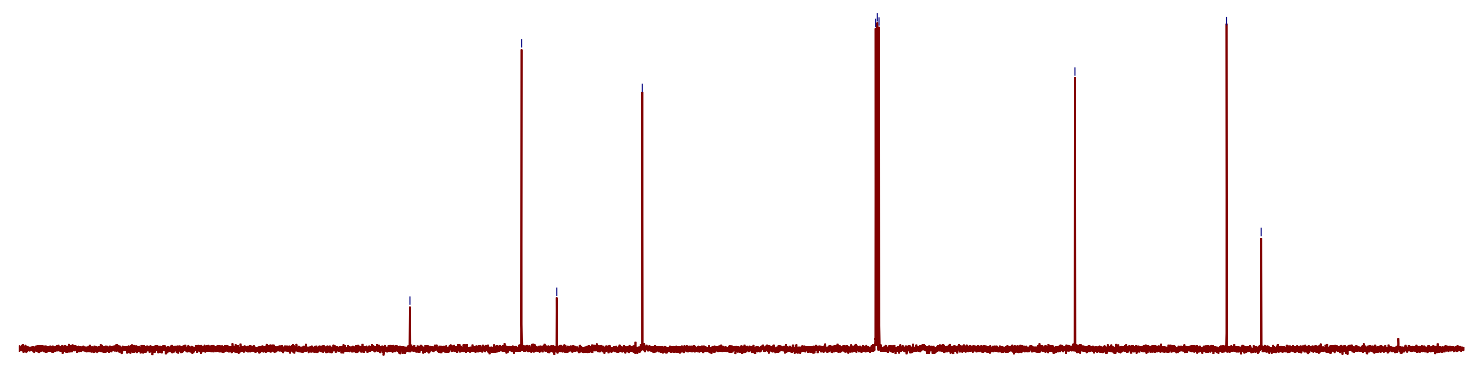

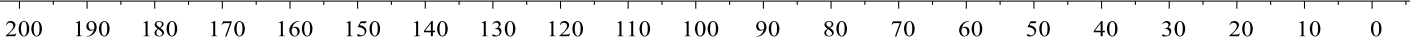


${ }^{1} \mathrm{H} \mathrm{NMR}\left(500 \mathrm{MHz}, \mathrm{CDCl}_{3}\right)$

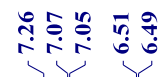

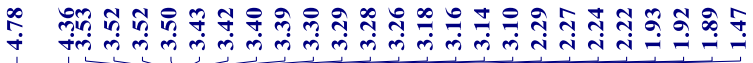

$\overbrace{3 c p}^{N}{ }_{N B O C}$

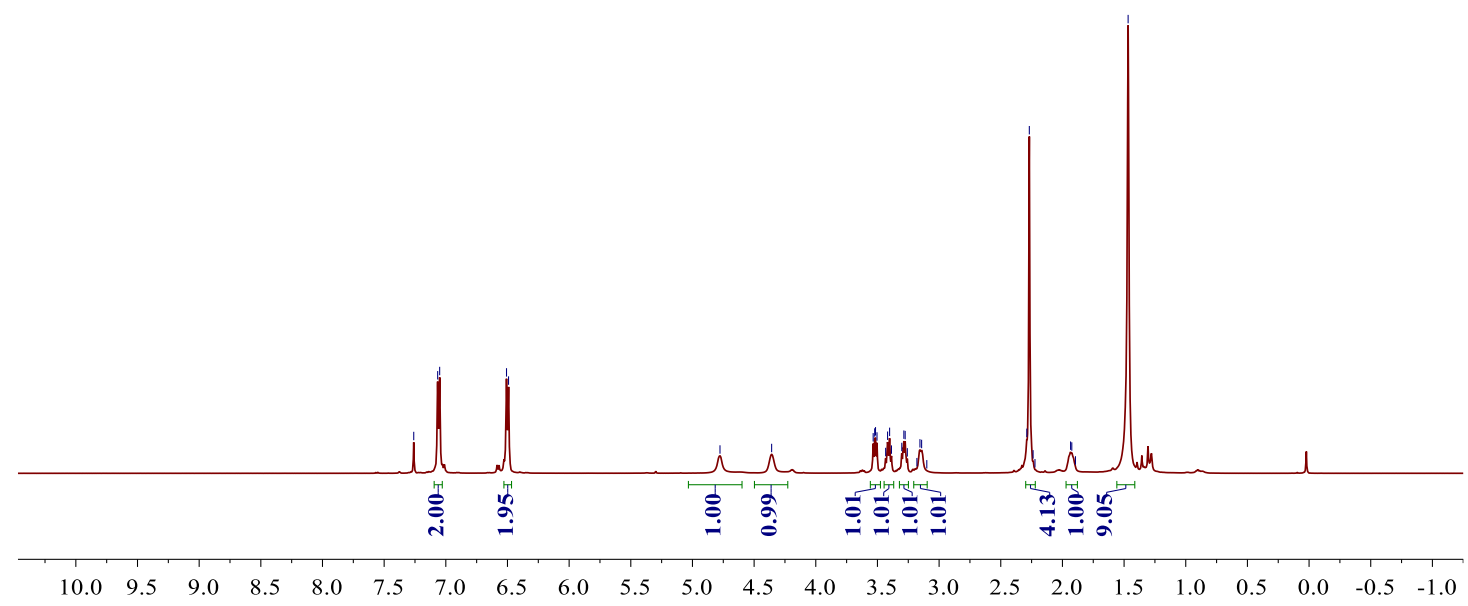

${ }^{13} \mathrm{C} \mathrm{NMR}\left(126 \mathrm{MHz}, \mathrm{CDCl}_{3}\right)$

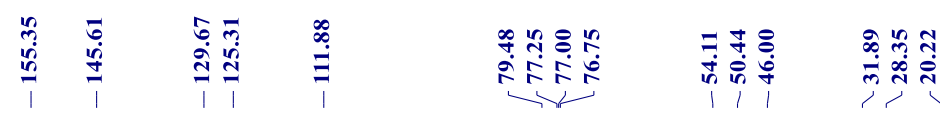<smiles>Cc1ccc(N2CCC(NC(=O)OCc3ccccc3)C2)cc1</smiles>

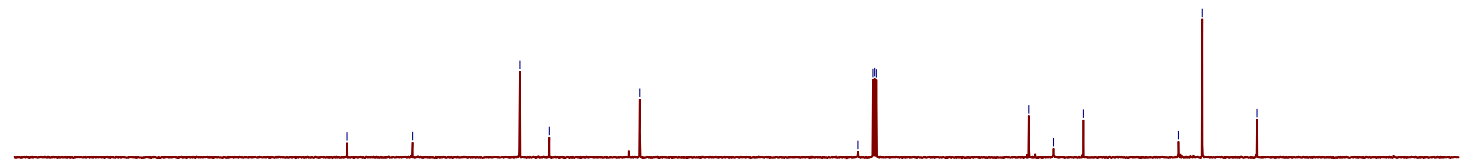

$\begin{array}{lllllllllllllllllllllll}200 & 190 & 180 & 170 & 160 & 150 & 140 & 130 & 120 & 110 & 100 & 90 & 80 & 70 & 60 & 50 & 40 & 30 & 20 & 10 & 0\end{array}$ 
${ }^{1} \mathrm{H}$ NMR $\left(500 \mathrm{MHz}, \mathrm{CDCl}_{3}\right)$
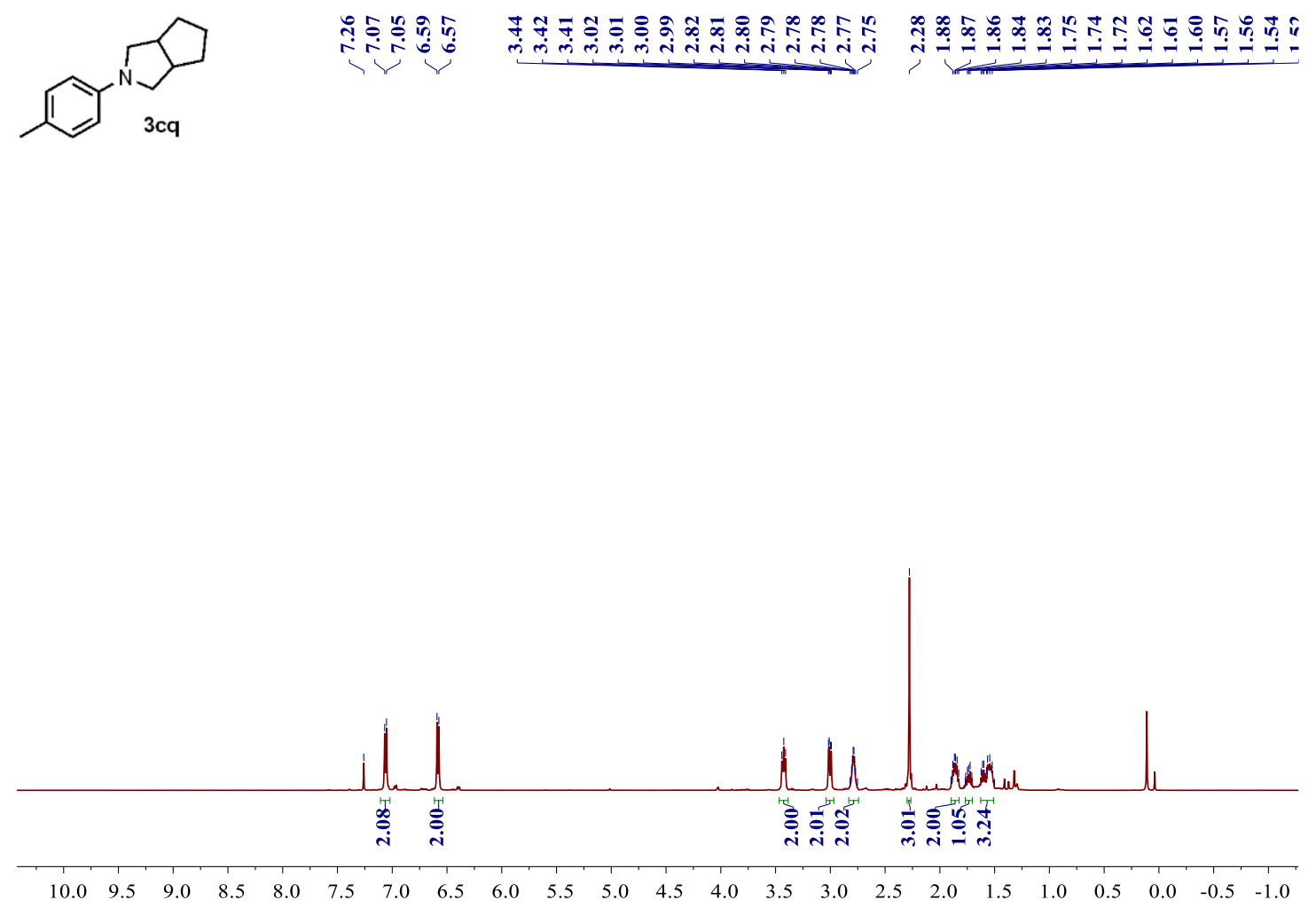

${ }^{13} \mathrm{C} \mathrm{NMR}\left(126 \mathrm{MHz}, \mathrm{CDCl}_{3}\right)$

>1

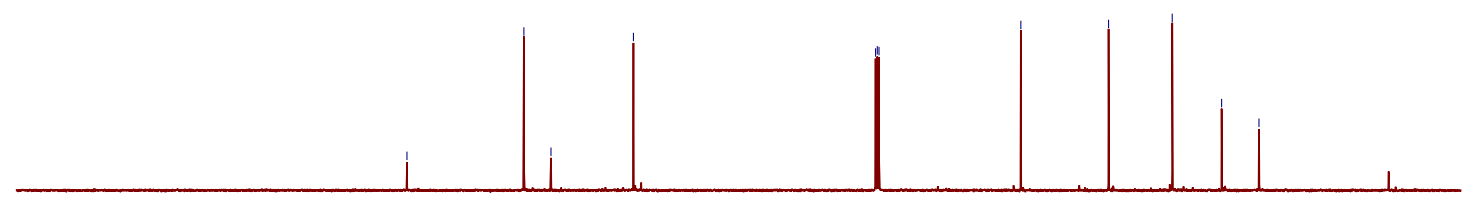

$\begin{array}{lllllllllllllllllllll}2 & 190 & 180 & 170 & 160 & 150 & 140 & 130 & 120 & 110 & 100 & 90 & 80 & 70 & 60 & 50 & 40 & 30 & 20 & 10 & 0\end{array}$ 
${ }^{1} \mathrm{H}$ NMR $\left(500 \mathrm{MHz}, \mathrm{CDCl}_{3}\right)$
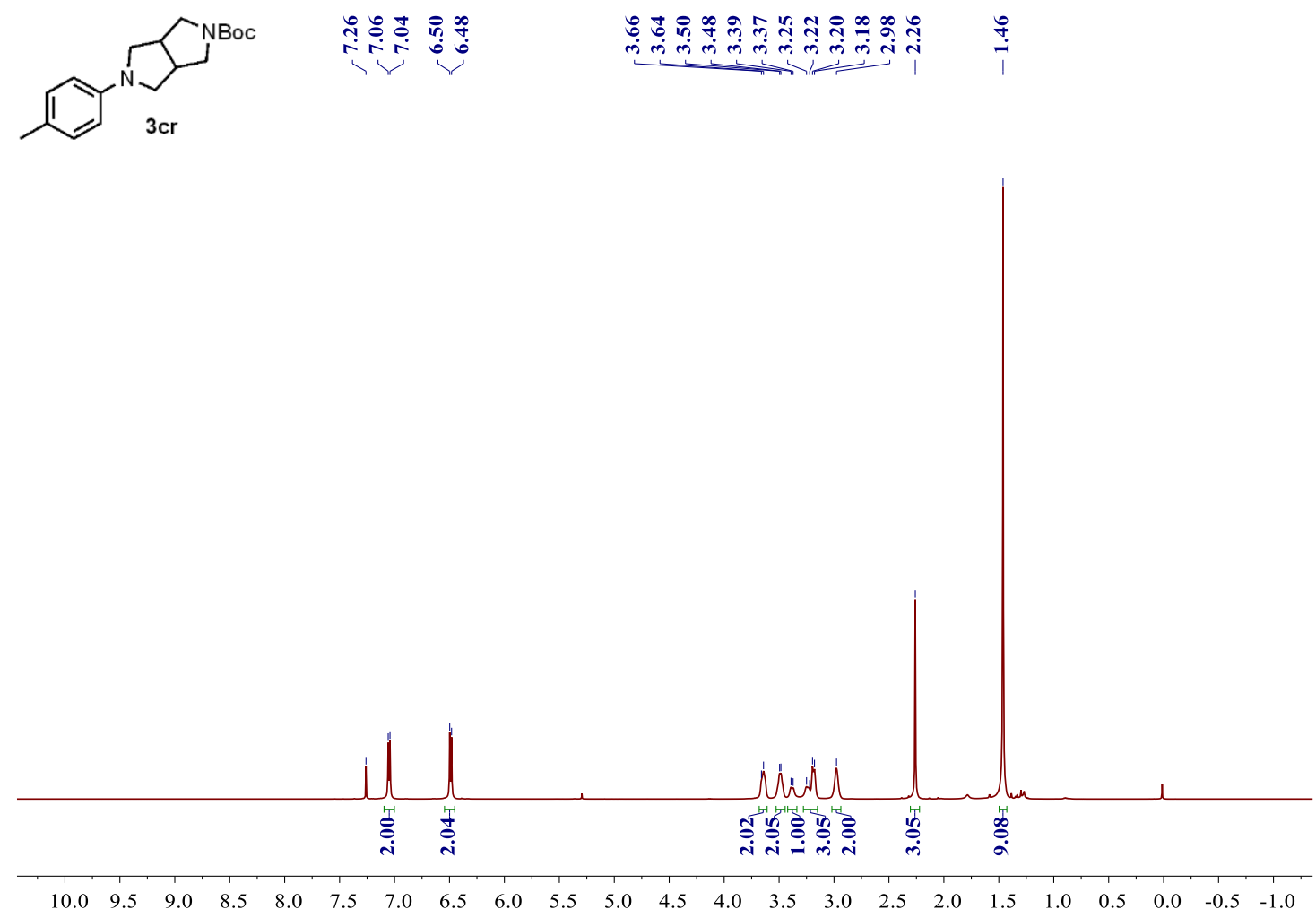

${ }^{13} \mathrm{C} \mathrm{NMR}\left(126 \mathrm{MHz}, \mathrm{CDCl}_{3}\right)$

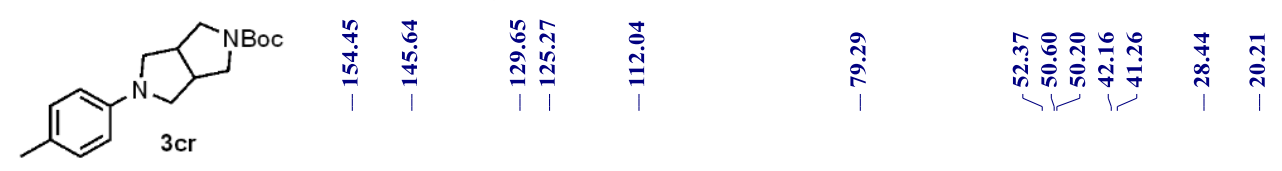

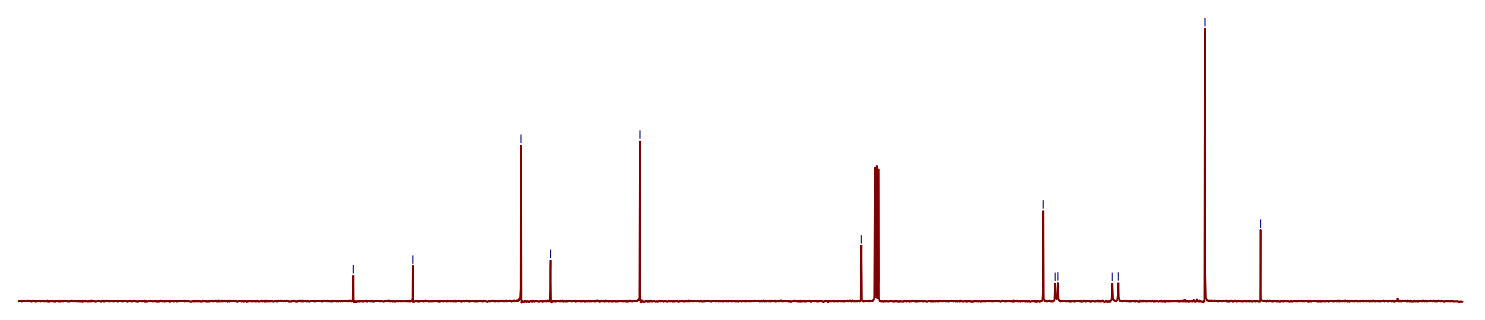

$\begin{array}{lllllllllllllllllllllll}200 & 190 & 180 & 170 & 160 & 150 & 140 & 130 & 120 & 110 & 100 & 90 & 80 & 70 & 60 & 50 & 40 & 30 & 20 & 10 & 0 & 10\end{array}$ 
${ }^{1} \mathrm{H}$ NMR $\left(500 \mathrm{MHz}, \mathrm{CDCl}_{3}\right)$
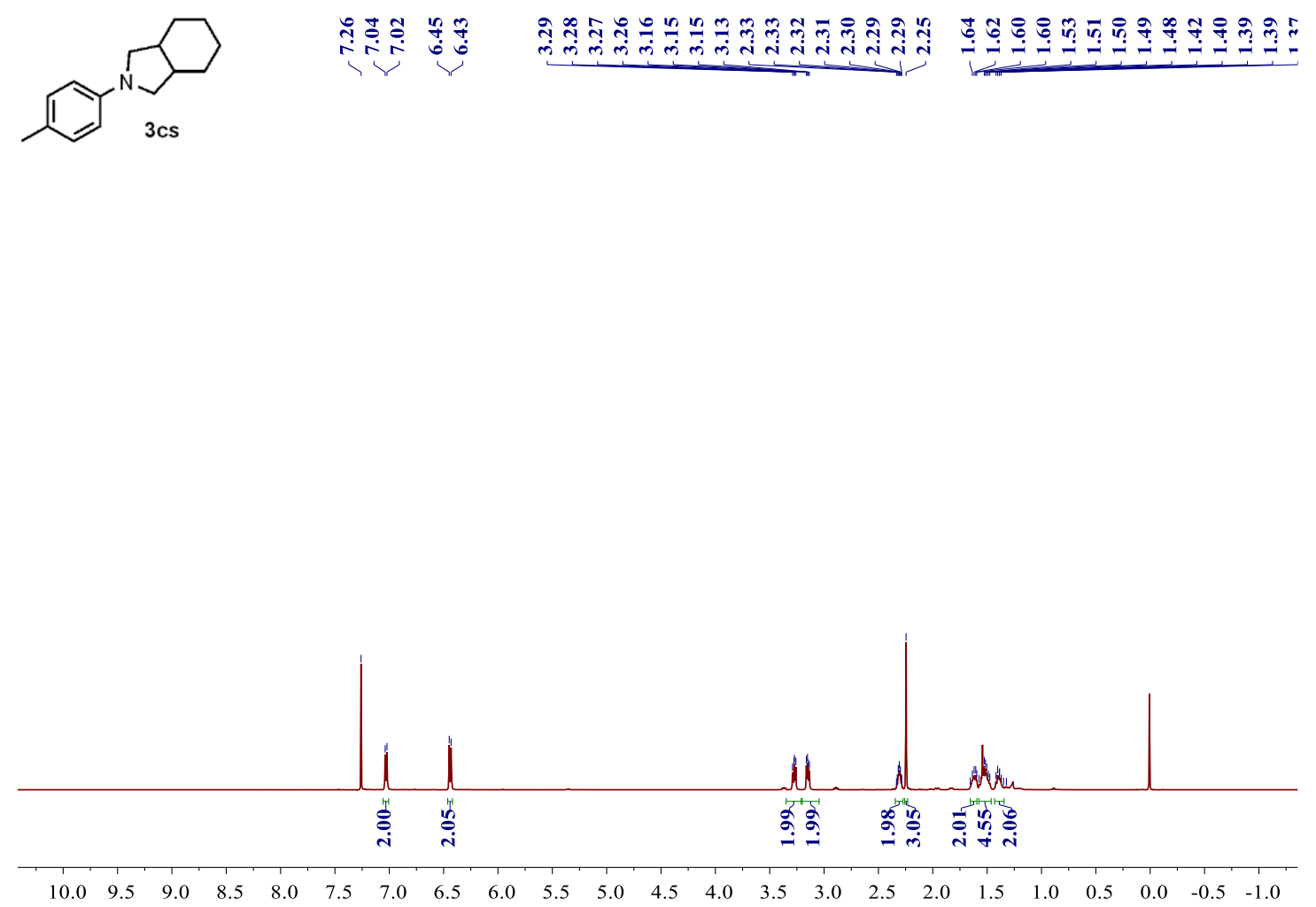

${ }^{13} \mathrm{C} \mathrm{NMR}\left(126 \mathrm{MHz}, \mathrm{CDCl}_{3}\right)$
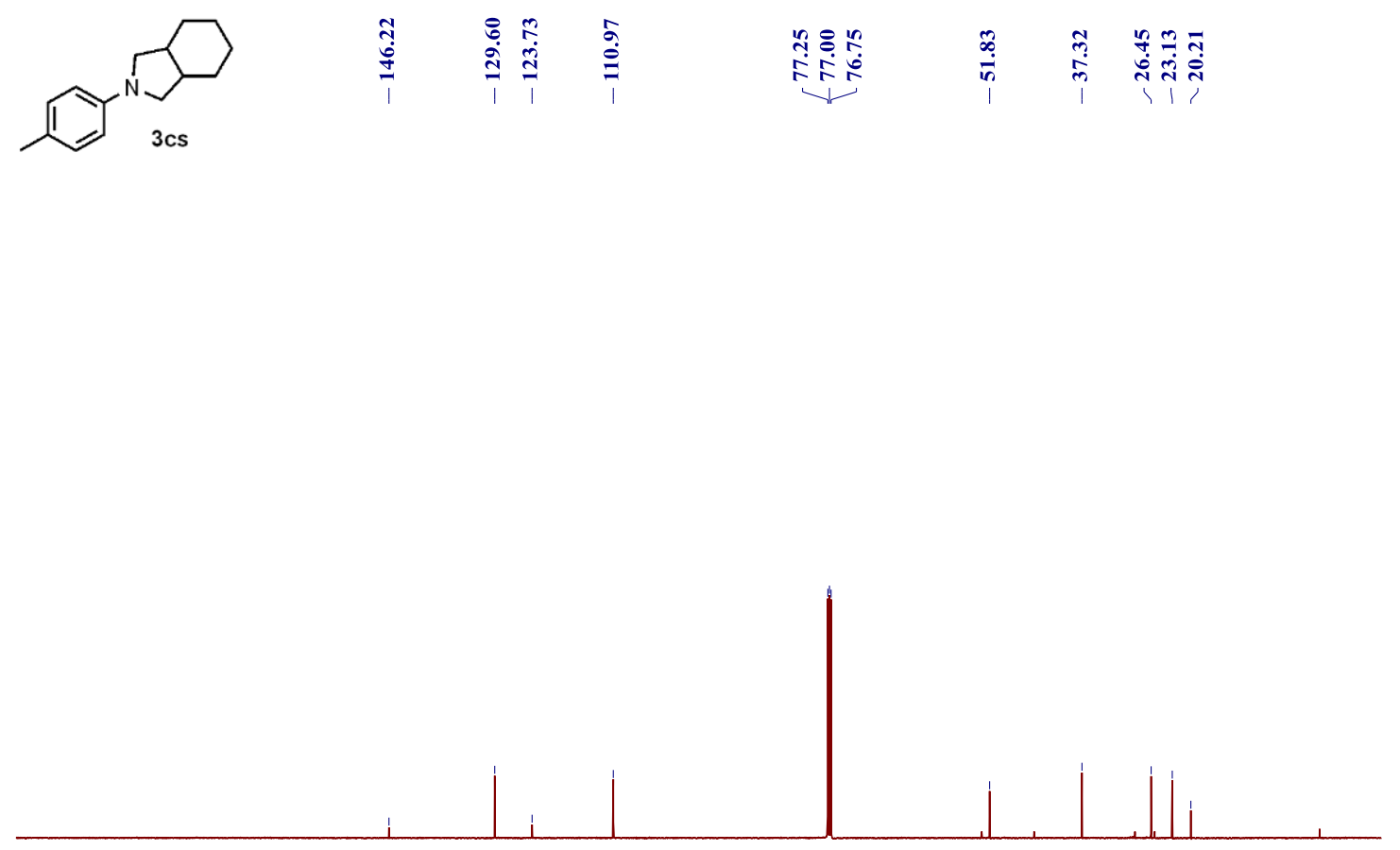

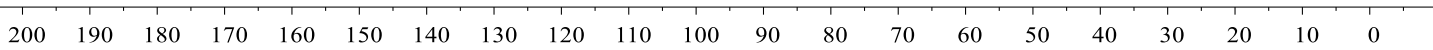


${ }^{1} \mathrm{H} \mathrm{NMR}\left(500 \mathrm{MHz}, \mathrm{CDCl}_{3}\right)$

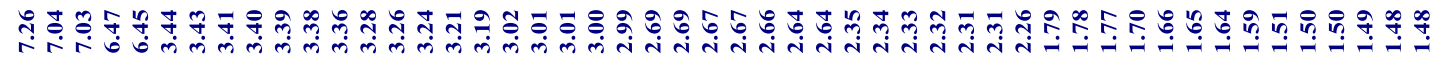<smiles>Cc1ccc(N2CC3CCCNC3C2)cc1</smiles>

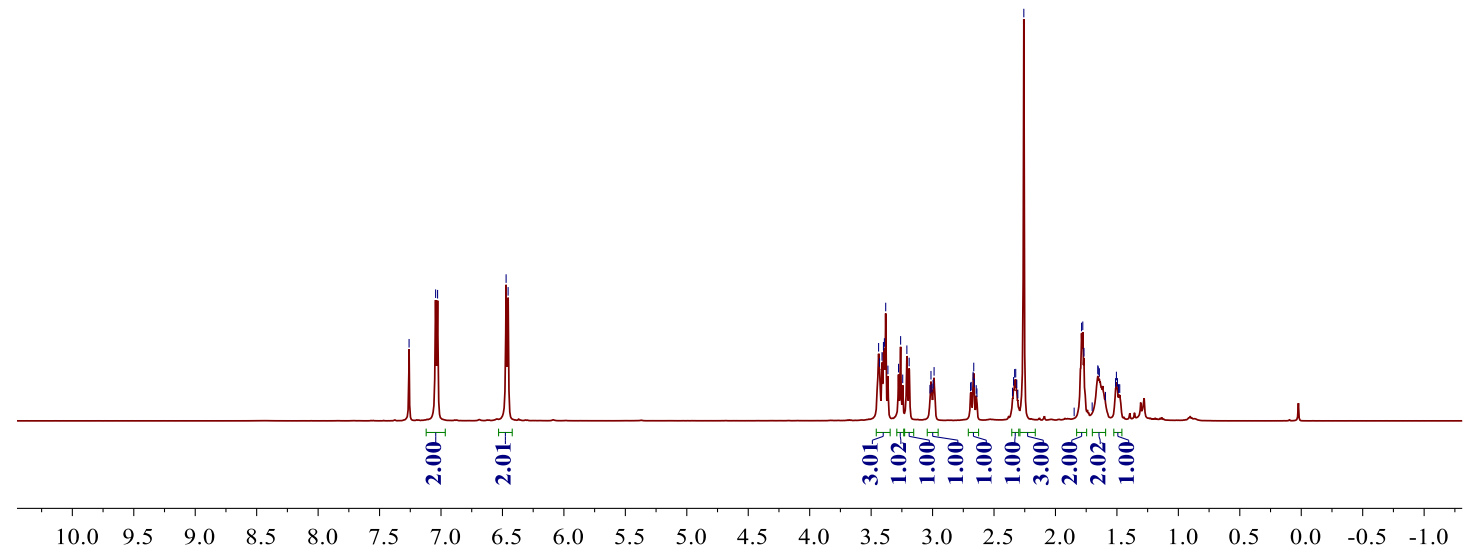

${ }^{13} \mathrm{C}$ NMR $\left(126 \mathrm{MHz}, \mathrm{CDCl}_{3}\right)$

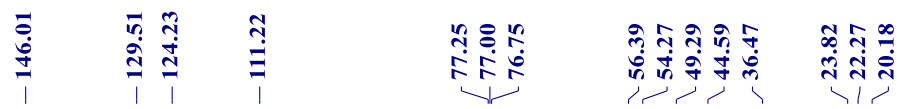<smiles>Cc1ccc(N2CC3CCCNC3C2)cc1</smiles>

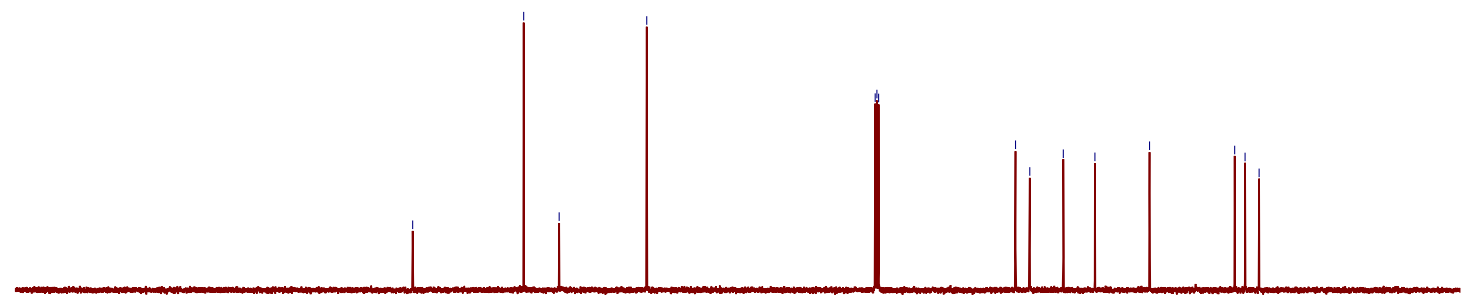

$\begin{array}{llllllllllllllllllll}200 & 190 & 180 & 170 & 160 & 150 & 140 & 130 & 120 & 110 & 100 & 90 & 80 & 70 & 60 & 50 & 40 & 30 & 20 & 10\end{array}$ 
${ }^{1} \mathrm{H} \mathrm{NMR}\left(500 \mathrm{MHz}, \mathrm{CDCl}_{3}\right)$
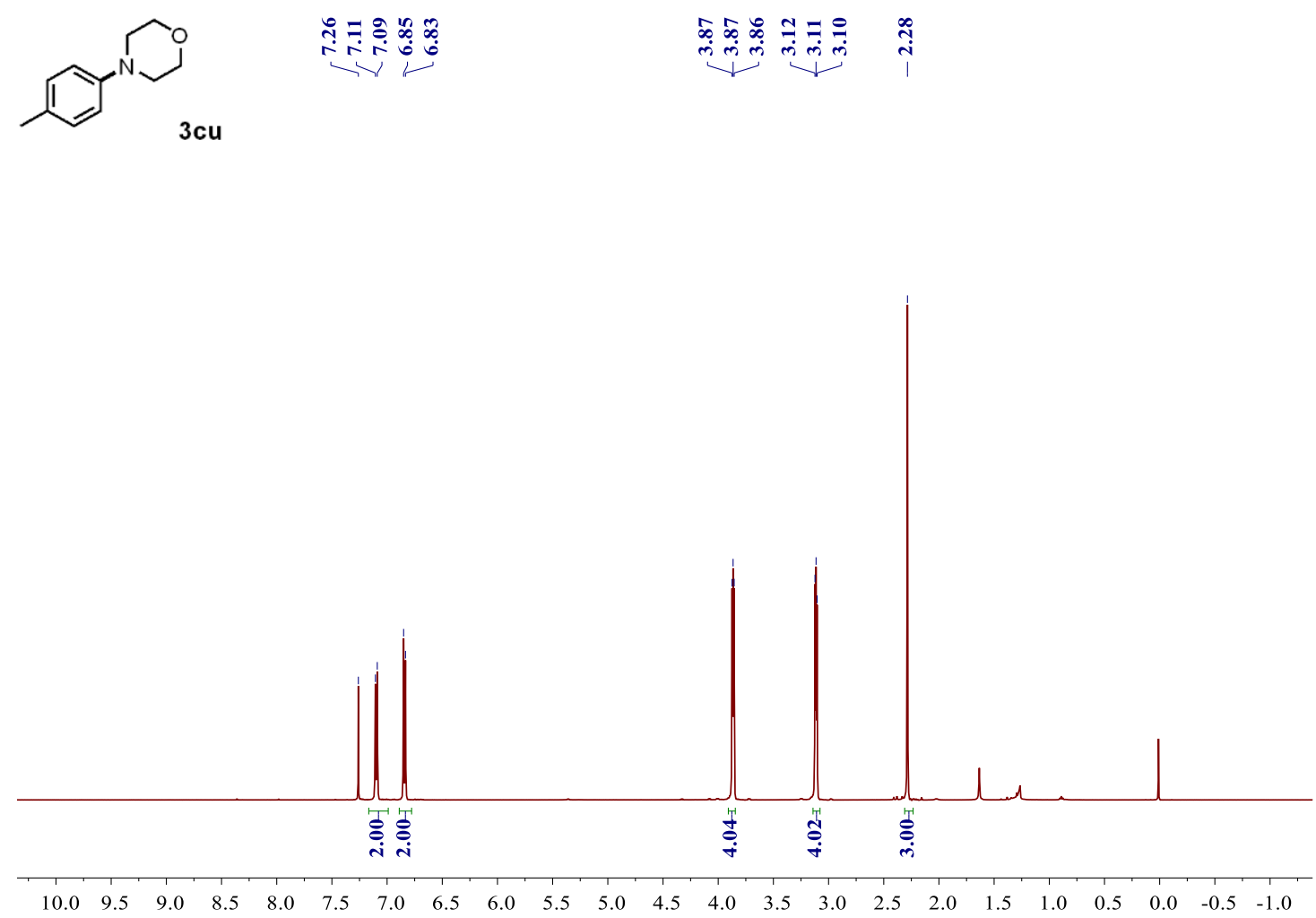

${ }^{13} \mathrm{C} \mathrm{NMR}\left(126 \mathrm{MHz}, \mathrm{CDCl}_{3}\right)$

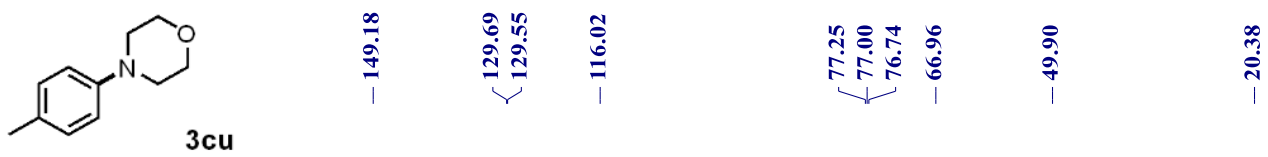

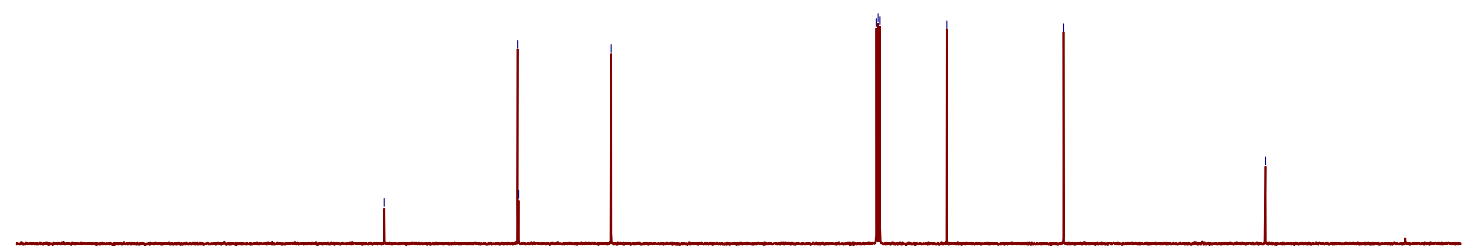

$\begin{array}{llllllllllllllllllllllll}200 & 190 & 180 & 170 & 160 & 150 & 140 & 130 & 120 & 110 & 100 & 90 & 80 & 70 & 60 & 50 & 40 & 30 & 20 & 10 & 0\end{array}$ 
${ }^{1} \mathrm{H}$ NMR $\left(500 \mathrm{MHz}, \mathrm{CDCl}_{3}\right)$

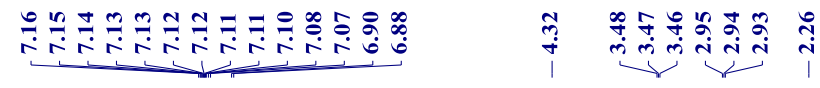<smiles>Cc1ccc(N2CCc3ccccc3C2)cc1</smiles>

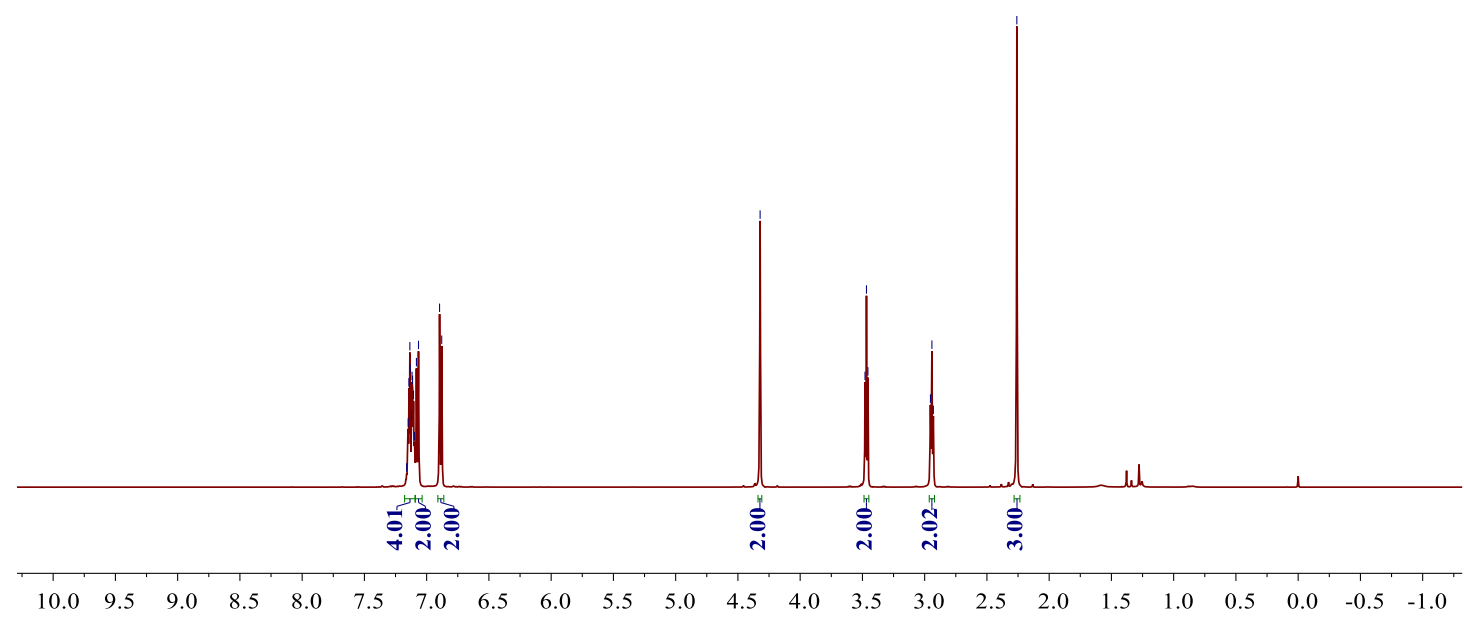

${ }^{13} \mathrm{C} \mathrm{NMR}\left(126 \mathrm{MHz}, \mathrm{CDCl}_{3}\right)$
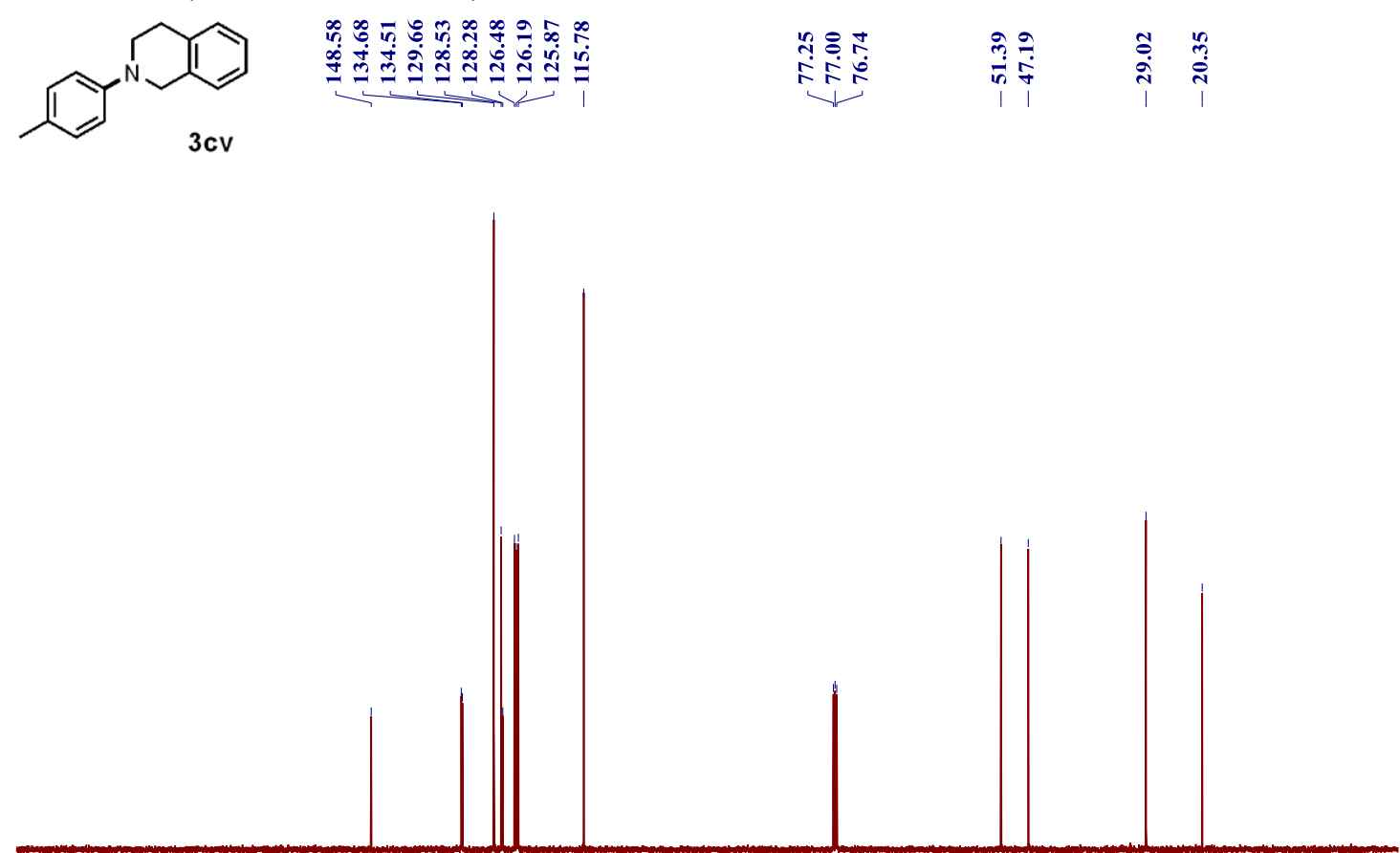

$\begin{array}{lllllllllllllllllllllll}200 & 190 & 180 & 170 & 160 & 150 & 140 & 130 & 120 & 110 & 100 & 90 & 80 & 70 & 60 & 50 & 40 & 30 & 20 & 10 & 0 & 0\end{array}$ 
${ }^{1} \mathrm{H}$ NMR $\left(500 \mathrm{MHz}, \mathrm{CDCl}_{3}\right)$
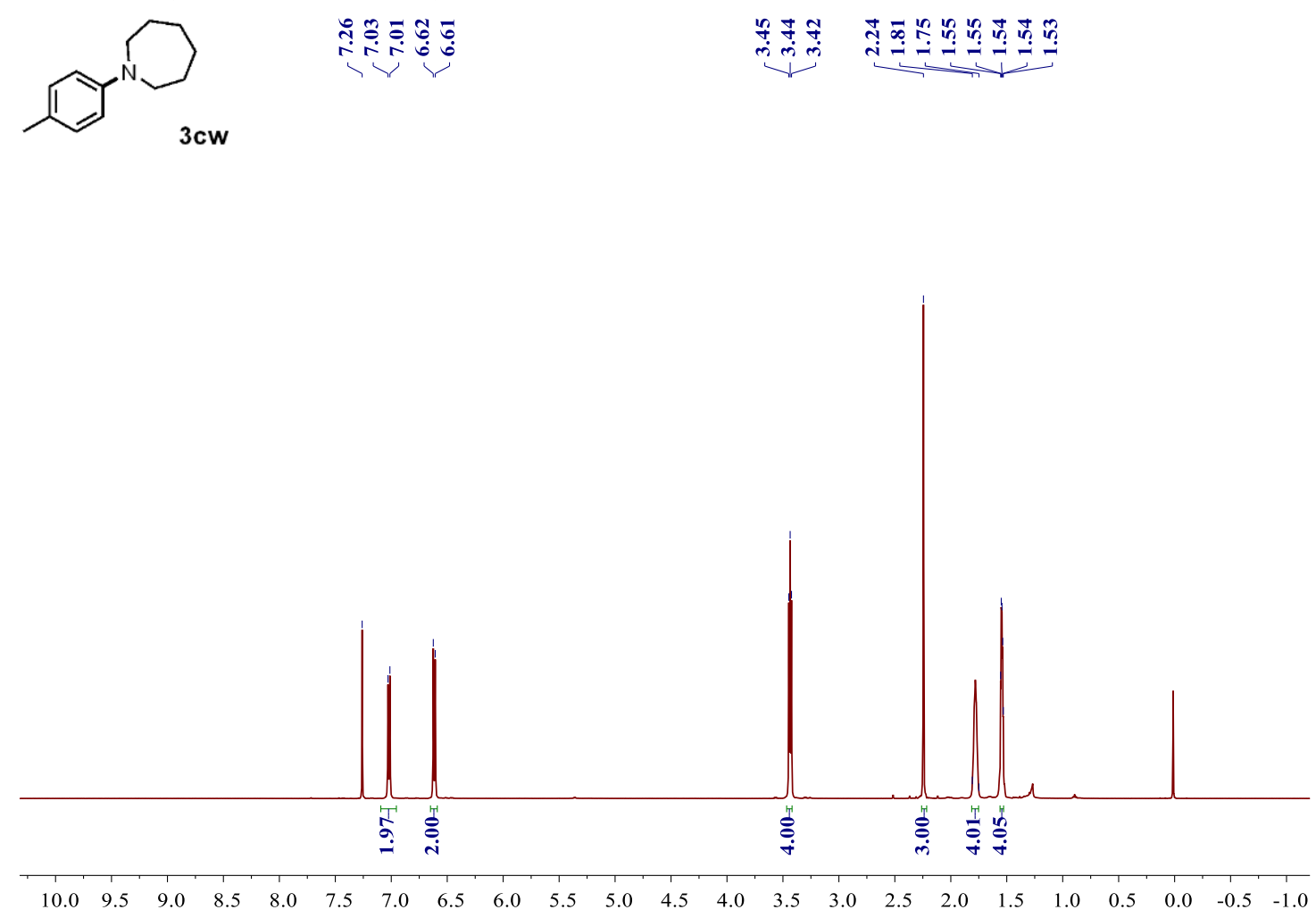

${ }^{13} \mathrm{C} \mathrm{NMR}\left(126 \mathrm{MHz}, \mathrm{CDCl}_{3}\right)$
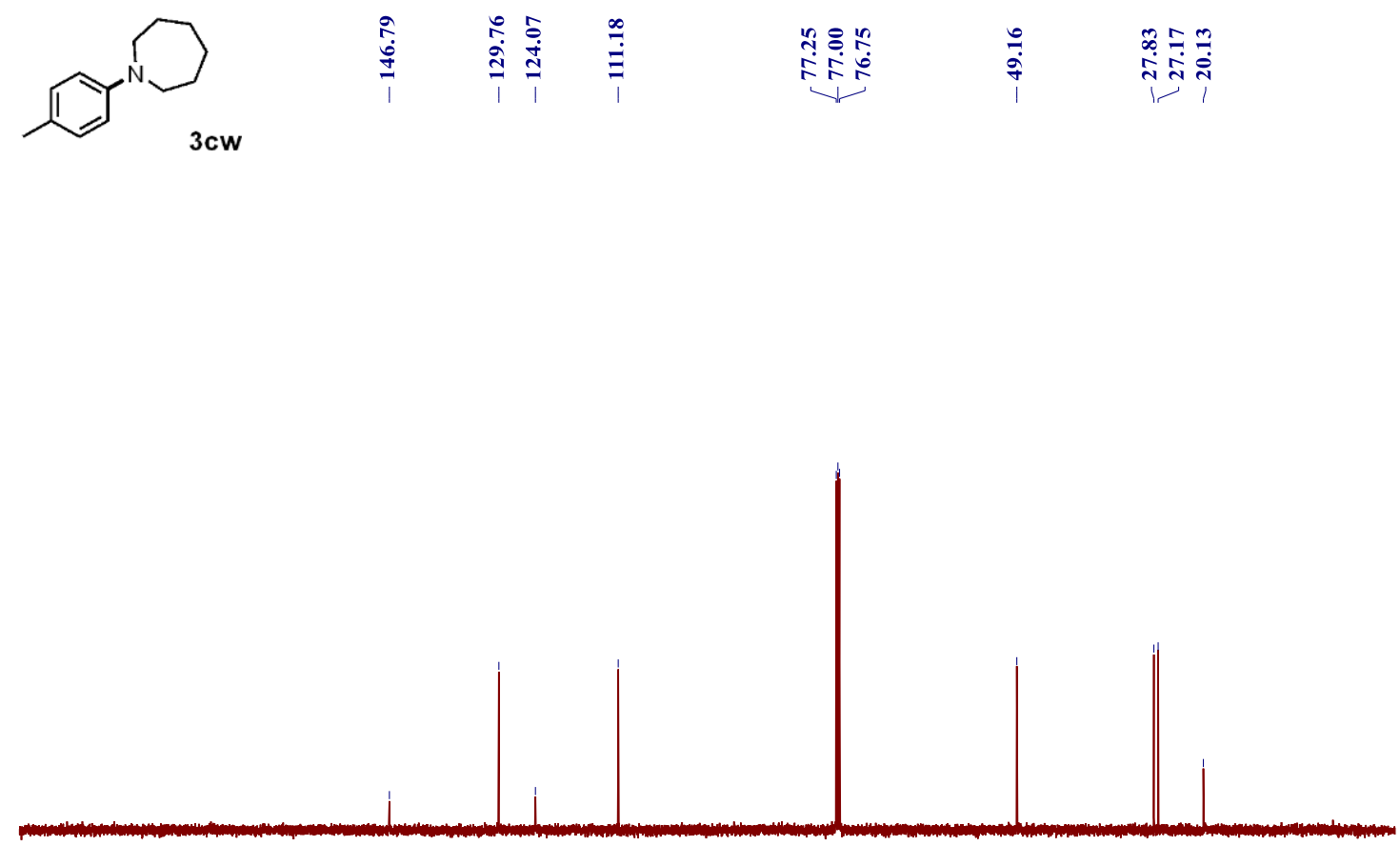

$\begin{array}{llllllllllllllllllllll}200 & 190 & 180 & 170 & 160 & 150 & 140 & 130 & 120 & 110 & 100 & 90 & 80 & 70 & 60 & 50 & 40 & 30 & 20 & 10 & 0 & 0\end{array}$ 
${ }^{1} \mathrm{H} \mathrm{NMR}\left(500 \mathrm{MHz}, \mathrm{CDCl}_{3}\right)$
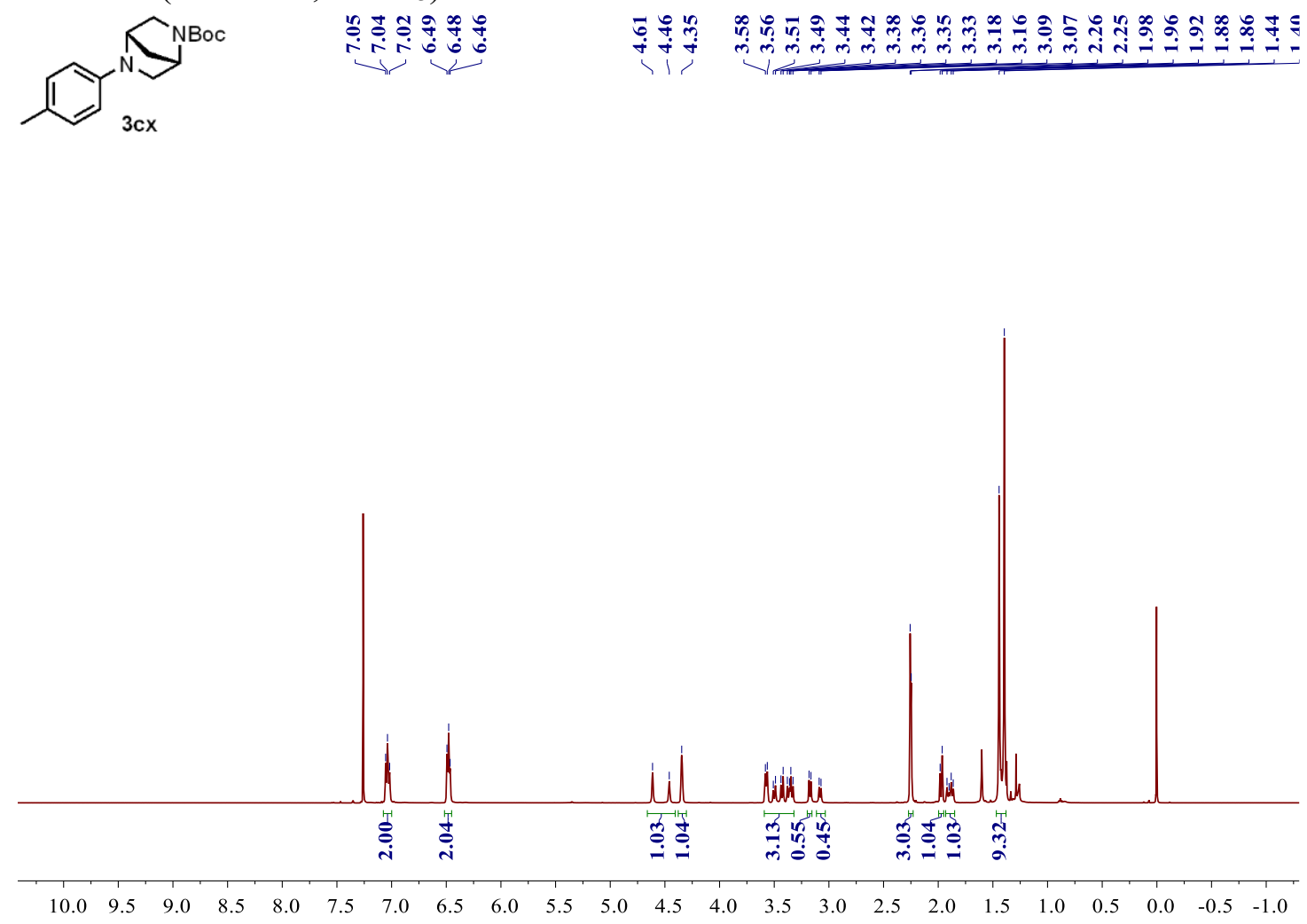

$\left.{ }^{13} \mathrm{C} \mathrm{NMR} \mathrm{(126} \mathrm{MHz,} \mathrm{CDCl}_{3}\right)$

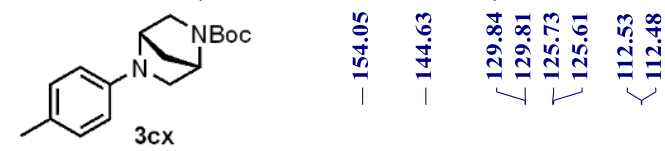

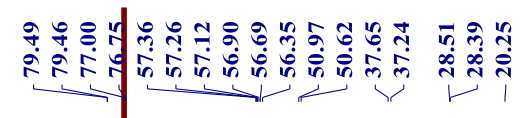

$\begin{array}{lllllllllllllllllllll}200 & 190 & 180 & 170 & 160 & 150 & 140 & 130 & 120 & 110 & 100 & 90 & 80 & 70 & 60 & 50 & 40 & 30 & 20 & 10 & 0\end{array}$ 
${ }^{1} \mathrm{H} \mathrm{NMR}\left(500 \mathrm{MHz}, \mathrm{CDCl}_{3}\right)$
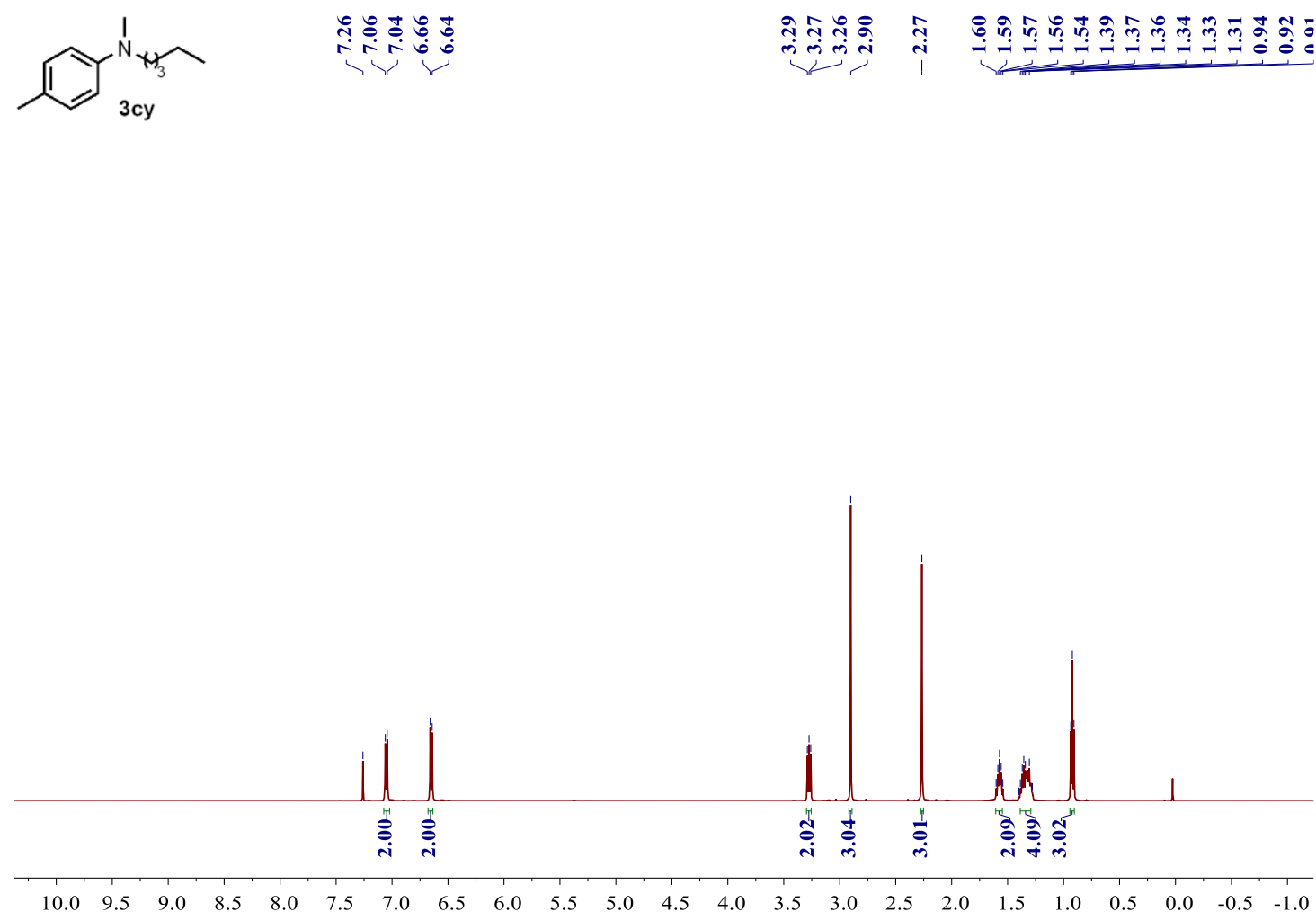

${ }^{13} \mathrm{C} \mathrm{NMR}\left(126 \mathrm{MHz}, \mathrm{CDCl}_{3}\right)$

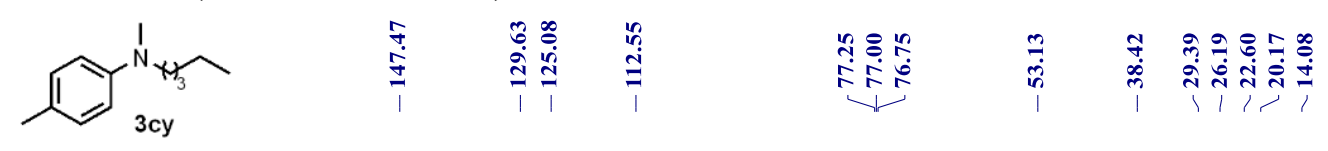

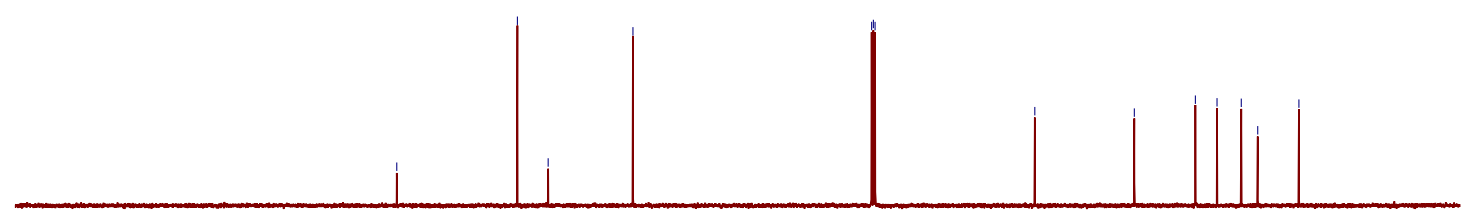

$\begin{array}{llllllllllllllllllllllll}200 & 190 & 180 & 170 & 160 & 150 & 140 & 130 & 120 & 110 & 100 & 90 & 80 & 70 & 60 & 50 & 40 & 30 & 20 & 10 & 0 & 0\end{array}$ 
${ }^{1} \mathrm{H} \mathrm{NMR}\left(500 \mathrm{MHz}, \mathrm{CDCl}_{3}\right)$

$\underbrace{\cdots}$<smiles>Cc1ccc(N(C)Cc2ccccc2)cc1</smiles>

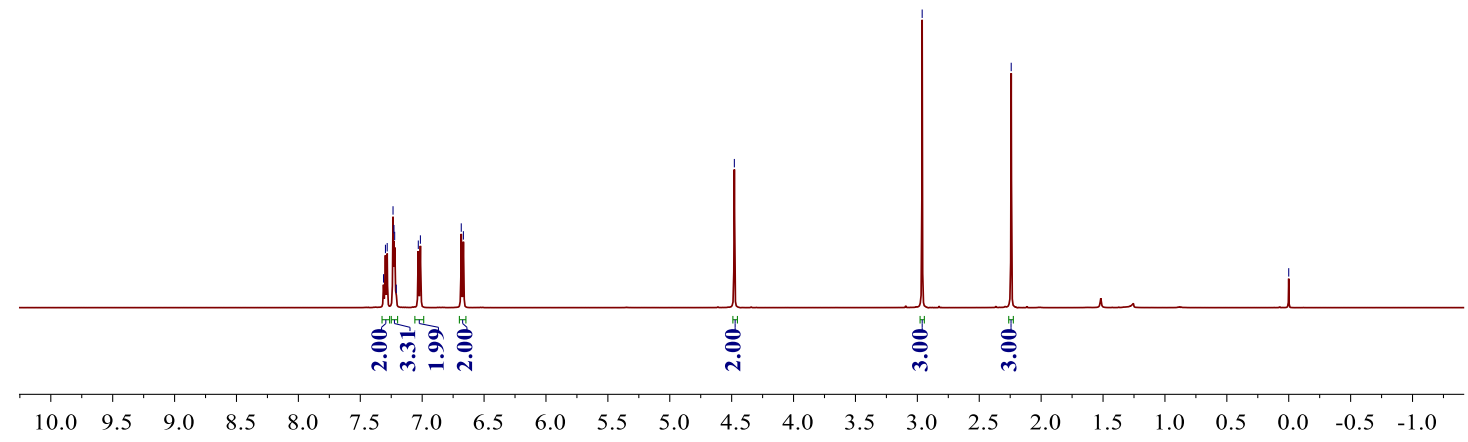

${ }^{13} \mathrm{C}$ NMR $\left(126 \mathrm{MHz}, \mathrm{CDCl}_{3}\right)$

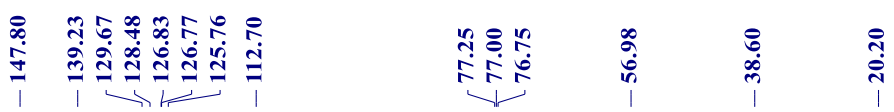<smiles>Cc1ccc(N(C)Cc2ccccc2)cc1</smiles>

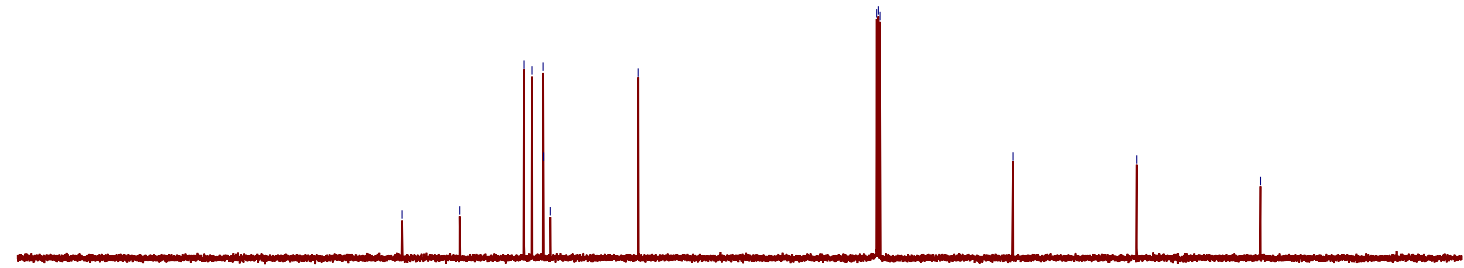

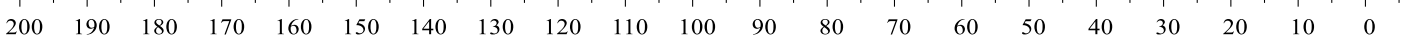


${ }^{1} \mathrm{H}$ NMR (500 MHz, $\left.\mathrm{CDCl}_{3}\right)$

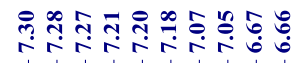

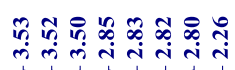

$\stackrel{8}{i}$

$\overbrace{3 d a}^{N}$

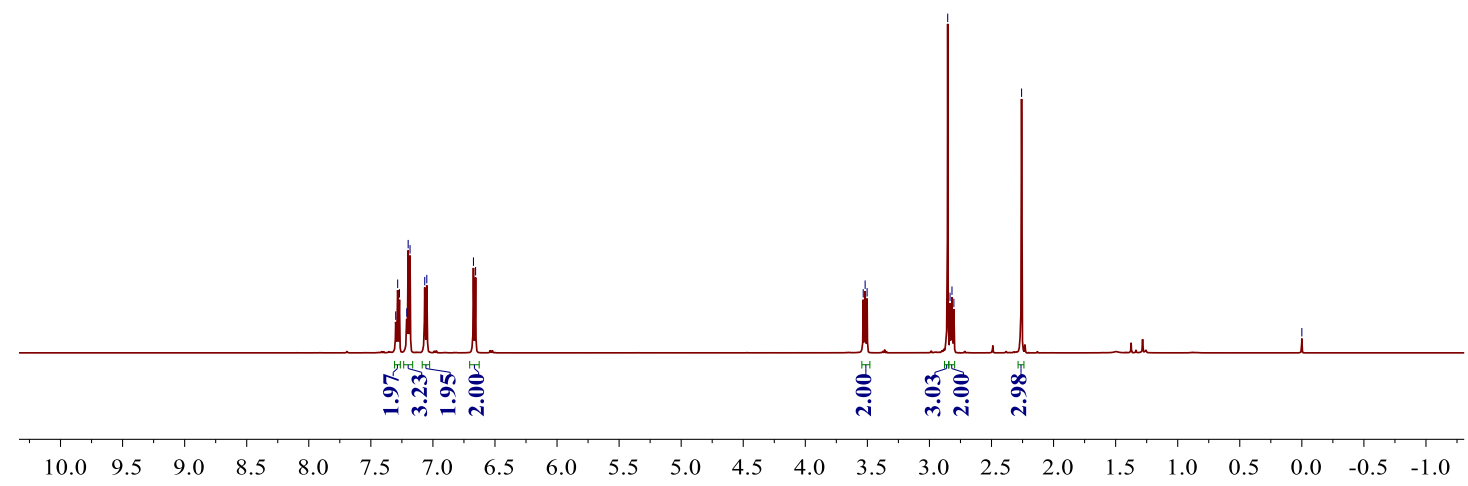

$\left.{ }^{13} \mathrm{C} \mathrm{NMR} \mathrm{(126} \mathrm{MHz,} \mathrm{CDCl}_{3}\right)$

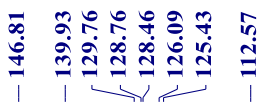

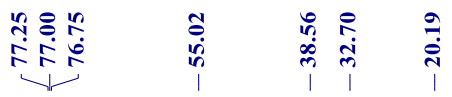<smiles>Cc1ccc(N(C)CCc2ccccc2)cc1</smiles>

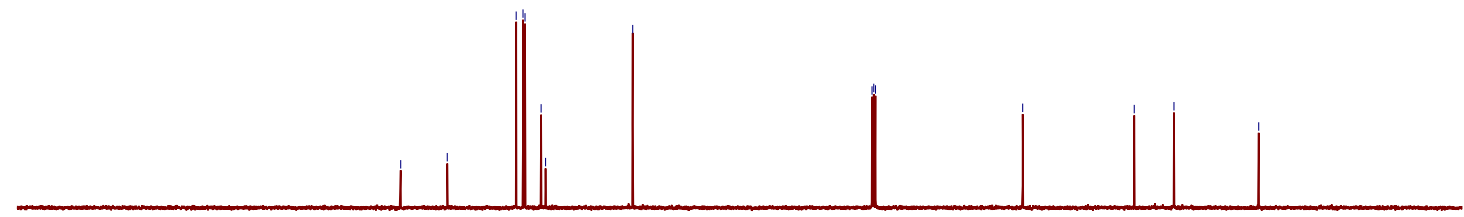

$\begin{array}{llllllllllllllllllllllll}200 & 190 & 180 & 170 & 160 & 150 & 140 & 130 & 120 & 110 & 100 & 90 & 80 & 70 & 60 & 50 & 40 & 30 & 20 & 10 & 0\end{array}$ 
${ }^{1} \mathrm{H}$ NMR $\left(500 \mathrm{MHz}, \mathrm{CDCl}_{3}\right)$
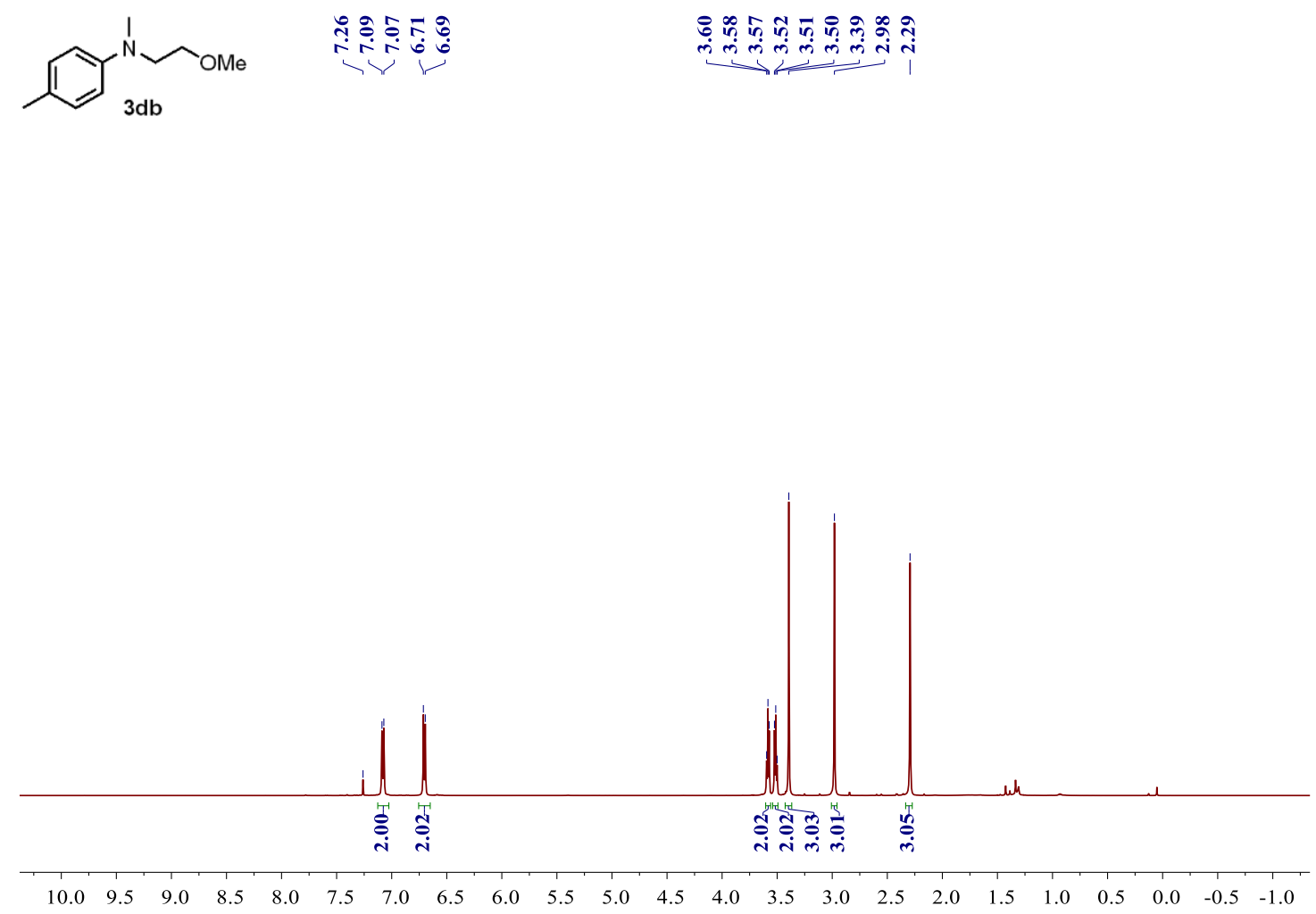

${ }^{13} \mathrm{C} \mathrm{NMR}\left(126 \mathrm{MHz}, \mathrm{CDCl}_{3}\right)$

$\overbrace{3 \mathrm{db}}^{\mathrm{N}} \mathrm{N}_{\mathrm{OMe}}$ 旁

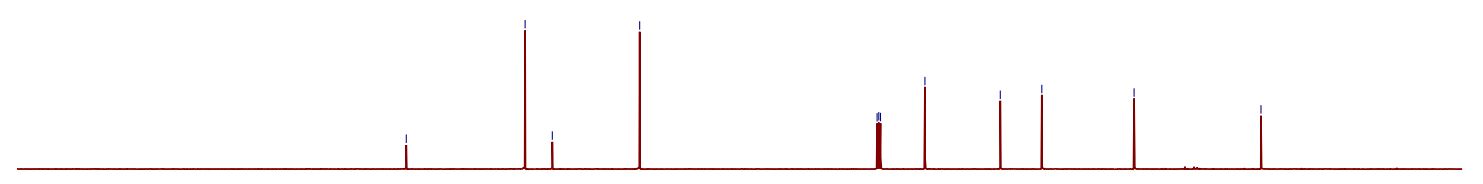

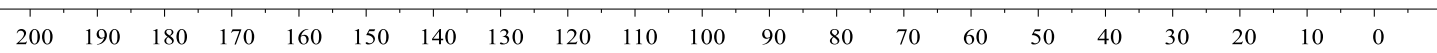


${ }^{1} \mathrm{H}$ NMR $\left(500 \mathrm{MHz}, \mathrm{CDCl}_{3}\right)$

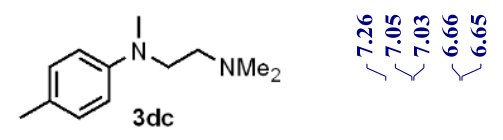

Fैं

$3 d c$

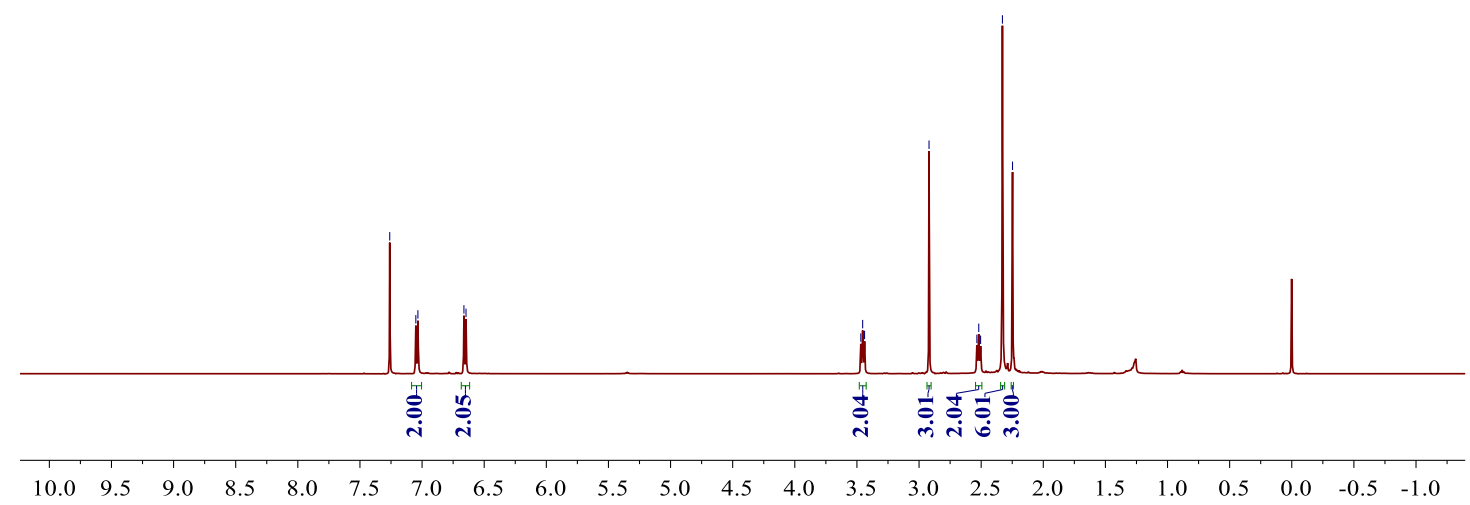

${ }^{13} \mathrm{C} \mathrm{NMR}\left(126 \mathrm{MHz}, \mathrm{CDCl}_{3}\right)$

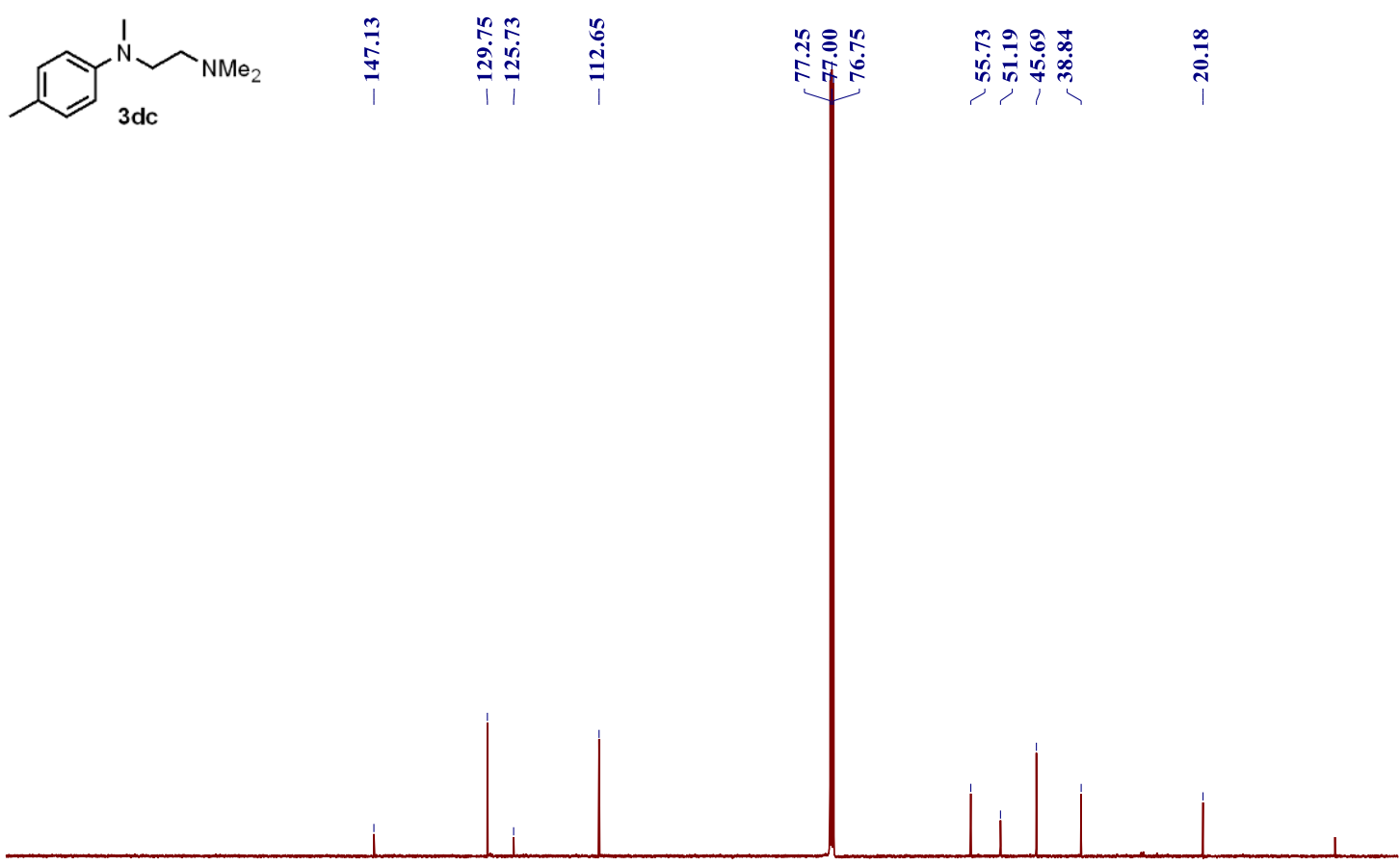

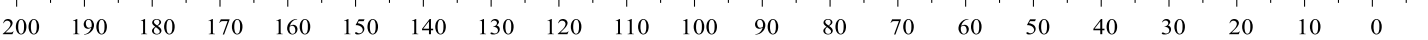


${ }^{1} \mathrm{H} \mathrm{NMR}\left(500 \mathrm{MHz}, \mathrm{CDCl}_{3}\right)$
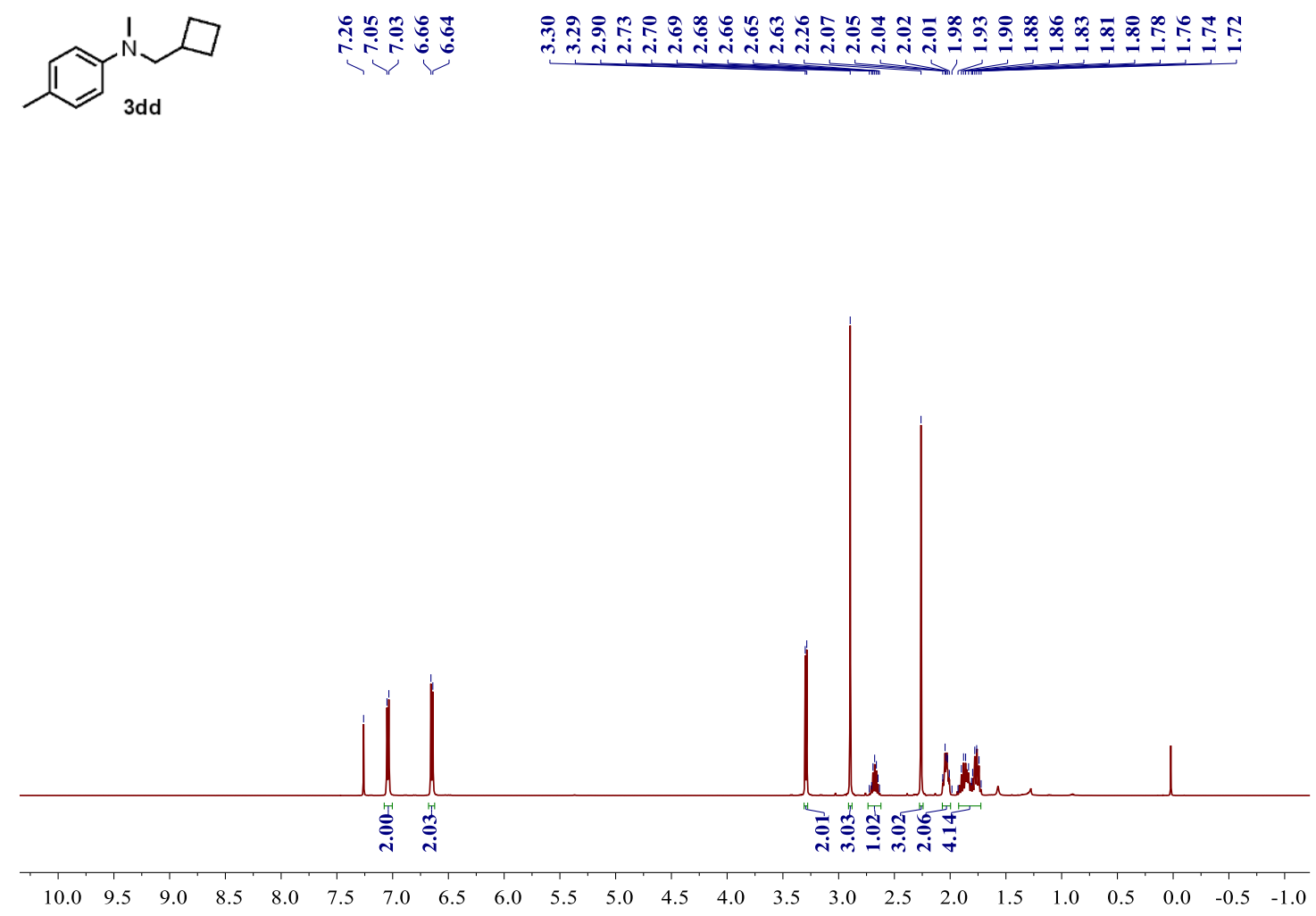

${ }^{13} \mathrm{C}$ NMR $\left(126 \mathrm{MHz}, \mathrm{CDCl}_{3}\right)$

$\overbrace{3 \mathrm{dd}}^{1}$

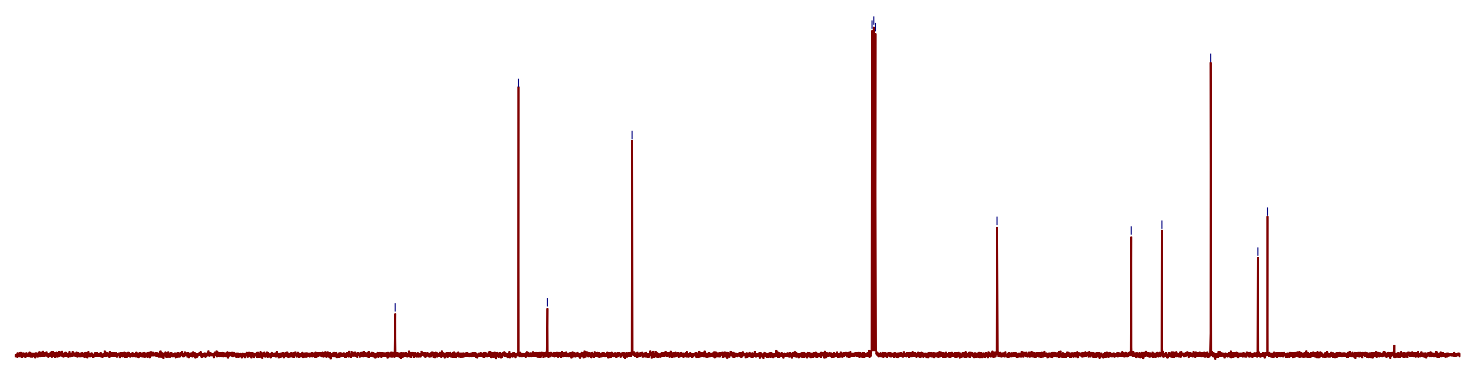

$\begin{array}{lllllllllllllllllllllll}200 & 190 & 180 & 170 & 160 & 150 & 140 & 130 & 120 & 110 & 100 & 90 & 80 & 70 & 60 & 50 & 40 & 30 & 20 & 10 & 0\end{array}$ 
${ }^{1} \mathrm{H}$ NMR $\left(500 \mathrm{MHz}, \mathrm{CDCl}_{3}\right)$
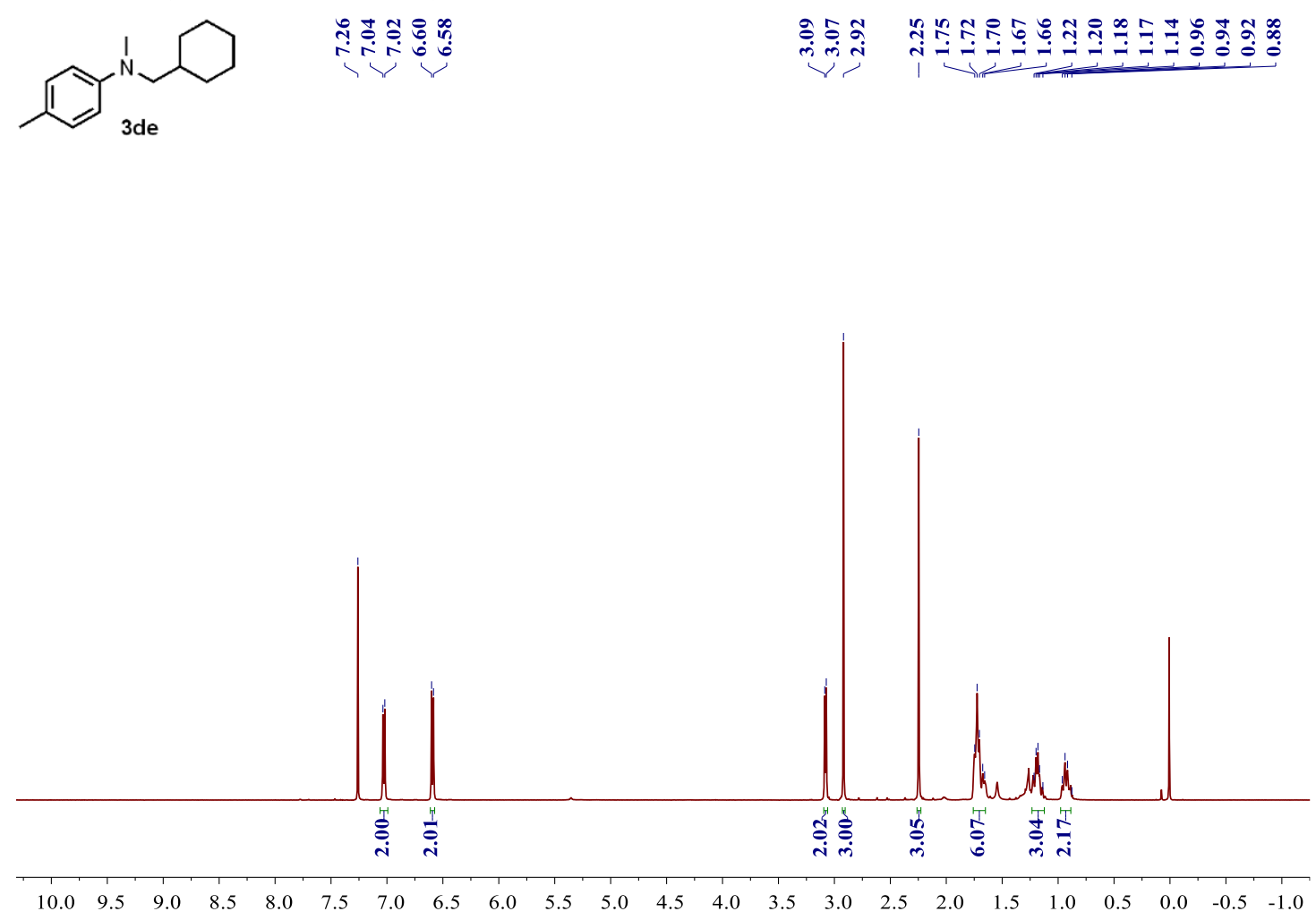

${ }^{13} \mathrm{C} \mathrm{NMR}\left(126 \mathrm{MHz}, \mathrm{CDCl}_{3}\right)$

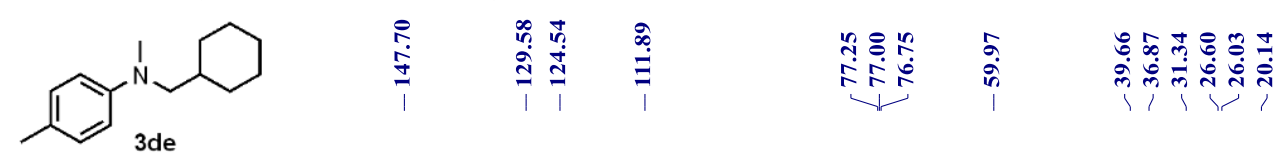

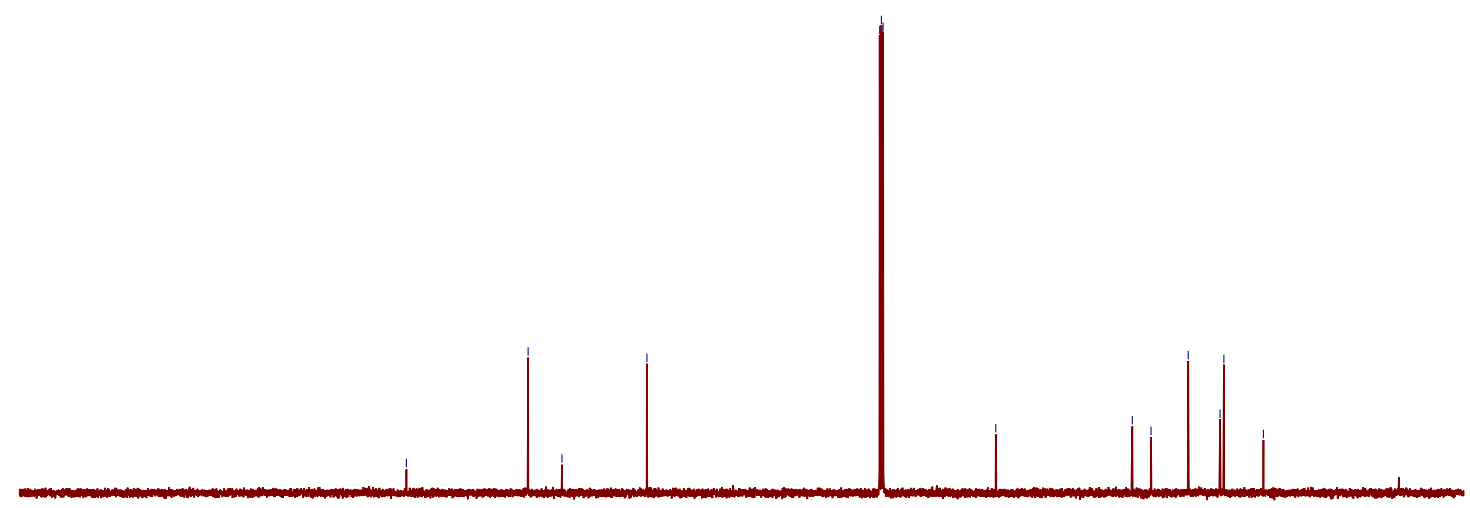

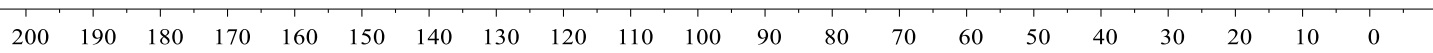


${ }^{1} \mathrm{H} \mathrm{NMR}\left(500 \mathrm{MHz}, \mathrm{CDCl}_{3}\right)$

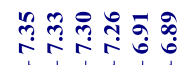

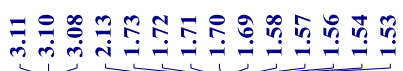

皮

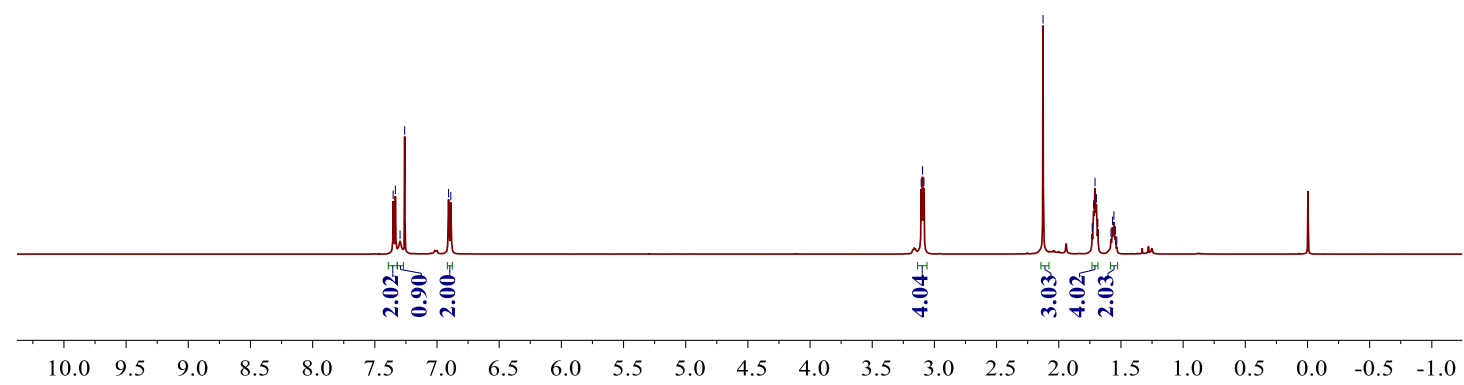

${ }^{13} \mathrm{C} \mathrm{NMR}\left(126 \mathrm{MHz}, \mathrm{CDCl}_{3}\right)$

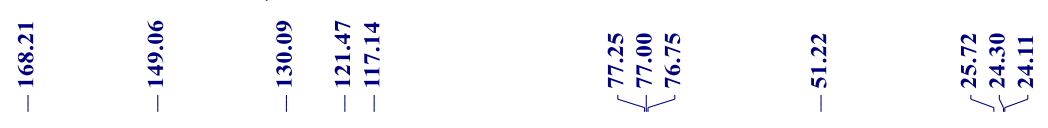

皮

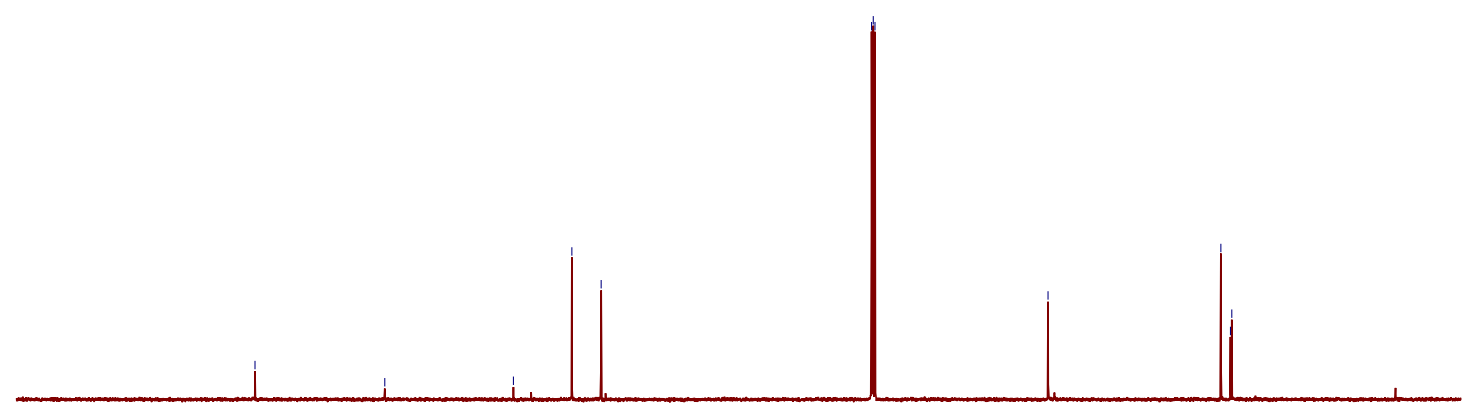

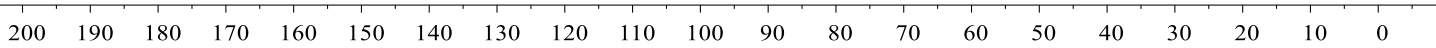


${ }^{1} \mathrm{H}$ NMR $\left(500 \mathrm{MHz}, \mathrm{CDCl}_{3}\right)$
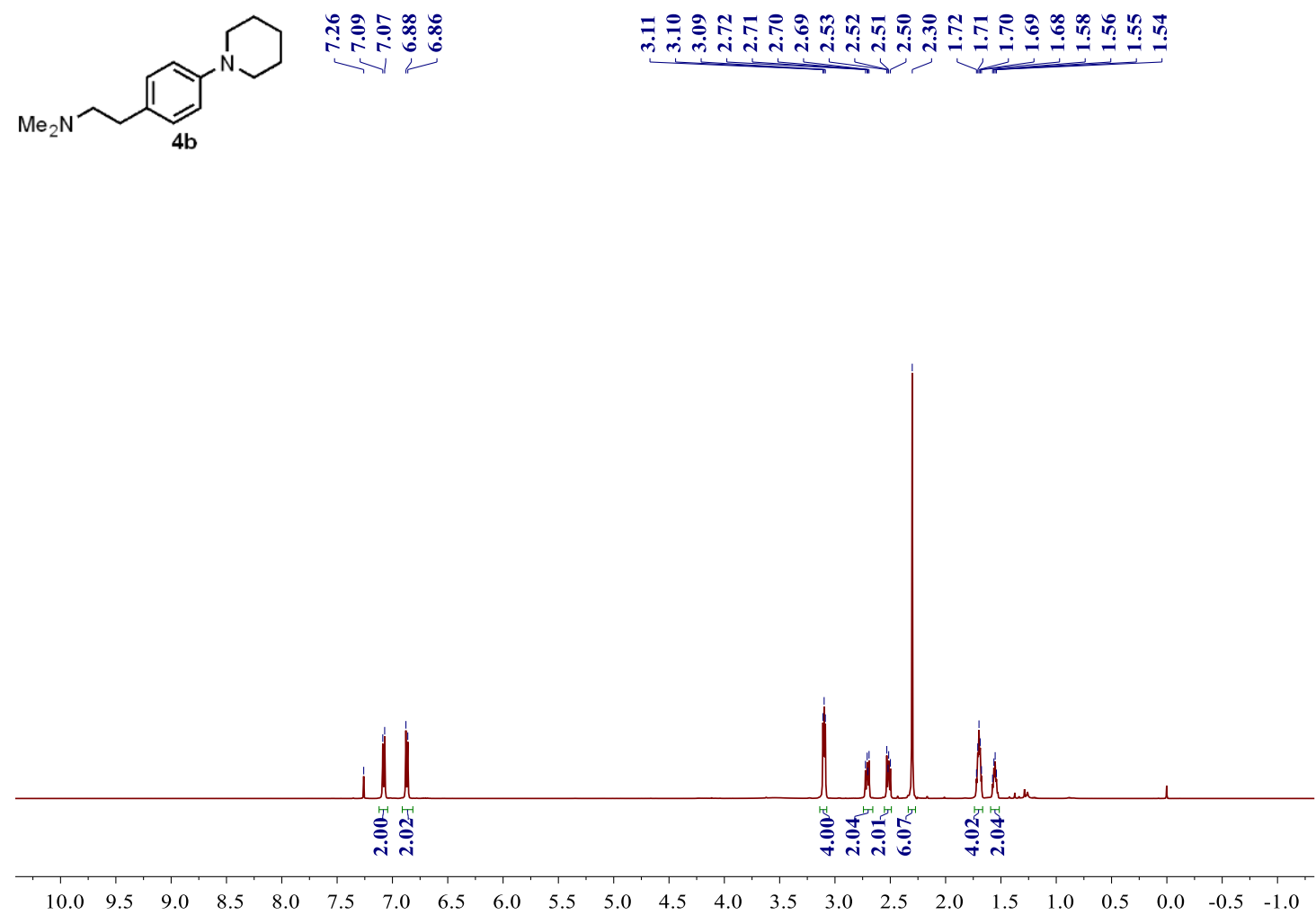

${ }^{13} \mathrm{C} \mathrm{NMR}\left(126 \mathrm{MHz}, \mathrm{CDCl}_{3}\right)$
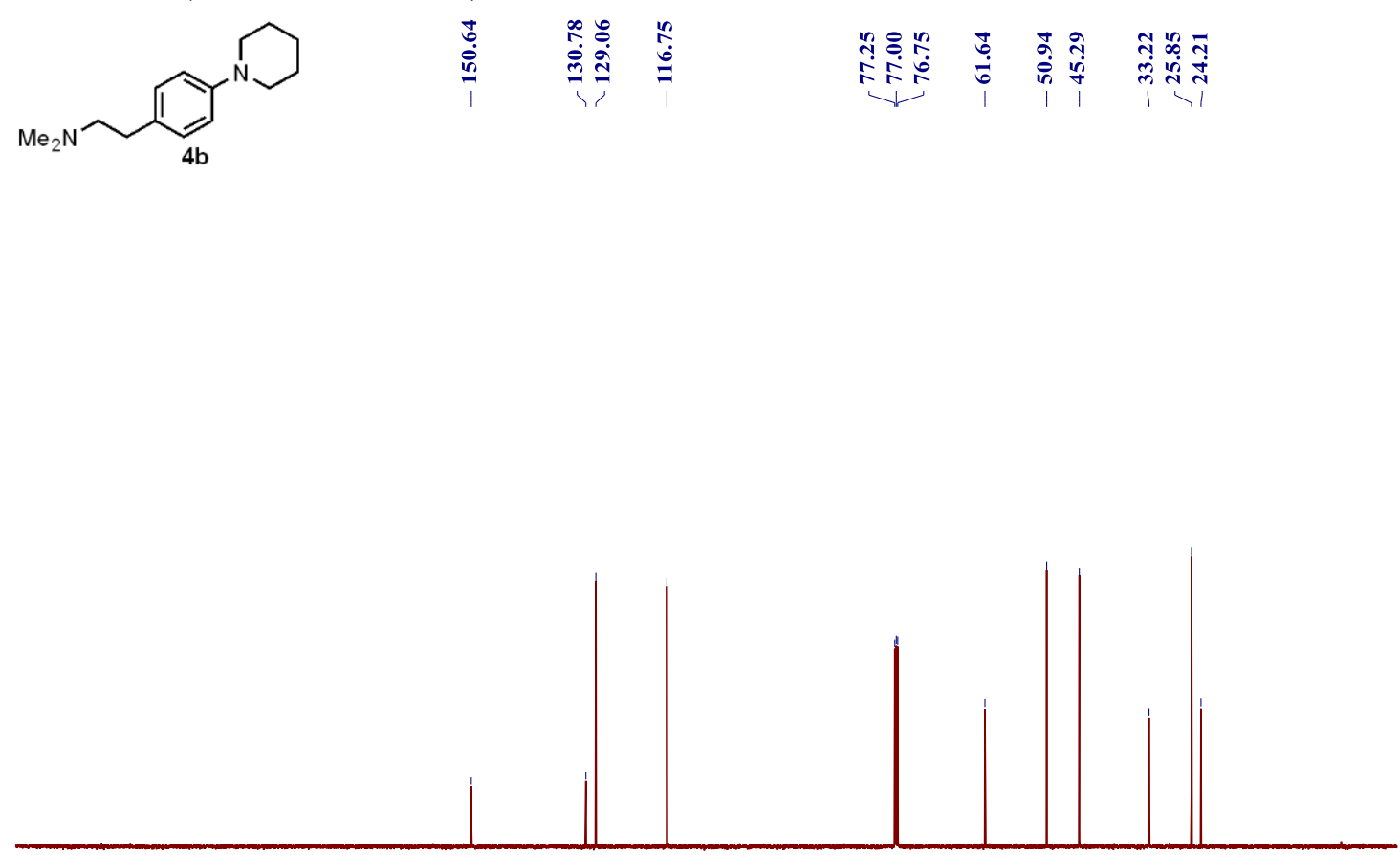

$\begin{array}{lllllllllllllllllllllllllll}220 & 210 & 200 & 190 & 180 & 170 & 160 & 150 & 140 & 130 & 120 & 110 & 100 & 90 & 80 & 70 & 60 & 50 & 40 & 30 & 20 & 10 & 0\end{array}$ 
${ }^{1} \mathrm{H}$ NMR $\left(500 \mathrm{MHz}, \mathrm{CDCl}_{3}\right)$

$$
\text { 年 }
$$<smiles>CC(C)(c1ccc(N2CCCCC2)cc1)C(Cl)(Cl)c1ccc(N2CCCCC2)cc1</smiles>

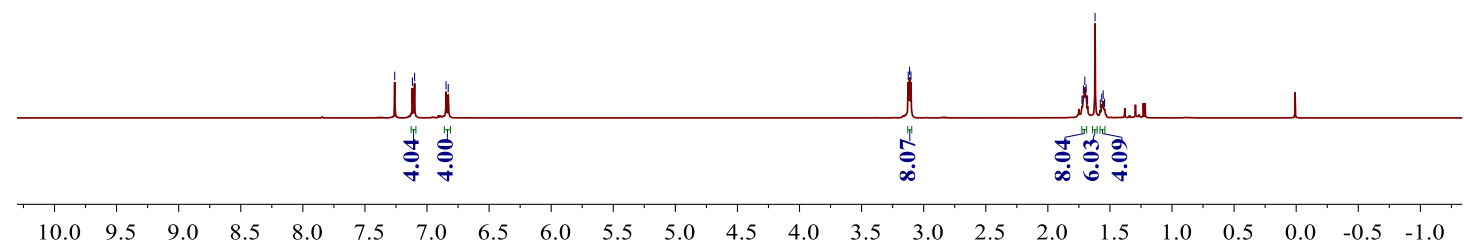

${ }^{13} \mathrm{C} \mathrm{NMR}\left(126 \mathrm{MHz}, \mathrm{CDCl}_{3}\right)$

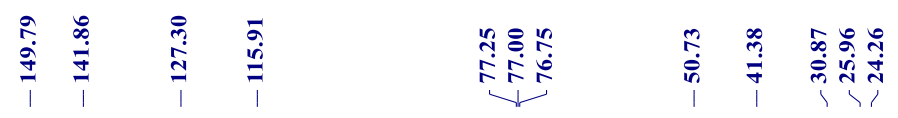<smiles>CC(C)(c1ccc(N2CCCCC2)cc1)c1ccc(N2CCCCC2)cc1</smiles>

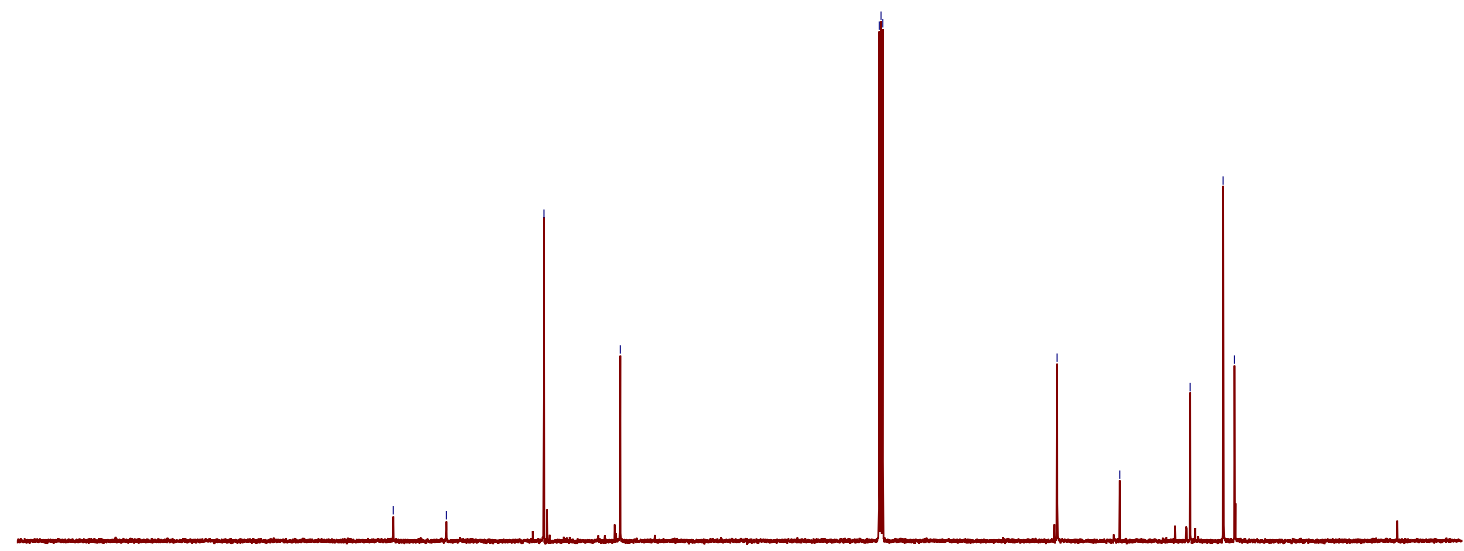

$\begin{array}{lllllllllllllllllllll}200 & 190 & 180 & 170 & 160 & 150 & 140 & 130 & 120 & 110 & 100 & 90 & 80 & 70 & 60 & 50 & 40 & 30 & 20 & 10 & 0\end{array}$ 
${ }^{1} \mathrm{H} \mathrm{NMR}\left(500 \mathrm{MHz}, \mathrm{CDCl}_{3}\right)$

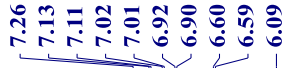

$\underbrace{ \pm}$
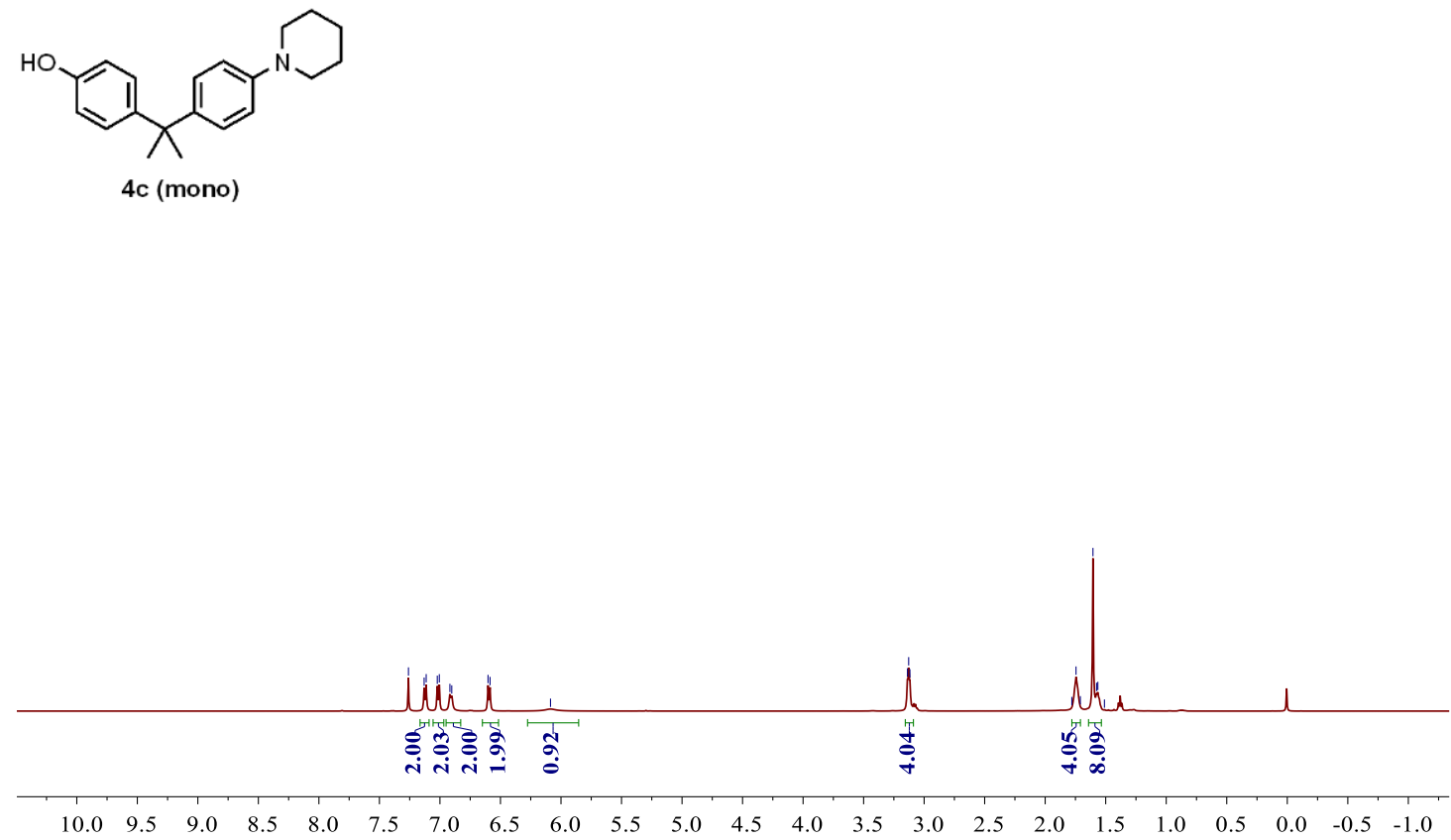

${ }^{13} \mathrm{C}$ NMR (126 MHz, $\left.\mathrm{CDCl}_{3}\right)$

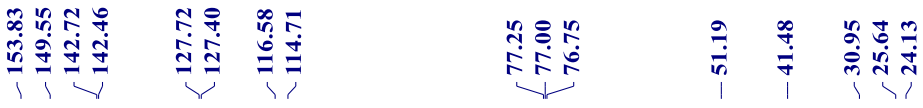<smiles>CC(C)(c1ccc(O)cc1)c1ccc(N2CCCCC2)cc1</smiles>

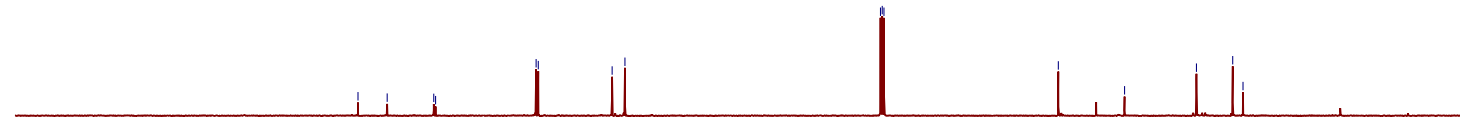

$\begin{array}{lllllllllllllllllllllll}200 & 190 & 180 & 170 & 160 & 150 & 140 & 130 & 120 & 110 & 100 & 90 & 80 & 70 & 60 & 50 & 40 & 30 & 20 & 10 & 0\end{array}$ 
${ }^{1} \mathrm{H}$ NMR $\left(500 \mathrm{MHz}, \mathrm{CDCl}_{3}\right)$
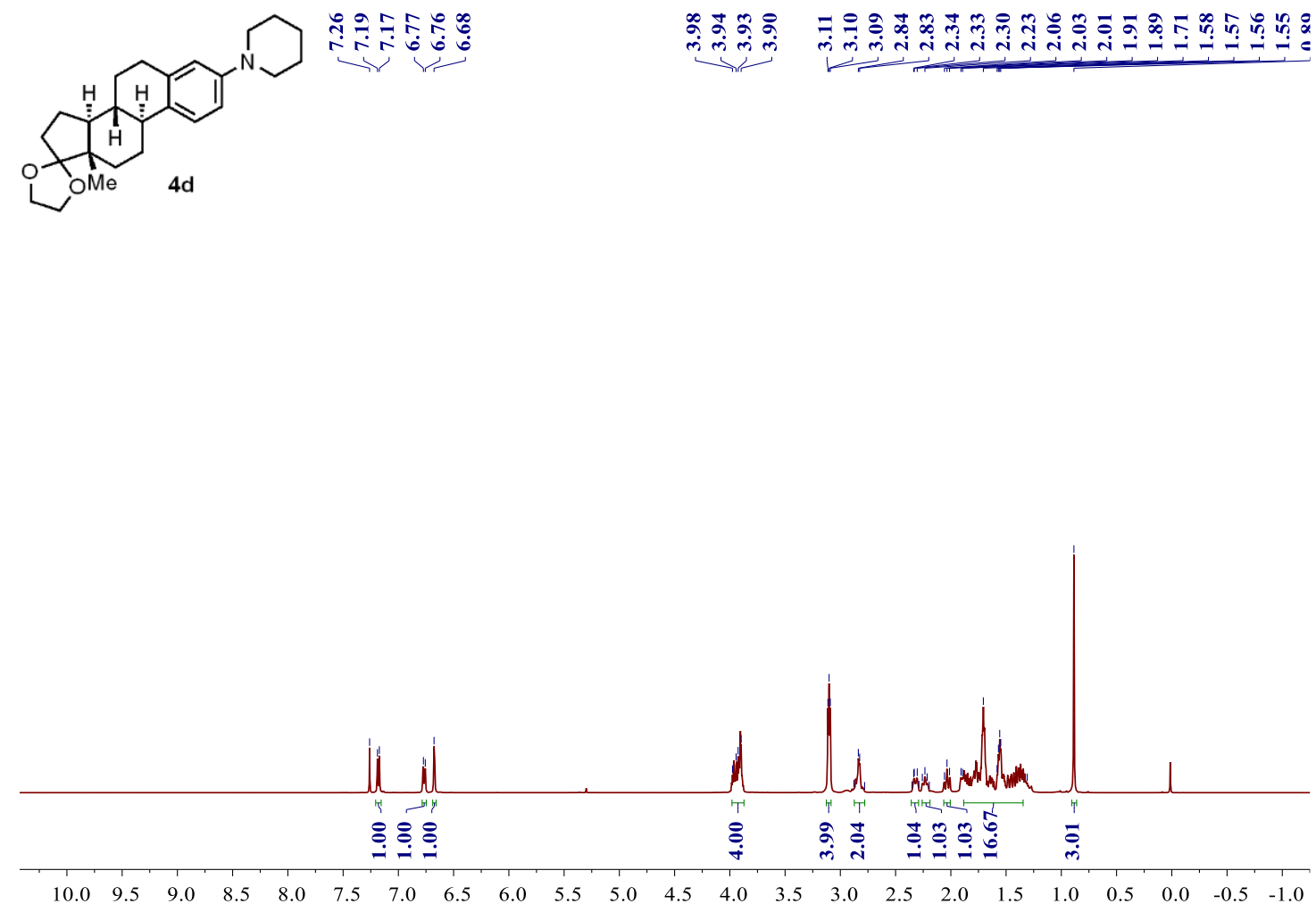

${ }^{13} \mathrm{C} \mathrm{NMR}\left(126 \mathrm{MHz}, \mathrm{CDCl}_{3}\right)$

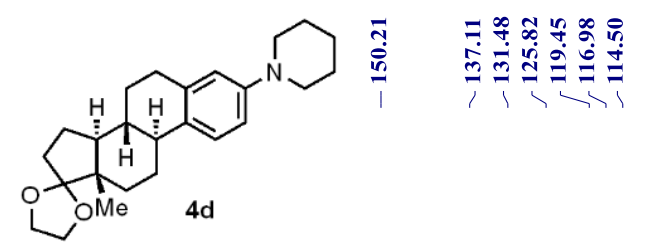

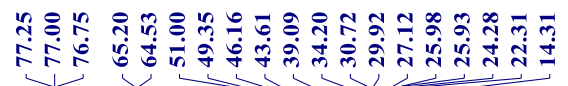

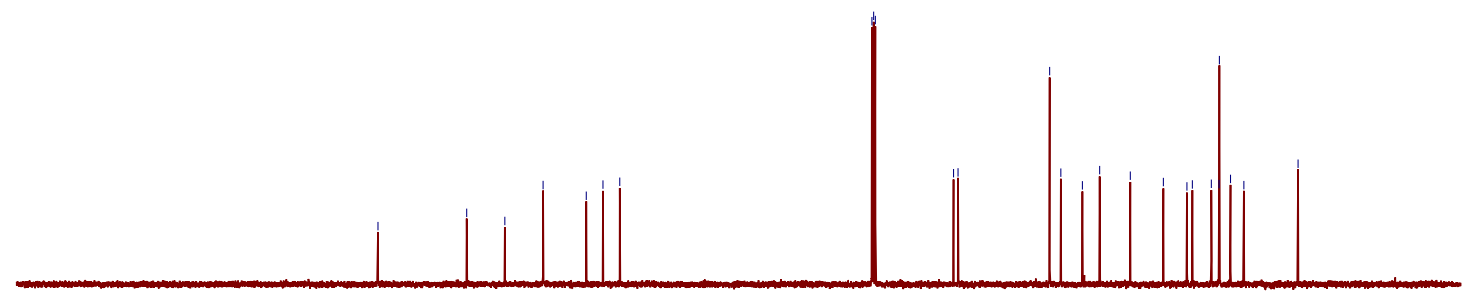

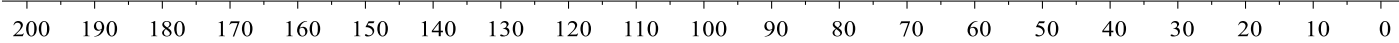


${ }^{1} \mathrm{H} \mathrm{NMR}\left(500 \mathrm{MHz}, \mathrm{CDCl}_{3}\right)$

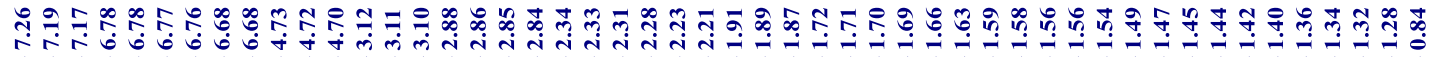
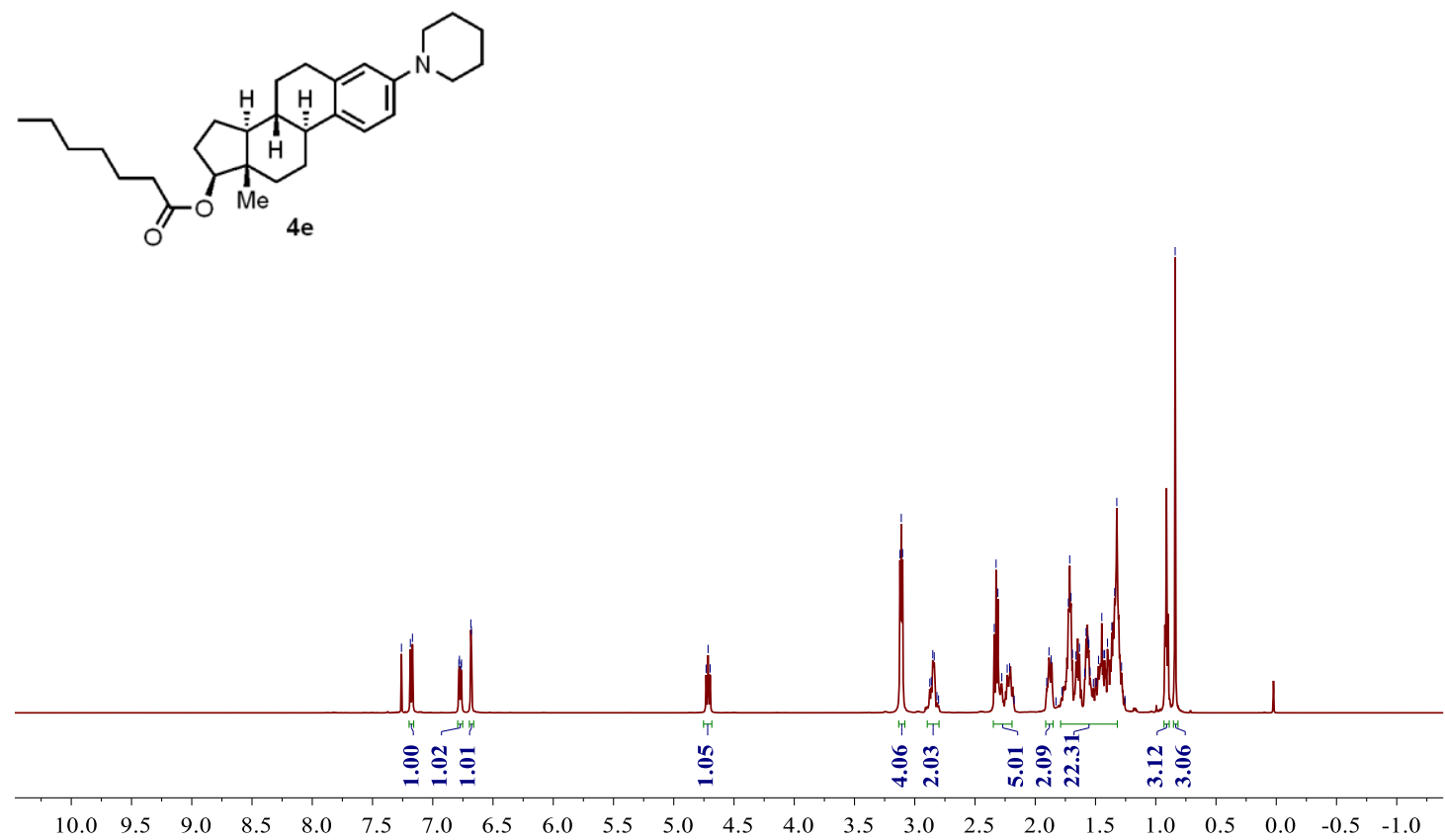

${ }^{13} \mathrm{C} \mathrm{NMR}\left(126 \mathrm{MHz}, \mathrm{CDCl}_{3}\right)$

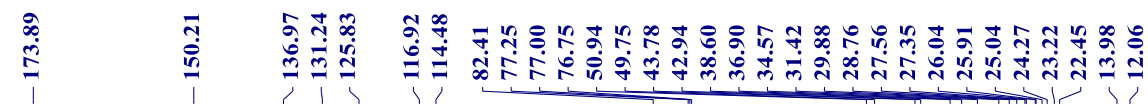
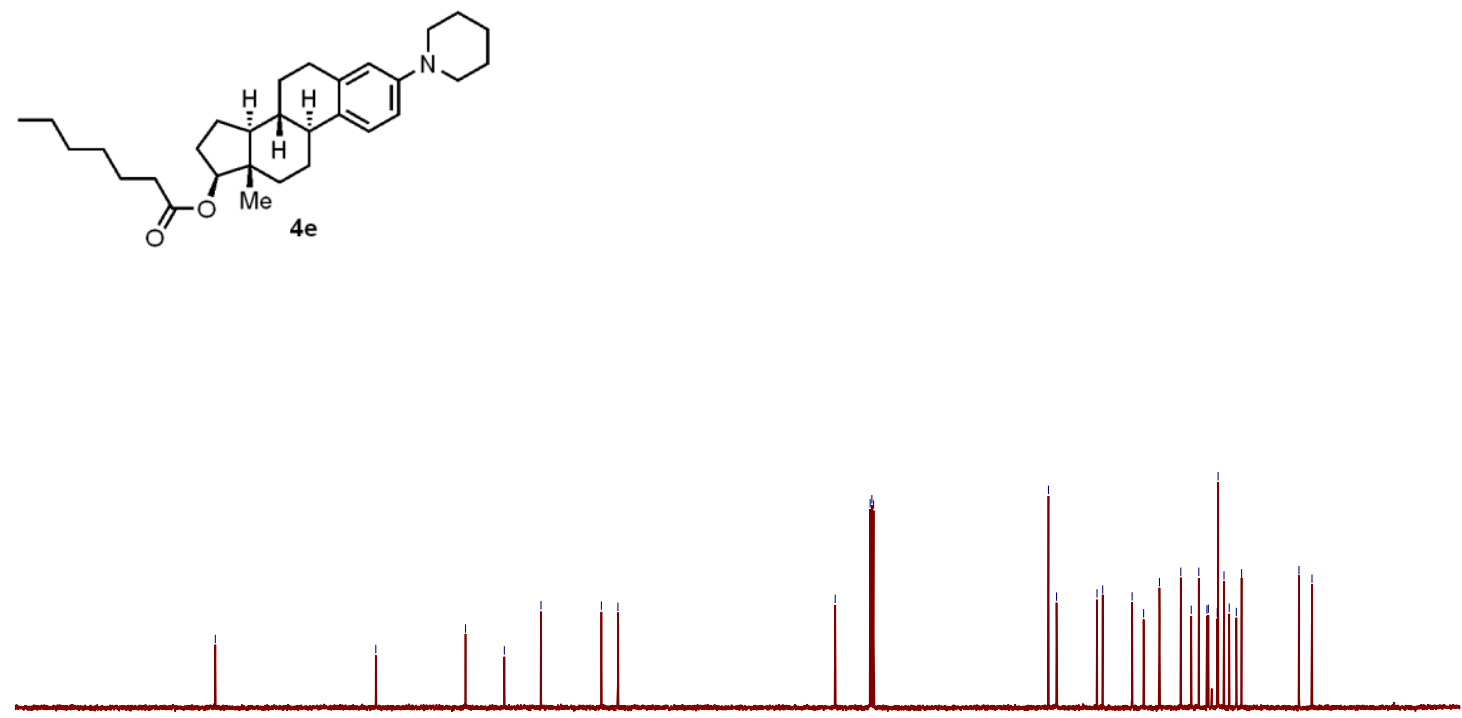

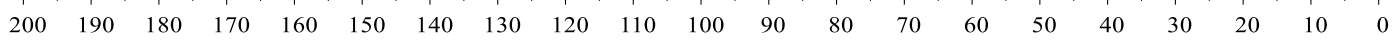


${ }^{1} \mathrm{H}$ NMR $\left(500 \mathrm{MHz}, \mathrm{CDCl}_{3}\right)$
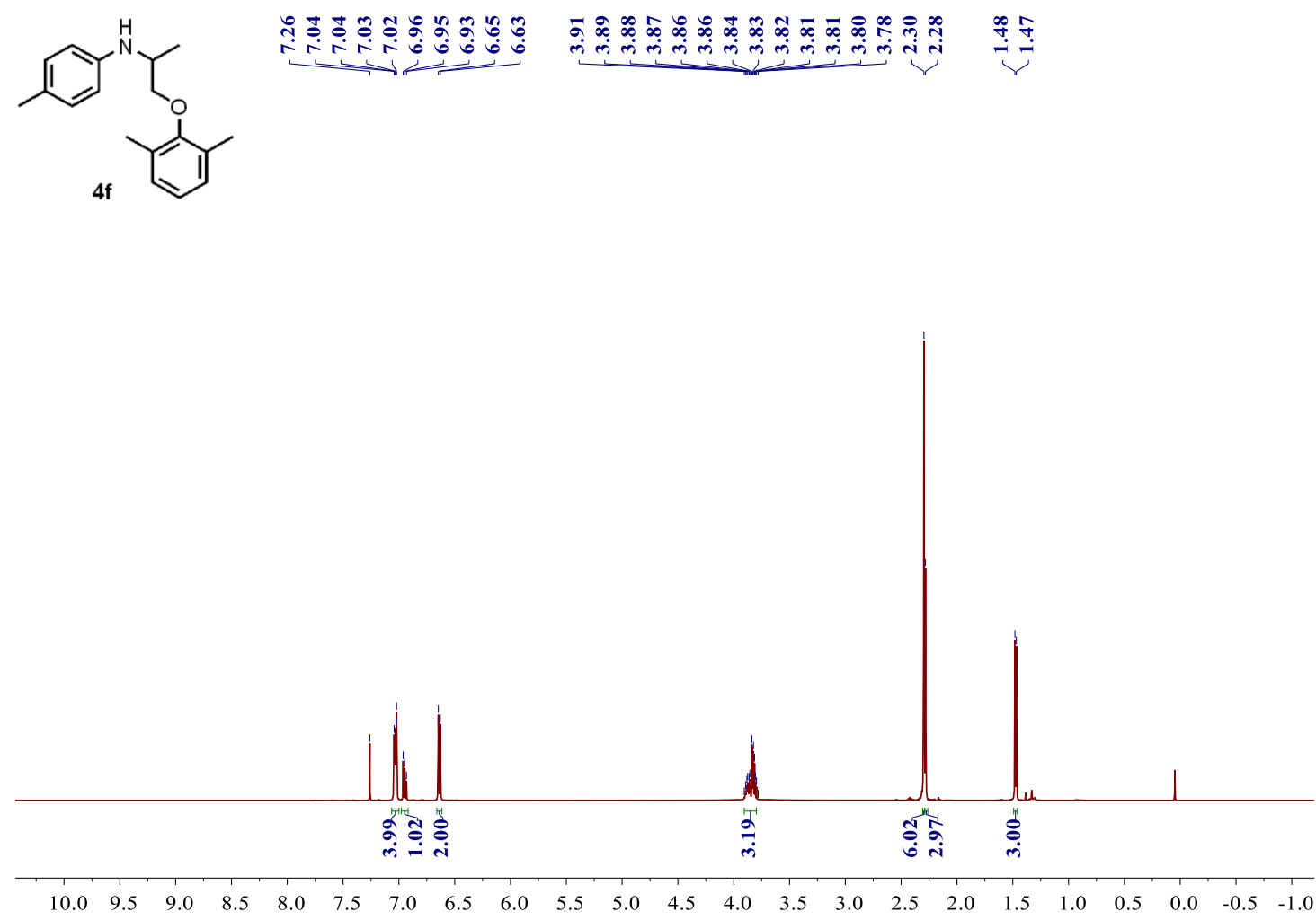

${ }^{13} \mathrm{C} \mathrm{NMR}\left(126 \mathrm{MHz}, \mathrm{CDCl}_{3}\right)$
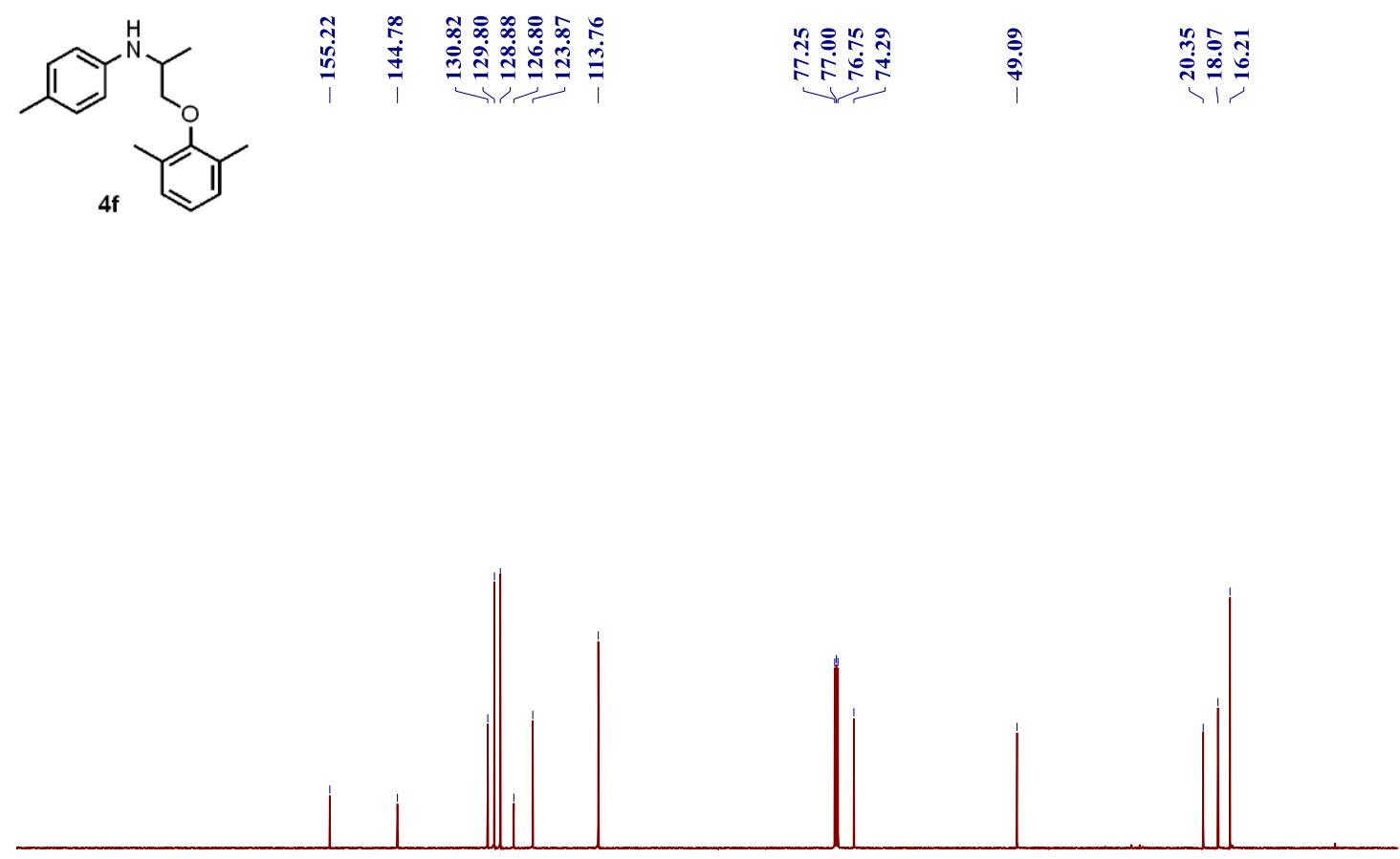

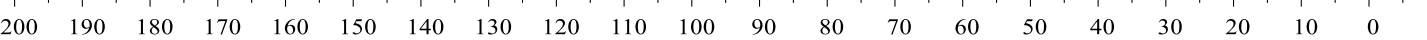


${ }^{1} \mathrm{H} \mathrm{NMR}\left(500 \mathrm{MHz}, \mathrm{CDCl}_{3}\right)$

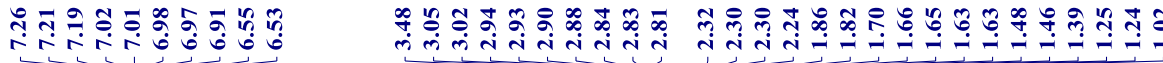
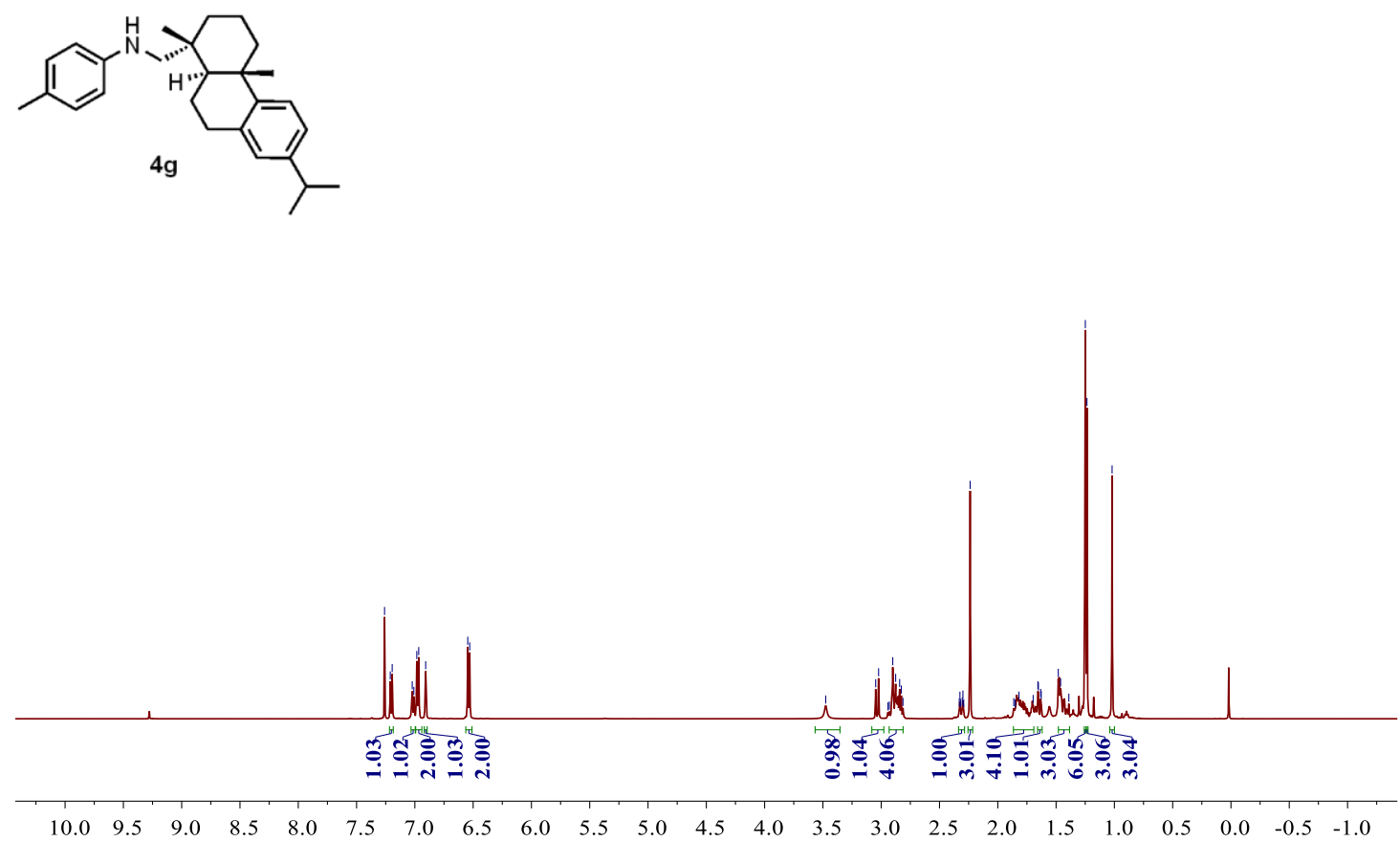

${ }^{13} \mathrm{C}$ NMR (126 MHz, $\left.\mathrm{CDCl}_{3}\right)$

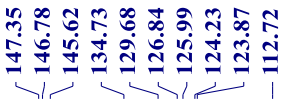

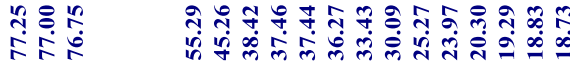

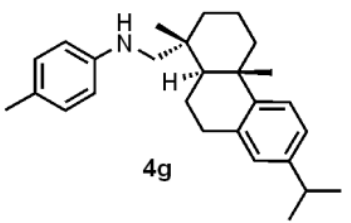


${ }^{1} \mathrm{H}$ NMR (500 MHz, $\left.\mathrm{CDCl}_{3}\right)$

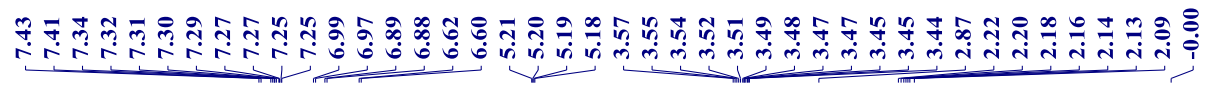
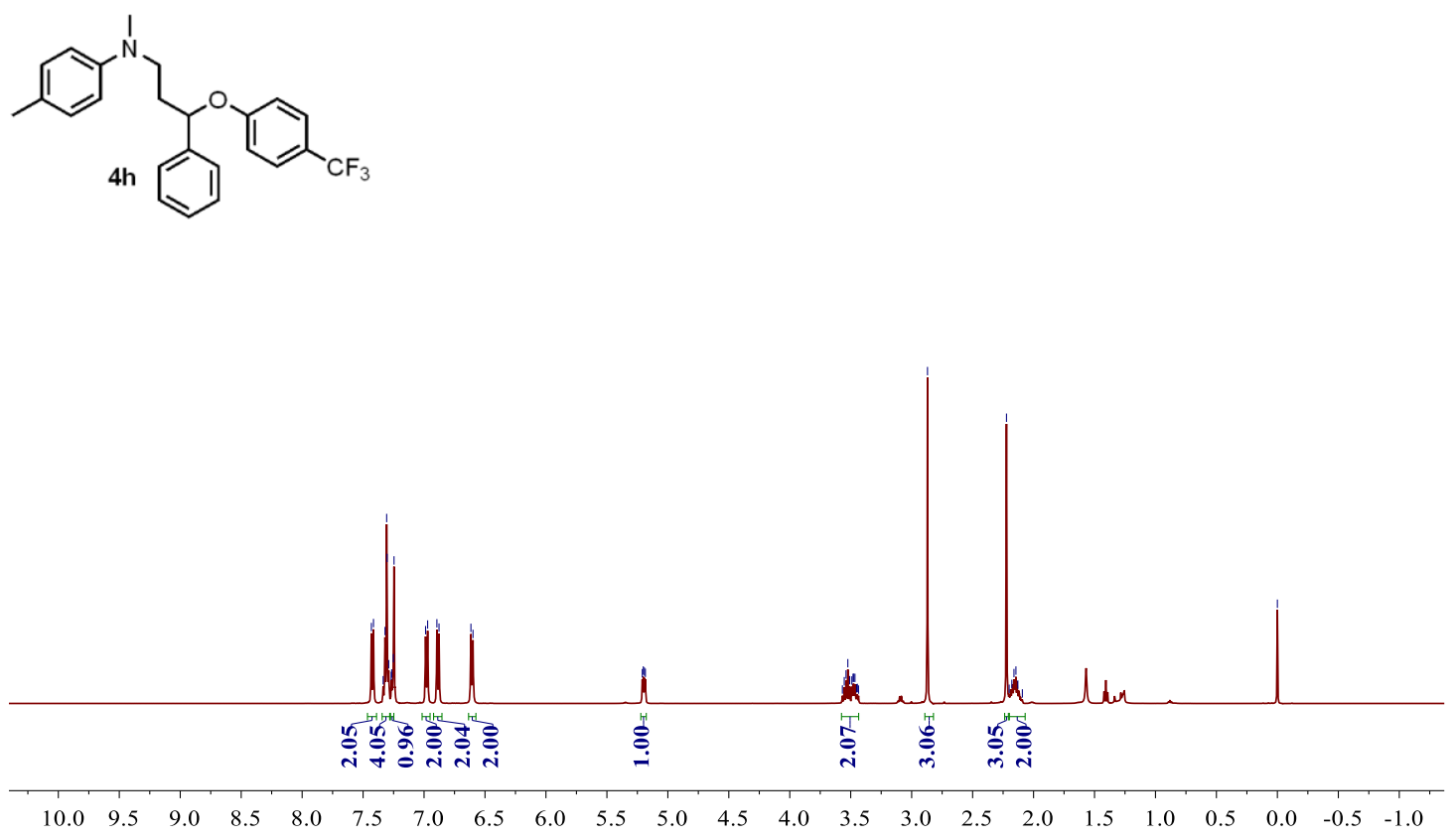

${ }^{13} \mathrm{C}$ NMR (126 MHz, $\left.\mathrm{CDCl}_{3}\right)$

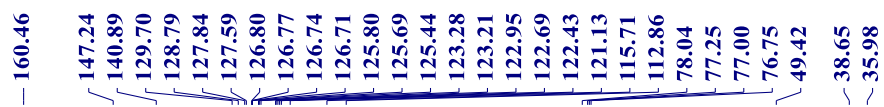

$\stackrel{ \pm}{ \pm}$
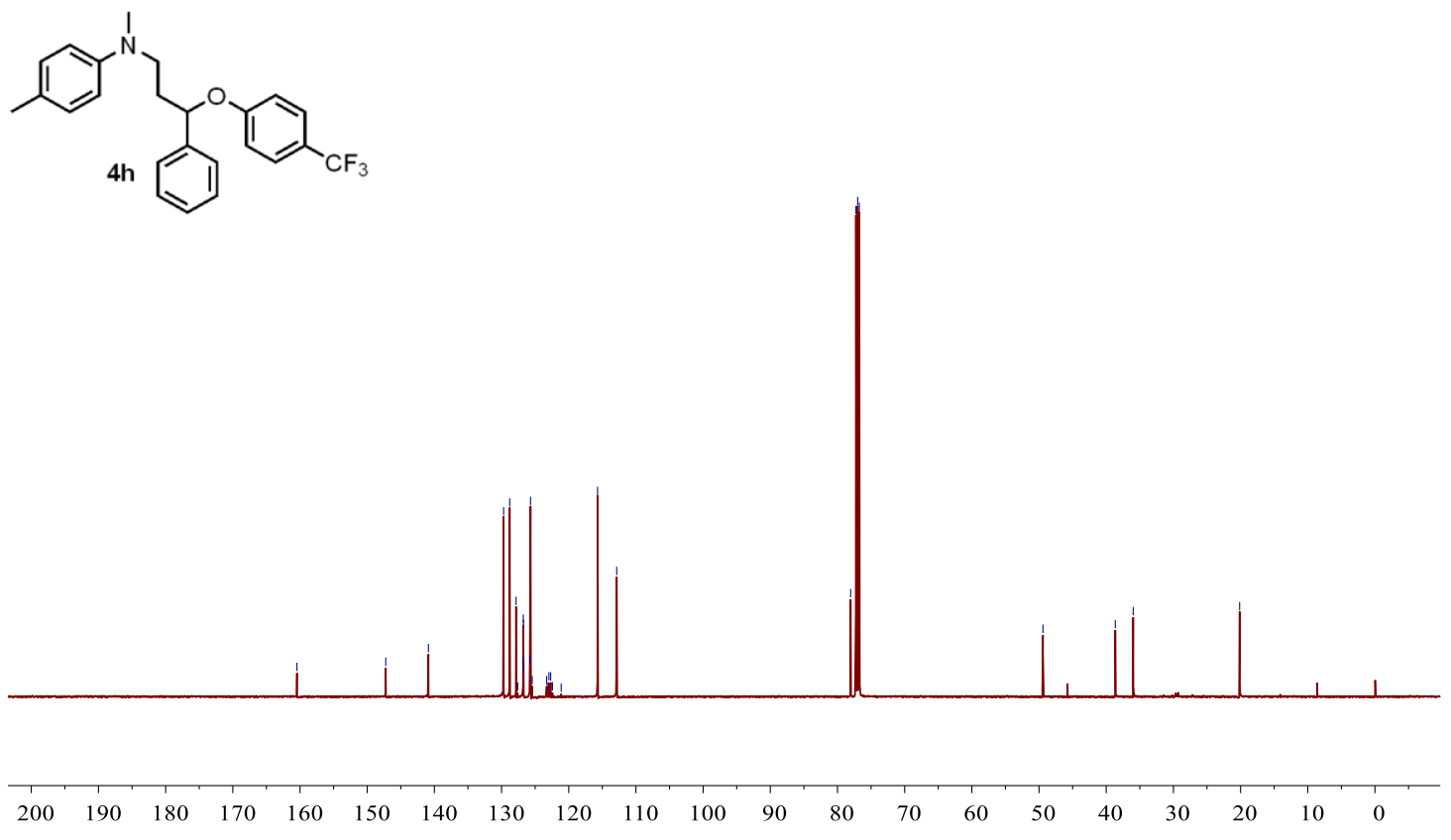

S170 
${ }^{19} \mathrm{~F}$ NMR (471 MHz, $\mathrm{CDCl}_{3}$ )

\section{in:}<smiles>Cc1ccc(N(C)CCC(Oc2ccc(C(F)(F)F)cc2)c2ccccc2)cc1</smiles>

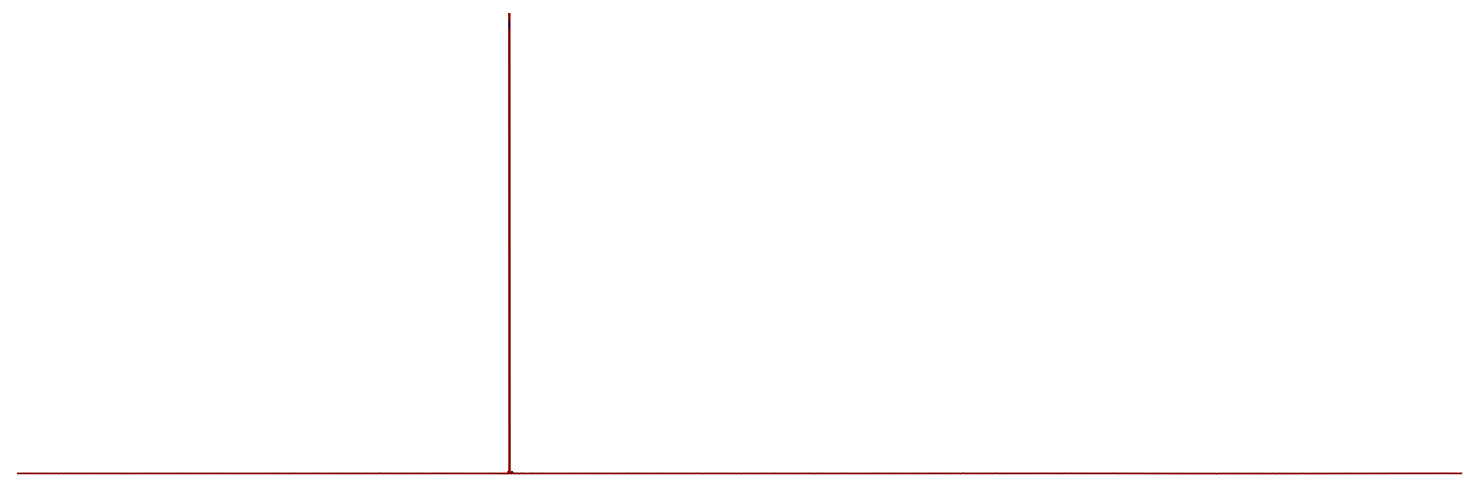

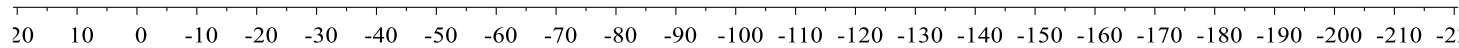

${ }^{1} \mathrm{H}$ NMR $\left(500 \mathrm{MHz}, \mathrm{CDCl}_{3}\right)$

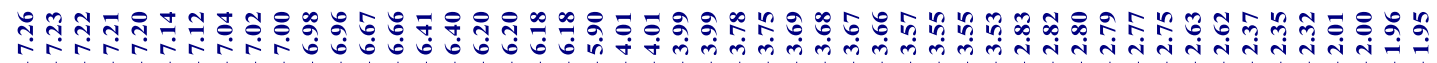<smiles>Cc1ccc(N2CCC(c3ccc(F)cc3)[C@H](COc3ccc4c(c3)OCO4)C2)cc1</smiles>

$4 \mathrm{i}$

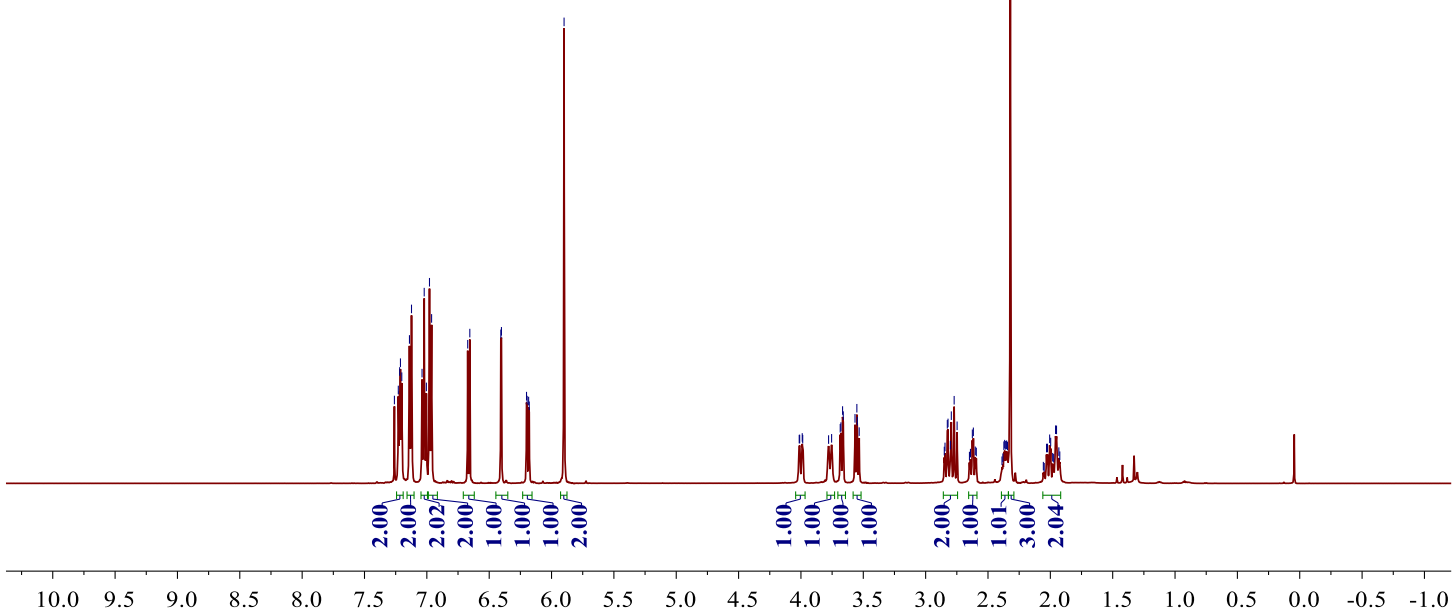


${ }^{13} \mathrm{C} \mathrm{NMR}\left(126 \mathrm{MHz}, \mathrm{CDCl}_{3}\right)$

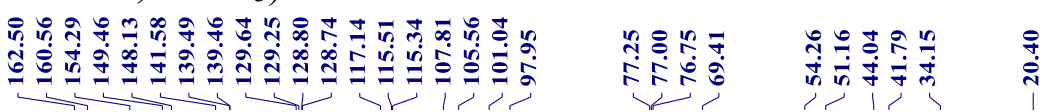<smiles>Cc1ccc(N2CCC(c3ccc(F)cc3)C(COc3ccc4c(c3)OCO4)C2)cc1</smiles>

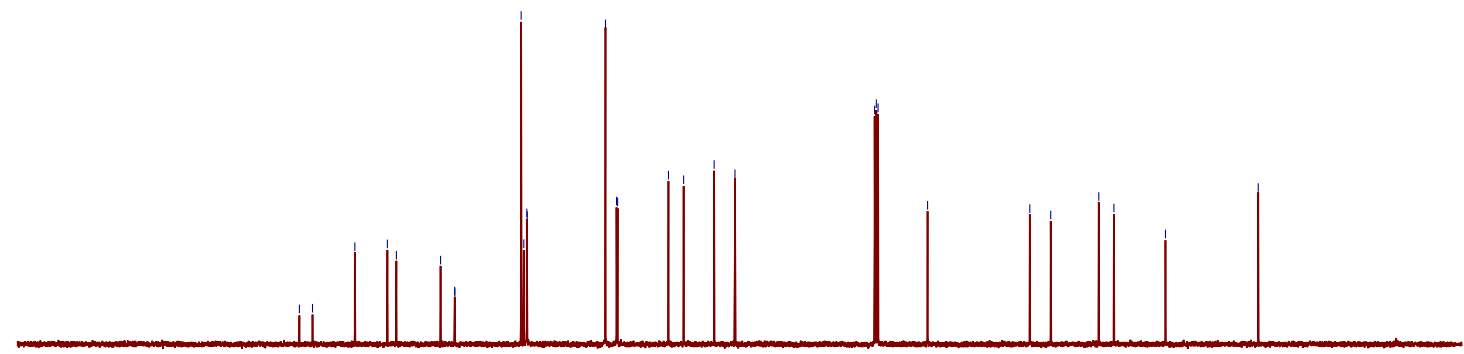

$\begin{array}{lllllllllllllllllllll}200 & 190 & 180 & 170 & 160 & 150 & 140 & 130 & 120 & 110 & 100 & 90 & 80 & 70 & 60 & 50 & 40 & 30 & 20 & 10 & 0\end{array}$

${ }^{19} \mathrm{~F}$ NMR $\left(471 \mathrm{MHz}, \mathrm{CDCl}_{3}\right)$

$$
\stackrel{\substack{n \\ 0}}{i}
$$<smiles>Cc1ccc(N2CCC(c3ccc(F)cc3)C(COc3ccc4c(c3)OCO4)C2)cc1</smiles>

$4 \mathrm{i}$

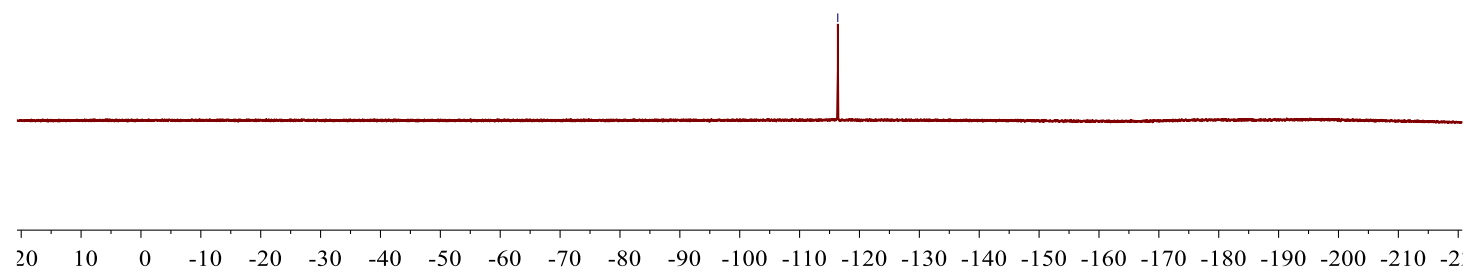


${ }^{1} \mathrm{H} \mathrm{NMR}\left(500 \mathrm{MHz}, \mathrm{CDCl}_{3}\right)$

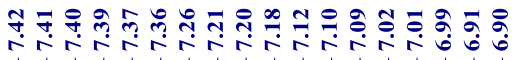

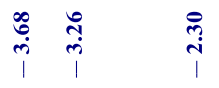<smiles>Cc1ccc(N2CCN(C3=Nc4ccccc4Oc4ccc(Cl)cc43)CC2)cc1</smiles>

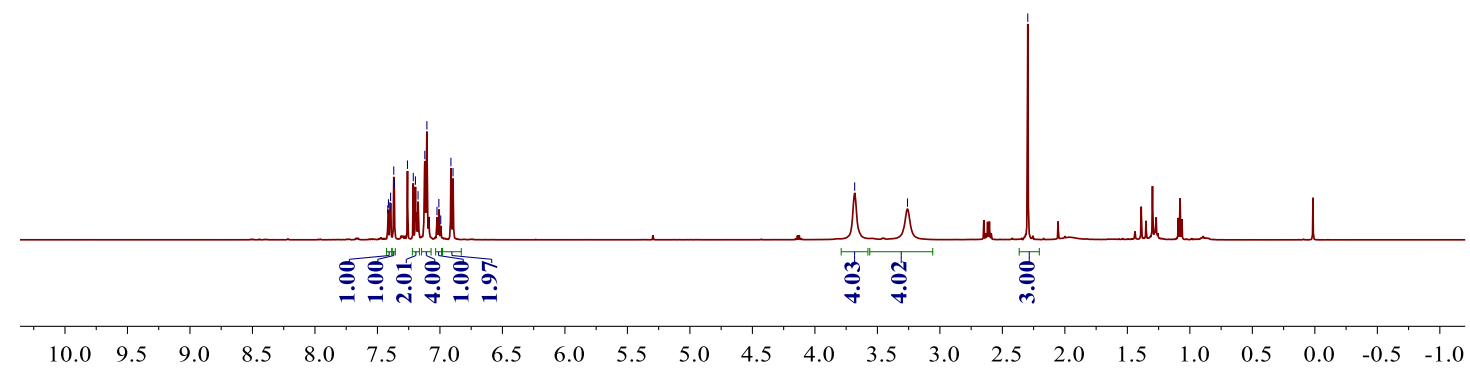

${ }^{13} \mathrm{C} \mathrm{NMR}\left(126 \mathrm{MHz}, \mathrm{CDCl}_{3}\right)$

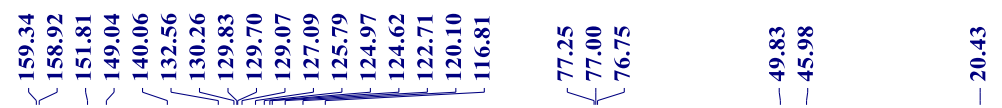<smiles>Cc1ccc(N2CCN(C3=Nc4ccccc4Oc4ccc(Cl)cc43)CC2)cc1</smiles>

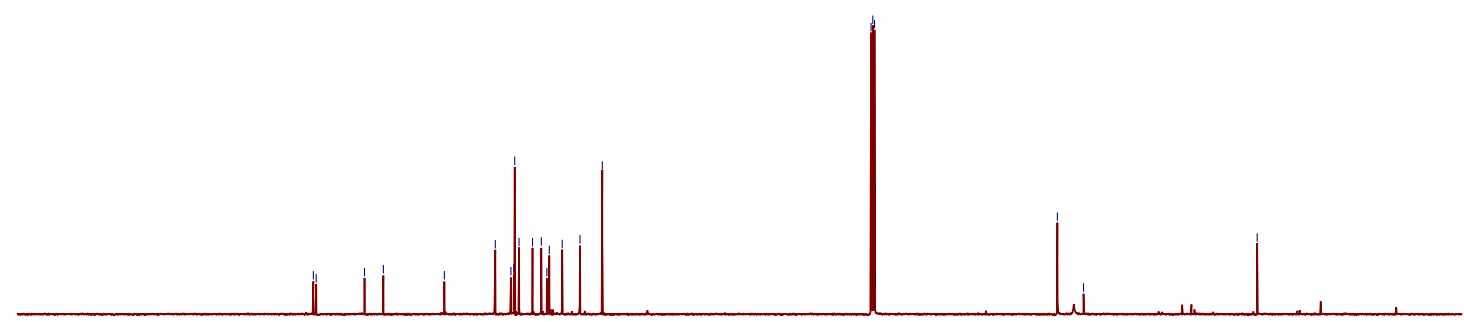

$\begin{array}{llllllllllllllllllllllll}200 & 190 & 180 & 170 & 160 & 150 & 140 & 130 & 120 & 110 & 100 & 90 & 80 & 70 & 60 & 50 & 40 & 30 & 20 & 10 & 0 & 10\end{array}$ 
${ }^{1} \mathrm{H}$ NMR $\left(500 \mathrm{MHz}\right.$, DMSO- $\left.d_{6}\right)$

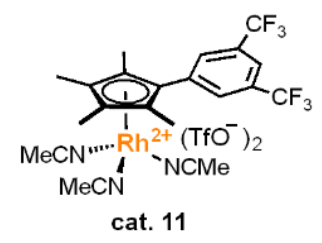

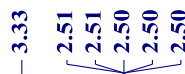

cat. 11

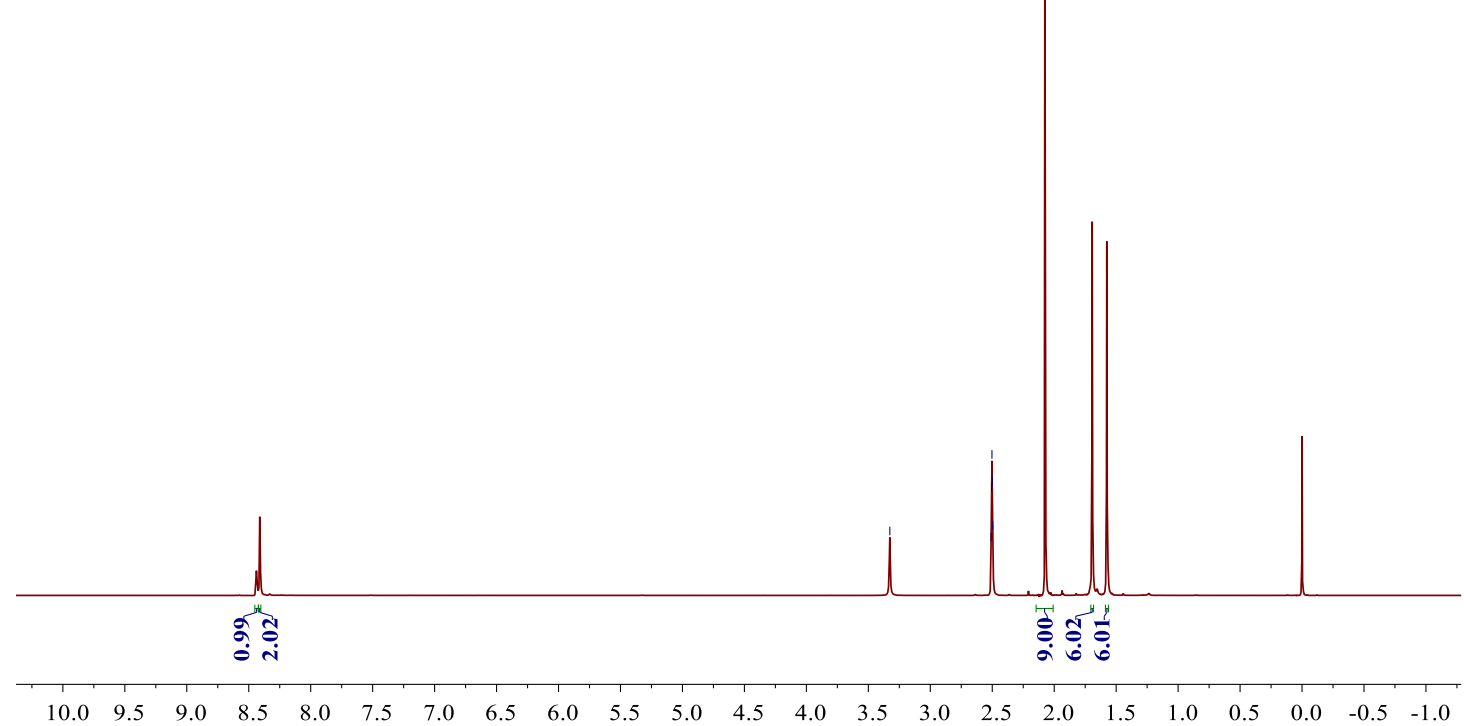

${ }^{13} \mathrm{C}$ NMR (126 MHz, DMSO- $\left.d_{6}\right)$

₹

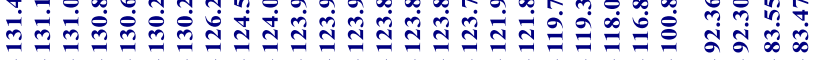

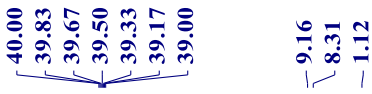

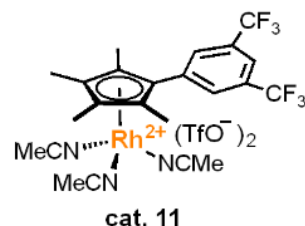

cat. 11 
${ }^{19} \mathrm{~F}$ NMR (471 MHz, DMSO- $\left.d_{6}\right)$

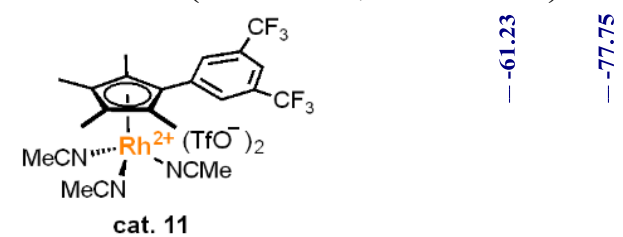

cat. 11

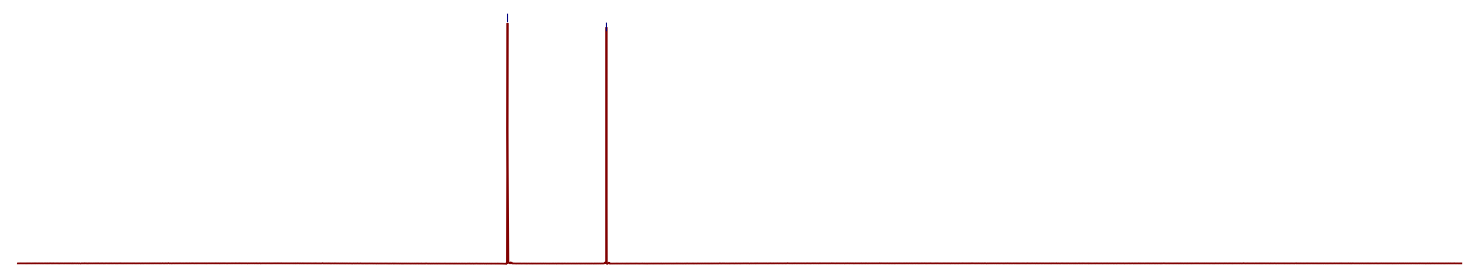

$\begin{array}{lllllllllllllllllllllllll}20 & 10 & 0 & -10 & -20 & -30 & -40 & -50 & -60 & -70 & -80 & -90 & -100 & -110 & -120 & -130 & -140 & -150 & -160 & -170 & -180 & -190 & -200 & -210 & -2\end{array}$ 